\title{
AN EXPERIMENTAL ASSESSMENT OF THE IMPACT OF SLUDGE VARIATION ON THE FRIT 202-A11 - SB3 GLASS SYSTEM
}

D.K. Peeler

K.M. Fox

T.B. Edwards

M.E. Smith

D.R. Best

I.A. Reamer

R.J. Workman

October 2007

Process Science and Engineering Section Savannah River National Laboratory Aiken, SC 29808 
WSRC-STI-2007-00616

Revision 0

\section{DISCLAIMER}

This report was prepared by Washington Savannah River Company (WSRC) for the United States Department of Energy under Contract No. DE-AC09-96SR18500 and is an account of work performed under that contract. Neither the United States Department of Energy, nor WSRC, nor any of their employees makes any warranty, expressed or implied, or assumes any legal liability or responsibility for the accuracy, completeness, or usefulness, of any information, apparatus, or product or process disclosed herein or represents that its use will not infringe privately owned rights. Reference herein to any specific commercial product, process, or service by trademark, name, manufacturer or otherwise does not necessarily constitute or imply endorsement, recommendation, or favoring of same by WSRC or by the United States Government or any agency thereof. The views and opinions of the authors expressed herein do not necessarily state or reflect those of the United States Government or any agency thereof.

Printed in the United States of America

Prepared For

U.S. Department of Energy

The Savannah River National Laboratory is operated for the U.S. Department of Energy by Washington Savannah River Company. 
Keywords: high level waste, glass, cold crucible induction melter

Retention: permanent

\section{AN EXPERIMENTAL ASSESSMENT OF THE IMPACT OF SLUDGE VARIATION ON THE FRIT 202-A11 - SB3 GLASS SYSTEM}

D.K. Peeler

K.M. Fox

T.B. Edwards

M.E. Smith

D.R. Best

I.A. Reamer

R.J. Workman

October 2007

Process Science and Engineering Section Savannah River National Laboratory Aiken, SC 29808 
WSRC-STI-2007-00616

Revision 0

\section{REVIEWS AND APPROVALS}

\section{AUTHORS:}

D.K. Peeler, Process Science and Engineering Section Date

K.M. Fox, Process Science and Engineering Section Date

\begin{tabular}{ll}
\hline T.B. Edwards, Statistical Consulting Section & Date
\end{tabular}

M.E. Smith, Process Science and Engineering Section Date

D.R. Best, Process Science and Engineering Section Date

I.A. Reamer, Process Science and Engineering Section Date

R.J. Workman, Process Science and Engineering Section Date

\section{TECHNICAL REVIEWER:}

J.D. Newell, Process Science and Engineering Section Date

\section{APPROVERS:}

C.C. Herman, Manager, Process Engineering Technology Group Date

R.E. Edwards, Manager, Process Science and Engineering Section Date 
WSRC-STI-2007-00616

Revision 0

\section{EXECUTIVE SUMMARY}

Twenty-seven glasses were designed to assess the impacts of both sludge variation ( \pm 5 or $\pm 10 \%$ for the major sludge components) and waste loading (WL) (50 or 52\%) on the Product Consistency Test (PCT) response after two thermal histories (quenching and a modified ccc schedule) within the Frit 202-A11 - Sludge Batch 3 (SB3) system. The PCT results of the quenched glasses (regardless of compositional view) indicate that all Frit 202-A11 - Sludge SB3 (referred to as HTLG) variability study glasses are very acceptable relative to the Environmental Assessment (EA) glass benchmark. More specifically, the normalized boron releases (NL [B] in $\mathrm{g} / \mathrm{L}$ ) range from $0.8 \mathrm{~g} / \mathrm{L}$ (for HTLG-60VS based on the measured composition) to $1.384 \mathrm{~g} / \mathrm{L}$ (HTLG-79VS based on the measured bias-corrected (bc) composition). These results can be compared to the NL [B] for the EA benchmark of $16.695 \mathrm{~g} / \mathrm{L}$. The PCT results of the quenched glasses are consistent with previous data in the Frit 202-A11 - SB3 system.

The PCT results for the ccc glasses are not as straight forward. The NL [B]s for the slow cooled glasses range from $0.607 \mathrm{~g} / \mathrm{L}$ (for HTLG-57ccc based on the measured composition) to $9.42 \mathrm{~g} / \mathrm{L}$ (for HTLG-67ccc based on the measured bc compositional view). Although these glasses would be classified as acceptable relative to the EA glass benchmark, the relatively high release of the slow cooled glasses would be of concern if this system were to be implemented into the Defense Waste Processing Facility (DWPF). The PCT responses for those glasses in which either nepheline or both nepheline and aegirine formed upon slow cooling lead to a significant reduction in PCT response. Although the formation of aegirine has (in general) a slightly negative impact on the PCT response, the formation of nepheline and aegirine is a combination that has a high probability of leading to a significant reduction in durability upon slow cooling.

With respect to the Cold Crucible Induction Melter (CCIM) demonstration, a clear cut delineation of sludge compositions and/or targeted WLs is desirable to avoid the formation of either of these phases. However, based on a statistical assessment of the PCT and X-ray diffraction (XRD) data, this direct relationship does not appear to exist for this sludge/frit system and identifying the nepheline and/or aegirine primary phase field is not readily apparent based on the limited data. In addition, the possibility to target higher WLs (>50\%) to avoid any negative impacts on durability as a result of crystallization, as previously done with the nominal SB3 composition, was dependent upon the sludge component combinations. Moreover, when composition variation is applied to the sludge, multiple sludge and frit combinations fall within the nepheline and/or aegirine phase fields even at 52\% WL, which ultimately lead to a negative impact on durability. Nonetheless, the PCT results do suggest that the probability of observing the negative impact is lower at the higher WLs. Only 2 of the inner layer, 52\% WL based glasses have NL [B] $>2 \mathrm{~g} / \mathrm{L}$ after slow cooling as compared to 4 of the 9 inner layer EVs targeted 50\% WL. 


\section{Table of Contents}

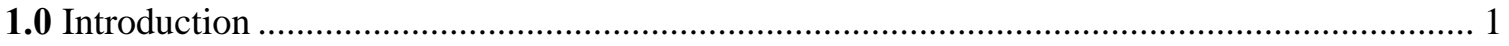

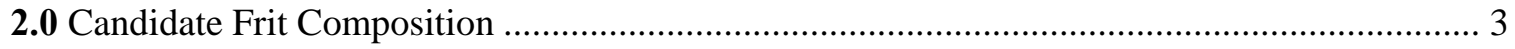

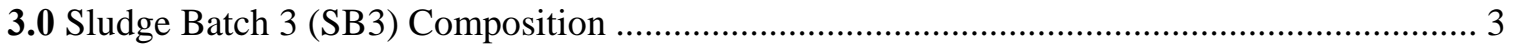

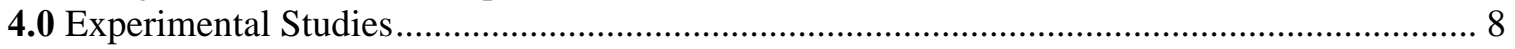

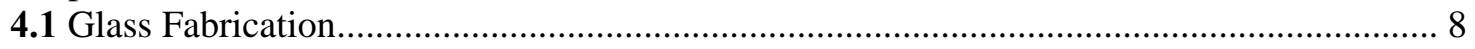

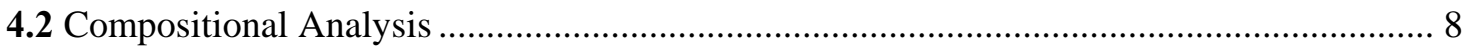

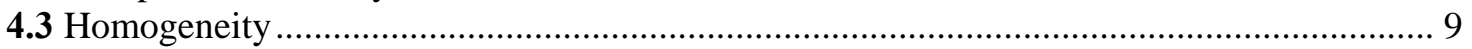

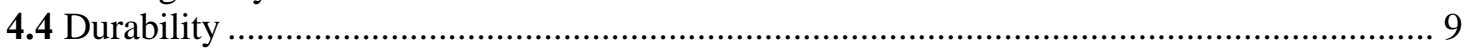

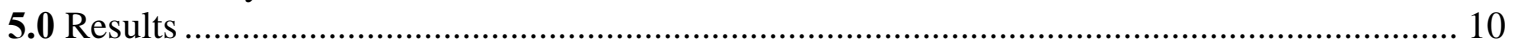

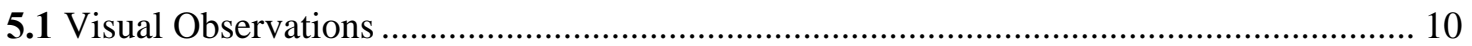

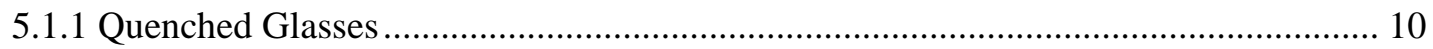

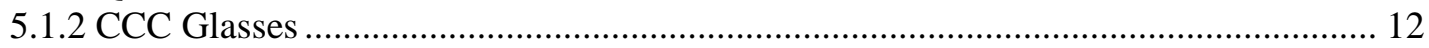

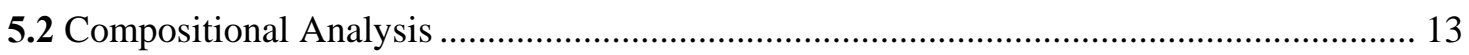

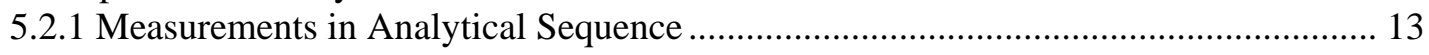

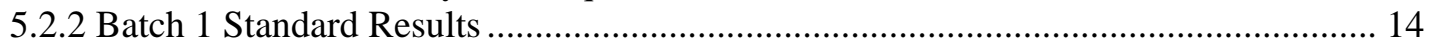

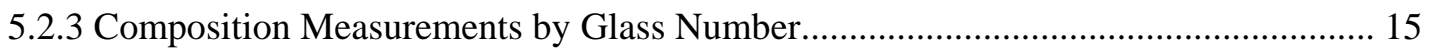

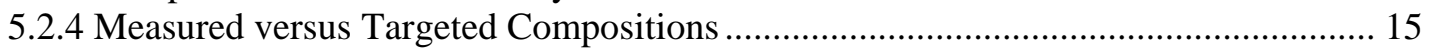

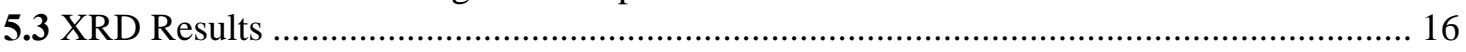

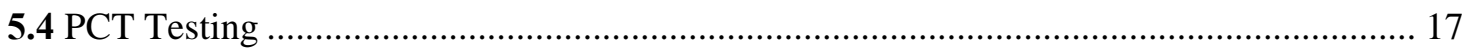

5.4.1 PCT Measurements in Analytical Sequence.............................................................. 18

5.4.2 Repeatability of the PCT Measurements for a Glass/Heat Treatment Combination ... 18

5.4.3 Results for the Samples of the Multi-Element Solution Standard ................................ 19

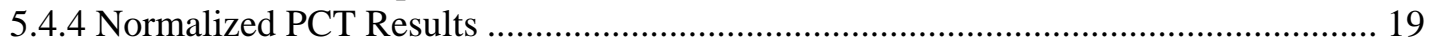

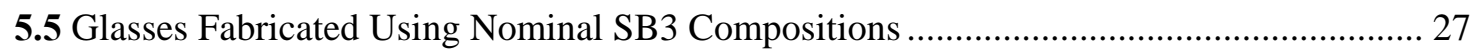

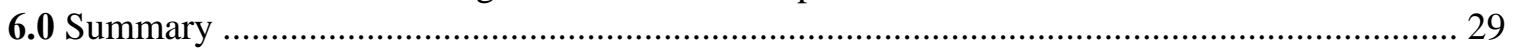

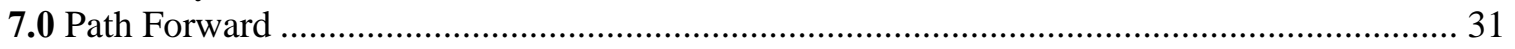

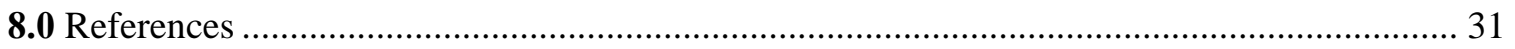

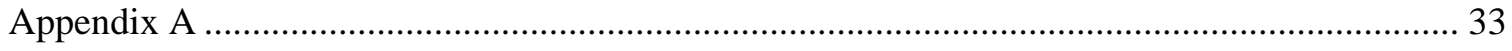

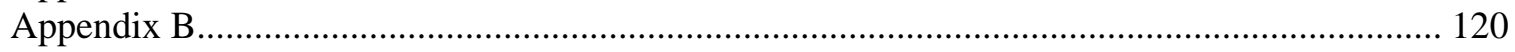




\section{List of Tables}

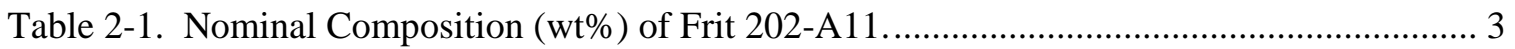

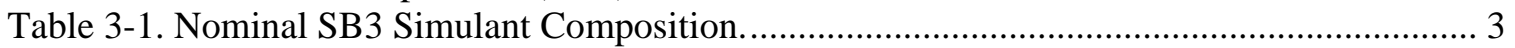

Table 3-2. Outer-Layer Intervals for the Major SB3 Oxides and Others ........................................ 4

Table 3-3. Composition (in mass fractions) of Others Grouping ............................................... 4

Table 3-4. Outer-Layer Sludge Compositions (as mass fractions)................................................ 5

Table 3-5. Inner-Layer Intervals for the Major SB3 Oxides and Others...................................... 6

Table 3-6. Inner-Layer Sludge Compositions (as mass fractions) ............................................. 6

Table 3-7. Target Compositions of the Frit 202-A11 Variability Study Glasses. .......................... 7

Table 5-1. Visual Observations of Quenched and CCC HTLG VS Glasses.............................. 11

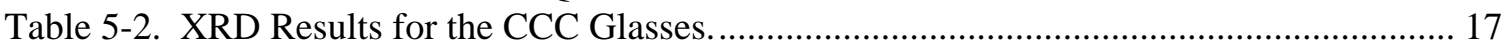

Table 5-3. Results from Samples of the Multi-Element Solution Standard by PCT Set.............. 19

Table 5-4. Normalized PCTs for Quenched Glasses by Compositional View............................. 21

Table 5-5. Normalized PCTs for CCC Glasses by Compositional View.................................... 23

Table 5-6. XRD and PCT results for the HTLG Variability Study Glasses................................. 26

Table 5-7. Targeted Compositions of Glasses at 45, 50, and 55 \%WL Based Upon Vendor's

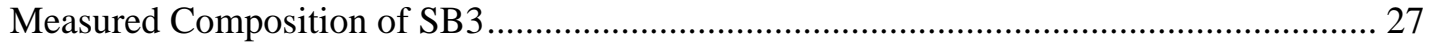

Table 5-8. PCT Results for HTLG-100, HTLG-101, and HTLG-102 ….................................... 28

Table 5-9. Normalized PCT Response Based upon Targeted Compositions for HTLG-100,

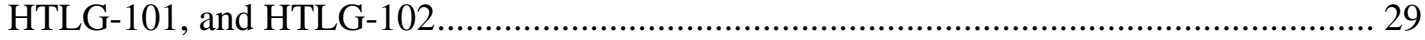




\section{LIST OF ABBREVIATIONS}

$\begin{array}{ll}\text { AD } & \text { Analytical Development } \\ \text { ARM } & \text { Approved Reference Material } \\ \text { ASTM } & \text { American Society of Testing and Materials } \\ \text { bc } & \text { bias corrected } \\ \text { cCC } & \text { Canister Centerline Cooling } \\ \text { CCIM } & \text { Cold Crucible Induction Melter } \\ \text { DOE } & \text { Department of Energy } \\ \text { DWPF } & \text { Defense Waste Processing Facility } \\ \text { EA } & \text { Environmental Assessment } \\ \text { EDS } & \text { Energy dispersive spectroscopy } \\ \text { HTLG } & \text { High temperature liquidus glasses } \\ \text { ICP-AES } & \text { Inductively Coupled Plasma-Atomic Emission } \\ \text { JHM } & \text { Spectroscopy } \\ \text { LM } & \text { Joule heated melter } \\ \text { PCT } & \text { Lithium metaborate } \\ \text { PF } & \text { Product Consistency Test } \\ \text { PSAL } & \text { Peroxide fusion } \\ \text { SB3 } & \text { Process Science Analytical Laboratory } \\ \text { SEM } & \text { Sludge Batch 3 } \\ \text { SRNL } & \text { Scanning Electron Microscopy } \\ \text { SRS } & \text { Savannah River National Laboratory } \\ T_{L} & \text { Savannah River Site } \\ \eta & \text { Liquidus temperature } \\ \text { WAPS } & \text { viscosity } \\ \text { WL } & \text { Waste Acceptance Product Specifications } \\ \text { XRD } & \text { waste loading } \\ & \end{array}$


WSRC-STI-2007-00616

Revision 0

\subsection{Introduction}

Initial operations at the Defense Waste Processing Facility (DWPF) targeted a nominal waste loading of $28 \%$ (on a calcined oxide, mass basis). Based on strategic glass formulation approaches and physical improvements to the Joule Heated Melter (JHM) system, operations with Sludge Batch 3 (SB3) transitioned to a nominal waste loading of 38\%. Although this has lead to a significant increase in the waste throughput for the site, additional increases are possible by coupling advanced melter technologies with glass formulation efforts. More specifically, advancements in melter technology have the potential to produce glass faster and at higher waste loadings (the two primary parameters that drive waste throughput) relative to current operations. One of these technologies is the Cold Crucible Induction Melter (CCIM) with bubbler and/or mechanical agitation.

Since any additional (sustained) improvement in waste loading and/or melt rate relative to current operations will help to reduce the life-cycle costs for the Department of Energy (DOE) at the Savannah River Site (SRS), there is interest in demonstrating the viability of CCIM technology to yield such an improvement. An initial CCIM demonstration is currently planned for the fall of 2007 and will be based on a DWPF waste slurry feed surrogate with a nominal operating temperature of approximately $1250^{\circ} \mathrm{C}$ targeting a $50 \%$ waste loading (WL) glass. ${ }^{1}$ The waste slurry feed (nominally 40 - 45 weight percent solids) surrogate will be representative of SB3 in order to allow a direct comparison to the DWPF joule heated melter performance during processing of this sludge waste. The pilot scale demonstration is being conducted to evaluate performance and identify potential processing issues with the existing CCIM technology and will include characterization of the resultant glass product to ensure current product performance (durability) specifications are met.

The CCIM glass product will be a borosilicate glass at a high waste loading (nominally at 50\%, based on a calcined oxide basis) that must meet current requirements for disposal at a Federal Repository. Specifically, the Waste Acceptance Product Specifications (WAPS Specification 1.3) define acceptance through the use of the American Society of Testing Materials (ASTM) Product Consistency Test (PCT) as the measure of waste form performance or durability (ASTM 2002). Acceptance relies upon the mean leachate concentrations of lithium, sodium and boron (after normalization for the glass composition) being less than those of the Environmental Assessment (EA) benchmark glass (Jantzen et al. 1993).

The CCIM demonstration, which will utilize a "constant” sludge composition (targeting a nominal SB3 composition) coupled with Frit 202-A11 at nominally 50\% WL, will theoretically yield a constant glass composition. Although this will support programmatic objectives, there is some need to assess the potential impacts of sludge variation and/or waste loading differences on potential processing and/or product performance properties. More specifically, the vendor will produce a sludge targeting the nominal SB3 composition. Recognizing the low probability of producing the exact targeted sludge composition and/or that actual WL could vary from $50 \pm 2 \%$ (around the nominal 50\% target), leads to questions regarding the impact of these variations on processing properties or perhaps more importantly on the durability of the final waste form. This is of particular importance given the nonlinear behavior previously observed between waste loading and critical processing / performance properties for this glass forming system. The

\footnotetext{
${ }^{1}$ Although this report is being issued after the CCIM demonstration, the activities documented here were developed to support, and were completed before, that demonstration. This report is being written from that perspective. The data presented in this report were used to support the decision to increase the WL in the CCIM demonstration from 50\% to $52 \%$.
} 
results from Peeler et al. (2007) suggested that a balance between WL and processing and product performance issues may be required for this CCIM demonstration. This latter statement is based on the fact that frit development efforts were not necessarily intended to optimize this glass system nor have these efforts accounted for the variation from the intended target that is likely to occur in the composition of the waste slurry feed surrogate that is being used in the study.

Given the identification of Frit 202-A11 as the preferred frit (Peeler et al. 2007), supplemental information was needed to provide a technical basis to facilitate potential decisions regarding the melter demonstration. Peeler et al. (2007) conducted an experimental study to provide technical data, which investigated the impact of WL on critical properties of interest for the Frit 202A11/SB3 glass system. That report documented the compositional and physical property information associated with two series of Frit 202-A11 based SB3 glasses. The first series (three glasses targeting 45, 50, and 55\% WL) was fabricated and characterized as part of the experimental support for the frit down-select process. In the second series (referred to as the "waste loading series”), eight Frit 202-A11 based glasses were produced and characterized targeting WLs from $44-58 \%$ in $2 \%$ increments. The primary intent of the second series was to cover a finer WL grid over which trends in critical properties could be assessed and decisions regarding the targeted WL could be made.

All of the glasses of the Peeler et al. (2007) study, regardless of heat treatment, were acceptable when their durabilities were compared to those of the EA glass. In general, for the nominal Frit 202-A11/SB3 glass system, as WL was increased, the durabilities for the quenched versions of the glasses tended to decrease due to the changing composition of the glass. For the glasses subjected to the canister centerline cooling (ccc) regime, the durability response appeared to be nonlinear as WL was increased. At WLs of $50 \%$ or less, X-ray diffraction (XRD) analysis indicated the potential for the presence of aegirine and/or nepheline crystalline phases, and when these phases were present, there was a decrease in the durability of the glass. As WL was increased above $50 \%$, there appeared to be a transition from the aegirine and/or nepheline primary phase fields to the spinel primary phase field leading to more durable ccc glasses.

Thus, the results for durability suggested that WLs of $50 \%$ or greater should be targeted for the CCIM demonstration, thus, avoiding the potential for the formation of aegirine and/or nepheline. However, if decisions to target WLs of $50 \%$ or greater are made, liquidus temperature $\left(\mathrm{T}_{\mathrm{L}}\right)$ measurements indicated that there could be some degree of crystallization within the melter if a nominal $1250^{\circ} \mathrm{C}$ temperature were to be used. The results from that study suggested that increasing WLs leads to higher liquidus temperatures in the glass forming system of interest. To minimize the potential of crystallization during processing, higher melt temperatures could be targeted which not only could allow for higher WLs to be obtained but could also result in a reduction in viscosity, which in itself could pose certain processing issues (e.g., the ability to control the pour and the possibility of increased volatility). The viscosity of the $50 \% \mathrm{WL}$ glass at 1250 and $1300^{\circ} \mathrm{C}$ was measured to be 20 and 13 Poise, respectively. Thus, the results from Peeler et al. (2007) suggested that a balance between processing and product performance issues may be required for the initial CCIM demonstration since the frit development efforts to date were not necessarily intended to optimize this glass system nor did they account for the variation from the intended target that is likely to occur in the composition of the waste slurry feed surrogate that is being used in the study.

To address this last issue, an additional experimental study was conducted to investigate the impact of sludge variation on select properties of the Frit 202-A11/SB3 glass system. The purpose of this report is to describe that investigation and to present the results that it generated. 
The composition of Frit 202-A11 is provided in Section 2. Section 3 describes the nominal composition of SB3 as well as the way variation in that composition was introduced in order to facilitate the selection of glass compositions for this phase of study. The selected glass compositions are presented in Section 3. Section 4 provides a description of the experimental aspects of this study. Section 5 presents the results of this experimental effort including visual observations of the study glasses, statistical reviews of the chemical composition and PCT measurements of the study glasses, and XRD assessments. Section 6 provides the conclusions that are drawn from these results.

\subsection{Candidate Frit Composition}

Based on preliminary model assessments as well as initial scoping tests which generated select physical property data, Frit 202-A11 was identified as the primary candidate for processing SB3 through the CCIM technology at an 50\% WL (Peeler et al. 2007). Table 2-1 summarizes the nominal composition of Frit 202-A11 (wt\%, calcined oxide basis).

Table 2-1. Nominal Composition (wt\%) of Frit 202-A11.

\begin{tabular}{|c|c|c|c|c||}
\hline Frit ID & $\mathbf{B}_{\mathbf{2}} \mathbf{O}_{\mathbf{3}}$ & $\mathbf{L i}_{\mathbf{2}} \mathbf{O}$ & $\mathbf{N a}_{\mathbf{2}} \mathbf{O}$ & $\mathbf{S i O}_{\mathbf{2}}$ \\
\hline $202-\mathrm{A} 11$ & 9 & 6 & 3 & 82 \\
\hline
\end{tabular}

\subsection{Sludge Batch 3 (SB3) Composition}

Table 3-1 summarizes the nominal SB3 simulant (non-radioactive) composition being used to support the glass formulation studies. This composition was based on the analytical data reported by the DWPF during processing of SB3 and represents a "running average" (renormalized after removing $\mathrm{U}_{3} \mathrm{O}_{8}$ and $\mathrm{ThO}_{2}$ ). ${ }^{2}$ It is recognized that the removal of $\mathrm{U}_{3} \mathrm{O}_{8}$ from the composition and the renormalization do increase the concentration of the other components. That being said, the primary concern with this renormalization process is the relative increase in the $\mathrm{Fe}_{2} \mathrm{O}_{3}, \mathrm{NiO}$, and $\mathrm{Cr}_{2} \mathrm{O}_{3}$ values as these components are spinel formers, and it is anticipated (and has been observed in preliminary testing) that spinel formation (or liquidus temperature) could be a significant technical issue for processing of SB3 at WLs of 50\% or greater. The artificial increase in these components and the targeting of higher WLs should increase the propensity for spinel formation. It should be noted that the $\mathrm{SO}_{4}{ }^{=}$concentration was fixed at $0.719 \mathrm{wt} \%$ (and was not renormalized).

Table 3-1. Nominal SB3 Simulant Composition.

\begin{tabular}{||c|c||}
\hline Oxide & $\begin{array}{c}\text { Nominal SB3 } \\
\text { Composition }\end{array}$ \\
\hline $\mathrm{Al}_{2} \mathrm{O}_{3}$ & 16.898 \\
\hline $\mathrm{CaO}$ & 3.305 \\
\hline $\mathrm{Cr}_{2} \mathrm{O}_{3}$ & 0.192 \\
\hline $\mathrm{CuO}$ & 0.075 \\
\hline $\mathrm{Fe}_{2} \mathrm{O}_{3}$ & 37.302 \\
\hline $\mathrm{K}_{2} \mathrm{O}$ & 0.339 \\
\hline
\end{tabular}

\footnotetext{
${ }^{2}$ The $\mathrm{U}_{3} \mathrm{O}_{8}$ concentration in SB3 was approximately $9 \mathrm{wt} \%$ (on a calcined oxide basis). $\mathrm{ThO}_{2}$ was less than $0.5 \mathrm{wt} \%$.
} 
WSRC-STI-2007-00616

Revision 0

\begin{tabular}{||c|c||}
\hline $\mathrm{MgO}$ & 3.813 \\
\hline $\mathrm{MnO}$ & 7.085 \\
\hline $\mathrm{Na}_{2} \mathrm{O}$ & 24.174 \\
\hline $\mathrm{NiO}$ & 1.921 \\
\hline $\mathrm{SiO}_{2}$ & 3.940 \\
\hline $\mathrm{TiO}_{2}$ & 0.057 \\
\hline $\mathrm{SO}_{4}{ }^{2}$ & 0.719 \\
\hline $\mathrm{ZrO}_{2}$ & 0.184 \\
\hline & 100.000 \\
\hline
\end{tabular}

Given the nominal SB3 composition, the primary objective of this task was to introduce compositional variation around the sludge and to assess the impact on specific properties of interest at WLs of interest. An interval of possible concentration values for each of the major oxides $\left(\mathrm{Al}_{2} \mathrm{O}_{3}, \mathrm{CaO}, \mathrm{Fe}_{2} \mathrm{O}_{3}, \mathrm{MgO}, \mathrm{MnO}, \mathrm{Na}_{2} \mathrm{O}, \mathrm{NiO}\right.$, and $\left.\mathrm{SiO}_{2}\right)$ of Table 3-1 is given in Table 3-2 and was determined by introducing variation around the nominal concentration for that oxide in the manner indicated in the latter table. As shown, the variation applied was $\pm 10 \%$ of the nominal value for $\mathrm{Al}_{2} \mathrm{O}_{3}, \mathrm{Fe}_{2} \mathrm{O}_{3}, \mathrm{MnO}$, and $\mathrm{Na}_{2} \mathrm{O}, \pm 0.5 \mathrm{wt} \%$ for $\mathrm{CaO}, \mathrm{MgO}, \mathrm{SiO}_{2}$, and "Others," and $\pm 0.25 \mathrm{wt} \%$ for $\mathrm{NiO}$. Application of the variation was intended to bound the possible compositional differences between the nominal SB3 composition and that produced by the vendor. For example, the nominal $\mathrm{Al}_{2} \mathrm{O}_{3}$ content in the sludge was anticipated to be $16.898 \mathrm{wt} \%$. With the applied variation of $\pm 10 \%$, values between 15.21 and $18.59 \mathrm{wt} \%$ could be realized in the actual SRAT product produced by the vendor.

Table 3-2. Outer-Layer Intervals for the Major SB3 Oxides and Others

\begin{tabular}{||c|c|c|c|c|c|c|c|c|c||}
\hline \hline \multirow{2}{*}{$\begin{array}{c}\text { Variation } \\
\text { Applied }\end{array}$} & \pm 10 & \pm 0.5 & \pm 10 & \pm 0.5 & \pm 10 & \pm 10 & \pm 0.25 & \pm 0.5 & \pm 0.5 \\
\cline { 2 - 11 } & $\%$ & wt $\%$ & $\%$ & wt $\%$ & wt\% & $\%$ & wt $\%$ & wt $\%$ & wt $\%$ \\
\hline Oxide & $\mathrm{Al}_{2} \mathrm{O}_{3}$ & $\mathrm{CaO}$ & $\mathrm{Fe}_{2} \mathrm{O}_{3}$ & $\mathrm{MgO}$ & $\mathrm{MnO}$ & $\mathrm{Na}_{2} \mathrm{O}$ & $\mathrm{NiO}$ & $\mathrm{SiO}_{2}$ & Others \\
\hline Min & 15.21 & 2.81 & 33.57 & 3.31 & 6.381 & 21.75 & 1.67 & 3.44 & 1.070 \\
\hline Max & 18.59 & 3.81 & 41.03 & 4.31 & 7.799 & 26.59 & 2.17 & 4.44 & 2.070 \\
\hline
\end{tabular}

The "Others" is a grouping of the minor oxides $\left(\mathrm{Cr}_{2} \mathrm{O}_{3}, \mathrm{CuO}, \mathrm{K}_{2} \mathrm{O}, \mathrm{SO}_{4}{ }^{=}, \mathrm{TiO}_{2}\right.$, and $\left.\mathrm{Zr}_{2} \mathrm{O}\right)$ that is shown in Table 3-2. The composition of "Others" is given in Table 3-3.

Table 3-3. Composition (in mass fractions) of Others Grouping

\begin{tabular}{|c|c|c|c|c|c|c|}
\hline $\mathrm{Cr}_{2} \mathrm{O}_{3}$ & $\mathrm{CuO}$ & $\mathrm{K}_{2} \mathrm{O}$ & $\mathrm{SO}_{4}{ }^{{ }^{2}}$ & $\mathrm{TiO}_{2}$ & $\mathrm{ZrO}_{2}$ & Others \\
\hline 0.12102 & 0.05096 & 0.21656 & 0.45860 & 0.03822 & 0.11465 & 1.00000 \\
\hline
\end{tabular}

Now, what constitutes a feasible SB3 composition for this variation study? A feasible composition is a combination of the oxides (including "Others") of Table 3-2 at concentrations in mass fractions such that each concentration is within the interval in that table for that oxide and such that the sum of the mass fractions over all of the oxides equals one. The problem of finding these feasible combinations may be considered as a mixture problem (Cornell 2002). Statistical software such as JMP Version 6.0.2 is available to assist in working with such problems (SAS 2006). 
One of the tools provided by JMP is its Custom Design routine within its Design of Experiments platform (SAS 2005). This routine allows the user to specify a mixture problem in a framework such as that described above, and with the problem defined in such a manner the user is then allowed to provide JMP with a model of interest, e.g., a model involving linear effects for each of the mixture components such as the one given by Equation 1, which serves as the basis for selecting an optimal set (relative to that model and the chosen optimality criterion) of design points.

Equation 1.

$$
\begin{aligned}
\mathrm{y}= & \mathrm{a}_{1} \times \mathrm{Al}_{2} \mathrm{O}_{3}+\mathrm{a}_{2} \times \mathrm{CaO}+\mathrm{a}_{3} \times \mathrm{Fe}_{2} \mathrm{O}_{3}+\mathrm{a}_{4} \times \mathrm{MgO}+\mathrm{a}_{5} \times \mathrm{MnO} \\
& +\mathrm{a}_{6} \times \mathrm{Na}_{2} \mathrm{O}+\mathrm{a}_{7} \times \mathrm{NiO}+\mathrm{a}_{8} \times \mathrm{SiO}_{2}+\mathrm{a}_{9} \times \text { Others }
\end{aligned}
$$

where y represents a "response" variable whose relationship to these sludge oxides is to be investigated as a linear function of their mass-fraction concentrations. While the actual fitting of Equation 1 is of no interest in this study, its use is very beneficial in selecting feasible compositions on the boundary of the region defined by the concentration intervals of Table 3-2.

There are several options available to the user in performing this optimization. One of the options is that the user can specify the target sum for the mixture. In this case, the sum of the concentrations was specified in JMP as 1 on a mass fraction basis. JMP also provides the user with a choice of optimality criteria; for this situation the D-optimality criterion (see SAS (2005) for a description of the available criteria) was selected to serve as the basis for optimizing the test matrix. Ten different starting conditions were also selected to drive the optimization process. Finally, the minimum number (i.e., 9 - one for each of the $a_{i}$ terms in Equation 1) of points was specified as the desired number for the test matrix. Using this framework and approach led to the test matrix (in mass fractions) that is provided in Table 3-4.

Table 3-4. Outer-Layer Sludge Compositions (as mass fractions).

\begin{tabular}{||c|c|c|c|c|c|c|c|c||}
\hline $\mathbf{A l}_{\mathbf{2}} \mathbf{O}_{\mathbf{3}}$ & $\mathbf{C a O}$ & $\mathbf{F e}_{\mathbf{2}} \mathbf{O}_{\mathbf{3}}$ & $\mathbf{M g O}$ & $\mathbf{M n O}$ & $\mathbf{N a}_{\mathbf{2}} \mathbf{O}$ & $\mathbf{N i O}$ & $\mathbf{S i O}_{\mathbf{2}}$ & Others \\
\hline 0.15536 & 0.0281 & 0.4103 & 0.0431 & 0.06381 & 0.21753 & 0.0167 & 0.0444 & 0.0207 \\
\hline 0.1521 & 0.0381 & 0.36022 & 0.0431 & 0.06381 & 0.26587 & 0.0217 & 0.0444 & 0.0107 \\
\hline 0.1859 & 0.0381 & 0.3357 & 0.0431 & 0.06381 & 0.26159 & 0.0167 & 0.0344 & 0.0207 \\
\hline 0.1521 & 0.0381 & 0.37522 & 0.0331 & 0.06381 & 0.26587 & 0.0167 & 0.0344 & 0.0207 \\
\hline 0.1859 & 0.0281 & 0.40476 & 0.0331 & 0.06381 & 0.21753 & 0.0217 & 0.0344 & 0.0107 \\
\hline 0.1521 & 0.0281 & 0.35604 & 0.0431 & 0.07799 & 0.26587 & 0.0217 & 0.0344 & 0.0207 \\
\hline 0.1521 & 0.0381 & 0.40938 & 0.0431 & 0.07799 & 0.21753 & 0.0167 & 0.0344 & 0.0107 \\
\hline 0.1859 & 0.0381 & 0.36058 & 0.0331 & 0.07799 & 0.21753 & 0.0217 & 0.0444 & 0.0207 \\
\hline 0.1859 & 0.0281 & 0.33724 & 0.0331 & 0.07799 & 0.26587 & 0.0167 & 0.0444 & 0.0107 \\
\hline
\end{tabular}

To provide additional coverage of the sludge region of interest for SB3, further points were selected for experimental testing. These additional sludge compositions were selected from the concentration intervals (i.e., an inner-layer set of intervals) defined by Table 3-5. The information given in that table indicates how variation was introduced to determine each of the intervals: variation was applied as $\pm 5 \%$ of the nominal value for $\mathrm{Al}_{2} \mathrm{O}_{3}, \mathrm{Fe}_{2} \mathrm{O}_{3}, \mathrm{MnO}$, and $\mathrm{Na}_{2} \mathrm{O}$ while $\pm 0.5 \mathrm{wt} \%$ variation was applied for $\mathrm{CaO}, \mathrm{MgO} \mathrm{SiO}_{2}$, and "Others," and $\pm 0.25 \mathrm{wt} \%$ for $\mathrm{NiO}$. Thus, while the intervals for $\mathrm{CaO}, \mathrm{MgO}, \mathrm{SiO}_{2}$, "Others," and $\mathrm{NiO}$ were the same as those 
WSRC-STI-2007-00616

Revision 0

of Table 3-2, the intervals for the other major oxides fall within those intervals defined in Table 3-2.

Table 3-5. Inner-Layer Intervals for the Major SB3 Oxides and Others

\begin{tabular}{||c|c|c|c|c|c|c|c|c|c||}
\hline \hline \multirow{2}{*}{$\begin{array}{c}\text { Variation } \\
\text { Applied }\end{array}$} & \pm 5 & \pm 0.5 & \pm 5 & \pm 0.5 & \pm 5 & \pm 5 & \pm 0.25 & \pm 0.5 & \pm 0.5 \\
\cline { 2 - 11 } & $\%$ & wt\% & $\%$ & wt\% & wt $\%$ & $\%$ & wt $\%$ & wt\% & wt $\%$ \\
\hline Oxide & $\mathrm{Al}_{2} \mathrm{O}_{3}$ & $\mathrm{CaO}$ & $\mathrm{Fe}_{2} \mathrm{O}_{3}$ & $\mathrm{MgO}$ & $\mathrm{MnO}$ & $\mathrm{Na}_{2} \mathrm{O}$ & $\mathrm{NiO}$ & $\mathrm{SiO}_{2}$ & Others \\
\hline Min & 16.055 & 2.81 & 35.435 & 3.31 & 6.736 & 22.962 & 1.67 & 3.44 & 1.07 \\
\hline Max & 17.745 & 3.81 & 39.165 & 4.31 & 7.445 & 25.379 & 2.17 & 4.44 & 2.07 \\
\hline
\end{tabular}

Using JMP's Custom Design routine in a manner similar to that used for the outer-layer, a set of 9 optimal sludge compositions was selected from the region defined by Table 3-5. These compositions are provided in Table 3-6.

Table 3-6. Inner-Layer Sludge Compositions (as mass fractions)

\begin{tabular}{||c|c|c|c|c|c|c|c|c||}
\hline $\mathbf{A l}_{\mathbf{2}} \mathbf{O}_{\mathbf{3}}$ & $\mathbf{C a O}$ & $\mathbf{F e}_{\mathbf{2}} \mathbf{O}_{\mathbf{3}}$ & $\mathbf{M g O}$ & $\mathbf{M n O}$ & $\mathbf{N a}_{\mathbf{2}} \mathbf{O}$ & $\mathbf{N i O}$ & $\mathbf{S i O}_{\mathbf{2}}$ & Others \\
\hline 0.16109 & 0.0281 & 0.37766 & 0.0331 & 0.07445 & 0.25379 & 0.0167 & 0.0344 & 0.0207 \\
\hline 0.17745 & 0.0281 & 0.39165 & 0.0431 & 0.06736 & 0.23054 & 0.0167 & 0.0344 & 0.0107 \\
\hline 0.16055 & 0.0381 & 0.3753 & 0.0331 & 0.06736 & 0.25379 & 0.0167 & 0.0444 & 0.0107 \\
\hline 0.17745 & 0.0372 & 0.35435 & 0.0331 & 0.06736 & 0.25379 & 0.0217 & 0.0344 & 0.0207 \\
\hline 0.16055 & 0.0281 & 0.3603 & 0.0431 & 0.06736 & 0.25379 & 0.0217 & 0.0444 & 0.0207 \\
\hline 0.17745 & 0.0381 & 0.35548 & 0.0431 & 0.07445 & 0.22962 & 0.0167 & 0.0444 & 0.0207 \\
\hline 0.17745 & 0.0281 & 0.38048 & 0.0331 & 0.07445 & 0.22962 & 0.0217 & 0.0444 & 0.0107 \\
\hline 0.16055 & 0.0381 & 0.39165 & 0.0359 & 0.06736 & 0.22962 & 0.0217 & 0.0344 & 0.0207 \\
\hline 0.16055 & 0.0381 & 0.36321 & 0.0431 & 0.07445 & 0.25379 & 0.0217 & 0.0344 & 0.0107 \\
\hline
\end{tabular}

The sludge compositions of Table 3-4 and Table 3-6 were expanded to cover all of the sludge oxides by exploding the mass fraction of "Others" into mass fractions for its components as given by Table 3-3. The resulting outer-layer sludge compositions were combined with Frit 202-A11 at a WL of $50 \%$ to generate 9 glass compositions. Each of the resulting inner-layer sludge compositions was combined with Frit 202-A11 at a WL of 50\% and 52\%, which yielded a total of 18 glass compositions.

The target glass compositions are provided in Table 3-7 and Table A1 of Appendix A. The 9 outer-layer points are labeled with glass identifiers from HTLG-53VS through HTLG-61VS, the 9 inner-layer points at 50\% WL are labeled as HTLG-62VS through HTLG-70VS, and the 9 inner-layer points at 52\% WL are labeled as HTLG-71VS through HTLG-79VS. 
Table 3-7. Target Compositions of the Frit 202-A11 Variability Study Glasses.

\begin{tabular}{|c|c|c|c|c|c|c|c|c|c|c|c|c|c|c|c|c|c|c|c|}
\hline Glass ID & $\overline{E V}$ & $\overline{\mathbf{W L}}$ & $\overline{\mathrm{Al}_{2} \mathbf{O}_{3}}$ & $\mathbf{B}_{2} \mathbf{O}_{3}$ & $\overline{\mathrm{CaO}}$ & $\mathrm{Cr}_{2} \mathrm{O}_{3}$ & $\overline{\mathrm{CuO}}$ & $\mathrm{Fe}_{2} \mathrm{O}_{3}$ & $\overline{K_{2} \mathbf{O}}$ & $\overline{\mathbf{L i}_{2} \mathbf{O}}$ & MgO & MnO & $\mathrm{Na}_{2} \mathrm{O}$ & NiO & $\mathrm{SO}_{4}$ & $\mathrm{SiO}_{2}$ & $\mathrm{TiO}_{2}$ & $\mathrm{ZrO}_{2}$ & Total \\
\hline HTLG-53VS & $\mathrm{OL}$ & 50 & 7.77 & 4.50 & 1.41 & 0.13 & 0.05 & 20.52 & 0.22 & 3.00 & 2.16 & 3.19 & 12.38 & 0.84 & 0.47 & 43.22 & 0.04 & 0.12 & 100.00 \\
\hline HTLG-54VS & OL & 50 & 7.61 & 4.50 & 1.91 & 0.06 & 0.03 & 18.01 & 0.12 & 3.00 & 2.16 & 3.19 & 14.79 & 1.09 & 0.25 & 43.22 & 0.02 & 0.06 & 100.00 \\
\hline HTLG-55VS & OL & 50 & 9.30 & 4.50 & 1.91 & 0.13 & 0.05 & 16.79 & 0.22 & 3.00 & 2.16 & 3.19 & 14.58 & 0.84 & 0.47 & 42.72 & 0.04 & 0.12 & 100.00 \\
\hline HTLG-56VS & $\mathrm{OL}$ & 50 & 7.61 & 4.50 & 1.91 & 0.13 & 0.05 & 18.76 & 0.22 & 3.00 & 1.66 & 3.19 & 14.79 & 0.84 & 0.47 & 42.72 & 0.04 & 0.12 & 100.00 \\
\hline HTLG-57VS & $\mathrm{OL}$ & 50 & 9.30 & 4.50 & 1.41 & 0.06 & 0.03 & 20.24 & 0.12 & 3.00 & 1.66 & 3.19 & 12.38 & 1.09 & 0.25 & 42.72 & 0.02 & 0.06 & 100.00 \\
\hline HTLG-58VS & $\overline{\mathrm{OL}}$ & 50 & 7.61 & 4.50 & 1.41 & 0.13 & 0.05 & 17.80 & 0.22 & 3.00 & 2.16 & 3.90 & 14.79 & 1.09 & 0.47 & 42.72 & 0.04 & 0.12 & 100.00 \\
\hline HTLG-59VS & $\mathrm{OL}$ & 50 & 7.61 & 4.50 & 1.91 & 0.06 & 0.03 & 20.47 & 0.12 & 3.00 & 2.16 & 3.90 & 12.38 & 0.84 & 0.25 & 42.72 & 0.02 & 0.06 & 100.00 \\
\hline HTLG-60VS & $\overline{\mathrm{OL}}$ & 50 & 9.30 & 4.50 & 1.91 & 0.13 & 0.05 & 18.03 & 0.22 & 3.00 & 1.66 & 3.90 & 12.38 & 1.09 & 0.47 & 43.22 & 0.04 & 0.12 & 100.00 \\
\hline HTLG-61VS & $\mathrm{OL}$ & 50 & 9.30 & 4.50 & 1.41 & 0.06 & 0.03 & 16.86 & 0.12 & 3.00 & 1.66 & 3.90 & 14.79 & 0.84 & 0.25 & 43.22 & 0.02 & 0.06 & 100.00 \\
\hline HTLG-62VS & IL & 50 & 8.05 & 4.50 & 1.41 & 0.13 & 0.05 & 18.88 & 0.22 & 3.00 & 1.66 & 3.72 & 14.19 & 0.84 & 0.47 & 42.72 & 0.04 & 0.12 & 100.00 \\
\hline HTLG-63VS & IL & 50 & 8.87 & 4.50 & 1.41 & 0.06 & 0.03 & 19.58 & 0.12 & 3.00 & 2.16 & 3.37 & 13.03 & 0.84 & 0.25 & 42.72 & 0.02 & 0.06 & 100.00 \\
\hline HTLG-64VS & IL & 50 & 8.03 & 4.50 & 1.91 & 0.06 & 0.03 & 18.77 & 0.12 & 3.00 & 1.66 & 3.37 & 14.19 & 0.84 & 0.25 & 43.22 & 0.02 & 0.06 & 100.00 \\
\hline HTLG-65VS & IL & 50 & 8.87 & 4.50 & 1.86 & 0.13 & 0.05 & 17.72 & 0.22 & 3.00 & 1.66 & 3.37 & 14.19 & 1.09 & 0.47 & 42.72 & 0.04 & 0.12 & 100.00 \\
\hline HTLG-66VS & IL & 50 & 8.03 & 4.50 & 1.41 & 0.13 & 0.05 & 18.02 & 0.22 & 3.00 & 2.16 & 3.37 & 14.19 & 1.09 & 0.47 & 43.22 & 0.04 & 0.12 & 100.00 \\
\hline HTLG-67VS & IL & 50 & 8.87 & 4.50 & 1.91 & 0.13 & 0.05 & 17.77 & 0.22 & 3.00 & 2.16 & 3.72 & 12.98 & 0.84 & 0.47 & 43.22 & 0.04 & 0.12 & 100.00 \\
\hline HTLG-68VS & IL & 50 & 8.87 & 4.50 & 1.41 & 0.06 & 0.03 & 19.02 & 0.12 & 3.00 & 1.66 & 3.72 & 12.98 & 1.09 & 0.25 & 43.22 & 0.02 & 0.06 & 100.00 \\
\hline HTLG-69VS & IL & 50 & 8.03 & 4.50 & 1.91 & 0.13 & 0.05 & 19.58 & 0.22 & 3.00 & 1.80 & 3.37 & 12.98 & 1.09 & 0.47 & 42.72 & 0.04 & 0.12 & 100.00 \\
\hline HTLG-70VS & IL & 50 & 8.03 & 4.50 & 1.91 & 0.06 & 0.03 & 18.16 & 0.12 & 3.00 & 2.16 & 3.72 & 14.19 & 1.09 & 0.25 & 42.72 & 0.02 & 0.06 & 100.00 \\
\hline HTLG-71VS & IL & 52 & 8.38 & 4.32 & 1.46 & 0.13 & 0.05 & 19.64 & 0.23 & 2.88 & 1.72 & 3.87 & 14.64 & 0.87 & 0.49 & 41.15 & 0.04 & 0.12 & 100.00 \\
\hline HTLG-72VS & IL & 52 & 9.23 & 4.32 & 1.46 & 0.07 & 0.03 & 20.37 & 0.12 & 2.88 & 2.24 & 3.50 & 13.43 & 0.87 & 0.26 & 41.15 & 0.02 & 0.06 & 100.00 \\
\hline HTLG-73VS & IL & 52 & 8.35 & 4.32 & 1.98 & 0.07 & 0.03 & 19.52 & 0.12 & 2.88 & 1.72 & 3.50 & 14.64 & 0.87 & 0.26 & 41.67 & 0.02 & 0.06 & 100.00 \\
\hline HTLG-74VS & IL & 52 & 9.23 & 4.32 & 1.93 & 0.13 & 0.05 & 18.43 & 0.23 & 2.88 & 1.72 & 3.50 & 14.64 & 1.13 & 0.49 & 41.15 & 0.04 & 0.12 & 100.00 \\
\hline HTLG-75VS & IL & 52 & 8.35 & 4.32 & 1.46 & 0.13 & 0.05 & 18.74 & 0.23 & 2.88 & 2.24 & 3.50 & 14.64 & 1.13 & 0.49 & 41.67 & 0.04 & 0.12 & 100.00 \\
\hline HTLG-76VS & IL & 52 & 9.23 & 4.32 & 1.98 & 0.13 & 0.05 & 18.48 & 0.23 & 2.88 & 2.24 & 3.87 & 13.38 & 0.87 & 0.49 & 41.67 & 0.04 & 0.12 & 100.00 \\
\hline HTLG-77VS & IL & 52 & 9.23 & 4.32 & 1.46 & 0.07 & 0.03 & 19.78 & 0.12 & 2.88 & 1.72 & 3.87 & 13.38 & 1.13 & 0.26 & 41.67 & 0.02 & 0.06 & 100.00 \\
\hline HTLG-78VS & IL & 52 & 8.35 & 4.32 & 1.98 & 0.13 & 0.05 & 20.37 & 0.23 & 2.88 & 1.87 & 3.50 & 13.38 & 1.13 & 0.49 & 41.15 & 0.04 & 0.12 & 100.00 \\
\hline HTLG-79VS & IL & 52 & 8.35 & 4.32 & 1.98 & 0.07 & 0.03 & 18.89 & 0.12 & 2.88 & 2.24 & 3.87 & 14.64 & 1.13 & 0.26 & 41.15 & 0.02 & 0.06 & 100.00 \\
\hline
\end{tabular}


WSRC-STI-2007-00616

Revision 0

\subsection{Experimental Studies}

The glass compositions presented in Table 3-7 (and Table A1 of Appendix A) were selected to allow for an assessment of the impact of possible variation in the sludge composition on properties of interest, specifically glass durability. As previously mentioned, the actual CCIM demonstration will utilize a sludge simulant produced by an off-site vendor. Although the vendor targeted the nominal SB3 composition as shown in Table 3-1, some variation from the target should be anticipated. Coupling the compositional uncertainty of the sludge simulant with potential uncertainties in the targeted WL may lead to a compositional region beyond that covered by technical data to date. That being the case, a series of glasses was fabricated and tested with the intent of bounding the compositional variation of the sludge simulant over a WL interval of interest. The glasses are referred to as the "HTLG" glasses indicating that these are high $\mathrm{T}_{\mathrm{L}}$ glasses. The numbering of variability study glasses, which began with the number 53 , was a continuation of the numbering scheme already established for the CCIM task with the "VS" indicating that these were "variability study" glasses.

\subsection{Glass Fabrication}

Each HTLG variability glass was prepared from the proper proportions of reagent-grade metal oxides, carbonates, $\mathrm{H}_{3} \mathrm{BO}_{3}$, and salts in $150 \mathrm{~g}$ batches. The raw materials were thoroughly mixed and placed into a $90 \%$ platinum / 10\% rhodium, $250 \mathrm{~mL}$ crucible. The batch was placed in a high-temperature furnace at the target melt temperature of $1250^{\circ} \mathrm{C}$. The crucible was removed from the furnace after an isothermal hold at $1250^{\circ} \mathrm{C}$ for 1 hour. The glass was poured onto a clean, stainless steel plate and allowed to air cool (quench). The glass pour patty was used as a sampling stock for the various property measurements, including chemical composition and durability testing.

Approximately $25 \mathrm{~g}$ of each HTLG glass was heat-treated to simulate cooling along the centerline of a DWPF-type canister to gauge the effects of thermal history on product performance. This cooling schedule is referred to as the ccc (canister centerline cooling) curve. It should be noted that the ccc schedule was altered to capture the increased nominal CCIM melt temperature of $1250^{\circ} \mathrm{C}$. That is, the nominal DWPF ccc schedule begins at $1150^{\circ} \mathrm{C}$ and initially ramps down at $8^{\circ} \mathrm{C} / \mathrm{min}$ into the $900^{\circ} \mathrm{C}$ range. The adjusted cooling schedule was initiated at $1250^{\circ} \mathrm{C}$ and was ramped down at $10^{\circ} \mathrm{C} / \mathrm{min}$ to $1150^{\circ} \mathrm{C}$ at which point the normal DWPF ccc schedule was followed. Also note that there was no isothermal hold at $1150^{\circ} \mathrm{C}$. This altered cooling curve may be extremely conservative relative to the actual cooling cycle. That is, the CCIM pour cycles may be vastly different than the nominal DWPF pours and could result in much quicker cool down cycle times than those being simulated.

\subsection{Compositional Analysis}

To confirm that the as-fabricated glasses corresponded to the defined target compositions of Table 3-7, a representative sample from each glass was submitted to the SRNL Process Science Analytical Laboratory (PSAL) for chemical analysis under the auspices of analytical plans ${ }^{f}$. Two dissolution techniques (i.e., sodium peroxide fusion [PF] and lithium-metaborate [LM]) were used. Each glass was prepared in duplicate for each cation dissolution technique (PF and LM). All of the prepared samples were analyzed (twice for each element of interest) by Inductively

\footnotetext{
${ }^{f}$ There were two analytical plans to guide these measurements as provided by TB Edwards in the memorandum SRNLSCS-2007-00030, “Analytical Plans for Measuring the Chemical Compositions of the HTL Variability Study Glasses (U),” dated June 18, 2007.
} 
Coupled Plasma - Atomic Emission Spectroscopy (ICP-AES). The measurements generated by these plans are presented and reviewed in the results section.

\subsection{Homogeneity}

Homogeneity in this report refers to the lack of any crystallization in the glass samples. Although visual observations for crystallization were performed, representative samples of all HTLG VS glasses were submitted to Analytical Development (AD) for X-ray diffraction (XRD) analysis. Samples were run under conditions providing a detection limit of approximately $0.5 \mathrm{vol} \%$. That is, if crystals (or undissolved solids) were present at 0.5 vol\% or greater, the diffractometer was not only capable of detecting the crystals but also allowed for a qualitative determination of the type of crystal(s) present. Otherwise, a characteristically high background devoid of crystalline peaks indicated that the glass product was amorphous, suggesting either a completely amorphous product or that the degree of crystallization was below the detection limit.

\subsection{Durability}

The ASTM PCT was used as the measure of waste form performance or durability. The PCT was performed in triplicate on each HTLG quenched and ccc glass. The experimental test matrix also included the EA glass, the Approved Reference Material (ARM) glass, and blanks from the sample cleaning batch. Samples were ground, washed, and prepared according to the standard procedure. Fifteen milliliters of Type I ASTM water were added to $1.5 \mathrm{~g}$ of glass in stainless steel vessels. The vessels were closed, sealed, and placed in an oven at $90 \pm 2{ }^{\circ} \mathrm{C}$ where the samples were maintained for 7 days. Once cooled, the resulting solutions were sampled (filtered and acidified) and then analyzed by PSAL under the auspices of two analytical plans ${ }^{f}$. These measurements and the corresponding normalized release rates that were calculated from them are presented and discussed in the next section.

\footnotetext{
${ }^{f}$ There were two analytical plans to guide these measurements as provided by TB Edwards in the memorandum SRNLSCS-2007-00021, “Analytical Plans for Measuring the PCT Solutions for the HTL Variability Study Glasses (U),” dated May 16, 2007.
} 
WSRC-STI-2007-00616

Revision 0

\subsection{Results}

In this section, the results from the variability study are presented and reviewed. Discussions are provided of the visual observations for both the quenched and ccc versions of the study glasses, the measurements of the chemical composition of the glasses, the crystallization of the glasses as assessed by XRD, and the measurements of the PCTs.

\subsection{Visual Observations}

\subsubsection{Quenched Glasses}

Prior to discussing the visual observations, a few words regarding the terminology used to describe the appearance of the glasses are warranted. The use of "clean" indicates that the sample was classified as a single-phase system (i.e., no evidence of crystallization either on the surface or within the bulk). The term "surface crystals" implies that the surface of the glass was characterized by the presence of crystallization while the cross-section of bulk glass appeared homogeneous (i.e., single-phase, black and shiny). Surface crystallization in the HTLG glasses was apparent through the presence of a "textured" surface that ranged in appearance from "dull" to "metallic". Most quenched glasses were also characterized by a metallic haze that covered the surface of the glass - indicative of surface crystallization. The haze was very light on some glasses but very extensive (to the point of being "mirror-like") on other glasses. On some pour patty surfaces, swirls or patches of crystallization could also be observed.

Table 5-1 and Table 5-2 summarize the visual observations on the quenched and ccc HTLG VS glasses, respectively. In general, the quenched glasses were characterized by varying degrees of surface crystallization with the pour patty cross-section being single phased or "clean" based on visual observations. With respect to the impact of inner versus outer layer at $50 \% \mathrm{WL}$ or inner layer at 50 versus 52\% WL, there is no clear distinction that can be made based on these visual observations. More specifically, surface crystallization is observed on all of the HTLG VS glasses independent of the WL or sludge layer from which the glass was based.

\subsubsection{Glasses}

Table 5-2 summarizes the visual observations of the ccc glasses. The results indicate a high degree of devitrification in all 202-A11 based HTLG glasses. All of the pour patty surfaces, as well as the cross sections or bulk, contain extensive devitrification or crystallization. Although visually unappealing, the primary concern to address is the possible impact of the devitrification on durability (i.e., Does the slow cooling promote the formation of a crystalline phase which results in a negative impact on the durability response relative to the equivalent quenched glass?). If there is no impact or the impact does not result in an unacceptable PCT response (as defined by the EA benchmark), the glasses would meet DWPF acceptance criteria. It is known that the formation of spinels has very little if any practical impact on the PCT response of DWPF-type glasses. However, nepheline and/or aegirine formation in these glasses is possible and could lead to a practical and/or significant reduction in durability as measured by the PCT. 
Table 5-1. Visual Observations of Quenched HTLG VS Glasses.

\begin{tabular}{|c|c|c|c|}
\hline Glass & 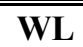 & Layer & Quenched \\
\hline HTLG-53VS & 50 & Outer & Light haze on pour patty surface; bulk clean \\
\hline HTLG-54VS & 50 & Outer & Light haze on pour patty surface; bulk clean \\
\hline HTLG-55VS & 50 & Outer & Haze or streaks on pour patty surface; bulk clean \\
\hline HTLG-56VS & 50 & Outer & Metallic swirls covering surface; bulk clean \\
\hline HTLG-57VS & 50 & Outer & Black shiny glass with a few light swirls on surface; bulk clean \\
\hline HTLG-58VS & 50 & Outer & Metallic sheen on surface with some metallic swirls; bulk clean \\
\hline HTLG-59VS & 50 & Outer & Dark shiny glass surface; bulk clean \\
\hline HTLG-60VS & 50 & Outer & Metallic swirls covering portions of surface; bulk clean \\
\hline HTLG-61VS & 50 & Outer & Haze with metallic swirls on surface; bulk clean \\
\hline HTLG-62VS & 50 & Inner & Haze with metallic swirls on surface; bulk clean \\
\hline HTLG-63VS & 50 & Inner & Dark brown glass with a haze on surface; bulk clean \\
\hline HTLG-64VS & 50 & Inner & Dark brown glass with a haze on surface; bulk clean \\
\hline HTLG-65VS & 50 & Inner & Dark brown glass with a haze on surface; bulk clean \\
\hline HTLG-66VS & 50 & Inner & Dark brown glass with a haze on surface, spots of crystals possible; bulk clean \\
\hline HTLG-67VS & 50 & Inner & Metallic/shiny haze on surface with crystals; bulk clean \\
\hline HTLG-68VS & 50 & Inner & Dark glass with light swirl haze on surface; bulk clean \\
\hline HTLG-69VS & 50 & Inner & Metallic/shiny haze on surface with crystals; bulk clean \\
\hline HTLG-70VS & 50 & Inner & Metallic/shiny haze on surface with crystals; bulk clean \\
\hline HTLG-71VS & 52 & Inner & Metallic/shiny haze on surface with metallic swirls; bulk clean \\
\hline HTLG-72VS & 52 & Inner & Black shiny glass with limited spots of crystals; bulk clean \\
\hline HTLG-73VS & 52 & Inner & Metallic/shiny haze on surface with crystals; bulk clean \\
\hline HTLG-74VS & 52 & Inner & Bronze with metallic swirls on surface; bulk clean \\
\hline HTLG-75VS & 52 & Inner & Bronze with metallic swirls on surface; bulk clean \\
\hline HTLG-76VS & 52 & Inner & Bronze with metallic swirls on surface; bulk clean \\
\hline HTLG-77VS & 52 & Inner & Metallic haze on surface; bulk clean \\
\hline HTLG-78VS & 52 & Inner & Metallic sheen or haze on surface with some metallic swirls, bulk clean \\
\hline HTLG-79VS & 52 & Inner & Metallic sheen or haze on surface with some metallic swirls, bulk clean \\
\hline
\end{tabular}


Table 5-3. Visual Observations of CCC HTLG VS Glasses.

\begin{tabular}{|c|c|c|c|}
\hline Glass & $\overline{\text { WL }}$ & Layer & $\mathbf{C C C}$ \\
\hline HTLG-53VS & 50 & Outer & Dull surface with crystals; bulk crystallized \\
\hline HTLG-54VS & 50 & Outer & Dull surface with crystals; bulk devitrified \\
\hline HTLG-55VS & 50 & Outer & Dull matte surface with crystals; bulk devitrified \\
\hline HTLG-56VS & 50 & Outer & Dull matte surface with crystals; bulk crystallized \\
\hline HTLG-57VS & 50 & Outer & Shiny/metallic surface with crystals, bulk crystallized \\
\hline HTLG-58VS & 50 & Outer & Dull matte, brown crystallized surface; bulk crystallized \\
\hline HTLG-59VS & 50 & Outer & Hazy surface with patches of crystals; bulk devitrified \\
\hline HTLG-60VS & 50 & Outer & Shiny/metallic surface with crystallization; bulk crystallized \\
\hline HTLG-61VS & 50 & Outer & Dull matte surface with crystals; bulk crystallized \\
\hline HTLG-62VS & 50 & Inner & Dull matte surface with major crystallization; bulk crystallized \\
\hline HTLG-63VS & 50 & Inner & Shiny/metallic surface with crystallization; bulk crystallized \\
\hline HTLG-64VS & 50 & Inner & Dull matte surface with crystals, some metallic spots; bulk crystallized \\
\hline HTLG-65VS & 50 & Inner & Dull matte surface with crystals; bulk crystallized \\
\hline HTLG-66VS & 50 & Inner & Dull matte surface with major crystallization; bulk crystallized \\
\hline HTLG-67VS & 50 & Inner & Dull matte surface with crystals; bulk crystallized \\
\hline HTLG-68VS & 50 & Inner & Dull matte surface with crystals; bulk crystallized \\
\hline HTLG-69VS & 50 & Inner & Dull matte surface with crystals; bulk crystallized \\
\hline HTLG-70VS & 50 & Inner & Dull matte surface with crystals; bulk crystallized \\
\hline HTLG-71VS & 52 & Inner & Crystallization on surface, bulk devitrified \\
\hline HTLG-72VS & 52 & Inner & Dull matte surface with crystals; bulk crystallized \\
\hline HTLG-73VS & 52 & Inner & Metallic crystallization on surface; bulk devitrified \\
\hline HTLG-74VS & 52 & Inner & Dull matte surface with patches of crystals; bulk crystallized \\
\hline HTLG-75VS & 52 & Inner & Dull matte surface with patches of crystals; bulk crystallized \\
\hline HTLG-76VS & 52 & Inner & Dull matte surface with crystals; bulk crystallized \\
\hline HTLG-77VS & 52 & Inner & Dull matte surface with crystals; bulk crystallized \\
\hline HTLG-78VS & 52 & Inner & Dull matte surface; bulk devitrified \\
\hline HTLG-79VS & 52 & Inner & Crystals over entire surface; bulk devitrified \\
\hline
\end{tabular}


WSRC-STI-2007-00616

Revision 0

\subsection{Compositional Analysis}

In this section, the measured versus targeted compositions of the 27 study glasses (HTLG-53VS through HTLG-79VS) are presented and compared. As stated earlier, the targeted compositions for these glasses are provided in Table 3-7 and in Table A1 of Appendix A. Chemical composition measurements for these glasses were conducted by the PSAL following two analytical plans, designated as series "P" and series "Q." $f$ Two dissolution methods were utilized in measuring these chemical compositions: samples prepared by lithium metaborate (LM) dissolution were used to measure elemental (cation) concentrations of aluminum (Al), calcium $(\mathrm{Ca})$, chromium $(\mathrm{Cr})$, copper $(\mathrm{Cu})$, potassium $(\mathrm{K})$, magnesium $(\mathrm{Mg})$, sodium $(\mathrm{Na})$, nickel $(\mathrm{Ni})$, sulfur (S), silicon (Si) for series "Q" only, titanium (Ti), and zirconium (Zr) concentrations, while samples from glasses prepared by peroxide fusion (PF) dissolution were used to measure elemental concentrations of boron (B), iron (Fe), lithium ( $\mathrm{Li}$ ), and silicon ( $\mathrm{Si}$ ) with Si being measured for both series "P" and series "Q.". All of the glasses were prepared twice by LM and by PF. The prepared samples were analyzed twice by ICP-AES with the instrumentation being re-calibrated between the two readings for each prepared glass.

Table A2 in Appendix A provides the elemental concentration measurements derived from the samples prepared using LM for series "P," Table A3 in Appendix A provides the series "P" measurements derived from the samples prepared using PF, Table A4 in Appendix A provides the elemental concentration measurements derived from the samples prepared using LM for series "Q," and Table A5 in Appendix A provides the series “Q” measurements derived from the samples prepared using PF. Measurements for the Batch 1 standard glass samples that were included in the PSAL analytical plans along with the study glasses are also provided in these tables.

The measured concentrations of the elements listed above were converted to oxide concentrations by multiplying the values for each element by the gravimetric factor for the corresponding oxide. During this process, an elemental concentration that was determined to be below the detection limit of the analytical procedures used by the PSAL was reduced to half of that detection limit as the oxide concentration was determined.

In the sub-sections that follow, the analytical sequences of the measurements are explored, the measurements of the standard samples are investigated and used for bias correction, the measurements for each glass are reviewed, the average chemical compositions (measured and bias-corrected) for each glass are determined, and comparisons are made between the measurements and the targeted compositions for the glasses.

\subsubsection{Measurements in Analytical Sequence}

Exhibit A1 in Appendix A provides plots of the measurements generated by the PSAL in Table A2 for samples prepared using the LM method. The plots are in analytical sequence with different symbols and colors being used to represent each of the study and standard glasses. Similar plots for the samples prepared using the PF method (i.e., the data in Table A3) are provided in Exhibit A2 in Appendix A. Exhibits A3 and A4 provide similar plots of the data in Tables A4 and A5 for the "Q" series of glasses. From the limited perspective of these plots, there appear to be no obvious outliers or other problems in these chemical composition measurements.

\footnotetext{
${ }^{f}$ The two analytical plans were provided by TB Edwards in the memorandum SRNL-SCS-2007-00030, “Analytical Plans for Measuring the Chemical Compositions of the HTL Variability Study Glasses (U),” dated June 18, 2007.
} 


\subsubsection{Batch 1 Standard Results}

In this section, the PSAL measurements of the chemical compositions of the Batch 1 samples are reviewed. These measurements are investigated across the ICP analytical blocks by series (i.e., "P" and "Q"), and the results are used to bias correct the measurements for the study glasses.

Exhibit A5 in Appendix A provides statistical analyses of the Batch 1 results generated by the LM prep method by block for each oxide of interest in series "P." The results include analysis of variance (ANOVA) investigations looking for statistically significant differences between the means of these groups for each of the oxides for each of the standards. The results from the statistical tests for the Batch 1 standard may be summarized as follows: $\mathrm{Cr}_{2} \mathrm{O}_{3}, \mathrm{MgO}, \mathrm{MnO}$, $\mathrm{Na}_{2} \mathrm{O}, \mathrm{NiO}$, and $\mathrm{SO}_{4}{ }^{\circ}$ have measurements that indicate a significant ICP-AES calibration effect on the block averages at the $5 \%$ significance level for the "P" series. The reference values for the oxide concentrations of the standards are given in the header for each set of measurements in the exhibit.

Exhibit A6 in Appendix A provides a similar set of analyses for the ICP-AES measurements derived from samples prepared via the PF method for the "P" series. There is no indication of a significant ICP-AES calibration effect on the block averages for these measurements at the $5 \%$ significance level. The reference values for the oxide concentrations of the standards are given in the header for each set of measurements in the exhibit.

Exhibit A7 in Appendix A provides a similar set of analyses for the ICP-AES measurements derived from samples prepared via the LM method for the " $Q$ " series. The results from the statistical tests for the Batch 1 standard may be summarized as follows: $\mathrm{MgO}, \mathrm{Na}_{2} \mathrm{O}, \mathrm{SO}_{4}{ }^{2}$, and $\mathrm{ZrO}_{2}$ have measurements that indicate a significant ICP-AES calibration effect on the block averages at the $5 \%$ significance level for the "Q" series. The reference values for the oxide concentrations of the standards are given in the header for each set of measurements in the exhibit.

Exhibit A8 in Appendix A provides a similar set of analyses for the ICP-AES measurements derived from samples prepared via the PF method for the "Q" series. The results from the statistical tests for the Batch 1 standard may be summarized as follows: $\mathrm{Fe}_{2} \mathrm{O}_{3}$ and $\mathrm{Li}_{2} \mathrm{O}$ have measurements that indicate a significant ICP-AES calibration effect on the block averages at the $5 \%$ significance level for the "Q" series. The reference values for the oxide concentrations of the standards are given in the header for each set of measurements in the exhibit.

Thus, some of these results provide incentive for adjusting the measurements by the effects of the ICP-AES calibrations. Bias-correction was conducted for an oxide as long as the reference value for the oxide concentration in the Batch 1 glass was greater than or equal to $0.1 \mathrm{wt} \%$. The basis for the bias correction is presented as part of Exhibits $\mathrm{A} 5$ through $\mathrm{A} 8$ - the average measurement for Batch 1 for each ICP-AES block for $\mathrm{Al}_{2} \mathrm{O}_{3}, \mathrm{~B}_{2} \mathrm{O}_{3}, \mathrm{CaO}, \mathrm{Cr}_{2} \mathrm{O}_{3}, \mathrm{CuO}, \mathrm{Fe}_{2} \mathrm{O}_{3}, \mathrm{~K}_{2} \mathrm{O}, \mathrm{MgO}$, $\mathrm{Na}_{2} \mathrm{O}, \mathrm{NiO}$, and $\mathrm{SiO}_{2}$. No bias correction was conducted for $\mathrm{SO}_{4}{ }^{\circ}$ and $\mathrm{ZrO}_{2}$.

The bias correction was conducted as follows. For each oxide, let $\overline{\mathrm{a}}_{\mathrm{ij}}$ be the average measurement for the $\mathrm{i}^{\text {th }}$ oxide at analytical block $\mathrm{j}$ for Batch 1 , and let $\mathrm{t}_{\mathrm{i}}$ be the reference value for the $\mathrm{i}^{\text {th }}$ oxide for Batch 1. (The averages and reference values are provided in Exhibits A5 through and A8.) Let $\bar{c}_{\mathrm{ijk}}$ be the average measurement for the $\mathrm{i}^{\text {th }}$ oxide at analytical block $\mathrm{j}$ for the $\mathrm{k}^{\text {th }}$ glass. The bias adjustment was conducted as follows 
WSRC-STI-2007-00616

Revision 0

$$
\bar{c}_{i j k} \bullet\left(1-\frac{\bar{a}_{i j}-t_{i}}{\bar{a}_{i j}}\right)=\bar{c}_{i j k} \bullet \frac{t_{i}}{\bar{a}_{i j}}
$$

Bias-corrected measurements are indicated by a "bc" suffix, and such adjustments were performed for all of the analytes of this study except for $\mathrm{SO}_{4}{ }^{=}$and $\mathrm{ZrO}_{2}$. Both measured and measured "bc" values are included in the discussion that follows. In these discussions biascorrected values for $\mathrm{SO}_{4}{ }^{=}$and $\mathrm{ZrO}_{2}$ are included for completeness (e.g., to allow a sum of oxides to be computed for the bias-corrected results). These bias-corrected values are the same as the original values for $\mathrm{SO}_{4}{ }{ }$ and $\mathrm{ZrO}_{2}$ (i.e., once again, no bias correction was performed for this pair of oxides).

\subsubsection{Composition Measurements by Glass Number}

Exhibits A9 and A10 in Appendix A provide plots of the oxide concentration measurements for the "P" series by Glass ID (including Batch 1 ) for the measured and bias-corrected (bc) values for the LM and PF preparation methods, respectively. The measurements are grouped by their Lab IDs for each Glass ID within their targeted concentrations for the indicated oxide. Exhibits A11 and A12 in Appendix A provide similar plots for the "Q" series results. Different symbols and colors are used to represent the different glasses. These plots show the individual measurements across the duplicate measurements of each preparation method. A review of the plots presented in these exhibits reveals the repeatability of the individual values for each analyte for each glass. There is no suggestion of any significant problems in the repeatability of the measurements in these plots. In comparing the measured values to their targeted concentrations, some differences are seen between the measured values and the targeted concentrations for some oxides for some of the glasses.

A review of these comparisons suggests that there is a pattern to the relationship between the targeted and measured concentrations for glasses numbered 65 and 66. It appears that these two glasses may have been switched at some point prior to the time these chemical composition measurements were conducted. A closer look at this possibility is provided in the next section.

\subsubsection{Measured versus Targeted Compositions}

The duplicate measurements for each oxide for each glass (over all of the preparation methods) were averaged to determine a representative chemical composition for each glass. These determinations were conducted both for the measured and for the bias-corrected data. A sum of oxides was also computed for each glass based upon both the measured and bias-corrected values. Exhibit A13 in Appendix A provides plots showing the results for each glass of series "P" for each oxide to help highlight the comparisons among the measured, bias-corrected, and targeted values. A similar plot for the series "Q" glasses is provided in Exhibit A14 of Appendix A.

Some general and specific observations from the plots of Exhibits A13 and A14 are offered below. In general, the measured and the measured bc compositional views for $\mathrm{CaO}$ appear to be higher than their targeted values for all study glasses. The measured and the measured bc compositional views for $\mathrm{Fe}_{2} \mathrm{O}_{3}$ and $\mathrm{MgO}$ appear to be lower than their targeted values for most of the study glasses. The measured $\mathrm{Cr}_{2} \mathrm{O}_{3}$ values also appear lower than targeted for most study glasses; however, these differences may be within analytical error of the ICP.

Specific observations include the measured $\mathrm{Li}_{2} \mathrm{O}$ value for HTLG-56VS which is slightly higher than its targeted value (3.16 versus $3.0 \mathrm{wt} \%$ in glass). The measured NiO value for HTLG-71VS is extremely low (0.002 $\mathrm{wt} \%$ - essentially a detection limit value) as compared to its targeted 
value (0.87 wt\%). A review of the batch sheet suggests that $\mathrm{NiO}$ was added to the glass. A comparison of the measured and targeted $\mathrm{SO}_{4}$ values suggests volatilization for most of the HTLG glasses was minimal (i.e., less then $\pm 10 \%$ difference between target and measured values). However, 7 of the 27 HTLG glasses (HTLG-67, -69, -71, 74, -75, -76, and -78) had relatively high differences between the target and measured $\mathrm{SO}_{4}$ values. The average difference was approximately $15 \%$ suggests some degree of volatilization in these seven glasses. Perhaps of some interest is that 5 of these 7 glasses are based on the 52\% (higher WL) inner layer EVs.

Perhaps of most significance, are the comparisons of the target and measured values for HTLG55VS and HTLG-66VS. Comparing the measured $\mathrm{Al}_{2} \mathrm{O}_{3}, \mathrm{CaO}$, and $\mathrm{MgO}$ values for these two glasses with their respective target concentrations, these two glasses appear to be switched. It is not known whether the sample switch occurred during the batching or melting processes, or during the labeling of the samples during preparation for compositional analysis. Regardless of where the error may have occurred, the data suggest that these two glasses were indeed switched at some point during the testing program. The sums of oxides for all of the glasses fall within the interval from $95 \%$ to $105 \%$.

Table A6 in Appendix A provides a summary of the average compositions as well as the targeted compositions and some associated differences and relative differences. Notice that the targeted sums of oxides for the standard glasses do not sum to $100 \%$ due to an incomplete coverage of the oxides in the Batch 1 and LRM glasses. Entries in Table A6 show the relative differences between the measured or bias-correct values and the targeted values. These differences are shaded when they are greater than or equal to $5 \%$.

With the exception of HTLG-65VS and HTLG-66VS, the analytical results suggest that the target compositions of the study glasses were met (at least to the extent of having no significant impact to the objectives of the study). With respect to HTLG-65VS and HTLG-66VS, the XRD and PCT data suggest the conclusions would not be different even if switching had occurred. More specifically, both glasses precipitated only magnetite upon ccc and the PCT responses are essentially identical regardless of the compositional view used to normalize.

\subsection{XRD Results}

Representative samples of the ccc HTLG glasses were submitted for XRD analysis and Table 5-2 summarizes the results. The formation of magnetite (spinel) in all of the HTLG variability study glasses is consistent with previous research at these higher WLs. Based on previous or historical data, the presence of spinel in the ccc based glasses will have no measurable effect on the durability response of these glasses. The same can not be said for the formation of nepheline and/or aegirine. Formation of these two phases has been linked to a negative impact on the durability of HLW glasses - the magnitude of that impact being dependent on the extent of devitrification (volume \%) and the ultimate change to the residual glass matrix.

At 50\% WL, the XRD results indicate the presence of nepheline and/or aegirine in 6 of the 9 outer layer glasses and in 7 of the 9 inner layer glasses. These results indicate if the vendor's SRAT product lies within the compositional variation applied and a 50\% WL is obtained, there is a high probability that aegirine and/or nepheline will be present in the resulting glass product upon slow cooling. However, as one transitions to higher WLs (52\%), only four of the nine inner layer based, HTLG VS glasses have nepheline or aegirine present after slow cooling. Although no outer layer, 52\% WL glasses were fabricated, the results appear to be consistent with previous trends indicating that targeting higher WLs (within the Frit 202-A11 - SB3 system) lowers the possibility of the formation of these two phases. However, the fact that both phases exist in some 
of the $52 \%$ WL glasses suggests that there are sludge oxide combinations that could still lead to their formation and a possible negative impact on durability. From a demonstration perspective, the questions at hand are: "What is the composition of the SRAT product produced by the vendor? and How does it compare to the EVs (inner or outer) being evaluated in the variability study"? If the actual SRAT product is compositionally similar to one of the EVs in which nepheline and/or aegirine formed, then targeting higher WLs (at least up to 52\%) may not be sufficient to avoid a negative response in durability space. However, it appears that one could lower (but not eliminate) the possibility of forming nepheline and/or aegirine in the slow cooled glasses (within the compositional region tested) by targeting higher WLs. Again, this is consistent with the observations of Peeler et al. (2007) in the Frit 202-A11 - nominal SB3 study in which as WL was increased above 50\%, there appeared to be a transition from the aegirine and/or nepheline primary phase fields to the spinel primary phase field, leading to more durable ccc glasses.

Table 5-2. XRD Results for the CCC Glasses.

\begin{tabular}{|c|c|c|c|}
\hline Glass & WL & $\begin{array}{c}\text { Outer/Inner } \\
\text { Layer }\end{array}$ & XRD Results \\
\hline HTLG-53VS & 50 & Outer & Magnetite, aegirine \\
\hline HTLG-54VS & 50 & Outer & Magnetite, aegirine, nepheline \\
\hline HTLG-55VS & 50 & Outer & Magnetite, aegirine, hematite, nepheline \\
\hline HTLG-56VS & 50 & Outer & Magnetite, aegirine, hematite, nepheline \\
\hline HTLG-57VS & 50 & Outer & Magnetite \\
\hline HTLG-58VS & 50 & Outer & Magnetite \\
\hline HTLG-59VS & 50 & Outer & Magnetite, aegirine \\
\hline HTLG-60VS & 50 & Outer & Magnetite, aegirine \\
\hline HTLG-61VS & 50 & Outer & Magnetite \\
\hline HTLG-62VS & 50 & Inner & Magnetite, nepheline \\
\hline HTLG-63VS & 50 & Inner & Magnetite, aegirine \\
\hline HTLG-64VS & 50 & Inner & Magnetite, aegirine \\
\hline HTLG-65VS & 50 & Inner & Magnetite \\
\hline HTLG-66VS & 50 & Inner & Magnetite \\
\hline HTLG-67VS & 50 & Inner & Magnetite, aegirine, nepheline, lithium silicate \\
\hline HTLG-68VS & 50 & Inner & Magnetite, aegirine \\
\hline HTLG-69VS & 50 & Inner & Magnetite, aegirine \\
\hline HTLG-70VS & 50 & Inner & Magnetite, aegirine \\
\hline HTLG-71VS & 52 & Inner & Magnetite, aegirine, nepheline \\
\hline HTLG-72VS & 52 & Inner & Magnetite \\
\hline HTLG-73VS & 52 & Inner & Magnetite, nepheline \\
\hline HTLG-74VS & 52 & Inner & Magnetite \\
\hline HTLG-75VS & 52 & Inner & Magnetite \\
\hline HTLG-76VS & 52 & Inner & Magnetite, aegirine \\
\hline HTLG-77VS & 52 & Inner & Magnetite \\
\hline HTLG-78VS & 52 & Inner & Magnetite, aegirine \\
\hline HTLG-79VS & 52 & Inner & Magnetite \\
\hline
\end{tabular}

\subsection{PCT Testing}

The study glasses, after being batched and fabricated, were subjected to the 7-day PCT to assess their durabilities. More specifically, Method A of the PCT (ASTM C-1285-2002 [see ASTM 
(2002)]) was used for these measurements. Durability is the critical product quality metric for DWPF glass studies. Two heat treatments (quenching and a modified ccc schedule) were used during the fabrication of each of the study glasses. Both heat treatments for each study glass were subjected to the PCT (in triplicate). PCTs were also conducted in triplicate for samples of the EA glass and for samples of the ARM glass as part of each PCT plan. Blanks (samples consisting only of ASTM Type I water) were also submitted as part of each PCT plan. Two plans were provided to PSAL to support the evaluation of the compositions of the solutions resulting from the PCTs ${ }^{f}$. Samples of a multi-element, standard solution were also included in each analytical plan (as a check on the accuracy of the ICP-AES used for the measurements). In this and the following sections, the measurements generated by the PSAL for these PCTs are presented and reviewed.

Table B1 in Appendix B provides the elemental leachate concentration measurements determined by the PSAL for the solution samples generated by the PCTs by set. Since there were two cleaning batches that supported the first set of PCTs (with a pair of blanks in each batch), the first set of PCTs contained 4 blanks while the second series contained the usual two. One of the quality control checkpoints for the PCT procedure is solution-weight loss over the course of the 7-day test. None of the PCT results for either set indicated a solution-weight loss problem. Any measurement in Table B1 below the detection limit of the analytical procedure (indicated by a “<”) was replaced by $1 / 2$ of the detection limit in subsequent analyses. In addition to adjustments for detection limits, the values were adjusted for the dilution factors: the values for the study glasses, the blanks, and the ARM glass in Table B1 were multiplied by 1.6667 to determine the values in parts per million (ppm) and the values for EA were multiplied by 16.6667. The last five columns of Table B1 provide the measurements resulting from these adjustments.

In the sub-sections that follow, the analytical sequence of the measurements is explored for each set of PCTs, the measurements of the standards are investigated and used to assess the overall accuracy of the ICP measurement process for each set, the measurements for each glass are reviewed, and plots are provided that explore the effects of heat treatment on the PCTs for these glasses.

\subsubsection{PCT Measurements in Analytical Sequence}

Exhibit B1 in Appendix B provides plots of the leachate (ppm) concentrations in analytical sequence as generated by the PSAL for all of the data by PCT set. Exhibit B2 in Appendix B provides plots of the leachate (ppm) concentrations in analytical sequence by set for only the study glasses (i.e., measurements for EA, ARM, blanks, and the solution standards are excluded). From the limited perspective provided by these plots, no problems are seen. More detailed investigations into these results are provided below.

\subsubsection{Repeatability of the PCT Measurements for a Glass/Heat Treatment Combination}

Exhibit B3 in Appendix B provides a closer look at the repeatability of the triplicate PCTs for each study glass and heat treatment combination. While there are some differences in the measurements for the two heat treatments for some of these glasses, there does appear to be good repeatability for the triplicates for each treatment for each of the glasses. A closer look at the differences between the heat treatments for each of the study glasses is provided in the discussions that follow.

\footnotetext{
${ }^{f}$ The two analytical plans were provided by TB Edwards in the memorandum SRNL-SCS-2007-00021, “Analytical Plans for Measuring the PCT Solutions for the HTL Variability Study Glasses (U),” dated May 16, 2007.
} 


\subsubsection{Results for the Samples of the Multi-Element Solution Standard}

Exhibit B4 in Appendix B provides analyses of the PSAL measurements of the samples of the multi-element solution standard by ICP-AES analytical (or calibration) block for each PCT set. An ANOVA investigating for statistically significant differences among the block averages for these samples for each element of interest is included in these exhibits. For the first set of PCTs, there is a statistically significant (at approximately a 5\% level) difference among the $\mathrm{Al}$ average measurements, the Fe average measurements, the $\mathrm{Li}$ average measurements, and the Na average measurements over the calibration blocks. For the second set of PCTs, there is a statistically significant (at approximately a 5\% level) difference among the Fe average measurements and the $\mathrm{Si}$ average measurements over the calibration blocks. However, no bias correction of the PCT results for the study glasses was conducted. This approach was taken since the triplicate PCTs for a single study glass were placed in different ICP-AES blocks. Averaging the ppm values for each set of triplicates helps to minimize the impact of the ICP-AES effects for both sets of PCTs.

Table 5-3 summarizes the average measurements and the reference values for the 3 primary elements present in the multi-element solution. The results indicate consistent and accurate measurements from the PSAL processes used to conduct these analyses.

Table 5-3. Results from Samples of the Multi-Element Solution Standard by PCT Set

\begin{tabular}{||c|c|c|c|c|c|c|c|c|c||}
\hline \multicolumn{9}{|c|}{ Set 1 } & \multicolumn{5}{c|}{ Set 2 } \\
\hline \begin{tabular}{c} 
Block \\
\hline 1
\end{tabular} & $\begin{array}{c}\mathrm{B} \\
(\mathrm{ppm})\end{array}$ & $\begin{array}{c}\mathrm{Li} \\
(\mathrm{ppm})\end{array}$ & $\begin{array}{c}\mathrm{Na} \\
(\mathrm{ppm})\end{array}$ & $\begin{array}{c}\mathrm{Si} \\
(\mathrm{ppm})\end{array}$ & Block & $\begin{array}{c}\mathrm{B} \\
(\mathrm{ppm})\end{array}$ & $\begin{array}{c}\mathrm{Li} \\
(\mathrm{ppm})\end{array}$ & $\begin{array}{c}\mathrm{Na} \\
(\mathrm{ppm})\end{array}$ & $\begin{array}{c}\text { Si } \\
(\mathrm{ppm})\end{array}$ \\
\hline 2 & 19.9 & 10.0 & 83.9 & 50.1 & 1 & 21.3 & 10.3 & 81.4 & 51.9 \\
\hline 3 & 20.2 & 9.7 & 78.8 & 49.0 & 2 & 20.5 & 10.2 & 84.3 & 48.9 \\
\hline 4 & 20.1 & 9.8 & 82.1 & 49.1 & 3 & 20.3 & 10.1 & 82.8 & 49.6 \\
\hline 5 & 20.0 & 9.7 & 84.9 & 48.8 & 5 & 20.2 & 10.1 & 83.1 & 48.8 \\
\hline 6 & 19.8 & 9.6 & 79.9 & 49.4 & 6 & 20.9 & 10.3 & 82.4 & 50.4 \\
\hline $\begin{array}{c}\text { Grand } \\
\text { Average }\end{array}$ & 20.1 & 9.8 & 81.9 & 49.4 & $\begin{array}{c}\text { Grand } \\
\text { Average }\end{array}$ & 20.5 & 10.2 & 82.6 & 50.0 \\
\hline $\begin{array}{c}\text { Reference } \\
\text { Value }\end{array}$ & 20 & 10 & 81 & 50 & $\begin{array}{c}\text { Reference } \\
\text { Value }\end{array}$ & 20 & 10 & 81 & 50 \\
\hline $\begin{array}{c}\text { \% } \\
\text { difference }\end{array}$ & $0.39 \%$ & $-2.41 \%$ & $1.13 \%$ & $-1.96 \%$ & $\begin{array}{c}\% \\
\text { difference }\end{array}$ & $2.58 \%$ & $1.96 \%$ & $1.93 \%$ & $-0.07 \%$ \\
\hline \hline
\end{tabular}

\subsubsection{Normalized PCT Results}

PCT leachate concentrations are typically normalized using the cation composition (expressed as a weight percent) in the glass to obtain a grams-per-liter $(\mathrm{g} / \mathrm{L})$ leachate concentration. The normalization of the PCTs is usually conducted using the measured compositions of the glasses. This is the preferred normalization process for the PCTs. For completeness, the targeted cation and the bias-corrected cation compositions were also used to conduct this normalization.

As is the usual convention, the common logarithm of the normalized PCT (normalized leachate, NL) for each element of interest was determined and used for comparison. To accomplish this computation, one must 
1.Determine the common logarithm of the elemental parts per million (ppm) leachate concentration for each of the triplicates and each of the elements of interest (these values are provided in Table B2 of Appendix B),

2.Average the common logarithms over the triplicates for each element of interest, and then

Normalizing Using Measured Composition (preferred method)

3. Subtract a quantity equal to 1 plus the common logarithm of the average cation measured concentration (expressed as a weight percent of the glass) from the average computed in step 2 .

Or Normalizing Using Target Composition

3. Subtract a quantity equal to 1 plus the common logarithm of the target cation concentration (expressed as a weight percent of the glass) from the average computed in step 2.

Or Normalizing Using Measured Bias-Corrected Composition

3. Subtract a quantity equal to 1 plus the common logarithm of the measured bias-corrected cation concentration (expressed as a weight percent of the glass) from the average computed in step 2.

Exhibit B5 in Appendix B provides scatter plots for these results and offers an opportunity to investigate the consistency in the leaching across the elements for the glasses of this study. All combinations of the normalizations of the PCTs (i.e., those generated using the targeted, measured, and bias-corrected compositional views) and both heat treatments are represented in a pair of scatter plot matrices: one matrix for each set of PCTs. Consistency in the leaching across the elements for a set of PCTs is typically demonstrated by a high degree of linear correlation among the values for pairs of these elements. The smallest correlation seen over both sets of PCTs is that for Li and $\mathrm{Na}$ in the first set, with a value of $\sim 94 \%$.

Table 5-4 and Table 5-5 summarize the normalized PCTs for the quenched and ccc glasses, respectively. The glasses are listed by glass identifier with normalized PCT data being presented for targeted, measured, and measured bc compositional views. The PCT results of the quenched glasses (regardless of compositional view) indicate that all HTLG variability study glasses are very acceptable relative to the EA glass benchmark. More specifically, the normalized boron releases (NL $[\mathrm{B}] \mathrm{in} \mathrm{g} / \mathrm{L}$ ) range from $0.8 \mathrm{~g} / \mathrm{L}$ (for HTLG-60VS based on the measured composition) to $1.384 \mathrm{~g} / \mathrm{L}$ (HTLG-79VS based on the measured bc composition). These results can be compared to the NL [B] for the EA benchmark of $16.695 \mathrm{~g} / \mathrm{L}$. Again, the PCT results of the quenched glasses are consistent with previous data in the Frit 202-A11 - SB3 system.

The PCT results for the ccc glasses are not as straight forward. The NL [B]s for the slow cooled glasses range from $0.607 \mathrm{~g} / \mathrm{L}$ (for HTLG-57ccc based on the measured composition) to $9.42 \mathrm{~g} / \mathrm{L}$ (for HTLG-67ccc based on the measured bc compositional view). Although these glasses would be classified as acceptable relative to the EA glass benchmark, the relatively high release of the slow cooled glasses would be of concern if this system were to be implemented into DWPF. This latter statement should be tempered with the fact that Frit 202-A11 was not optimized for the SB3 system but was simply developed within the time constraints to meet programmatic objectives. If the CCIM technology were to be implemented into DWPF, more detailed glass formulation studies would be performed leading to a frit recommendation that would address this concern. 
Table 5-4. Normalized PCTs for Quenched Glasses by Compositional View.

\begin{tabular}{|c|c|c|c|c|c|}
\hline Glass ID & Composition & $\begin{array}{c}\text { NL } \\
{[B(g / L)]}\end{array}$ & $\begin{array}{c}\mathrm{NL} \\
{[\mathrm{Li}(\mathrm{g} / \mathrm{L})]}\end{array}$ & $\begin{array}{c}\text { NL } \\
{[\mathrm{Na}(\mathrm{g} / \mathrm{L})]}\end{array}$ & $\begin{array}{c}\text { NL } \\
{[\mathrm{Si}(\mathrm{g} / \mathrm{L})]}\end{array}$ \\
\hline ARM & reference & 0.487 & 0.572 & 0.525 & 0.277 \\
\hline EA & reference & 17.602 & 9.546 & 13.774 & 3.953 \\
\hline ARM & reference & 0.500 & 0.583 & 0.509 & 0.274 \\
\hline EA & reference & 18.262 & 10.023 & 13.728 & 4.004 \\
\hline HTLG-53VS & targeted & 1.074 & 0.925 & 0.976 & 0.489 \\
\hline HTLG-54VS & targeted & 1.259 & 0.998 & 1.200 & 0.578 \\
\hline HTLG-55VS & targeted & 0.985 & 0.854 & 1.025 & 0.482 \\
\hline HTLG-56VS & targeted & 1.272 & 1.132 & 1.245 & 0.599 \\
\hline HTLG-57VS & targeted & 0.815 & 0.834 & 0.846 & 0.468 \\
\hline HTLG-58VS & targeted & 1.236 & 0.984 & 1.217 & 0.579 \\
\hline HTLG-59VS & targeted & 1.151 & 0.993 & 1.093 & 0.517 \\
\hline HTLG-60VS & targeted & 0.826 & 0.823 & 0.869 & 0.440 \\
\hline HTLG-61VS & targeted & 0.980 & 0.863 & 0.996 & 0.503 \\
\hline HTLG-62VS & targeted & 1.108 & 0.934 & 1.061 & 0.534 \\
\hline HTLG-63VS & targeted & 0.974 & 0.872 & 0.936 & 0.487 \\
\hline HTLG-64VS & targeted & 1.142 & 0.995 & 1.109 & 0.544 \\
\hline HTLG-65VS & targeted & 1.107 & 0.946 & 1.092 & 0.541 \\
\hline HTLG-66VS & targeted & 0.971 & 0.848 & 1.007 & 0.476 \\
\hline HTLG-67VS & targeted & 0.944 & 0.848 & 0.904 & 0.431 \\
\hline HTLG-68VS & targeted & 0.942 & 0.844 & 0.882 & 0.463 \\
\hline HTLG-69VS & targeted & 0.980 & 0.898 & 0.987 & 0.464 \\
\hline HTLG-70VS & targeted & 1.276 & 1.041 & 1.216 & 0.557 \\
\hline HTLG-71VS & targeted & 1.222 & 1.010 & 1.134 & 0.544 \\
\hline HTLG-72VS & targeted & 1.044 & 0.903 & 0.984 & 0.504 \\
\hline HTLG-73VS & targeted & 1.291 & 1.031 & 1.169 & 0.537 \\
\hline HTLG-74VS & targeted & 1.069 & 0.915 & 1.076 & 0.495 \\
\hline HTLG-75VS & targeted & 1.196 & 0.956 & 1.136 & 0.530 \\
\hline HTLG-76VS & targeted & 0.997 & 0.893 & 1.023 & 0.462 \\
\hline HTLG-77VS & targeted & 0.991 & 0.895 & 0.988 & 0.496 \\
\hline HTLG-78VS & targeted & 1.081 & 0.962 & 1.080 & 0.506 \\
\hline HTLG-79VS & targeted & 1.362 & 1.087 & 1.267 & 0.573 \\
\hline HTLG-53VS & measured & 1.080 & 0.946 & 0.994 & 0.483 \\
\hline HTLG-54VS & measured & 1.266 & 1.019 & 1.237 & 0.585 \\
\hline HTLG-55VS & measured & 0.984 & 0.870 & 1.064 & 0.476 \\
\hline HTLG-56VS & measured & 1.293 & 1.075 & 1.277 & 0.608 \\
\hline HTLG-57VS & measured & 0.812 & 0.842 & 0.835 & 0.463 \\
\hline HTLG-58VS & measured & 1.230 & 1.014 & 1.254 & 0.576 \\
\hline HTLG-59VS & measured & 1.168 & 1.015 & 1.101 & 0.515 \\
\hline HTLG-60VS & measured & 0.803 & 0.828 & 0.830 & 0.439 \\
\hline HTLG-61VS & measured & 0.995 & 0.881 & 1.000 & 0.509 \\
\hline HTLG-62VS & measured & 1.100 & 0.943 & 1.074 & 0.538 \\
\hline HTLG-63VS & measured & 0.979 & 0.884 & 0.917 & 0.493 \\
\hline HTLG-64VS & measured & 1.172 & 1.006 & 1.106 & 0.540 \\
\hline HTLG-65VS & measured & 1.123 & 0.947 & 1.095 & 0.541 \\
\hline HTLG-66VS & measured & 0.971 & 0.872 & 1.000 & 0.484 \\
\hline HTLG-67VS & measured & 0.965 & 0.847 & 0.907 & 0.441 \\
\hline HTLG-68VS & measured & 0.956 & 0.842 & 0.895 & 0.477 \\
\hline HTLG-69VS & measured & 0.973 & 0.898 & 0.983 & 0.468 \\
\hline HTLG-70VS & measured & 1.281 & 1.063 & 1.225 & 0.578 \\
\hline HTLG-71VS & measured & 1.204 & 0.994 & 1.135 & 0.547 \\
\hline HTLG-72VS & measured & 1.061 & 0.903 & 0.986 & 0.516 \\
\hline HTLG-73VS & measured & 1.332 & 1.037 & 1.178 & 0.555 \\
\hline HTLG-74VS & measured & 1.127 & 0.931 & 1.075 & 0.514 \\
\hline HTLG-75VS & measured & 1.228 & 0.956 & 1.145 & 0.544 \\
\hline HTLG-76VS & measured & 0.998 & 0.903 & 1.017 & 0.472 \\
\hline HTLG-77VS & measured & 1.002 & 0.895 & 0.976 & 0.504 \\
\hline HTLG-78VS & measured & 1.071 & 0.966 & 1.080 & 0.512 \\
\hline
\end{tabular}


WSRC-STI-2007-00616

Revision 0

\begin{tabular}{||l|c|c|c|c|c||}
\hline \multicolumn{1}{|c|}{ Glass ID } & Composition & $\begin{array}{c}\mathbf{N L} \\
{[\mathbf{B}(\mathbf{g} / \mathbf{L})]}\end{array}$ & $\begin{array}{c}\mathbf{N L} \\
{[\mathbf{L i}(\mathbf{g} / \mathbf{L})]}\end{array}$ & $\begin{array}{c}\mathbf{N L} \\
{[\mathbf{N a}(\mathbf{g} / \mathbf{L})]}\end{array}$ & $\begin{array}{c}\mathbf{N L} \\
{[\mathbf{S i} \mathbf{( g / L )}]}\end{array}$ \\
\hline HTLG-79VS & measured & 1.353 & 1.102 & 1.265 & 0.585 \\
\hline HTLG-53VS & measured bc & 1.103 & 0.941 & 1.055 & 0.473 \\
\hline HTLG-54VS & measured bc & 1.284 & 1.015 & 1.313 & 0.561 \\
\hline HTLG-55VS & measured bc & 1.005 & 0.866 & 1.129 & 0.467 \\
\hline HTLG-56VS & measured bc & 1.312 & 1.071 & 1.355 & 0.583 \\
\hline HTLG-57VS & measured bc & 0.830 & 0.838 & 0.911 & 0.454 \\
\hline HTLG-58VS & measured bc & 1.256 & 1.009 & 1.331 & 0.564 \\
\hline HTLG-59VS & measured bc & 1.193 & 1.011 & 1.169 & 0.505 \\
\hline HTLG-60VS & measured bc & 0.814 & 0.825 & 0.905 & 0.421 \\
\hline HTLG-61VS & measured bc & 1.009 & 0.878 & 1.090 & 0.488 \\
\hline HTLG-62VS & measured bc & 1.116 & 0.940 & 1.140 & 0.516 \\
\hline HTLG-63VS & measured bc & 0.993 & 0.881 & 1.000 & 0.473 \\
\hline HTLG-64VS & measured bc & 1.197 & 1.002 & 1.206 & 0.530 \\
\hline HTLG-65VS & measured bc & 1.140 & 0.943 & 1.194 & 0.519 \\
\hline HTLG-66VS & measured bc & 0.991 & 0.868 & 1.091 & 0.474 \\
\hline HTLG-67VS & measured bc & 0.987 & 0.853 & 0.969 & 0.426 \\
\hline HTLG-68VS & measured bc & 0.947 & 0.838 & 0.955 & 0.456 \\
\hline HTLG-69VS & measured bc & 0.995 & 0.904 & 1.049 & 0.454 \\
\hline HTLG-70VS & measured bc & 1.269 & 1.057 & 1.309 & 0.550 \\
\hline HTLG-71VS & measured bc & 1.231 & 1.000 & 1.213 & 0.529 \\
\hline HTLG-72VS & measured bc & 1.052 & 0.898 & 1.052 & 0.492 \\
\hline HTLG-73VS & measured bc & 1.320 & 1.032 & 1.257 & 0.529 \\
\hline HTLG-74VS & measured bc & 1.117 & 0.927 & 1.148 & 0.488 \\
\hline HTLG-75VS & measured bc & 1.216 & 0.951 & 1.224 & 0.517 \\
\hline HTLG-76VS & measured bc & 1.021 & 0.909 & 1.087 & 0.456 \\
\hline HTLG-77VS & measured bc & 0.993 & 0.891 & 1.041 & 0.481 \\
\hline HTLG-78VS & measured bc & 1.095 & 0.972 & 1.152 & 0.497 \\
\hline HTLG-79VS & measured bc & 1.384 & 1.109 & 1.350 & 0.568 \\
\hline \hline
\end{tabular}


Table 5-5. Normalized PCTs for CCC Glasses by Compositional View.

\begin{tabular}{|c|c|c|c|c|c|}
\hline Glass ID & Composition & $\begin{array}{c}\mathrm{NL} \\
{[\mathrm{B}(\mathrm{g} / \mathrm{L})]}\end{array}$ & $\begin{array}{c}\mathrm{NL} \\
{[\mathrm{Li}(\mathrm{g} / \mathrm{L})]}\end{array}$ & $\begin{array}{c}\mathrm{NL} \\
{[\mathrm{Na}(\mathrm{g} / \mathrm{L})]}\end{array}$ & $\begin{array}{c}\mathrm{NL} \\
{[\mathrm{Si}(\mathrm{g} / \mathrm{L})]}\end{array}$ \\
\hline ARM & reference & 0.487 & 0.572 & 0.525 & 0.277 \\
\hline EA & reference & 17.602 & 9.546 & 13.774 & 3.953 \\
\hline ARM & reference & 0.500 & 0.583 & 0.509 & 0.274 \\
\hline EA & reference & 18.262 & 10.023 & 13.728 & 4.004 \\
\hline HTLG-53VS & targeted & 0.826 & 0.916 & 0.915 & 0.500 \\
\hline HTLG-54VS & targeted & 4.102 & 2.576 & 2.106 & 0.919 \\
\hline HTLG-55VS & targeted & 6.657 & 5.274 & 3.061 & 1.357 \\
\hline HTLG-56VS & targeted & 2.599 & 2.615 & 1.817 & 0.968 \\
\hline HTLG-57VS & targeted & 0.609 & 0.677 & 0.716 & 0.413 \\
\hline HTLG-58VS & targeted & 0.865 & 0.890 & 1.040 & 0.548 \\
\hline HTLG-59VS & targeted & 1.805 & 1.965 & 1.356 & 0.677 \\
\hline HTLG-60VS & targeted & 0.643 & 0.769 & 0.763 & 0.431 \\
\hline HTLG-61VS & targeted & 0.896 & 0.918 & 0.948 & 0.531 \\
\hline HTLG-62VS & targeted & 2.347 & 2.049 & 1.596 & 0.836 \\
\hline HTLG-63VS & targeted & 0.726 & 0.768 & 0.891 & 0.467 \\
\hline HTLG-64VS & targeted & 6.717 & 5.625 & 3.135 & 1.400 \\
\hline HTLG-65VS & targeted & 0.945 & 1.070 & 1.023 & 0.551 \\
\hline HTLG-66VS & targeted & 0.875 & 1.036 & 0.966 & 0.501 \\
\hline HTLG-67VS & targeted & 9.013 & 7.403 & 4.217 & 1.663 \\
\hline HTLG-68VS & targeted & 0.666 & 0.683 & 0.744 & 0.424 \\
\hline HTLG-69VS & targeted & 0.705 & 0.725 & 0.804 & 0.429 \\
\hline HTLG-70VS & targeted & 2.977 & 2.273 & 1.689 & 0.833 \\
\hline HTLG-71VS & targeted & 8.287 & 6.163 & 4.751 & 1.877 \\
\hline HTLG-72VS & targeted & 0.730 & 0.703 & 0.792 & 0.434 \\
\hline HTLG-73VS & targeted & 3.888 & 3.924 & 2.244 & 1.215 \\
\hline HTLG-74VS & targeted & 0.903 & 0.882 & 0.937 & 0.489 \\
\hline HTLG-75VS & targeted & 0.832 & 0.805 & 0.971 & 0.497 \\
\hline HTLG-76VS & targeted & 1.556 & 1.153 & 1.109 & 0.529 \\
\hline HTLG-77VS & targeted & 0.685 & 0.706 & 0.758 & 0.418 \\
\hline HTLG-78VS & targeted & 0.723 & 0.838 & 0.892 & 0.448 \\
\hline HTLG-79VS & targeted & 1.387 & 1.354 & 1.199 & 0.607 \\
\hline HTLG-53VS & measured & 0.831 & 0.937 & 0.932 & 0.493 \\
\hline HTLG-54VS & measured & 4.125 & 2.629 & 2.170 & 0.929 \\
\hline HTLG-55VS & measured & 6.645 & 5.375 & 3.176 & 1.342 \\
\hline HTLG-56VS & measured & 2.641 & 2.483 & 1.863 & 0.982 \\
\hline HTLG-57VS & measured & 0.607 & 0.684 & 0.707 & 0.409 \\
\hline HTLG-58VS & measured & 0.861 & 0.917 & 1.072 & 0.544 \\
\hline HTLG-59VS & measured & 1.831 & 2.010 & 1.366 & 0.675 \\
\hline HTLG-60VS & measured & 0.625 & 0.774 & 0.729 & 0.430 \\
\hline HTLG-61VS & measured & 0.909 & 0.938 & 0.952 & 0.537 \\
\hline HTLG-62VS & measured & 2.330 & 2.069 & 1.615 & 0.842 \\
\hline HTLG-63VS & measured & 0.730 & 0.778 & 0.873 & 0.473 \\
\hline HTLG-64VS & measured & 6.889 & 5.690 & 3.128 & 1.391 \\
\hline HTLG-65VS & measured & 0.959 & 1.071 & 1.026 & 0.551 \\
\hline HTLG-66VS & measured & 0.875 & 1.065 & 0.959 & 0.509 \\
\hline HTLG-67VS & measured & 9.211 & 7.395 & 4.231 & 1.704 \\
\hline HTLG-68VS & measured & 0.676 & 0.681 & 0.754 & 0.438 \\
\hline HTLG-69VS & measured & 0.700 & 0.725 & 0.802 & 0.432 \\
\hline HTLG-70VS & measured & 2.988 & 2.320 & 1.701 & 0.866 \\
\hline HTLG-71VS & measured & 8.160 & 6.062 & 4.756 & 1.885 \\
\hline HTLG-72VS & measured & 0.742 & 0.703 & 0.794 & 0.444 \\
\hline HTLG-73VS & measured & 4.013 & 3.947 & 2.262 & 1.255 \\
\hline HTLG-74VS & measured & 0.952 & 0.898 & 0.936 & 0.508 \\
\hline HTLG-75VS & measured & 0.853 & 0.806 & 0.978 & 0.510 \\
\hline HTLG-76VS & measured & 1.558 & 1.166 & 1.103 & 0.539 \\
\hline HTLG-77VS & measured & 0.692 & 0.706 & 0.749 & 0.425 \\
\hline HTLG-78VS & measured & 0.715 & 0.841 & 0.892 & 0.453 \\
\hline
\end{tabular}


WSRC-STI-2007-00616

Revision 0

\begin{tabular}{||c|c|c|c|c|c||}
\hline \multicolumn{1}{|c|}{ Glass ID } & Composition & $\begin{array}{c}\mathbf{N L} \\
{[\mathbf{B}(\mathbf{g} / \mathbf{L})]}\end{array}$ & $\begin{array}{c}\mathbf{N L} \\
[\mathbf{L i} \mathbf{( g / L})]\end{array}$ & $\begin{array}{c}\mathbf{N L} \\
[\mathbf{N a} \mathbf{( g / L})]\end{array}$ & $\begin{array}{c}\mathbf{N L} \\
{[\mathbf{S i} \mathbf{( g / \mathbf { L } ) ]}}\end{array}$ \\
\hline HTLG-79VS & measured & 1.378 & 1.372 & 1.198 & 0.619 \\
\hline HTLG-53VS & measured bc & 0.848 & 0.933 & 0.989 & 0.484 \\
\hline HTLG-54VS & measured bc & 4.184 & 2.620 & 2.304 & 0.891 \\
\hline HTLG-55VS & measured bc & 6.787 & 5.351 & 3.372 & 1.316 \\
\hline HTLG-56VS & measured bc & 2.679 & 2.474 & 1.978 & 0.942 \\
\hline HTLG-57VS & measured bc & 0.620 & 0.681 & 0.771 & 0.401 \\
\hline HTLG-58VS & measured bc & 0.879 & 0.913 & 1.138 & 0.533 \\
\hline HTLG-59VS & measured bc & 1.870 & 2.001 & 1.450 & 0.662 \\
\hline HTLG-60VS & measured bc & 0.634 & 0.771 & 0.795 & 0.412 \\
\hline HTLG-61VS & measured bc & 0.923 & 0.934 & 1.038 & 0.515 \\
\hline HTLG-62VS & measured bc & 2.364 & 2.062 & 1.715 & 0.808 \\
\hline HTLG-63VS & measured bc & 0.740 & 0.776 & 0.952 & 0.453 \\
\hline HTLG-64VS & measured bc & 7.036 & 5.666 & 3.411 & 1.364 \\
\hline HTLG-65VS & measured bc & 0.973 & 1.067 & 1.118 & 0.529 \\
\hline HTLG-66VS & measured bc & 0.893 & 1.061 & 1.046 & 0.499 \\
\hline HTLG-67VS & measured bc & 9.420 & 7.441 & 4.522 & 1.648 \\
\hline HTLG-68VS & measured bc & 0.669 & 0.677 & 0.805 & 0.418 \\
\hline HTLG-69VS & measured bc & 0.716 & 0.730 & 0.856 & 0.420 \\
\hline HTLG-70VS & measured bc & 2.961 & 2.308 & 1.818 & 0.823 \\
\hline HTLG-71VS & measured bc & 8.345 & 6.100 & 5.083 & 1.824 \\
\hline HTLG-72VS & measured bc & 0.736 & 0.699 & 0.847 & 0.423 \\
\hline HTLG-73VS & measured bc & 3.976 & 3.928 & 2.414 & 1.198 \\
\hline HTLG-74VS & measured bc & 0.943 & 0.893 & 1.000 & 0.483 \\
\hline HTLG-75VS & measured bc & 0.846 & 0.802 & 1.046 & 0.485 \\
\hline HTLG-76VS & measured bc & 1.593 & 1.174 & 1.179 & 0.522 \\
\hline HTLG-77VS & measured bc & 0.685 & 0.703 & 0.799 & 0.405 \\
\hline HTLG-78VS & measured bc & 0.732 & 0.846 & 0.952 & 0.440 \\
\hline HTLG-79VS & measured bc & 1.410 & 1.381 & 1.278 & 0.601 \\
\hline
\end{tabular}

Table 5-6 provides a summary of the XRD results for the ccc glasses and the PCT responses for the quenched and ccc glasses (normalized based on the targeted glass composition). The information is presented in this manner to provide insight into the effect of the various crystalline phases that formed upon slow cooling on the PCT response. A comparison of the quenched and cCC PCT response provides insight into the magnitude of the crystallization effect (assuming that the quenched glasses contained only magnetite which should have no impact on the PCT response).

Consider the ccc glasses which precipitated magnetite as the only crystalline phase upon slow cooling (i.e., HLTG-57, -58, 65, -66, -72, -74, -75, -77, and -79). There is very little, if any, practical difference in PCT response between the quenched and ccc versions of these glasses. The NL [B]s are all below $\sim 1.4 \mathrm{~g} / \mathrm{L}$. Next consider the formation of aegirine upon slow cooling (i.e., HTLG-53, -59, -60, -63, -64, -68, -69, -70, -76, and -78) and its impact on the PCT response. With the exception of HTLG-64ccc (an inner layer, 50\% WL based glass), all of the NL [B]s are less than $3 \mathrm{~g} / \mathrm{L}$. The NL [B] of HTLG-64ccc is reported to be $6.7 \mathrm{~g} / \mathrm{L}$. For those glasses precipitating only aegirine after slow cooling, the PCT data are mixed which is related to the effect of this crystalline phase on the response. More specifically, in some glasses (HTLG-53, $60,-63,-68,-69$, and -78 ), the formation of this phase appears to have no practical impact on the PCT response. Aegirine formation in the slowly cooled versions of HTLG-59, -70, and -76 appears to have a measurable, negative impact on the PCT response - but the glasses still have very acceptable NL [B] values as compared to the EA benchmark. The troubling data point for aegirine formation is HTLG-64 (an inner layer, EV targeted 50\% WL). The formation of aegirine in this glass has a significant, negative impact on the PCT response ( $\sim 6 \mathrm{x}$ decrease in durability). Although this is still "acceptable", it would be of concern with respect to DWPF operations. The 
aegirine based data suggest that the impact of its formation within the composition region studied is rather unpredictable.

Next consider those glasses which formed either nepheline or both nepheline and aegirine upon slow cooling (i.e., HTLG-54, -55, -56, -67, and -71). The NL [B]s for these ccc based glasses range from $\sim 2.3 \mathrm{~g} / \mathrm{L}$ (HTLG-62) to $\sim 9.1 \mathrm{~g} / \mathrm{L}$ (HTLG-67). Although the formation of aegirine has (in general) a slightly negative impact on the PCT response (as previously discussed), the formation of nepheline and aegirine is a combination that has a high probability of leading to a significant reduction in durability upon slow cooling.

With respect to the CCIM demonstration, one would hope that there is a clear cut delineation of sludge compositions and/or targeted WLs that could avoid the formation of either of these phases within the sludge composition region being evaluated. However, based on a statistical assessment of the PCT and XRD data, there does not appear to be a direct relationship among the sludge components and their combinations that can be used to identify a nepheline and/or aegirine primary phase field. In addition, the ability to avoid the formation of these phases within the sludge compositional region and WL interval used is not available. More specifically, based on previous data, the possibility to target higher WLs (> 50\%) to avoid any negative impacts on durability as a result of crystallization was observed when the nominal SB3 composition was utilized. However, when composition variation is applied to the sludge, there are multiple combinations of sludge components that fall within the nepheline and/or aegirine phase fields even at 52\% WL which ultimately lead to a negative impact on durability. Having said that, the PCT results do suggest that the probability of observing that negative impact is lower at the higher WLs (only 2 of the inner layer, 52\% WL based glasses have NL [B] $>2 \mathrm{~g} / \mathrm{L}$ after slow cooling as compared to 4 of the 9 inner layer EVs targeted 50\% WL). 
Table 5-6. XRD and PCT results for the HTLG Variability Study Glasses.

(PCT normalized to target compositions).

\begin{tabular}{|c|c|c|c|c|c|}
\hline Glass & $\overline{W L}$ & $\begin{array}{c}\text { Outer/Inner } \\
\text { Layer }\end{array}$ & XRD Results for CCC & $\begin{array}{c}\text { NL }[B](g / L) \\
\text { for Quenched }\end{array}$ & $\begin{array}{c}\mathrm{NL}[\mathrm{B}](\mathrm{g} / \mathrm{L}) \\
\text { for } \mathrm{CCC} \\
\end{array}$ \\
\hline HTLG-53VS & 50 & Outer & Magnetite, aegirine & 1.074 & 0.826 \\
\hline HTLG-54VS & 50 & Outer & Magnetite, aegirine, nepheline & 1.259 & 4.102 \\
\hline HTLG-55VS & 50 & Outer & Magnetite, aegirine, hematite, nepheline & 0.985 & 6.657 \\
\hline HTLG-56VS & 50 & Outer & Magnetite, aegirine, hematite, nepheline & 1.272 & 2.599 \\
\hline HTLG-57VS & 50 & Outer & Magnetite & 0.815 & 0.609 \\
\hline HTLG-58VS & 50 & Outer & Magnetite & 1.236 & 0.865 \\
\hline HTLG-59VS & 50 & Outer & Magnetite, aegirine & 1.151 & 1.805 \\
\hline HTLG-60VS & 50 & Outer & Magnetite, aegirine & 0.826 & 0.643 \\
\hline HTLG-61VS & 50 & Outer & Magnetite & 0.980 & 0.896 \\
\hline HTLG-62VS & 50 & Inner & Magnetite, nepheline & 1.108 & 2.347 \\
\hline HTLG-63VS & 50 & Inner & Magnetite, aegirine & 0.974 & 0.726 \\
\hline HTLG-64VS & 50 & Inner & Magnetite, aegirine & 1.142 & 6.717 \\
\hline HTLG-65VS & 50 & Inner & Magnetite & 1.107 & 0.945 \\
\hline HTLG-66VS & 50 & Inner & Magnetite & 0.971 & 0.875 \\
\hline HTLG-67VS & 50 & Inner & Magnetite, aegirine, nepheline, lithium silicate & 0.944 & 9.013 \\
\hline HTLG-68VS & 50 & Inner & Magnetite, aegirine & 0.942 & 0.666 \\
\hline HTLG-69VS & 50 & Inner & Magnetite, aegirine & 0.980 & 0.705 \\
\hline HTLG-70VS & 50 & Inner & Magnetite, aegirine & 1.276 & 2.977 \\
\hline HTLG-71VS & 52 & Inner & Magnetite, aegirine, nepheline & 1.222 & 8.287 \\
\hline HTLG-72VS & 52 & Inner & Magnetite & 1.044 & 0.730 \\
\hline HTLG-73VS & 52 & Inner & Magnetite, nepheline & 1.291 & 3.888 \\
\hline HTLG-74VS & 52 & Inner & Magnetite & 1.069 & 0.903 \\
\hline HTLG-75VS & 52 & Inner & Magnetite & 1.196 & 0.832 \\
\hline HTLG-76VS & 52 & Inner & Magnetite, aegirine & 0.997 & 1.556 \\
\hline HTLG-77VS & 52 & Inner & Magnetite & 0.991 & 0.685 \\
\hline HTLG-78VS & 52 & Inner & Magnetite, aegirine & 1.081 & 0.723 \\
\hline HTLG-79VS & 52 & Inner & Magnetite & 1.362 & 1.387 \\
\hline
\end{tabular}




\subsection{Glasses Fabricated Using Nominal SB3 Compositions}

As this variability study was being completed, measurements on the composition of the SB3 sludge simulant that was prepared by the vendor became available. Three glasses were made based upon these measurements targeting WLs of 45, 50, and 55\%; the glasses were designated as HTLG-100, HTLG-101, and HTLG-102, respectively. The targeted compositions of these glasses are provided in Table 5-7. Glasses were prepared from the proper proportions of reagentgrade metal oxides, carbonates, $\mathrm{H}_{3} \mathrm{BO}_{3}$, and salts in $150 \mathrm{~g}$ batches as described in Section 4.1.

Table 5-7. Targeted Compositions of Glasses at 45, 50, and $55 \% \mathrm{WL}$ Based Upon Vendor's Measured Composition of SB3

\begin{tabular}{|c|c|c|c|}
\hline & $\begin{array}{c}\text { HTLG- } \\
\mathbf{1 0 0}\end{array}$ & $\begin{array}{c}\text { HTLG- } \\
\mathbf{1 0 1}\end{array}$ & $\begin{array}{c}\text { HTLG- } \\
\mathbf{1 0 2}\end{array}$ \\
\hline $\mathrm{WL}$ & 45 & 50 & 55 \\
\hline $\mathrm{Al}_{2} \mathrm{O}_{3}$ & 7.83 & 8.70 & 9.56 \\
\hline $\mathrm{B}_{2} \mathrm{O}_{3}$ & 4.95 & 4.50 & 4.05 \\
\hline $\mathrm{CaO}$ & 1.26 & 1.40 & 1.53 \\
\hline $\mathrm{Cr}_{2} \mathrm{O}_{3}$ & 0.09 & 0.10 & 0.11 \\
\hline $\mathrm{CuO}$ & 0.00 & 0.00 & 0.00 \\
\hline $\mathrm{Fe}_{2} \mathrm{O}_{3}$ & 15.77 & 17.52 & 19.27 \\
\hline $\mathrm{K}_{2} \mathrm{O}$ & 0.33 & 0.37 & 0.41 \\
\hline $\mathrm{Li}_{2} \mathrm{O}$ & 3.30 & 3.00 & 2.70 \\
\hline $\mathrm{MgO}^{2}$ & 1.86 & 2.04 & 2.24 \\
\hline $\mathrm{MnO}^{2}$ & 4.07 & 4.53 & 4.98 \\
\hline $\mathrm{Na}_{2} \mathrm{O}$ & 11.80 & 12.78 & 13.75 \\
\hline $\mathrm{NiO}^{2}$ & 0.58 & 0.65 & 0.71 \\
\hline $\mathrm{SO}_{4}$ & 0.40 & 0.44 & 0.49 \\
\hline $\mathrm{SiO}_{2}$ & 46.88 & 42.98 & 39.07 \\
\hline $\mathrm{TiO}_{2}$ & 0.00 & 0.00 & 0.00 \\
\hline $\mathrm{ZrO}_{2}$ & 0.08 & 0.09 & 0.09 \\
\hline $\mathrm{Total}$ & 99.19 & 99.07 & 98.98 \\
\hline
\end{tabular}

The XRD assessments of the ccc versions of these glasses indicated magnetite, aegirine, nepheline, and lithium silicate for HTLG-100 but only magnetite for HTLG-101 and HTLG-102. These data suggest that targeting higher WLs (between 50 and 55\%) should result in very acceptable glasses regardless of thermal heat treatment (quenched or cсc).

PCTs were conducted for these glasses and the resulting solutions were submitted to PSAL for measurement. The PCTs were conducted in triplicate for both quenched and ccc versions of these three glasses along with a blank and with triplicate EA and triplicate ARM samples. The raw data from these analyses are provided in Table 5-8 for documentation purposes. 
Table 5-8. PCT Results for HTLG-100, HTLG-101, and HTLG-102

\begin{tabular}{|c|c|c|c|c|c|c|c|c|c|c|c|c|c|}
\hline Glass ID & SRTC-ML ID & Al ar & B ar & Fe ar & Li ar & Na ar & Si ar & Al (ppm) & B (ppm) & $\mathrm{Fe}(\mathrm{ppm})$ & Li (ppm) & $\mathrm{Na}(\mathrm{ppm})$ & Si (ppm) \\
\hline Blank-1 & $07-2935$ & $<0.100$ & $<0.100$ & $<0.100$ & $<1.00$ & $<1.00$ & $<0.100$ & 0.083 & 0.083 & 0.083 & 0.833 & 0.833 & 0.083 \\
\hline Blank-2 & $07-2936$ & $<0.100$ & $<0.100$ & $<0.100$ & $<1.00$ & $<1.00$ & $<0.100$ & 0.083 & 0.083 & 0.083 & 0.833 & 0.833 & 0.083 \\
\hline ARM-1 & $07-2937$ & 2.98 & 9.93 & 0.329 & 8.5 & 21.8 & 36.8 & 4.967 & 16.550 & 0.548 & 14.167 & 36.334 & 61.335 \\
\hline ARM-2 & $07-2938$ & 2.95 & 10 & $<0.100$ & 8.53 & 22.6 & 36.9 & 4.917 & 16.667 & 0.083 & 14.217 & 37.667 & 61.501 \\
\hline ARM-3 & $07-2939$ & 3.19 & 9.77 & $<0.100$ & 8.41 & 21.5 & 36.8 & 5.317 & 16.284 & 0.083 & 14.017 & 35.834 & 61.335 \\
\hline EA-1 & $07-2940$ & 0.279 & 38.6 & $<0.100$ & 11.9 & 104 & 56 & 4.650 & 643.335 & 0.833 & 198.334 & 1733.337 & 933.335 \\
\hline EA-2 & $07-2941$ & 0.276 & 39.3 & $<0.100$ & 11.9 & 104 & 56.3 & 4.600 & 655.001 & 0.833 & 198.334 & 1733.337 & 938.335 \\
\hline EA-3 & $07-2942$ & 0.3 & 39.8 & $<0.100$ & 11.9 & 104 & 56.9 & 5.000 & 663.335 & 0.833 & 198.334 & 1733.337 & 948.335 \\
\hline HTLG-100-1 & $07-2943$ & 8.76 & 7.64 & 2.72 & 7.34 & 40 & 59.8 & 14.600 & 12.734 & 4.533 & 12.234 & 66.668 & 99.669 \\
\hline HTLG-100-2 & $07-2944$ & 8.45 & 6.58 & 3.25 & 7.07 & 38.5 & 57.6 & 14.084 & 10.967 & 5.417 & 11.784 & 64.168 & 96.002 \\
\hline HTLG-100-3 & $07-2945$ & 8.47 & 6.56 & 3.22 & 7 & 39 & 58.1 & 14.117 & 10.934 & 5.367 & 11.667 & 65.001 & 96.835 \\
\hline HTLG-100-ссC-1 & $07-2946$ & 10.8 & 50.1 & 12.6 & 42 & 116 & 152 & 18.000 & 83.502 & 21.000 & 70.001 & 193.337 & 253.338 \\
\hline HTLG-100-ссс-2 & $07-2947$ & 10.7 & 50.8 & 12.7 & 42.4 & 115 & 161 & 17.834 & 84.668 & 21.167 & 70.668 & 191.671 & 268.339 \\
\hline HTLG-100-сcс-3 & $07-2948$ & 10.4 & 49.6 & 11.8 & 41.7 & 114 & 154 & 17.334 & 82.668 & 19.667 & 69.501 & 190.004 & 256.672 \\
\hline HTLG-101-1 & $07-2949$ & 10.7 & 7.79 & 3.15 & 7.13 & 52.8 & 56 & 17.834 & 12.984 & 5.250 & 11.884 & 88.002 & 93.335 \\
\hline HTLG-101-2 & $07-2950$ & 10.9 & 8.06 & 2.3 & 7.53 & 54.4 & 58.8 & 18.167 & 13.434 & 3.833 & 12.550 & 90.668 & 98.002 \\
\hline HTLG-101-3 & $07-2951$ & 11 & 7.89 & 2.38 & 7.59 & 56.1 & 59.1 & 18.334 & 13.150 & 3.967 & 12.650 & 93.502 & 98.502 \\
\hline HTLG-101-сCC-1 & $07-2952$ & 10.5 & 14.7 & 2.81 & 15.1 & 72.3 & 82.6 & 17.500 & 24.500 & 4.683 & 25.167 & 120.502 & 137.669 \\
\hline HTLG-101-ссс-2 & $07-2953$ & 10.1 & 14.4 & 2.99 & 15 & 70.8 & 82.6 & 16.834 & 24.000 & 4.983 & 25.001 & 118.002 & 137.669 \\
\hline HTLG-101-ссс-3 & $07-2954$ & 9.81 & 14 & 3.2 & 14.5 & 68.4 & 79.1 & 16.350 & 23.334 & 5.333 & 24.167 & 114.002 & 131.836 \\
\hline HTLG-102-1 & $07-2955$ & 15.1 & 8.27 & 7.46 & 7.45 & 70.8 & 61.4 & 25.167 & 13.784 & 12.434 & 12.417 & 118.002 & 102.335 \\
\hline HTLG-102-2 & $07-2956$ & 15 & 8.13 & 6.8 & 7.43 & 71.2 & 59.8 & 25.001 & 13.550 & 11.334 & 12.384 & 118.669 & 99.669 \\
\hline HTLG-102-3 & $07-2957$ & 15 & 8.07 & 6.63 & 7.51 & 72.2 & 60 & 25.001 & 13.450 & 11.050 & 12.517 & 120.336 & 100.002 \\
\hline HTLG-102-сcс-1 & $07-2958$ & 11.9 & 4.97 & 1.83 & 6.06 & 56.3 & 51.3 & 19.834 & 8.283 & 3.050 & 10.100 & 93.835 & 85.502 \\
\hline HTLG-102-сcс-2 & $07-2959$ & 12.1 & 5.03 & 1.86 & 6.2 & 58.1 & 52 & 20.167 & 8.384 & 3.100 & 10.334 & 96.835 & 86.668 \\
\hline HTLG-102-сCC-3 & $07-2960$ & 12.3 & 5.07 & 1.95 & 6.23 & 58.4 & 53.1 & 20.500 & 8.450 & 3.250 & 10.384 & 97.335 & 88.502 \\
\hline
\end{tabular}


Using the targeted compositions for these three glasses, the PCT responses were normalized as in the earlier discussion. This yielded the results presented in Table 5-9. The trends in the quenched glasses indicate that the durability does gradually decrease as WL increases. Assuming the formation of magnetite in these glasses, this trend is consistent with previous data and reflects a changing durability response as a function of a changing glass composition. With respect to the ccc based glasses, the durability trend is opposite to that of their quenched counterparts. As WL increases for the ccc glasses, the durability increases. This is primarily a function of the transition from the nepheline, aegirine, lithium silicate phase fields experienced at $45 \%$ WL to a magnetite only phase field at 50\% WL and higher as determined by XRD analyses. The results indicate that glasses based on the measured composition of the vendor's sludge simulant, which target $50 \%$ WL or higher, produce very acceptable glasses - regardless of thermal history.

Table 5-9. Normalized PCT Response Based upon Targeted Compositions for HTLG-100, HTLG-101, and HTLG-102

\begin{tabular}{||c|c|c|c|c|c|c||}
\hline \hline WL & Glass ID & $\begin{array}{c}\text { Heat } \\
\text { Treatment }\end{array}$ & $\begin{array}{c}\text { NL B } \\
(\mathbf{g} / \mathbf{L})\end{array}$ & $\begin{array}{c}\text { NL Li } \\
(\mathbf{g} / \mathbf{L})\end{array}$ & $\begin{array}{c}\text { NL Na } \\
(\mathbf{g} / \mathbf{L})\end{array}$ & $\begin{array}{c}\text { NL Si } \\
(\mathbf{g} / \mathbf{L})\end{array}$ \\
\hline & ARM & & 0.47 & 0.60 & 0.51 & 0.28 \\
\hline & EA & ref & 18.63 & 10.02 & 13.91 & 4.13 \\
\hline 45 & HTLG-100 & cCC & 5.44 & 4.57 & 2.19 & 1.18 \\
\hline 50 & HTLG-101 & ccC & 1.71 & 1.78 & 1.24 & 0.68 \\
\hline 55 & HTLG-102 & cCC & 0.67 & 0.82 & 0.94 & 0.48 \\
\hline 45 & HTLG-100 & quenched & 0.75 & 0.78 & 0.75 & 0.44 \\
\hline 50 & HTLG-101 & quenched & 0.94 & 0.89 & 0.96 & 0.48 \\
\hline 55 & HTLG-102 & quenched & 1.08 & 0.99 & 1.17 & 0.55 \\
\hline
\end{tabular}

\subsection{Summary}

Strategic glass formulation approaches and physical improvements to DWPF's existing Joule Heated Melter (JHM) have lead to a significant increase in the waste throughput for the Savannah River Site. Although WLs have transitioned from a nominal 28\% to 38\% WL with SB3 while maintaining melt rate, additional increases are possible by coupling advanced melter technologies with glass formulation efforts. More specifically, advancements in melter technology have the potential to produce glass faster and at higher waste loadings (the two primary parameters that drive waste throughput) relative to current operations. One of these technologies is the Cold Crucible Induction Melter (CCIM) with bubbler and/or mechanical agitation.

Since any additional (sustained) improvement in waste loading and/or melt rate relative to current operations will help to reduce the life-cycle costs for the Department of Energy (DOE) at the Savannah River Site (SRS), there is interest in demonstrating the viability of CCIM technology to yield such an improvement. A demonstration is currently planned for the fall of 2007 and will be based on a DWPF waste slurry feed surrogate with a nominal operating temperature of approximately $1250^{\circ} \mathrm{C}$. The CCIM glass product will be a borosilicate glass at a high waste loading (about $50 \%$, based on a calcined oxide basis) that must meet current requirements for disposal at a Federal Repository.

Given the identification of Frit 202-A11 as the preferred frit (Peeler et al. 2007), supplemental information was needed to provide a technical basis to facilitate decisions regarding the melter demonstration. More specifically, initial testing to identify the candidate frit was based on a 
nominal sludge composition without accounting for variation that could result based on the vendor's ability to produce the targeted composition. The CCIM demonstration, which will utilize a "constant" sludge composition (targeting a nominal SB3 composition) coupled with Frit 202-A11 at 50\% waste loading (WL), will theoretically yield a constant glass composition. Although this will support programmatic objectives, there is some need to assess the potential impacts of sludge variation and/or waste loading differences on potential processing and/or product performance properties. Recognizing the low probability of producing the exact targeted nominal sludge composition and/or that targeted WL could vary from $50 \pm 2 \%$ (around the nominal 50\%), the impact of these variations must be considered on processing properties or perhaps, more importantly, on the durability of the final waste form. This is of particular importance given the nonlinear behavior previously observed between waste loading and critical processing / performance properties for this glass forming system. The results from Peeler et al. (2007) suggested that a balance between WL and processing and product performance issues may be required for this CCIM demonstration. This latter statement is based on the fact that frit development efforts were not necessarily intended to optimize this glass system nor have these efforts accounted for the variation from the intended target that is likely to occur in the composition of the waste slurry feed surrogate that is being used in the study.

Twenty-seven glasses were designed to assess the impacts of both sludge variation ( \pm 5 or $\pm 10 \%$ for the major sludge components) and WL (50 or 52\%) on the PCT response after two thermal histories (quenching and a modified ccc schedule). The PCT results of the quenched glasses (regardless of compositional view) indicate that all HTLG variability study glasses are very acceptable relative to the EA glass benchmark. More specifically, the normalized boron releases (NL [B] in g/L) range from $0.8 \mathrm{~g} / \mathrm{L}$ (for HTLG-60VS based on the measured composition) to $1.384 \mathrm{~g} / \mathrm{L}$ (HTLG-79VS based on the measured bc composition). These results can be compared to the NL [B] for the EA benchmark of $16.695 \mathrm{~g} / \mathrm{L}$. Again, the PCT results of the quenched glasses are consistent with previous data in the Frit 202-A11 - SB3 system.

The PCT results for the ccc glasses are not as straight forward. The NL [B]s for the slow cooled glasses range from $0.607 \mathrm{~g} / \mathrm{L}$ (for HTLG-57ccc based on the measured composition) to $9.42 \mathrm{~g} / \mathrm{L}$ (for HTLG-67ccc based on the measured bc compositional view). Although these glasses would be classified as acceptable relative to the EA glass benchmark, the relatively high release of the slow cooled glasses would be of concern if this system were to be implemented into DWPF. The PCT responses for those glasses in which either nepheline or both nepheline and aegirine formed upon slow cooling lead to a significant reduction in PCT response. Although the formation of aegirine has (in general) a slightly negative impact on the PCT response (as previously discussed), the formation of nepheline and aegirine is a combination that has a high probability of leading to a significant reduction in durability upon slow cooling.

With respect to the CCIM demonstration, a clear cut delineation of sludge compositions and/or targeted WLs is desirable to avoid the formation of either of these phases within the sludge composition region being evaluated. However, based on a statistical assessment of the PCT and $\mathrm{XRD}$ data, there does not appear to be a direct relationship among the sludge components and their combinations that can be used to identify a nepheline and/or aegirine primary phase field. In addition, the ability to avoid the formation of these phases within the sludge compositional region and WL interval used is not available. More specifically, based on previous data, the possibility to target higher WLs (> 50\%) to avoid any negative impacts on durability as a result of crystallization was observed when the nominal SB3 composition was utilized. However, when composition variation is applied to the sludge, there are multiple combinations of sludge components that fall within the nepheline and/or aegirine phase fields even at 52\% WL, which ultimately lead to a negative impact on the durability. Nevertheless, the PCT results do suggest 
that the probability of observing the negative impact is lower at the higher WLs (only 2 of the inner layer, 52\% WL based glasses have NL [B] $>2 \mathrm{~g} / \mathrm{L}$ after slow cooling as compared to 4 of the 9 inner layer EVs that targeted 50\% WL).

\subsection{Path Forward}

(1) Future glass formulation studies should utilize alternative process control models (even if preliminary) which may be more applicable to the higher WL regions of interest to the CCIM technology. This is not a critical statement against the current DWPF process control models (which have been very successful in controlling the DWPF process) but an acknowledgement that the compositional region of interest for these higher WL glasses may stretch the applicability of the current models.

(2) Perform specific studies to identify the compositional regions in which nepheline and/or aegirine develop and methods which frit development efforts can implement to avoid their formation.

(3) Optimize the frit selection process for the systems of interest. Although the current study did identify Frit 202-A11 as a candidate for the SB3 system, the formation of nepheline and/or aegirine in the slowly cooled glasses would be a concern for DWPF implementation. More specifically, having a system that is sensitive to sludge variation with respect to durability is a positioning which DWPF may wish to avoid. Development of more robust frits capable of handling anticipated variation would be desired - even more so, if the limiting parameter was associated with a processing constraint (i.e., $T_{L}$ or viscosity) and not a durability related issue.

\subsection{References}

ASTM. 2002. "Standard Method for Determining Chemical Durability of Nuclear Waste Glasses: The Product Consistency Test (PCT),” ASTM C-1285-2002.

Cornell, JA. 2002. Experiments with Mixtures: Designs, Models, and the Analysis of Mixture Data, John Wiley \& Sons, Inc., New York.

Edwards, TB, KG Brown, RL Postles. 2006. SME Acceptability Determination for DWPF Process Control, WSRC-TR-95-00364. Revision 5, Washington Savannah River Company, Aiken, South Carolina.

Jantzen, CM, NE Bibler, DC Beam, CL Crawford and MA Pickett. 1993. Characterization of the Defense Waste Processing Facility (DWPF) Environmental Assessment (EA) Glass Standard Reference Material, WSRC-TR-92-346, Revision 1, Westinghouse Savannah River Company, Aiken, South Carolina.

Jantzen CM, JB Pickett, KG Brown, TB Edwards, and DC Beam. 1995. Process/Product Models for the Defense Waste Processing Facility (DWPF): Part I. Predicting Glass Durability from Composition Using a Thermodynamic Hydration Energy Reaction Model (THERMO), WSRC-TR-93-672, Revision 1, Westinghouse Savannah River Company, Aiken, South Carolina. 
Peeler, DK, TB Edwards, CC Herman, IA Reamer, RJ Workman, JD Vienna, JV Crum, DE Smith, and DS Kim. 2002. Development of High Waste Loading Glasses for Advanced Melter Technologies, WSRC-TR-2002-00426, Revision 0, Westinghouse Savannah River Company, Aiken, South Carolina.

Peeler, DK. 2007. CCIM Testing: Status of Glass Formulation Activities, SRNL-PSE2007-00070, Washington Savannah River Company, Aiken, South Carolina.

Peeler, DK, KM Fox, TB Edwards, DR Best, IA Reamer, and RJ Workman. 2007. Data Packet for the Frit 202-A11 - SB3 Glass System: A Candidate for the Cold Crucible Induction Melter (CCIM) Demonstration, WSRC-STI-2007-00302, Revision 0, Washington Savannah River Company, Aiken, South Carolina.

SAS 2005. JMP Design of Experiments, Release 6, SAS Institute, Inc., Cary, North Carolina.

SAS 2006. JMP Version 6.0.2, SAS Institute, Inc., Cary, North Carolina. 


\section{Appendix A}

\section{Tables and Exhibits Supporting the Review of the Chemical Composition Measurements}


Table A1. Targeted Oxide Concentrations (as wt\%'s) for the HTL Study Glasses

\begin{tabular}{|c|c|c|c|c|c|c|c|c|c|c|c|c|c|c|c|c|c|c|}
\hline Glass ID & $\mathrm{WL}$ & $\mathrm{Al} 2 \mathrm{O} 3$ & $\mathrm{~B} 2 \mathrm{O} 3$ & $\mathrm{CaO}$ & Cr2O3 & $\mathrm{CuO}$ & $\mathrm{Fe} 2 \mathrm{O} 3$ & $\mathrm{~K} 2 \mathrm{O}$ & $\mathrm{Li} 2 \mathrm{O}$ & $\mathrm{MgO}$ & $\mathrm{MnO}$ & $\mathrm{Na} 2 \mathrm{O}$ & $\mathrm{NiO}$ & $\mathrm{SO} 4$ & $\mathrm{SiO} 2$ & $\mathrm{TiO} 2$ & $\mathrm{ZrO} 2$ & Total \\
\hline HTLG-53 & 50 & 7.77 & 4.50 & 1.41 & 0.13 & 0.05 & 20.52 & 0.22 & 3.00 & 2.16 & 3.19 & 12.38 & 0.84 & 0.47 & 43.22 & 0.04 & 0.12 & 100.00 \\
\hline HTLG-54 & 50 & 7.61 & 4.50 & 1.91 & 0.06 & 0.03 & 18.01 & 0.12 & 3.00 & 2.16 & 3.19 & 14.79 & 1.09 & 0.25 & 43.22 & 0.02 & 0.06 & 100.00 \\
\hline HTLG-55 & 50 & 9.30 & 4.50 & 1.91 & 0.13 & 0.05 & 16.79 & 0.22 & 3.00 & 2.16 & 3.19 & 14.58 & 0.84 & 0.47 & 42.72 & 0.04 & 0.12 & 100.00 \\
\hline HTLG-56 & 50 & 7.61 & 4.50 & 1.91 & 0.13 & 0.05 & 18.76 & 0.22 & 3.00 & 1.66 & 3.19 & 14.79 & 0.84 & 0.47 & 42.72 & 0.04 & 0.12 & 100.00 \\
\hline HTLG-57 & 50 & 9.30 & 4.50 & 1.41 & 0.06 & 0.03 & 20.24 & 0.12 & 3.00 & 1.66 & 3.19 & 12.38 & 1.09 & 0.25 & 42.72 & 0.02 & 0.06 & 100.00 \\
\hline HTLG-58 & 50 & 7.61 & 4.50 & 1.41 & 0.13 & 0.05 & 17.80 & 0.22 & 3.00 & 2.16 & 3.90 & 14.79 & 1.09 & 0.47 & 42.72 & 0.04 & 0.12 & 100.00 \\
\hline HTLG-59 & 50 & 7.61 & 4.50 & 1.91 & 0.06 & 0.03 & 20.47 & 0.12 & 3.00 & 2.16 & 3.90 & 12.38 & 0.84 & 0.25 & 42.72 & 0.02 & 0.06 & 100.00 \\
\hline HTLG-60 & 50 & 9.30 & 4.50 & 1.91 & 0.13 & 0.05 & 18.03 & 0.22 & 3.00 & 1.66 & 3.90 & 12.38 & 1.09 & 0.47 & 43.22 & 0.04 & 0.12 & 100.00 \\
\hline HTLG-61 & 50 & 9.30 & 4.50 & 1.41 & 0.06 & 0.03 & 16.86 & 0.12 & 3.00 & 1.66 & 3.90 & 14.79 & 0.84 & 0.25 & 43.22 & 0.02 & 0.06 & 100.00 \\
\hline HTLG-62 & 50 & 8.05 & 4.50 & 1.41 & 0.13 & 0.05 & 18.88 & 0.22 & 3.00 & 1.66 & 3.72 & 14.19 & 0.84 & 0.47 & 42.72 & 0.04 & 0.12 & 100.00 \\
\hline HTLG-63 & 50 & 8.87 & 4.50 & 1.41 & 0.06 & 0.03 & 19.58 & 0.12 & 3.00 & 2.16 & 3.37 & 13.03 & 0.84 & 0.25 & 42.72 & 0.02 & 0.06 & 100.00 \\
\hline HTLG-64 & 50 & 8.03 & 4.50 & 1.91 & 0.06 & 0.03 & 18.77 & 0.12 & 3.00 & 1.66 & 3.37 & 14.19 & 0.84 & 0.25 & 43.22 & 0.02 & 0.06 & 100.00 \\
\hline HTLG-65 & 50 & 8.87 & 4.50 & 1.86 & 0.13 & 0.05 & 17.72 & 0.22 & 3.00 & 1.66 & 3.37 & 14.19 & 1.09 & 0.47 & 42.72 & 0.04 & 0.12 & 100.00 \\
\hline HTLG-66 & 50 & 8.03 & 4.50 & 1.41 & 0.13 & 0.05 & 18.02 & 0.22 & 3.00 & 2.16 & 3.37 & 14.19 & 1.09 & 0.47 & 43.22 & 0.04 & 0.12 & 100.00 \\
\hline HTLG-67 & 50 & 8.87 & 4.50 & 1.91 & 0.13 & 0.05 & 17.77 & 0.22 & 3.00 & 2.16 & 3.72 & 12.98 & 0.84 & 0.47 & 43.22 & 0.04 & 0.12 & 100.00 \\
\hline HTLG-68 & 50 & 8.87 & 4.50 & 1.41 & 0.06 & 0.03 & 19.02 & 0.12 & 3.00 & 1.66 & 3.72 & 12.98 & 1.09 & 0.25 & 43.22 & 0.02 & 0.06 & 100.00 \\
\hline HTLG-69 & 50 & 8.03 & 4.50 & 1.91 & 0.13 & 0.05 & 19.58 & 0.22 & 3.00 & 1.80 & 3.37 & 12.98 & 1.09 & 0.47 & 42.72 & 0.04 & 0.12 & 100.00 \\
\hline HTLG-70 & 50 & 8.03 & 4.50 & 1.91 & 0.06 & 0.03 & 18.16 & 0.12 & 3.00 & 2.16 & 3.72 & 14.19 & 1.09 & 0.25 & 42.72 & 0.02 & 0.06 & 100.00 \\
\hline HTLG-71 & 52 & 8.38 & 4.32 & 1.46 & 0.13 & 0.05 & 19.64 & 0.23 & 2.88 & 1.72 & 3.87 & 14.64 & 0.87 & 0.49 & 41.15 & 0.04 & 0.12 & 100.00 \\
\hline HTLG-72 & 52 & 9.23 & 4.32 & 1.46 & 0.07 & 0.03 & 20.37 & 0.12 & 2.88 & 2.24 & 3.50 & 13.43 & 0.87 & 0.26 & 41.15 & 0.02 & 0.06 & 100.00 \\
\hline HTLG-73 & 52 & 8.35 & 4.32 & 1.98 & 0.07 & 0.03 & 19.52 & 0.12 & 2.88 & 1.72 & 3.50 & 14.64 & 0.87 & 0.26 & 41.67 & 0.02 & 0.06 & 100.00 \\
\hline HTLG-74 & 52 & 9.23 & 4.32 & 1.93 & 0.13 & 0.05 & 18.43 & 0.23 & 2.88 & 1.72 & 3.50 & 14.64 & 1.13 & 0.49 & 41.15 & 0.04 & 0.12 & 100.00 \\
\hline HTLG-75 & 52 & 8.35 & 4.32 & 1.46 & 0.13 & 0.05 & 18.74 & 0.23 & 2.88 & 2.24 & 3.50 & 14.64 & 1.13 & 0.49 & 41.67 & 0.04 & 0.12 & 100.00 \\
\hline HTLG-76 & 52 & 9.23 & 4.32 & 1.98 & 0.13 & 0.05 & 18.48 & 0.23 & 2.88 & 2.24 & 3.87 & 13.38 & 0.87 & 0.49 & 41.67 & 0.04 & 0.12 & 100.00 \\
\hline HTLG-77 & 52 & 9.23 & 4.32 & 1.46 & 0.07 & 0.03 & 19.78 & 0.12 & 2.88 & 1.72 & 3.87 & 13.38 & 1.13 & 0.26 & 41.67 & 0.02 & 0.06 & 100.00 \\
\hline HTLG-78 & 52 & 8.35 & 4.32 & 1.98 & 0.13 & 0.05 & 20.37 & 0.23 & 2.88 & 1.87 & 3.50 & 13.38 & 1.13 & 0.49 & 41.15 & 0.04 & 0.12 & 100.00 \\
\hline HTLG-79 & 52 & 8.35 & 4.32 & 1.98 & 0.07 & 0.03 & 18.89 & 0.12 & 2.88 & 2.24 & 3.87 & 14.64 & 1.13 & 0.26 & 41.15 & 0.02 & 0.06 & 100.00 \\
\hline
\end{tabular}


Table A2. Measured Elemental Concentrations (wt\%) for Series "P" Samples Prepared Using Lithium Metaborate

\begin{tabular}{|c|c|c|c|c|c|c|c|c|c|c|c|c|c|c|c|c|}
\hline $\begin{array}{l}\text { Glass ID } \\
\end{array}$ & Block & Sub-Block & $\begin{array}{l}\text { Sequence } \\
\end{array}$ & $\begin{array}{l}\text { Lab ID } \\
\end{array}$ & $\begin{array}{l}\mathrm{Al}(\mathrm{wt} \%) \\
\end{array}$ & $\begin{array}{l}\mathrm{Ca}(\mathrm{wt} \%) \\
\end{array}$ & $\begin{array}{l}\mathrm{Cr}(\mathrm{wt} \%) \\
\end{array}$ & $\begin{array}{l}\mathrm{Cu}(\mathrm{wt} \%) \\
\end{array}$ & $\overline{~ K ~(w t \%) ~}$ & Mg (wt\%) & Mn (wt\%) & $\begin{array}{l}\mathrm{Na}(\mathrm{wt} \%) \\
\end{array}$ & Ni (wt\%) & S (wt\%) & $\begin{array}{l}\text { Ti (wt\%) } \\
\end{array}$ & $\overline{Z Z r(w t \%)}$ \\
\hline Batch 1 & 1 & 1 & 1 & BCHLM1111 & 2.65 & 0.873 & 0.079 & 0.314 & 2.54 & 0.830 & 1.40 & 7.24 & 0.529 & 0.022 & 0.372 & 0.066 \\
\hline $\begin{array}{l}\text { HTL-57VS } \\
\end{array}$ & 1 & 1 & 2 & P06LM21 & 5.02 & 1.04 & 0.039 & 0.024 & 0.088 & 0.941 & 2.52 & 9.27 & 0.717 & 0.085 & 0.017 & 0.049 \\
\hline HTLG-66VS & 1 & 1 & 3 & $\begin{array}{l}\text { P11LM11 } \\
\end{array}$ & 4.77 & 1.42 & 0.073 & 0.042 & 0.207 & 0.916 & 2.62 & 10.5 & 0.749 & 0.147 & 0.027 & 0.079 \\
\hline $\begin{array}{l}\text { HTLG-65VS } \\
\end{array}$ & 1 & 1 & 4 & $\begin{array}{l}\text { P08LM21 } \\
\end{array}$ & 4.35 & 1.05 & 0.076 & 0.042 & 0.179 & 1.22 & 2.71 & 10.5 & 0.773 & 0.146 & 0.029 & 0.087 \\
\hline $\begin{array}{l}\text { HTLG-64VS } \\
\end{array}$ & 1 & 1 & 5 & P05LM11 & 4.35 & 1.47 & 0.047 & 0.024 & 0.097 & 0.934 & 2.65 & 10.6 & 0.597 & 0.088 & 0.016 & 0.054 \\
\hline $\begin{array}{l}\text { HTLG-60VS } \\
\end{array}$ & 1 & 1 & 6 & $\begin{array}{l}\text { P01LM21 } \\
\end{array}$ & 5.06 & 1.48 & 0.079 & 0.047 & 0.205 & 0.953 & 3.03 & 9.42 & 0.803 & 0.149 & 0.027 & 0.086 \\
\hline HTLG-61VS & 1 & 1 & 7 & P03LM21 & 5.04 & 1.07 & 0.045 & 0.023 & 0.093 & 0.942 & 3.06 & 11.0 & 0.606 & 0.089 & 0.015 & 0.049 \\
\hline HTLG-61VS & 1 & 1 & 8 & $\begin{array}{l}\text { P03LM11 } \\
\end{array}$ & 5.02 & 1.07 & 0.044 & 0.022 & 0.094 & 0.927 & 3.00 & 11.0 & 0.601 & 0.087 & 0.015 & 0.046 \\
\hline Batch 1 & 1 & 1 & 9 & BCHLM1112 & 2.69 & 0.867 & 0.080 & 0.313 & 2.53 & 0.833 & 1.39 & 7.39 & 0.529 & 0.026 & 0.369 & 0.065 \\
\hline $\begin{array}{l}\text { HTLG-64VS } \\
\end{array}$ & 1 & 1 & 10 & P05LM21 & 4.37 & 1.48 & 0.046 & 0.023 & 0.098 & 0.915 & 2.52 & 10.7 & 0.579 & 0.089 & 0.016 & 0.049 \\
\hline $\begin{array}{l}\text { HTL-63VS } \\
\end{array}$ & 1 & 1 & 11 & $\begin{array}{l}\text { P13LM21 } \\
\end{array}$ & 4.85 & 1.09 & 0.038 & 0.023 & 0.098 & 1.17 & 2.54 & 9.93 & 0.543 & 0.088 & 0.016 & 0.052 \\
\hline HTLG-60VS & 1 & 1 & 12 & $\begin{array}{l}\text { P01LM11 } \\
\end{array}$ & 5.18 & 1.53 & 0.078 & 0.042 & 0.199 & 0.934 & 3.02 & 10.2 & 0.755 & 0.154 & 0.026 & 0.083 \\
\hline $\begin{array}{l}\text { HTL-57VS } \\
\end{array}$ & 1 & 1 & 13 & $\begin{array}{l}\text { P06LM11 } \\
\end{array}$ & 5.08 & 1.08 & 0.041 & 0.023 & 0.093 & 0.941 & 2.37 & 9.53 & 0.725 & 0.090 & 0.016 & 0.049 \\
\hline HTLG-66VS & 1 & 1 & 14 & P11LM21 & 4.94 & 1.49 & 0.072 & 0.041 & 0.207 & 0.903 & 2.49 & 11.0 & 0.735 & 0.147 & 0.027 & 0.077 \\
\hline HTLG-65VS & 1 & 1 & 15 & $\begin{array}{l}\text { P08LM11 } \\
\end{array}$ & 4.41 & 1.09 & 0.074 & 0.042 & 0.185 & 1.21 & 2.52 & 10.7 & 0.744 & 0.155 & 0.028 & 0.086 \\
\hline HTLG-63VS & 1 & 1 & 16 & P13LM11 & 4.82 & 1.08 & 0.039 & 0.022 & 0.089 & 1.19 & 2.50 & 10.1 & 0.570 & 0.086 & 0.016 & 0.048 \\
\hline Batch 1 & 1 & 1 & 17 & BCHLM1113 & 2.69 & 0.851 & 0.081 & 0.310 & 2.50 & 0.852 & 1.32 & 7.51 & 0.541 & 0.024 & 0.371 & 0.065 \\
\hline Batch 1 & 1 & 2 & 1 & BCHLM1121 & 2.63 & 0.865 & 0.077 & 0.310 & 2.50 & 0.845 & 1.35 & 7.18 & 0.535 & 0.022 & 0.369 & 0.064 \\
\hline $\begin{array}{l}\text { HTLG-66VS } \\
\end{array}$ & 1 & 2 & 2 & P11LM22 & 4.76 & 1.41 & 0.070 & 0.040 & 0.211 & 0.908 & 2.67 & 10.3 & 0.744 & 0.147 & 0.025 & 0.076 \\
\hline HTLG-64VS & 1 & 2 & 3 & $\begin{array}{l}\text { P05LM22 } \\
\end{array}$ & 4.35 & 1.47 & 0.043 & 0.022 & 0.103 & 0.925 & 2.64 & 10.4 & 0.589 & 0.088 & 0.013 & 0.048 \\
\hline $\begin{array}{l}\text { HTLG-65VS } \\
\end{array}$ & 1 & 2 & 4 & $\begin{array}{l}\text { P08LM22 } \\
\end{array}$ & 4.31 & 1.05 & 0.073 & 0.041 & 0.182 & 1.23 & 2.66 & 10.3 & 0.781 & 0.154 & 0.026 & 0.086 \\
\hline $\begin{array}{l}\text { HTL-57VS } \\
\end{array}$ & 1 & 2 & 5 & $\begin{array}{l}\text { P06LM12 } \\
\end{array}$ & 4.98 & 1.06 & 0.037 & 0.022 & 0.099 & 0.927 & 2.49 & 9.13 & 0.719 & 0.089 & 0.014 & 0.047 \\
\hline HTLG-61VS & 1 & 2 & 6 & P03LM12 & 4.98 & 1.07 & 0.042 & 0.022 & 0.101 & 0.941 & 3.06 & 10.9 & 0.611 & 0.092 & 0.013 & 0.045 \\
\hline HTLG-57VS & 1 & 2 & 7 & $\begin{array}{l}\text { P06LM22 } \\
\end{array}$ & 5.04 & 1.08 & 0.036 & 0.023 & 0.092 & 0.951 & 2.45 & 9.29 & 0.725 & 0.088 & 0.014 & 0.047 \\
\hline HTLG-61VS & 1 & 2 & 8 & $\begin{array}{l}\text { P03LM22 } \\
\end{array}$ & 4.96 & 1.06 & 0.043 & 0.022 & 0.096 & 0.952 & 3.06 & 10.8 & 0.617 & 0.090 & 0.013 & 0.047 \\
\hline Batch 1 & 1 & 2 & 9 & BCHLM1123 & 2.62 & 0.866 & 0.079 & 0.313 & 2.52 & 0.861 & 1.31 & 7.09 & 0.548 & 0.024 & 0.374 & 0.064 \\
\hline HTLG-63VS & 1 & 2 & 10 & P13LM22 & 4.81 & 1.07 & 0.036 & 0.022 & 0.101 & 1.20 & 2.68 & 9.75 & 0.559 & 0.093 & 0.015 & 0.051 \\
\hline $\begin{array}{l}\text { HTLG-60VS } \\
\end{array}$ & 1 & 2 & 11 & $\begin{array}{l}\text { P01LM12 } \\
\end{array}$ & 4.97 & 1.47 & 0.075 & 0.041 & 0.201 & 0.937 & 3.02 & 9.67 & 0.751 & 0.147 & 0.024 & 0.081 \\
\hline HTLG-64VS & 1 & 2 & 12 & $\begin{array}{l}\text { P05LM12 } \\
\end{array}$ & 4.30 & 1.46 & 0.044 & 0.023 & 0.099 & 0.941 & 2.65 & 10.5 & 0.597 & 0.091 & 0.014 & 0.053 \\
\hline HTLG-60VS & 1 & 2 & 13 & $\begin{array}{l}\text { P01LM22 } \\
\end{array}$ & 4.95 & 1.44 & 0.078 & 0.047 & 0.212 & 0.967 & 3.12 & 9.18 & 0.813 & 0.151 & 0.025 & 0.084 \\
\hline HTLG-63VS & 1 & 2 & 14 & P13LM12 & 4.72 & 1.05 & 0.037 & 0.022 & 0.094 & 1.22 & 2.66 & 9.68 & 0.577 & 0.092 & 0.014 & 0.047 \\
\hline HTLG-65VS & 1 & 2 & 15 & P08LM12 & 4.35 & 1.07 & 0.072 & 0.042 & 0.189 & 1.23 & 2.61 & 10.5 & 0.751 & 0.151 & 0.026 & 0.085 \\
\hline HTLG-66VS & 1 & 2 & 16 & $\begin{array}{l}\text { P11LM12 } \\
\end{array}$ & 4.80 & 1.45 & 0.071 & 0.042 & 0.212 & 0.934 & 2.51 & 10.6 & 0.759 & 0.149 & 0.025 & 0.079 \\
\hline Batch 1 & 1 & 2 & 17 & BCHLM1123 & 2.64 & 0.887 & 0.079 & 0.324 & 2.54 & 0.857 & 1.30 & 7.29 & 0.543 & 0.023 & 0.380 & 0.065 \\
\hline Batch 1 & 2 & 1 & 1 & BCHLM1211 & 2.63 & 0.855 & 0.077 & 0.310 & 2.45 & 0.828 & 1.29 & 7.17 & 0.526 & 0.024 & 0.368 & 0.064 \\
\hline HTLG-62VS & 2 & 1 & 2 & $\begin{array}{l}\text { P07LM21 } \\
\end{array}$ & 4.29 & 1.06 & 0.075 & 0.043 & 0.189 & 0.942 & 2.82 & 10.2 & 0.604 & $\begin{array}{ll}0.149 \\
\end{array}$ & 0.025 & 0.085 \\
\hline $\begin{array}{l}\text { HTLG-59VS } \\
\end{array}$ & 2 & 1 & 3 & $\begin{array}{l}\text { P14LM21 } \\
\end{array}$ & 4.09 & 1.46 & 0.038 & 0.025 & 0.102 & 1.15 & 2.93 & 8.96 & 0.558 & 0.090 & 0.014 & 0.051 \\
\hline HTLG-58VS & 2 & 1 & 4 & $\begin{array}{l}\text { P09LM21 } \\
\end{array}$ & 4.08 & 1.06 & 0.076 & 0.044 & 0.179 & 1.24 & 2.93 & 10.5 & 0.797 & 0.166 & 0.026 & 0.082 \\
\hline $\begin{array}{l}\text { HTLG-56VS } \\
\end{array}$ & 2 & 1 & 5 & P12LM11 & 4.07 & 1.46 & 0.077 & 0.044 & 0.197 & 0.932 & 2.33 & 10.6 & 0.589 & 0.153 & 0.026 & 0.085 \\
\hline $\begin{array}{l}\text { HTLG-55VS } \\
\end{array}$ & 2 & 1 & 6 & $\begin{array}{l}\text { P04LN11 } \\
\end{array}$ & 5.01 & 1.46 & 0.097 & 0.046 & 0.191 & 1.15 & 2.36 & 10.3 & 0.589 & 0.147 & 0.025 & 0.090 \\
\hline HTLG-53VS & 2 & 1 & 7 & $\begin{array}{l}\text { P02LM11 } \\
\end{array}$ & 4.11 & 1.05 & 0.062 & 0.146 & $\begin{array}{ll}0.179 \\
\end{array}$ & 1.19 & 2.40 & 8.78 & 0.575 & 0.144 & 0.027 & 0.089 \\
\hline HTLG-56VS & 2 & 1 & 8 & $\begin{array}{l}\text { P12LM21 } \\
\end{array}$ & 4.09 & 1.46 & 0.076 & 0.043 & 0.213 & 0.903 & 2.37 & 10.6 & 0.561 & 0.147 & 0.024 & 0.084 \\
\hline Batch 1 & 2 & 1 & 9 & BCHLM1212 & 2.61 & 0.858 & 0.077 & 0.314 & 2.48 & 0.840 & 1.29 & 7.00 & 0.526 & 0.019 & 0.369 & 0.065 \\
\hline HTLG-54VS & 2 & 1 & 10 & $\begin{array}{l}\text { P10LM21 } \\
\end{array}$ & 4.06 & 1.45 & 0.040 & 0.036 & 0.103 & 1.15 & 2.34 & 10.5 & 0.739 & 0.087 & 0.017 & 0.046 \\
\hline $\begin{array}{l}\text { HTLG-54VS } \\
\end{array}$ & 2 & 1 & 11 & $\begin{array}{l}\text { P10LM11 } \\
\end{array}$ & 4.04 & 1.43 & 0.042 & 0.036 & 0.091 & 1.20 & 2.43 & 10.4 & 0.776 & 0.085 & 0.018 & 0.047 \\
\hline HTLG-59VS & 2 & 1 & 12 & $\begin{array}{l}\text { P14LM11 } \\
\end{array}$ & 4.12 & 1.47 & 0.038 & 0.025 & 0.102 & 1.17 & 3.02 & 8.97 & 0.570 & 0.084 & 0.015 & 0.046 \\
\hline
\end{tabular}


Table A2. Measured Elemental Concentrations (wt\%) for Series "P" Samples Prepared Using Lithium Metaborate

\begin{tabular}{|c|c|c|c|c|c|c|c|c|c|c|c|c|c|c|c|c|}
\hline $\begin{array}{l}\text { Glass ID } \\
\end{array}$ & Block & S Sub-Block & $\begin{array}{l}\text { Sequence } \\
\end{array}$ & $\begin{array}{ll}\text { Lab ID } \\
\end{array}$ & $\overline{\mathrm{Al} \text { (wt\%) }}$ & "Ca (wt\%) & $\bar{~} \mathrm{Cr}$ (wt\%) & " Cu (wt\%) & 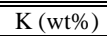 & "Mg (wt\%) & " Mn (wt\%) & $\begin{array}{l}\mathrm{Na} \text { (wt\%) } \\
\end{array}$ & " Ni (wt\%) & 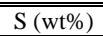 & Ti (wt\%) & $\overline{~ Z r ~(w t \%) ~}$ \\
\hline HTLG-55VS & 2 & 1 & 13 & $\begin{array}{l}\text { P04LM21 } \\
\end{array}$ & 5.00 & 1.45 & 0.092 & 0.045 & 0.199 & 1.14 & 2.49 & 10.3 & 0.564 & 0.149 & 0.026 & 0.086 \\
\hline HTLG-62VS & 2 & 1 & 14 & P07LM11 & 4.39 & 1.05 & 0.075 & 0.047 & 0.230 & 0.923 & 3.00 & 10.3 & 0.643 & 0.148 & 0.024 & 0.086 \\
\hline HTLG-58VS & 2 & 1 & 15 & $\begin{array}{l}\text { P09LM11 } \\
\end{array}$ & 4.10 & 1.05 & 0.073 & 0.044 & 0.208 & 1.15 & 3.09 & 10.5 & 0.739 & 0.143 & 0.025 & 0.080 \\
\hline HTLG-53VS & 2 & 1 & 16 & P02LM21 & 4.13 & 1.04 & 0.060 & 0.147 & 0.197 & 1.15 & 2.52 & 8.85 & 0.551 & 0.144 & 0.027 & 0.090 \\
\hline Batch 1 & 2 & 1 & 17 & BCHLM1213 & 2.61 & 0.884 & 0.073 & 0.310 & 2.52 & 0.821 & 1.34 & 6.96 & 0.508 & 0.025 & 0.369 & 0.065 \\
\hline Batch 1 & 2 & 2 & 1 & BCHLM1221 & 2.61 & 0.882 & 0.076 & 0.318 & 2.51 & 0.824 & 1.38 & 7.07 & 0.522 & 0.016 & 0.370 & 0.065 \\
\hline HTLG-62VS & 2 & 2 & 2 & $\begin{array}{l}\text { P07LM22 } \\
\end{array}$ & 4.33 & 1.06 & 0.071 & 0.041 & 0.193 & 0.925 & 2.98 & 10.4 & 0.588 & 0.141 & 0.025 & 0.084 \\
\hline HTLG-58VS & 2 & 2 & 3 & $\begin{array}{l}\text { P09LM22 } \\
\end{array}$ & 4.11 & 1.06 & 0.076 & 0.043 & 0.185 & 1.21 & 3.20 & 10.7 & 0.787 & 0.163 & 0.027 & 0.082 \\
\hline HTLG-58VS & 2 & 2 & 4 & P09LM12 & 4.14 & 1.06 & 0.072 & 0.042 & 0.207 & 1.14 & 3.17 & 10.9 & 0.739 & 0.142 & 0.025 & 0.079 \\
\hline HTLG-56VS & 2 & 2 & 5 & $\begin{array}{l}\text { P12LM12 } \\
\end{array}$ & 4.09 & 1.44 & 0.076 & 0.043 & 0.205 & 0.923 & 2.66 & 10.7 & 0.586 & 0.150 & 0.026 & 0.085 \\
\hline HTLG-55VS & 2 & 2 & 6 & P04LM22 & 5.04 & 1.44 & 0.092 & 0.044 & 0.200 & 1.14 & 2.70 & 10.5 & 0.563 & 0.143 & 0.026 & 0.086 \\
\hline HTLG-59VS & 2 & 2 & 7 & $\begin{array}{l}\text { P14LM12 } \\
\end{array}$ & 4.18 & 1.48 & 0.037 & 0.024 & 0.105 & 1.15 & 3.25 & 9.28 & 0.566 & 0.088 & 0.015 & 0.046 \\
\hline HTLG-54VS & 2 & 2 & 8 & P10LM22 & 4.14 & 1.46 & 0.039 & 0.036 & 0.108 & 1.12 & 2.60 & 10.8 & 0.725 & 0.082 & 0.017 & 0.045 \\
\hline Batch 1 & 2 & 2 & 9 & BCHLM1222 & 2.65 & 0.874 & 0.075 & 0.310 & 2.50 & 0.805 & 1.41 & 7.15 & 0.513 & 0.017 & 0.366 & 0.063 \\
\hline HTLG-53VS & 2 & 2 & 10 & $\begin{array}{l}\text { P02LM22 } \\
\end{array}$ & 4.22 & 1.06 & 0.059 & 0.145 & 0.199 & 1.15 & 2.64 & 9.22 & 0.553 & 0.140 & 0.027 & 0.090 \\
\hline HTLG-56VS & 2 & 2 & 11 & P12LM22 & 4.14 & 1.45 & 0.075 & 0.042 & 0.220 & 0.878 & 2.71 & 10.9 & 0.553 & 0.138 & 0.025 & 0.083 \\
\hline HTLG-55VS & 2 & 2 & 12 & $\begin{array}{l}\text { P04LM12 } \\
\end{array}$ & 5.11 & 1.45 & 0.096 & 0.045 & 0.199 & 1.13 & 2.79 & 10.6 & 0.583 & 0.139 & 0.026 & 0.091 \\
\hline HTLG-59VS & 2 & 2 & 13 & P14LM22 & 4.15 & 1.46 & 0.037 & 0.024 & 0.105 & 1.13 & 3.31 & 9.27 & 0.546 & 0.080 & 0.014 & 0.051 \\
\hline HTLG-54VS & 2 & 2 & 14 & $\begin{array}{l}\text { P10LM12 } \\
\end{array}$ & 4.17 & 1.46 & 0.041 & 0.035 & 0.092 & 1.18 & 2.71 & 10.9 & 0.765 & 0.083 & 0.018 & 0.047 \\
\hline HTLG-53VS & 2 & 2 & 15 & $\begin{array}{l}\text { P02LM12 } \\
\end{array}$ & 4.21 & 1.06 & 0.061 & 0.145 & 0.181 & 1.17 & 2.74 & 9.21 & 0.563 & 0.138 & 0.027 & 0.087 \\
\hline HTLG-62VS & 2 & 2 & 16 & $\begin{array}{l}\text { P07LM12 } \\
\end{array}$ & 4.46 & 1.06 & 0.074 & 0.045 & 0.227 & 0.918 & 3.23 & 10.7 & 0.637 & 0.139 & 0.024 & 0.085 \\
\hline Batch 1 & 2 & 2 & 17 & BCHLM1223 & 2.68 & 0.865 & 0.075 & 0.310 & 2.47 & 0.827 & 1.48 & 7.19 & 0.519 & 0.018 & 0.368 & 0.064 \\
\hline
\end{tabular}




\section{Table A3. Measured Elemental Concentrations (wt\%) for Series "P" Samples Prepared Using Peroxide Fusion}

\begin{tabular}{|c|c|c|c|c|c|c|c|c|}
\hline Glass ID & Block & Sub-Block & Sequence & Lab ID & B (wt\%) & Fe (wt\%) & $\mathrm{Li}(\mathrm{wt} \%)$ & Si (wt\%) \\
\hline Batch 1 & 1 & 1 & 1 & BCHPF1111 & 2.54 & 8.96 & 2.06 & 22.3 \\
\hline HTLG-65VS & 1 & 1 & 2 & P08PF11 & 1.37 & 11.6 & 1.39 & 19.8 \\
\hline HTLG-62VS & 1 & 1 & 3 & P07PF11 & 1.37 & 12.2 & 1.38 & 19.6 \\
\hline HTLG-63VS & 1 & 1 & 4 & P13PF21 & 1.38 & 12.9 & 1.35 & 19.4 \\
\hline HTLG-65VS & 1 & 1 & 5 & P08PF21 & 1.35 & 11.3 & 1.38 & 19.6 \\
\hline HTLG-54VS & 1 & 1 & 6 & P10PF11 & 1.35 & 11.4 & 1.35 & 19.5 \\
\hline HTLG-61VS & 1 & 1 & 7 & P03PF11 & 1.35 & 10.7 & 1.36 & 19.5 \\
\hline HTLG-56VS & 1 & 1 & 8 & P12PF11 & 1.34 & 11.9 & 1.45 & 19.2 \\
\hline Batch 1 & 1 & 1 & 9 & BCHPF1112 & 2.37 & 8.56 & 2.04 & 22.1 \\
\hline HTLG-62VS & 1 & 1 & 10 & P07PF21 & 1.42 & 12.1 & 1.38 & 19.5 \\
\hline HTLG-60VS & 1 & 1 & 11 & P01PF21 & 1.43 & 11.8 & 1.38 & 19.9 \\
\hline HTLG-60VS & 1 & 1 & 12 & P01PF11 & 1.41 & 11.7 & 1.37 & 19.8 \\
\hline HTLG-56VS & 1 & 1 & 13 & P12PF21 & 1.37 & 11.9 & 1.48 & 19.5 \\
\hline HTLG-63VS & 1 & 1 & 14 & P13PF11 & 1.37 & 12.4 & 1.39 & 19.5 \\
\hline HTLG-54VS & 1 & 1 & 15 & P10PF21 & 1.4 & 11.9 & 1.38 & 20.1 \\
\hline HTLG-61VS & 1 & 1 & 16 & P03PF21 & 1.4 & 11.3 & 1.37 & 20 \\
\hline Batch 1 & 1 & 1 & 17 & BCHPF1113 & 2.39 & 8.54 & 2.05 & 22.3 \\
\hline Batch 1 & 1 & 2 & 1 & BCHPF1121 & 2.56 & 9.31 & 2.05 & 23 \\
\hline HTLG-62VS & 1 & 2 & 2 & P07PF12 & 1.44 & 12.7 & 1.38 & 20.1 \\
\hline HTLG-54VS & 1 & 2 & 3 & P10PF22 & 1.42 & 12.4 & 1.36 & 20.2 \\
\hline HTLG-56VS & 1 & 2 & 4 & P12PF12 & 1.41 & 12.7 & 1.46 & 19.9 \\
\hline HTLG-54VS & 1 & 2 & 5 & P10PF12 & 1.39 & 12.3 & 1.37 & 20.1 \\
\hline HTLG-65VS & 1 & 2 & 6 & P08PF12 & 1.39 & 11.9 & 1.4 & 20.2 \\
\hline HTLG-60VS & 1 & 2 & 7 & P01PF22 & 1.46 & 12.9 & 1.4 & 20.7 \\
\hline HTLG-63VS & 1 & 2 & 8 & P13PF22 & 1.41 & 13.7 & 1.37 & 20.1 \\
\hline Batch 1 & 1 & 2 & 9 & BCHPF1123 & 2.45 & 9.16 & 2.05 & 23 \\
\hline HTLG-63VS & 1 & 2 & 10 & P13PF12 & 1.4 & 12.5 & 1.39 & 19.9 \\
\hline HTLG-65VS & 1 & 2 & 11 & P08PF22 & 1.4 & 11.9 & 1.4 & 20.2 \\
\hline HTLG-60VS & 1 & 2 & 12 & P01PF12 & 1.45 & 12.6 & 1.39 & 20.6 \\
\hline HTLG-56VS & 1 & 2 & 13 & P12PF22 & 1.38 & 12.5 & 1.48 & 20.1 \\
\hline HTLG-62VS & 1 & 2 & 14 & P07PF22 & 1.4 & 12.9 & 1.38 & 20.1 \\
\hline HTLG-61VS & 1 & 2 & 15 & P03PF22 & 1.39 & 11.7 & 1.36 & 20.3 \\
\hline HTLG-61VS & 1 & 2 & 16 & P03PF12 & 1.37 & 11.3 & 1.37 & 20.1 \\
\hline Batch 1 & 1 & 2 & 17 & BCHPF1123 & 2.39 & 8.86 & 2.05 & 22.4 \\
\hline Batch 1 & 2 & 1 & 1 & BCHPF1211 & 2.56 & 8.98 & 2.05 & 23 \\
\hline HTLG-66VS & 2 & 1 & 2 & P11PF21 & 1.45 & 12.1 & 1.35 & 19.9 \\
\hline HTLG-66VS & 2 & 1 & 3 & P11PF11 & 1.38 & 11.6 & 1.35 & 19.6 \\
\hline HTLG-59VS & 2 & 1 & 4 & P14PF21 & 1.4 & 13.8 & 1.37 & 20 \\
\hline HTLG-57VS & 2 & 1 & 5 & P06PF11 & 1.4 & 13.4 & 1.38 & 20 \\
\hline HTLG-64VS & 2 & 1 & 6 & P05PF11 & 1.39 & 12.8 & 1.37 & 20.3 \\
\hline HTLG-59VS & 2 & 1 & 7 & P14PF11 & 1.4 & 14.1 & 1.36 & 20.1 \\
\hline HTLG-58VS & 2 & 1 & 8 & P09PF11 & 1.39 & 12.1 & 1.35 & 20.1 \\
\hline Batch 1 & 2 & 1 & 9 & BCHPF1212 & 2.44 & 9.09 & 2.05 & 22.8 \\
\hline HTLG-64VS & 2 & 1 & 10 & P05PF21 & 1.36 & 12 & 1.37 & 20.1 \\
\hline HTLG-55VS & 2 & 1 & 11 & P04PF21 & 1.42 & 11.9 & 1.36 & 20.1 \\
\hline HTLG-53VS & 2 & 1 & 12 & P02PF21 & 1.41 & 14.4 & 1.36 & 20.4 \\
\hline HTLG-57VS & 2 & 1 & 13 & P06PF21 & 1.37 & 13.4 & 1.38 & 20.1 \\
\hline HTLG-58VS & 2 & 1 & 14 & P09PF21 & 1.4 & 12.3 & 1.35 & 20 \\
\hline HTLG-53VS & 2 & 1 & 15 & P02PF11 & 1.4 & 14.3 & 1.36 & 20.4 \\
\hline HTLG-55VS & 2 & 1 & 16 & P04PF11 & 1.39 & 11.7 & 1.37 & 20.1 \\
\hline Batch 1 & 2 & 1 & 17 & BCHPF1213 & 2.43 & 9.12 & 2.03 & 22.9 \\
\hline Batch 1 & 2 & 2 & 1 & BCHPF1221 & 2.71 & 9.95 & 2.22 & 24.8 \\
\hline HTLG-57VS & 2 & 2 & 2 & P06PF22 & 1.45 & 13.8 & 1.38 & 20.4 \\
\hline HTLG-58VS & 2 & 2 & 3 & P09PF22 & 1.44 & 12.6 & 1.35 & 20.2 \\
\hline HTLG-64VS & 2 & 2 & 4 & P05PF22 & 1.35 & 12.3 & 1.39 & 20.4 \\
\hline HTLG-57VS & 2 & 2 & 5 & P06PF12 & 1.39 & 13.7 & 1.38 & 20.2 \\
\hline HTLG-58VS & 2 & 2 & 6 & P09PF12 & 1.39 & 12.2 & 1.36 & 20.1 \\
\hline HTLG-55VS & 2 & 2 & 7 & P04PF22 & 1.39 & 11.9 & 1.37 & 20.2 \\
\hline
\end{tabular}


Table A3. Measured Elemental Concentrations (wt\%) for Series "P”' Samples Prepared Using Peroxide Fusion

\begin{tabular}{|c|c|c|c|c|c|c|c|c|}
\hline Glass ID & Block & Sub-Block & Sequence & Lab ID & B (wt\%) & Fe (wt\%) & Li (wt\%) & Si (wt\%) \\
\hline HTLG-53VS & 2 & 2 & 8 & P02PF22 & 1.39 & 14.5 & 1.37 & 20.8 \\
\hline Batch 1 & 2 & 2 & 9 & BCHPF1222 & 2.43 & 9.26 & 2.06 & 23.2 \\
\hline HTLG-66VS & 2 & 2 & 10 & P11PF12 & 1.38 & 11.9 & 1.35 & 19.9 \\
\hline HTLG-59VS & 2 & 2 & 11 & P14PF12 & 1.37 & 14 & 1.35 & 20 \\
\hline HTLG-66VS & 2 & 2 & 12 & P11PF22 & 1.38 & 12.3 & 1.37 & 20.2 \\
\hline HTLG-64VS & 2 & 2 & 13 & P05PF12 & 1.35 & 12.7 & 1.38 & 20.5 \\
\hline HTLG-55VS & 2 & 2 & 14 & P04PF12 & 1.4 & 12.1 & 1.37 & 20.4 \\
\hline HTLG-53VS & 2 & 2 & 15 & P02PF12 & 1.36 & 14.3 & 1.36 & 20.3 \\
\hline HTLG-59VS & 2 & 2 & 16 & P14PF22 & 1.34 & 13.5 & 1.37 & 20 \\
\hline Batch 1 & 2 & 2 & 17 & BCHPF1223 & 2.23 & 8.69 & 1.88 & 21.4 \\
\hline
\end{tabular}


Table A4. Measured Elemental Concentrations (wt\%) for Series “Q” Samples Prepared Using Lithium Metaborate

\begin{tabular}{|c|c|c|c|c|c|c|c|c|c|c|c|c|c|c|c|c|}
\hline Glass ID & $\begin{array}{c}\text { Block } \\
\text { Sub-Blk }\end{array}$ & Sequence & Lab ID & $\begin{array}{c}\mathrm{Al} \\
\text { (wt\%) }\end{array}$ & $\begin{array}{c}\mathrm{Ca} \\
\text { (wt\%) }\end{array}$ & $\begin{array}{c}\mathrm{Cr} \\
\text { (wt\%) }\end{array}$ & $\begin{array}{c}\mathrm{Cu} \\
\text { (wt\%) }\end{array}$ & $\begin{array}{c}\mathrm{K} \\
\text { (wt\%) }\end{array}$ & $\begin{array}{c}\mathrm{Mg} \\
\text { (wt\%) }\end{array}$ & $\begin{array}{c}\mathrm{Mn} \\
\text { (wt\%) }\end{array}$ & $\begin{array}{c}\mathrm{Na} \\
\text { (wt\%) }\end{array}$ & $\begin{array}{c}\mathrm{Ni} \\
\text { (wt\%) }\end{array}$ & $\begin{array}{c}\mathrm{S} \\
\text { (wt\%) }\end{array}$ & $\begin{array}{c}\mathrm{Si} \\
\text { (wt\%) }\end{array}$ & $\begin{array}{c}\mathrm{Ti} \\
\text { (wt\%) }\end{array}$ & $\begin{array}{c}\mathrm{Zr} \\
\text { (wt\%) }\end{array}$ \\
\hline Batch 1 & $1-1$ & 1 & BCHLM1111 & 2.64 & 0.885 & 0.078 & 0.315 & 2.54 & 0.871 & 1.33 & 7.13 & 0.544 & 0.015 & 22.4 & 0.380 & 0.063 \\
\hline HTLG-72VS & $1-1$ & 2 & Q02LM11 & 4.95 & 1.10 & 0.042 & 0.025 & 0.098 & 1.28 & 2.90 & 9.83 & 0.581 & 0.087 & 18.9 & 0.014 & 0.047 \\
\hline HTLG-77VS & $1-1$ & 3 & Q09LM21 & 5.00 & 1.11 & 0.037 & 0.024 & 0.105 & 0.963 & 3.22 & 10.0 & 0.750 & 0.091 & 19.3 & 0.015 & 0.052 \\
\hline 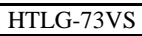 & $1-1$ & 4 & Q05LM11 & 4.46 & 1.52 & 0.041 & 0.024 & 0.112 & 0.944 & 2.82 & 10.8 & 0.599 & 0.084 & 18.8 & 0.014 & 0.044 \\
\hline $\begin{array}{l}\text { HTLG-69VS } \\
\end{array}$ & $1-1$ & 5 & Q10LM21 & 4.34 & 1.48 & 0.067 & 0.043 & 0.197 & 1.02 & 2.73 & 9.65 & 0.751 & 0.137 & 19.5 & 0.024 & 0.083 \\
\hline $\begin{array}{l}\text { HTLG-69VS } \\
\end{array}$ & $1-1$ & 6 & Q10LM11 & 4.34 & 1.47 & 0.066 & 0.041 & 0.195 & 1.02 & 2.72 & 9.67 & 0.741 & 0.136 & 19.5 & 0.024 & 0.083 \\
\hline HTLG-72VS & $1-1$ & 7 & Q02LM21 & 4.91 & 1.09 & 0.041 & 0.026 & 0.102 & 1.25 & 2.81 & 9.96 & 0.579 & 0.086 & 18.6 & 0.014 & 0.047 \\
\hline $\begin{array}{l}\text { HTLG-77VS } \\
\end{array}$ & $1-1$ & 8 & Q09LM11 & 5.00 & 1.12 & 0.038 & 0.027 & 0.115 & 0.964 & 3.10 & 10.0 & 0.794 & 0.086 & 18.9 & 0.014 & 0.045 \\
\hline Batch 1 & $1-1$ & 9 & BCHLM1112 & 2.64 & 0.875 & 0.075 & 0.308 & 2.47 & 0.852 & 1.34 & 7.15 & 0.528 & 0.013 & 22.6 & 0.374 & 0.061 \\
\hline HTLG-68VS & $1-1$ & 10 & Q04LM21 & 4.70 & 1.04 & 0.037 & 0.028 & 0.093 & 0.941 & 2.87 & 9.46 & 0.740 & 0.081 & 19.0 & 0.014 & 0.044 \\
\hline $\begin{array}{l}\text { HTLG-78VS } \\
\end{array}$ & $1-1$ & 11 & Q08LM21 & 4.47 & 1.51 & 0.070 & 0.045 & 0.205 & 1.02 & 2.77 & 9.76 & 0.764 & 0.137 & 18.4 & 0.025 & 0.083 \\
\hline HTLG-73VS & $1-1$ & 12 & Q05LM21 & 4.43 & 1.51 & 0.042 & 0.023 & 0.099 & 0.983 & 2.72 & 10.7 & 0.607 & 0.087 & 18.4 & 0.014 & 0.047 \\
\hline $\begin{array}{l}\text { HTLG-68VS } \\
\end{array}$ & $1-1$ & 13 & Q04LM11 & 4.72 & 1.06 & 0.039 & 0.023 & 0.093 & 0.953 & 2.89 & 9.53 & 0.757 & 0.088 & 18.9 & 0.013 & 0.045 \\
\hline HTLG-79VS & $1-1$ & 14 & Q13LM11 & 4.55 & 1.56 & 0.040 & 0.029 & 0.113 & 1.22 & 2.97 & 11.1 & 0.751 & 0.084 & 18.3 & 0.014 & 0.042 \\
\hline $\begin{array}{l}\text { HTLG-79VS } \\
\end{array}$ & $1-1$ & 15 & Q13LM21 & 4.47 & 1.51 & 0.039 & 0.027 & 0.114 & 1.22 & 3.07 & 10.6 & 0.751 & 0.088 & 18.3 & 0.014 & 0.044 \\
\hline HTLG-78VS & $1-1$ & 16 & Q08LM11 & 4.44 & 1.48 & 0.071 & 0.046 & 0.202 & 1.04 & 2.78 & 9.85 & 0.769 & 0.133 & 18.3 & 0.025 & 0.086 \\
\hline Batch 1 & $1-1$ & 17 & BCHLM1113 & 2.60 & 0.875 & 0.076 & 0.310 & 2.47 & 0.847 & 1.35 & 7.06 & 0.526 & 0.018 & 22.2 & 0.370 & 0.062 \\
\hline Batch 1 & $1-2$ & 1 & BCHLM1121 & 2.62 & 0.868 & 0.077 & 0.309 & 2.48 & 0.836 & 1.36 & 7.07 & 0.522 & 0.020 & 22.2 & 0.366 & 0.065 \\
\hline HTLG-68VS & $1-2$ & 2 & Q04LM22 & 4.71 & 1.04 & 0.039 & 0.028 & 0.092 & 0.939 & 2.87 & 9.41 & 0.735 & 0.085 & 19.0 & 0.014 & 0.048 \\
\hline $\begin{array}{l}\text { HTLG-69VS } \\
\end{array}$ & $1-2$ & 3 & Q10LM12 & 4.33 & 1.46 & 0.066 & 0.040 & 0.193 & 0.985 & 2.59 & 9.61 & 0.723 & 0.137 & 18.8 & 0.023 & 0.086 \\
\hline HTLG-73VS & $1-2$ & 4 & Q05LM12 & 4.46 & 1.51 & 0.042 & 0.023 & 0.106 & 0.942 & 2.68 & 10.7 & 0.590 & 0.088 & 18.3 & 0.014 & 0.049 \\
\hline HTLG-72VS & $1-2$ & 5 & Q02LM22 & 4.94 & 1.10 & 0.041 & 0.024 & 0.096 & 1.22 & 2.66 & 10.0 & 0.565 & 0.084 & 18.1 & 0.014 & 0.050 \\
\hline HTLG-79VS & $1-2$ & 6 & Q13LM22 & 4.50 & 1.53 & 0.039 & 0.027 & 0.111 & 1.19 & 3.04 & 10.7 & 0.736 & 0.085 & 18.2 & 0.014 & 0.047 \\
\hline HTLG-77VS & $1-2$ & 7 & Q09LM12 & 5.05 & 1.12 & 0.039 & 0.026 & 0.111 & 0.955 & 3.08 & 10.1 & 0.783 & 0.088 & 18.8 & 0.014 & 0.049 \\
\hline HTLG-78VS & $1-2$ & 8 & Q08LM22 & 4.51 & 1.52 & 0.071 & 0.044 & 0.203 & 1.02 & 2.77 & 9.89 & 0.753 & 0.133 & 18.5 & 0.025 & 0.088 \\
\hline Batch 1 & $1-2$ & 9 & BCHLM1123 & 2.62 & 0.875 & 0.075 & 0.310 & 2.49 & 0.819 & 1.35 & 7.17 & 0.515 & 0.015 & 22.2 & 0.368 & 0.067 \\
\hline HTLG-68VS & $1-2$ & 10 & Q04LM12 & 4.74 & 1.05 & 0.040 & 0.022 & 0.094 & 0.933 & 2.91 & 9.57 & 0.738 & 0.082 & 19.0 & 0.014 & 0.047 \\
\hline HTLG-79VS & $1-2$ & 11 & Q13LM12 & 4.57 & 1.57 & 0.039 & 0.028 & 0.115 & 1.18 & 2.95 & 11.1 & 0.731 & 0.086 & 18.4 & 0.014 & 0.045 \\
\hline HTLG-72VS & $1-2$ & 12 & Q02LM12 & 4.95 & 1.11 & 0.040 & 0.025 & 0.099 & 1.21 & 2.67 & 9.98 & 0.552 & 0.084 & 18.1 & 0.014 & 0.051 \\
\hline HTLG-69VS & $1-2$ & 13 & Q10LM22 & 4.35 & 1.48 & 0.066 & 0.042 & 0.196 & 0.993 & 2.63 & 9.71 & 0.730 & 0.135 & 19.0 & 0.024 & 0.087 \\
\hline HTLG-73VS & $1-2$ & 14 & Q05LM22 & 4.49 & 1.53 & 0.042 & 0.023 & 0.104 & 0.948 & 2.67 & 10.9 & 0.591 & 0.089 & 18.3 & 0.014 & 0.049 \\
\hline HTLG-78VS & $1-2$ & 15 & Q08LM12 & 4.53 & 1.53 & 0.070 & 0.046 & 0.205 & 1.02 & 2.70 & 10.2 & 0.752 & 0.133 & 18.2 & 0.025 & 0.089 \\
\hline HTLG-77VS & $1-2$ & 16 & Q09LM22 & 4.98 & 1.10 & 0.038 & 0.024 & 0.105 & 0.938 & 2.97 & 10.1 & 0.729 & 0.084 & 18.4 & 0.014 & 0.057 \\
\hline Batch 1 & $1-2$ & 17 & BCHLM1123 & 2.63 & 0.879 & 0.075 & 0.308 & 2.50 & 0.826 & 1.28 & 7.18 & 0.511 & 0.019 & 21.8 & 0.368 & 0.065 \\
\hline Batch 1 & $2-1$ & 1 & BCHLM1211 & 2.64 & 0.868 & 0.077 & 0.312 & 2.47 & 0.838 & 1.33 & 7.18 & 0.522 & 0.024 & 22.1 & 0.368 & 0.068 \\
\hline HTLG-71VS & $2-1$ & 2 & Q11LM21 & 4.54 & 1.10 & 0.086 & 0.050 & 0.216 & 0.980 & 3.08 & 10.8 & 0.002 & 0.142 & 18.3 & 0.027 & 0.091 \\
\hline HTLG-70VS & $2-1$ & 3 & Q06LM11 & 4.26 & 1.43 & 0.038 & 0.025 & 0.102 & 1.19 & 2.91 & 10.5 & 0.746 & 0.083 & 18.7 & 0.015 & 0.050 \\
\hline HTLG-67VS & $2-1$ & 4 & Q12LM11 & 4.79 & 1.46 & 0.069 & 0.046 & 0.205 & 1.17 & 2.97 & 9.64 & 0.558 & 0.140 & 19.3 & 0.025 & 0.088 \\
\hline HTLG-67VS & $2-1$ & 5 & Q12LM21 & 4.76 & 1.44 & 0.069 & 0.046 & 0.207 & 1.16 & 2.97 & 9.60 & 0.565 & 0.141 & 19.2 & 0.025 & 0.088 \\
\hline $\begin{array}{l}\text { HTLG-75VS } \\
\end{array}$ & $2-1$ & 6 & Q03LM11 & 4.54 & 1.11 & 0.072 & 0.046 & 0.203 & 1.23 & 2.84 & 10.8 & 0.758 & 0.129 & 18.7 & 0.027 & 0.088 \\
\hline HTLG-71VS & $2-1$ & 7 & Q11LM11 & 4.59 & 1.12 & 0.087 & 0.049 & 0.208 & 0.974 & 3.14 & 11.1 & 0.002 & 0.148 & 18.5 & 0.027 & 0.090 \\
\hline HTLG-70VS & $2-1$ & 8 & Q06LM21 & 4.32 & 1.48 & 0.038 & 0.036 & 0.098 & 1.19 & 2.80 & 10.7 & 0.761 & 0.084 & 18.6 & 0.014 & 0.052 \\
\hline Batch 1 & $2-1$ & 9 & BCHLM1212 & 2.62 & 0.883 & 0.076 & 0.315 & 2.49 & 0.833 & 1.35 & 7.23 & 0.519 & 0.021 & 22.0 & 0.371 & 0.069 \\
\hline HTLG-76VS & $2-1$ & 10 & Q01LM11 & 5.05 & 1.60 & 0.078 & 0.046 & 0.209 & 1.21 & 2.90 & 10.2 & 0.576 & 0.143 & 18.1 & 0.027 & 0.093 \\
\hline HTLG-75VS & $2-1$ & 11 & Q03LM21 & 4.55 & 1.11 & 0.075 & 0.047 & 0.204 & 1.23 & 2.74 & 11.0 & 0.790 & 0.124 & 18.4 & 0.027 & 0.086 \\
\hline
\end{tabular}


Table A4. Measured Elemental Concentrations (wt\%) for Series “Q” Samples Prepared Using Lithium Metaborate

\begin{tabular}{|c|c|c|c|c|c|c|c|c|c|c|c|c|c|c|c|c|}
\hline Glass ID & $\begin{array}{c}\text { Block } \\
\text { Sub-Blk }\end{array}$ & Sequence & Lab ID & $\begin{array}{c}\mathrm{Al} \\
(\mathrm{wt} \%)\end{array}$ & $\begin{array}{c}\mathrm{Ca} \\
\text { (wt\%) }\end{array}$ & $\begin{array}{c}\mathrm{Cr} \\
\text { (wt\%) }\end{array}$ & $\begin{array}{c}\mathrm{Cu} \\
\text { (wt\%) }\end{array}$ & $\begin{array}{c}\mathrm{K} \\
\text { (wt\%) }\end{array}$ & $\begin{array}{c}\mathrm{Mg} \\
(\mathrm{wt} \%)\end{array}$ & $\begin{array}{c}\mathrm{Mn} \\
\text { (wt\%) }\end{array}$ & $\begin{array}{c}\mathrm{Na} \\
(\mathrm{wt} \%)\end{array}$ & $\begin{array}{c}\mathrm{Ni} \\
\text { (wt\%) }\end{array}$ & $\begin{array}{c}\mathrm{S} \\
\text { (wt\%) }\end{array}$ & $\begin{array}{c}\mathrm{Si} \\
(\mathrm{wt} \%)\end{array}$ & $\begin{array}{c}\mathrm{Ti} \\
\text { (wt\%) }\end{array}$ & $\begin{array}{c}\mathrm{Zr} \\
\text { (wt\%) }\end{array}$ \\
\hline HTLG-74VS & $2-1$ & 12 & Q07LM11 & 5.02 & 1.51 & 0.072 & 0.050 & 0.227 & 0.952 & 2.74 & 11.0 & 0.763 & 0.133 & 18.3 & 0.025 & 0.087 \\
\hline $\begin{array}{l}\text { HTL-74VS } \\
\end{array}$ & $2-1$ & 13 & Q07LM21 & 5.01 & $\begin{array}{l}1.49 \\
\end{array}$ & 0.073 & 0.049 & 0.218 & 0.972 & 2.78 & 10.9 & 0.779 & 0.134 & $\begin{array}{ll}18.4 \\
\end{array}$ & 0.026 & 0.091 \\
\hline HTLG-76VS & $2-1$ & 14 & Q01LM21 & 5.00 & 1.54 & 0.076 & 0.047 & 0.211 & 1.23 & 3.00 & 10.0 & 0.578 & 0.143 & 18.5 & 0.027 & 0.093 \\
\hline Batch 1 & $2-1$ & 15 & BCHLM1213 & 2.67 & 0.874 & 0.077 & 0.312 & 2.49 & 0.835 & 1.33 & 7.29 & 0.520 & 0.020 & $\begin{array}{ll}22.1 \\
\end{array}$ & 0.368 & 0.069 \\
\hline Batch 1 & $2-2$ & 1 & BCHLM1221 & 2.62 & 0.878 & 0.077 & 0.314 & 2.51 & 0.831 & 1.33 & 7.04 & 0.524 & 0.022 & 22.2 & 0.367 & 0.068 \\
\hline $\begin{array}{l}\text { HTG-67VS } \\
\end{array}$ & $2-2$ & 2 & Q12LM22 & 4.77 & 1.47 & 0.070 & 0.045 & 0.206 & 1.17 & 2.86 & 9.63 & 0.570 & 0.139 & 19.1 & 0.025 & 0.086 \\
\hline $\begin{array}{l}\text { HTLG-76VS } \\
\end{array}$ & $2-2$ & 3 & Q01LM22 & 4.93 & 1.55 & 0.076 & 0.046 & 0.209 & 1.22 & 2.93 & 9.91 & 0.583 & 0.142 & $\begin{array}{l}18.1 \\
\end{array}$ & 0.027 & 0.092 \\
\hline HTLG-70VS & $2-2$ & 4 & Q06LM12 & 4.22 & 1.45 & 0.037 & 0.025 & 0.102 & 1.18 & 2.77 & 10.3 & 0.743 & 0.087 & 18.4 & 0.014 & 0.049 \\
\hline HTLG-71VS & $2-2$ & 5 & Q11LM12 & 4.54 & 1.13 & 0.086 & 0.048 & 0.210 & 0.981 & 3.08 & $\begin{array}{ll}10.8 \\
\end{array}$ & 0.002 & 0.146 & $\begin{array}{l}18.3 \\
\end{array}$ & 0.027 & 0.089 \\
\hline HTLG-75VS & $2-2$ & 6 & Q03LM12 & 4.50 & 1.12 & 0.073 & 0.046 & 0.204 & 1.24 & 2.75 & 10.6 & 0.765 & 0.128 & 18.4 & 0.027 & 0.089 \\
\hline $\begin{array}{l}\text { HTL-75VS } \\
\end{array}$ & $2-2$ & 7 & Q03LM22 & 4.49 & 1.11 & 0.076 & 0.047 & 0.205 & 1.24 & 2.85 & 10.7 & 0.797 & 0.122 & 18.6 & 0.027 & 0.087 \\
\hline HTLG-67VS & $2-2$ & 8 & Q12LM12 & 4.74 & 1.46 & 0.069 & 0.045 & 0.204 & 1.16 & 2.87 & 9.52 & 0.556 & 0.141 & 19.0 & 0.025 & 0.087 \\
\hline Batch 1 & $2-2$ & 9 & BCHLM1222 & 2.62 & 0.875 & 0.072 & 0.305 & 2.56 & 0.817 & 1.31 & 7.11 & 0.511 & 0.020 & 21.9 & 0.391 & 0.070 \\
\hline HTLG-74VS & $2-2$ & 10 & Q07LM12 & 4.98 & 1.51 & 0.070 & 0.049 & 0.228 & 0.932 & 2.62 & 10.9 & 0.759 & 0.134 & 18.0 & 0.026 & 0.086 \\
\hline HTLG-71VS & $2-2$ & 11 & Q11LM22 & 4.53 & 1.12 & 0.084 & 0.049 & 0.217 & 0.953 & 3.04 & 10.7 & 0.001 & 0.141 & 18.2 & 0.026 & 0.091 \\
\hline $\begin{array}{l}\text { HTL-70VS } \\
\end{array}$ & $2-2$ & 12 & Q06LM22 & 4.22 & 1.43 & 0.037 & 0.036 & 0.102 & 1.16 & 2.91 & 10.3 & 0.748 & 0.082 & $\begin{array}{l}18.7 \\
\end{array}$ & 0.014 & 0.050 \\
\hline $\begin{array}{l}\text { HTLG-74VS } \\
\text { HTLG }\end{array}$ & $2-2$ & 13 & Q07LM22 & 4.93 & 1.48 & 0.071 & 0.048 & 0.221 & 0.949 & 2.80 & 10.7 & 0.771 & 0.131 & 18.3 & 0.026 & 0.089 \\
\hline $\begin{array}{l}\text { HTLG-76VS } \\
\end{array}$ & $2-2$ & 14 & Q01LM12 & 4.95 & 1.55 & 0.076 & 0.045 & 0.217 & 1.20 & 3.07 & 9.80 & 0.567 & 0.141 & 18.4 & 0.026 & 0.095 \\
\hline Batch 1 & $2-2$ & 15 & BCHLM1223 & 2.58 & 0.871 & 0.068 & 0.304 & 2.61 & 0.790 & 1.33 & 6.98 & 0.493 & 0.020 & 22.0 & 0.386 & 0.068 \\
\hline
\end{tabular}


Table A5. Measured Elemental Concentrations (wt\%) for Series "Q" Samples Prepared Using Peroxide Fusion

\begin{tabular}{|c|c|c|c|c|c|c|c|c|}
\hline Glass ID & Block & Sub-Block & Sequence & Lab ID & B (wt\%) & Fe (wt\%) & Li (wt\%) & Si (wt\%) \\
\hline Batch 1 & 1 & 1 & 1 & BCHLM1111 & 2.51 & 8.94 & 2.04 & 22.9 \\
\hline HTLG-77VS & 1 & 1 & 2 & Q09PF21 & 1.37 & 12.9 & 1.32 & 20 \\
\hline HTLG-68VS & 1 & 1 & 3 & Q04PF21 & 1.39 & 12.4 & 1.36 & 20.1 \\
\hline HTLG-70VS & 1 & 1 & 4 & Q06PF21 & 1.41 & 12.5 & 1.35 & 20.1 \\
\hline HTLG-72VS & 1 & 1 & 5 & Q02PF21 & 1.35 & 13.4 & 1.33 & 19.6 \\
\hline HTLG-75VS & 1 & 1 & 6 & Q03PF11 & 1.29 & 11.5 & 1.3 & 19 \\
\hline HTLG-75VS & 1 & 1 & 7 & Q03PF21 & 1.29 & 11.6 & 1.3 & 19.3 \\
\hline HTLG-74VS & 1 & 1 & 8 & Q07PF21 & 1.25 & 11 & 1.28 & 18.5 \\
\hline Batch 1 & 1 & 1 & 9 & BCHLM1112 & 2.3 & 8.31 & 1.98 & 21.9 \\
\hline HTLG-77VS & 1 & 1 & 10 & Q09PF11 & 1.31 & 11.9 & 1.28 & 19 \\
\hline HTLG-70VS & 1 & 1 & 11 & Q06PF11 & 1.36 & 11.8 & 1.32 & 19.3 \\
\hline HTLG-72VS & 1 & 1 & 12 & Q02PF11 & 1.26 & 12.3 & 1.28 & 18.6 \\
\hline HTLG-68VS & 1 & 1 & 13 & Q04PF11 & 1.32 & 11.8 & 1.34 & 19.7 \\
\hline HTLG-73VS & 1 & 1 & 14 & Q05PF21 & 1.29 & 12.5 & 1.29 & 19 \\
\hline HTLG-74VS & 1 & 1 & 15 & Q07PF11 & 1.27 & 11.2 & 1.29 & 18.7 \\
\hline HTLG-73VS & 1 & 1 & 16 & Q05PF11 & 1.27 & 12.1 & 1.29 & 19 \\
\hline Batch 1 & 1 & 1 & 17 & BCHLM1113 & 2.27 & 8.09 & 1.99 & 22.1 \\
\hline Batch 1 & 1 & 2 & 1 & BCHLM1121 & 2.51 & 8.95 & 2.07 & 22.5 \\
\hline HTLG-73VS & 1 & 2 & 2 & Q05PF12 & 1.33 & 13.5 & 1.36 & 19.4 \\
\hline HTLG-72VS & 1 & 2 & 3 & Q02PF12 & 1.34 & 13.3 & 1.35 & 19 \\
\hline HTLG-70VS & 1 & 2 & 4 & Q06PF22 & 1.4 & 12.4 & 1.38 & 19.9 \\
\hline HTLG-74VS & 1 & 2 & 5 & Q07PF12 & 1.27 & 12.4 & 1.32 & 18.6 \\
\hline HTLG-75VS & 1 & 2 & 6 & Q03PF12 & 1.33 & 12.1 & 1.37 & 19.6 \\
\hline HTLG-75VS & 1 & 2 & 7 & Q03PF22 & 1.32 & 11.9 & 1.38 & 19.7 \\
\hline HTLG-74VS & 1 & 2 & 8 & Q07PF22 & 1.3 & 11.8 & 1.37 & 19.3 \\
\hline Batch 1 & 1 & 2 & 9 & BCHLM1123 & 2.34 & 8.52 & 2.06 & 22.6 \\
\hline HTLG-68VS & 1 & 2 & 10 & Q04PF22 & 1.42 & 12.6 & 1.45 & 20.4 \\
\hline HTLG-70VS & 1 & 2 & 11 & Q06PF12 & 1.4 & 12.4 & 1.41 & 20 \\
\hline HTLG-77VS & 1 & 2 & 12 & Q09PF22 & 1.31 & 12.3 & 1.37 & 19.4 \\
\hline HTLG-73VS & 1 & 2 & 13 & Q05PF22 & 1.31 & 12.8 & 1.38 & 19.6 \\
\hline HTLG-77VS & 1 & 2 & 14 & Q09PF12 & 1.32 & 12.6 & 1.38 & 19.7 \\
\hline HTLG-72VS & 1 & 2 & 15 & Q02PF22 & 1.33 & 13.4 & 1.39 & 19.5 \\
\hline HTLG-68VS & 1 & 2 & 16 & Q04PF12 & 1.38 & 12.8 & 1.44 & 20.5 \\
\hline Batch 1 & 1 & 2 & 17 & BCHLM1123 & 2.43 & 8.96 & 2.14 & 23.4 \\
\hline Batch 1 & 2 & 1 & 1 & BCHLM1211 & 2.5 & 9.01 & 2.06 & 22.7 \\
\hline HTLG-76VS & 2 & 1 & 2 & Q01PF11 & 1.35 & 13 & 1.35 & 19.9 \\
\hline HTLG-79VS & 2 & 1 & 3 & Q13PF21 & 1.36 & 12.1 & 1.31 & 19 \\
\hline HTLG-71VS & 2 & 1 & 4 & Q11PF11 & 1.35 & 12.7 & 1.38 & 19.7 \\
\hline HTLG-67VS & 2 & 1 & 5 & Q12PF11 & 1.4 & 11.8 & 1.41 & 20.1 \\
\hline HTLG-79VS & 2 & 1 & 6 & Q13PF11 & 1.35 & 11.9 & 1.38 & 19.7 \\
\hline HTLG-69VS & 2 & 1 & 7 & Q10PF11 & 1.41 & 13.1 & 1.42 & 20.3 \\
\hline HTLG-67VS & 2 & 1 & 8 & Q12PF21 & 1.38 & 11.9 & 1.42 & 20.3 \\
\hline Batch 1 & 2 & 1 & 9 & BCHLM1212 & 2.48 & 9.37 & 2.12 & 23.5 \\
\hline HTLG-71VS & 2 & 1 & 10 & Q11PF21 & 1.39 & 13.4 & 1.38 & 19.8 \\
\hline HTLG-78VS & 2 & 1 & 11 & Q08PF11 & 1.37 & 13.7 & 1.35 & 19.6 \\
\hline HTLG-78VS & 2 & 1 & 12 & Q08PF21 & 1.39 & 14.1 & 1.36 & 19.7 \\
\hline HTLG-69VS & 2 & 1 & 13 & Q10PF21 & 1.42 & 13.3 & 1.42 & 20.4 \\
\hline HTLG-76VS & 2 & 1 & 14 & Q01PF21 & 1.37 & 12.7 & 1.37 & 20.1 \\
\hline Batch 1 & 2 & 1 & 15 & BCHLM1213 & 2.48 & 9.45 & 2.11 & 23.6 \\
\hline Batch 1 & 2 & 2 & 1 & BCHLM1221 & 2.54 & 9.07 & 2.04 & 23.4 \\
\hline HTLG-79VS & 2 & 2 & 2 & Q13PF22 & 1.34 & 12 & 1.26 & 19.2 \\
\hline HTLG-79VS & 2 & 2 & 3 & Q13PF12 & 1.35 & 11.9 & 1.33 & 19.8 \\
\hline HTLG-76VS & 2 & 2 & 4 & Q01PF12 & 1.3 & 13.1 & 1.26 & 19.5 \\
\hline HTLG-78VS & 2 & 2 & 5 & Q08PF22 & 1.35 & 13.9 & 1.31 & 19.9 \\
\hline HTLG-69VS & 2 & 2 & 6 & Q10PF22 & 1.38 & 13 & 1.36 & 20.4 \\
\hline HTLG-76VS & 2 & 2 & 7 & Q01PF22 & 1.34 & 12.7 & 1.31 & 20.2 \\
\hline HTLG-71VS & 2 & 2 & 8 & Q11PF22 & 1.33 & 13.5 & 1.33 & 20.1 \\
\hline Batch 1 & 2 & 2 & 9 & BCHLM1222 & 2.45 & 9.29 & 2.04 & 23.5 \\
\hline HTLG-71VS & 2 & 2 & 10 & Q11PF12 & 1.38 & 13.4 & 1.35 & 20.3 \\
\hline HTLG-69VS & 2 & 2 & 11 & Q10PF12 & 1.42 & 13.4 & 1.37 & 20.7 \\
\hline
\end{tabular}


Table A5. Measured Elemental Concentrations (wt\%) for Series “Q” Samples Prepared Using Peroxide Fusion

\begin{tabular}{|c|c|c|c|c|c|c|c|c|}
\hline Glass ID & Block & Sub-Block & Sequence & Lab ID & B (wt\%) & Fe (wt\%) & Li (wt\%) & Si (wt\%) \\
\hline HTLG-78VS & 2 & 2 & 12 & Q08PF12 & 1.31 & 13.3 & 1.31 & 19.7 \\
\hline HTLG-67VS & 2 & 2 & 13 & Q12PF22 & 1.34 & 11.6 & 1.37 & 20.4 \\
\hline HTLG-67VS & 2 & 2 & 14 & Q12PF12 & 1.35 & 11.9 & 1.38 & 20.4 \\
\hline Batch 1 & 2 & 2 & 15 & BCHLM1223 & 2.37 & 8.92 & 2.05 & 23.4 \\
\hline
\end{tabular}


Table A6. Average Measured and Bias-Corrected Chemical Compositions Versus Targeted Compositions by Oxide for the HTL Study Glasses

\begin{tabular}{|c|c|c|c|c|c|c|c|c|}
\hline & & & Measured & & & & & \\
\hline & & Measured & Bias-Corrected & Targeted & Diff of & Diff of & $\%$ Diff of & $\%$ Diff of \\
\hline Glass ID & Oxide & (wt\%) & (wt\%) & $(\mathrm{wt} \%)$ & Measured & Meas BC & Measured & Meas BC \\
\hline Batch 1 & $\mathrm{Al} 2 \mathrm{O} 3$ (wt\%) & 4.9930 & 4.8770 & 4.8770 & 0.1160 & 0.0000 & $2.4 \%$ & $0.0 \%$ \\
\hline Batch 1 & B2O3 (wt\%) & 7.9156 & 7.7770 & 7.7770 & 0.1386 & 0.0000 & $1.8 \%$ & $0.0 \%$ \\
\hline Batch 1 & $\mathrm{CaO}$ (wt\%) & 1.2158 & 1.2200 & 1.2200 & -0.0042 & 0.0000 & $-0.3 \%$ & $0.0 \%$ \\
\hline Batch 1 & Cr2O3 (wt\%) & 0.1130 & 0.1070 & 0.1070 & 0.0060 & 0.0000 & $5.6 \%$ & $0.0 \%$ \\
\hline Batch 1 & $\mathrm{CuO}$ (wt\%) & 0.3918 & 0.3990 & 0.3990 & -0.0072 & 0.0000 & $-1.8 \%$ & $0.0 \%$ \\
\hline Batch 1 & Fe2O3 (wt\%) & 12.9245 & 12.8390 & 12.8390 & 0.0855 & 0.0000 & $0.7 \%$ & $0.0 \%$ \\
\hline Batch 1 & K2O (wt\%) & 3.0175 & 3.3270 & 3.3270 & -0.3095 & 0.0000 & $-9.3 \%$ & $0.0 \%$ \\
\hline Batch 1 & Li2O (wt\%) & 4.4117 & 4.4290 & 4.4290 & -0.0173 & 0.0000 & $-0.4 \%$ & $0.0 \%$ \\
\hline Batch 1 & MgO (wt\%) & 1.3851 & 1.4190 & 1.4190 & & & $-2.4 \%$ & $0.0 \%$ \\
\hline Batch 1 & $\mathrm{MnO}(\mathrm{wt} \%)$ & 1.7496 & 1.7260 & 1.7260 & 0.0236 & 0.0000 & $1.4 \%$ & $0.0 \%$ \\
\hline Batch 1 & $\mathrm{Na} 2 \mathrm{O}$ (wt\%) & 9.6876 & 9.0030 & 9.0030 & 0.6846 & 0.0000 & $7.6 \%$ & $0.0 \%$ \\
\hline Batch 1 & $\mathrm{NiO}$ (wt\%) & 0.6722 & 0.7510 & 0.7510 & -0.0788 & 0.0000 & $-10.5 \%$ & $0.0 \%$ \\
\hline Batch 1 & SiO2 (wt\%) & 48.7047 & 50.2200 & 50.2200 & -1.5153 & 0.0000 & $-3.0 \%$ & $0.0 \%$ \\
\hline Batch 1 & SO4 (wt\%) & 0.0649 & 0.0649 & 0.0000 & 0.0649 & 0.0649 & & \\
\hline Batch 1 & TiO2 (wt\%) & 0.6179 & 0.6770 & 0.6770 & -0.0591 & 0.0000 & $-8.7 \%$ & $0.0 \%$ \\
\hline Batch 1 & $\mathrm{ZrO} 2$ (wt\%) & 0.0872 & 0.0872 & 0.0980 & -0.0108 & -0.0108 & $-11.0 \%$ & $-11.0 \%$ \\
\hline Batch 1 & Oxide Sum (wt\%) & 97.9521 & 98.9232 & 98.8690 & -0.9169 & 0.0542 & $-0.9 \%$ & $0.1 \%$ \\
\hline HTLG-53VS & $\mathrm{Al} 2 \mathrm{O} 3(\mathrm{wt} \%)$ & 7.8745 & 7.7230 & 7.7700 & 0.1045 & -0.0470 & $1.3 \%$ & $-0.6 \%$ \\
\hline HTLG-53VS & B2O3 (wt\%) & 4.4757 & 4.3823 & 4.5000 & -0.0243 & -0.1177 & $-0.5 \%$ & $-2.6 \%$ \\
\hline HTLG-53VS & $\mathrm{CaO}(\mathrm{wt} \%)$ & 1.4727 & 1.4765 & 1.4100 & 0.0627 & 0.0665 & $4.4 \%$ & $4.7 \%$ \\
\hline HTLG-53VS & Cr2O3 (wt\%) & 0.0884 & 0.0857 & 0.1300 & -0.0416 & -0.0443 & $-32.0 \%$ & $-34.0 \%$ \\
\hline HTLG-53VS & $\mathrm{CuO}(\mathrm{wt} \%)$ & 0.1824 & 0.1864 & 0.0500 & 0.1324 & 0.1364 & $264.9 \%$ & $272.8 \%$ \\
\hline HTLG-53VS & Fe2O3 (wt\%) & 20.5519 & 20.1039 & 20.5200 & 0.0319 & -0.4161 & $0.2 \%$ & $-2.0 \%$ \\
\hline HTLG-53VS & K2O (wt\%) & 0.2277 & 0.2527 & 0.2200 & 0.0077 & 0.0327 & $3.5 \%$ & $14.9 \%$ \\
\hline HTLG-53VS & Li2O (wt\%) & 2.9333 & 2.9461 & 3.0000 & -0.0667 & -0.0539 & $-2.2 \%$ & $-1.8 \%$ \\
\hline HTLG-53VS & MgO (wt\%) & 1.9319 & 2.0059 & 2.1600 & -0.2281 & -0.1541 & $-10.6 \%$ & $-7.1 \%$ \\
\hline HTLG-53VS & $\mathrm{MnO}(\mathrm{wt} \%)$ & 3.3248 & 3.2557 & 3.1900 & 0.1348 & 0.0657 & $4.2 \%$ & $2.1 \%$ \\
\hline HTLG-53VS & Na2O (wt\%) & 12.1522 & 11.4462 & 12.3800 & -0.2278 & -0.9338 & $-1.8 \%$ & $-7.5 \%$ \\
\hline HTLG-53VS & $\mathrm{NiO}$ (wt\%) & 0.7132 & 0.8110 & 0.8400 & -0.1268 & -0.0290 & $-15.1 \%$ & $-3.4 \%$ \\
\hline HTLG-53VS & $\mathrm{SiO} 2(\mathrm{wt} \%)$ & 43.8022 & 44.6747 & 43.2200 & 0.5822 & 1.4547 & $1.3 \%$ & $3.4 \%$ \\
\hline HTLG-53VS & SO4 (wt\%) & 0.4239 & 0.4239 & 0.4700 & -0.0461 & -0.0461 & $-9.8 \%$ & $-9.8 \%$ \\
\hline HTLG-53VS & TiO2 (wt\%) & 0.0450 & 0.0496 & 0.0400 & 0.0050 & 0.0096 & $12.6 \%$ & $24.1 \%$ \\
\hline HTLG-53VS & $\mathrm{ZrO} 2$ (wt\%) & 0.1202 & 0.1202 & 0.1200 & 0.0002 & 0.0002 & $0.2 \%$ & $0.2 \%$ \\
\hline HTLG-53VS & Oxide Sum (wt\%) & 100.3202 & 99.9438 & 100.0200 & 0.3002 & -0.0762 & $0.3 \%$ & $-0.1 \%$ \\
\hline HTLG-54VS & Al2O3 (wt\%) & 7.7517 & 7.6024 & 7.6100 & 0.1417 & -0.0076 & $1.9 \%$ & $-0.1 \%$ \\
\hline HTLG-54VS & B2O3 (wt\%) & 4.4757 & 4.4121 & 4.5000 & -0.0243 & -0.0879 & $-0.5 \%$ & $-2.0 \%$ \\
\hline HTLG-54VS & $\mathrm{CaO}$ (wt\%) & 2.0288 & 2.0341 & 1.9100 & 0.1188 & 0.1241 & $6.2 \%$ & $6.5 \%$ \\
\hline HTLG-54VS & Cr2O3 (wt\%) & 0.0592 & 0.0574 & 0.0600 & -0.0008 & -0.0026 & $-1.3 \%$ & $-4.3 \%$ \\
\hline HTLG-54VS & $\mathrm{CuO}(\mathrm{wt} \%)$ & 0.0448 & 0.0457 & 0.0300 & 0.0148 & 0.0157 & $49.2 \%$ & $52.4 \%$ \\
\hline HTLG-54VS & Fe2O3 (wt\%) & 17.1564 & 17.3120 & 18.0100 & -0.8536 & -0.6980 & $-4.7 \%$ & $-3.9 \%$ \\
\hline HTLG-54VS & K2O (wt\%) & 0.1187 & 0.1317 & 0.1200 & -0.0013 & 0.0117 & $-1.1 \%$ & $9.7 \%$ \\
\hline HTLG-54VS & Li2O (wt\%) & 2.9387 & 2.9491 & 3.0000 & -0.0613 & -0.0509 & $-2.0 \%$ & $-1.7 \%$ \\
\hline HTLG-54VS & MgO (wt\%) & 1.9278 & 2.0015 & 2.1600 & -0.2322 & -0.1585 & $-10.8 \%$ & $-7.3 \%$ \\
\hline HTLG-54VS & $\mathrm{MnO}(\mathrm{wt} \%)$ & 3.2538 & 3.1850 & 3.1900 & 0.0638 & -0.0050 & $2.0 \%$ & $-0.2 \%$ \\
\hline HTLG-54VS & $\mathrm{Na} 2 \mathrm{O}$ (wt\%) & 14.3562 & 13.5225 & 14.7900 & -0.4338 & -1.2675 & $-2.9 \%$ & $-8.6 \%$ \\
\hline HTLG-54VS & $\mathrm{NiO}(\mathrm{wt} \%)$ & 0.9560 & 1.0871 & 1.0900 & -0.1340 & -0.0029 & $-12.3 \%$ & $-0.3 \%$ \\
\hline HTLG-54VS & SiO2 (wt\%) & 42.7325 & 44.5533 & 43.2200 & -0.4875 & 1.3333 & $-1.1 \%$ & $3.1 \%$ \\
\hline HTLG-54VS & SO4 (wt\%) & 0.2524 & 0.2524 & 0.2500 & 0.0024 & 0.0024 & $1.0 \%$ & $1.0 \%$ \\
\hline HTLG-54VS & TiO2 (wt\%) & 0.0292 & 0.0322 & 0.0200 & 0.0092 & 0.0122 & $46.0 \%$ & $60.8 \%$ \\
\hline HTLG-54VS & $\mathrm{ZrO} 2(\mathrm{wt} \%)$ & 0.0625 & 0.0625 & 0.0600 & 0.0025 & 0.0025 & $4.1 \%$ & $4.1 \%$ \\
\hline HTLG-54VS & Oxide Sum (wt\%) & 98.1442 & 99.2409 & 100.0200 & -1.8758 & -0.7791 & $-1.9 \%$ & $-0.8 \%$ \\
\hline HTLG-55VS & $\mathrm{Al} 2 \mathrm{O} 3(\mathrm{wt} \%)$ & 9.5231 & 9.3401 & 9.3000 & 0.2231 & 0.0401 & $2.4 \%$ & $0.4 \%$ \\
\hline HTLG-55VS & B2O3 (wt\%) & 4.5079 & 4.4140 & 4.5000 & 0.0079 & -0.0860 & $0.2 \%$ & $-1.9 \%$ \\
\hline HTLG-55VS & $\mathrm{CaO}(\mathrm{wt} \%)$ & 2.0288 & 2.0342 & 1.9100 & 0.1188 & 0.1242 & $6.2 \%$ & $6.5 \%$ \\
\hline HTLG-55VS & Cr2O3 (wt\%) & 0.1378 & 0.1336 & 0.1300 & 0.0078 & 0.0036 & $6.0 \%$ & $2.7 \%$ \\
\hline HTLG-55VS & $\mathrm{CuO}(\mathrm{wt} \%)$ & 0.0563 & 0.0575 & 0.0500 & 0.0063 & 0.0075 & $12.7 \%$ & $15.1 \%$ \\
\hline HTLG-55VS & Fe2O3 (wt\%) & 17.0134 & 16.6411 & 16.7900 & 0.2234 & -0.1489 & $1.3 \%$ & $-0.9 \%$ \\
\hline HTLG-55VS & K2O (wt\%) & 0.2376 & 0.2637 & 0.2200 & 0.0176 & 0.0437 & $8.0 \%$ & $19.9 \%$ \\
\hline HTLG-55VS & Li2O (wt\%) & 2.9441 & 2.9569 & 3.0000 & -0.0559 & -0.0431 & $-1.9 \%$ & $-1.4 \%$ \\
\hline HTLG-55VS & MgO (wt\%) & 1.8905 & 1.9628 & 2.1600 & -0.2695 & -0.1972 & $-12.5 \%$ & $-9.1 \%$ \\
\hline HTLG-55VS & $\mathrm{MnO}$ (wt\%) & 3.3378 & 3.2660 & 3.1900 & 0.1478 & 0.0760 & $4.6 \%$ & $2.4 \%$ \\
\hline HTLG-55VS & $\mathrm{Na} 2 \mathrm{O}(\mathrm{wt} \%)$ & 14.0529 & 13.2374 & 14.5800 & -0.5271 & -1.3426 & $-3.6 \%$ & $-9.2 \%$ \\
\hline HTLG-55VS & $\mathrm{NiO}$ (wt\%) & 0.7314 & 0.8317 & 0.8400 & -0.1086 & -0.0083 & $-12.9 \%$ & $-1.0 \%$ \\
\hline HTLG-55VS & $\mathrm{SiO} 2$ (wt\%) & 43.2139 & 44.0743 & 42.7200 & 0.4939 & 1.3543 & $1.2 \%$ & $3.2 \%$ \\
\hline HTLG-55VS & SO4 (wt\%) & 0.4329 & 0.4329 & 0.4700 & -0.0371 & -0.0371 & $-7.9 \%$ & $-7.9 \%$ \\
\hline HTLG-55VS & TiO2 (wt\%) & 0.0430 & 0.0473 & 0.0400 & 0.0030 & 0.0073 & $7.4 \%$ & $18.3 \%$ \\
\hline HTLG-55VS & $\mathrm{ZrO} 2$ (wt $\%)$ & 0.1192 & 0.1192 & 0.1200 & -0.0008 & -0.0008 & $-0.7 \%$ & $-0.7 \%$ \\
\hline HTLG-55VS & Oxide Sum (wt\%) & 100.2704 & 99.8126 & 100.0200 & 0.2504 & -0.2074 & $0.3 \%$ & $-0.2 \%$ \\
\hline
\end{tabular}


Table A6. Average Measured and Bias-Corrected Chemical Compositions Versus Targeted Compositions by Oxide for the HTL Study Glasses

\begin{tabular}{|c|c|c|c|c|c|c|c|c|}
\hline & & & Measured & & & & & \\
\hline & & Measured & Bias-Corrected & Targeted & Diff of & Diff of & $\%$ Diff of & $\%$ Diff of \\
\hline Glass ID & Oxide & (wt\%) & (wt\%) & $(\mathrm{wt} \%)$ & Measured & Meas BC & Measured & Meas BC \\
\hline HTLG-56VS & $\mathrm{Al} 2 \mathrm{O} 3$ (wt\%) & 7.7422 & 7.5935 & 7.6100 & 0.1322 & -0.0165 & $1.7 \%$ & $-0.2 \%$ \\
\hline HTLG-56VS & B2O3 (wt\%) & 4.4274 & 4.3644 & 4.5000 & -0.0726 & -0.1356 & $-1.6 \%$ & $-3.0 \%$ \\
\hline HTLG-56VS & $\mathrm{CaO}$ (wt\%) & 2.0323 & 2.0377 & 1.9100 & 0.1223 & 0.1277 & $6.4 \%$ & $6.7 \%$ \\
\hline HTLG-56VS & Cr2O3 (wt\%) & 0.1111 & 0.1077 & 0.1300 & -0.0189 & -0.0223 & $-14.6 \%$ & $-17.1 \%$ \\
\hline HTLG-56VS & $\mathrm{CuO}$ (wt\%) & 0.0538 & 0.0550 & 0.0500 & 0.0038 & 0.0050 & $7.7 \%$ & $10.0 \%$ \\
\hline HTLG-56VS & Fe2O3 (wt\%) & 17.5138 & 17.6730 & 18.7600 & -1.2462 & -1.0870 & $-6.6 \%$ & $-5.8 \%$ \\
\hline HTLG-56VS & K2O (wt\%) & 0.2515 & 0.2791 & 0.2200 & 0.0315 & 0.0591 & $14.3 \%$ & $26.9 \%$ \\
\hline HTLG-56VS & Li2O (wt\%) & 3.1594 & 3.1705 & 3.0000 & 0.1594 & 0.1705 & $5.3 \%$ & $5.7 \%$ \\
\hline HTLG-56VS & MgO (wt\%) & 1.5074 & 1.5650 & 1.6600 & -0.1526 & -0.0950 & $-9.2 \%$ & $-5.7 \%$ \\
\hline HTLG-56VS & $\mathrm{MnO}$ (wt\%) & 3.2506 & 3.1801 & 3.1900 & 0.0606 & -0.0099 & $1.9 \%$ & $-0.3 \%$ \\
\hline HTLG-56VS & $\mathrm{Na} 2 \mathrm{O}$ (wt\%) & 14.4236 & 13.5868 & 14.7900 & -0.3664 & -1.2032 & $-2.5 \%$ & $-8.1 \%$ \\
\hline HTLG-56VS & $\mathrm{NiO}$ (wt\%) & 0.7282 & 0.8280 & 0.8400 & -0.1118 & -0.0120 & $-13.3 \%$ & $-1.4 \%$ \\
\hline HTLG-56VS & SiO2 (wt\%) & 42.0907 & 43.8799 & 42.7200 & -0.6293 & 1.1599 & $-1.5 \%$ & $2.7 \%$ \\
\hline HTLG-56VS & SO4 (wt\%) & 0.4404 & 0.4404 & 0.4700 & -0.0296 & -0.0296 & $-6.3 \%$ & $-6.3 \%$ \\
\hline HTLG-56VS & TiO2 (wt\%) & 0.0421 & 0.0464 & 0.0400 & 0.0021 & 0.0064 & $5.3 \%$ & $16.0 \%$ \\
\hline HTLG-56VS & $\mathrm{ZrO} 2$ (wt\%) & 0.1138 & 0.1138 & 0.1200 & -0.0062 & -0.0062 & $-5.2 \%$ & $-5.2 \%$ \\
\hline HTLG-56VS & Oxide Sum (wt\%) & 97.8883 & 98.9214 & 100.0100 & -2.1217 & -1.0886 & $-2.1 \%$ & $-1.1 \%$ \\
\hline HTLG-57VS & $\mathrm{Al} 2 \mathrm{O} 3(\mathrm{wt} \%)$ & 9.5042 & 9.2459 & 9.3000 & 0.2042 & -0.0541 & $2.2 \%$ & $-0.6 \%$ \\
\hline HTLG-57VS & B2O3 (wt\%) & 4.5159 & 4.4222 & 4.5000 & 0.0159 & -0.0778 & $0.4 \%$ & $-1.7 \%$ \\
\hline HTLG-57VS & $\mathrm{CaO}(\mathrm{wt} \%)$ & 1.4901 & 1.4966 & 1.4100 & 0.0801 & 0.0866 & $5.7 \%$ & $6.1 \%$ \\
\hline HTLG-57VS & Cr2O3 (wt\%) & 0.0559 & 0.0517 & 0.0600 & -0.0041 & -0.0083 & $-6.8 \%$ & $-13.9 \%$ \\
\hline HTLG-57VS & $\mathrm{CuO}(\mathrm{wt} \%)$ & 0.0288 & 0.0292 & 0.0300 & -0.0012 & -0.0008 & $-4.0 \%$ & $-2.6 \%$ \\
\hline HTLG-57VS & Fe2O3 (wt\%) & 19.4082 & 18.9823 & 20.2400 & -0.8318 & -1.2577 & $-4.1 \%$ & $-6.2 \%$ \\
\hline HTLG-57VS & K2O (wt\%) & 0.1120 & 0.1227 & 0.1200 & -0.0080 & 0.0027 & $-6.6 \%$ & $2.3 \%$ \\
\hline HTLG-57VS & Li2O (wt\%) & 2.9710 & 2.9839 & 3.0000 & -0.0290 & -0.0161 & $-1.0 \%$ & $-0.5 \%$ \\
\hline HTLG-57VS & MgO (wt\%) & 1.5588 & 1.5762 & 1.6600 & -0.1012 & -0.0838 & $-6.1 \%$ & $-5.0 \%$ \\
\hline HTLG-57VS & $\mathrm{MnO}(\mathrm{wt} \%)$ & 3.1731 & 3.1550 & 3.1900 & -0.0169 & -0.0350 & $-0.5 \%$ & $-1.1 \%$ \\
\hline HTLG-57VS & Na2O (wt\%) & 12.5431 & 11.5025 & 12.3800 & 0.1631 & -0.8775 & $1.3 \%$ & $-7.1 \%$ \\
\hline HTLG-57VS & $\mathrm{NiO}$ (wt\%) & 0.9181 & 1.0082 & 1.0900 & -0.1719 & -0.0818 & $-15.8 \%$ & $-7.5 \%$ \\
\hline HTLG-57VS & $\mathrm{SiO} 2(\mathrm{wt} \%)$ & 43.1604 & 44.0195 & 42.7200 & 0.4404 & 1.2995 & $1.0 \%$ & $3.0 \%$ \\
\hline HTLG-57VS & SO4 (wt\%) & 0.2636 & 0.2636 & 0.2500 & 0.0136 & 0.0136 & $5.5 \%$ & $5.5 \%$ \\
\hline HTLG-57VS & TiO2 (wt\%) & 0.0254 & 0.0277 & 0.0200 & 0.0054 & 0.0077 & $27.2 \%$ & $38.6 \%$ \\
\hline HTLG-57VS & ZrO2 (wt\%) & 0.0648 & 0.0648 & 0.0600 & 0.0048 & 0.0048 & $8.1 \%$ & $8.1 \%$ \\
\hline HTLG-57VS & Oxide Sum (wt\%) & 99.7936 & 98.9521 & 100.0300 & -0.2364 & -1.0779 & $-0.2 \%$ & $-1.1 \%$ \\
\hline HTLG-58VS & Al2O3 (wt\%) & 7.7611 & 7.6121 & 7.6100 & 0.1511 & 0.0021 & $2.0 \%$ & $0.0 \%$ \\
\hline HTLG-58VS & B2O3 (wt\%) & 4.5240 & 4.4299 & 4.5000 & 0.0240 & -0.0701 & $0.5 \%$ & $-1.6 \%$ \\
\hline HTLG-58VS & $\mathrm{CaO}$ (wt\%) & 1.4797 & 1.4835 & 1.4100 & 0.0697 & 0.0735 & $4.9 \%$ & $5.2 \%$ \\
\hline HTLG-58VS & Cr2O3 (wt $\%)$ & 0.1085 & 0.1052 & 0.1300 & -0.0215 & -0.0248 & $-16.5 \%$ & $-19.1 \%$ \\
\hline HTLG-58VS & $\mathrm{CuO}(\mathrm{wt} \%)$ & 0.0541 & 0.0553 & 0.0500 & 0.0041 & 0.0053 & $8.3 \%$ & $10.6 \%$ \\
\hline HTLG-58VS & Fe2O3 (wt\%) & 17.5853 & 17.2005 & 17.8000 & -0.2147 & -0.5995 & $-1.2 \%$ & $-3.4 \%$ \\
\hline HTLG-58VS & K2O (wt\%) & 0.2346 & 0.2604 & 0.2200 & 0.0146 & 0.0404 & $6.6 \%$ & $18.4 \%$ \\
\hline HTLG-58VS & Li2O (wt\%) & 2.9118 & 2.9244 & 3.0000 & -0.0882 & -0.0756 & $-2.9 \%$ & $-2.5 \%$ \\
\hline HTLG-58VS & MgO (wt\%) & 1.9651 & 2.0402 & 2.1600 & -0.1949 & -0.1198 & $-9.0 \%$ & $-5.5 \%$ \\
\hline HTLG-58VS & $\mathrm{MnO}(\mathrm{wt} \%)$ & 3.9995 & 3.9191 & 3.9000 & 0.0995 & 0.0191 & $2.6 \%$ & $0.5 \%$ \\
\hline HTLG-58VS & $\mathrm{Na} 2 \mathrm{O}$ (wt\%) & 14.3562 & 13.5229 & 14.7900 & -0.4338 & -1.2671 & $-2.9 \%$ & $-8.6 \%$ \\
\hline HTLG-58VS & $\mathrm{NiO}(\mathrm{wt} \%)$ & 0.9741 & 1.1077 & 1.0900 & -0.1159 & 0.0177 & $-10.6 \%$ & $1.6 \%$ \\
\hline HTLG-58VS & $\mathrm{SiO} 2(\mathrm{wt} \%)$ & 42.9999 & 43.8567 & 42.7200 & 0.2799 & 1.1367 & $0.7 \%$ & $2.7 \%$ \\
\hline HTLG-58VS & SO4 (wt\%) & 0.4599 & 0.4599 & 0.4700 & -0.0101 & -0.0101 & $-2.2 \%$ & $-2.2 \%$ \\
\hline HTLG-58VS & TiO2 (wt\%) & 0.0430 & 0.0473 & 0.0400 & 0.0030 & 0.0073 & $7.4 \%$ & $18.3 \%$ \\
\hline HTLG-58VS & ZrO2 (wt\%) & 0.1091 & 0.1091 & 0.1200 & -0.0109 & -0.0109 & $-9.1 \%$ & $-9.1 \%$ \\
\hline HTLG-58VS & Oxide Sum (wt\%) & 99.5658 & 99.1343 & 100.0100 & -0.4442 & -0.8757 & $-0.4 \%$ & $-0.9 \%$ \\
\hline HTLG-59VS & $\mathrm{Al} 2 \mathrm{O} 3(\mathrm{wt} \%)$ & 7.8131 & 7.6629 & 7.6100 & 0.2031 & 0.0529 & $2.7 \%$ & $0.7 \%$ \\
\hline HTLG-59VS & B2O3 (wt\%) & 4.4354 & 4.3428 & 4.5000 & -0.0646 & -0.1572 & $-1.4 \%$ & $-3.5 \%$ \\
\hline HTLG-59VS & $\mathrm{CaO}(\mathrm{wt} \%)$ & 2.0533 & 2.0587 & 1.9100 & 0.1433 & 0.1487 & $7.5 \%$ & $7.8 \%$ \\
\hline HTLG-59VS & Cr2O3 (wt\%) & 0.0548 & 0.0531 & 0.0600 & -0.0052 & -0.0069 & $-8.7 \%$ & $-11.4 \%$ \\
\hline HTLG-59VS & $\mathrm{CuO}(\mathrm{wt} \%)$ & 0.0307 & 0.0313 & 0.0300 & 0.0007 & 0.0013 & $2.2 \%$ & $4.4 \%$ \\
\hline HTLG-59VS & Fe2O3 (wt\%) & 19.8013 & 19.3719 & 20.4700 & -0.6687 & -1.0981 & $-3.3 \%$ & $-5.4 \%$ \\
\hline HTLG-59VS & K2O (wt\%) & 0.1247 & 0.1384 & 0.1200 & 0.0047 & 0.0184 & $3.9 \%$ & $15.3 \%$ \\
\hline HTLG-59VS & Li2O (wt\%) & 2.9333 & 2.9461 & 3.0000 & -0.0667 & -0.0539 & $-2.2 \%$ & $-1.8 \%$ \\
\hline HTLG-59VS & MgO (wt\%) & 1.9070 & 1.9800 & 2.1600 & -0.2530 & -0.1800 & $-11.7 \%$ & $-8.3 \%$ \\
\hline HTLG-59VS & $\mathrm{MnO}$ (wt\%) & 4.0382 & 3.9536 & 3.9000 & 0.1382 & 0.0536 & $3.5 \%$ & $1.4 \%$ \\
\hline HTLG-59VS & $\mathrm{Na} 2 \mathrm{O}(\mathrm{wt} \%)$ & 12.2938 & 11.5799 & 12.3800 & -0.0862 & -0.8001 & $-0.7 \%$ & $-6.5 \%$ \\
\hline HTLG-59VS & $\mathrm{NiO}$ (wt\%) & 0.7126 & 0.8103 & 0.8400 & -0.1274 & -0.0297 & $-15.2 \%$ & $-3.5 \%$ \\
\hline HTLG-59VS & $\mathrm{SiO} 2$ (wt\%) & 42.8395 & 43.6939 & 42.7200 & 0.1195 & 0.9739 & $0.3 \%$ & $2.3 \%$ \\
\hline HTLG-59VS & SO4 (wt\%) & 0.2561 & 0.2561 & 0.2500 & 0.0061 & 0.0061 & $2.5 \%$ & $2.5 \%$ \\
\hline HTLG-59VS & TiO2 (wt\%) & 0.0242 & 0.0267 & 0.0200 & 0.0042 & 0.0067 & $20.9 \%$ & $33.3 \%$ \\
\hline HTLG-59VS & $\mathrm{ZrO} 2$ (wt\%) & 0.0655 & 0.0655 & 0.0600 & 0.0055 & 0.0055 & $9.2 \%$ & $9.2 \%$ \\
\hline HTLG-59VS & Oxide Sum (wt\%) & 99.3836 & 98.9713 & 100.0300 & -0.6464 & -1.0587 & $-0.6 \%$ & $-1.1 \%$ \\
\hline
\end{tabular}


Table A6. Average Measured and Bias-Corrected Chemical Compositions Versus Targeted Compositions by Oxide for the HTL Study Glasses

\begin{tabular}{|c|c|c|c|c|c|c|c|c|}
\hline & & & Measured & & & & & \\
\hline & & Measured & Bias-Corrected & Targeted & Diff of & Diff of & $\%$ Diff of & $\%$ Diff of \\
\hline Glass ID & Oxide & (wt\%) & (wt\%) & $(\mathrm{wt} \%)$ & Measured & Meas BC & Measured & Meas BC \\
\hline HTLG-60VS & $\mathrm{Al} 2 \mathrm{O} 3$ (wt\%) & 9.5231 & 9.2633 & 9.3000 & 0.2231 & -0.0367 & $2.4 \%$ & $-0.4 \%$ \\
\hline HTLG-60VS & B2O3 (wt\%) & 4.6286 & 4.5629 & 4.5000 & 0.1286 & 0.0629 & $2.9 \%$ & $1.4 \%$ \\
\hline HTLG-60VS & $\mathrm{CaO}$ (wt\%) & 2.0708 & 2.0800 & 1.9100 & 0.1608 & 0.1700 & $8.4 \%$ & $8.9 \%$ \\
\hline HTLG-60VS & Cr2O3 (wt\%) & 0.1133 & 0.1047 & 0.1300 & -0.0167 & -0.0253 & $-12.9 \%$ & $-19.4 \%$ \\
\hline HTLG-60VS & $\mathrm{CuO}(\mathrm{wt} \%)$ & 0.0554 & 0.0562 & 0.0500 & 0.0054 & 0.0062 & $10.8 \%$ & $12.5 \%$ \\
\hline HTLG-60VS & Fe2O3 (wt\%) & 17.5138 & 17.6678 & 18.0300 & -0.5162 & -0.3622 & $-2.9 \%$ & $-2.0 \%$ \\
\hline HTLG-60VS & K2O (wt\%) & 0.2460 & 0.2695 & 0.2200 & 0.0260 & 0.0495 & $11.8 \%$ & $22.5 \%$ \\
\hline HTLG-60VS & Li2O (wt\%) & 2.9818 & 2.9923 & 3.0000 & -0.0182 & -0.0077 & $-0.6 \%$ & $-0.3 \%$ \\
\hline HTLG-60VS & MgO (wt\%) & 1.5717 & 1.5891 & 1.6600 & -0.0883 & -0.0709 & $-5.3 \%$ & $-4.3 \%$ \\
\hline HTLG-60VS & $\mathrm{MnO}$ (wt\%) & 3.9349 & 3.9127 & 3.9000 & 0.0349 & 0.0127 & $0.9 \%$ & $0.3 \%$ \\
\hline HTLG-60VS & $\mathrm{Na} 2 \mathrm{O}$ (wt\%) & 12.9644 & 11.8872 & 12.3800 & 0.5844 & -0.4928 & $4.7 \%$ & $-4.0 \%$ \\
\hline HTLG-60VS & $\mathrm{NiO}$ (wt\%) & 0.9932 & 1.0906 & 1.0900 & -0.0968 & 0.0006 & $-8.9 \%$ & $0.1 \%$ \\
\hline HTLG-60VS & SiO2 (wt\%) & 43.3208 & 45.1605 & 43.2200 & 0.1008 & 1.9405 & $0.2 \%$ & $4.5 \%$ \\
\hline HTLG-60VS & SO4 (wt\%) & 0.4501 & 0.4501 & 0.4700 & -0.0199 & -0.0199 & $-4.2 \%$ & $-4.2 \%$ \\
\hline HTLG-60VS & TiO2 (wt\%) & 0.0425 & 0.0464 & 0.0400 & 0.0025 & 0.0064 & $6.3 \%$ & $15.9 \%$ \\
\hline HTLG-60VS & $\mathrm{ZrO} 2$ (wt\%) & 0.1128 & 0.1128 & 0.1200 & -0.0072 & -0.0072 & $-6.0 \%$ & $-6.0 \%$ \\
\hline HTLG-60VS & Oxide Sum (wt\%) & 100.5232 & 101.2460 & 100.0200 & 0.5032 & 1.2260 & $0.5 \%$ & $1.2 \%$ \\
\hline HTLG-61VS & $\mathrm{Al} 2 \mathrm{O} 3(\mathrm{wt} \%)$ & 9.4475 & 9.1906 & 9.3000 & 0.1475 & -0.1094 & $1.6 \%$ & $-1.2 \%$ \\
\hline HTLG-61VS & B2O3 (wt\%) & 4.4354 & 4.3727 & 4.5000 & -0.0646 & -0.1273 & $-1.4 \%$ & $-2.8 \%$ \\
\hline HTLG-61VS & $\mathrm{CaO}(\mathrm{wt} \%)$ & 1.4936 & 1.5002 & 1.4100 & 0.0836 & 0.0902 & $5.9 \%$ & $6.4 \%$ \\
\hline HTLG-61VS & Cr2O3 (wt\%) & 0.0636 & 0.0588 & 0.0600 & 0.0036 & -0.0012 & $6.0 \%$ & $-2.0 \%$ \\
\hline HTLG-61VS & $\mathrm{CuO}(\mathrm{wt} \%)$ & 0.0279 & 0.0283 & 0.0300 & -0.0021 & -0.0017 & $-7.2 \%$ & $-5.7 \%$ \\
\hline HTLG-61VS & Fe2O3 (wt\%) & 16.0841 & 16.2327 & 16.8600 & -0.7759 & -0.6273 & $-4.6 \%$ & $-3.7 \%$ \\
\hline HTLG-61VS & K2O (wt\%) & 0.1156 & 0.1267 & 0.1200 & -0.0044 & 0.0067 & $-3.6 \%$ & $5.6 \%$ \\
\hline HTLG-61VS & Li2O (wt\%) & 2.9387 & 2.9491 & 3.0000 & -0.0613 & -0.0509 & $-2.0 \%$ & $-1.7 \%$ \\
\hline HTLG-61VS & MgO (wt\%) & 1.5596 & 1.5769 & 1.6600 & -0.1004 & -0.0831 & $-6.0 \%$ & $-5.0 \%$ \\
\hline HTLG-61VS & $\mathrm{MnO}(\mathrm{wt} \%)$ & 3.9317 & 3.9093 & 3.9000 & 0.0317 & 0.0093 & $0.8 \%$ & $0.2 \%$ \\
\hline HTLG-61VS & Na2O (wt\%) & 14.7269 & 13.5056 & 14.7900 & -0.0631 & -1.2844 & $-0.4 \%$ & $-8.7 \%$ \\
\hline HTLG-61VS & $\mathrm{NiO}$ (wt\%) & 0.7746 & 0.8505 & 0.8400 & -0.0654 & 0.0105 & $-7.8 \%$ & $1.3 \%$ \\
\hline HTLG-61VS & $\mathrm{SiO} 2(\mathrm{wt} \%)$ & 42.7325 & 44.5519 & 43.2200 & -0.4875 & 1.3319 & $-1.1 \%$ & $3.1 \%$ \\
\hline HTLG-61VS & SO4 (wt\%) & 0.2681 & 0.2681 & 0.2500 & 0.0181 & 0.0181 & $7.3 \%$ & $7.3 \%$ \\
\hline HTLG-61VS & TiO2 (wt\%) & 0.0234 & 0.0255 & 0.0200 & 0.0034 & 0.0055 & $16.8 \%$ & $27.3 \%$ \\
\hline HTLG-61VS & ZrO2 (wt\%) & 0.0631 & 0.0631 & 0.0600 & 0.0031 & 0.0031 & $5.2 \%$ & $5.2 \%$ \\
\hline HTLG-61VS & Oxide Sum (wt\%) & 98.6865 & 99.2100 & 100.0200 & -1.3335 & -0.8100 & $-1.3 \%$ & $-0.8 \%$ \\
\hline HTLG-62VS & $\mathrm{Al} 2 \mathrm{O} 3(\mathrm{wt} \%)$ & 8.2524 & 8.0938 & 8.0500 & 0.2024 & 0.0438 & $2.5 \%$ & $0.5 \%$ \\
\hline HTLG-62VS & B2O3 (wt\%) & 4.5320 & 4.4677 & 4.5000 & 0.0320 & -0.0323 & $0.7 \%$ & $-0.7 \%$ \\
\hline HTLG-62VS & $\mathrm{CaO}$ (wt\%) & 1.4797 & 1.4835 & 1.4100 & 0.0697 & 0.0735 & $4.9 \%$ & $5.2 \%$ \\
\hline HTLG-62VS & Cr2O3 (wt $\%)$ & 0.1078 & 0.1045 & 0.1300 & -0.0222 & -0.0255 & $-17.1 \%$ & $-19.6 \%$ \\
\hline HTLG-62VS & $\mathrm{CuO}(\mathrm{wt} \%)$ & 0.0551 & 0.0563 & 0.0500 & 0.0051 & 0.0063 & $10.2 \%$ & $12.5 \%$ \\
\hline HTLG-62VS & Fe2O3 (wt\%) & 17.8355 & 17.9986 & 18.8800 & -1.0445 & -0.8814 & $-5.5 \%$ & $-4.7 \%$ \\
\hline HTLG-62VS & K2O (wt\%) & 0.2527 & 0.2804 & 0.2200 & 0.0327 & 0.0604 & $14.8 \%$ & $27.5 \%$ \\
\hline HTLG-62VS & Li2O (wt\%) & 2.9710 & 2.9815 & 3.0000 & -0.0290 & -0.0185 & $-1.0 \%$ & $-0.6 \%$ \\
\hline HTLG-62VS & MgO (wt\%) & 1.5372 & 1.5961 & 1.6600 & -0.1228 & -0.0639 & $-7.4 \%$ & $-3.9 \%$ \\
\hline HTLG-62VS & $\mathrm{MnO}$ (wt\%) & 3.8833 & 3.8046 & 3.7200 & 0.1633 & 0.0846 & $4.4 \%$ & $2.3 \%$ \\
\hline HTLG-62VS & $\mathrm{Na} 2 \mathrm{O}$ (wt\%) & 14.0192 & 13.2054 & 14.1900 & -0.1708 & -0.9846 & $-1.2 \%$ & $-6.9 \%$ \\
\hline HTLG-62VS & $\mathrm{NiO}$ (wt\%) & 0.7864 & 0.8942 & 0.8400 & -0.0536 & 0.0542 & $-6.4 \%$ & $6.5 \%$ \\
\hline HTLG-62VS & $\mathrm{SiO} 2(\mathrm{wt} \%)$ & 42.4116 & 44.2159 & 42.7200 & -0.3084 & 1.4959 & $-0.7 \%$ & $3.5 \%$ \\
\hline HTLG-62VS & SO4 (wt\%) & 0.4322 & 0.4322 & 0.4700 & -0.0378 & -0.0378 & $-8.1 \%$ & $-8.1 \%$ \\
\hline HTLG-62VS & TiO2 (wt\%) & 0.0409 & 0.0450 & 0.0400 & 0.0009 & 0.0050 & $2.2 \%$ & $12.6 \%$ \\
\hline HTLG-62VS & $\mathrm{ZrO} 2$ (wt\%) & 0.1148 & 0.1148 & 0.1200 & -0.0052 & -0.0052 & $-4.3 \%$ & $-4.3 \%$ \\
\hline HTLG-62VS & Oxide Sum (wt\%) & 98.7117 & 99.7746 & 100.0000 & -1.2883 & -0.2254 & $-1.3 \%$ & $-0.2 \%$ \\
\hline HTLG-63VS & $\mathrm{Al} 2 \mathrm{O} 3(\mathrm{wt} \%)$ & 9.0696 & 8.8228 & 8.8700 & 0.1996 & -0.0472 & $2.3 \%$ & $-0.5 \%$ \\
\hline HTLG-63VS & B2O3 (wt\%) & 4.4757 & 4.4121 & 4.5000 & -0.0243 & -0.0879 & $-0.5 \%$ & $-2.0 \%$ \\
\hline HTLG-63VS & $\mathrm{CaO}(\mathrm{wt} \%)$ & 1.5006 & 1.5073 & 1.4100 & 0.0906 & 0.0973 & $6.4 \%$ & $6.9 \%$ \\
\hline HTLG-63VS & Cr2O3 (wt\%) & 0.0548 & 0.0507 & 0.0600 & -0.0052 & -0.0093 & $-8.7 \%$ & $-15.5 \%$ \\
\hline HTLG-63VS & $\mathrm{CuO}(\mathrm{wt} \%)$ & 0.0279 & 0.0283 & 0.0300 & -0.0021 & -0.0017 & $-7.2 \%$ & $-5.7 \%$ \\
\hline HTLG-63VS & Fe2O3 (wt $\%)$ & 18.4074 & 18.5795 & 19.5800 & -1.1726 & -1.0005 & $-6.0 \%$ & $-5.1 \%$ \\
\hline HTLG-63VS & K2O (wt\%) & 0.1150 & 0.1260 & 0.1200 & -0.0050 & 0.0060 & $-4.1 \%$ & $5.0 \%$ \\
\hline HTLG-63VS & Li2O (wt\%) & 2.9602 & 2.9707 & 3.0000 & -0.0398 & -0.0293 & $-1.3 \%$ & $-1.0 \%$ \\
\hline HTLG-63VS & MgO (wt\%) & 1.9817 & 2.0035 & 2.1600 & -0.1783 & -0.1565 & $-8.3 \%$ & $-7.2 \%$ \\
\hline HTLG-63VS & $\mathrm{MnO}$ (wt\%) & 3.3507 & 3.3330 & 3.3700 & -0.0193 & -0.0370 & $-0.6 \%$ & $-1.1 \%$ \\
\hline HTLG-63VS & $\mathrm{Na} 2 \mathrm{O}$ (wt\%) & 13.2980 & 12.1939 & 13.0300 & 0.2680 & -0.8361 & $2.1 \%$ & $-6.4 \%$ \\
\hline HTLG-63VS & $\mathrm{NiO}$ (wt\%) & 0.7155 & 0.7856 & 0.8400 & -0.1245 & -0.0544 & $-14.8 \%$ & $-6.5 \%$ \\
\hline HTLG-63VS & $\mathrm{SiO} 2$ (wt\%) & 42.1977 & 43.9929 & 42.7200 & -0.5223 & 1.2729 & $-1.2 \%$ & $3.0 \%$ \\
\hline HTLG-63VS & SO4 (wt\%) & 0.2689 & 0.2689 & 0.2500 & 0.0189 & 0.0189 & $7.6 \%$ & $7.6 \%$ \\
\hline HTLG-63VS & TiO2 (wt\%) & 0.0254 & 0.0277 & 0.0200 & 0.0054 & 0.0077 & $27.2 \%$ & $38.6 \%$ \\
\hline HTLG-63VS & $\mathrm{ZrO} 2$ (wt\%) & 0.0669 & 0.0669 & 0.0600 & 0.0069 & 0.0069 & $11.4 \%$ & $11.4 \%$ \\
\hline HTLG-63VS & Oxide Sum (wt\%) & 98.5159 & 99.1698 & 100.0200 & -1.5041 & -0.8502 & $-1.5 \%$ & $-0.9 \%$ \\
\hline
\end{tabular}


WSRC-STI-2007-00616

Revision 0

Table A6. Average Measured and Bias-Corrected Chemical Compositions Versus Targeted Compositions by Oxide for the HTL Study Glasses

\begin{tabular}{|c|c|c|c|c|c|c|c|c|}
\hline & & & Measured & & & & & \\
\hline & & Measured & Bias-Corrected & Targeted & Diff of & Diff of & \% Diff of & \% Diff of \\
\hline Glass ID & Oxide & (wt\%) & (wt\%) & (wt\%) & Measured & Meas BC & Measured & Meas BC \\
\hline HTLG-64VS & $\mathrm{Al} 2 \mathrm{O} 3$ (wt\%) & 8.2052 & 7.9821 & 8.0300 & 0.1752 & -0.0479 & $2.2 \%$ & $-0.6 \%$ \\
\hline HTLG-64VS & B2O3 (wt\%) & 4.3871 & 4.2957 & 4.5000 & -0.1129 & -0.2043 & $-2.5 \%$ & $-4.5 \%$ \\
\hline HTLG-64VS & $\mathrm{CaO}$ (wt\%) & 2.0568 & 2.0658 & 1.9100 & 0.1468 & 0.1558 & $7.7 \%$ & $8.2 \%$ \\
\hline HTLG-64VS & Cr2O3 (wt\%) & 0.0658 & 0.0608 & 0.0600 & 0.0058 & 0.0008 & $9.6 \%$ & $1.3 \%$ \\
\hline HTLG-64VS & $\mathrm{CuO}(\mathrm{wt} \%)$ & 0.0288 & 0.0292 & 0.0300 & -0.0012 & -0.0008 & $-4.0 \%$ & $-2.6 \%$ \\
\hline HTLG-64VS & Fe2O3 (wt\%) & 17.7998 & 17.4112 & 18.7700 & -0.9702 & -1.3588 & $-5.2 \%$ & $-7.2 \%$ \\
\hline HTLG-64VS & K2O (wt\%) & 0.1196 & 0.1309 & 0.1200 & -0.0004 & 0.0109 & $-0.4 \%$ & $9.1 \%$ \\
\hline HTLG-64VS & $\mathrm{Li} 2 \mathrm{O}(\mathrm{wt} \%)$ & 2.9656 & 2.9785 & 3.0000 & -0.0344 & -0.0215 & $-1.1 \%$ & $-0.7 \%$ \\
\hline HTLG-64VS & MgO (wt\%) & 1.5401 & 1.5573 & 1.6600 & -0.1199 & -0.1027 & $-7.2 \%$ & $-6.2 \%$ \\
\hline HTLG-64VS & $\mathrm{MnO}(\mathrm{wt} \%)$ & 3.3765 & 3.3576 & 3.3700 & 0.0065 & -0.0124 & $0.2 \%$ & $-0.4 \%$ \\
\hline HTLG-64VS & Na2O (wt\%) & 14.2214 & 13.0416 & 14.1900 & 0.0314 & -1.1484 & $0.2 \%$ & $-8.1 \%$ \\
\hline HTLG-64VS & $\mathrm{NiO}$ (wt\%) & 0.7514 & 0.8251 & 0.8400 & -0.0886 & -0.0149 & $-10.5 \%$ & $-1.8 \%$ \\
\hline HTLG-64VS & $\mathrm{SiO} 2(\mathrm{wt} \%)$ & 43.4813 & 44.3468 & 43.2200 & 0.2613 & 1.1268 & $0.6 \%$ & $2.6 \%$ \\
\hline HTLG-64VS & SO4 (wt\%) & 0.2666 & 0.2666 & 0.2500 & 0.0166 & 0.0166 & $6.7 \%$ & $6.7 \%$ \\
\hline HTLG-64VS & $\mathrm{TiO} 2$ (wt\%) & 0.0246 & 0.0268 & 0.0200 & 0.0046 & 0.0068 & $23.0 \%$ & $34.1 \%$ \\
\hline HTLG-64VS & ZrO2 (wt\%) & 0.0689 & 0.0689 & 0.0600 & 0.0089 & 0.0089 & $14.8 \%$ & $14.8 \%$ \\
\hline HTLG-64VS & Oxide Sum (wt\%) & 99.3594 & 98.4450 & 100.0300 & -0.6706 & -1.5850 & $-0.7 \%$ & $-1.6 \%$ \\
\hline HTLG-65VS & $\mathrm{Al} 2 \mathrm{O} 3(\mathrm{wt} \%)$ & 8.2288 & 8.0050 & 8.8700 & -0.6412 & -0.8650 & $-7.2 \%$ & $-9.8 \%$ \\
\hline HTLG-65VS & B2O3 (wt\%) & 4.4354 & 4.3724 & 4.5000 & -0.0646 & -0.1276 & $-1.4 \%$ & $-2.8 \%$ \\
\hline HTLG-65VS & $\mathrm{CaO}(\mathrm{wt} \%)$ & 1.4901 & 1.4967 & 1.8600 & -0.3699 & -0.3633 & $-19.9 \%$ & $-19.5 \%$ \\
\hline HTLG-65VS & Cr2O3 (wt\%) & 0.1078 & 0.0997 & 0.1300 & -0.0222 & -0.0303 & $-17.1 \%$ & $-23.3 \%$ \\
\hline HTLG-65VS & $\mathrm{CuO}(\mathrm{wt} \%)$ & 0.0523 & 0.0531 & 0.0500 & 0.0023 & 0.0031 & $4.5 \%$ & $6.1 \%$ \\
\hline HTLG-65VS & Fe2O3 (wt\%) & 16.6917 & 16.8471 & 17.7200 & -1.0283 & -0.8729 & $-5.8 \%$ & $-4.9 \%$ \\
\hline HTLG-65VS & K2O (wt\%) & 0.2213 & 0.2424 & 0.2200 & 0.0013 & 0.0224 & $0.6 \%$ & $10.2 \%$ \\
\hline HTLG-65VS & Li2O (wt\%) & 2.9979 & 3.0085 & 3.0000 & -0.0021 & 0.0085 & $-0.1 \%$ & $0.3 \%$ \\
\hline HTLG-65VS & MgO (wt\%) & 2.0273 & 2.0498 & 1.6600 & 0.3673 & 0.3898 & $22.1 \%$ & $23.5 \%$ \\
\hline HTLG-65VS & $\mathrm{MnO}(\mathrm{wt} \%)$ & 3.3894 & 3.3700 & 3.3700 & 0.0194 & 0.0000 & $0.6 \%$ & $0.0 \%$ \\
\hline HTLG-65VS & $\mathrm{Na} 2 \mathrm{O}$ (wt\%) & 14.1540 & 12.9798 & 14.1900 & -0.0360 & -1.2102 & $-0.3 \%$ & $-8.5 \%$ \\
\hline HTLG-65VS & $\mathrm{NiO}$ (wt\%) & 0.9700 & 1.0651 & 1.0900 & -0.1200 & -0.0249 & $-11.0 \%$ & $-2.3 \%$ \\
\hline HTLG-65VS & $\mathrm{SiO} 2(\mathrm{wt} \%)$ & 42.6790 & 44.4955 & 42.7200 & -0.0410 & 1.7755 & $-0.1 \%$ & $4.2 \%$ \\
\hline HTLG-65VS & SO4 (wt\%) & 0.4539 & 0.4539 & 0.4700 & -0.0161 & -0.0161 & $-3.4 \%$ & $-3.4 \%$ \\
\hline HTLG-65VS & $\mathrm{TiO} 2(\mathrm{wt} \%)$ & 0.0455 & 0.0495 & 0.0400 & 0.0055 & 0.0095 & $13.6 \%$ & $23.8 \%$ \\
\hline HTLG-65VS & ZrO2 (wt\%) & 0.1162 & 0.1162 & 0.1200 & -0.0038 & -0.0038 & $-3.2 \%$ & $-3.2 \%$ \\
\hline HTLG-65VS & Oxide Sum (wt\%) & 98.0606 & 98.7045 & 100.0100 & -1.9494 & -1.3055 & $-1.9 \%$ & $-1.3 \%$ \\
\hline HTLG-66VS & $\mathrm{Al} 2 \mathrm{O} 3$ (wt\%) & 9.1027 & 8.8550 & 8.0300 & 1.0727 & 0.8250 & $13.4 \%$ & $10.3 \%$ \\
\hline HTLG-66VS & B2O3 (wt\%) & 4.4998 & 4.4059 & 4.5000 & -0.0002 & -0.0941 & $0.0 \%$ & $-2.1 \%$ \\
\hline HTLG-66VS & $\mathrm{CaO}$ (wt\%) & 2.0183 & 2.0272 & 1.4100 & 0.6083 & 0.6172 & $43.1 \%$ & $43.8 \%$ \\
\hline HTLG-66VS & Cr2O3 (wt\%) & 0.1045 & 0.0966 & 0.1300 & -0.0255 & -0.0334 & $-19.6 \%$ & $-25.7 \%$ \\
\hline HTLG-66VS & $\mathrm{CuO}(\mathrm{wt} \%)$ & 0.0516 & 0.0524 & 0.0500 & 0.0016 & 0.0024 & $3.3 \%$ & $4.8 \%$ \\
\hline HTLG-66VS & Fe2O3 (wt\%) & 17.1207 & 16.7455 & 18.0200 & -0.8993 & -1.2745 & $-5.0 \%$ & $-7.1 \%$ \\
\hline HTLG-66VS & K2O (wt\%) & 0.2521 & 0.2761 & 0.2200 & 0.0321 & 0.0561 & $14.6 \%$ & $25.5 \%$ \\
\hline HTLG-66VS & $\mathrm{Li} 2 \mathrm{O}(\mathrm{wt} \%)$ & 2.9172 & 2.9298 & 3.0000 & -0.0828 & -0.0702 & $-2.8 \%$ & $-2.3 \%$ \\
\hline HTLG-66VS & MgO (wt\%) & 1.5178 & 1.5346 & 2.1600 & -0.6422 & -0.6254 & $-29.7 \%$ & $-29.0 \%$ \\
\hline HTLG-66VS & $\mathrm{MnO}(\mathrm{wt} \%)$ & 3.3216 & 3.3028 & 3.3700 & -0.0484 & -0.0672 & $-1.4 \%$ & $-2.0 \%$ \\
\hline HTLG-66VS & $\mathrm{Na} 2 \mathrm{O}$ (wt\%) & 14.2888 & 13.1026 & 14.1900 & 0.0988 & -1.0874 & $0.7 \%$ & $-7.7 \%$ \\
\hline HTLG-66VS & $\mathrm{NiO}$ (wt\%) & 0.9502 & 1.0434 & 1.0900 & -0.1398 & -0.0466 & $-12.8 \%$ & $-4.3 \%$ \\
\hline HTLG-66VS & SiO2 (wt\%) & 42.5721 & 43.4192 & 43.2200 & -0.6479 & 0.1992 & $-1.5 \%$ & $0.5 \%$ \\
\hline HTLG-66VS & SO4 (wt\%) & 0.4419 & 0.4419 & 0.4700 & -0.0281 & -0.0281 & $-6.0 \%$ & $-6.0 \%$ \\
\hline HTLG-66VS & TiO2 (wt\%) & 0.0434 & 0.0473 & 0.0400 & 0.0034 & 0.0073 & $8.4 \%$ & $18.2 \%$ \\
\hline HTLG-66VS & $\mathrm{ZrO} 2$ (wt\%) & 0.1050 & 0.1050 & 0.1200 & -0.0150 & -0.0150 & $-12.5 \%$ & $-12.5 \%$ \\
\hline HTLG-66VS & Oxide Sum (wt\%) & 99.3076 & 98.3854 & 100.0200 & -0.7124 & -1.6346 & $-0.7 \%$ & $-1.6 \%$ \\
\hline
\end{tabular}


WSRC-STI-2007-00616

Revision 0

Table A6. Average Measured and Bias-Corrected Chemical Compositions Versus Targeted Compositions by Oxide for the HTL Study Glasses

\begin{tabular}{|c|c|c|c|c|c|c|c|c|}
\hline & & & Measured & & & & & \\
\hline & & Measured & Bias-Corrected & Targeted & Diff of & Diff of & \% Diff of & \% Diff of \\
\hline Glass ID & Oxide & (wt\%) & (wt\%) & (wt\%) & Measured & Meas BC & Measured & Meas BC \\
\hline Batch 1 & $\mathrm{Al} 2 \mathrm{O} 3$ (wt\%) & 4.9599 & 4.8770 & 4.8770 & 0.0829 & 0.0000 & $1.7 \%$ & $0.0 \%$ \\
\hline Batch 1 & $\mathrm{~B} 2 \mathrm{O} 3(\mathrm{wt} \%)$ & 7.8297 & 7.7770 & 7.7770 & 0.0527 & 0.0000 & $0.7 \%$ & $0.0 \%$ \\
\hline Batch 1 & $\mathrm{CaO}(\mathrm{wt} \%)$ & 1.2250 & 1.2200 & 1.2200 & 0.0050 & 0.0000 & $0.4 \%$ & $0.0 \%$ \\
\hline Batch 1 & Cr2O3 (wt\%) & 0.1100 & 0.1070 & 0.1070 & 0.0030 & 0.0000 & $2.8 \%$ & $0.0 \%$ \\
\hline Batch 1 & $\mathrm{CuO}(\mathrm{wt} \%)$ & 0.3883 & 0.3990 & 0.3990 & -0.0107 & 0.0000 & $-2.7 \%$ & $0.0 \%$ \\
\hline Batch 1 & $\mathrm{Fe} 2 \mathrm{O} 3(\mathrm{wt} \%)$ & 12.7339 & 12.8390 & 12.8390 & -0.1051 & 0.0000 & $-0.8 \%$ & $0.0 \%$ \\
\hline Batch 1 & K2O (wt\%) & 3.0195 & 3.3270 & 3.3270 & -0.3075 & 0.0000 & $-9.2 \%$ & $0.0 \%$ \\
\hline Batch 1 & Li2O (wt\%) & 4.4314 & 4.4290 & 4.4290 & 0.0024 & 0.0000 & $0.1 \%$ & $0.0 \%$ \\
\hline Batch 1 & MgO (wt\%) & 1.3812 & 1.4190 & 1.4190 & -0.0378 & 0.0000 & $-2.7 \%$ & $0.0 \%$ \\
\hline Batch 1 & $\mathrm{MnO}(\mathrm{wt} \%)$ & 1.7205 & 1.7260 & 1.7260 & -0.0055 & 0.0000 & $-0.3 \%$ & $0.0 \%$ \\
\hline Batch 1 & $\mathrm{Na} 2 \mathrm{O}$ (wt\%) & 9.6146 & 9.0030 & 9.0030 & 0.6116 & 0.0000 & $6.8 \%$ & $0.0 \%$ \\
\hline Batch 1 & $\mathrm{NiO}$ (wt\%) & 0.6612 & 0.7510 & 0.7510 & -0.0898 & 0.0000 & $-12.0 \%$ & $0.0 \%$ \\
\hline Batch 1 & $\mathrm{SiO} 2$ (wt\%) & 48.2412 & 50.2200 & 50.2200 & -1.9788 & 0.0000 & $-3.9 \%$ & $0.0 \%$ \\
\hline Batch 1 & SO4 (wt\%) & 0.0567 & 0.0567 & 0.0000 & 0.0567 & 0.0567 & & \\
\hline Batch 1 & TiO2 (wt\%) & 0.6223 & 0.6770 & 0.6770 & -0.0547 & 0.0000 & $-8.1 \%$ & $0.0 \%$ \\
\hline Batch 1 & $\mathrm{ZrO} 2$ (wt\%) & 0.0895 & 0.0895 & 0.0980 & -0.0085 & -0.0085 & $-8.7 \%$ & $-8.7 \%$ \\
\hline Batch 1 & Oxide Sum (wt\%) & 97.0849 & 98.9172 & 98.8690 & -1.7841 & 0.0482 & $-1.8 \%$ & $0.0 \%$ \\
\hline HTLG-67VS & $\mathrm{Al} 2 \mathrm{O} 3(\mathrm{wt} \%)$ & 9.0035 & 8.8532 & 8.8700 & 0.1335 & -0.0168 & $1.5 \%$ & $-0.2 \%$ \\
\hline HTLG-67VS & B2O3 (wt\%) & 4.4032 & 4.3054 & 4.5000 & -0.0968 & -0.1946 & $-2.2 \%$ & $-4.3 \%$ \\
\hline HTLG-67VS & $\mathrm{CaO}(\mathrm{wt} \%)$ & 2.0393 & 2.0326 & 1.9100 & 0.1293 & 0.1226 & $6.8 \%$ & $6.4 \%$ \\
\hline HTLG-67VS & Cr2O3 (wt $\%)$ & 0.1012 & 0.0996 & 0.1300 & -0.0288 & -0.0304 & $-22.1 \%$ & $-23.4 \%$ \\
\hline HTLG-67VS & $\mathrm{CuO}(\mathrm{wt} \%)$ & 0.0570 & 0.0585 & 0.0500 & 0.0070 & 0.0085 & $13.9 \%$ & $17.0 \%$ \\
\hline HTLG-67VS & Fe2O3 (wt\%) & 16.8705 & 16.4953 & 17.7700 & -0.8995 & -1.2747 & $-5.1 \%$ & $-7.2 \%$ \\
\hline HTLG-67VS & K2O (wt\%) & 0.2475 & 0.2712 & 0.2200 & 0.0275 & 0.0512 & $12.5 \%$ & $23.3 \%$ \\
\hline HTLG-67VS & Li2O (wt\%) & 3.0033 & 2.9847 & 3.0000 & 0.0033 & -0.0153 & $0.1 \%$ & $-0.5 \%$ \\
\hline HTLG-67VS & MgO (wt\%) & 1.9319 & 2.0066 & 2.1600 & -0.2281 & -0.1534 & $-10.6 \%$ & $-7.1 \%$ \\
\hline HTLG-67VS & $\mathrm{MnO}(\mathrm{wt} \%)$ & 3.7671 & 3.7859 & 3.7200 & 0.0471 & 0.0659 & $1.3 \%$ & $1.8 \%$ \\
\hline HTLG-67VS & $\mathrm{Na} 2 \mathrm{O}$ (wt\%) & 12.9374 & 12.1063 & 12.9800 & -0.0426 & -0.8737 & $-0.3 \%$ & $-6.7 \%$ \\
\hline HTLG-67VS & $\mathrm{NiO}$ (wt\%) & 0.7155 & 0.8203 & 0.8400 & -0.1245 & -0.0197 & $-14.8 \%$ & $-2.3 \%$ \\
\hline HTLG-67VS & SiO2 (wt\%) & 42.1977 & 43.6375 & 43.2200 & -1.0223 & 0.4175 & $-2.4 \%$ & $1.0 \%$ \\
\hline HTLG-67VS & SO4 (wt\%) & 0.4202 & 0.4202 & 0.4700 & -0.0498 & -0.0498 & $-10.6 \%$ & $-10.6 \%$ \\
\hline HTLG-67VS & TiO2 (wt\%) & 0.0417 & 0.0451 & 0.0400 & 0.0017 & 0.0051 & $4.3 \%$ & $12.8 \%$ \\
\hline HTLG-67VS & ZrO2 (wt\%) & 0.1179 & 0.1179 & 0.1200 & -0.0021 & -0.0021 & $-1.8 \%$ & $-1.8 \%$ \\
\hline HTLG-67VS & Oxide Sum (wt\%) & 97.8548 & 98.0402 & 100.0000 & -2.1452 & -1.9598 & $-2.1 \%$ & $-2.0 \%$ \\
\hline HTLG-68VS & $\mathrm{Al} 2 \mathrm{O} 3$ (wt\%) & 8.9137 & 8.7647 & 8.8700 & 0.0437 & -0.1053 & $0.5 \%$ & $-1.2 \%$ \\
\hline HTLG-68VS & B2O3 (wt \%) & 4.4354 & 4.4760 & 4.5000 & -0.0646 & -0.0240 & $-1.4 \%$ & $-0.5 \%$ \\
\hline HTLG-68VS & $\mathrm{CaO}$ (wt\%) & 1.4657 & 1.4586 & 1.4100 & 0.0557 & 0.0486 & $3.9 \%$ & $3.4 \%$ \\
\hline HTLG-68VS & Cr2O3 (wt\%) & 0.0566 & 0.0546 & 0.0600 & -0.0034 & -0.0054 & $-5.6 \%$ & $-9.1 \%$ \\
\hline HTLG-68VS & $\mathrm{CuO}(\mathrm{wt} \%)$ & 0.0316 & 0.0325 & 0.0300 & 0.0016 & 0.0025 & $5.4 \%$ & $8.3 \%$ \\
\hline HTLG-68VS & Fe2O3 (wt\%) & 17.7283 & 18.4500 & 19.0200 & -1.2917 & -0.5700 & $-6.8 \%$ & $-3.0 \%$ \\
\hline HTLG-68VS & K2O (wt\%) & 0.1120 & 0.1242 & 0.1200 & -0.0080 & 0.0042 & $-6.6 \%$ & $3.5 \%$ \\
\hline HTLG-68VS & Li2O (wt\%) & 3.0087 & 3.0234 & 3.0000 & 0.0087 & 0.0234 & $0.3 \%$ & $0.8 \%$ \\
\hline HTLG-68VS & $\mathrm{MgO}(\mathrm{wt} \%)$ & 1.5613 & 1.5873 & 1.6600 & -0.0987 & -0.0727 & $-5.9 \%$ & $-4.4 \%$ \\
\hline HTLG-68VS & $\mathrm{MnO}(\mathrm{wt} \%)$ & 3.7251 & 3.7300 & 3.7200 & 0.0051 & 0.0100 & $0.1 \%$ & $0.3 \%$ \\
\hline HTLG-68VS & $\mathrm{Na} 2 \mathrm{O}$ (wt\%) & 12.7959 & 11.9918 & 12.9800 & -0.1841 & -0.9882 & $-1.4 \%$ & $-7.6 \%$ \\
\hline HTLG-68VS & $\mathrm{NiO}(\mathrm{wt} \%)$ & 0.9448 & 1.0636 & 1.0900 & -0.1452 & -0.0264 & $-13.3 \%$ & $-2.4 \%$ \\
\hline HTLG-68VS & SiO2 (wt\%) & 41.8768 & 43.8798 & 43.2200 & -1.3432 & 0.6598 & $-3.1 \%$ & $1.5 \%$ \\
\hline HTLG-68VS & SO4 (wt\%) & 0.2517 & 0.2517 & 0.2500 & 0.0017 & 0.0017 & $0.7 \%$ & $0.7 \%$ \\
\hline HTLG-68VS & TiO2 (wt\%) & 0.0229 & 0.0251 & 0.0200 & 0.0029 & 0.0051 & $14.7 \%$ & $25.5 \%$ \\
\hline HTLG-68VS & $\mathrm{ZrO} 2$ (wt\%) & 0.0621 & 0.0621 & 0.0600 & 0.0021 & 0.0021 & $3.6 \%$ & $3.6 \%$ \\
\hline HTLG-68VS & Oxide Sum (wt\%) & 96.9927 & 98.9753 & 100.0100 & -3.0173 & -1.0347 & $-3.0 \%$ & $-1.0 \%$ \\
\hline HTLG-69VS & $\mathrm{Al} 2 \mathrm{O} 3$ (wt\%) & 8.2004 & 8.0633 & 8.0300 & 0.1704 & 0.0333 & $2.1 \%$ & $0.4 \%$ \\
\hline HTLG-69VS & $\mathrm{B} 2 \mathrm{O} 3(\mathrm{wt} \%)$ & 4.5320 & 4.4317 & 4.5000 & 0.0320 & -0.0683 & $0.7 \%$ & $-1.5 \%$ \\
\hline HTLG-69VS & $\mathrm{CaO}$ (wt\%) & 2.0603 & 2.0504 & 1.9100 & 0.1503 & 0.1404 & $7.9 \%$ & $7.3 \%$ \\
\hline HTLG-69VS & Cr2O3 (wt\%) & 0.0968 & 0.0933 & 0.1300 & -0.0332 & -0.0367 & $-25.5 \%$ & $-28.3 \%$ \\
\hline HTLG-69VS & $\mathrm{CuO}(\mathrm{wt} \%)$ & 0.0519 & 0.0534 & 0.0500 & 0.0019 & 0.0034 & $3.9 \%$ & $6.8 \%$ \\
\hline HTLG-69VS & $\mathrm{Fe} 2 \mathrm{O} 3(\mathrm{wt} \%)$ & 18.8720 & 18.4531 & 19.5800 & -0.7080 & -1.1269 & $-3.6 \%$ & $-5.8 \%$ \\
\hline HTLG-69VS & K2O (wt\%) & 0.2352 & 0.2607 & 0.2200 & 0.0152 & 0.0407 & $6.9 \%$ & $18.5 \%$ \\
\hline HTLG-69VS & Li2O (wt\%) & 2.9979 & 2.9791 & 3.0000 & -0.0021 & -0.0209 & $-0.1 \%$ & $-0.7 \%$ \\
\hline HTLG-69VS & $\mathrm{MgO}(\mathrm{wt} \%)$ & 1.6658 & 1.6933 & 1.8000 & -0.1342 & -0.1067 & $-7.5 \%$ & $-5.9 \%$ \\
\hline HTLG-69VS & $\mathrm{MnO}$ (wt\%) & 3.4443 & 3.4485 & 3.3700 & 0.0743 & 0.0785 & $2.2 \%$ & $2.3 \%$ \\
\hline HTLG-69VS & $\mathrm{Na} 2 \mathrm{O}$ (wt\%) & 13.0217 & 12.2034 & 12.9800 & 0.0417 & -0.7766 & $0.3 \%$ & $-6.0 \%$ \\
\hline HTLG-69VS & $\mathrm{NiO}$ (wt\%) & 0.9369 & 1.0546 & 1.0900 & -0.1531 & -0.0354 & $-14.0 \%$ & $-3.3 \%$ \\
\hline HTLG-69VS & $\mathrm{SiO} 2(\mathrm{wt} \%)$ & 42.4116 & 43.6742 & 42.7200 & -0.3084 & 0.9542 & $-0.7 \%$ & $2.2 \%$ \\
\hline HTLG-69VS & SO4 (wt\%) & 0.4082 & 0.4082 & 0.4700 & -0.0618 & -0.0618 & $-13.2 \%$ & $-13.2 \%$ \\
\hline HTLG-69VS & TiO2 (wt\%) & 0.0396 & 0.0433 & 0.0400 & -0.0004 & 0.0033 & $-1.0 \%$ & $8.3 \%$ \\
\hline HTLG-69VS & $\mathrm{ZrO} 2$ (wt\%) & 0.1145 & 0.1145 & 0.1200 & -0.0055 & -0.0055 & $-4.6 \%$ & $-4.6 \%$ \\
\hline HTLG-69VS & Oxide Sum (wt\%) & 99.0892 & 99.0249 & 100.0100 & -0.9208 & -0.9851 & $-0.9 \%$ & $-1.0 \%$ \\
\hline
\end{tabular}


WSRC-STI-2007-00616

Revision 0

Table A6. Average Measured and Bias-Corrected Chemical Compositions Versus Targeted Compositions by Oxide for the HTL Study Glasses

\begin{tabular}{|c|c|c|c|c|c|c|c|c|}
\hline & & & Measured & & & & & \\
\hline & & Measured & Bias-Corrected & Targeted & Diff of & Diff of & \% Diff of & \% Diff of \\
\hline Glass ID & Oxide & (wt\%) & (wt\%) & (wt\%) & Measured & Meas BC & Measured & Meas BC \\
\hline HTLG-70VS & $\mathrm{Al} 2 \mathrm{O} 3$ (wt\%) & 8.0398 & 7.9053 & 8.0300 & 0.0098 & -0.1247 & $0.1 \%$ & $-1.6 \%$ \\
\hline HTLG-70VS & B2O3 (wt\%) & 4.4837 & 4.5254 & 4.5000 & -0.0163 & 0.0254 & $-0.4 \%$ & $0.6 \%$ \\
\hline HTLG-70VS & $\mathrm{CaO}$ (wt\%) & 2.0253 & 2.0186 & 1.9100 & 0.1153 & 0.1086 & $6.0 \%$ & $5.7 \%$ \\
\hline HTLG-70VS & Cr2O3 (wt\%) & 0.0548 & 0.0539 & 0.0600 & -0.0052 & -0.0061 & $-8.7 \%$ & $-10.2 \%$ \\
\hline HTLG-70VS & $\mathrm{CuO}(\mathrm{wt} \%)$ & 0.0382 & 0.0392 & 0.0300 & 0.0082 & 0.0092 & $27.3 \%$ & $30.7 \%$ \\
\hline HTLG-70VS & $\mathrm{Fe} 2 \mathrm{O} 3(\mathrm{wt} \%)$ & 17.5496 & 18.2694 & 18.1600 & -0.6104 & 0.1094 & $-3.4 \%$ & $0.6 \%$ \\
\hline HTLG-70VS & K2O (wt\%) & 0.1217 & 0.1333 & 0.1200 & 0.0017 & 0.0133 & $1.4 \%$ & $11.1 \%$ \\
\hline HTLG-70VS & Li2O (wt\%) & 2.9387 & 2.9538 & 3.0000 & -0.0613 & -0.0462 & $-2.0 \%$ & $-1.5 \%$ \\
\hline HTLG-70VS & MgO (wt\%) & 1.9568 & 2.0322 & 2.1600 & -0.2032 & -0.1278 & $-9.4 \%$ & $-5.9 \%$ \\
\hline HTLG-70VS & $\mathrm{MnO}(\mathrm{wt} \%)$ & 3.6767 & 3.6954 & 3.7200 & -0.0433 & -0.0246 & $-1.2 \%$ & $-0.7 \%$ \\
\hline HTLG-70VS & $\mathrm{Na} 2 \mathrm{O}$ (wt\%) & 14.0866 & 13.1796 & 14.1900 & -0.1034 & -1.0104 & $-0.7 \%$ & $-7.1 \%$ \\
\hline HTLG-70VS & $\mathrm{NiO}$ (wt\%) & 0.9537 & 1.0934 & 1.0900 & -0.1363 & 0.0034 & $-12.5 \%$ & $0.3 \%$ \\
\hline HTLG-70VS & $\mathrm{SiO} 2$ (wt\%) & 41.1013 & 43.2420 & 42.7200 & -1.6187 & 0.5220 & $-3.8 \%$ & $1.2 \%$ \\
\hline HTLG-70VS & SO4 (wt\%) & 0.2517 & 0.2517 & 0.2500 & 0.0017 & 0.0017 & $0.7 \%$ & $0.7 \%$ \\
\hline HTLG-70VS & TiO2 (wt\%) & 0.0238 & 0.0257 & 0.0200 & 0.0038 & 0.0057 & $18.8 \%$ & $28.6 \%$ \\
\hline HTLG-70VS & $\mathrm{ZrO} 2$ (wt\%) & 0.0679 & 0.0679 & 0.0600 & 0.0079 & 0.0079 & $13.1 \%$ & $13.1 \%$ \\
\hline HTLG-70VS & Oxide Sum (wt\%) & 97.3702 & 99.4867 & 100.0200 & -2.6498 & -0.5333 & $-2.6 \%$ & $-0.5 \%$ \\
\hline HTLG-71VS & $\mathrm{Al} 2 \mathrm{O} 3(\mathrm{wt} \%)$ & 8.5972 & 8.4537 & 8.3800 & 0.2172 & 0.0737 & $2.6 \%$ & $0.9 \%$ \\
\hline HTLG-71VS & B2O3 (wt\%) & 4.3871 & 4.2900 & 4.3200 & 0.0671 & -0.0300 & $1.6 \%$ & $-0.7 \%$ \\
\hline HTLG-71VS & $\mathrm{CaO}(\mathrm{wt} \%)$ & 1.5636 & 1.5584 & 1.4600 & 0.1036 & 0.0984 & $7.1 \%$ & $6.7 \%$ \\
\hline HTLG-71VS & Cr2O3 (wt $\%)$ & 0.1253 & 0.1232 & 0.1300 & -0.0047 & -0.0068 & $-3.6 \%$ & $-5.2 \%$ \\
\hline HTLG-71VS & $\mathrm{CuO}(\mathrm{wt} \%)$ & 0.0613 & 0.0630 & 0.0500 & 0.0113 & 0.0130 & $22.7 \%$ & $26.0 \%$ \\
\hline HTLG-71VS & Fe2O3 (wt\%) & 18.9435 & 18.5258 & 19.6400 & -0.6965 & -1.1142 & $-3.5 \%$ & $-5.7 \%$ \\
\hline HTLG-71VS & K2O (wt\%) & 0.2563 & 0.2807 & 0.2300 & 0.0263 & 0.0507 & $11.4 \%$ & $22.1 \%$ \\
\hline HTLG-71VS & Li2O (wt\%) & 2.9279 & 2.9098 & 2.8800 & 0.0479 & 0.0298 & $1.7 \%$ & $1.0 \%$ \\
\hline HTLG-71VS & $\mathrm{MgO}(\mathrm{wt} \%)$ & 1.6119 & 1.6741 & 1.7200 & -0.1081 & -0.0459 & $-6.3 \%$ & $-2.7 \%$ \\
\hline HTLG-71VS & $\mathrm{MnO}(\mathrm{wt} \%)$ & 3.9834 & 4.0035 & 3.8700 & 0.1134 & 0.1335 & $2.9 \%$ & $3.4 \%$ \\
\hline HTLG-71VS & $\mathrm{Na} 2 \mathrm{O}$ (wt\%) & 14.6258 & 13.6850 & 14.6400 & -0.0142 & -0.9550 & $-0.1 \%$ & $-6.5 \%$ \\
\hline HTLG-71VS & $\mathrm{NiO}$ (wt\%) & 0.0022 & 0.0025 & 0.8700 & -0.8678 & -0.8675 & $-99.7 \%$ & $-99.7 \%$ \\
\hline HTLG-71VS & SiO2 (wt\%) & 40.9676 & 42.3480 & 41.1500 & -0.1824 & 1.1980 & $-0.4 \%$ & $2.9 \%$ \\
\hline HTLG-71VS & SO4 (wt\%) & 0.4322 & 0.4322 & 0.4900 & -0.0578 & -0.0578 & $-11.8 \%$ & $-11.8 \%$ \\
\hline HTLG-71VS & TiO2 (wt\%) & 0.0446 & 0.0483 & 0.0400 & 0.0046 & 0.0083 & $11.5 \%$ & $20.7 \%$ \\
\hline HTLG-71VS & ZrO2 (wt\%) & 0.1219 & 0.1219 & 0.1200 & 0.0019 & 0.0019 & $1.6 \%$ & $1.6 \%$ \\
\hline HTLG-71VS & Oxide Sum (wt\%) & 98.6519 & 98.5201 & 99.9900 & -1.3381 & -1.4699 & $-1.3 \%$ & $-1.5 \%$ \\
\hline HTLG-72VS & Al2O3 (wt\%) & 9.3294 & 9.1734 & 9.2300 & 0.0994 & -0.0566 & $1.1 \%$ & $-0.6 \%$ \\
\hline HTLG-72VS & B2O3 (wt\%) & 4.2503 & 4.2894 & 4.3200 & -0.0697 & -0.0306 & $-1.6 \%$ & $-0.7 \%$ \\
\hline HTLG-72VS & $\mathrm{CaO}$ (wt\%) & 1.5391 & 1.5317 & 1.4600 & 0.0791 & 0.0717 & $5.4 \%$ & $4.9 \%$ \\
\hline HTLG-72VS & Cr2O3 (wt\%) & 0.0599 & 0.0577 & 0.0700 & -0.0101 & -0.0123 & $-14.4 \%$ & $-17.5 \%$ \\
\hline HTLG-72VS & $\mathrm{CuO}(\mathrm{wt} \%)$ & 0.0313 & 0.0322 & 0.0300 & 0.0013 & 0.0022 & $4.3 \%$ & $7.3 \%$ \\
\hline HTLG-72VS & Fe2O3 (wt\%) & 18.7291 & 19.4937 & 20.3700 & -1.6409 & -0.8763 & $-8.1 \%$ & $-4.3 \%$ \\
\hline HTLG-72VS & K2O (wt\%) & 0.1190 & 0.1319 & 0.1200 & -0.0010 & 0.0119 & $-0.9 \%$ & $9.9 \%$ \\
\hline HTLG-72VS & Li2O (wt\%) & 2.8795 & 2.8942 & 2.8800 & -0.0005 & 0.0142 & $0.0 \%$ & $0.5 \%$ \\
\hline HTLG-72VS & $\mathrm{MgO}(\mathrm{wt} \%)$ & 2.0563 & 2.0901 & 2.2400 & -0.1837 & -0.1499 & $-8.2 \%$ & $-6.7 \%$ \\
\hline HTLG-72VS & $\mathrm{MnO}(\mathrm{wt} \%)$ & 3.5637 & 3.5679 & 3.5000 & 0.0637 & 0.0679 & $1.8 \%$ & $1.9 \%$ \\
\hline HTLG-72VS & $\mathrm{Na} 2 \mathrm{O}$ (wt\%) & 13.4025 & 12.5601 & 13.4300 & -0.0275 & -0.8699 & $-0.2 \%$ & $-6.5 \%$ \\
\hline HTLG-72VS & $\mathrm{NiO}$ (wt\%) & 0.7244 & 0.8153 & 0.8700 & -0.1456 & -0.0547 & $-16.7 \%$ & $-6.3 \%$ \\
\hline HTLG-72VS & SiO2 (wt\%) & 40.2188 & 42.1454 & 41.1500 & -0.9312 & 0.9954 & $-2.3 \%$ & $2.4 \%$ \\
\hline HTLG-72VS & SO4 (wt\%) & 0.2554 & 0.2554 & 0.2600 & -0.0046 & -0.0046 & $-1.8 \%$ & $-1.8 \%$ \\
\hline HTLG-72VS & TiO2 (wt\%) & 0.0234 & 0.0255 & 0.0200 & 0.0034 & 0.0055 & $16.8 \%$ & $27.7 \%$ \\
\hline HTLG-72VS & $\mathrm{ZrO} 2$ (wt\%) & 0.0659 & 0.0659 & 0.0600 & 0.0059 & 0.0059 & $9.8 \%$ & $9.8 \%$ \\
\hline HTLG-72VS & Oxide Sum (wt\%) & 97.2479 & 99.1298 & 100.0100 & -2.7621 & -0.8802 & $-2.8 \%$ & $-0.9 \%$ \\
\hline HTLG-73VS & $\mathrm{Al} 2 \mathrm{O} 3$ (wt\%) & 8.4272 & 8.2863 & 8.3500 & 0.0772 & -0.0637 & $0.9 \%$ & $-0.8 \%$ \\
\hline HTLG-73VS & B2O3 (wt $\%)$ & 4.1859 & 4.2242 & 4.3200 & -0.1341 & -0.0958 & $-3.1 \%$ & $-2.2 \%$ \\
\hline HTLG-73VS & $\mathrm{CaO}$ (wt\%) & 2.1233 & 2.1130 & 1.9800 & 0.1433 & 0.1330 & $7.2 \%$ & $6.7 \%$ \\
\hline HTLG-73VS & Cr2O3 (wt\%) & 0.0610 & 0.0588 & 0.0700 & -0.0090 & -0.0112 & $-12.8 \%$ & $-16.0 \%$ \\
\hline HTLG-73VS & $\mathrm{CuO}(\mathrm{wt} \%)$ & 0.0291 & 0.0299 & 0.0300 & -0.0009 & -0.0001 & $-3.0 \%$ & $-0.3 \%$ \\
\hline HTLG-73VS & Fe2O3 (wt\%) & 18.1929 & 18.9299 & 19.5200 & -1.3271 & -0.5901 & $-6.8 \%$ & $-3.0 \%$ \\
\hline HTLG-73VS & K2O (wt\%) & 0.1268 & 0.1405 & 0.1200 & 0.0068 & 0.0205 & $5.7 \%$ & $17.1 \%$ \\
\hline HTLG-73VS & Li2O (wt\%) & 2.8634 & 2.8776 & 2.8800 & -0.0166 & -0.0024 & $-0.6 \%$ & $-0.1 \%$ \\
\hline HTLG-73VS & $\mathrm{MgO}(\mathrm{wt} \%)$ & 1.5824 & 1.6087 & 1.7200 & -0.1376 & -0.1113 & $-8.0 \%$ & $-6.5 \%$ \\
\hline HTLG-73VS & $\mathrm{MnO}$ (wt\%) & 3.5153 & 3.5197 & 3.5000 & 0.0153 & 0.0197 & $0.4 \%$ & $0.6 \%$ \\
\hline HTLG-73VS & $\mathrm{Na} 2 \mathrm{O}(\mathrm{wt} \%)$ & 14.5247 & 13.6119 & 14.6400 & -0.1153 & -1.0281 & $-0.8 \%$ & $-7.0 \%$ \\
\hline HTLG-73VS & $\mathrm{NiO}$ (wt\%) & 0.7594 & 0.8548 & 0.8700 & -0.1106 & -0.0152 & $-12.7 \%$ & $-1.7 \%$ \\
\hline HTLG-73VS & $\mathrm{SiO} 2(\mathrm{wt} \%)$ & 40.3258 & 42.2563 & 41.6700 & -1.3442 & 0.5863 & $-3.2 \%$ & $1.4 \%$ \\
\hline HTLG-73VS & SO4 (wt\%) & 0.2606 & 0.2606 & 0.2600 & 0.0006 & 0.0006 & $0.2 \%$ & $0.2 \%$ \\
\hline HTLG-73VS & TiO2 (wt\%) & 0.0234 & 0.0255 & 0.0200 & 0.0034 & 0.0055 & $16.8 \%$ & $27.7 \%$ \\
\hline HTLG-73VS & $\mathrm{ZrO} 2(\mathrm{wt} \%)$ & 0.0638 & 0.0638 & 0.0600 & 0.0038 & 0.0038 & $6.4 \%$ & $6.4 \%$ \\
\hline HTLG-73VS & Oxide Sum (wt\%) & 97.0649 & 98.8617 & 100.0100 & -2.9451 & -1.1483 & $-2.9 \%$ & $-1.1 \%$ \\
\hline
\end{tabular}


WSRC-STI-2007-00616

Revision 0

Table A6. Average Measured and Bias-Corrected Chemical Compositions Versus Targeted Compositions by Oxide for the HTL Study Glasses

\begin{tabular}{|c|c|c|c|c|c|c|c|c|}
\hline & & & Measured & & & & & \\
\hline & & Measured & Bias-Corrected & Targeted & Diff of & Diff of & \% Diff of & \% Diff of \\
\hline Glass ID & Oxide & (wt\%) & (wt\%) & (wt\%) & Measured & Meas BC & Measured & Meas BC \\
\hline HTLG-74VS & $\mathrm{Al} 2 \mathrm{O} 3$ (wt\%) & 9.4192 & 9.2617 & 9.2300 & 0.1892 & 0.0317 & $2.0 \%$ & $0.3 \%$ \\
\hline HTLG-74VS & B2O3 (wt\%) & 4.0973 & 4.1352 & 4.3200 & -0.2227 & -0.1848 & $-5.2 \%$ & $-4.3 \%$ \\
\hline HTLG-74VS & $\mathrm{CaO}$ (wt\%) & 2.0953 & 2.0883 & 1.9300 & 0.1653 & 0.1583 & $8.6 \%$ & $8.2 \%$ \\
\hline HTLG-74VS & Cr2O3 (wt\%) & 0.1045 & 0.1027 & 0.1300 & -0.0255 & -0.0273 & $-19.6 \%$ & $-21.0 \%$ \\
\hline HTLG-74VS & $\mathrm{CuO}(\mathrm{wt} \%)$ & 0.0613 & 0.0630 & 0.0500 & 0.0113 & 0.0130 & $22.7 \%$ & $26.0 \%$ \\
\hline HTLG-74VS & $\mathrm{Fe} 2 \mathrm{O} 3(\mathrm{wt} \%)$ & 16.5845 & 17.2528 & 18.4300 & -1.8455 & -1.1772 & $-10.0 \%$ & $-6.4 \%$ \\
\hline HTLG-74VS & K2O (wt\%) & 0.2692 & 0.2949 & 0.2300 & 0.0392 & 0.0649 & $17.1 \%$ & $28.2 \%$ \\
\hline HTLG-74VS & Li2O (wt\%) & 2.8311 & 2.8456 & 2.8800 & -0.0489 & -0.0344 & $-1.7 \%$ & $-1.2 \%$ \\
\hline HTLG-74VS & MgO (wt\%) & 1.5775 & 1.6382 & 1.7200 & -0.1425 & -0.0818 & $-8.3 \%$ & $-4.8 \%$ \\
\hline HTLG-74VS & $\mathrm{MnO}(\mathrm{wt} \%)$ & 3.5314 & 3.5493 & 3.5000 & 0.0314 & 0.0493 & $0.9 \%$ & $1.4 \%$ \\
\hline HTLG-74VS & $\mathrm{Na} 2 \mathrm{O}$ (wt\%) & 14.6595 & 13.7169 & 14.6400 & 0.0195 & -0.9231 & $0.1 \%$ & $-6.3 \%$ \\
\hline HTLG-74VS & $\mathrm{NiO}$ (wt\%) & 0.9773 & 1.1204 & 1.1300 & -0.1527 & -0.0096 & $-13.5 \%$ & $-0.9 \%$ \\
\hline HTLG-74VS & $\mathrm{SiO} 2$ (wt\%) & 39.6038 & 41.6742 & 41.1500 & -1.5462 & 0.5242 & $-3.8 \%$ & $1.3 \%$ \\
\hline HTLG-74VS & SO4 (wt\%) & 0.3985 & 0.3985 & 0.4900 & -0.0915 & -0.0915 & $-18.7 \%$ & $-18.7 \%$ \\
\hline HTLG-74VS & TiO2 (wt\%) & 0.0430 & 0.0465 & 0.0400 & 0.0030 & 0.0065 & $7.4 \%$ & $16.2 \%$ \\
\hline HTLG-74VS & ZrO2 (wt\%) & 0.1192 & 0.1192 & 0.1200 & -0.0008 & -0.0008 & $-0.7 \%$ & $-0.7 \%$ \\
\hline HTLG-74VS & Oxide Sum (wt\%) & 96.3725 & 98.3074 & 99.9900 & -3.6175 & -1.6826 & $-3.6 \%$ & $-1.7 \%$ \\
\hline HTLG-75VS & $\mathrm{Al} 2 \mathrm{O} 3(\mathrm{wt} \%)$ & 8.5405 & 8.3978 & 8.3500 & 0.1905 & 0.0478 & $2.3 \%$ & $0.6 \%$ \\
\hline HTLG-75VS & B2O3 (wt\%) & 4.2100 & 4.2487 & 4.3200 & -0.1100 & -0.0713 & $-2.5 \%$ & $-1.7 \%$ \\
\hline HTLG-75VS & $\mathrm{CaO}(\mathrm{wt} \%)$ & 1.5566 & 1.5514 & 1.4600 & 0.0966 & 0.0914 & $6.6 \%$ & $6.3 \%$ \\
\hline HTLG-75VS & Cr2O3 (wt $\%)$ & 0.1082 & 0.1064 & 0.1300 & -0.0218 & -0.0236 & $-16.8 \%$ & $-18.2 \%$ \\
\hline HTLG-75VS & $\mathrm{CuO}(\mathrm{wt} \%)$ & 0.0582 & 0.0598 & 0.0500 & 0.0082 & 0.0098 & $16.4 \%$ & $19.6 \%$ \\
\hline HTLG-75VS & Fe2O3 (wt\%) & 16.8347 & 17.5220 & 18.7400 & -1.9053 & -1.2180 & $-10.2 \%$ & $-6.5 \%$ \\
\hline HTLG-75VS & K2O (wt\%) & 0.2457 & 0.2692 & 0.2300 & 0.0157 & 0.0392 & $6.8 \%$ & $17.0 \%$ \\
\hline HTLG-75VS & Li2O (wt\%) & 2.8795 & 2.8939 & 2.8800 & -0.0005 & 0.0139 & $0.0 \%$ & $0.5 \%$ \\
\hline HTLG-75VS & $\mathrm{MgO}(\mathrm{wt} \%)$ & 2.0480 & 2.1273 & 2.2400 & -0.1920 & -0.1127 & $-8.6 \%$ & $-5.0 \%$ \\
\hline HTLG-75VS & $\mathrm{MnO}(\mathrm{wt} \%)$ & 3.6089 & 3.6273 & 3.5000 & 0.1089 & 0.1273 & $3.1 \%$ & $3.6 \%$ \\
\hline HTLG-75VS & $\mathrm{Na} 2 \mathrm{O}$ (wt\%) & 14.5247 & 13.5899 & 14.6400 & -0.1153 & -1.0501 & $-0.8 \%$ & $-7.2 \%$ \\
\hline HTLG-75VS & $\mathrm{NiO}$ (wt\%) & 0.9894 & 1.1343 & 1.1300 & -0.1406 & 0.0043 & $-12.4 \%$ & $0.4 \%$ \\
\hline HTLG-75VS & SiO2 (wt\%) & 40.5665 & 42.6820 & 41.6700 & -1.1035 & 1.0120 & $-2.6 \%$ & $2.4 \%$ \\
\hline HTLG-75VS & SO4 (wt\%) & 0.3767 & 0.3767 & 0.4900 & -0.1133 & -0.1133 & $-23.1 \%$ & $-23.1 \%$ \\
\hline HTLG-75VS & TiO2 (wt\%) & 0.0450 & 0.0487 & 0.0400 & 0.0050 & 0.0087 & $12.6 \%$ & $21.8 \%$ \\
\hline HTLG-75VS & ZrO2 (wt\%) & 0.1182 & 0.1182 & 0.1200 & -0.0018 & -0.0018 & $-1.5 \%$ & $-1.5 \%$ \\
\hline HTLG-75VS & Oxide Sum (wt\%) & 96.7109 & 98.7538 & 99.9900 & -3.2791 & -1.2362 & $-3.3 \%$ & $-1.2 \%$ \\
\hline HTLG-76VS & Al2O3 (wt\%) & 9.4144 & 9.2569 & 9.2300 & 0.1844 & 0.0269 & $2.0 \%$ & $0.3 \%$ \\
\hline HTLG-76VS & B2O3 (wt\%) & 4.3147 & 4.2189 & 4.3200 & -0.0053 & -0.1011 & $-0.1 \%$ & $-2.3 \%$ \\
\hline HTLG-76VS & $\mathrm{CaO}$ (wt\%) & 2.1828 & 2.1755 & 1.9800 & 0.2028 & 0.1955 & $10.2 \%$ & $9.9 \%$ \\
\hline HTLG-76VS & Cr2O3 (wt\%) & 0.1118 & 0.1099 & 0.1300 & -0.0182 & -0.0201 & $-14.0 \%$ & $-15.4 \%$ \\
\hline HTLG-76VS & $\mathrm{CuO}(\mathrm{wt} \%)$ & 0.0576 & 0.0591 & 0.0500 & 0.0076 & 0.0091 & $15.2 \%$ & $18.3 \%$ \\
\hline HTLG-76VS & Fe2O3 (wt\%) & 18.4074 & 17.9991 & 18.4800 & -0.0726 & -0.4809 & $-0.4 \%$ & $-2.6 \%$ \\
\hline HTLG-76VS & K2O (wt\%) & 0.2548 & 0.2791 & 0.2300 & 0.0248 & 0.0491 & $10.8 \%$ & $21.3 \%$ \\
\hline HTLG-76VS & Li2O (wt\%) & 2.8472 & 2.8291 & 2.8800 & -0.0328 & -0.0509 & $-1.1 \%$ & $-1.8 \%$ \\
\hline HTLG-76VS & $\mathrm{MgO}(\mathrm{wt} \%)$ & 2.0148 & 2.0926 & 2.2400 & -0.2252 & -0.1474 & $-10.1 \%$ & $-6.6 \%$ \\
\hline HTLG-76VS & $\mathrm{MnO}(\mathrm{wt} \%)$ & 3.8413 & 3.8610 & 3.8700 & -0.0287 & -0.0090 & $-0.7 \%$ & $-0.2 \%$ \\
\hline HTLG-76VS & $\mathrm{Na} 2 \mathrm{O}$ (wt\%) & 13.4497 & 12.5840 & 13.3800 & 0.0697 & -0.7960 & $0.5 \%$ & $-5.9 \%$ \\
\hline HTLG-76VS & $\mathrm{NiO}$ (wt\%) & 0.7330 & 0.8403 & 0.8700 & -0.1370 & -0.0297 & $-15.8 \%$ & $-3.4 \%$ \\
\hline HTLG-76VS & SiO2 (wt\%) & 40.8606 & 42.2385 & 41.6700 & -0.8094 & 0.5685 & $-1.9 \%$ & $1.4 \%$ \\
\hline HTLG-76VS & SO4 (wt\%) & 0.4262 & 0.4262 & 0.4900 & -0.0638 & -0.0638 & $-13.0 \%$ & $-13.0 \%$ \\
\hline HTLG-76VS & TiO2 (wt\%) & 0.0446 & 0.0483 & 0.0400 & 0.0046 & 0.0083 & $11.5 \%$ & $20.7 \%$ \\
\hline HTLG-76VS & $\mathrm{ZrO} 2$ (wt\%) & 0.1260 & 0.1260 & 0.1200 & 0.0060 & 0.0060 & $5.0 \%$ & $5.0 \%$ \\
\hline HTLG-76VS & Oxide Sum (wt\%) & 99.0868 & 99.1445 & 99.9800 & -0.8932 & -0.8355 & $-0.9 \%$ & $-0.8 \%$ \\
\hline HTLG-77VS & $\mathrm{Al} 2 \mathrm{O} 3$ (wt\%) & 9.4617 & 9.3035 & 9.2300 & 0.2317 & 0.0735 & $2.5 \%$ & $0.8 \%$ \\
\hline HTLG-77VS & $\mathrm{B} 2 \mathrm{O} 3(\mathrm{wt} \%)$ & 4.2744 & 4.3150 & 4.3200 & -0.0456 & -0.0050 & $-1.1 \%$ & $-0.1 \%$ \\
\hline HTLG-77VS & $\mathrm{CaO}$ (wt\%) & 1.5566 & 1.5491 & 1.4600 & 0.0966 & 0.0891 & $6.6 \%$ & $6.1 \%$ \\
\hline HTLG-77VS & Cr2O3 (wt\%) & 0.0555 & 0.0535 & 0.0700 & -0.0145 & -0.0165 & $-20.7 \%$ & $-23.6 \%$ \\
\hline HTLG-77VS & $\mathrm{CuO}(\mathrm{wt} \%)$ & 0.0316 & 0.0325 & 0.0300 & 0.0016 & 0.0025 & $5.4 \%$ & $8.3 \%$ \\
\hline HTLG-77VS & Fe2O3 (wt\%) & 17.7640 & 18.4959 & 19.7800 & -2.0160 & -1.2841 & $-10.2 \%$ & $-6.5 \%$ \\
\hline HTLG-77VS & K2O (wt\%) & 0.1313 & 0.1455 & 0.1200 & 0.0113 & 0.0255 & $9.4 \%$ & $21.3 \%$ \\
\hline HTLG-77VS & Li2O (wt\%) & 2.8795 & 2.8939 & 2.8800 & -0.0005 & 0.0139 & $0.0 \%$ & $0.5 \%$ \\
\hline HTLG-77VS & $\mathrm{MgO}(\mathrm{wt} \%)$ & 1.5837 & 1.6100 & 1.7200 & -0.1363 & -0.1100 & $-7.9 \%$ & $-6.4 \%$ \\
\hline HTLG-77VS & $\mathrm{MnO}$ (wt\%) & 3.9930 & 3.9980 & 3.8700 & 0.1230 & 0.1280 & $3.2 \%$ & $3.3 \%$ \\
\hline HTLG-77VS & $\mathrm{Na} 2 \mathrm{O}$ (wt\%) & 13.5474 & 12.6959 & 13.3800 & 0.1674 & -0.6841 & $1.3 \%$ & $-5.1 \%$ \\
\hline HTLG-77VS & $\mathrm{NiO}$ (wt\%) & 0.9722 & 1.0944 & 1.1300 & -0.1578 & -0.0356 & $-14.0 \%$ & $-3.2 \%$ \\
\hline HTLG-77VS & $\mathrm{SiO} 2(\mathrm{wt} \%)$ & 41.0478 & 43.0162 & 41.6700 & -0.6222 & 1.3462 & $-1.5 \%$ & $3.2 \%$ \\
\hline HTLG-77VS & SO4 (wt\%) & 0.2614 & 0.2614 & 0.2600 & 0.0014 & 0.0014 & $0.5 \%$ & $0.5 \%$ \\
\hline HTLG-77VS & TiO2 (wt\%) & 0.0238 & 0.0260 & 0.0200 & 0.0038 & 0.0060 & $18.8 \%$ & $30.0 \%$ \\
\hline HTLG-77VS & $\mathrm{ZrO} 2(\mathrm{wt} \%)$ & 0.0686 & 0.0686 & 0.0600 & 0.0086 & 0.0086 & $14.3 \%$ & $14.3 \%$ \\
\hline HTLG-77VS & Oxide Sum (wt\%) & 97.6525 & 99.5594 & 100.0000 & -2.3475 & -0.4406 & $-2.3 \%$ & $-0.4 \%$ \\
\hline
\end{tabular}


WSRC-STI-2007-00616

Revision 0

Table A6. Average Measured and Bias-Corrected Chemical Compositions Versus Targeted Compositions by Oxide for the HTL Study Glasses

\begin{tabular}{|c|c|c|c|c|c|c|c|c|}
\hline & & & Measured & & & & & \\
\hline & & Measured & Bias-Corrected & Targeted & Diff of & Diff of & \% Diff of & \% Diff of \\
\hline Glass ID & Oxide & $(\mathrm{wt} \%)$ & $(\mathrm{wt} \%)$ & $(\mathrm{wt} \%)$ & Measured & Meas BC & Measured & Meas BC \\
\hline HTLG-78VS & $\mathrm{Al} 2 \mathrm{O} 3$ (wt\%) & 8.4791 & 8.3374 & 8.3500 & 0.1291 & -0.0126 & $1.5 \%$ & $-0.2 \%$ \\
\hline HTLG-78VS & $\mathrm{B} 2 \mathrm{O} 3(\mathrm{wt} \%)$ & 4.3630 & 4.2660 & 4.3200 & 0.0430 & -0.0540 & $1.0 \%$ & $-1.3 \%$ \\
\hline HTLG-78VS & $\mathrm{CaO}$ (wt\%) & 2.1128 & 2.1026 & 1.9800 & 0.1328 & 0.1226 & $6.7 \%$ & $6.2 \%$ \\
\hline HTLG-78VS & Cr2O3 (wt\%) & 0.1030 & 0.0993 & 0.1300 & -0.0270 & -0.0307 & $-20.7 \%$ & $-23.6 \%$ \\
\hline HTLG-78VS & $\mathrm{CuO}(\mathrm{wt} \%)$ & 0.0566 & 0.0582 & 0.0500 & 0.0066 & 0.0082 & $13.3 \%$ & $16.5 \%$ \\
\hline HTLG-78VS & $\mathrm{Fe} 2 \mathrm{O} 3$ (wt\%) & 19.6584 & 19.2199 & 20.3700 & -0.7116 & -1.1501 & $-3.5 \%$ & $-5.6 \%$ \\
\hline HTLG-78VS & K2O (wt\%) & 0.2454 & 0.2721 & 0.2300 & 0.0154 & 0.0421 & $6.7 \%$ & $18.3 \%$ \\
\hline HTLG-78VS & Li2O (wt\%) & 2.8687 & 2.8509 & 2.8800 & -0.0113 & -0.0291 & $-0.4 \%$ & $-1.0 \%$ \\
\hline HTLG-78VS & $\mathrm{MgO}(\mathrm{wt} \%)$ & 1.6998 & 1.7281 & 1.8700 & -0.1702 & -0.1419 & $-9.1 \%$ & $-7.6 \%$ \\
\hline HTLG-78VS & $\mathrm{MnO}(\mathrm{wt} \%)$ & 3.5573 & 3.5618 & 3.5000 & 0.0573 & 0.0618 & $1.6 \%$ & $1.8 \%$ \\
\hline HTLG-78VS & $\mathrm{Na} 2 \mathrm{O}$ (wt\%) & 13.3789 & 12.5378 & 13.3800 & -0.0011 & -0.8422 & $0.0 \%$ & $-6.3 \%$ \\
\hline HTLG-78VS & $\mathrm{NiO}$ (wt\%) & 0.9665 & 1.0879 & 1.1300 & -0.1635 & -0.0421 & $-14.5 \%$ & $-3.7 \%$ \\
\hline HTLG-78VS & $\mathrm{SiO} 2$ (wt\%) & 40.7269 & 41.9371 & 41.1500 & -0.4231 & 0.7871 & $-1.0 \%$ & $1.9 \%$ \\
\hline HTLG-78VS & SO4 (wt\%) & 0.4015 & 0.4015 & 0.4900 & -0.0885 & -0.0885 & $-18.1 \%$ & $-18.1 \%$ \\
\hline HTLG-78VS & $\mathrm{TiO} 2(\mathrm{wt} \%)$ & 0.0417 & 0.0456 & 0.0400 & 0.0017 & 0.0056 & $4.3 \%$ & $14.1 \%$ \\
\hline HTLG-78VS & $\mathrm{ZrO} 2$ (wt\%) & 0.1168 & 0.1168 & 0.1200 & -0.0032 & -0.0032 & $-2.6 \%$ & $-2.6 \%$ \\
\hline HTLG-78VS & Oxide Sum (wt\%) & 98.7764 & 98.6232 & 99.9900 & -1.2136 & -1.3668 & $-1.2 \%$ & $-1.4 \%$ \\
\hline HTLG-79VS & $\mathrm{Al} 2 \mathrm{O} 3$ (wt\%) & 8.5453 & 8.4024 & 8.3500 & 0.1953 & 0.0524 & $2.3 \%$ & $0.6 \%$ \\
\hline HTLG-79VS & B2O3 (wt\%) & 4.3469 & 4.2507 & 4.3200 & 0.0269 & -0.0693 & $0.6 \%$ & $-1.6 \%$ \\
\hline HTLG-79VS & $\mathrm{CaO}(\mathrm{wt} \%)$ & 2.1583 & 2.1479 & 1.9800 & 0.1783 & 0.1679 & $9.0 \%$ & $8.5 \%$ \\
\hline HTLG-79VS & Cr2O3 (wt\%) & 0.0574 & 0.0553 & 0.0700 & -0.0126 & -0.0147 & $-18.0 \%$ & $-21.1 \%$ \\
\hline HTLG-79VS & $\mathrm{CuO}(\mathrm{wt} \%)$ & 0.0347 & 0.0357 & 0.0300 & 0.0047 & 0.0057 & $15.8 \%$ & $19.1 \%$ \\
\hline HTLG-79VS & Fe2O3 (wt\%) & 17.1207 & 16.7402 & 18.8900 & -1.7693 & -2.1498 & $-9.4 \%$ & $-11.4 \%$ \\
\hline HTLG-79VS & K2O (wt\%) & 0.1364 & 0.1512 & 0.1200 & 0.0164 & 0.0312 & $13.7 \%$ & $26.0 \%$ \\
\hline HTLG-79VS & Li2O (wt\%) & 2.8418 & 2.8241 & 2.8800 & -0.0382 & -0.0559 & $-1.3 \%$ & $-1.9 \%$ \\
\hline HTLG-79VS & MgO (wt\%) & 1.9941 & 2.0271 & 2.2400 & -0.2459 & -0.2129 & $-11.0 \%$ & $-9.5 \%$ \\
\hline HTLG-79VS & $\mathrm{MnO}(\mathrm{wt} \%)$ & 3.8833 & 3.8883 & 3.8700 & 0.0133 & 0.0183 & $0.3 \%$ & $0.5 \%$ \\
\hline HTLG-79VS & $\mathrm{Na} 2 \mathrm{O}(\mathrm{wt} \%)$ & 14.6595 & 13.7382 & 14.6400 & 0.0195 & -0.9018 & $0.1 \%$ & $-6.2 \%$ \\
\hline HTLG-79VS & $\mathrm{NiO}(\mathrm{wt} \%)$ & 0.9445 & 1.0632 & 1.1300 & -0.1855 & -0.0668 & $-16.4 \%$ & $-5.9 \%$ \\
\hline HTLG-79VS & SiO2 (wt\%) & 40.3525 & 41.5580 & 41.1500 & -0.7975 & 0.4080 & $-1.9 \%$ & $1.0 \%$ \\
\hline HTLG-79VS & SO4 (wt\%) & 0.2569 & 0.2569 & 0.2600 & -0.0031 & -0.0031 & $-1.2 \%$ & $-1.2 \%$ \\
\hline HTLG-79VS & $\mathrm{TiO} 2(\mathrm{wt} \%)$ & 0.0234 & 0.0255 & 0.0200 & 0.0034 & 0.0055 & $16.8 \%$ & $27.7 \%$ \\
\hline HTLG-79VS & $\mathrm{ZrO} 2$ (wt\%) & 0.0601 & 0.0601 & 0.0600 & 0.0001 & 0.0001 & $0.2 \%$ & $0.2 \%$ \\
\hline HTLG-79VS & Oxide Sum (wt\%) & 97.4157 & 97.2248 & 100.0100 & -2.5943 & -2.7852 & $-2.6 \%$ & $-2.8 \%$ \\
\hline
\end{tabular}




\section{Exhibit A1. Oxide Measurements in Analytical Sequence for Series "P" Samples Prepared Using the LM Method}
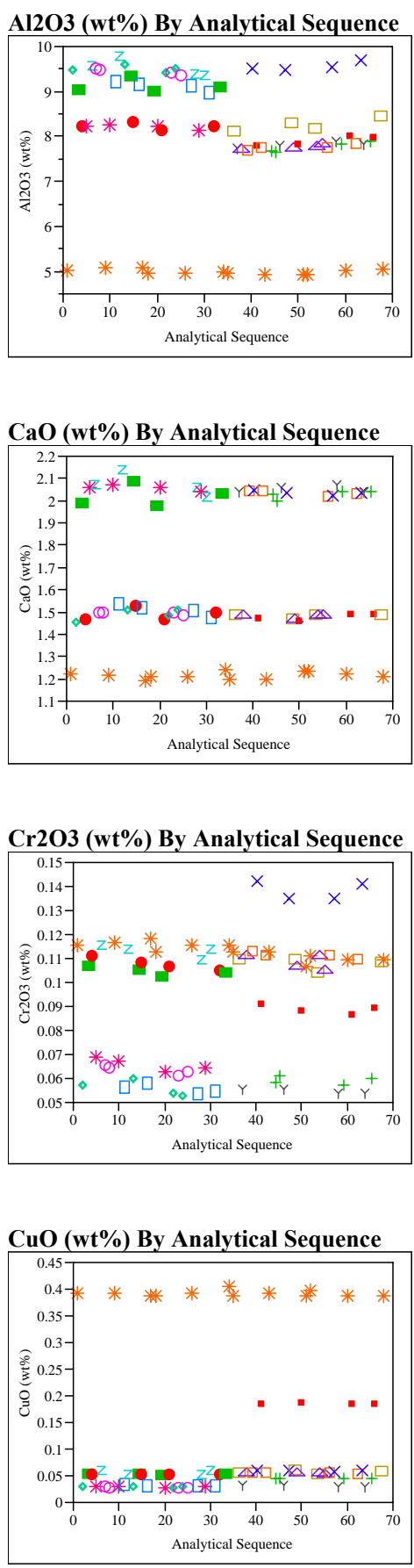
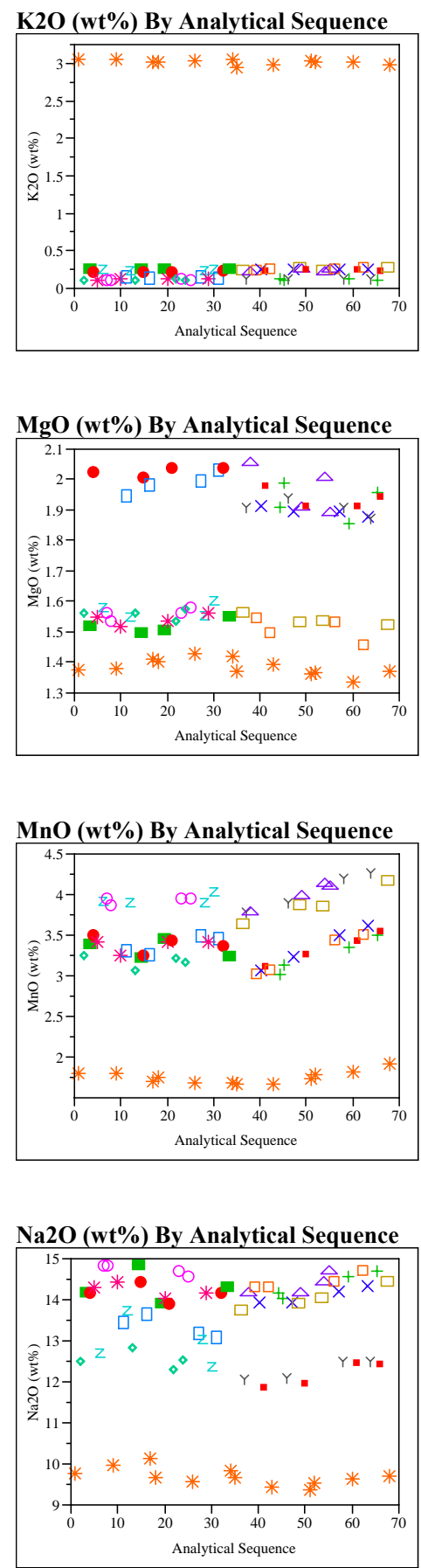
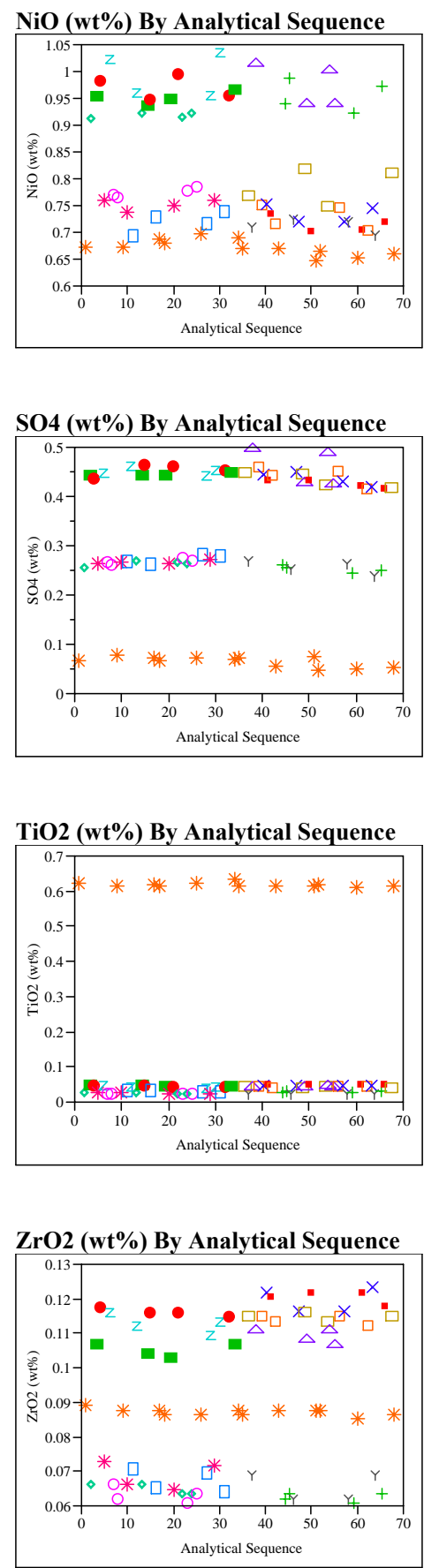


\section{Exhibit A2. Measurements in Analytical Sequence for Series "P" Samples Prepared Using the PF Method}
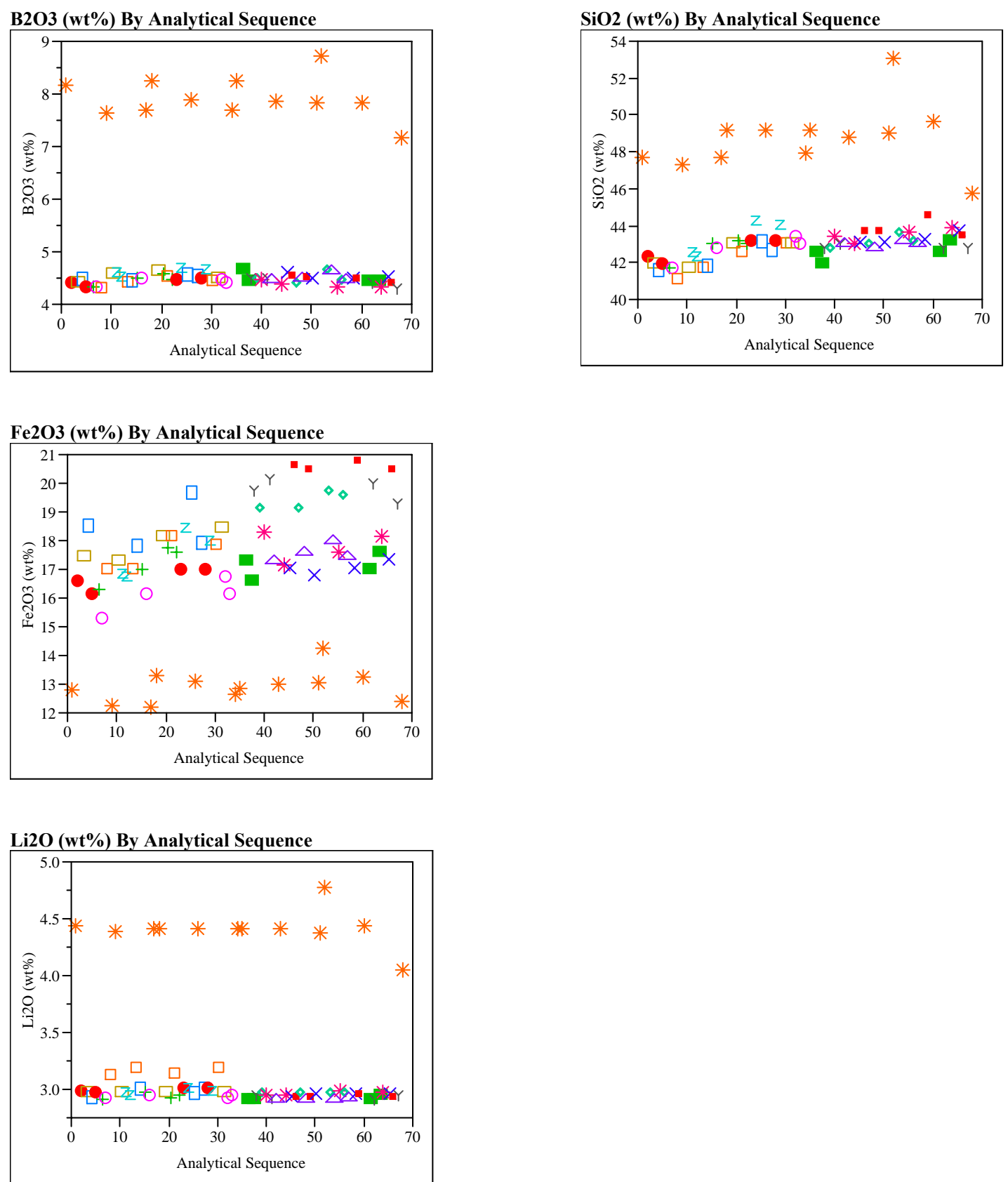
WSRC-STI-2007-00616

Revision 0

\section{Exhibit A3. Measurements in Analytical Sequence for Series "Q" Samples Prepared Using the LM Method}

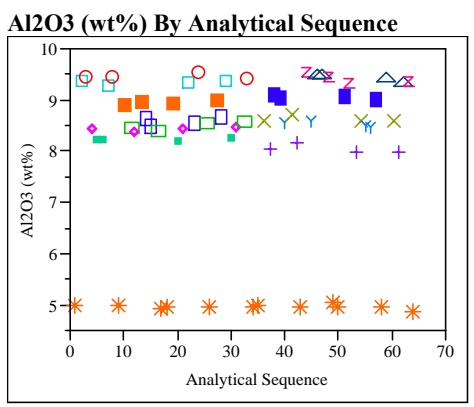

$\mathrm{CaO}(w \mathrm{t} \%)$ By Analytical Sequence

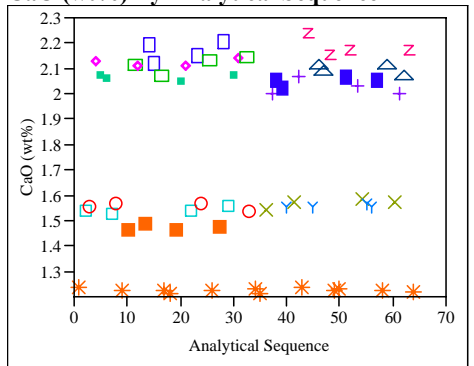

Cr2O3 (wt\%) By Analytical Sequence

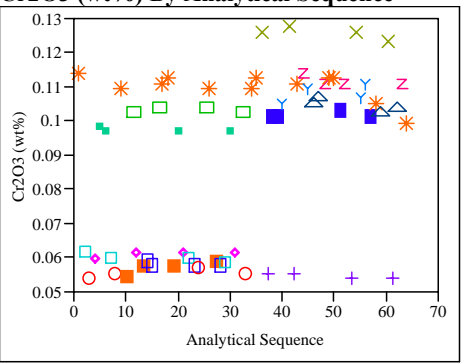

$\mathrm{CuO}(\mathrm{wt} \%)$ By Analytical Sequence

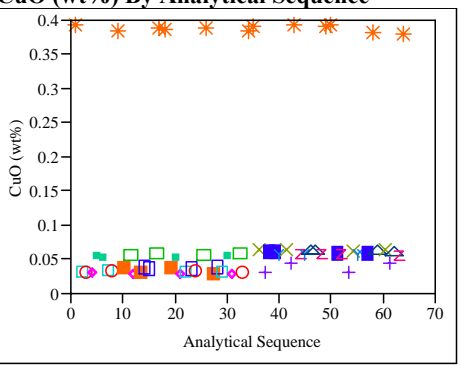

K2O (wt\%) By Analytical Sequence

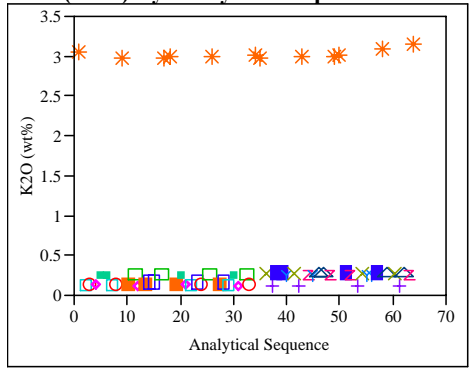

MgO (wt\%) By Analytical Sequence

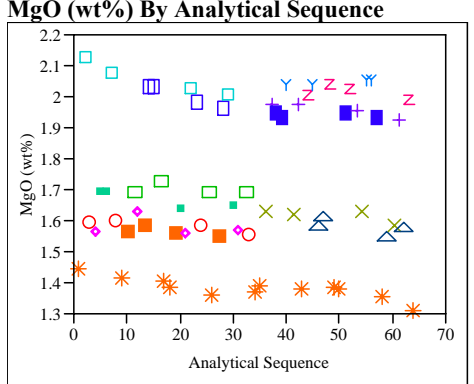

MnO (wt\%) By Analytical Sequence

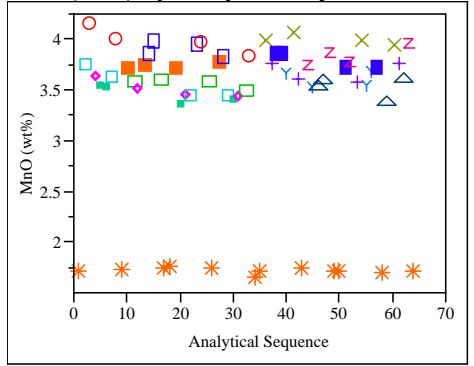

Na2O (wt\%) By Analytical Sequence

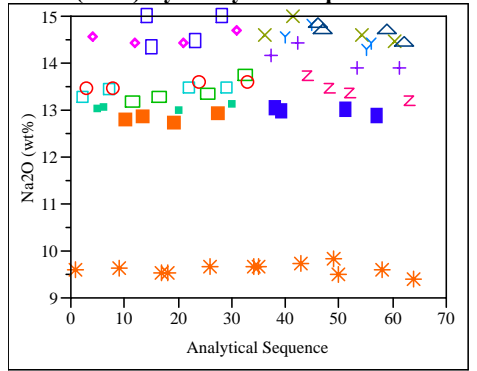

NiO (wt\%) By Analytical Sequence

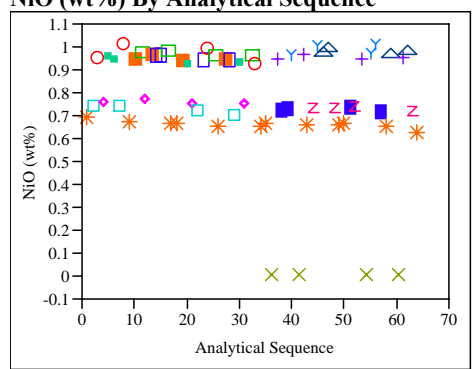

SO4 (wt\%) By Analytical Sequence

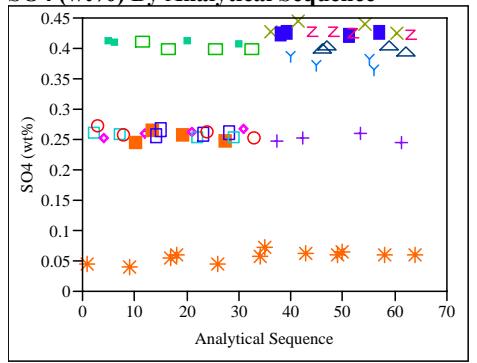

SiO2 (wt\%) By Analytical Sequence

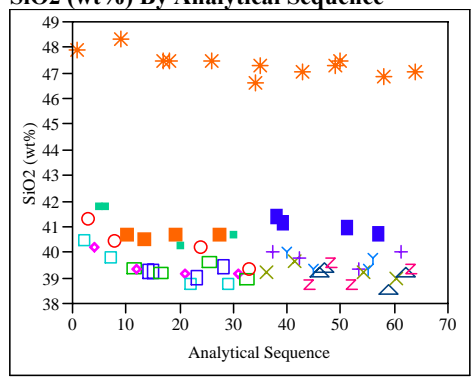

TiO2 (wt\%) By Analytical Sequence

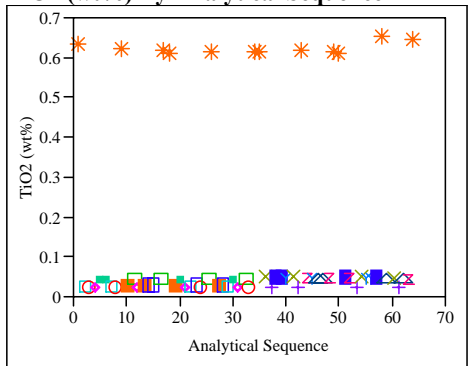

ZrO2 (wt\%) By Analytical Sequence

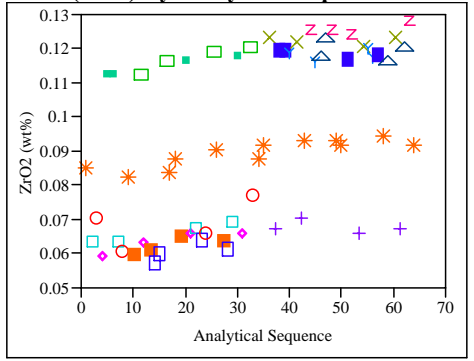




\section{Exhibit A4. Measurements in Analytical Sequence for Series "Q" Samples Prepared Using the PF Method}

Bivariate Fit of B2O3 (wt\%) By Analytical Sequence

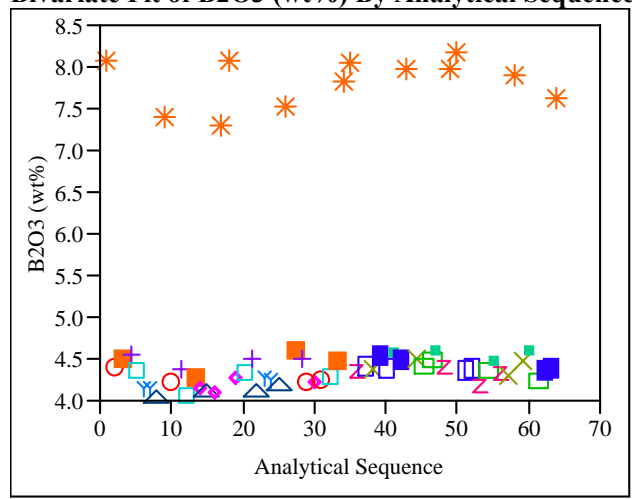

Bivariate Fit of Fe2O3 (wt\%) By Analytical Sequence

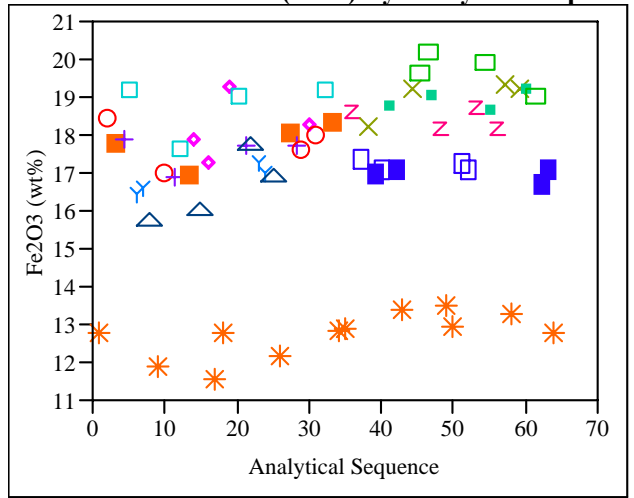

Bivariate Fit of Li2O (wt\%) By Analytical Sequence

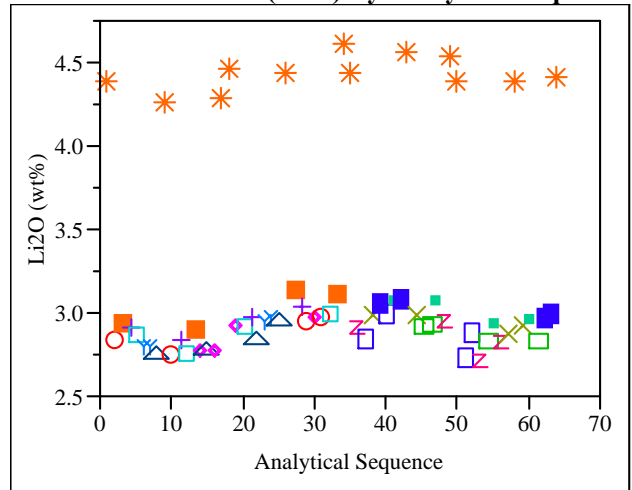

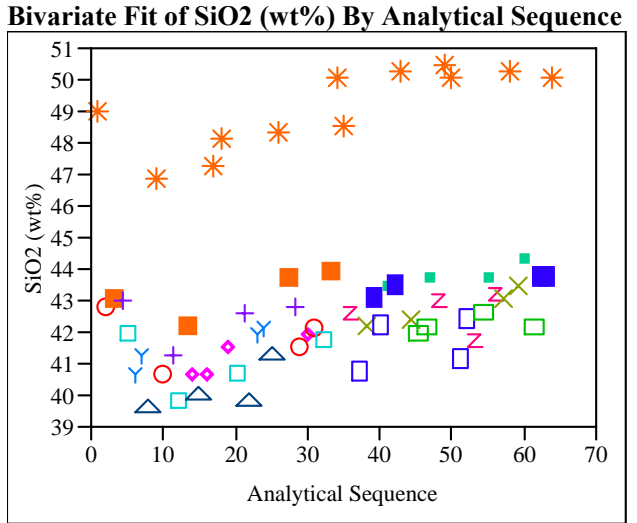




\section{Exhibit A5. PSAL Measurements by Analytical Block for Series "Q" Samples of the Batch 1 Standard Glass Prepared Using the LM Method}

Oneway Analysis of Al2O3 (wt\%) By Block/Sub-Block

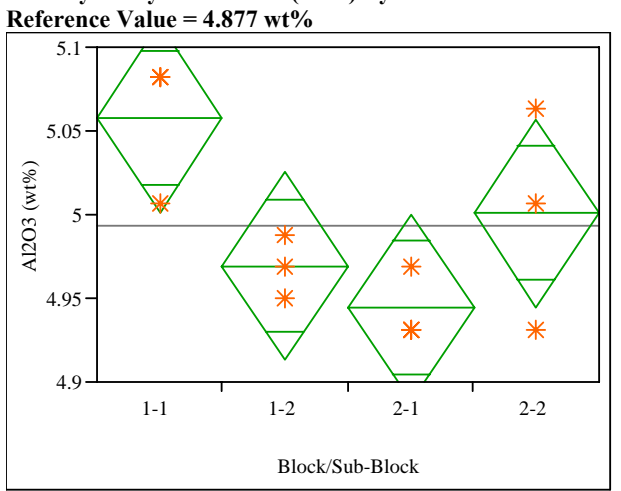

Oneway Anova

Summary of Fit

$\begin{array}{lr}\text { Rsquare } & 0.600998 \\ \text { Adj Rsquare } & 0.451372 \\ \text { Root Mean Square Error } & 0.042251 \\ \text { Mean of Response } & 4.993004 \\ \text { Observations (or Sum Wgts) } & 12\end{array}$

Observations (or Sum Wgts)

Source DF Sum of Squares Mean Square F Ratio Prob $>$ F $\begin{array}{lll}\text { Error } & 8 & 0.02151052 \\ \text { C. Block } & 3 & 0.01428084\end{array}$

C. Total

$11 \quad 0.03579136$

Means for Oneway Anova

Level Number Mean Std Error Lower 95\% Upper 95\%

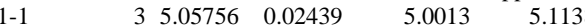

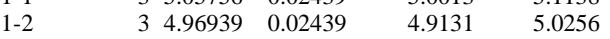

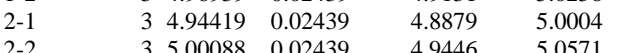

Std Error uses a pooled estimate of error variance
Oneway Analysis of $\mathrm{CaO}(\mathrm{wt} \%)$ By Block/Sub-Block Reference Value $=1.220 \mathrm{wt} \%$

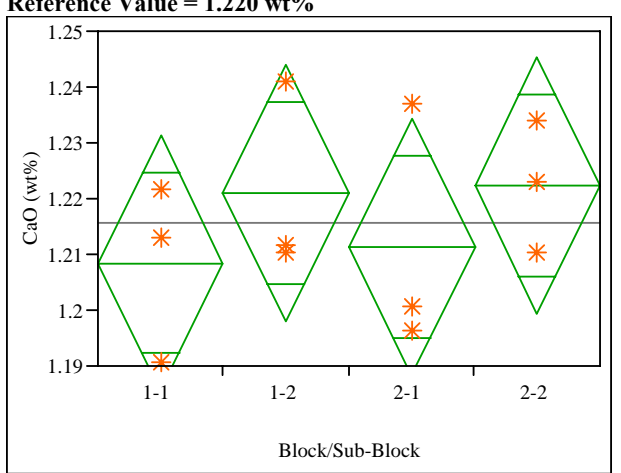

Oneway Anova

Summary of Fit

$\begin{array}{lr}\text { Rsquare } & 0.155199 \\ \text { Adj Rsquare } & -0.1616 \\ \text { Root Mean Square Error } & 0.017284 \\ \text { Mean of Response } & 1.215788 \\ \text { Observations (or Sum Wgts) } & 12\end{array}$

Analysis of Variance

Source DF Sum of Squares Mean Square F Ratio Prob > F $\begin{array}{llllll}\text { Block/Sub-Block } & 3 & 0.00043903 & 0.000146 & 0.4899 & 0.6989\end{array}$

$\begin{array}{lrrr}\text { Error } & 8 & 0.00238977 & 0.000299 \\ \text { C. Total } & 11 & 0.00282880 & \end{array}$

Level Number Mean Std Error Lower 95\% Upper 95\%

$\begin{array}{llllll}1-1 & 3 & 1.20844 & 0.00998 & 1.1854 & 1.2315\end{array}$

$\begin{array}{llllll}1-2 & 3 & 1.22104 & 0.00998 & 1.1980 & 1.2440\end{array}$

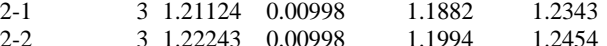

Std Error uses a pooled estimate of error variance
Oneway Analysis of $\mathrm{Cr} 203(\mathrm{wt} \%)$ By Block/Sub-Block Reference Value $=0.107 \mathbf{w t} \%$

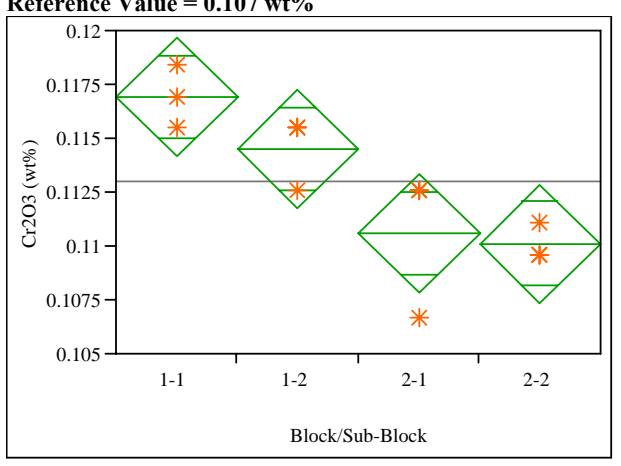

Oneway Anova

Summary of Fit

$\begin{array}{lr}\text { Rsquare } & 0.736264 \\ \text { Adj Rsquare } & 0.637363 \\ \text { Root Mean Square Error } & 0.002067 \\ \text { Mean of Response } & 0.11303 \\ \text { Observations (or Sum Wgts) } & 12\end{array}$

Analysis of Variance

Source DF Sum of Squares Mean Square F Ratio Prob > F $\begin{array}{lrrrrr}\text { Source } & \text { DF } & \text { Sum of Squares } & \text { Mean Square } & \text { F Ratio } & \text { Prob > F } \\ \text { Block/Sub-Block } & 3 & 0.00009542 & 0.000032 & 7.4444 & 0.0106\end{array}$ $\begin{array}{llll}\text { Error } & 8 & 0.00003418 & 4.273 \mathrm{e}-6\end{array}$

$\begin{array}{lll} & 11 & 0.0003418 \\ \text { C. Total } & 11 & 0.00012960\end{array}$

Means for Oneway Anova

Level Number Mean Std Error Lower 95\% Upper 95\% $\begin{array}{llllll}1-1 & 3 & 0.116928 & 0.00119 & 0.11418 & 0.11968\end{array}$

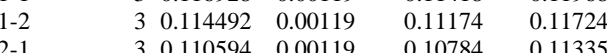

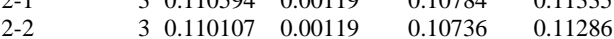

Std Error uses a pooled estimate of error variance 


\section{Exhibit A5. PSAL Measurements by Analytical Block for Series "Q" Samples of the Batch 1 Standard Glass Prepared Using the LM Method}

Oneway Analysis of $\mathrm{CuO}$ (wt\%) By Block/Sub-Block

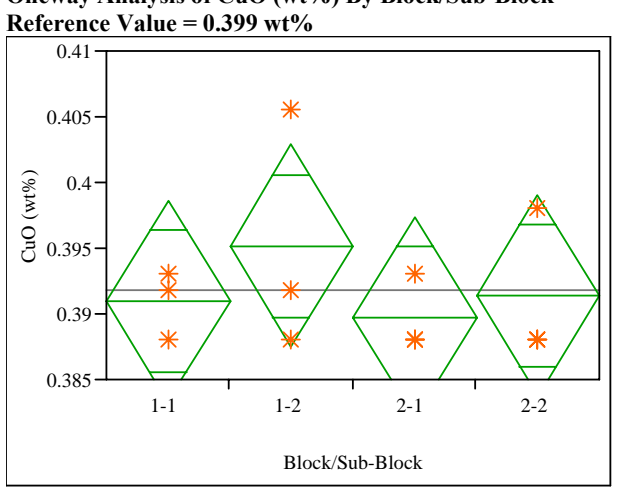

Oneway Anova

Summary of Fit

$\begin{array}{lr}\text { Rsquare } & 0.155116 \\ \text { Adj Rsquare } & -0.16172 \\ \text { Root Mean Square Error } & 0.005782 \\ \text { Mean of Response } & 0.391813 \\ \text { Observations (or Sum Wgts) } & 12\end{array}$

Observations (or Sum Wgts)

Analysis of Variance

Source DF Sum of Squares Mean Square F Ratio Prob > $\begin{array}{llllll}\text { Block/Sub-Block } & 3 & 0.00004910 & 0.000016 & 0.4896 & 0.699\end{array}$ Error $\quad 8 \quad 0.00026744$

C. Total $1 \quad 0.00031653$

Means for Oneway Anova

Level Number Mean Std Error Lower 95\% Upper 95\%

$\begin{array}{lllllll}1-1 & & 3 & 0.390979 & 0.00334 & 0.38328 & 0.39868\end{array}$

$\begin{array}{llllll}1-2 & 3 & 0.395152 & 0.00334 & 0.38745 & 0.40285\end{array}$

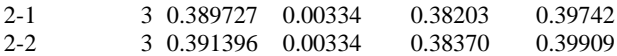

Std Error uses a pooled estimate of error variance
Oneway Analysis of K2O (wt\%) By Block/Sub-Block Reference Value $=3.327 \mathrm{wt} \%$

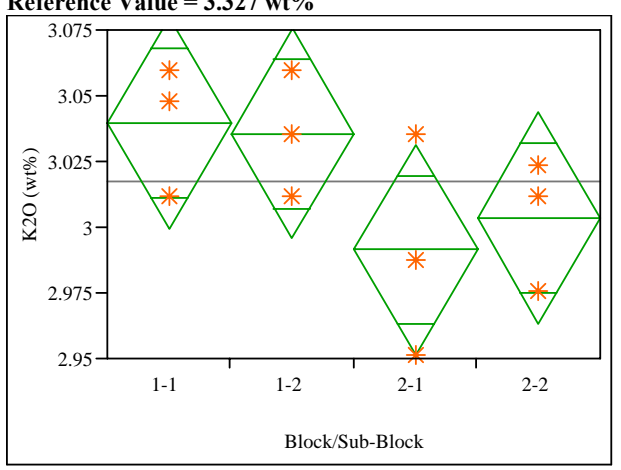

\section{Oneway Anova}

Summary of Fit

$\begin{array}{lr}\text { Rsquare } & 0.411765 \\ \text { Adj Rsquare } & 0.191176 \\ \text { Root Mean Square Error } & 0.030115 \\ \text { Mean of Response } & 3.017523 \\ \text { Observations (or Sum Wgts) } & 12\end{array}$

Analysis of Variance

Source DF Sum of Squares Mean Square F Ratio Prob $>$ F $\begin{array}{lllllll}\text { Block/Sub-Block } & 3 & 0.00507871 & 0.001693 & 1.8667 & 0.2136\end{array}$ Error 80.00725531 0.000907

C. Total $11 \quad 0.01233402$

Means for Oneway Anova

Level Number Mean Std Error Lower 95\% Upper 95\%

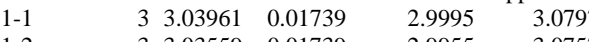

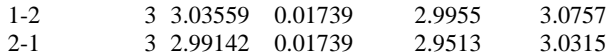

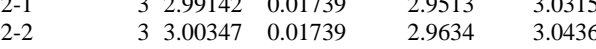

Std Error uses a pooled estimate of error variance
Oneway Analysis of $\mathrm{MgO}(\mathrm{wt} \%)$ By Block/Sub-Block Reference Value $=1.419 \mathrm{wt} \%$

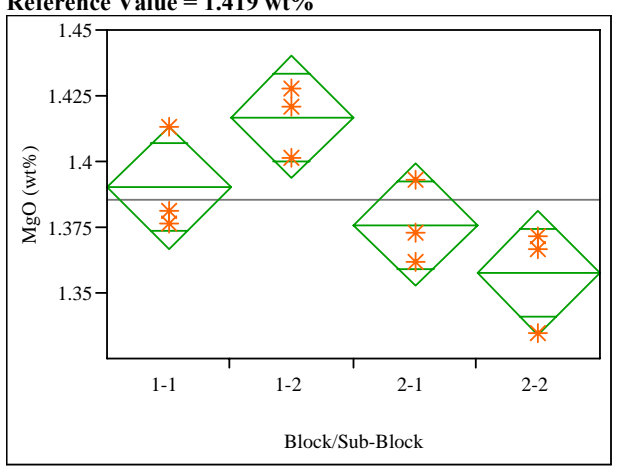

Oneway Anova

Summary of Fit

$\begin{array}{ll} & 0.695569 \\ \text { Adj Rsquare } & 0.581408\end{array}$

Root Mean Square Error $\quad 0.017517$

Mean of Response 1.385095

Observations (or Sum Wgts) 12

Analysis of Variance

DF Sum of Squares Mean Square F Ratio Prob > F $\begin{array}{lllllll}\text { Block/Sub-Block } & 3 & 0.00560877 & 0.001870 & 6.0929 & 0.0184\end{array}$ $\begin{array}{lll}\text { Error } & 8 & 0.00245480\end{array}$

$\begin{array}{lll}\text { C. Total } & 11 & 0.00806357\end{array}$

Means for Oneway Anova

Level Number Mean Std Error Lower 95\% Upper 95\%

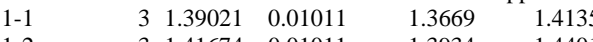

$\begin{array}{llllll}1-2 & 3 & 1.41674 & 0.01011 & 1.3934 & 1.4401\end{array}$

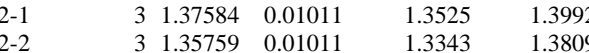

Std Error uses a pooled estimate of error variance 


\section{Exhibit A5. PSAL Measurements by Analytical Block for Series "Q" Samples of the Batch 1 Standard Glass Prepared Using the LM Method}

Oneway Analysis of MnO (wt\%) By Block/Sub-Block Reference Value $=1.726 \mathrm{wt} \%$

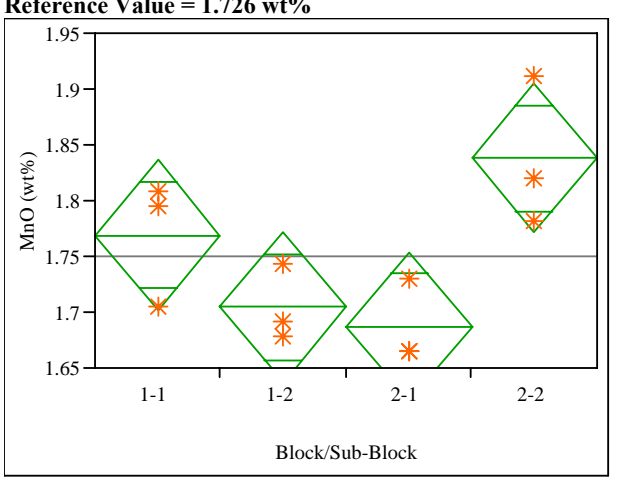

Oneway Anova

Summary of Fit

$\begin{array}{lr}\text { Rsquare } & 0.676444 \\ \text { Adj Rsquare } & 0.555111 \\ \text { Root Mean Square Error } & 0.050285 \\ \text { Mean of Response } & 1.749576 \\ \text { Observations (or Sum Wgts) } & 12\end{array}$

Analysis of Variance

Source DF Sum of Squares Mean Square F Ratio Prob > F $\begin{array}{llllll}\text { Block/Sub-Block } & 3 & 0.04229124 & 0.014097 & 5.5751 & 0.0232\end{array}$ $\begin{array}{lrrr}\text { Error } & 8 & 0.02022866 & 0.002529\end{array}$ 10.06251990

Means for Oneway Anova

Level Number Mean Std Error Lower 95\% Upper 95\%

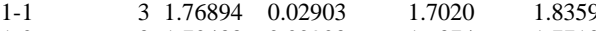

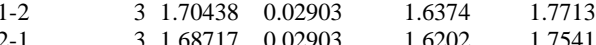

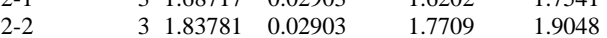

Std Error uses a pooled estimate of error variance
Oneway Analysis of $\mathrm{Na2O}(\mathrm{wt} \%)$ By Block/Sub-Block Reference Value $=9.003 \mathbf{w t} \%$

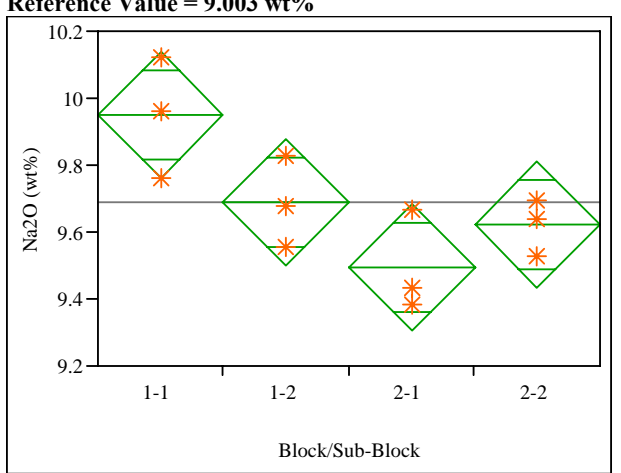

\section{Oneway Anova}

Summary of Fit

$\begin{array}{lr}\text { Rsquare } & 0.670696 \\ \text { Adj Rsquare } & 0.547206 \\ \text { Root Mean Square Error } & 0.14218 \\ \text { Mean of Response } & 9.687627 \\ \text { Observations (or Sum Wgts) } & 12\end{array}$

Analysis of Variance

Source DF Sum of Squares Mean Square F Ratio Prob > F $\begin{array}{lllllll}\text { Block/Sub-Block } & 3 & 0.32938039 & 0.109793 & 5.4312 & 0.0248\end{array}$ $\begin{array}{lrrr}\text { Error } & 8 & 0.16172226 & 0.020215\end{array}$

Means for Oneway Anova

Level Number Mean Std Error Lower 95\% Upper 95\% $\begin{array}{llllll}1-1 & 3 & 9.94824 & 0.08209 & 9.7589 & 10.138\end{array}$ $\begin{array}{llllll}1-2 & 3 & 9.68763 & 0.08209 & 9.4983 & 9.877\end{array}$

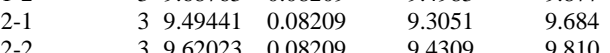

Std Error uses a pooled estimate of error variance
Oneway Analysis of NiO (wto) By Block/Sub-Block Reference Value $=0.751 \mathrm{wt} \%$

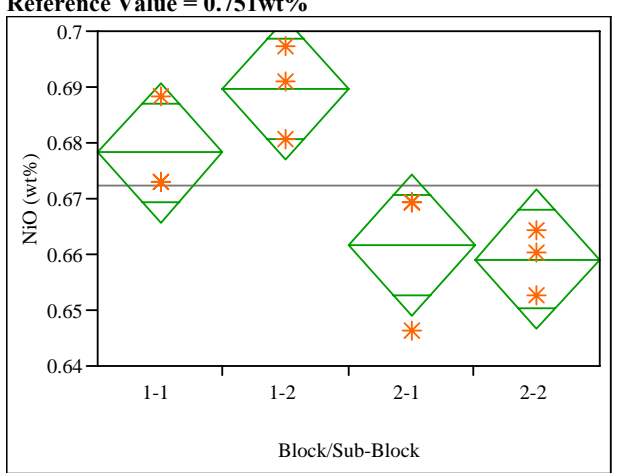

Oneway Anova

Summary of Fit

$\begin{array}{lr}\text { Rsquare } & 0.724008 \\ \text { Adj Rsquare } & 0.620511 \\ \text { Root Mean Square Error } & 0.009437 \\ \text { Mean of Response } & 0.672198 \\ \text { Observations (or Sum Wgts) } & 12\end{array}$

Observations (or Sum Wgts)

Analysis of Variance

Source DF Sum of Squares Mean Square F Ratio Prob > F $\begin{array}{llllll}\text { Block/Sub-Block } 3 & 0.00186903 & 0.000623 & 6.9955 & 0.0126\end{array}$ $\begin{array}{lrr}\text { Error } & 8 & 0.00071247 \\ \text { C. Total } & 11 & 0.00258150\end{array}$

Means for Oneway Anova

Level Number Mean Std Error Lower 95\% Upper 95\% $\begin{array}{llllll}1-1 & 3 & 0.678243 & 0.00545 & 0.66568 & 0.69081\end{array}$ $\begin{array}{llllll}1-2 & 3 & 0.689695 & 0.00545 & 0.67713 & 0.70226\end{array}$ $\begin{array}{llllll}2-1 & 3 & 0.661700 & 0.00545 & 0.64914 & 0.67426\end{array}$ $\begin{array}{llllll}2-2 & 3 & 0.659155 & 0.00545 & 0.64659 & 0.67172\end{array}$

Std Error uses a pooled estimate of error variance 


\section{Exhibit A5. PSAL Measurements by Analytical Block for Series "Q" Samples of the Batch 1 Standard Glass Prepared Using the LM Method}

Oneway Analysis of SO4 (wt\%) By Block/Sub-Block

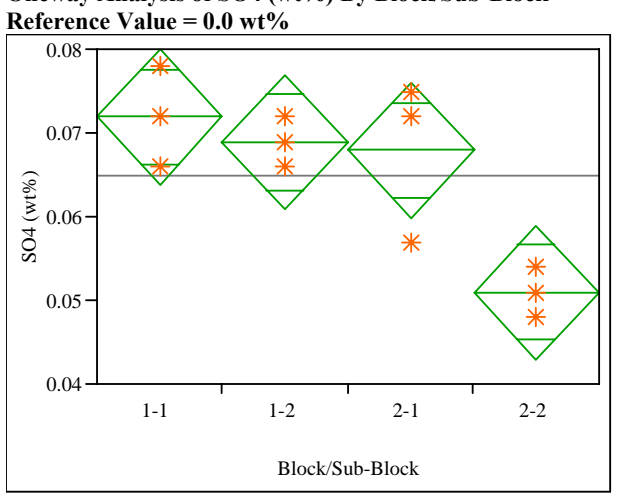

Oneway Anova

Summary of Fit

$\begin{array}{lr}\text { Rsquare } & 0.733696 \\ \text { Adj Rsquare } & 0.633832 \\ \text { Root Mean Square Error } & 0.006054 \\ \text { Mean of Response } & 0.064911 \\ \text { Observations (or Sum Wgts) } & 12\end{array}$

Analysis of Variance

Source DF Sum of Squares Mean Square F Ratio Prob $>$ F $\begin{array}{lrr}\text { Block/Sub-Block } & 3 & 0.00080779 \\ \text { Error } & 8 & 0.00029320\end{array}$

C. Total $11 \quad 0.00110098$

Means for Oneway Anova

Level Number Mean Std Error Lower 95\% Upper 95\% $\begin{array}{llllll}1-1 & 3 & 0.071902 & 0.00350 & 0.06384 & 0.07996\end{array}$

$\begin{array}{llllll}1-2 & 3 & 0.068906 & 0.00350 & 0.06085 & 0.07697\end{array}$

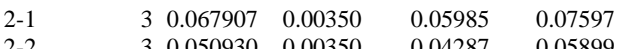

Std Error uses a pooled estimate of error variance
Oneway Analysis of TiO2 (wt\%) By Block/Sub-Block Reference Value $=0.677 \mathrm{wt} \%$

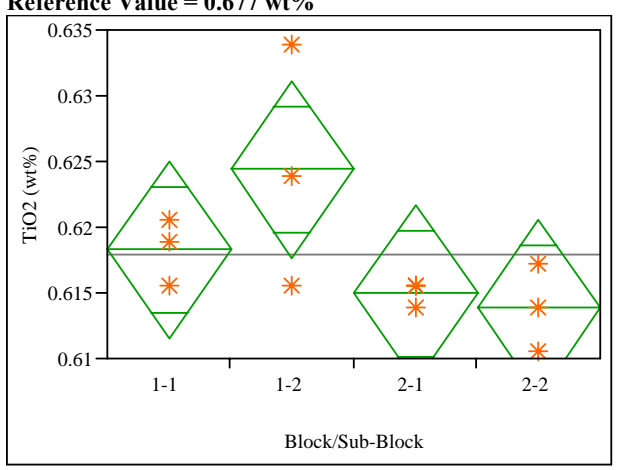

Oneway Anova

Summary of Fit

Adj Rsquare

0.307431

square Error $\quad 0.005073$

$\begin{array}{lr} & 0.617855 \\ \text { Observations (or Sum Wgts) } & 12\end{array}$

Analysis of Variance

Source DF Sum of Squares Mean Square F Ratio Prob > F $\begin{array}{llllll}\text { Block/Sub-Block } & 3 & 0.00020287 & 0.000068 & 2.6276 & 0.1221\end{array}$ $\begin{array}{lll}\text { Error } & 8 & 0.00020588\end{array}$

C. Total

$11 \quad 0.00040876$ 0.000026

Means for Oneway Anova

Level Number Mean Std Error Lower 95\% Upper 95\%

$\begin{array}{llllll}1-1 & 3 & 0.618272 & 0.00293 & 0.61152 & 0.62503\end{array}$

$\begin{array}{llllll}1-2 & 3 & 0.624388 & 0.00293 & 0.61763 & 0.63114\end{array}$

$\begin{array}{llllll}2-1 & 3 & 0.614936 & 0.00293 & 0.60818 & 0.62169\end{array}$

$\begin{array}{llllll}2-2 & 3 & 0.613824 & 0.00293 & 0.60707 & 0.62058\end{array}$

Std Error uses a pooled estimate of error variance
Oneway Analysis of ZrO2 (wt\%) By Block/Sub-Block Reference Value $=0.098 \mathrm{wt} \%$

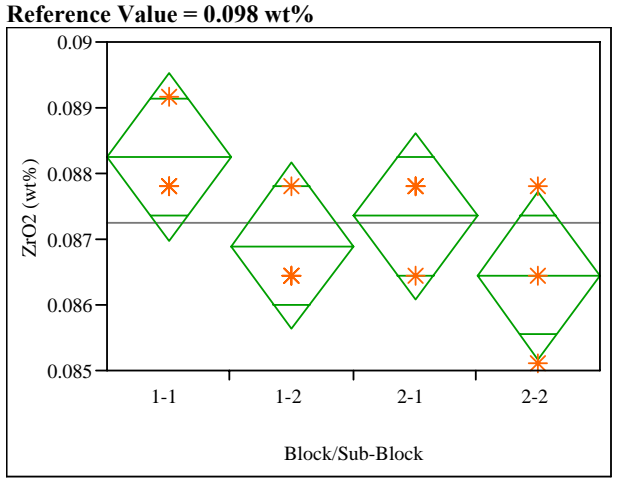

Oneway Anova

Summary of Fit

Adj Rsquare $\quad 0.204819$

Root Mean Square Error $\quad 0.000955$

Mean of Response 0.087239

Observations (or Sum Wgts) 12

Analysis of Variance

DF Sum of Squares Mean Square F Ratio Prob $>$ F $\begin{array}{llllll}\text { Block/Sub-Block } & 3 & 0.00000532 & 1.774 \mathrm{e}-6 & 1.9444 & 0.2011\end{array}$ $\begin{array}{lll}\text { Error } & 8 & 0.00000730\end{array}$

$\begin{array}{lll}\text { C. Total } & 11 & 0.00001262\end{array}$

Means for Oneway Anova

Level Number Mean Std Error Lower 95\% Upper 95\%

$\begin{array}{lllllll}1-1 & & 3 & 0.088252 & 0.00055 & 0.08698 & 0.08952\end{array}$

$\begin{array}{llllll}1-2 & 3 & 0.086901 & 0.00055 & 0.08563 & 0.08817\end{array}$

$\begin{array}{llllll}2-1 & 3 & 0.087352 & 0.00055 & 0.08608 & 0.08862\end{array}$

$\begin{array}{llllll}2-2 & 3 & 0.086451 & 0.00055 & 0.08518 & 0.08772\end{array}$

Std Error uses a pooled estimate of error variance 
Exhibit A6. PSAL Measurements by Analytical Block for Series "Q" Samples of the Batch 1 Standard Glass Prepared Using the PF Method

Oneway Analysis of B2O3 (wt\%) By Block/Sub-Block

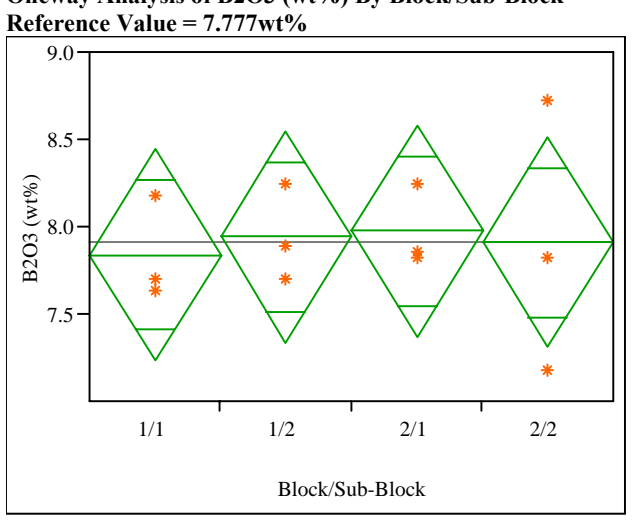

Oneway Anova

Summary of Fit

Adj Rsquare $\quad 0.01914$

Root Mean Square Error $\quad \begin{array}{r}-0.34868 \\ 0.453747\end{array}$

Mean of Response $\quad 7.915588$

Observations (or Sum Wgts) 12

Analysis of Variance

Source DF Sum of Squares Mean Square F Ratio Prob $>$ F

$\begin{array}{llllll}\text { Block/Sub-Block } & 3 & 0.0321400 & 0.010713 & 0.0520 & 0.9832\end{array}$

$\begin{array}{lrr}\text { Error } & 8 & 1.6470908\end{array}$

C. Total

$11 \quad 1.6792309$

Means for Oneway Anova

Level Number Mean Std Error Lower 95\% Upper 95\%

$\begin{array}{llllll}1 / 1 & 3 & 7.83509 & 0.26197 & 7.2310 & 8.4392\end{array}$

$\begin{array}{llllll}1 / 2 & 3 & 7.94242 & 0.26197 & 7.3383 & 8.5465 \\ & 3 & 7.97462 & 0.26197 & 7.3705 & 8.5787\end{array}$

$\begin{array}{llllll}2 / 1 & 3 & 7.97462 & 0.26197 & 7.3705 & 8.578\end{array}$

$\begin{array}{llllll}2 / 2 & 3 & 7.91022 & 0.26197 & 7.3061 & 8.5143\end{array}$

Std Error uses a pooled estimate of error variance
Oneway Analysis of Fe2O3 (wt\%) By Block/Sub-Block Reference Value $=\mathbf{1 2 . 8 3 9} \mathbf{w t} \%$

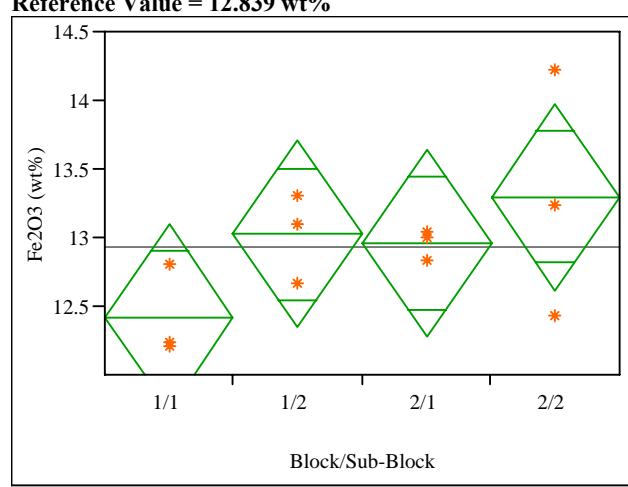

Oneway Anova

Summary of Fi

Rsquare

$\begin{array}{ll} & 0.366914 \\ \text { Adj Rsquare } & 0.129507\end{array}$

Adj Rsquare $\quad 0.129507$

Reot

Mean of Response 12.92449

Analysis of Variance

$\begin{array}{lrrrrr} & & & & \\ \text { Source } & \text { DF } & \text { Sum of Squares } & \text { Mean Square } & \text { F Ratio } & \text { Prob }>\text { F } \\ \text { Block/Sub-Block } & 3 & 1.2134797 & 0.404493 & 1.5455 & 0.2763\end{array}$

$\begin{array}{lrrr}\text { Block/Sub-Block } & 3 & 1.2134797 & 0.404493 \\ \text { Error } & 8 & 2.0937804 & 0.261723\end{array}$

$11 \quad 3.3072601$

Means for Oneway Anova

Level Number Mean Std Error Lower 95\% Upper 95\%

$\begin{array}{llllll}1 / 1 & 3 & 12.4193 & 0.29537 & 11.738 & 13.100\end{array}$

$\begin{array}{llllll}1 / 2 & 3 & 13.0246 & 0.29537 & 12.343 & 13.706 \\ 2 / 1 & 3 & 12.9578 & 0.29537 & 12.277 & 13.639\end{array}$

$\begin{array}{llllll}2 / 2 & 3 & 13.2962 & 0.29537 & 12.615 & 13.977\end{array}$

Std Error uses a pooled estimate of error variance 
Exhibit A6. PSAL Measurements by Analytical Block for Series "Q" Samples of the Batch 1 Standard Glass Prepared Using the PF Method

Oneway Analysis of Li2O (wt\%) By Block/Sub-Block

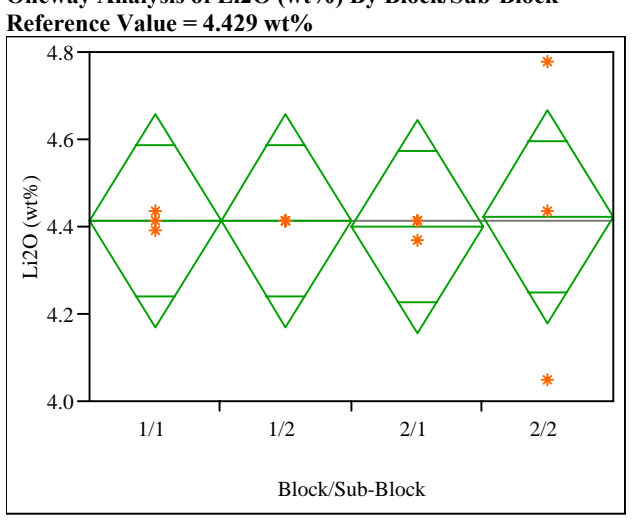

Oneway Anova

Summary of Fit

$\begin{array}{lr}\text { Rsquare } & 0.002707 \\ \text { Adj Rsquare } & -0.37128 \\ \text { Root Mean Square Error } & 0.183839 \\ \text { Mean of Response } & 4.411651 \\ \text { Observations (or Sum Wgts) } & 12\end{array}$

$\begin{array}{lll}\text { Analysis of Variance } & & \\ \text { Source } & \text { DF Sum of Squares Mean Square F Ratio Prob }>F\end{array}$

$\begin{array}{llllll}\text { Block/Sub-Block } & 3 & 0.00073387 & 0.000245 & 0.0072 & 0.9991\end{array}$

$\begin{array}{lll}\text { Error } & 8 & 0.27037374\end{array}$

0.033797

Means for Oneway Anova

Level Number Mean Std Error Lower 95\% Upper 95\%

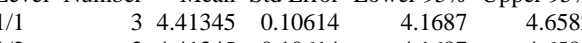

$\begin{array}{llllll}1 / 2 & 3 & 4.41345 & 0.10614 & 4.1687 & 4.6582\end{array}$

$\begin{array}{llllll}2 / 1 & 3 & 4.39909 & 0.10614 & 4.1543 & 4.6439\end{array}$

$\begin{array}{llllll}2 / 2 & 3 & 4.42062 & 0.10614 & 4.1759 & 4.6654\end{array}$

Std Error uses a pooled estimate of error variance
Oneway Analysis of SiO2 (wt\%) By Block/Sub-Block Reference Value $=\mathbf{5 0 . 2 2} \mathbf{w t} \%$

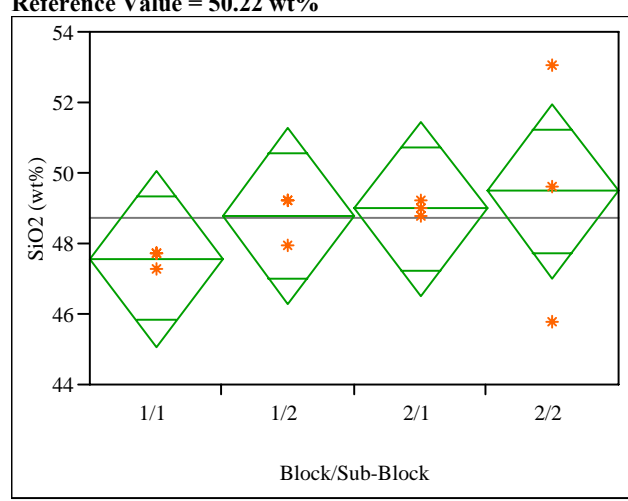

Oneway Anova

Summary of Fi

$\begin{array}{lr}\text { Rsquare } & 0.177798 \\ \text { Adj Rsquare } & -0.13053 \\ \text { Root Mean Square Error } & 1.863976 \\ \text { Mean of Response } & 48.70473 \\ \text { Observations (or Sum Wgts) } & 12\end{array}$

$\begin{array}{ll}\text { Mean of Response } & 48.70473 \\ \text { Observations (or Sum Wgts) }\end{array}$

Analysis of Variance

Source DF Sum of Squares Mean Square F Ratio Prob $>$ F

$\begin{array}{llllll}\text { Block/Sub-Block } & 3 & 6.010607 & 2.00354 & 0.5767 & 0.6464\end{array}$

$\begin{array}{lrrr}\text { Error } & 8 & 27.795245 & 3.47441 \\ \text { C. Total } & 11 & 33.805852 & \end{array}$

Level Number Mean Std Error Lower 95\% Upper 95\%

$\begin{array}{llllll}1 / 1 & 3 & 47.5638 & 1.0762 & 45.082 & 50.045\end{array}$

$\begin{array}{llllll}1 / 2 & 3 & 48.7760 & 1.0762 & 46.294 & 51.258 \\ 2 / 1 & 3 & 48.9900 & 1.0762 & 46.508 & 51.472\end{array}$

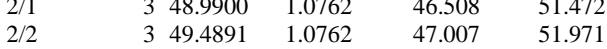

Std Error uses a pooled estimate of error variance 


\section{Exhibit A7. PSAL Measurements by Analytical Block for Series "P" Samples of the Batch 1 Standard Glass Prepared by LM}

Oneway Analysis of Al2O3 (wt\%) By Block/Sub-Block Reference Value $=4.8777 \mathrm{wt} \%$

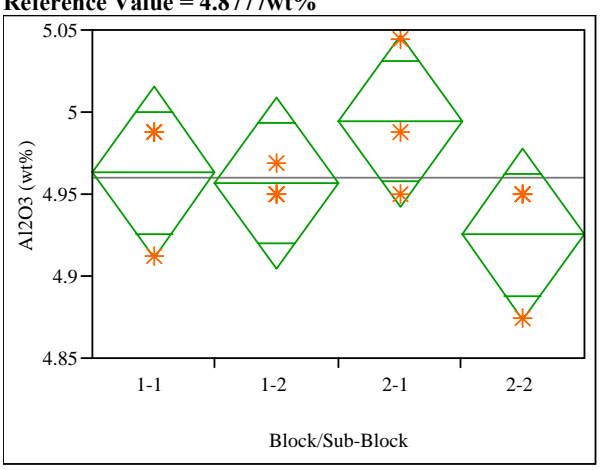

Oneway Anova

Summary of Fit

$\begin{array}{lr}\text { Rsquare } & 0.369697 \\ \text { Adj Rsquare } & 0.133333 \\ \text { Root Mean Square Error } & 0.039333 \\ \text { Mean of Response } & 4.959938 \\ \text { Observations (or Sum Wgts) } & 12\end{array}$

\begin{tabular}{|c|c|c|c|c|c|}
\hline \multicolumn{6}{|c|}{ Analysis of Variance } \\
\hline Source & DF & $\begin{array}{r}\text { Sum of } \\
\text { Squares }\end{array}$ & $\begin{array}{r}\text { Mean } \\
\text { Square }\end{array}$ & $\begin{array}{r}F \\
\text { Ratio }\end{array}$ & $\begin{array}{r}\text { Prob }> \\
F\end{array}$ \\
\hline $\begin{array}{l}\text { Block/Sub- } \\
\text { Block }\end{array}$ & 3 & 0.00725943 & 0.002420 & 1.5641 & 0.2721 \\
\hline Error & 8 & 0.01237673 & 0.001547 & & \\
\hline C. Total & 11 & 0.01963616 & & & \\
\hline
\end{tabular}

Means for Oneway Anova

Level Number Mean Std Error Lower 95\% Upper 95\%

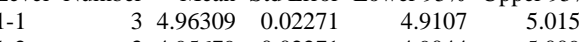

$\begin{array}{llllll}1-2 & 3 & 4.95679 & 0.02271 & 4.9044 & 5.0092\end{array}$

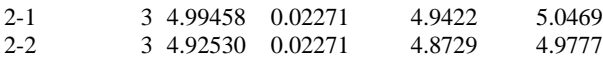

Std Error uses a pooled estimate of error variance
Oneway Analysis of $\mathrm{CaO}$ (wt\%) By Block/Sub-Block Reference Value $=1.220 \mathrm{wt} \%$

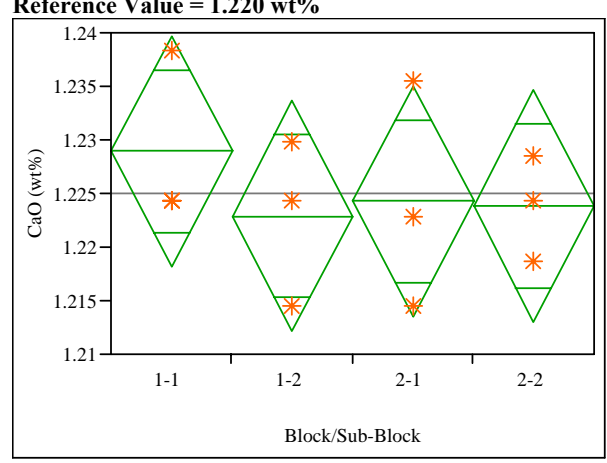

Oneway Anova

Summary of Fit

Rsquare

Adj Rsquare

0.22121
0.08088

Mean of Response $\quad 1.225$

Observations (or Sum Wgts) $\quad 12$

Analysis of Variance

$\begin{array}{llllll}\text { Source } & \text { DF } & \text { Sum of } & \text { Mean } & F & \text { Prob > }\end{array}$

$\begin{array}{lrrrrr} & & \text { Squares } & \text { Square } & \text { Ratio } & \text { F } \\ \text { Block/Sub- } & 3 & 0.00006591 & 0.000022 & 0.3358 & 0.8001\end{array}$

$\begin{array}{llll}\text { Block } & & & \\ \text { Error } & 8 & 0.00052337 & 0.000065\end{array}$

$\begin{array}{lrr}\text { Error } & 8 & 0.00052337 \\ \text { C. Total } & 11 & 0.00058929\end{array}$

Means for Oneway Anova

Level Number Mean Std Error Lower 95\% Upper 95\%

$\begin{array}{lrrrrr}\text { Level } & \text { Number } & \text { Mean } & \text { Std Error } & \text { Lower 95\% } & \text { Upper 95\% } \\ 1-1 & 3 & 1.22896 & 0.00467 & 1.2182 & 1.2397\end{array}$

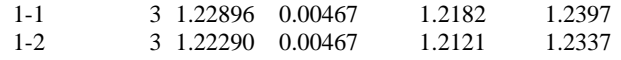

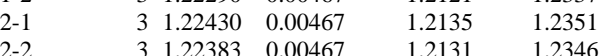

Std Error uses a pooled estimate of error variance
Oneway Analysis of Cr203 (wt\%) By Block/Sub-Block Reference Value $=0.1 .07 \mathrm{wt} \%$

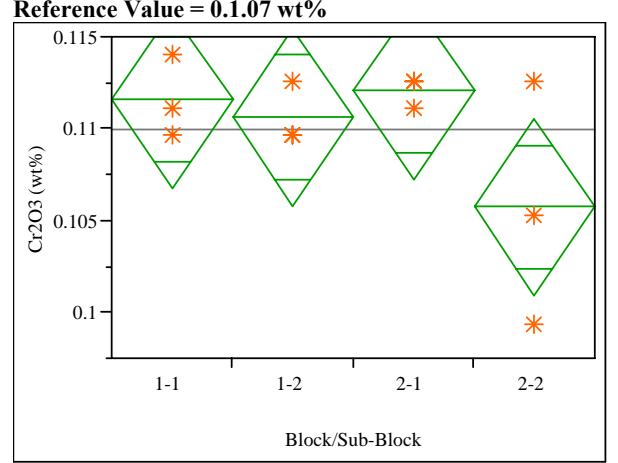

Oneway Anova

Summary of Fit

Rsquare

Adj Rsquare $\quad 0.422354$

Root Mean Square Error $\quad 0.205737$

Mean of Response 0.109985

Mean of Resporse

Analysis of Variance

$\begin{array}{lllll}\text { Source DF } & \text { Sum of Mean F Prob > }\end{array}$

$\begin{array}{llrrrr} & & \text { Squares } & \text { Square } & \text { Ratio } & \text { F } \\ \text { Block/Sub- } & 3 & 0.00007602 & 0.000025 & 1.9498 & 0.2002 \\ \text { Block } & & & & & \end{array}$

$\begin{array}{lllll}\text { Block } & 8 & 0.00010397 & 0.000013 \\ \text { Error } & 8 & \end{array}$

$\begin{array}{lrr}\text { C. Total } & 11 & 0.00017998\end{array}$

Means for Oneway Anova

Level Number Mean Std Error Lower 95\% Upper 95\%

$\begin{array}{llllll}1-1 & 3 & 0.111569 & 0.00208 & 0.10677 & 0.11637\end{array}$

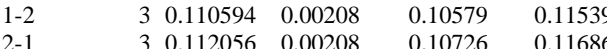

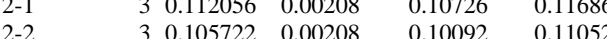

Std Error uses a pooled estimate of error variance 


\section{Exhibit A7. PSAL Measurements by Analytical Block for Series "P" Samples of the Batch 1 Standard Glass Prepared by LM}

Oneway Analysis of $\mathrm{CuO}(\mathrm{wt} \%)$ By Block/Sub-Block

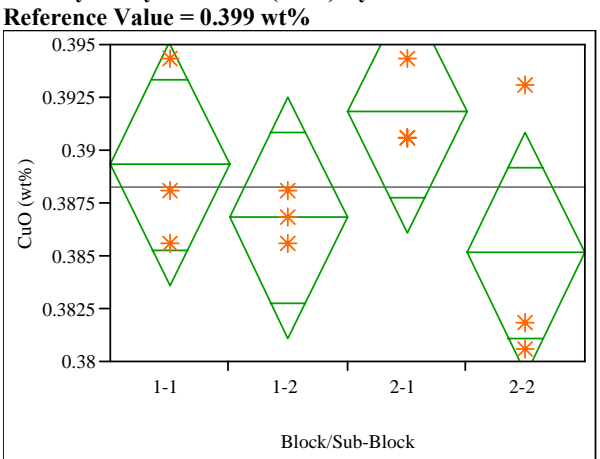

\section{Oneway Anova}

Summary of Fit

$\begin{array}{lr}\text { Rsquare } & 0.341067 \\ \text { Adj Rsquare } & 0.093968 \\ \text { Root Mean Square Error } & 0.004306 \\ \text { Mean of Response } & 0.388267 \\ \text { Observations (or Sum Wgts) } & 12\end{array}$

Analysis of Variance

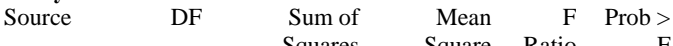

$\begin{array}{llrrrr} & & \text { Squares } & \text { Square } & \text { Ratio } & \text { F } \\ \text { Block/Sub- } & 3 & 0.00007678 & 0.000026 & 1.3803 & 0.3171\end{array}$

Block/S

Block

Crror

$\begin{array}{lll}8 & 0.00014834 & 0.000019\end{array}$

\section{Means for Oneway Anova}

Level Number Mean Std Error Lower 95\% Upper 95\%

$\begin{array}{llllll}1-1 & 3 & 0.389310 & 0.00249 & 0.38358 & 0.39504\end{array}$

$\begin{array}{llllll}1-2 & 3 & 0.386806 & 0.00249 & 0.38107 & 0.39254\end{array}$

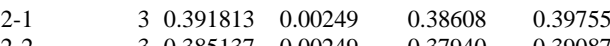

Std Error uses a pooled estimate of error variance
Oneway Analysis of K2O (wt\%) By Block/Sub-Block

Reference Value $=3.327 \mathrm{wt} \%$

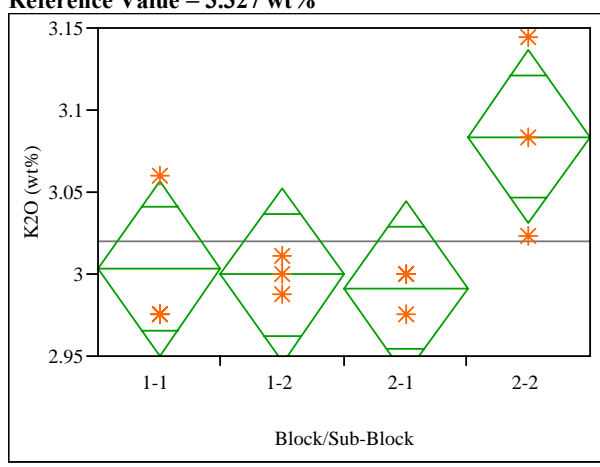

\section{Oneway Anova}

Summary of Fit

$\begin{array}{lr}\text { Rsquare } & 0.569079 \\ \text { Adj Rsquare } & 0.407484 \\ \text { Root Mean Square Error } & 0.0398 \\ \text { Mean of Response } & 3.019531 \\ \text { Observations (or Sum Wgts) } & 12\end{array}$

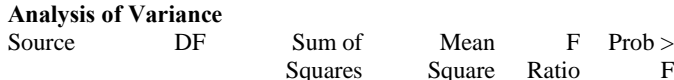

$\begin{array}{llrrrr} & & \text { Squares } & \text { Square } & \text { Ratio } & \text { F } \\ \text { Block/Sub- } & 3 & 0.01673557 & 0.005579 & 3.5216 & 0.0686\end{array}$

Block

Error

0.01673557

C. Total

$\begin{array}{rr}8 & 0.01267260 \\ 11 & 0.02940817\end{array}$

Means for Oneway Anova

Level Number Mean Std Error Lower 95\% Upper 95\%

$\begin{array}{llllll}1-1 & 3 & 3.00347 & 0.02298 & 2.9505 & 3.0565\end{array}$

$\begin{array}{llllll}1-2 & 3 & 2.99945 & 0.02298 & 2.9465 & 3.0524\end{array}$

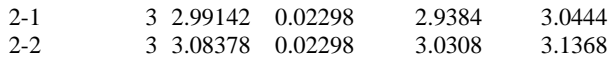

Std Error uses a pooled estimate of error variance
Oneway Analysis of $\mathrm{MgO}(\mathrm{wt} \%)$ By Block/Sub-Block Reference Value $=1.419 \mathrm{wt} \%$

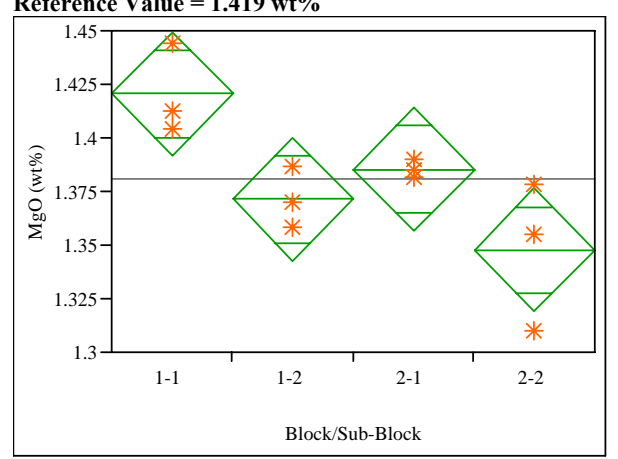

Oneway Anova

Summary of Fit

Rsquare

$\begin{array}{ll}\text { Adj Rsquare } & 0.693142 \\ & 0.578071\end{array}$

Root Mean Square Error $\quad 0.57807$

Mean of Response 1.381226

Mean of Resonse

Analysis of Variance

$\begin{array}{lllll}\text { Source DF } & \text { Sum of } & \text { Mean } & \text { P } & \text { Prob > }\end{array}$ $\begin{array}{lrrrrr} & & \text { Squares } & \text { Square } & \text { Ratio } & \text { F } \\ \text { Block/Sub- } & 3 & 0.00837340 & 0.002791 & 6.0236 & 0.0189\end{array}$

Block $\quad 8 \quad 0.00370694 \quad 0.000463$

$\begin{array}{lrr}\text { Error } & 8 & 0.00370694 \\ \text { C. Total } & 11 & 0.01208034\end{array}$

Means for Oneway Anova

Level Number Mean Std Error Lower 95\% Upper 95\%

$\begin{array}{llllll}1-1 & 3 & 1.42061 & 0.01243 & 1.3920 & 1.4493\end{array}$

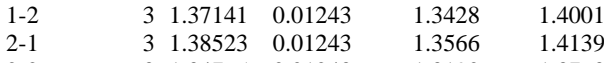

$\begin{array}{llllll}2-2 & 3 & 1.34765 & 0.01243 & 1.3190 & 1.3763\end{array}$

Std Error uses a pooled estimate of error variance 


\section{Exhibit A7. PSAL Measurements by Analytical Block for Series "P" Samples of the Batch 1 Standard Glass Prepared by LM}

Oneway Analysis of MnO (wt\%) By Block/Sub-Block

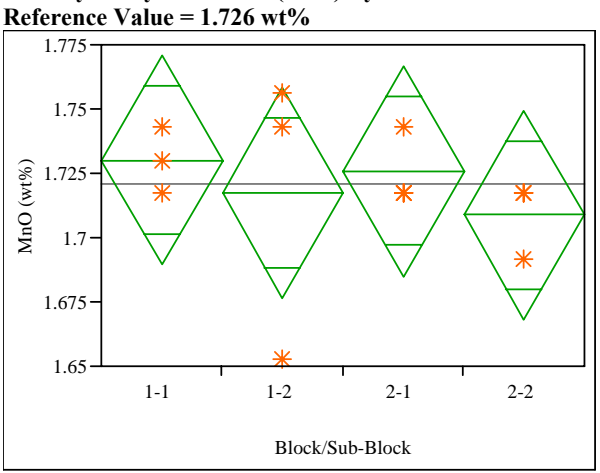

Oneway Anova

Summary of Fit

\begin{tabular}{lr} 
Rsquare & 0.097844 \\
Adj Rsquare & -0.24046 \\
Root Mean Square Error & 0.030737 \\
Mean of Response & 1.720524 \\
\hline
\end{tabular}

\section{Analysis of Variance}

$\begin{array}{llllll}\text { Source DF } & \text { Sum of Mean F } & \text { Prob > }\end{array}$

$\begin{array}{llrrrr} & & \text { Squares } & \text { Square } & \text { Ratio } & \text { F } \\ \text { Block/Sub- } & 3 & 0.00081971 & 0.000273 & 0.2892 & 0.8321\end{array}$

\begin{tabular}{lllll} 
Block & & & & \\
Error & 8 & 0.00755796 & 0.000945 \\
\hline
\end{tabular}

$\begin{array}{lrr}\text { Error } & 8 & 0.00755796 \\ \text { C. Total } & 11 & 0.00837767\end{array}$

Means for Oneway Anova

Level Number Mean Std Error Lower 95\% Upper 95\%

$\begin{array}{rrrrrr}1-1 & 3 & 1.73021 & 0.01775 & 1.6893 & 1.7711\end{array}$

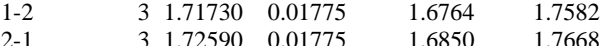

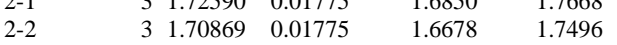

Std Error uses a pooled estimate of error variance
Oneway Analysis of $\mathrm{Na2O}$ (wt\%) By Block/Sub-Block Reference Value $=9.003 \mathrm{wt} \%$

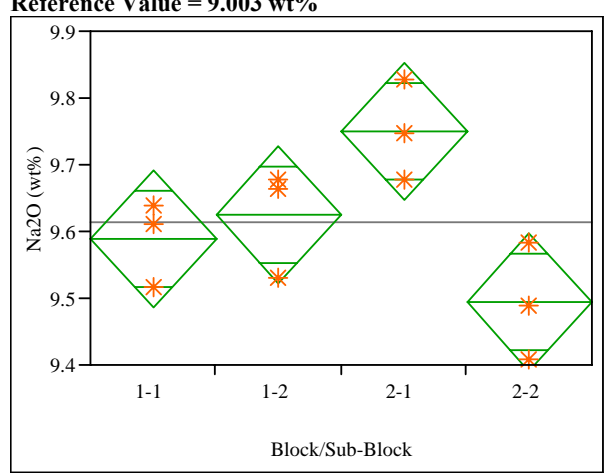

Oneway Anova

Summary of Fit

$\begin{array}{lr}\text { Rsquare } & 0.678147 \\ \text { Adj Rsquare } & 0.557452 \\ \text { Root Mean Square Error } & 0.077437 \\ \text { Mean of Response } & 9.61461 \\ \text { Observations (or Sum Wgts) } & 12\end{array}$

$\begin{array}{lrrrrr}\begin{array}{l}\text { Analysis of Variance } \\ \text { Source }\end{array} & \text { DF } & \begin{array}{r}\text { Sum of } \\ \text { Squares }\end{array} & \begin{array}{r}\text { Mean } \\ \text { Square }\end{array} & \begin{array}{r}\text { Ratio } \\ \text { Prob > }\end{array} & \text { F } \\ \begin{array}{l}\text { Block/Sub- } \\ \text { Block }\end{array} & 3 & 0.10107641 & 0.033692 & 5.6187 & 0.0227 \\ \text { Error } & 8 & & & & \\ \text { C. Total } & 11 & 0.14904795 & 0.005996 & & \\ & & & & \end{array}$

Means for Oneway Anova

Level Number Mean Std Error Lower 95\% Upper 95\%

$\begin{array}{llllll}1-1 & 3 & 9.58877 & 0.04471 & 9.4857 & 9.6919\end{array}$

$\begin{array}{llllll}1-2 & 3 & 9.62472 & 0.04471 & 9.5216 & 9.7278\end{array}$

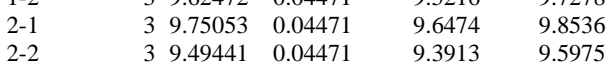

Std Error uses a pooled estimate of error variance
Oneway Analysis of NiO (wt\%) By Block/Sub-Block Reference Value $=0.751 \mathbf{w t}^{\%}$

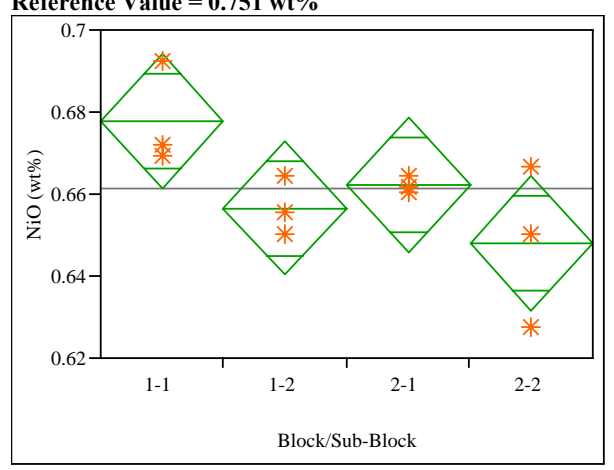

Oneway Anova

Summary of Fit

Rsquare

Root Mean Square Error $\quad 0.012288$

$\begin{array}{lr}\text { Mean of Response } & 0.66117 \\ \text { Observations (or Sum Wgts) } & 12\end{array}$

Analysis of Variance

Source DF Sum of

Block/Sub- $\quad 3 \quad \begin{array}{r}\text { Squares } \\ 0.00140700\end{array}$

Block

Error

C. Total

$8 \quad 0.00120797$

$11 \quad 0.00261496$

Means for Oneway Anova

Level Number Mean Std Error Lower 95\% Upper 95\%

$\begin{array}{llllll}1-1 & 3 & 0.677818 & 0.00709 & 0.66146 & 0.69418\end{array}$

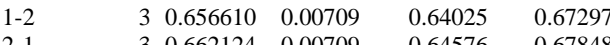

$\begin{array}{lllllll}2-1 & & 3 & 0.662124 & 0.00709 & 0.64576 & 0.67848 \\ 2-2 & & 3 & 0.648127 & 0.00709 & 0.63177 & 0.66449\end{array}$

$\begin{array}{lllllll}2-2 & 3 & 0.648127 & 0.00709 & 0.63177 & 0.66449\end{array}$

Std Error uses a pooled estimate of error variance 
Exhibit A7. PSAL Measurements by Analytical Block for Series "P" Samples of the Batch 1 Standard Glass Prepared by LM

Oneway Analysis of SO4 (wt\%) By Block/Sub-Block

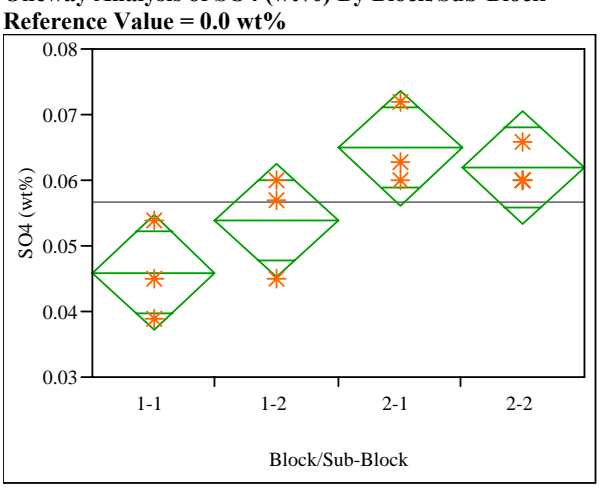

Oneway Anova

Summary of Fit

$\begin{array}{lr}\text { Rsquare } & 0.6574 \\ \text { Adj Rsquare } & 0.528926 \\ \text { Root Mean Square Error } & 0.006529 \\ \text { Mean of Response } & 0.056672 \\ \text { Observations (or Sum Wgts) } & 12\end{array}$

\begin{tabular}{|c|c|c|c|c|c|}
\hline \multicolumn{6}{|c|}{ Analysis of Variance } \\
\hline Source & DF & $\begin{array}{r}\text { Sum of } \\
\text { Squares }\end{array}$ & $\begin{array}{r}\text { Mean } \\
\text { Square }\end{array}$ & $\begin{array}{r}F \\
\text { Ratio }\end{array}$ & $\begin{array}{r}\text { Prob }> \\
F\end{array}$ \\
\hline $\begin{array}{l}\text { Block/Sub- } \\
\text { Block }\end{array}$ & 3 & 0.00065446 & 0.000218 & 5.1170 & 0.0289 \\
\hline Error & 8 & 0.00034107 & 0.000043 & & \\
\hline C. Total & 11 & 0.00099552 & & & \\
\hline
\end{tabular}

Means for Oneway Anova

Level Number Mean Std Error Lower 95\% Upper 95\%

$\begin{array}{llllll}1-1 & 3 & 0.045937 & 0.00377 & 0.03724 & 0.05463\end{array}$

$\begin{array}{llllll}1-2 & 3 & 0.053926 & 0.00377 & 0.04523 & 0.06262\end{array}$

$\begin{array}{lllllll}2-1 & & 3 & 0.064911 & 0.00377 & 0.05622 & 0.07360 \\ 2-2 & & 3 & 0.061915 & 0.00377 & 0.05322 & 0.07061\end{array}$

Std Error uses a pooled estimate of error variance
Oneway Analysis of SiO2 (wt\%) By Block/Sub-Block Reference Value $=\mathbf{5 0 . 2 2} \mathbf{w t} \%$

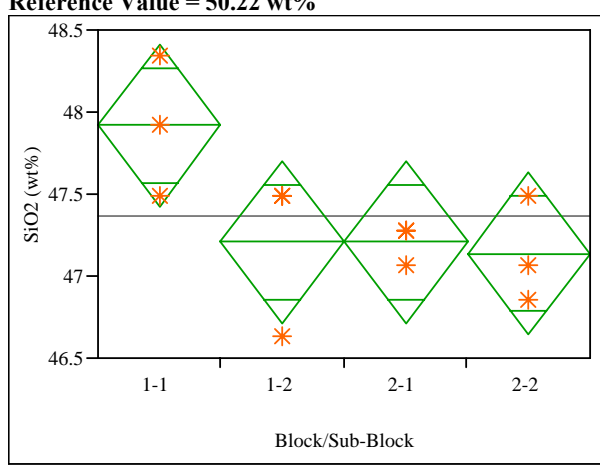

\section{Oneway Anova}

Summary of Fit

$\begin{array}{lr}\text { Rsquare } & 0.528642 \\ \text { Adj Rsquare } & 0.351882 \\ \text { Root Mean Square Error } & 0.370538 \\ \text { Mean of Response } & 47.36767 \\ \text { Observations (or Sum Wgts) } & 12\end{array}$

$\begin{array}{lrrrrr}\begin{array}{l}\text { Analysis of Variance } \\ \text { Source }\end{array} & \text { DF } & \begin{array}{r}\text { Sum of } \\ \text { Squares }\end{array} & \begin{array}{r}\text { Mean } \\ \text { Square }\end{array} & \begin{array}{r}\text { Ratio } \\ \text { Prob > }\end{array} \\ \begin{array}{l}\text { Block/Sub- } \\ \text { Block }\end{array} & 3 & 1.2318694 & 0.410623 & 2.9907 & 0.0957 \\ \text { Error } & 8 & 1.0983851 & 0.137298 & & \\ \text { C. Total } & 11 & 2.3302545 & & & \end{array}$

Means for Oneway Anova

Level Number Mean Std Error Lower 95\% Upper 95\%

$\begin{array}{llllrr}1-1 & 3 & 47.9203 & 0.21393 & 47.427 & 48.414\end{array}$

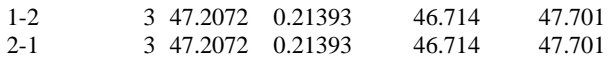

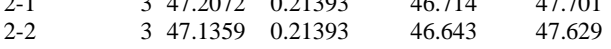

Std Error uses a pooled estimate of error variance
Oneway Analysis of TiO2 (wt\%) By Block/Sub-Block Reference Value $=0.677 \mathbf{w t}^{\circ}$

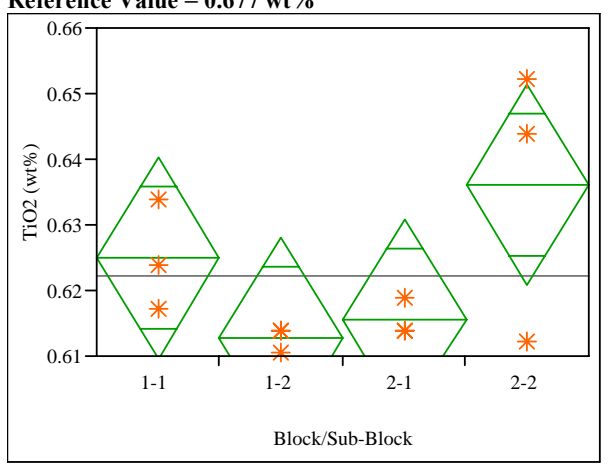

Oneway Anova

Summary of Fit

$\begin{array}{ll}\text { Rsquare } & 0.487122 \\ \text { Adj Rsquare } & 0.294792\end{array}$

Root Mean Square Error $\quad 0.011496$

$\begin{array}{lr}\text { Mean of Response } & 0.622303 \\ \text { Observations (or Sum Wgts) } & 12\end{array}$

$\begin{array}{rrrr}\text { Suman of } & F & \text { Mean } \\ \text { Squares } & \text { Square Ratio } & \end{array}$

$\begin{array}{llrrrr}\text { Block/Sub- } & 3 & 0.00100415 & 0.000335 & 2.5327 & 0.1304\end{array}$

$\begin{array}{lllll}\text { Block } & 8 & 0.00105725 & 0.000132 \\ \text { Error } & 8 & 0.00206140 & \end{array}$

$\begin{array}{lrr} & 11 & 0.00105725 \\ \text { C. Total } & 11 & 0.00206140\end{array}$

Means for Oneway Anova

Level Number Mean Std Error Lower 95\% Upper 95\%

$\begin{array}{lllrrr}1-1 & 3 & 0.624944 & 0.00664 & 0.60964 & 0.64025\end{array}$

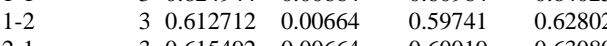

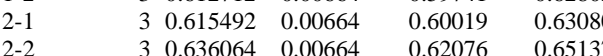

$\begin{array}{llllll}2-2 & 3 & 0.636064 & 0.00664 & 0.62076 & 0.65137\end{array}$

Std Error uses a pooled estimate of error variance 
Exhibit A7. PSAL Measurements by Analytical Block for Series "P" Samples of the

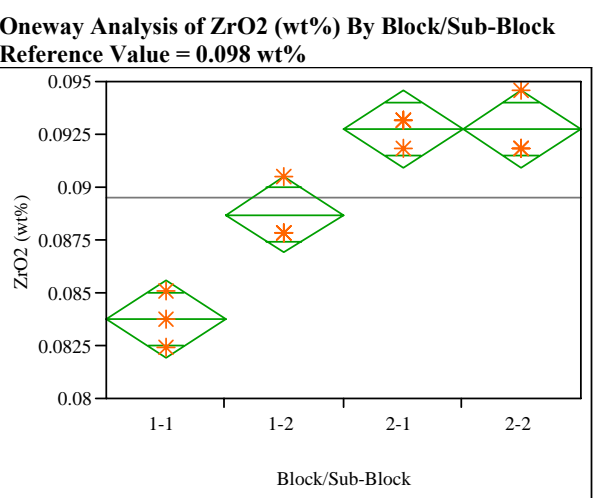

Oneway Anova

Summary of Fit

$\begin{array}{lr}\text { Rsquare } & 0.918575 \\ \text { Adj Rsquare } & 0.888041 \\ \text { Root Mean Square Error } & 0.001351 \\ \text { Mean of Response } & 0.089491 \\ \text { Observations (or Sum Wgts) } & 12\end{array}$

Analysis of Variance

Source DF Sum of Mean F Ratio Prob >

$\begin{array}{lrrrrr} & & \text { Squares } & \text { Square } & \text { F } \\ \text { Block/Sub- } & 3 & 0.00016468 & 0.000055 & 30.0833 & 0.0001\end{array}$

Block

$\begin{array}{lrrrr}\text { Elock } & 8 & 0.00001460 & 1.825 \mathrm{e}-6\end{array}$

$\begin{array}{lrl}\text { C. Total } & 11 & 0.00017927\end{array}$

Means for Oneway Anova

Level Number Mean Std Error Lower 95\% Upper 95\%

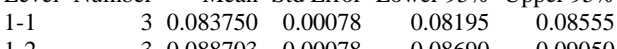

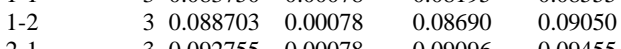

$\begin{array}{llllll}2-1 & 3 & 0.092755 & 0.00078 & 0.09096 & 0.09455 \\ 2-2 & 3 & 0.092755 & 0.00078 & 0.09096 & 0.09455\end{array}$

Std Error uses a pooled estimate of error variance

\section{Batch 1 Standard Glass Prepared by LM}


Exhibit A8. PSAL Measurements by Analytical Block for Series "P" Samples of the Batch 1 Standard Glass Prepared by PF

Oneway Analysis of B2O3 (wt\%) By Block/Sub-Block

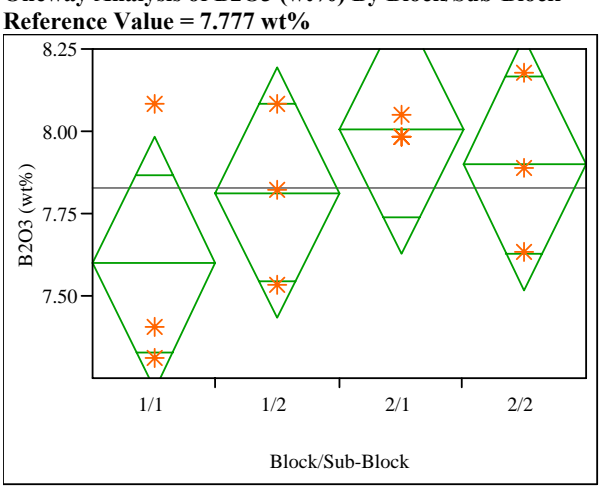

Oneway Anova

Summary of Fit

$\begin{array}{lr}\text { Rsquare } & 0.290563 \\ \text { Adj Rsquare } & 0.024524 \\ \text { Root Mean Square Error } & 0.286643 \\ \text { Mean of Response } & 7.829724 \\ \text { Observations (or Sum Wgts) } & 12\end{array}$$$
\text { Analysis of Varian }
$$$$
\text { Source DF Sum of Squares Mean Square F Ratio Prob > F }
$$$$
\begin{array}{llllll}
\text { Block/Sub-Block } & 3 & 0.26921606 & 0.089739 & 1.0922 & 0.4066
\end{array}
$$$$
\begin{array}{lll}
\text { Error } & 8 & 0.65731573
\end{array}
$$$$
0.08216
$$

C. Total $11 \quad 0.92653180$

Means for Oneway Anova

Level Number Mean Std Error Lower 95\% Upper 95\%

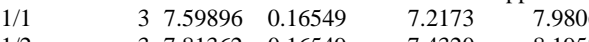

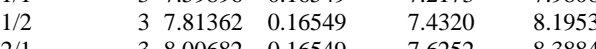

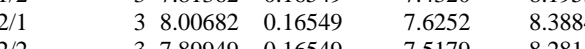

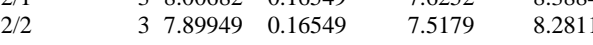

Std Error uses a pooled estimate of error variance
Oneway Analysis of Fe2O3 (wt\%) By Block/Sub-Block Reference Value $=12.839 \mathrm{wt} \%$

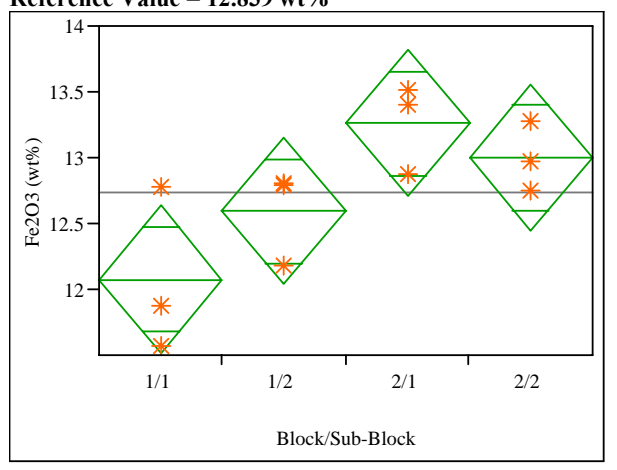

Oneway Anova

Summary of Fit

$\begin{array}{lr}\text { Rsquare } & 0.629085 \\ \text { Adj Rsquare } & 0.489992 \\ \text { Root Mean Square Error } & 0.421277 \\ \text { Mean of Response } & 12.73386 \\ \text { Observations (or Sum Wgts) } & 12\end{array}$

Observations (or Sum Wgts) 12.73386

Analysis of Varianc

Source DF Sum of Squares Mean Square F Ratio Prob > F

$\begin{array}{lllllll}\text { Block/Sub-Block } & 3 & 2.4080179 & 0.802673 & 4.5228 & 0.0390\end{array}$

$\begin{array}{lrr}\text { Error } & 8 & 1.4197916\end{array}$

0.177474

Means for Oneway Anova

Level Number Mean Std Error Lower 95\% Upper 95\%

$\begin{array}{llllll}1 / 1 & 3 & 12.0762 & 0.24322 & 11.515 & 12.637\end{array}$

$\begin{array}{llllll}1 / 2 & 3 & 12.5957 & 0.24322 & 12.035 & 13.157\end{array}$

$\begin{array}{llllll}2 / 1 & 3 & 13.2629 & 0.24322 & 12.702 & 13.824 \\ 2 / 2 & 3 & 13.0007 & 0.24322 & 12.440 & 13.562\end{array}$

Std Error uses a pooled estimate of error variance 
Exhibit A8. PSAL Measurements by Analytical Block for Series "P" Samples of the Batch 1 Standard Glass Prepared by PF

Oneway Analysis of Li2O (wt\%) By Block/Sub-Block

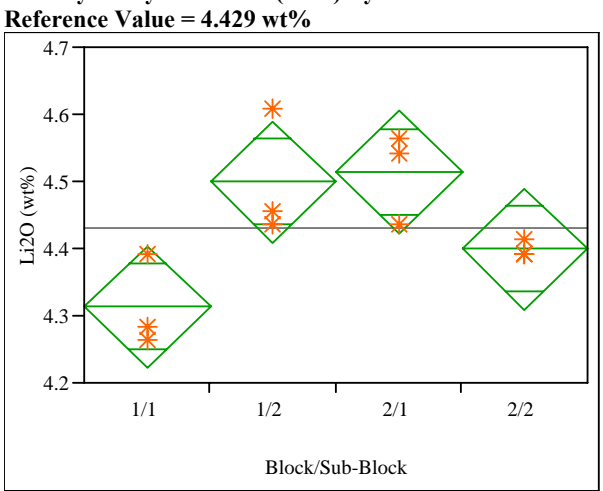

Oneway Anova

Summary of Fit

$\begin{array}{lr}\text { Rsquare } & 0.682119 \\ \text { Adj Rsquare } & 0.562914 \\ \text { Root Mean Square Error } & 0.068081 \\ \text { Mean of Response } & 4.431386 \\ \text { Observations (or Sum Wgts) } & 12\end{array}$

\section{Analysis of Variance}

Source DF Sum of Squares Mean Square F Ratio Prob $>$ F

$\begin{array}{lrrrrr}\text { Block/Sub-Block } & 3 & 0.07956713 & 0.026522 & 5.7222 & 0.0217 \\ \text { Error } & 8 & 0.03707983 & 0.004635 & & \end{array}$

Error

0.004635

Means for Oneway Anova

Level Number Mean Std Error Lower 95\% Upper 95\%

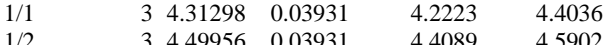

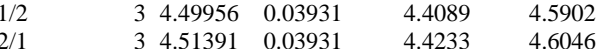

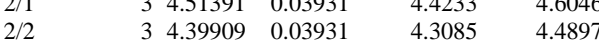

Std Error uses a pooled estimate of error variance
Oneway Analysis of $\mathrm{SiO} 2(\mathrm{wt} \%)$ By Block/Sub-Block Reference Value $=\mathbf{5 0 . 2 2} \mathrm{wt}^{\%} \%$

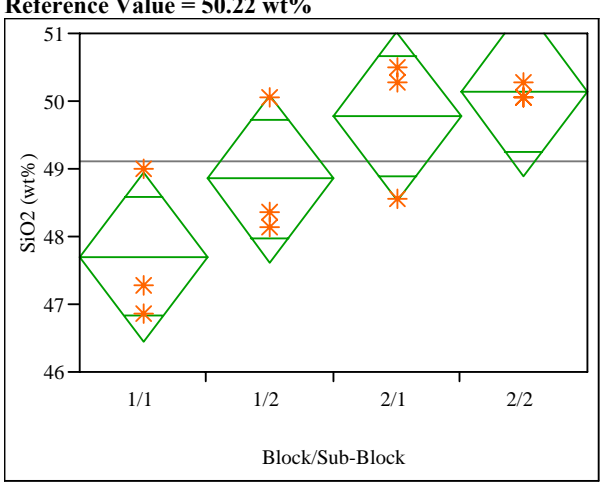

Oneway Anova

Summary of Fit

$\begin{array}{lr}\text { Rsquare } & 0.599913 \\ \text { Adj Rsquare } & 0.449881 \\ \text { Root Mean Square Error } & 0.938614 \\ \text { Mean of Response } & 49.11476 \\ \text { Observations (or Sum Wgts) } & 12\end{array}$

Mean of Response $\quad 49.11476$

Analysis of Variance

Source DF Sum of Squares Mean Square F Ratio Prob > F

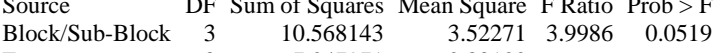

$\begin{array}{lrrr}\text { Error } & 8 & 7.047971 & 0.88100 \\ \text { C. } & 11 & 17.616113 & \end{array}$

$\begin{array}{lrr}\text { C. Total } & 11 & 17.616113\end{array}$

Means for Oneway Anova

Level Number Mean Std Error Lower 95\% Upper 95\%

$\begin{array}{llllll}1 / 1 & 3 & 47.7064 & 0.54191 & 46.457 & 48.956\end{array}$

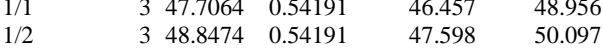

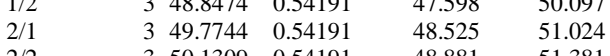

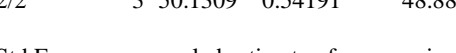

Std Error uses a pooled estimate of error variance 
Exhibit A9. Measured and Measured Bias-Corrected Oxide Weight Percents by Glass ID by Targeted Value for the Series "P" Glasses Prepared Using the LM Method

Al2O3 (wt\%)

Variability Chart for Measured

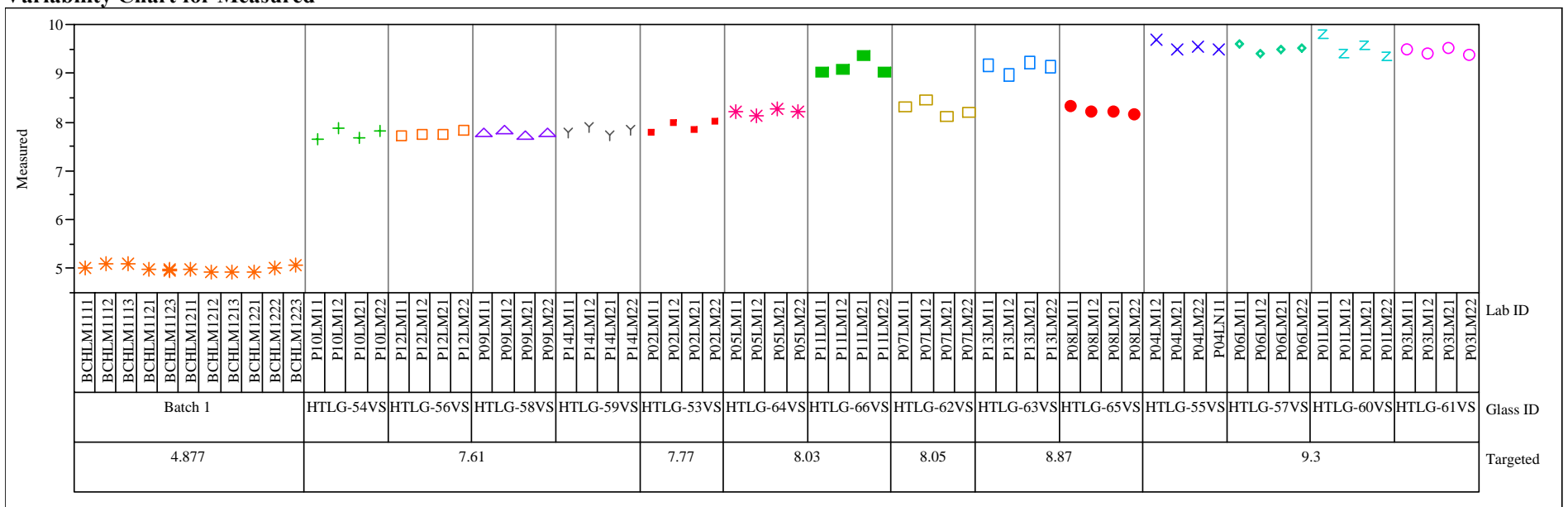

Variability Chart for Measured bc

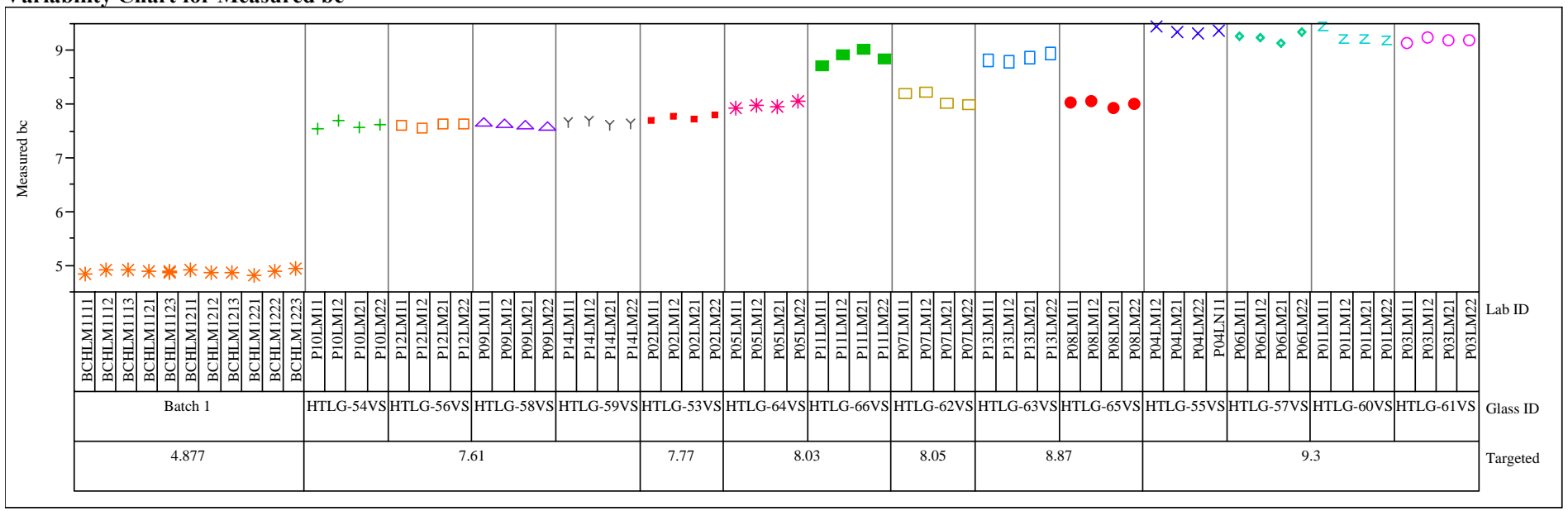


Exhibit A9. Measured and Measured Bias-Corrected Oxide Weight Percents by Glass ID by Targeted Value for the Series "P" Glasses Prepared Using the LM Method

$\mathrm{CaO}(\mathrm{wt} \%)$

Variability Chart for Measured

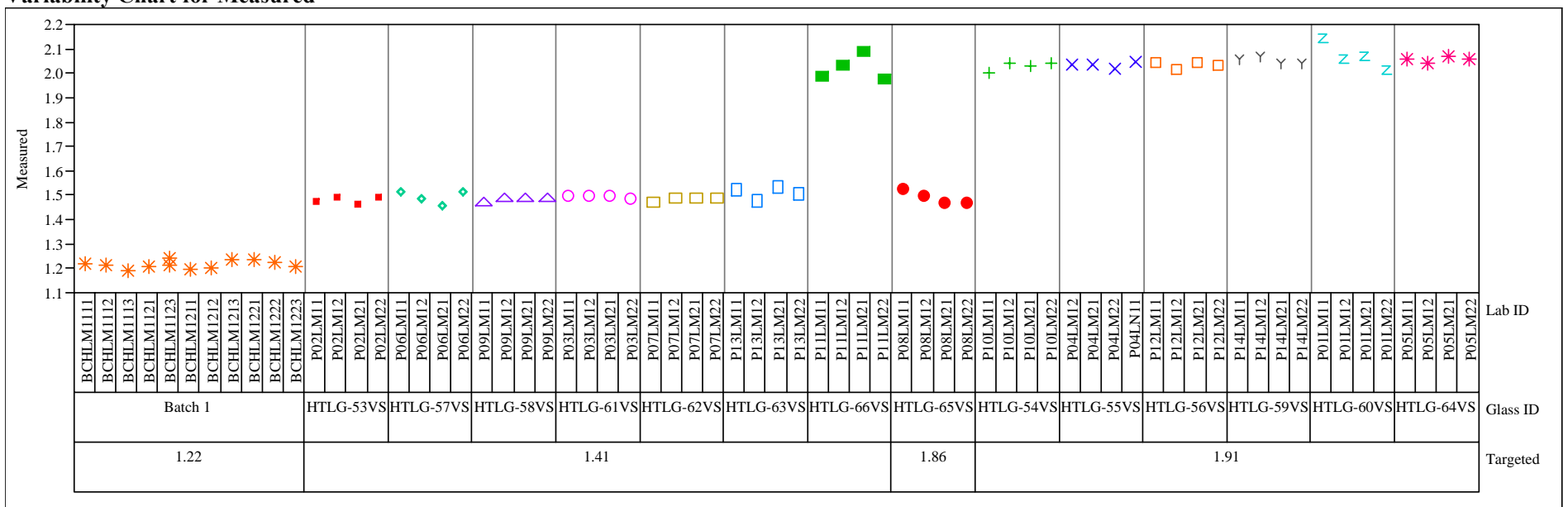

Variability Chart for Measured bc

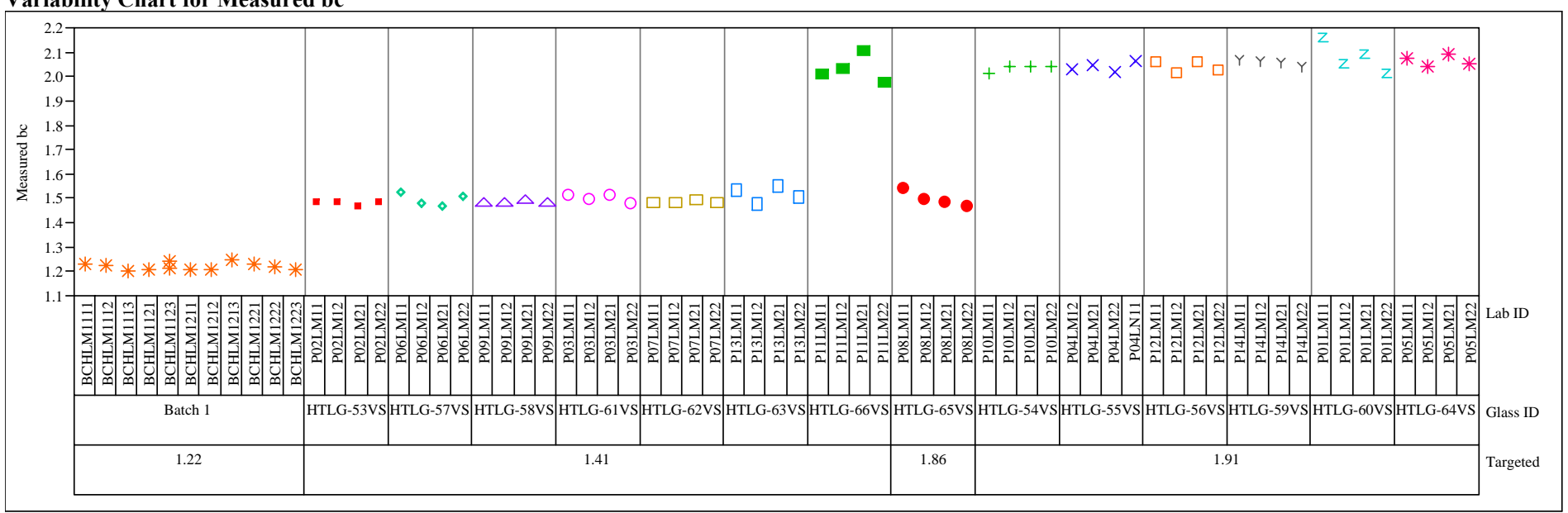


Exhibit A9. Measured and Measured Bias-Corrected Oxide Weight Percents

by Glass ID by Targeted Value for the Series "P" Glasses Prepared Using the LM Method

$\mathrm{Cr} 2 \mathrm{O3}(\mathrm{wt} \%)$

Variability Chart for Measured

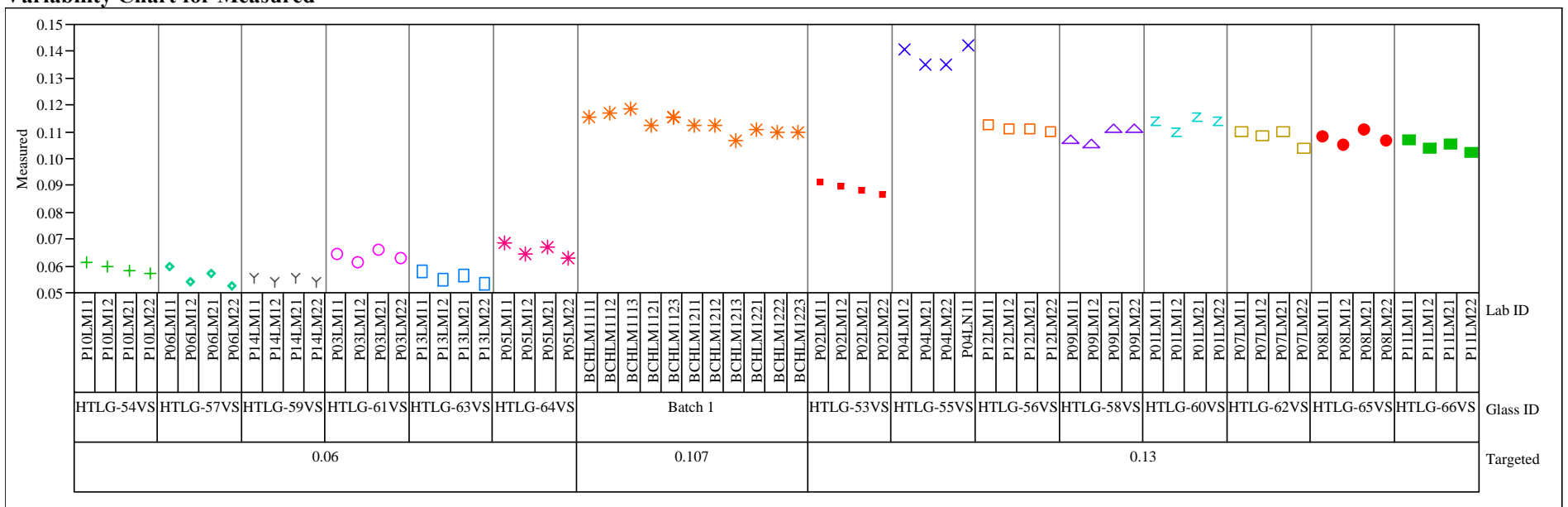

Variability Chart for Measured bc

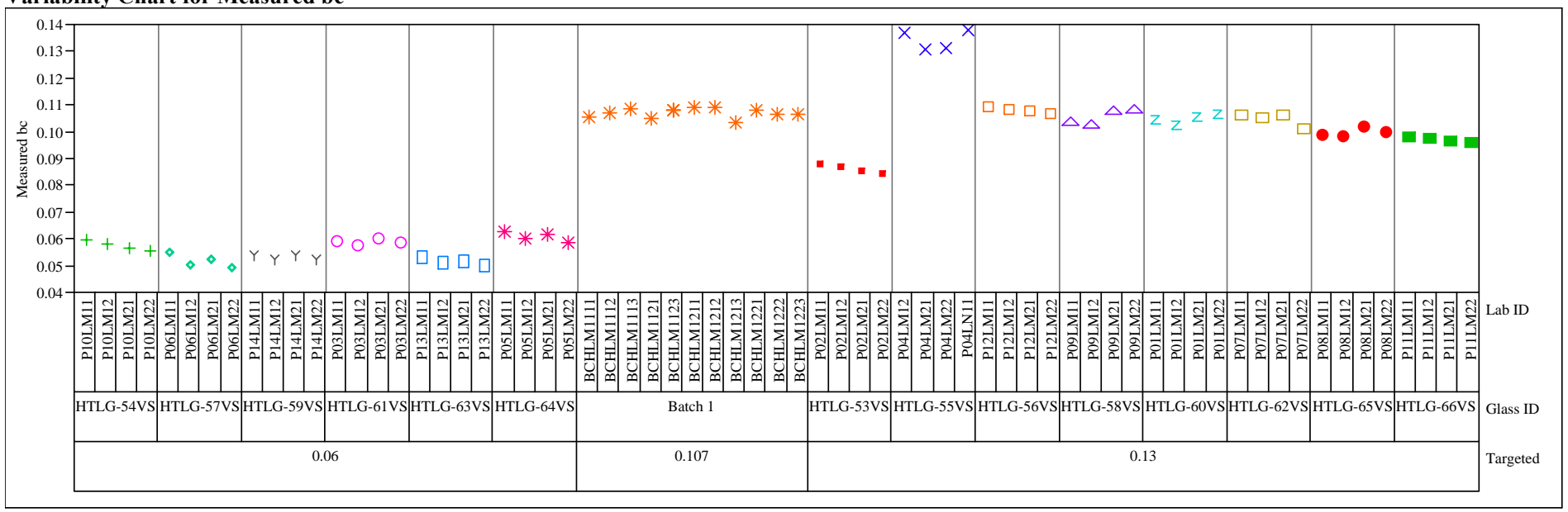


Exhibit A9. Measured and Measured Bias-Corrected Oxide Weight Percents

by Glass ID by Targeted Value for the Series "P" Glasses Prepared Using the LM Method

$\mathrm{CuO}(\mathrm{wt} \%)$

Variability Chart for Measured

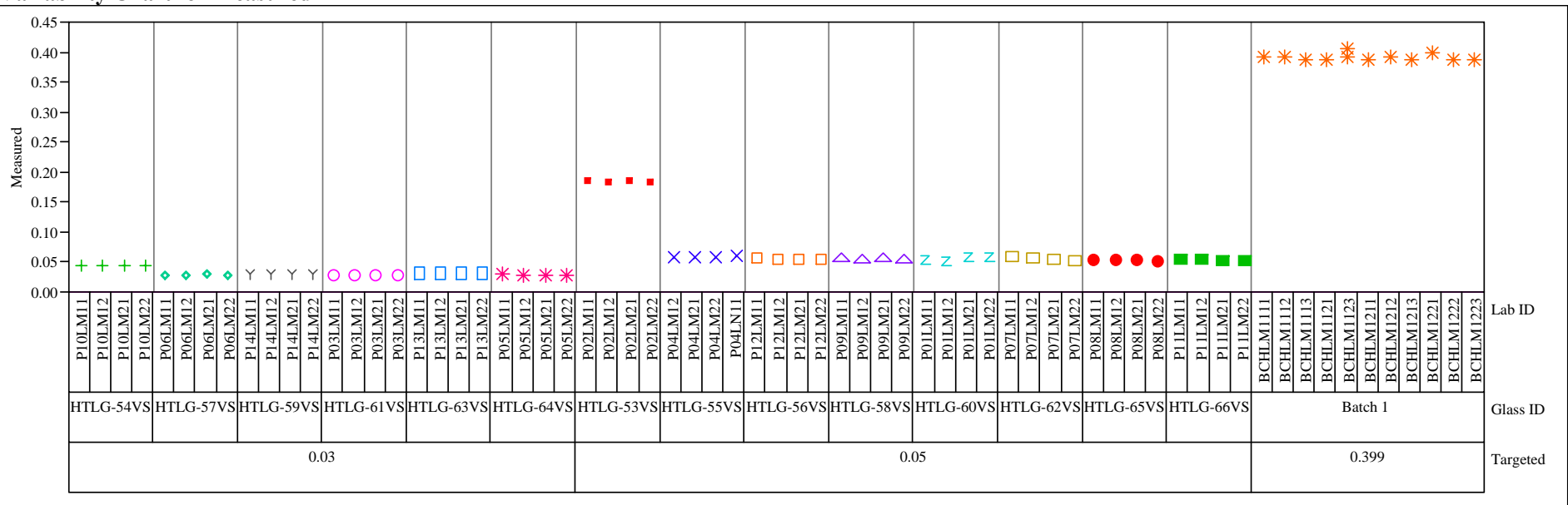

Variability Chart for Measured bc

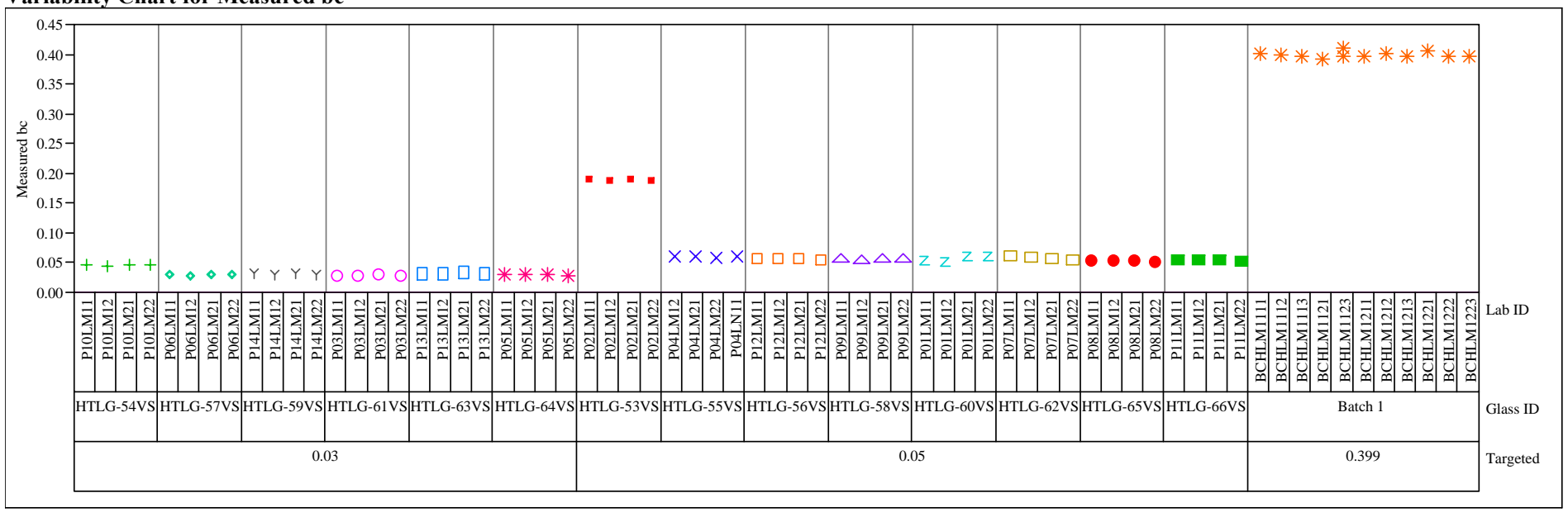


Exhibit A9. Measured and Measured Bias-Corrected Oxide Weight Percents

by Glass ID by Targeted Value for the Series "P" Glasses Prepared Using the LM Method

$\mathrm{K} 2 \mathrm{O}(\mathrm{wt} \%)$

Variability Chart for Measured

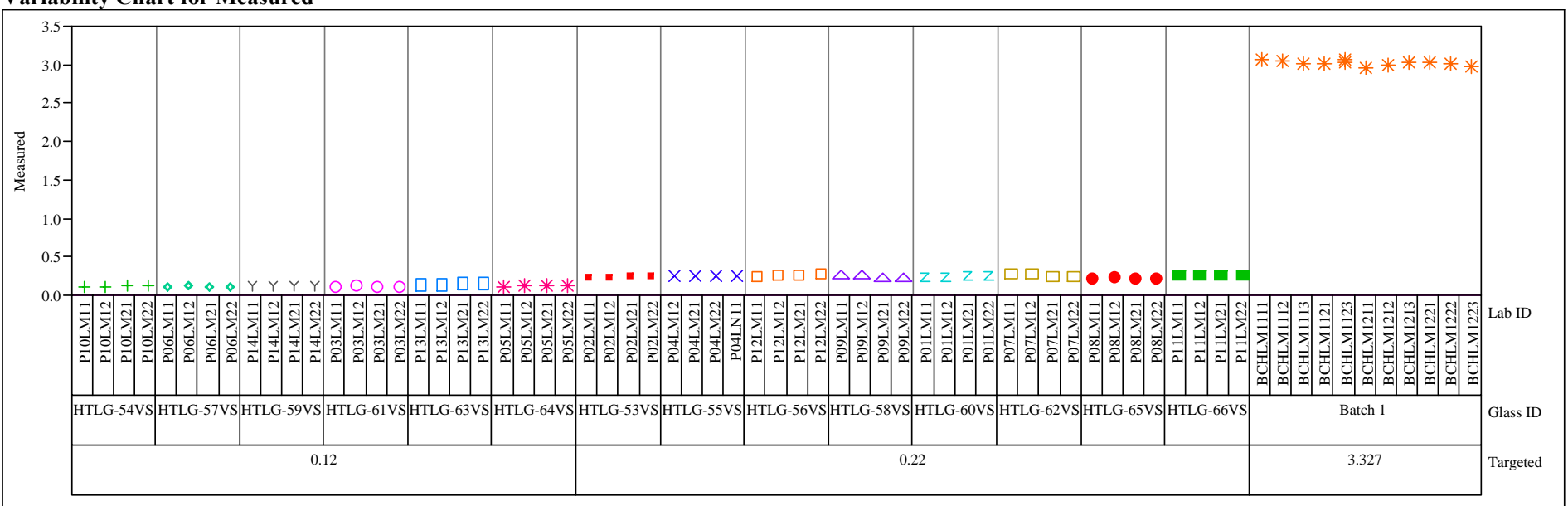

Variability Chart for Measured bc

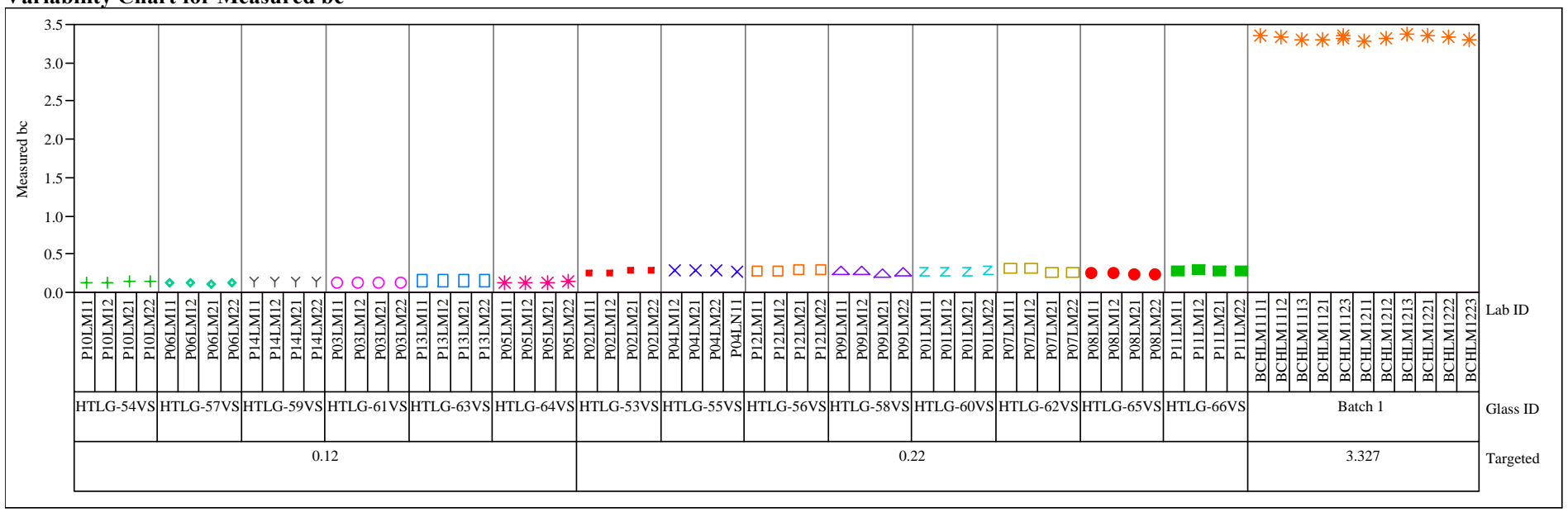


Exhibit A9. Measured and Measured Bias-Corrected Oxide Weight Percents

by Glass ID by Targeted Value for the Series "P" Glasses Prepared Using the LM Method

$\mathrm{MgO}$ (wt\%)

Variability Chart for Measured

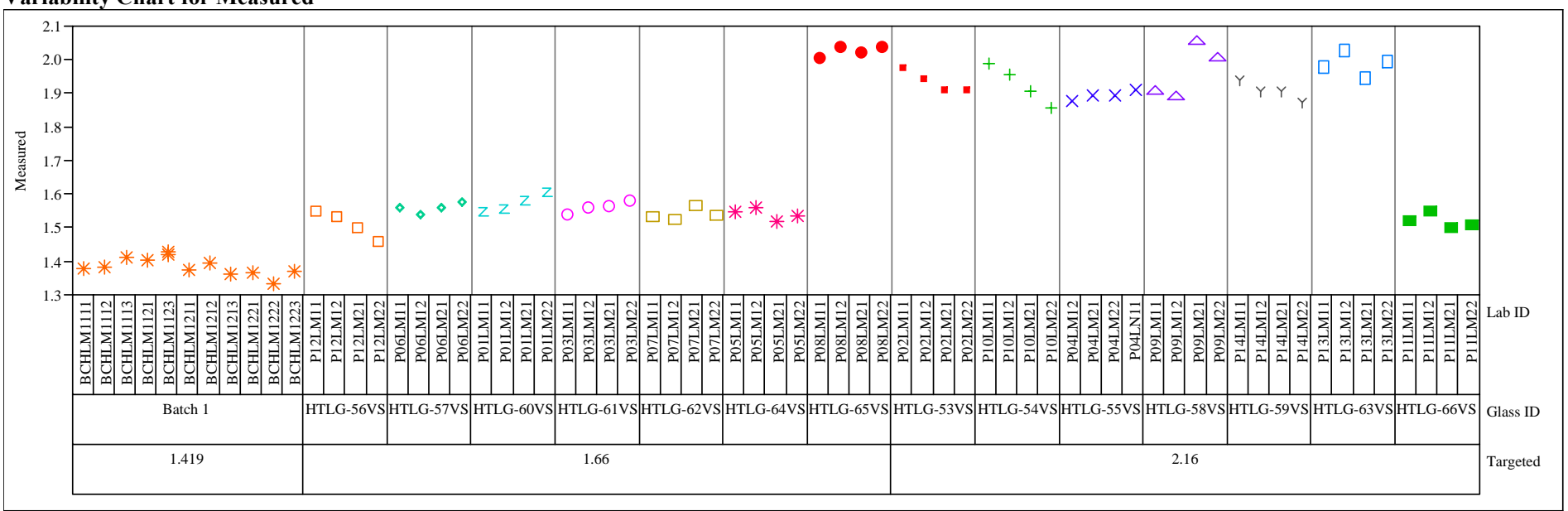

Variability Chart for Measured bc

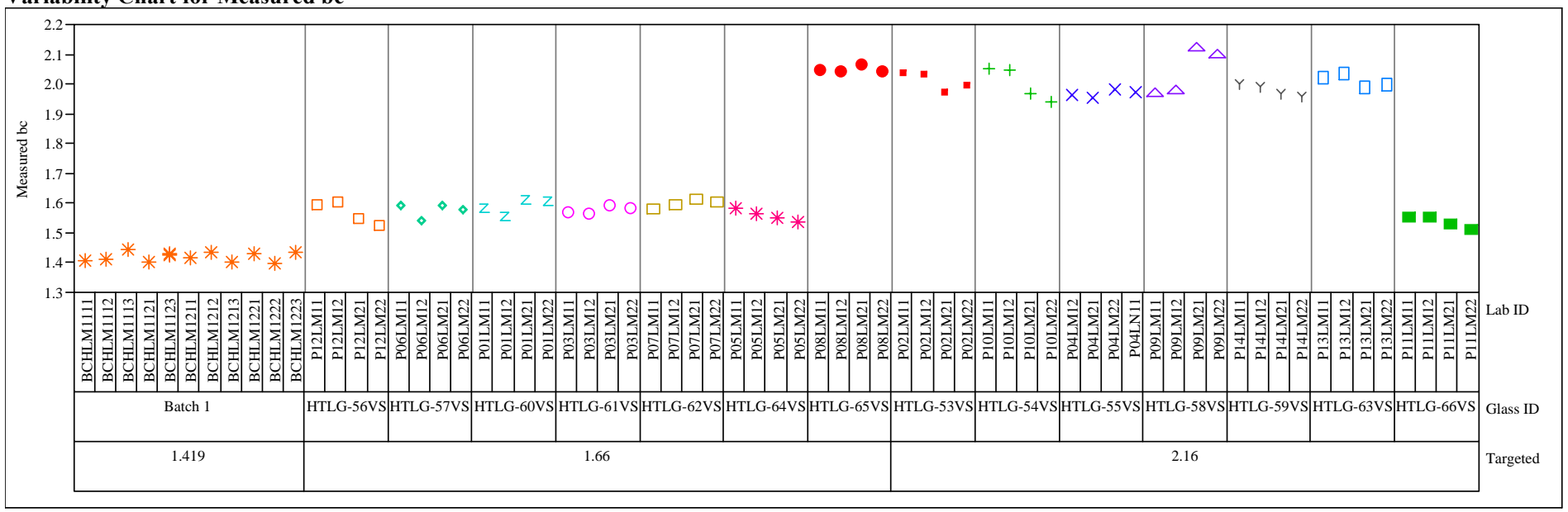


Exhibit A9. Measured and Measured Bias-Corrected Oxide Weight Percents

by Glass ID by Targeted Value for the Series "P" Glasses Prepared Using the LM Method

MnO (wt\%)

Variability Chart for Measured

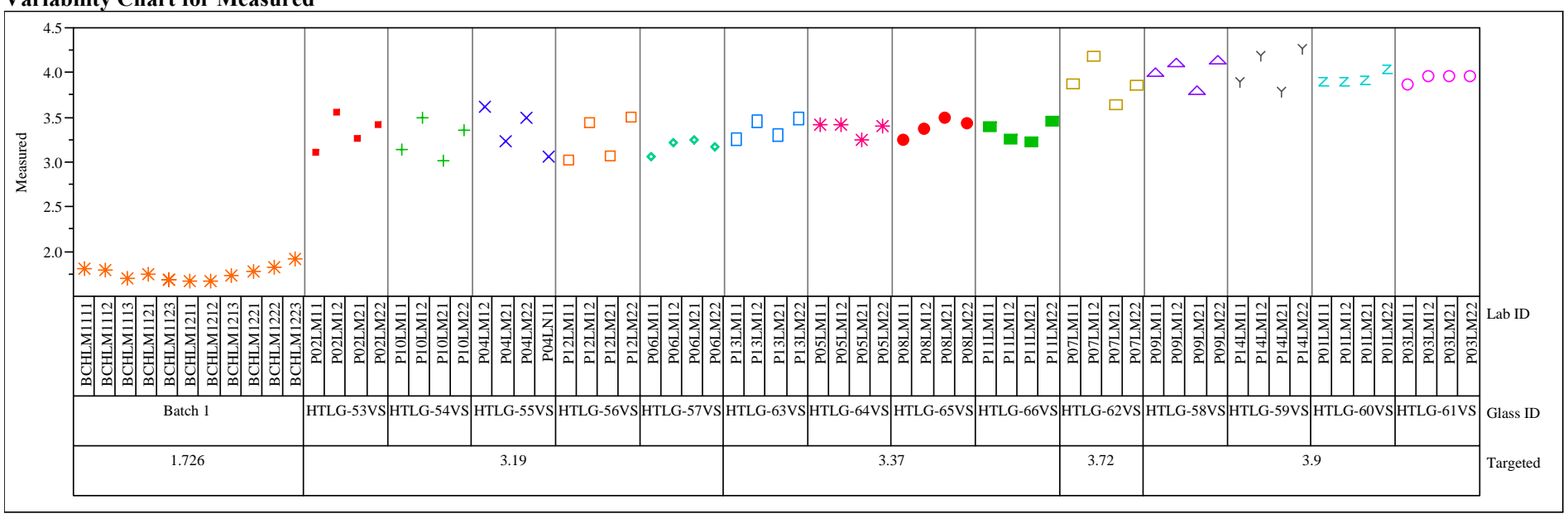

Variability Chart for Measured bc

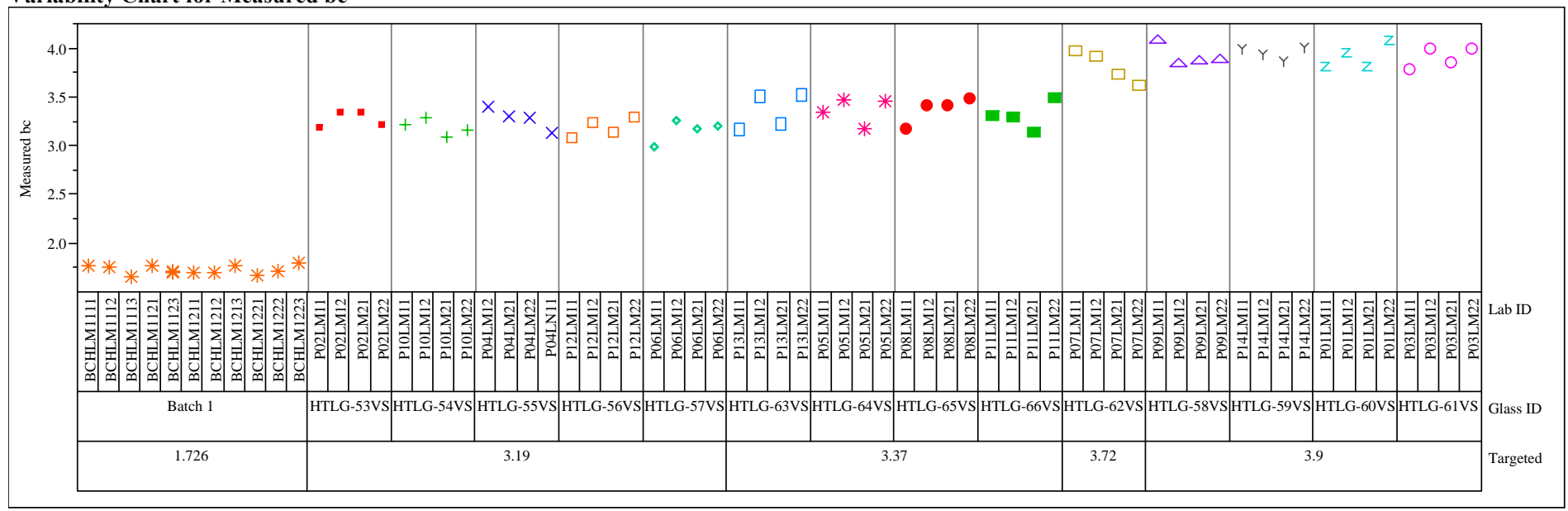


Exhibit A9. Measured and Measured Bias-Corrected Oxide Weight Percents by Glass ID by Targeted Value for the Series "P" Glasses Prepared Using the LM Method

$\mathrm{Na2O}$ (wt\%)

Variability Chart for Measured

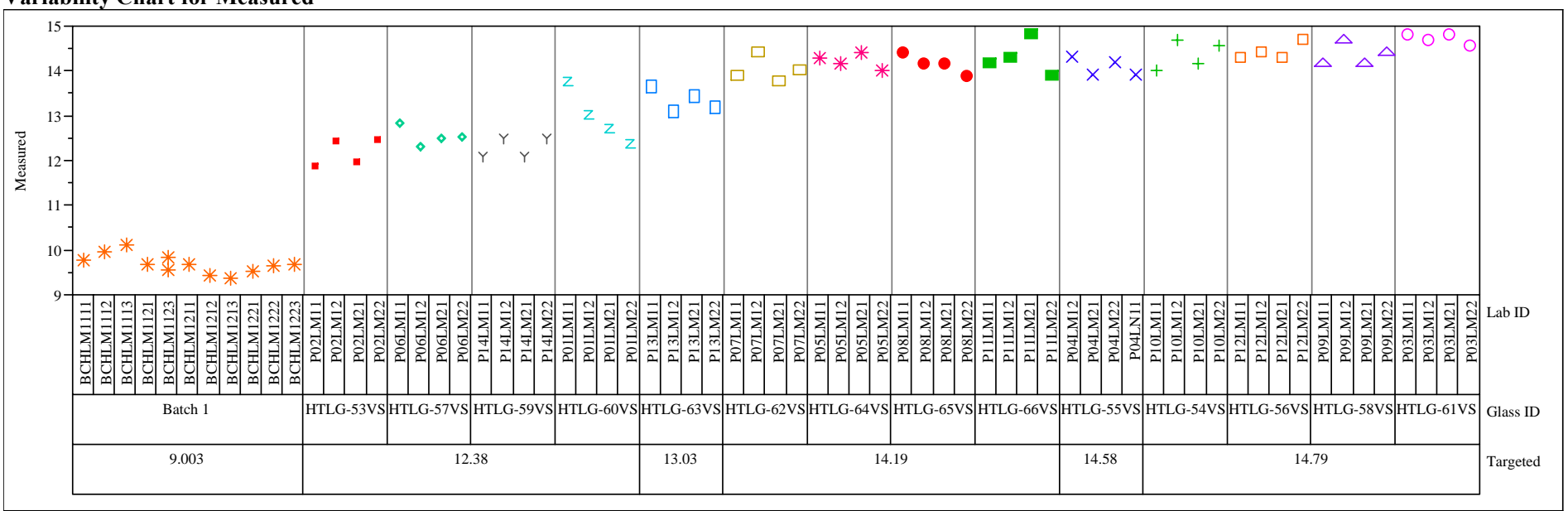

Variability Chart for Measured bc

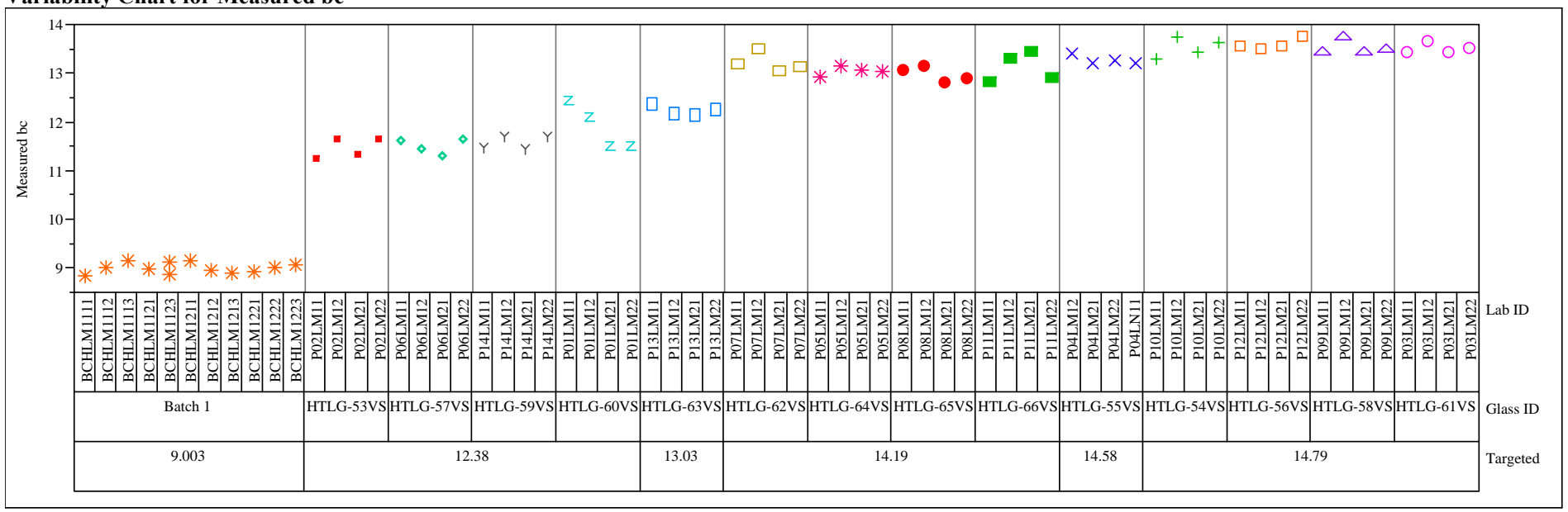


Exhibit A9. Measured and Measured Bias-Corrected Oxide Weight Percents

by Glass ID by Targeted Value for the Series "P" Glasses Prepared Using the LM Method

NiO (wt\%)

Variability Chart for Measured

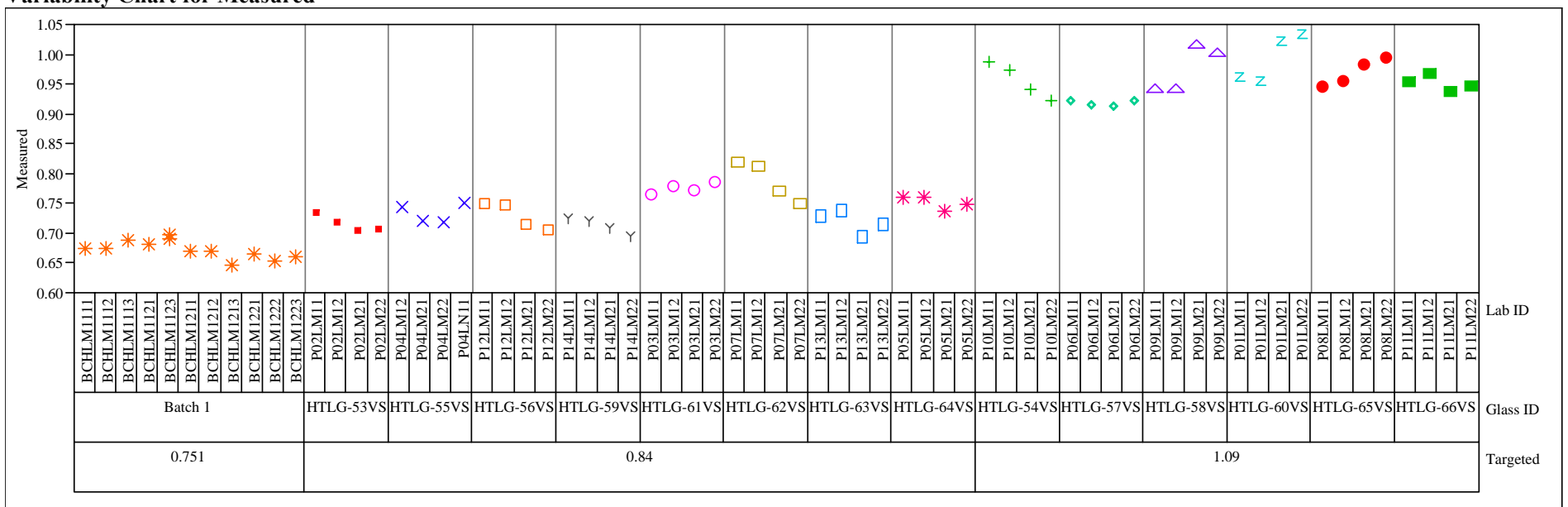

Variability Chart for Measured bc

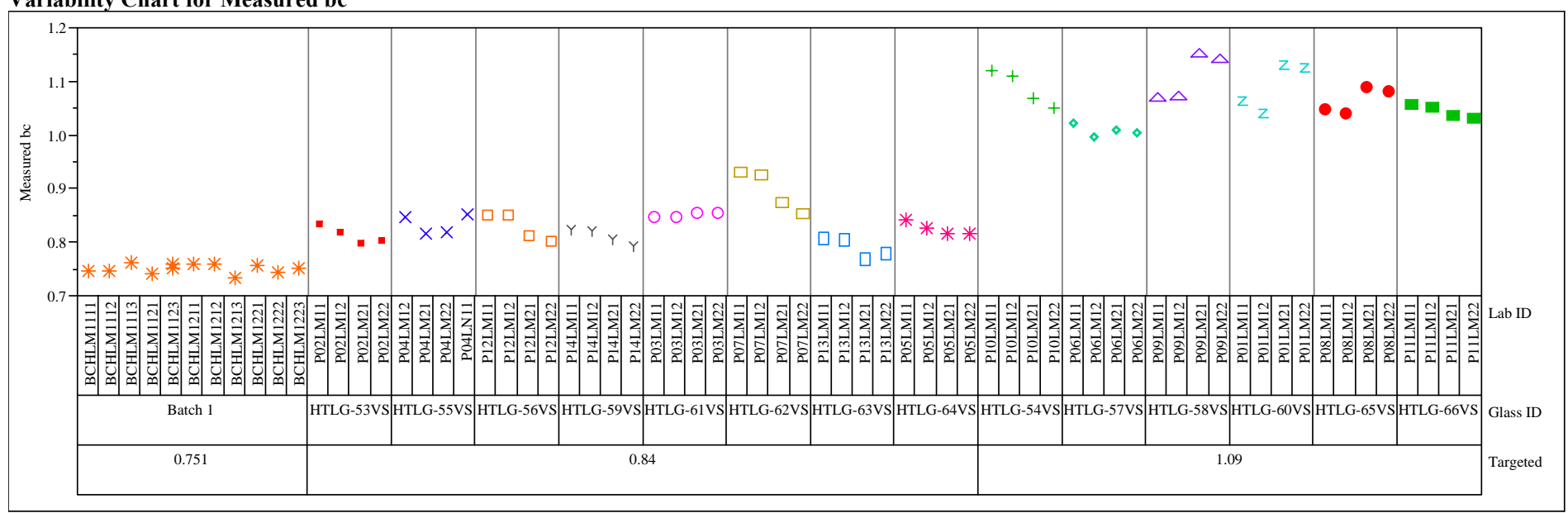


Exhibit A9. Measured and Measured Bias-Corrected Oxide Weight Percents

by Glass ID by Targeted Value for the Series "P" Glasses Prepared Using the LM Method

SO4 (wt\%)

Variability Chart for Measured

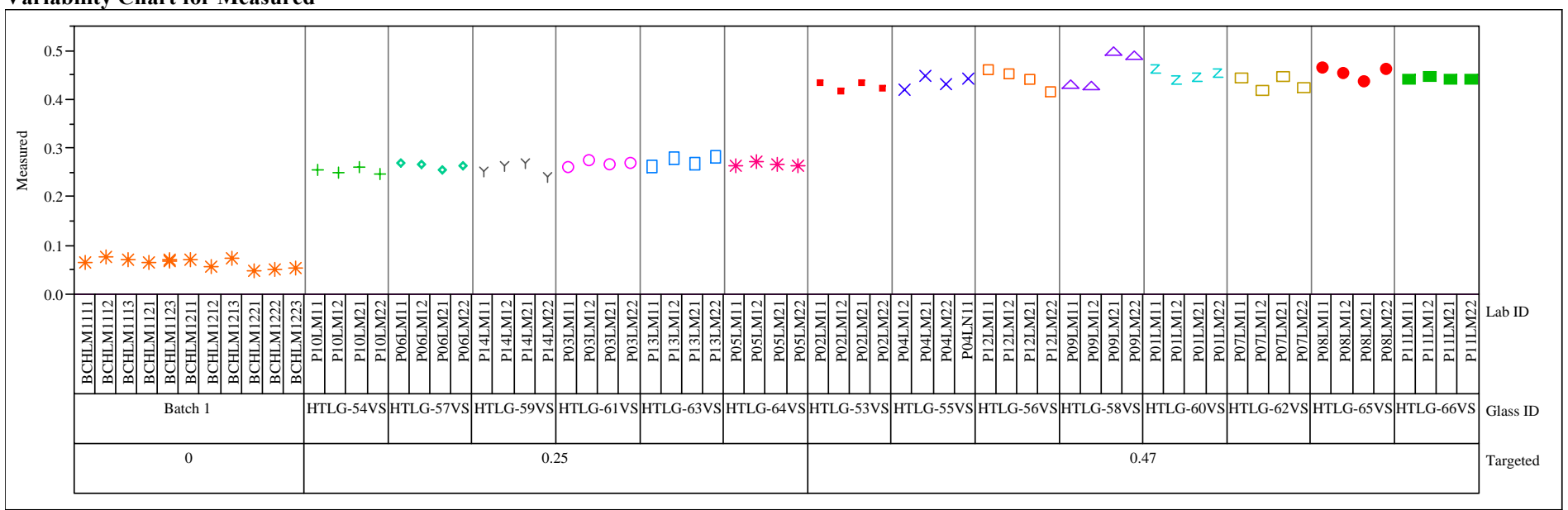

Variability Chart for Measured bc

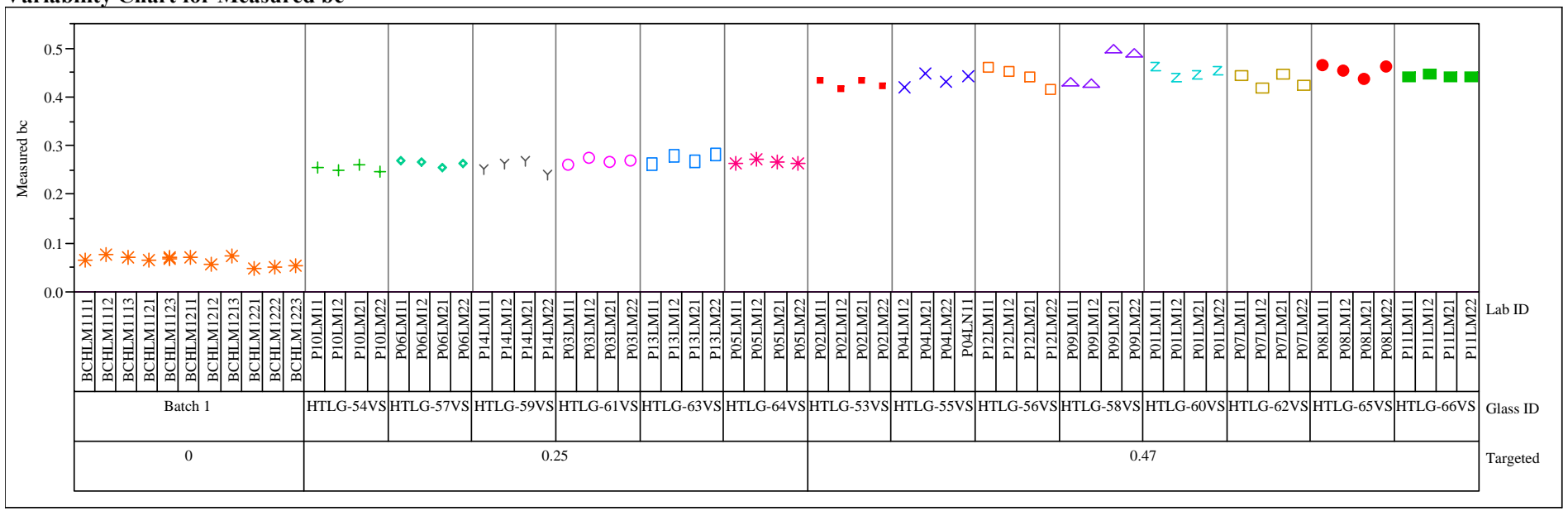


Exhibit A9. Measured and Measured Bias-Corrected Oxide Weight Percents

by Glass ID by Targeted Value for the Series "P" Glasses Prepared Using the LM Method

TiO2 (wt\%)

Variability Chart for Measured

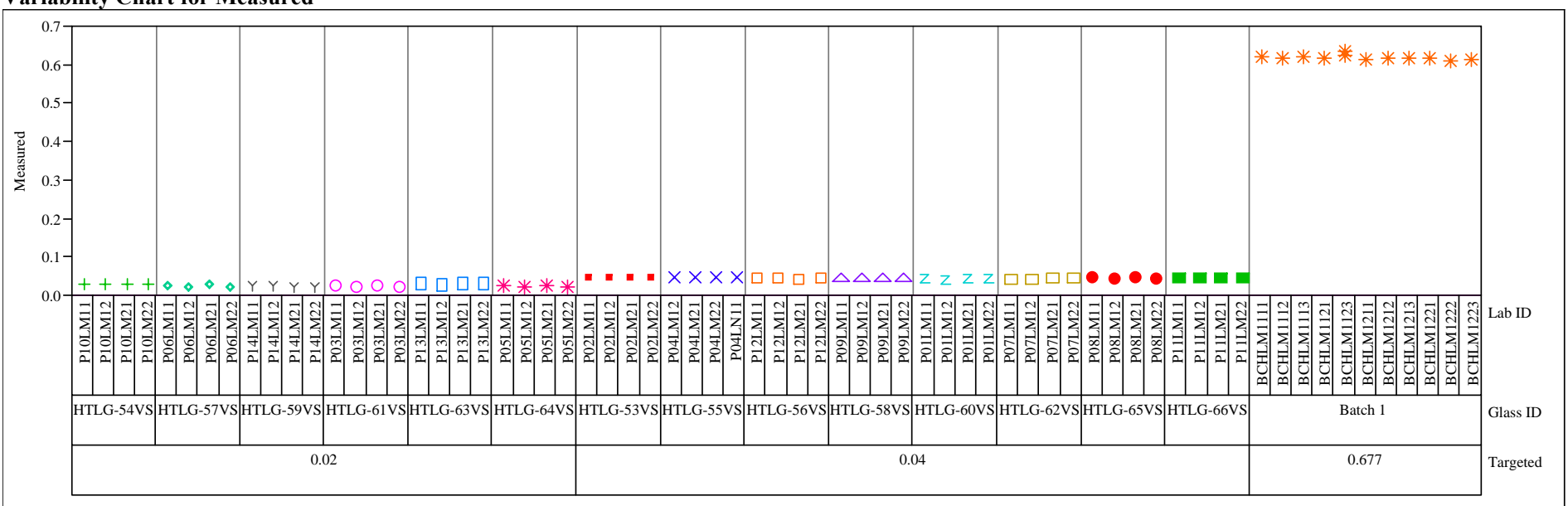

Variability Chart for Measured bc

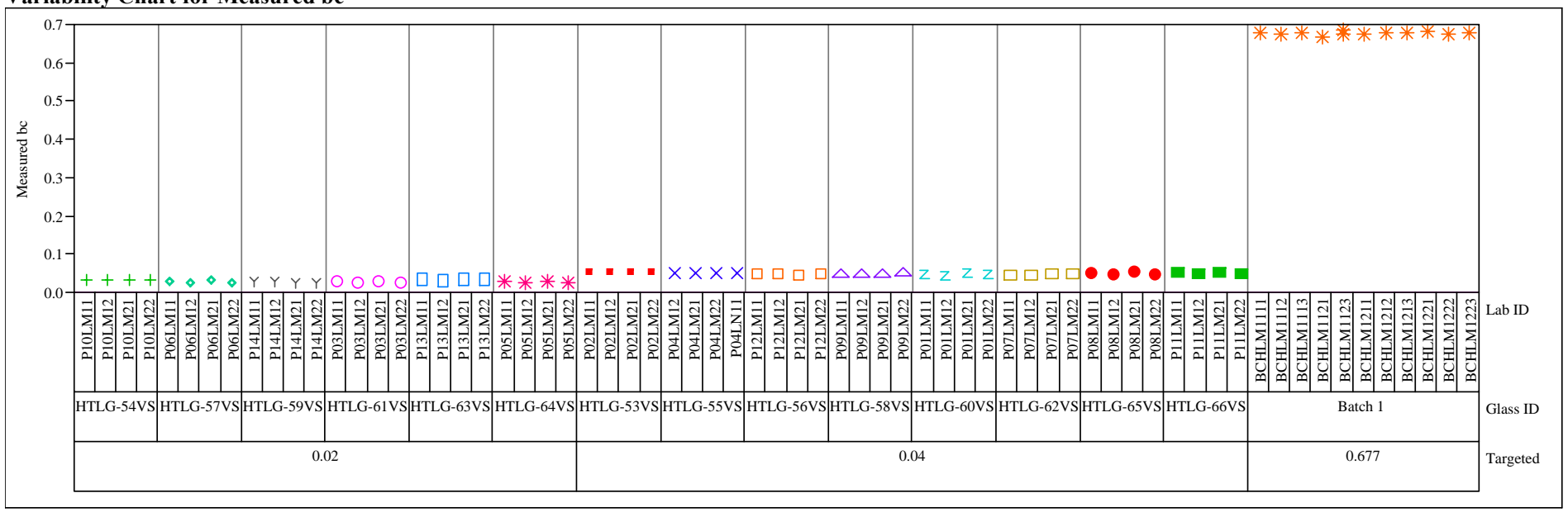


Exhibit A9. Measured and Measured Bias-Corrected Oxide Weight Percents

by Glass ID by Targeted Value for the Series "P" Glasses Prepared Using the LM Method

$\mathrm{ZrO2}(\mathrm{wt} \%)$

Variability Chart for Measured

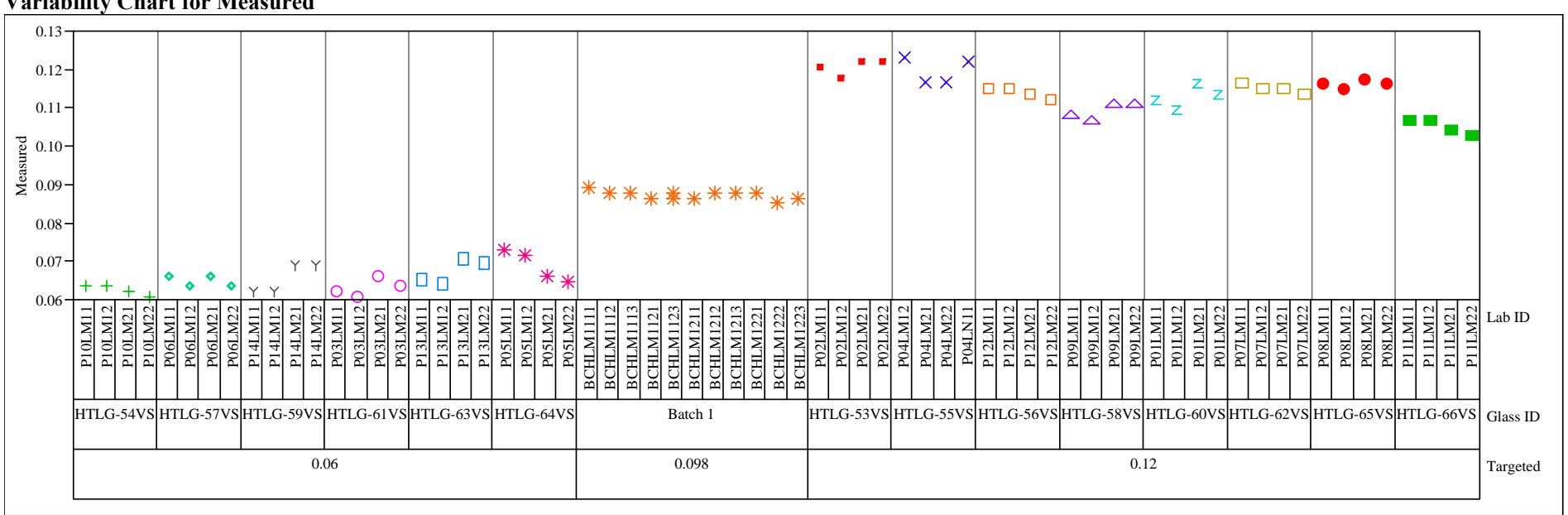

Variability Chart for Measured bc

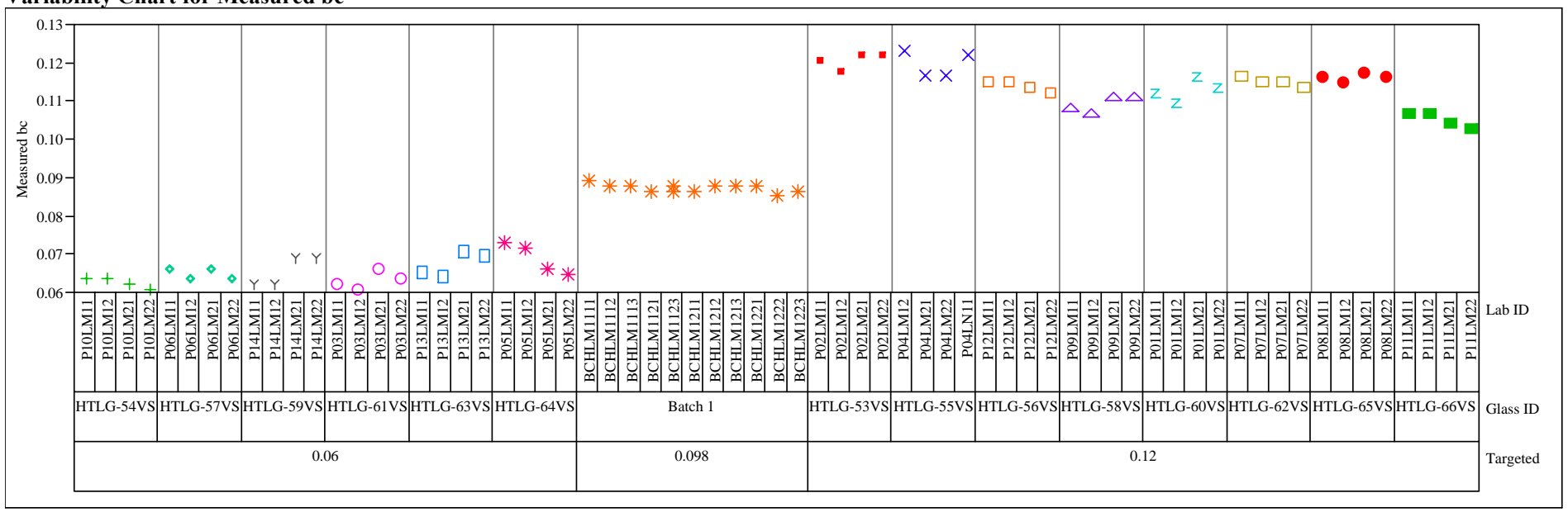


Exhibit A10. Measured and Measured Bias-Corrected Oxide Weight Percents by Glass ID by Targeted Value for the Series "P" Glasses Prepared Using the PF Method

B2O3 (wt\%)

Variability Chart for Measured

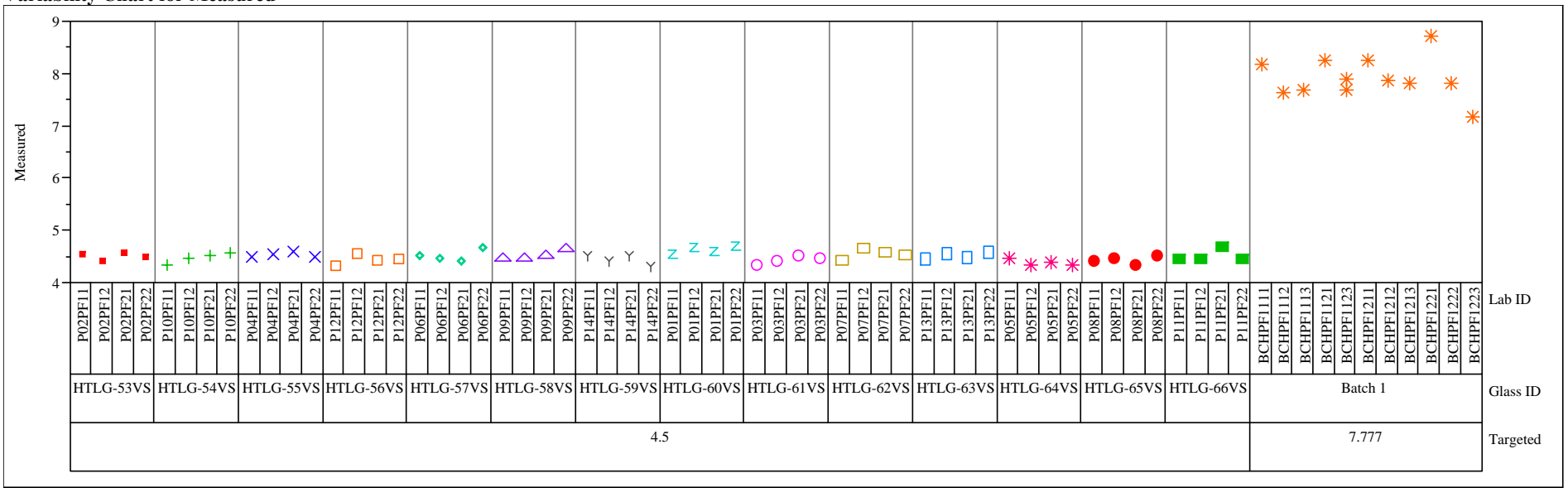

Variability Chart for Measured bc

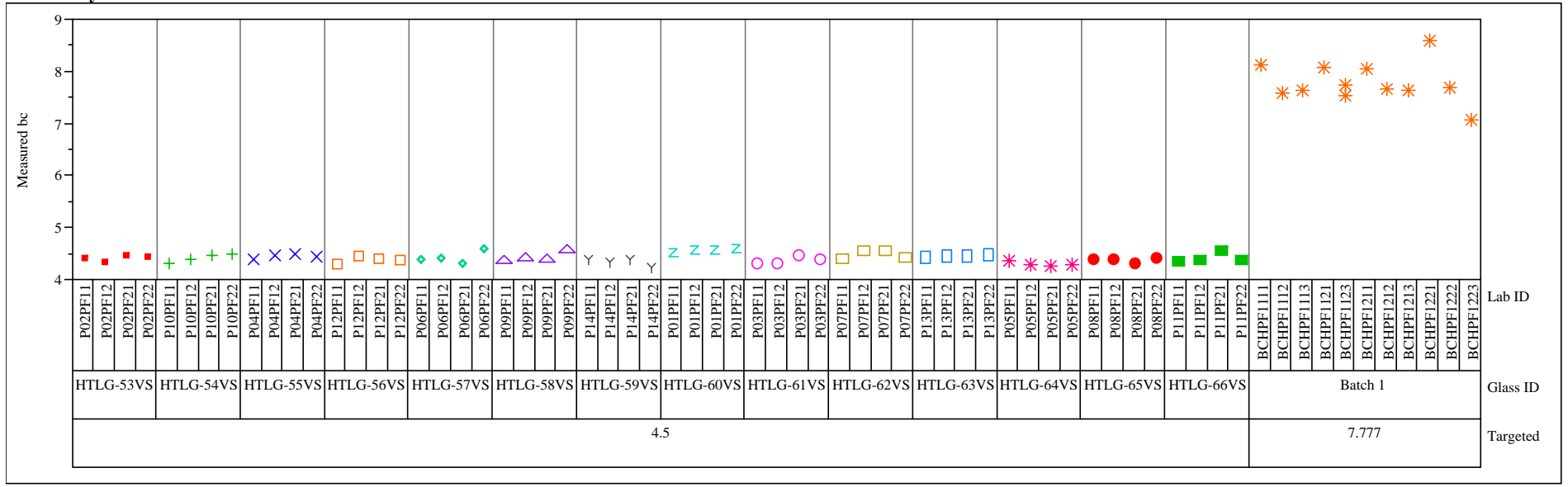


Exhibit A10. Measured and Measured Bias-Corrected Oxide Weight Percents by Glass ID by Targeted Value for the Series "P" Glasses Prepared Using the PF Method

$\mathrm{Fe} 2 \mathrm{O3}(\mathrm{wt} \%)$

Variability Chart for Measured

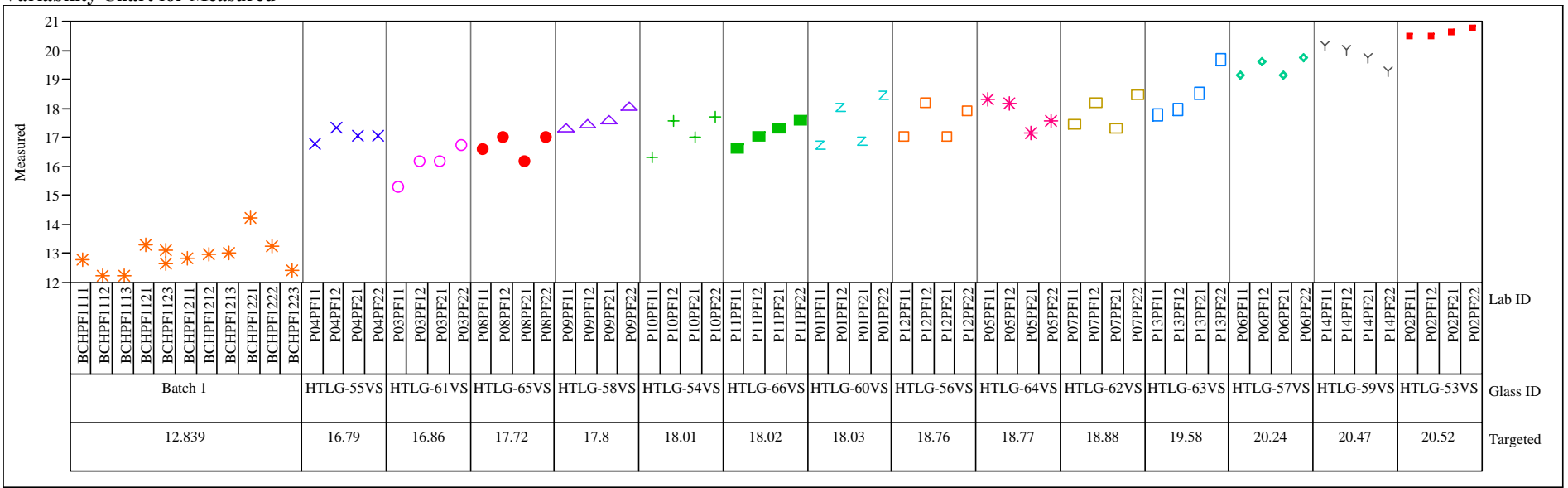

Variability Chart for Measured be

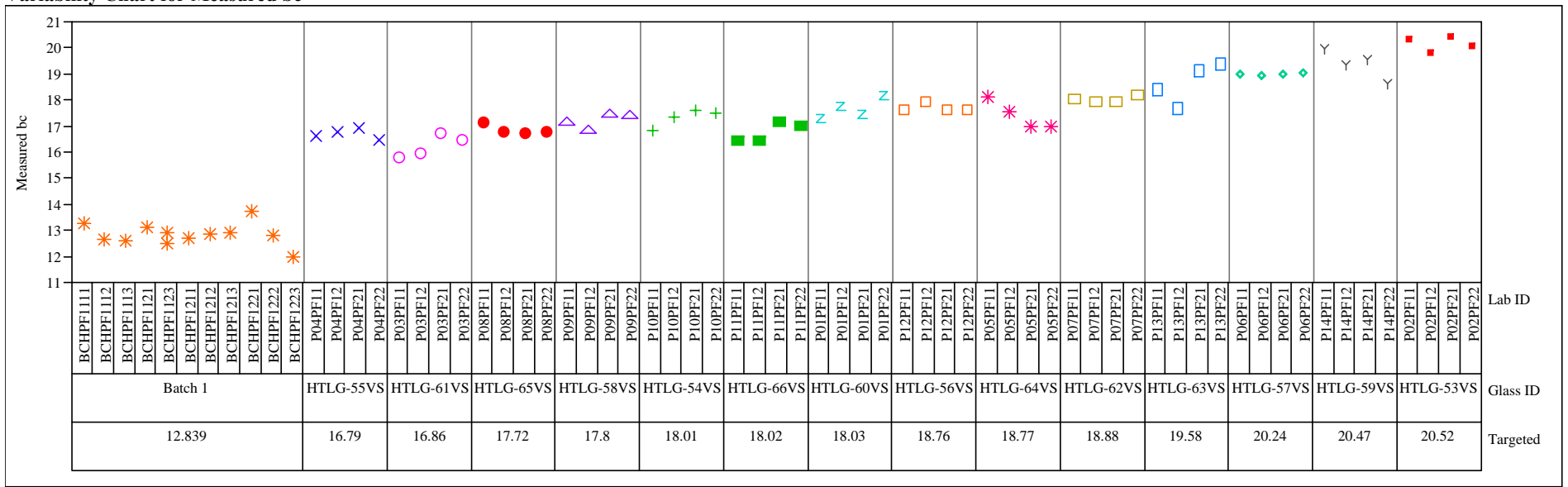


Exhibit A10. Measured and Measured Bias-Corrected Oxide Weight Percents by Glass ID by Targeted Value for the Series "P" Glasses Prepared Using the PF Method

Li2O (wt\%)

Variability Chart for Measured

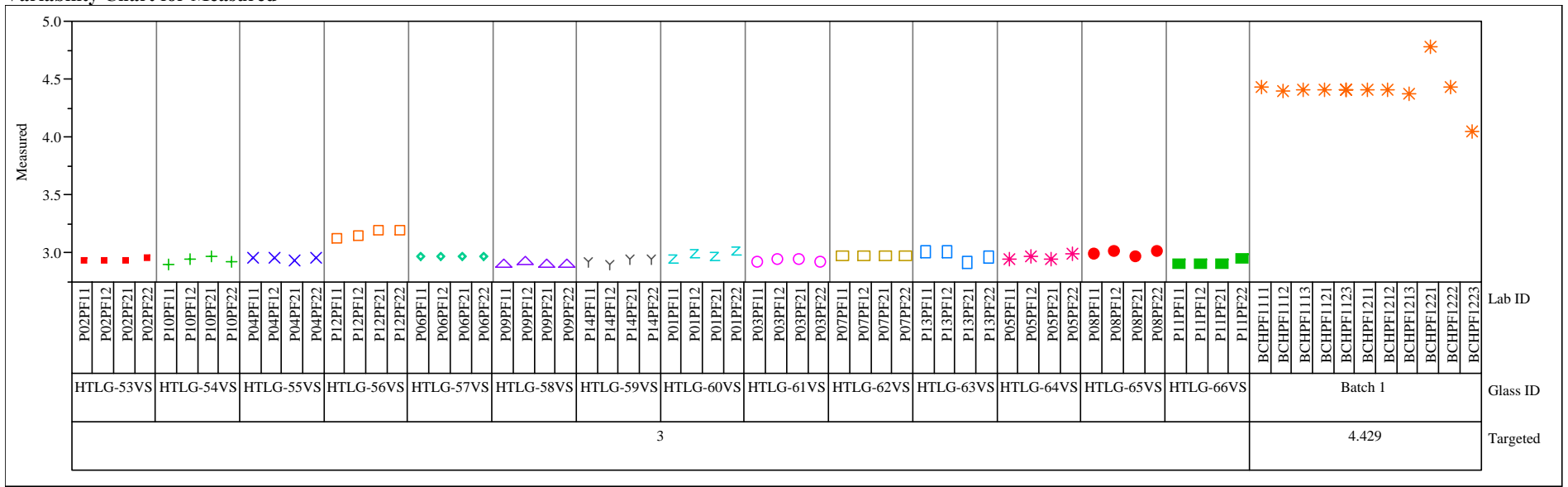

Variability Chart for Measured bc

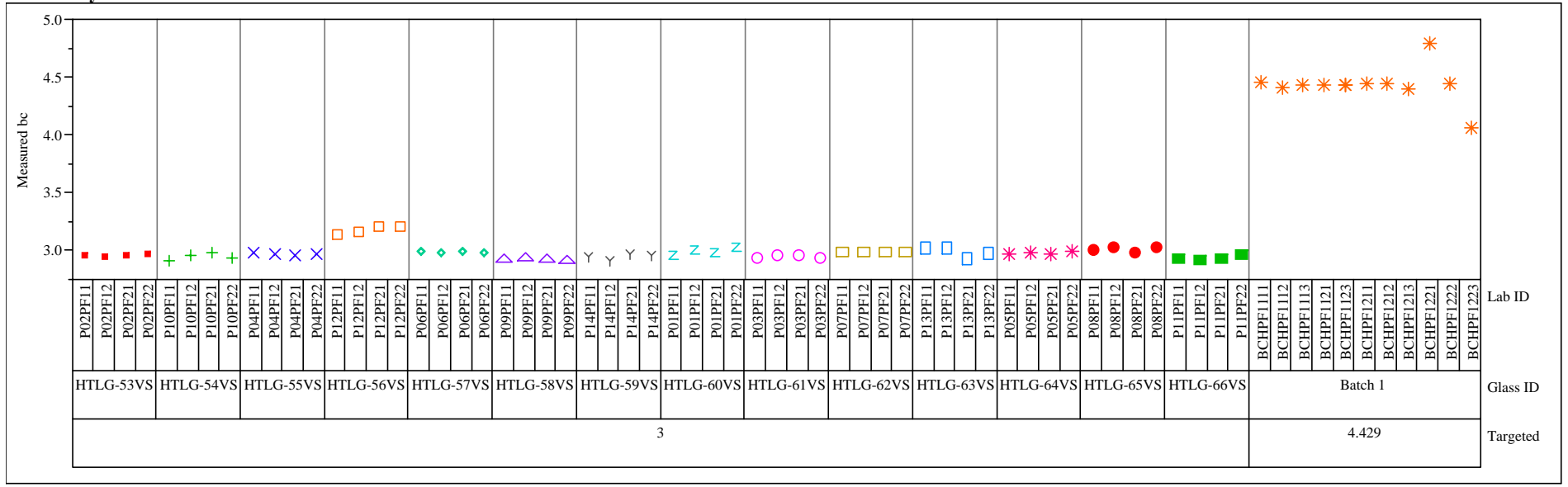


Exhibit A10. Measured and Measured Bias-Corrected Oxide Weight Percents by Glass ID by Targeted Value for the Series "P" Glasses Prepared Using the PF Method

$\mathrm{SiO} 2(\mathrm{wt} \%)$

Variability Chart for Measured

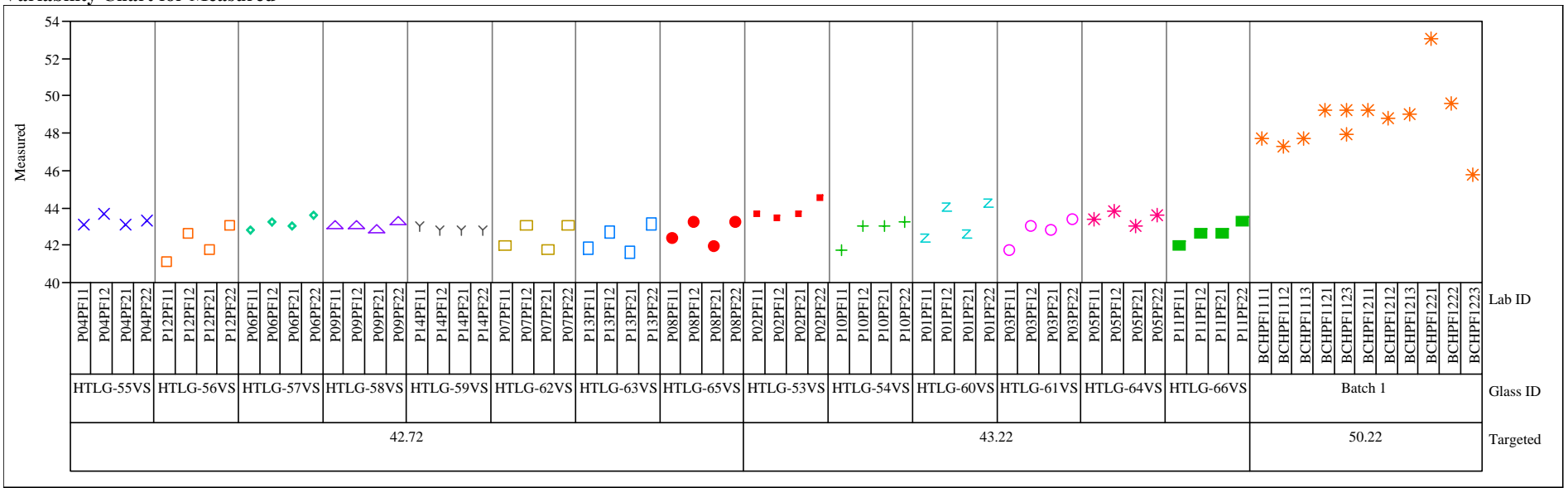

Variability Chart for Measured bc

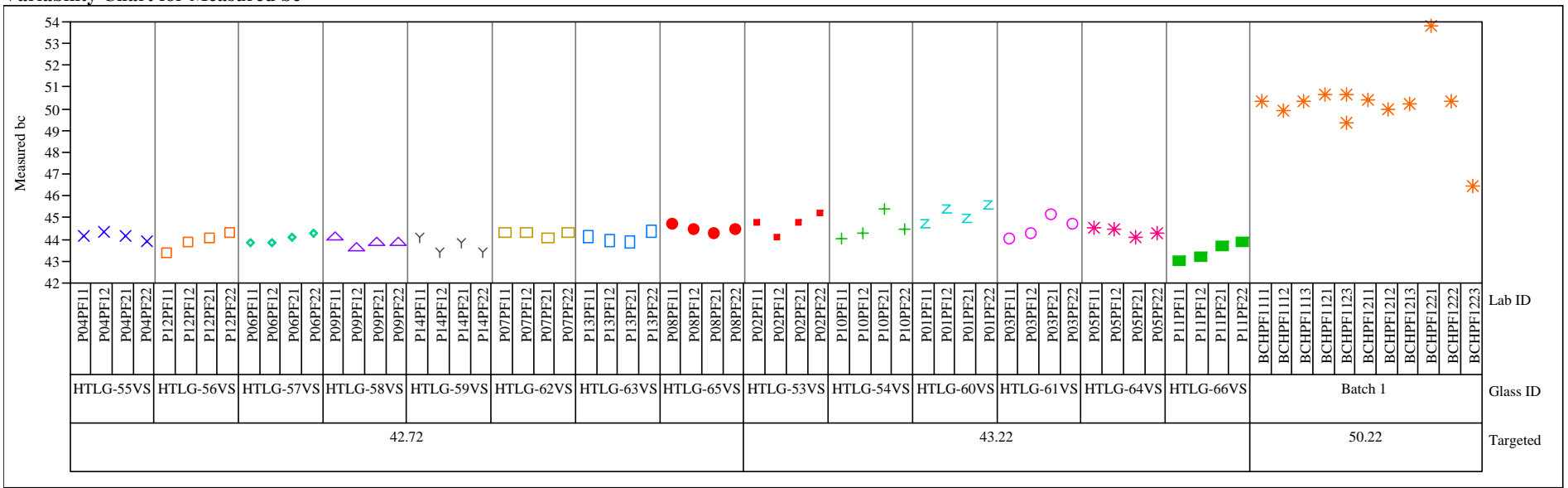


Exhibit A11. Measured and Measured Bias-Corrected Oxide Weight Percents by Glass ID by Targeted Value for the Series "Q" Glasses Prepared Using the LM Method

$\mathrm{Al2O3}(\mathrm{wt} \%)$

Variabilty Chart for Measured

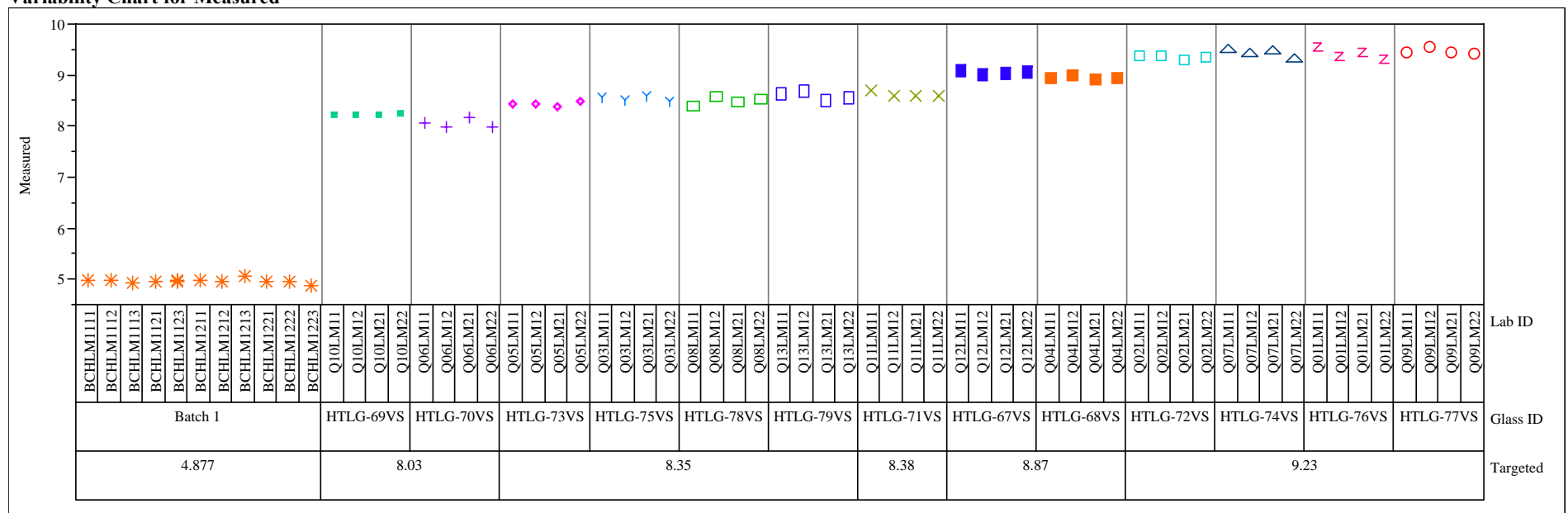

Variability Chart for Measured be

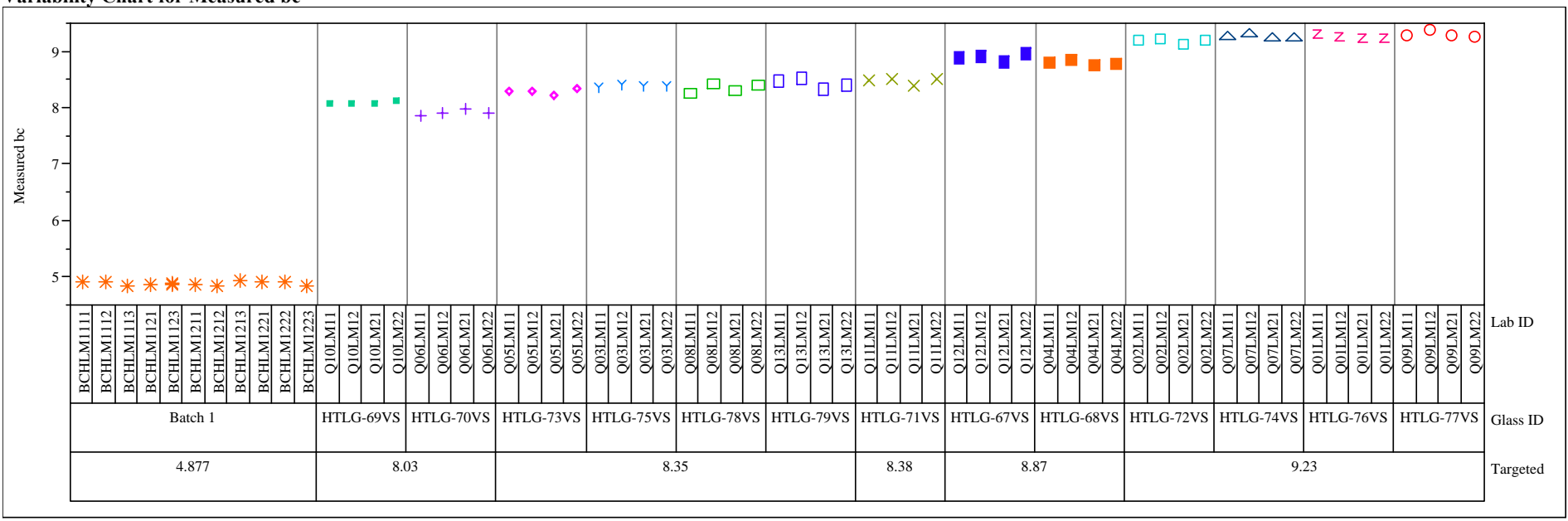


Exhibit A11. Measured and Measured Bias-Corrected Oxide Weight Percents by Glass ID by Targeted Value for the Series "Q" Glasses Prepared Using the LM Method

$\mathrm{CaO}$ (wt\%)

Variability Chart for Measure

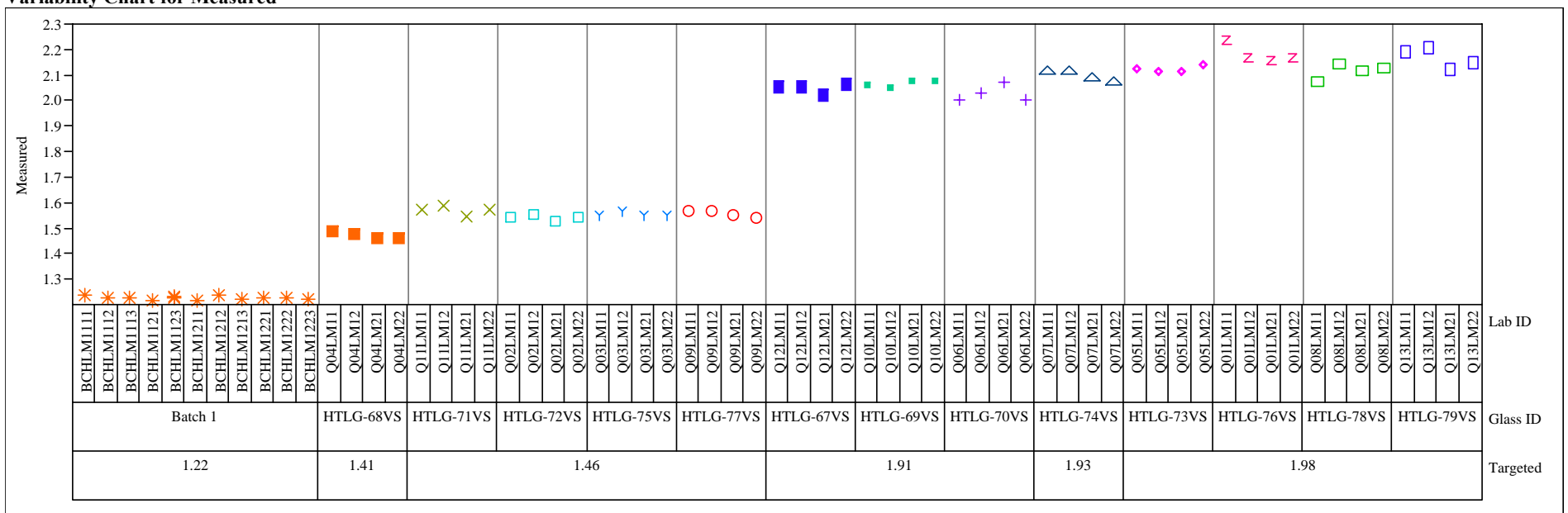

Variability Chart for Measured be

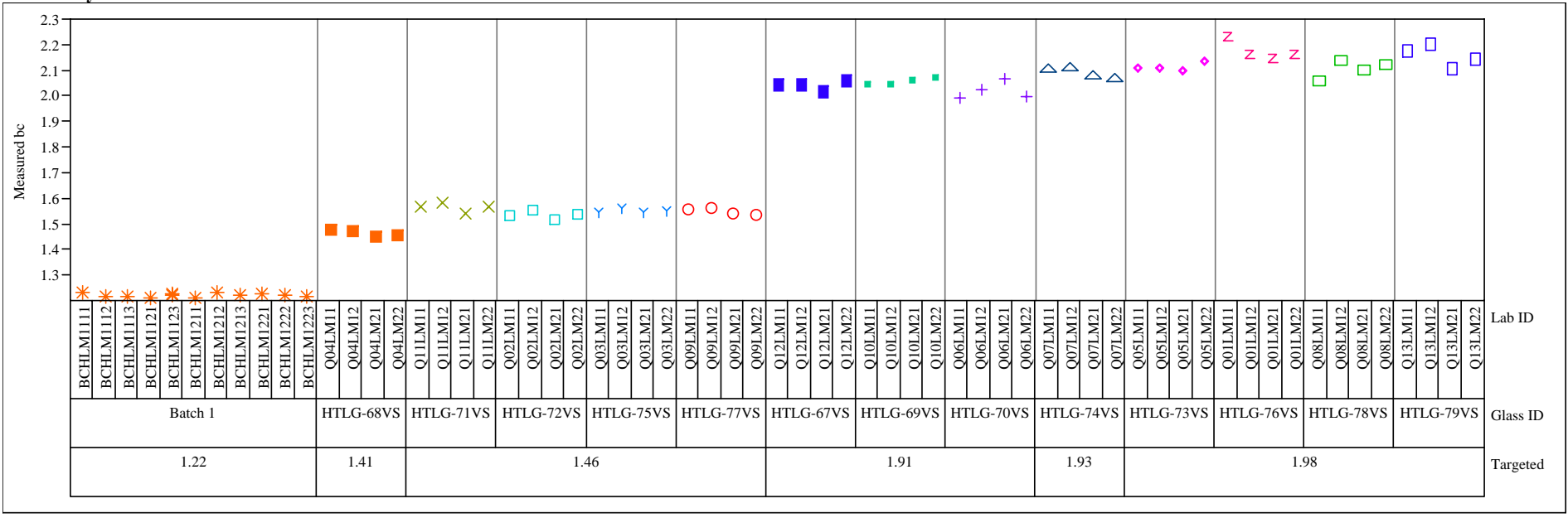


Exhibit A11. Measured and Measured Bias-Corrected Oxide Weight Percents by Glass ID by Targeted Value for the Series "Q" Glasses Prepared Using the LM Method

$\mathrm{Cr} 2 \mathrm{O3}(\mathrm{wt} \%)$

Variability Chart for Measured

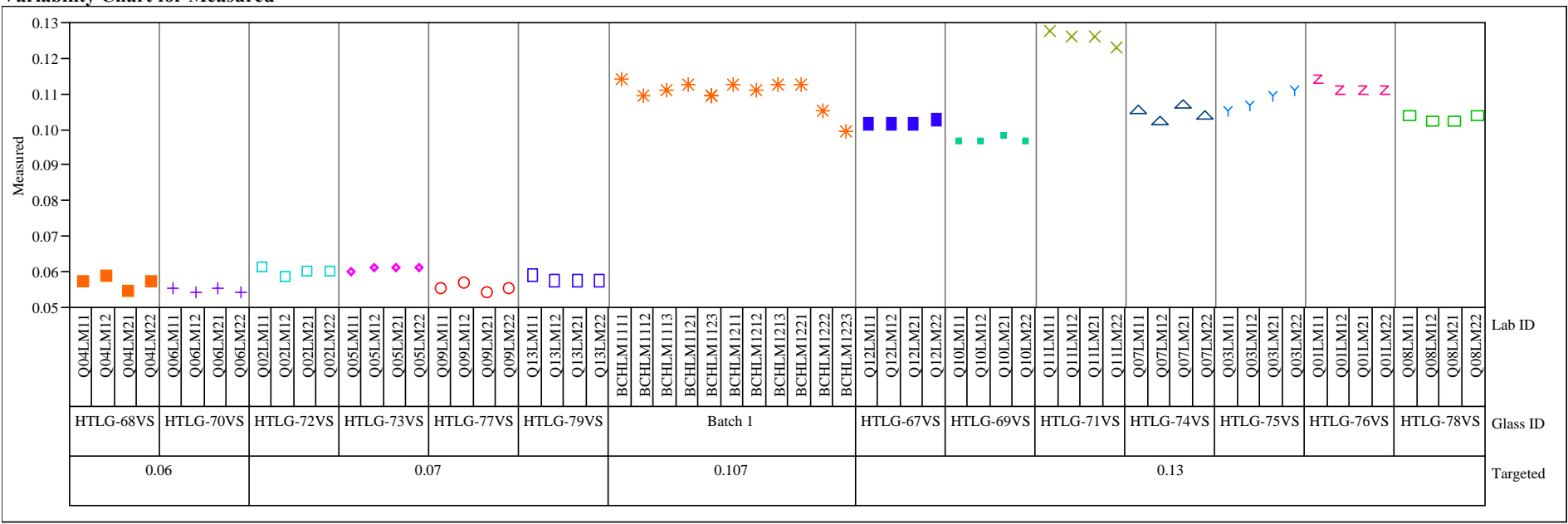

Variability Chart for Measured bc

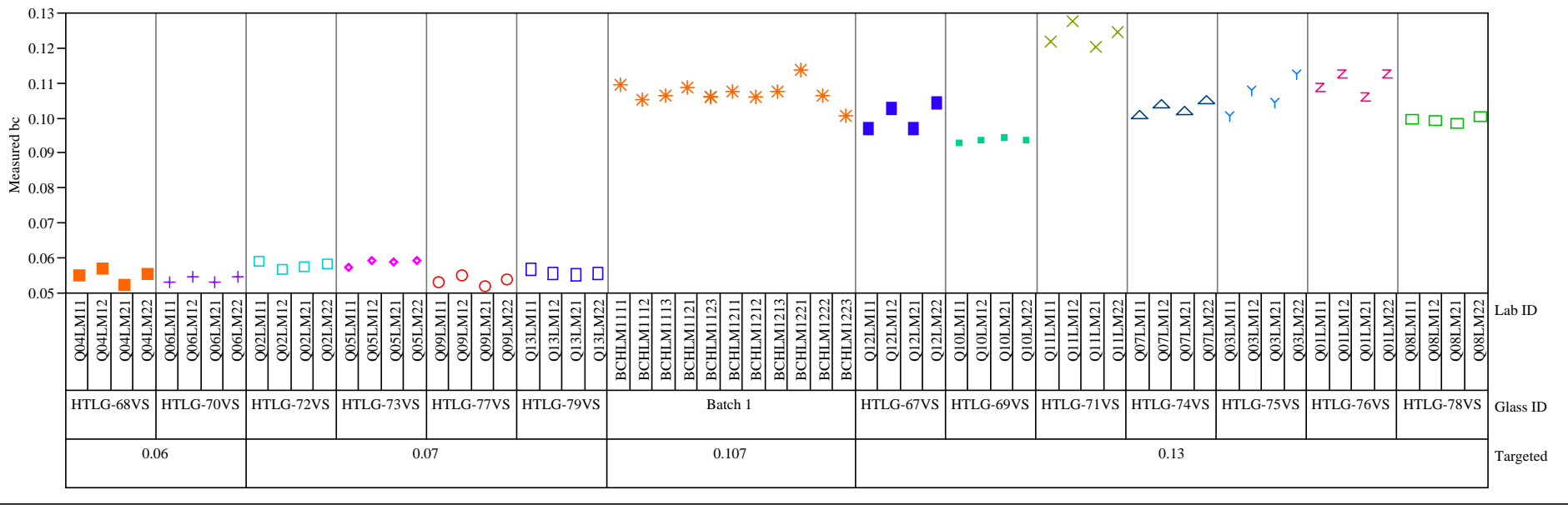


Exhibit A11. Measured and Measured Bias-Corrected Oxide Weight Percents by Glass ID by Targeted Value for the Series "Q" Glasses Prepared Using the LM Method

$\mathrm{CuO}$ (wt\%)

Variability Chart for Measured
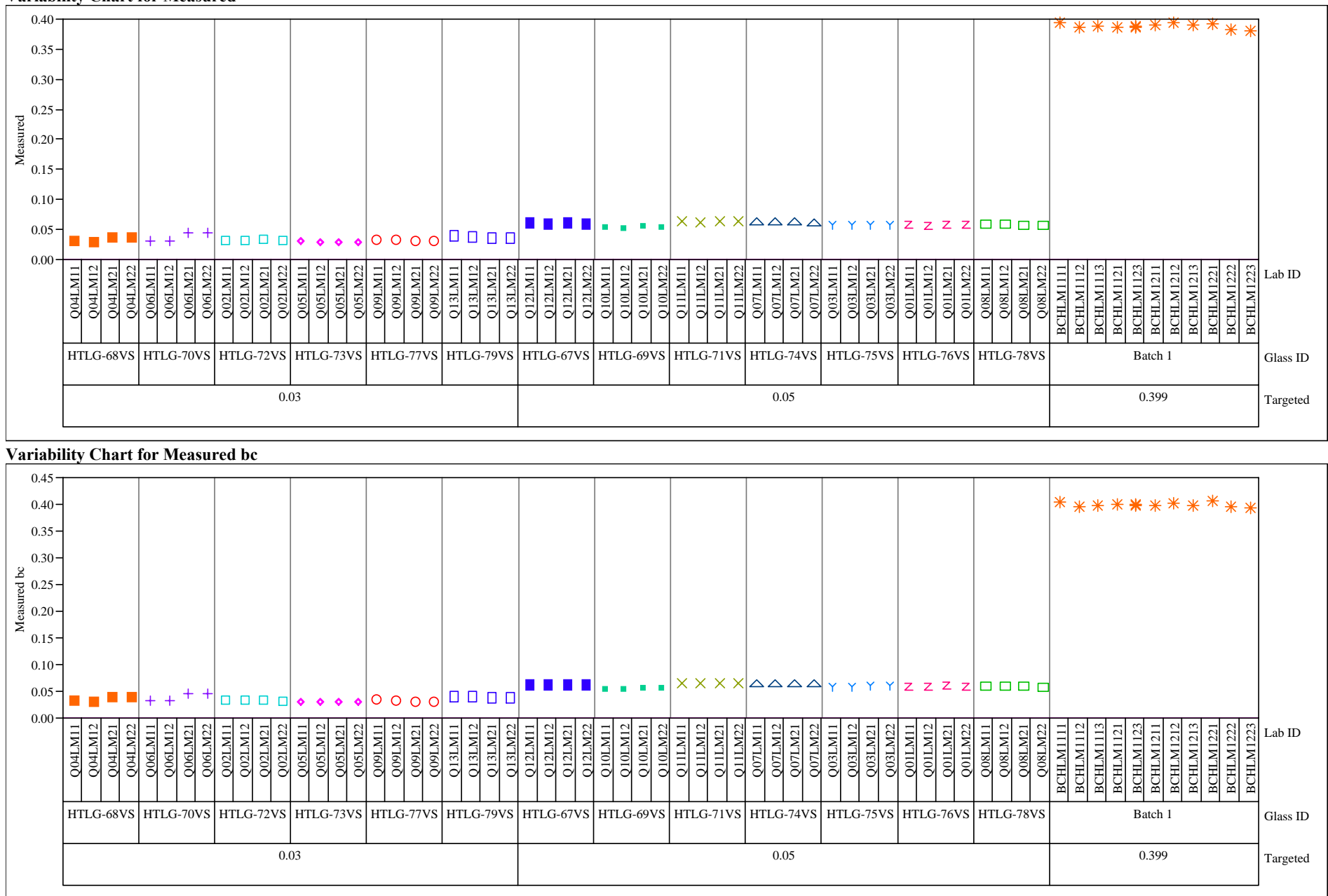
Exhibit A11. Measured and Measured Bias-Corrected Oxide Weight Percents by Glass ID by Targeted Value for the Series " $Q$ " Glasses Prepared Using the LM Method

K2O (wt\%)

Variability Chart for Measured
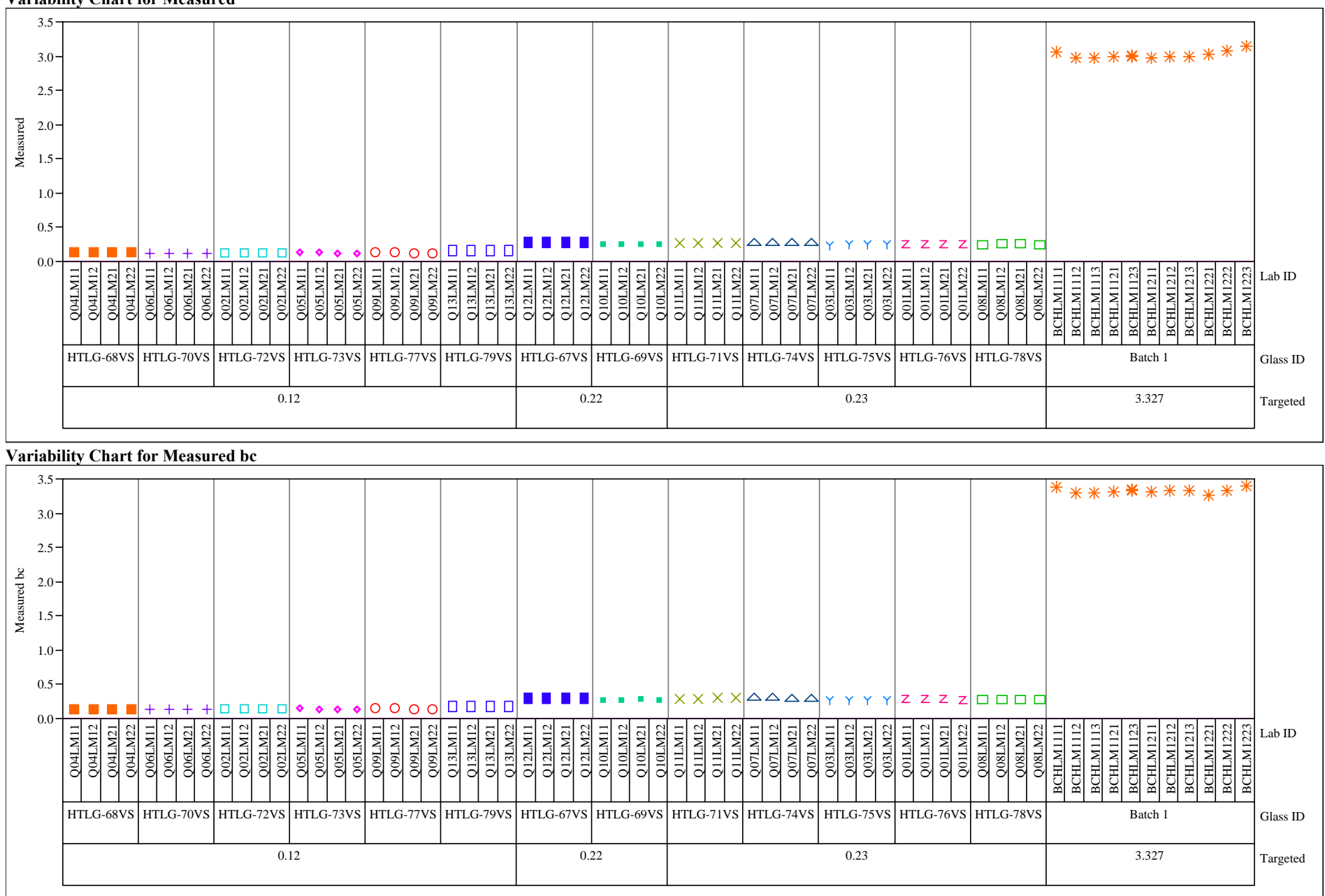
Exhibit A11. Measured and Measured Bias-Corrected Oxide Weight Percents by Glass ID by Targeted Value for the Series " $Q$ " Glasses Prepared Using the LM Method

$\operatorname{MgO}(\mathbf{w t} \%)$

Variability Chart for Measure
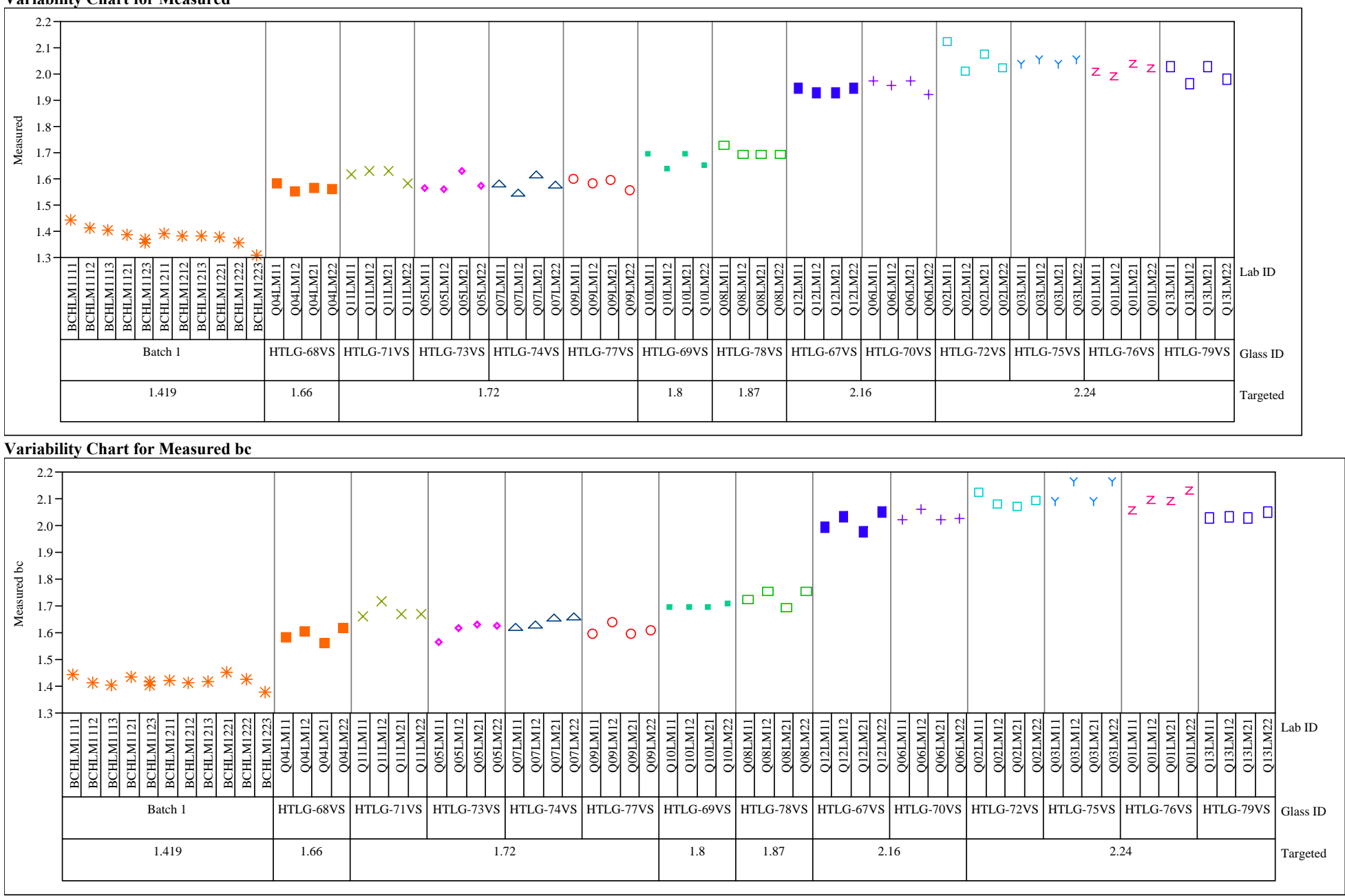
Exhibit A11. Measured and Measured Bias-Corrected Oxide Weight Percents by Glass ID by Targeted Value for the Series " $Q$ " Glasses Prepared Using the LM Method

MnO (wt\%)

Variability Chart for Measured

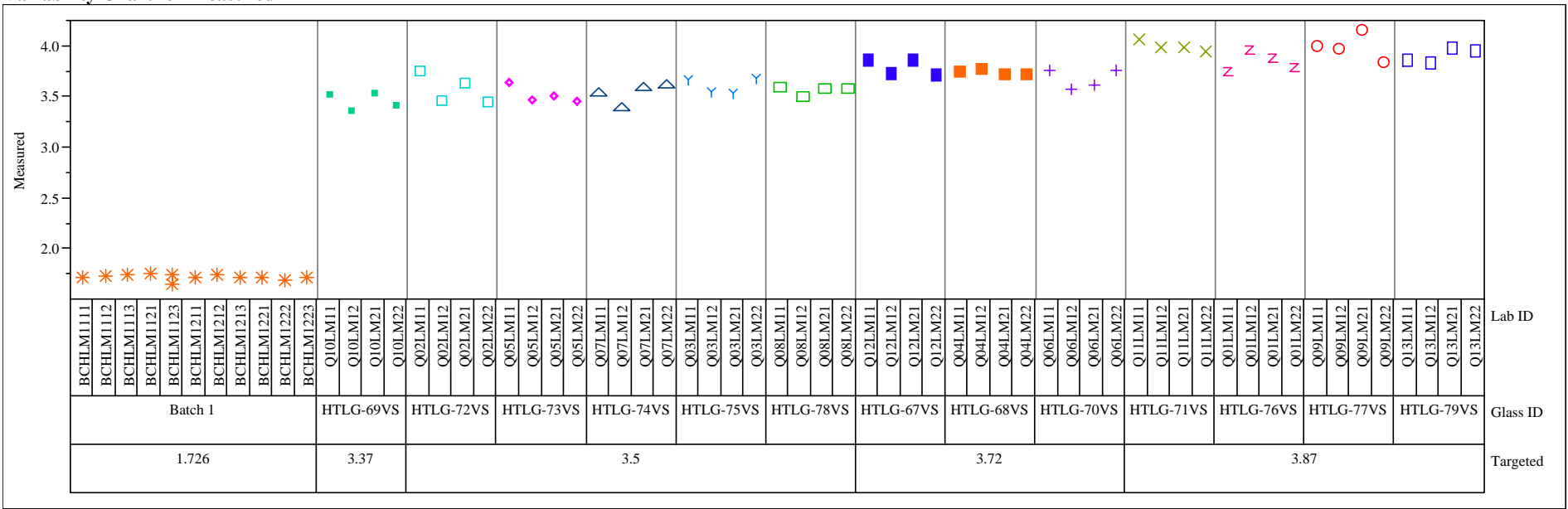

Variability Chart for Measured be

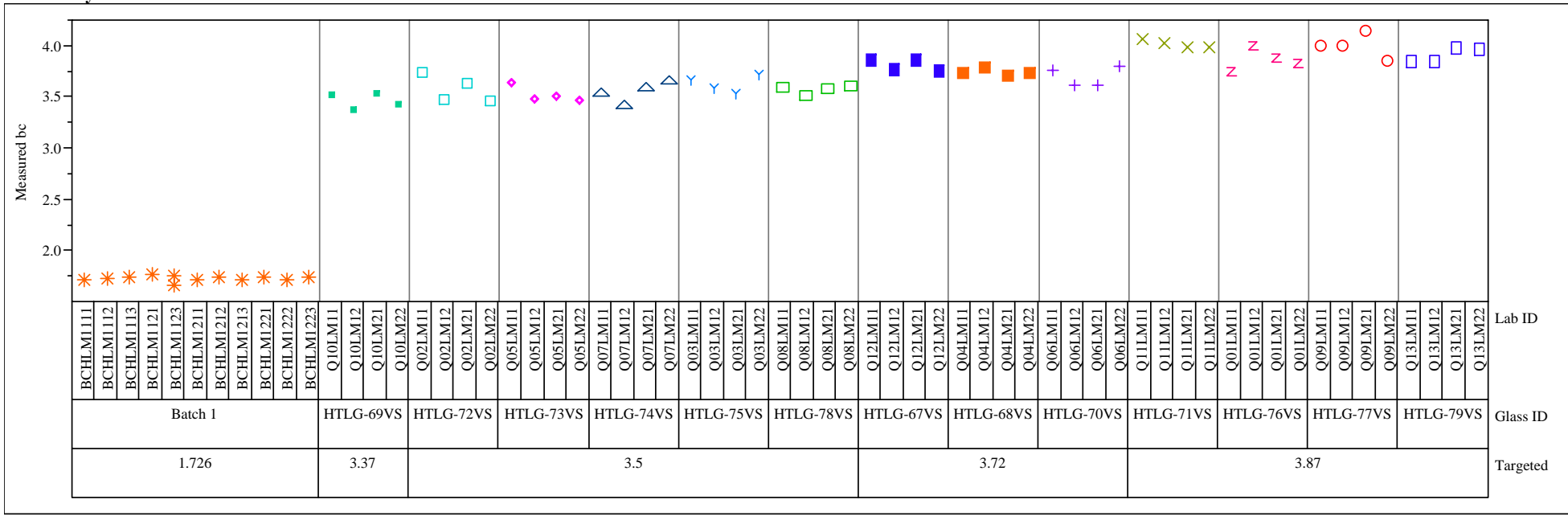


Exhibit A11. Measured and Measured Bias-Corrected Oxide Weight Percents by Glass ID by Targeted Value for the Series "Q" Glasses Prepared Using the LM Method

$\mathrm{Na2O}$ (wt\%)

Variability Chart for Measured

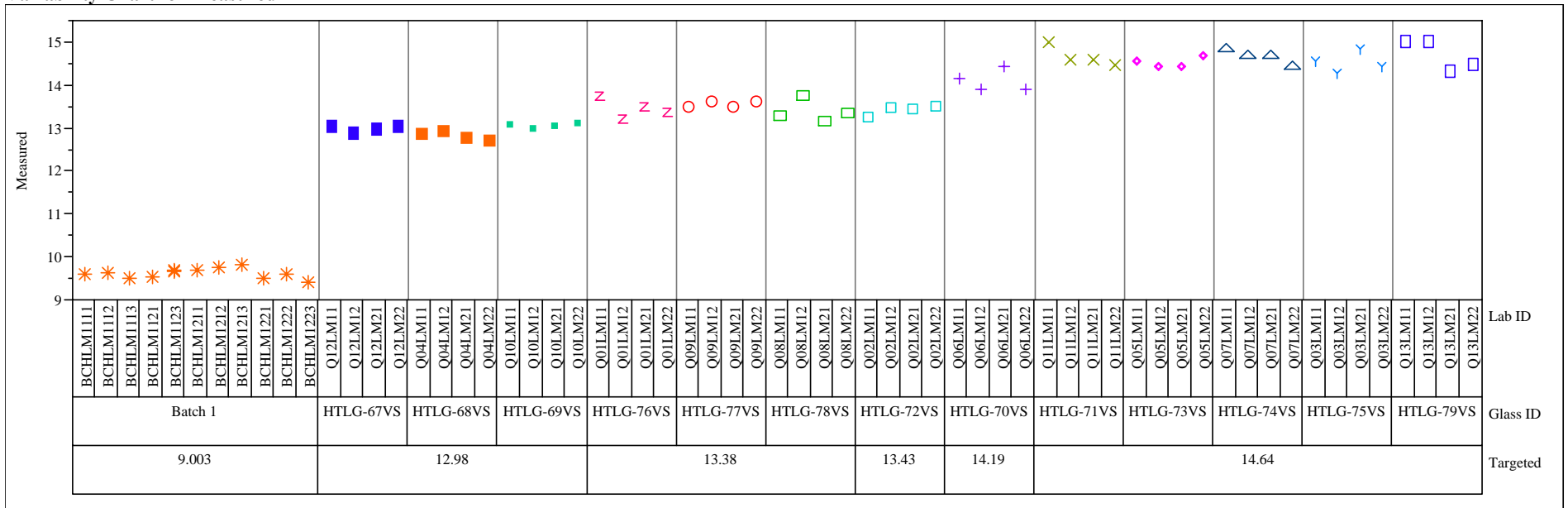

Variability Chart for Measured bc

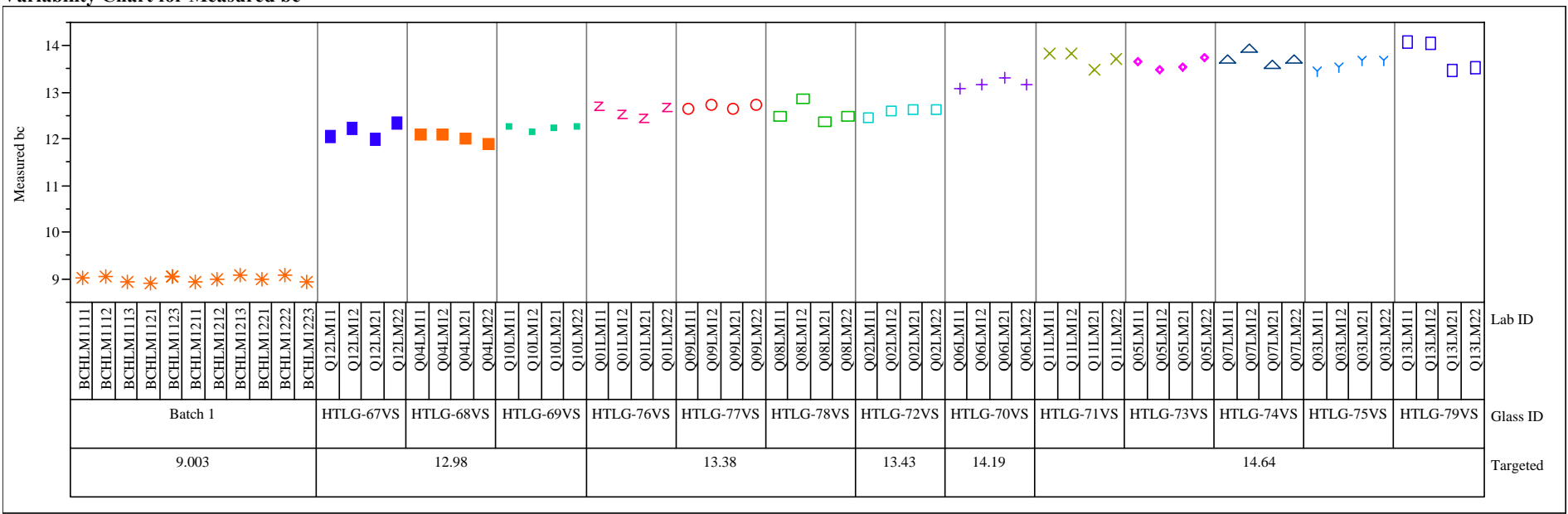


Exhibit A11. Measured and Measured Bias-Corrected Oxide Weight Percents by Glass ID by Targeted Value for the Series "Q" Glasses Prepared Using the LM Method

$\mathrm{NiO}(\mathrm{wt} \%)$

Variability Chart for Measured
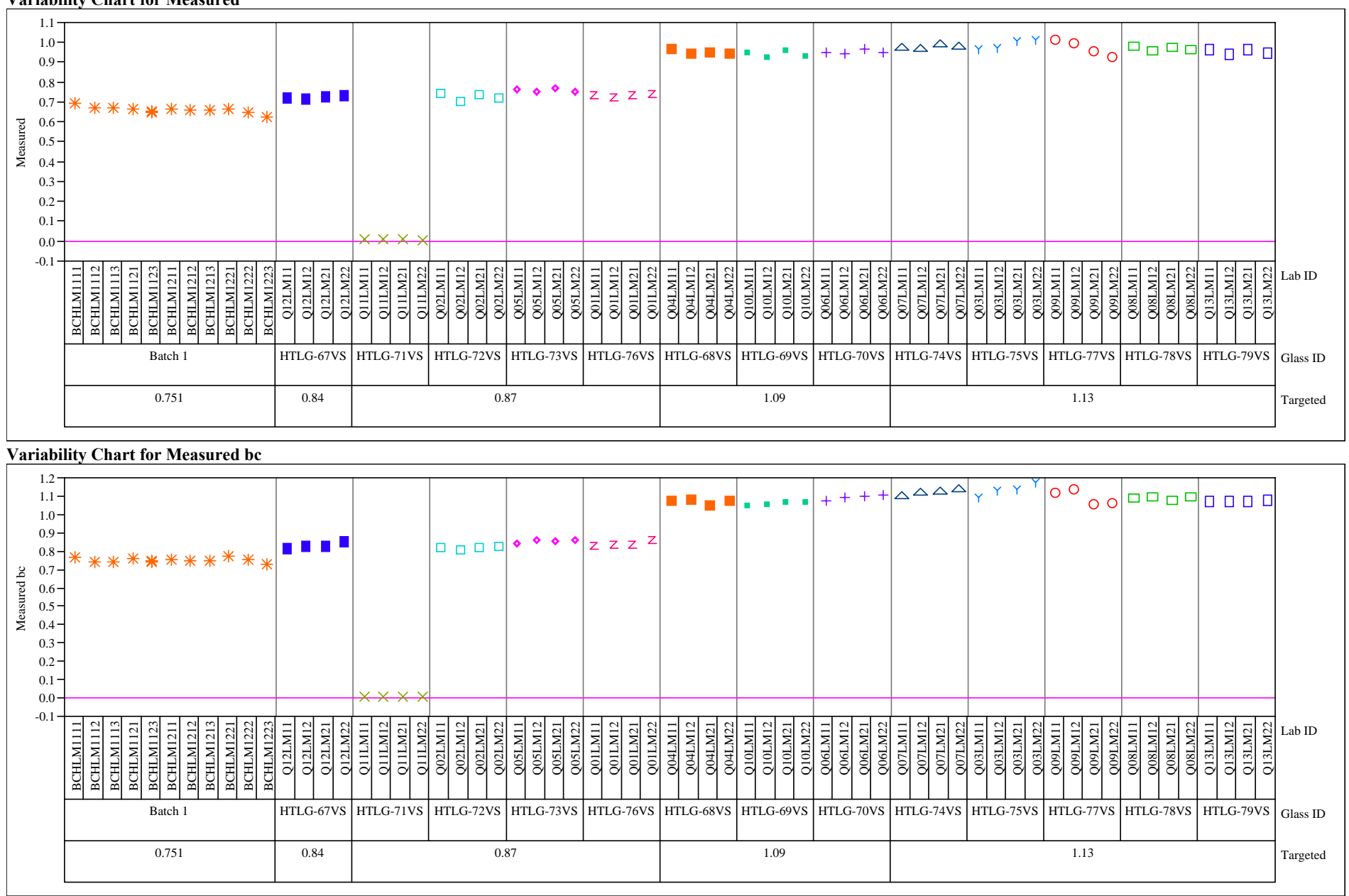
Exhibit A11. Measured and Measured Bias-Corrected Oxide Weight Percents by Glass ID by Targeted Value for the Series " $Q$ " Glasses Prepared Using the LM Method

$\mathrm{SiO2}(\mathrm{wt} \%)$

Variability Chart for Measured
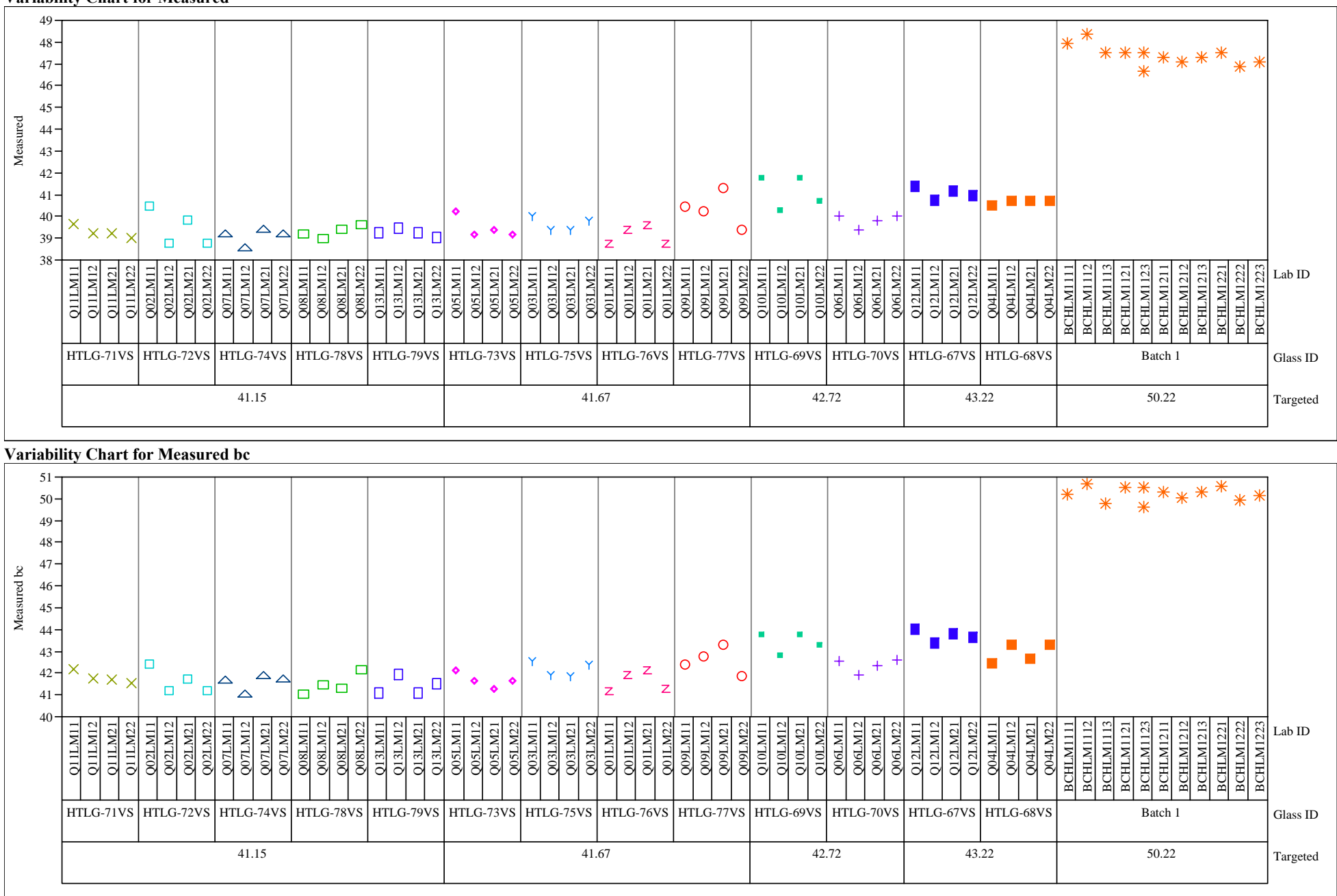
Exhibit A11. Measured and Measured Bias-Corrected Oxide Weight Percents by Glass ID by Targeted Value for the Series " $Q$ " Glasses Prepared Using the LM Method

SO4 (wt\%)

Variability Chart for Measured
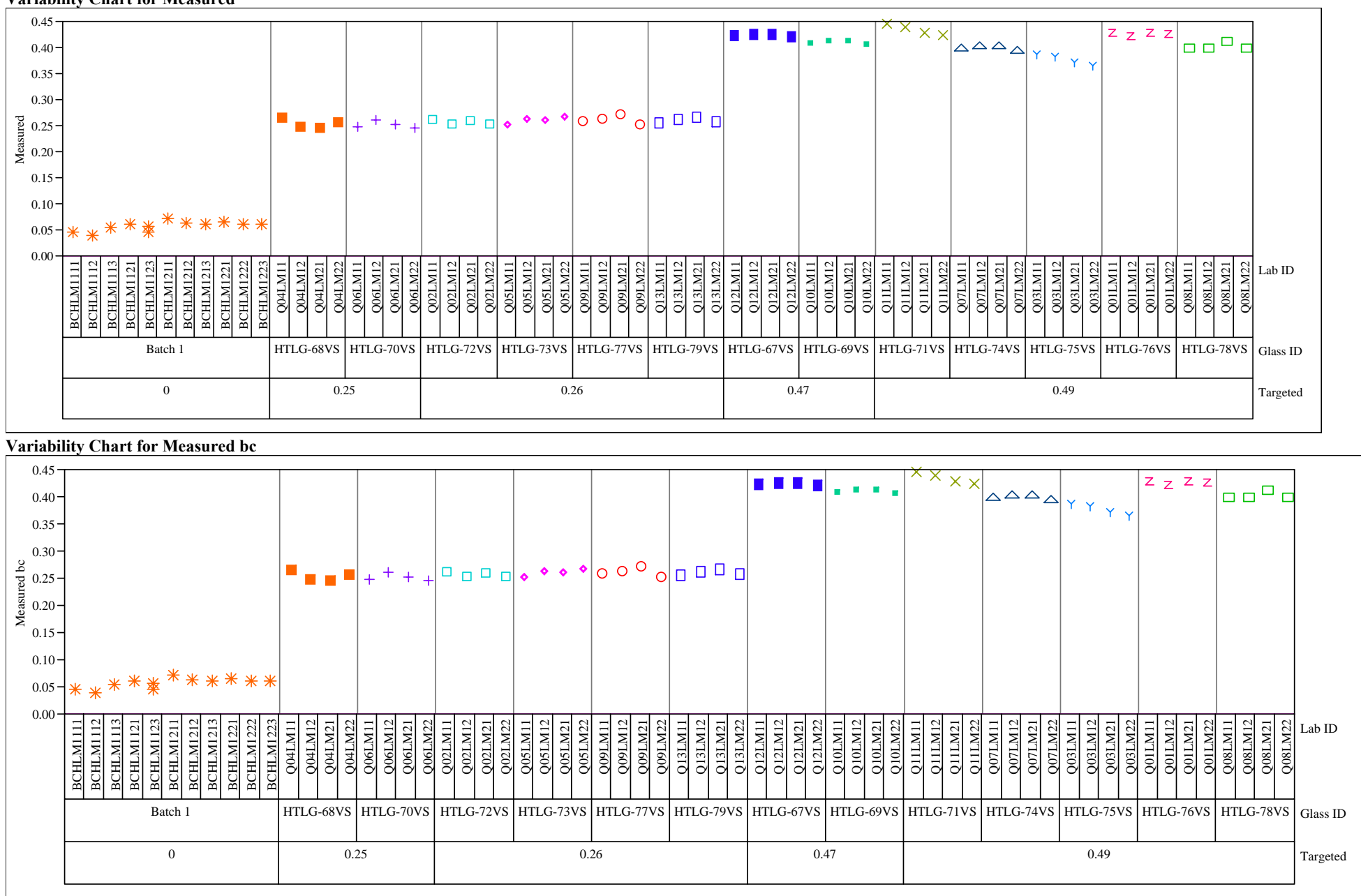
Exhibit A11. Measured and Measured Bias-Corrected Oxide Weight Percents by Glass ID by Targeted Value for the Series " $Q$ " Glasses Prepared Using the LM Method

TiO2 (wt\%)

Variability Chart for Measured

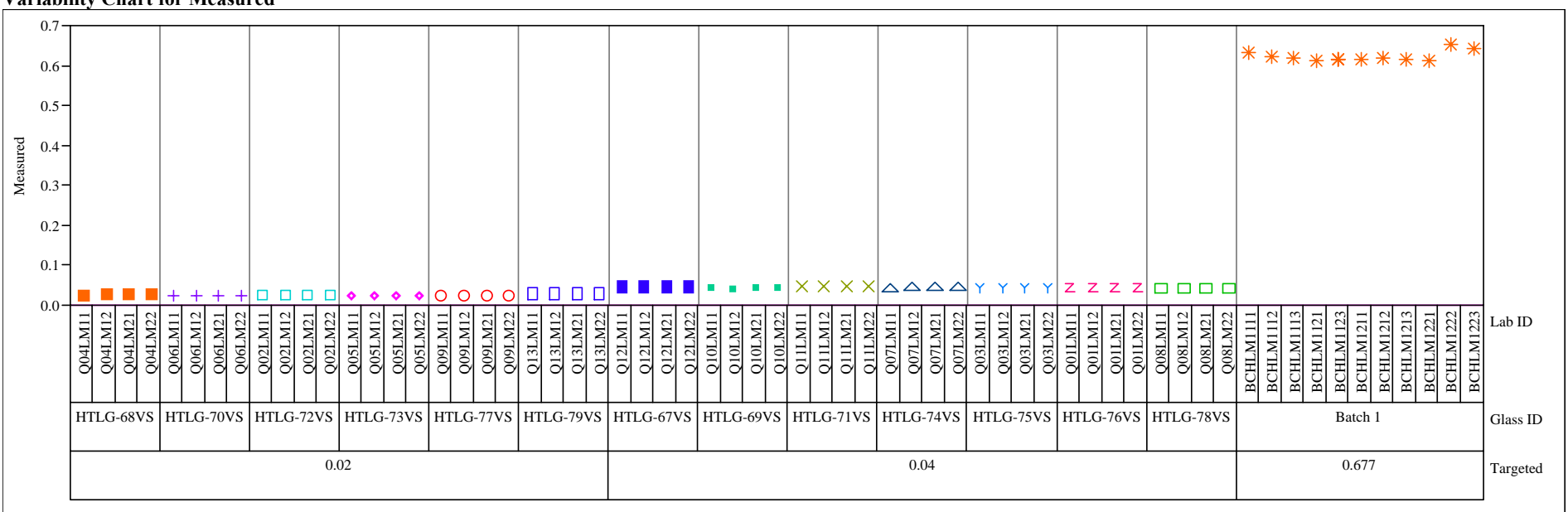

Variability Chart for Measured bc

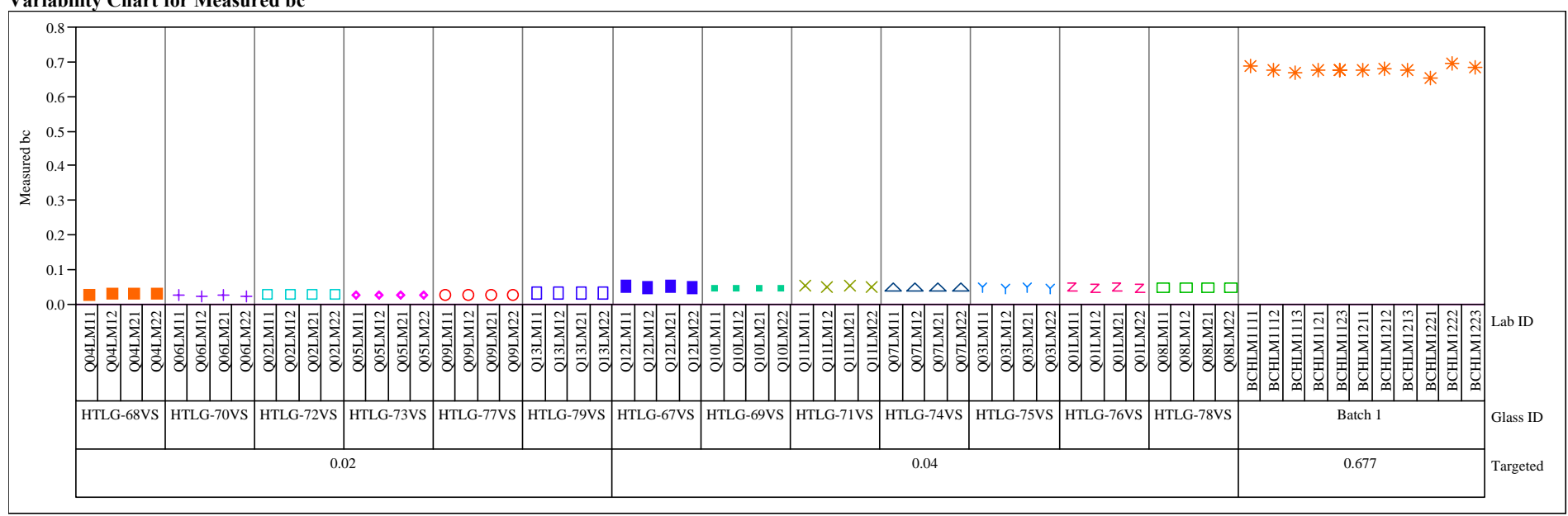


Exhibit A11. Measured and Measured Bias-Corrected Oxide Weight Percents by Glass ID by Targeted Value for the Series " $Q$ " Glasses Prepared Using the LM Method

$\mathrm{ZrO2}(\mathrm{wt} \%)$

Variability Chart for Measured

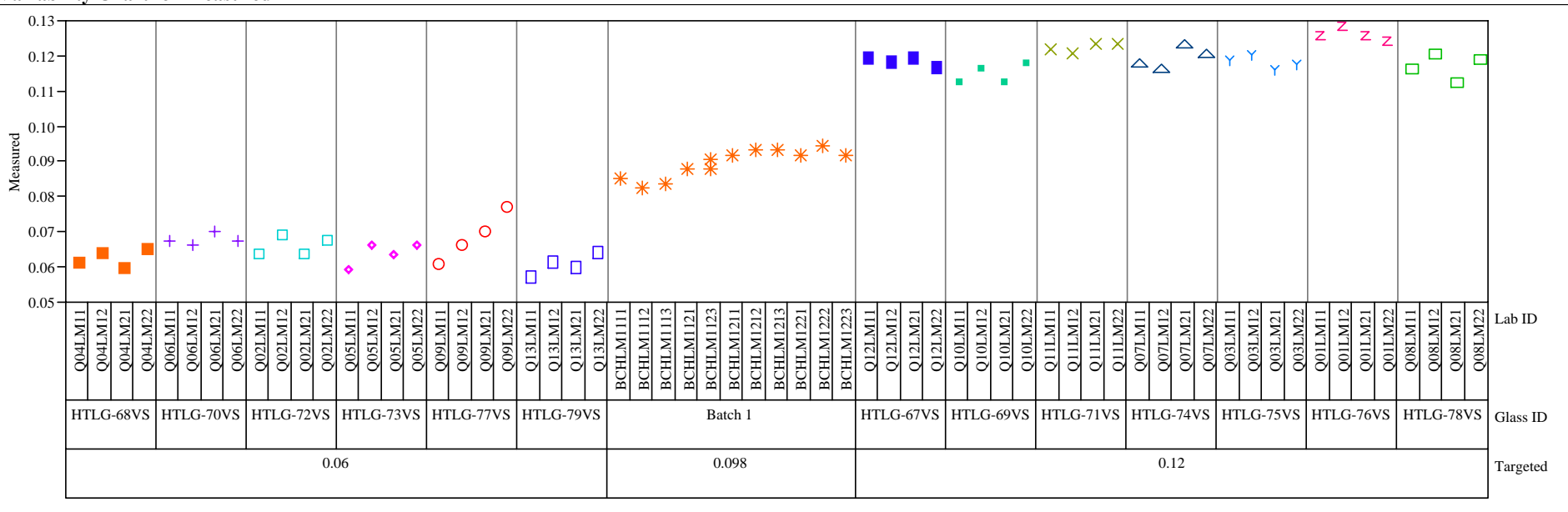

Variability Chart for Measured be

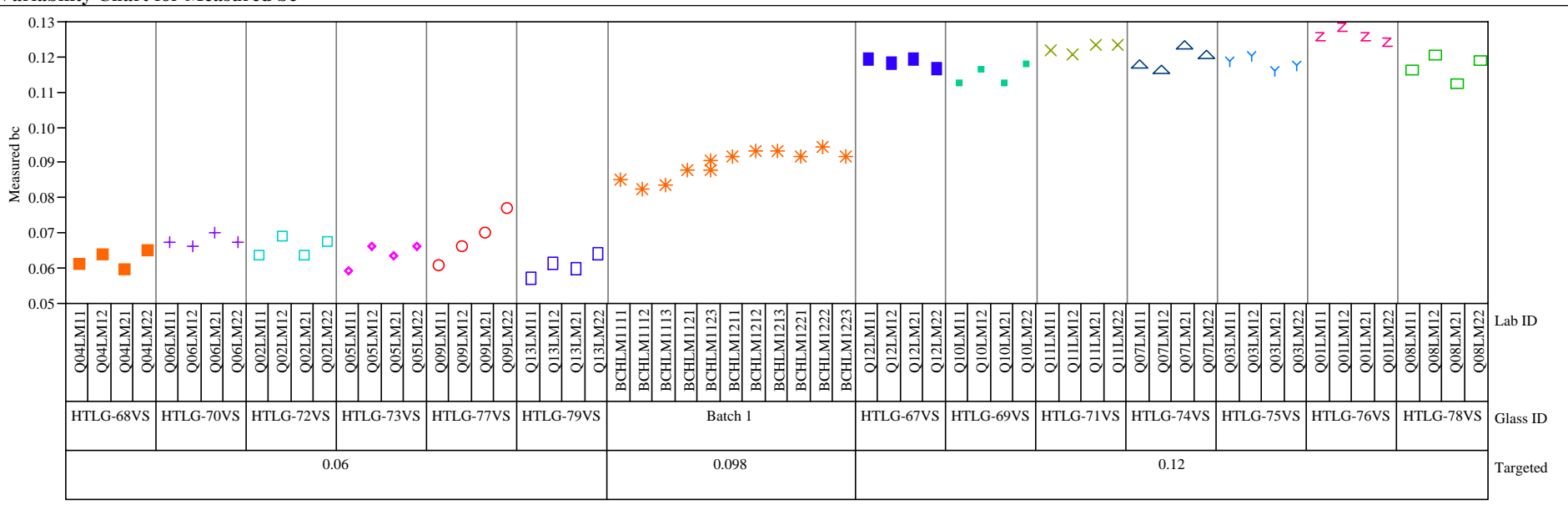


Exhibit A12. Measured and Measured Bias-Corrected Oxide Weight Percents by Glass ID by Targeted Value for the Series "Q" Glasses Prepared Using the PF Method

B2O3 (wt\%)

Variability Chart for Measured

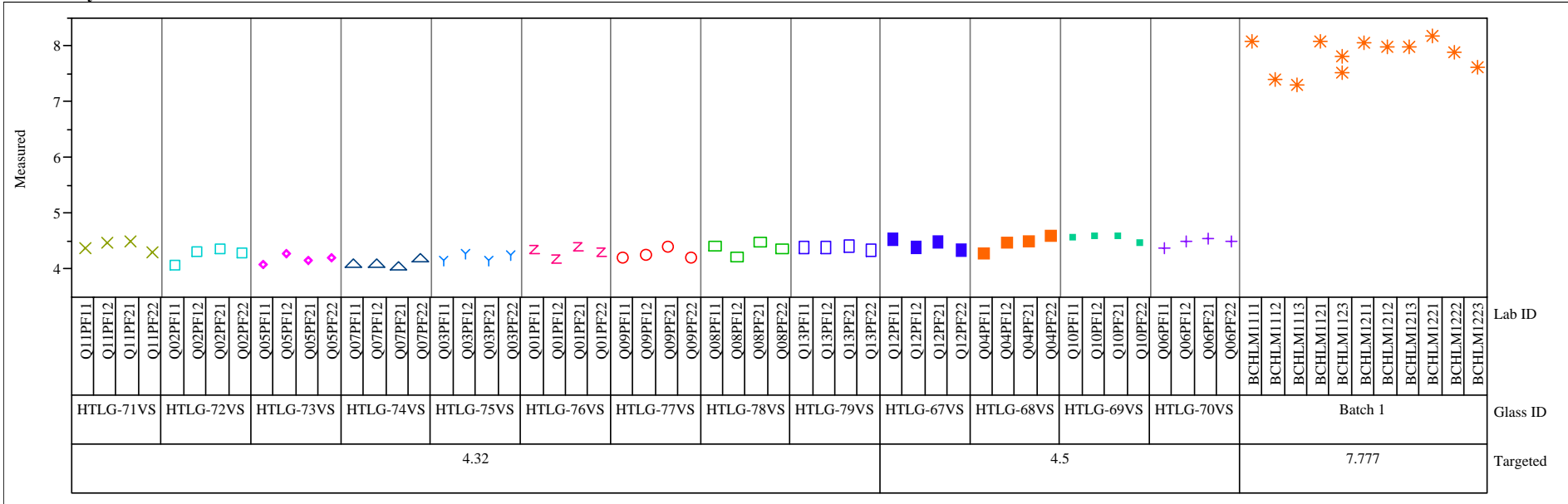

Variability Chart for Measured bc

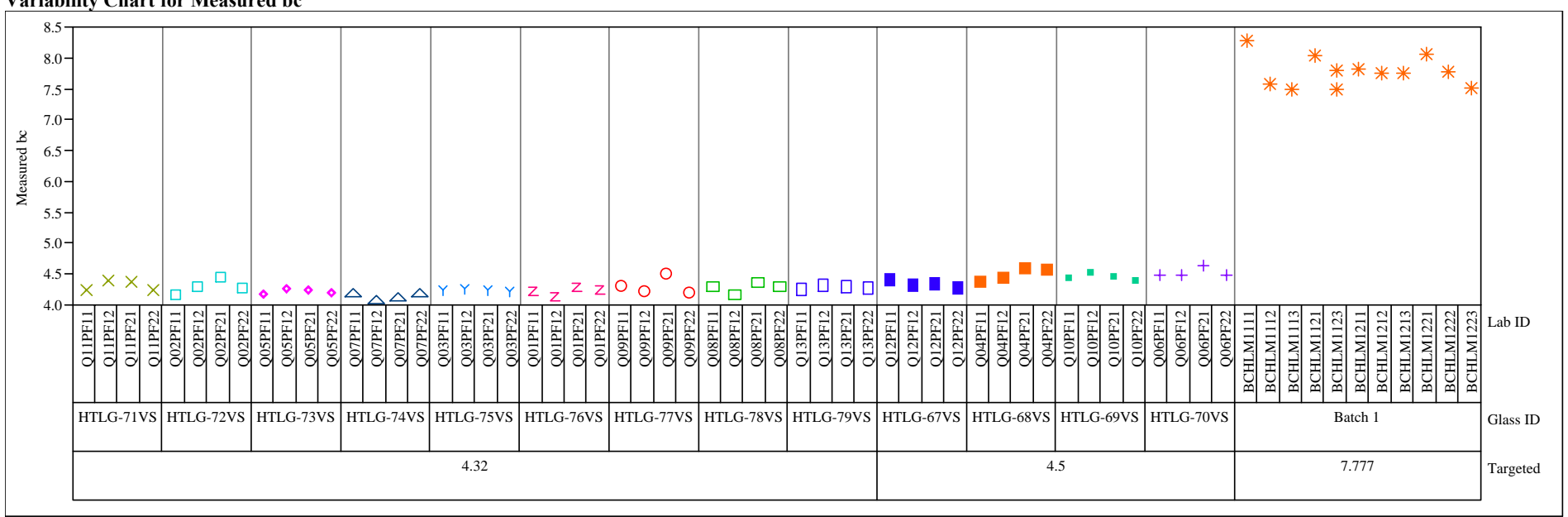


Exhibit A12. Measured and Measured Bias-Corrected Oxide Weight Percents by Glass ID by Targeted Value for the Series "Q" Glasses Prepared Using the PF Method

$\mathrm{Fe} 2 \mathrm{O} 3(\mathrm{wt} \%)$

Variability Chart for Measured

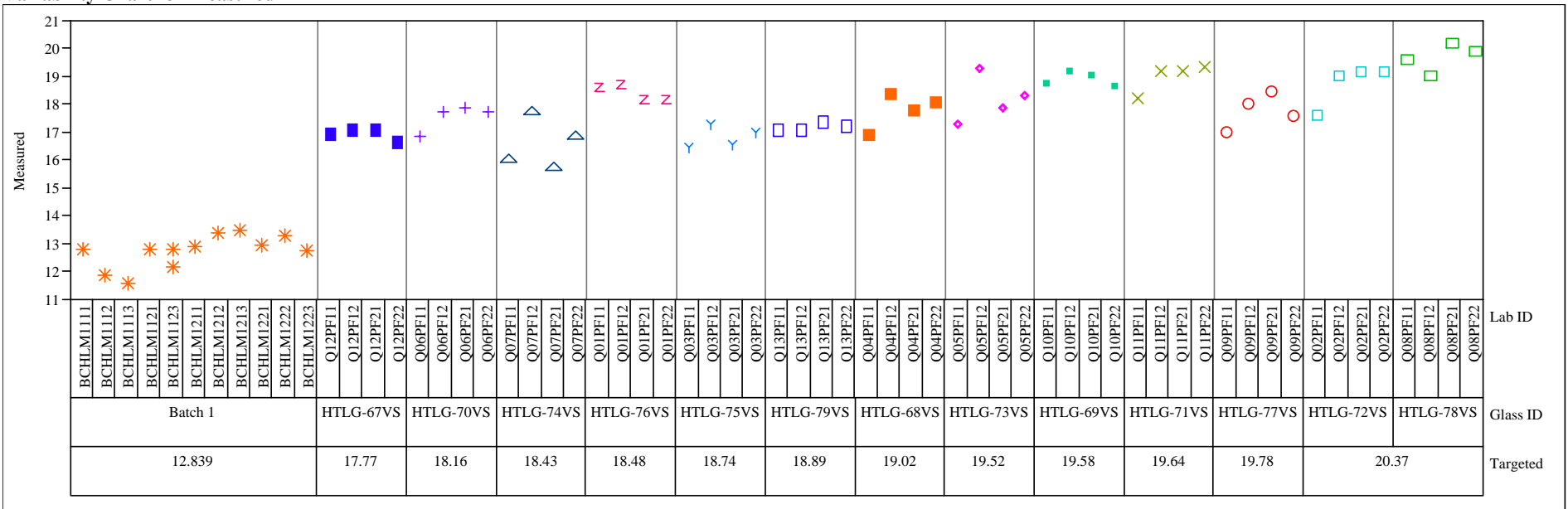

Variability Chart for Measured bc

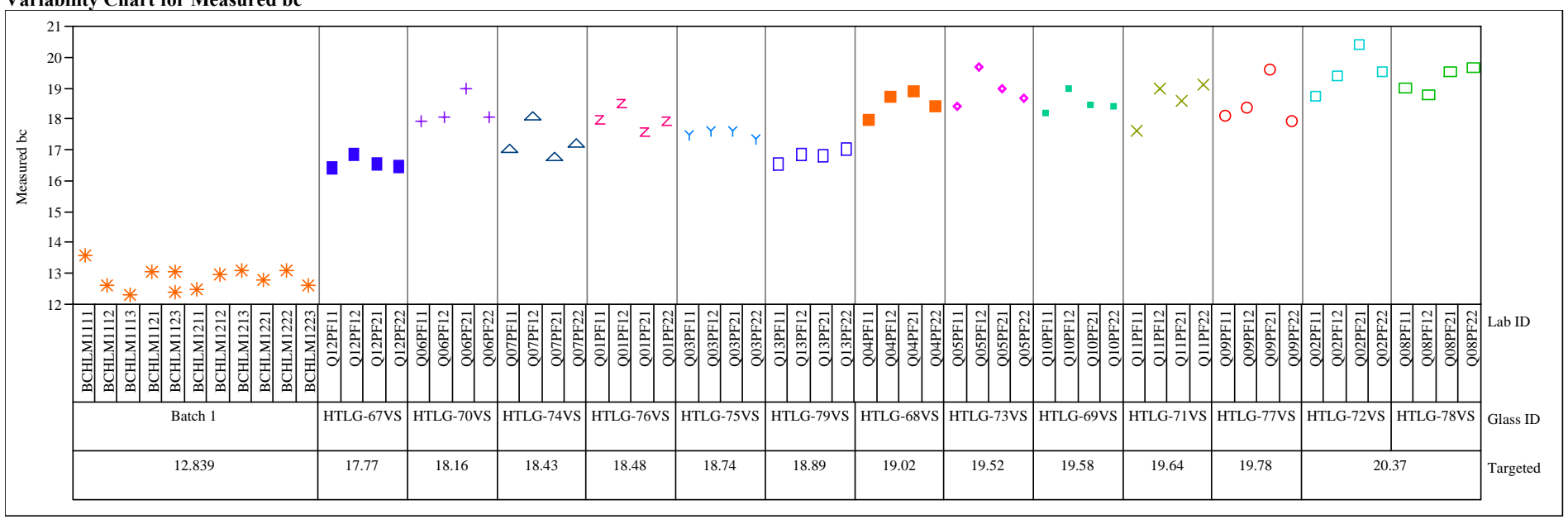


Exhibit A12. Measured and Measured Bias-Corrected Oxide Weight Percents by Glass ID by Targeted Value for the Series "Q" Glasses Prepared Using the PF Method

Li2O (wt\%)

Variability Chart for Measured

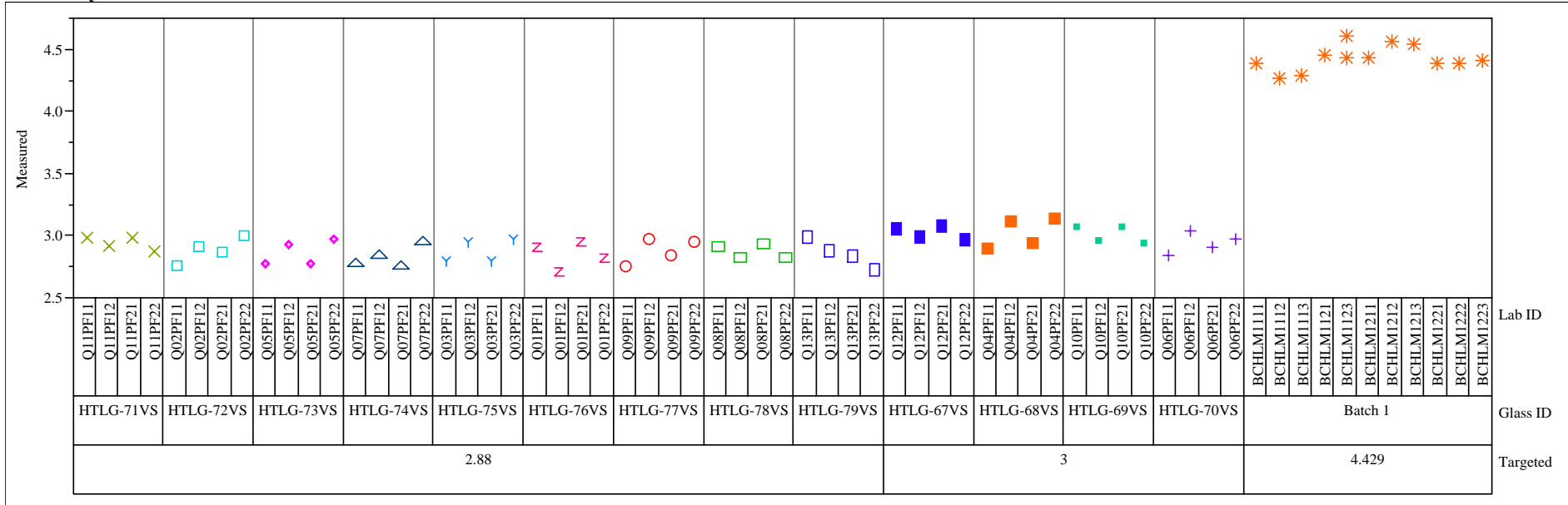

Variability Chart for Measured bc

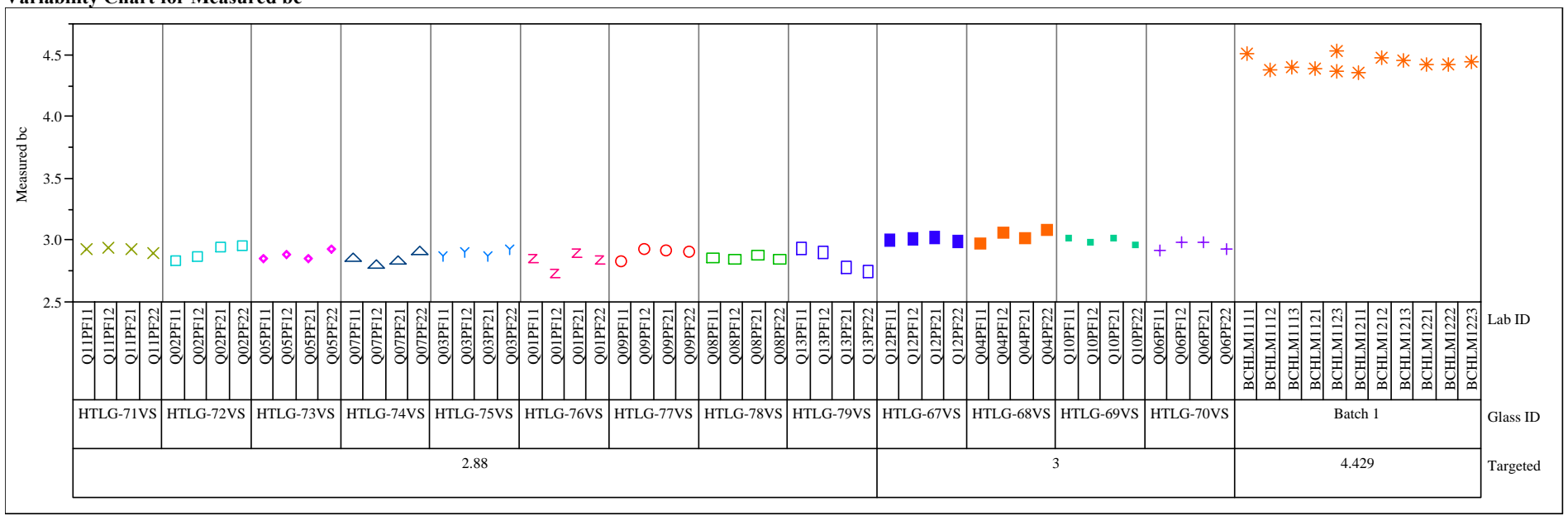


Exhibit A12. Measured and Measured Bias-Corrected Oxide Weight Percents by Glass ID by Targeted Value for the Series "Q" Glasses Prepared Using the PF Method

$\mathrm{SiO} 2(\mathrm{wt} \%)$

Variability Chart for Measured

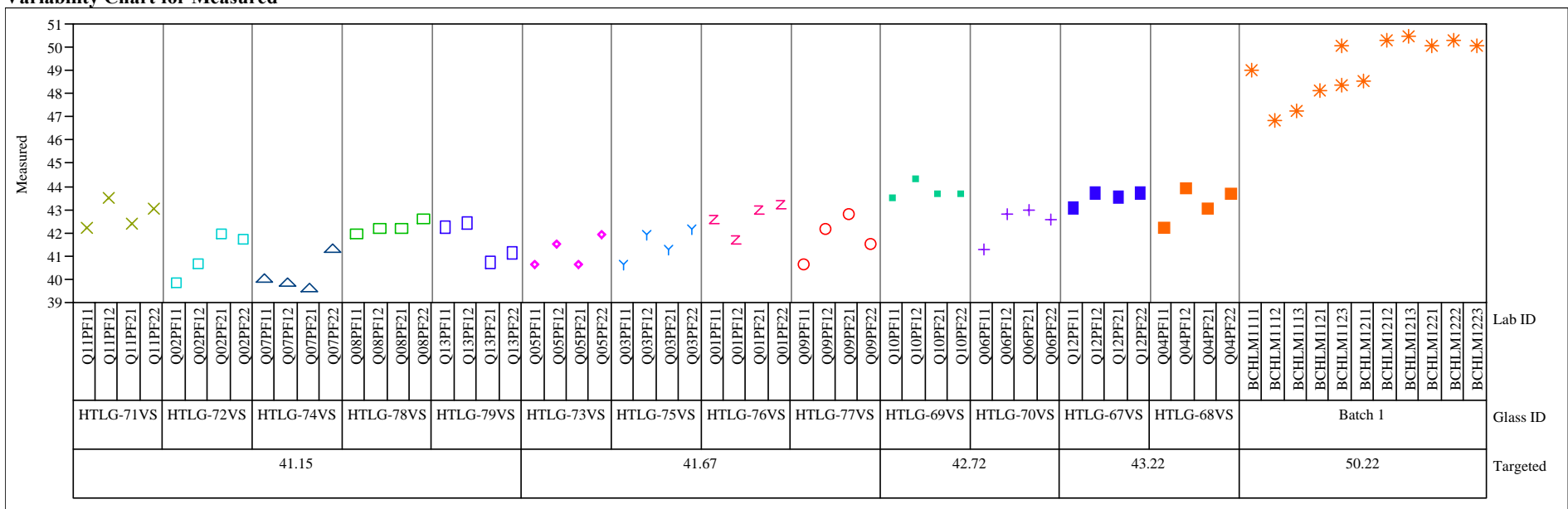

Variability Chart for Measured bc

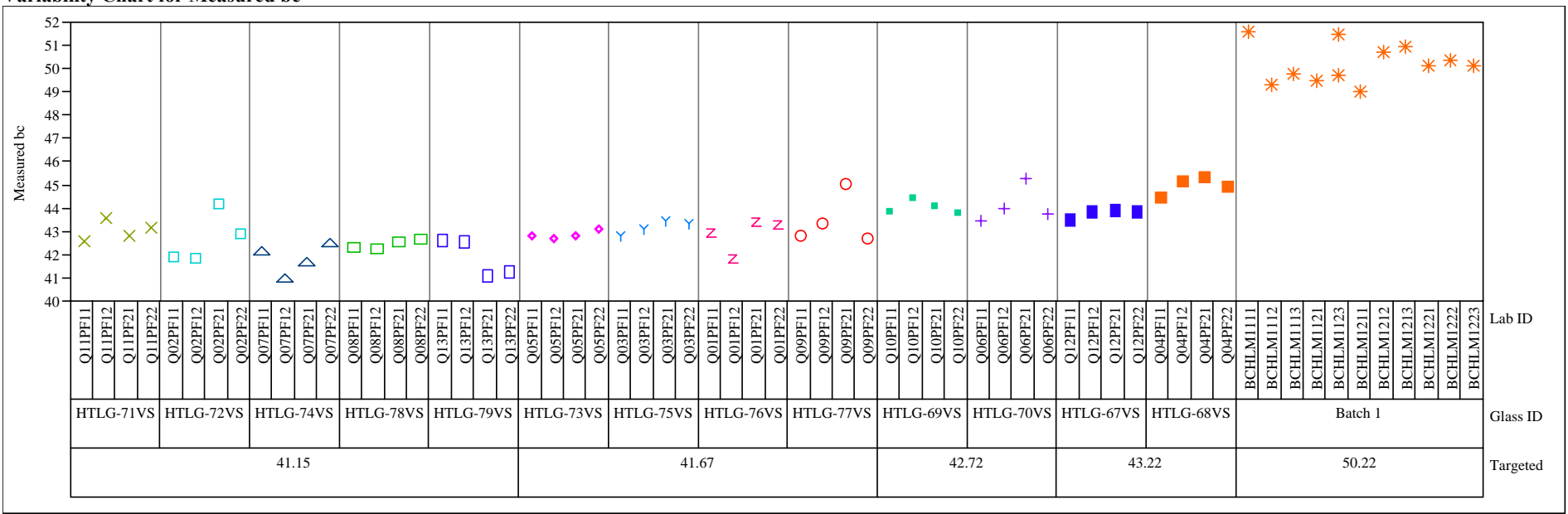


Exhibit A13. Average Measured and Bias-Corrected (bc) Versus Targeted Compositions by Glass ID by Analyte for the "P" Series

Overlay Plot Oxide=Al2O3 (wt \%)

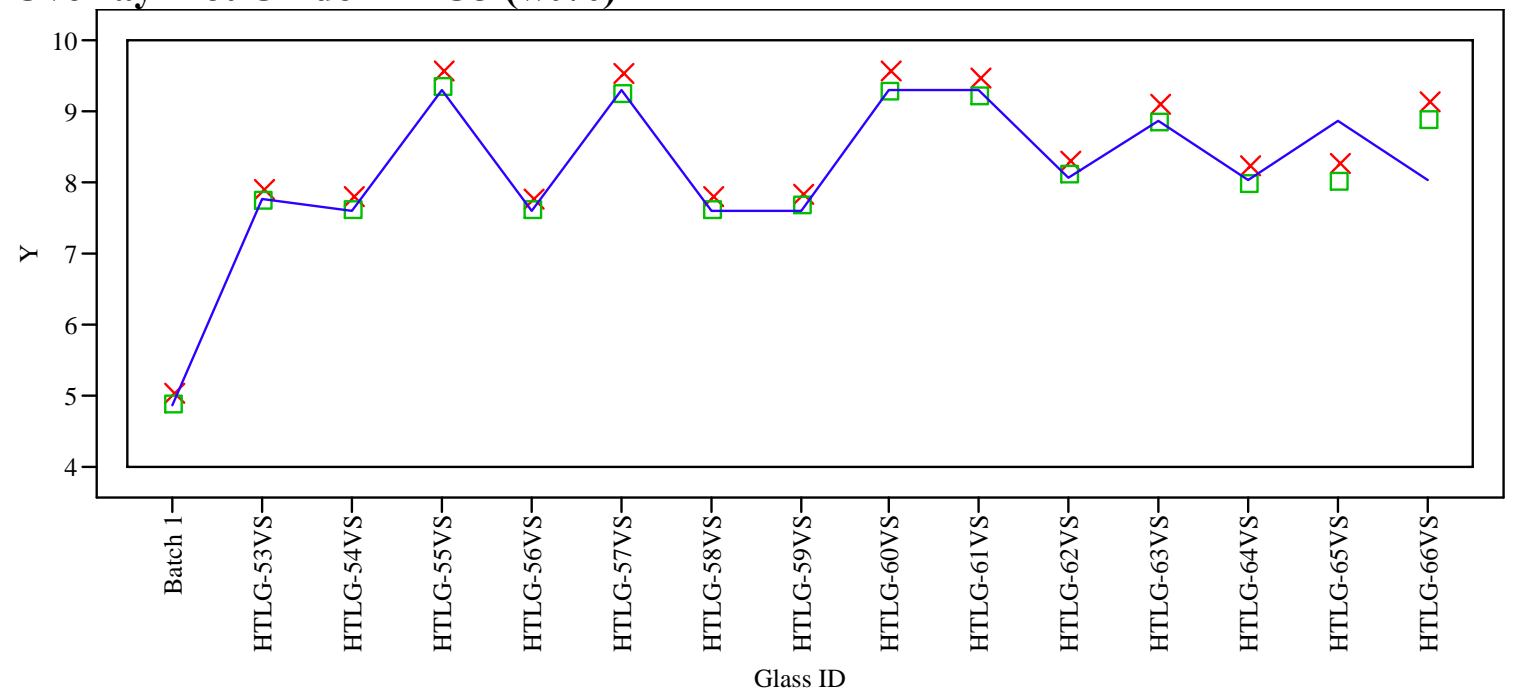

\section{Overlay Plot Oxide $=$ B2O3 $($ wt $\%)$}

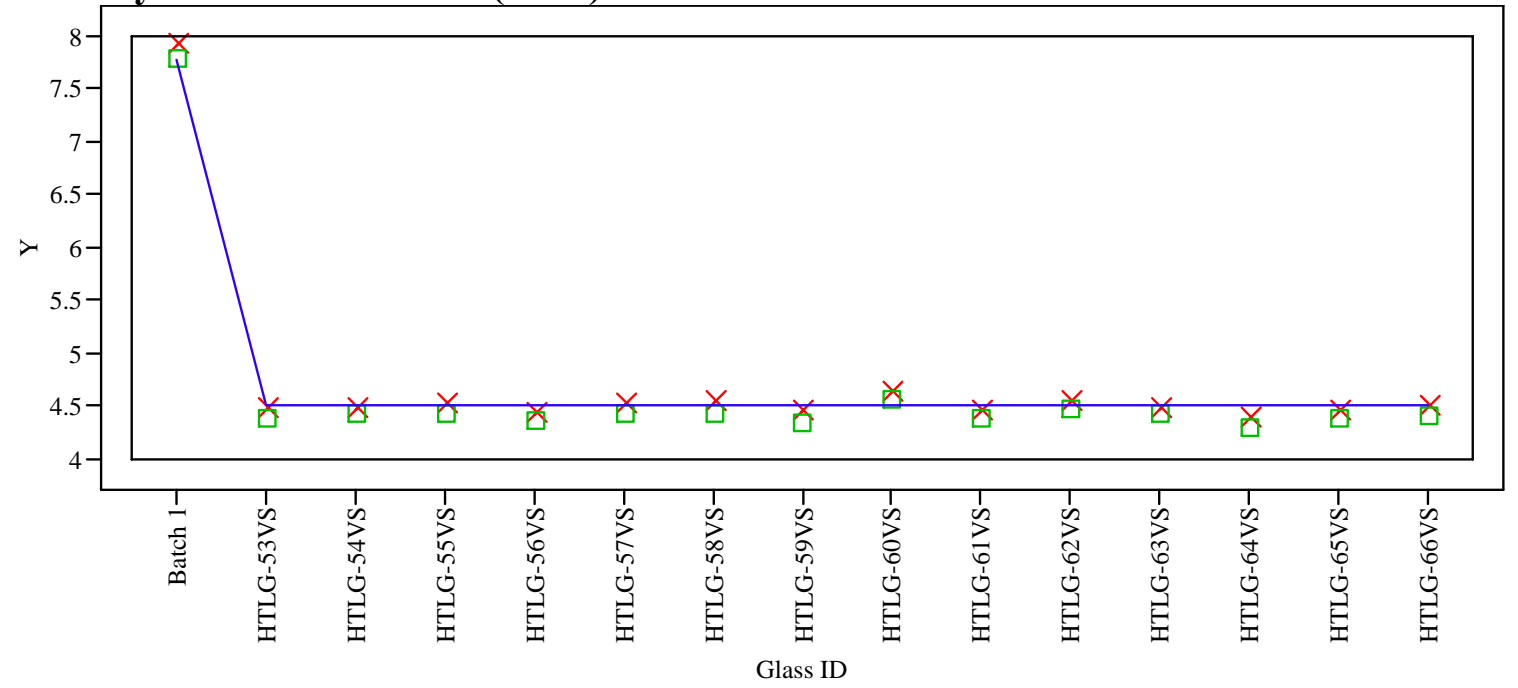

Y $\times$ Measured

- Measured bc Targeted 


\section{Exhibit A13. Average Measured and Bias-Corrected (bc) Versus Targeted Compositions by Glass ID by Analyte for the "P" Series}

Overlay Plot Oxide $=\mathrm{CaO}\left(\mathrm{wt}_{\mathrm{t}} \%\right)$

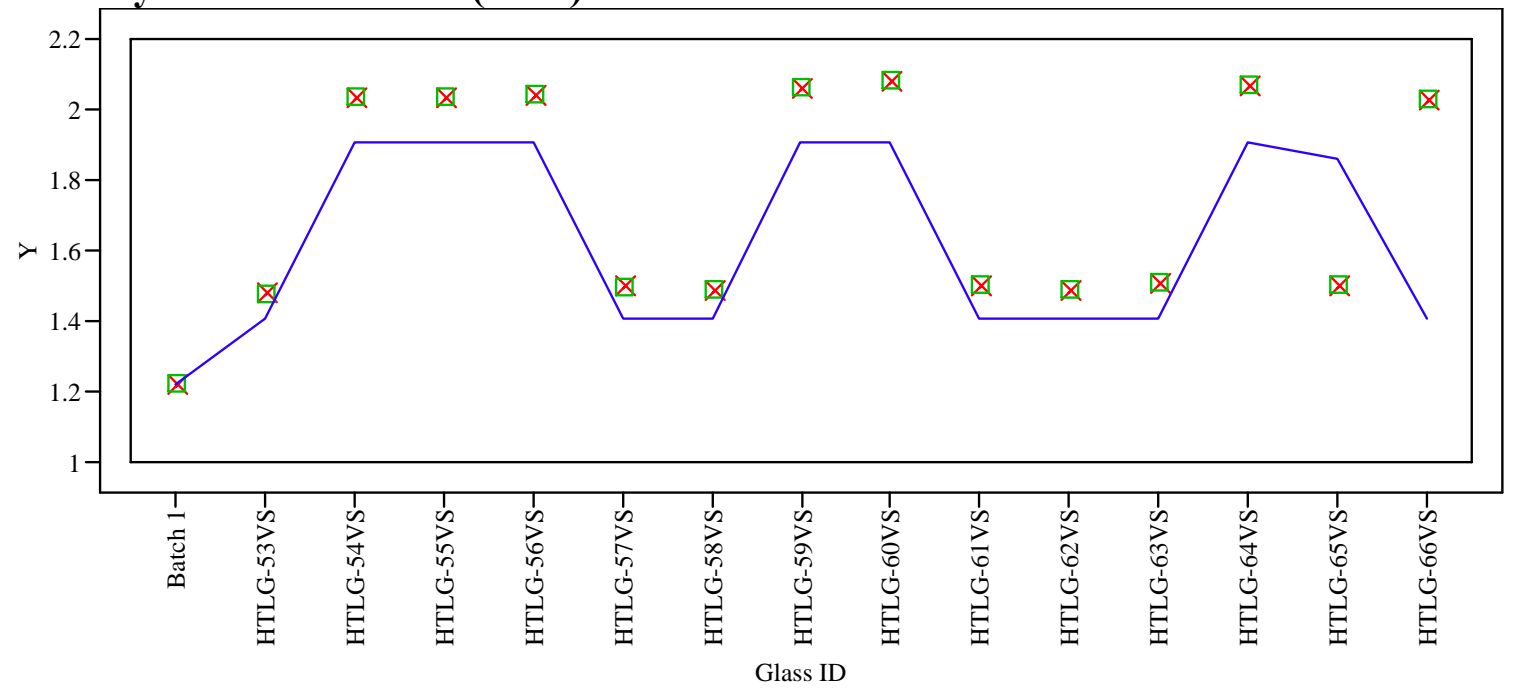

Overlay Plot Oxide $=\mathrm{Cr} 2 \mathrm{O3}(\mathbf{w t} \%)$

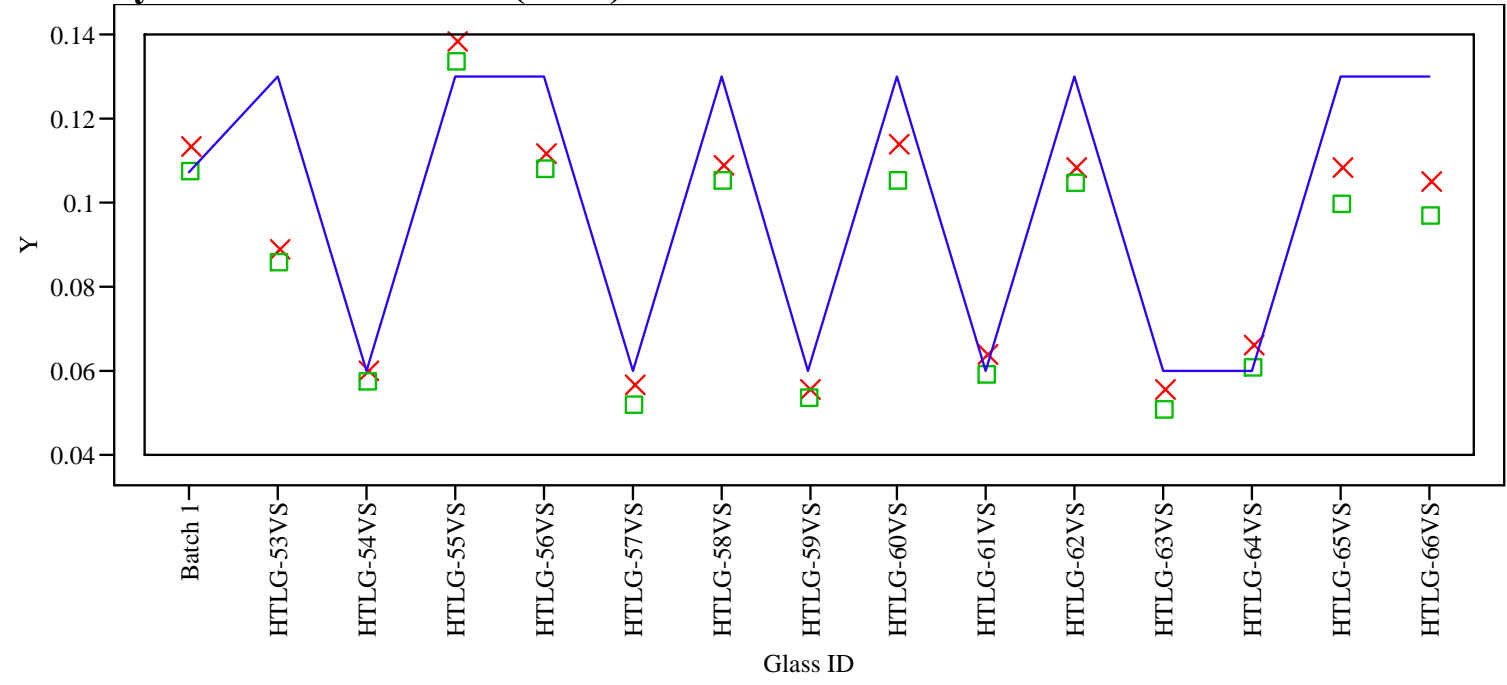

Y $\times$ Measured

- Measured bc — Targeted 


\section{Exhibit A13. Average Measured and Bias-Corrected (bc) Versus Targeted Compositions by Glass ID by Analyte for the "P" Series}

Overlay Plot Oxide $=\mathrm{CuO}\left(\mathrm{wt}_{\mathrm{o}} \%\right)$

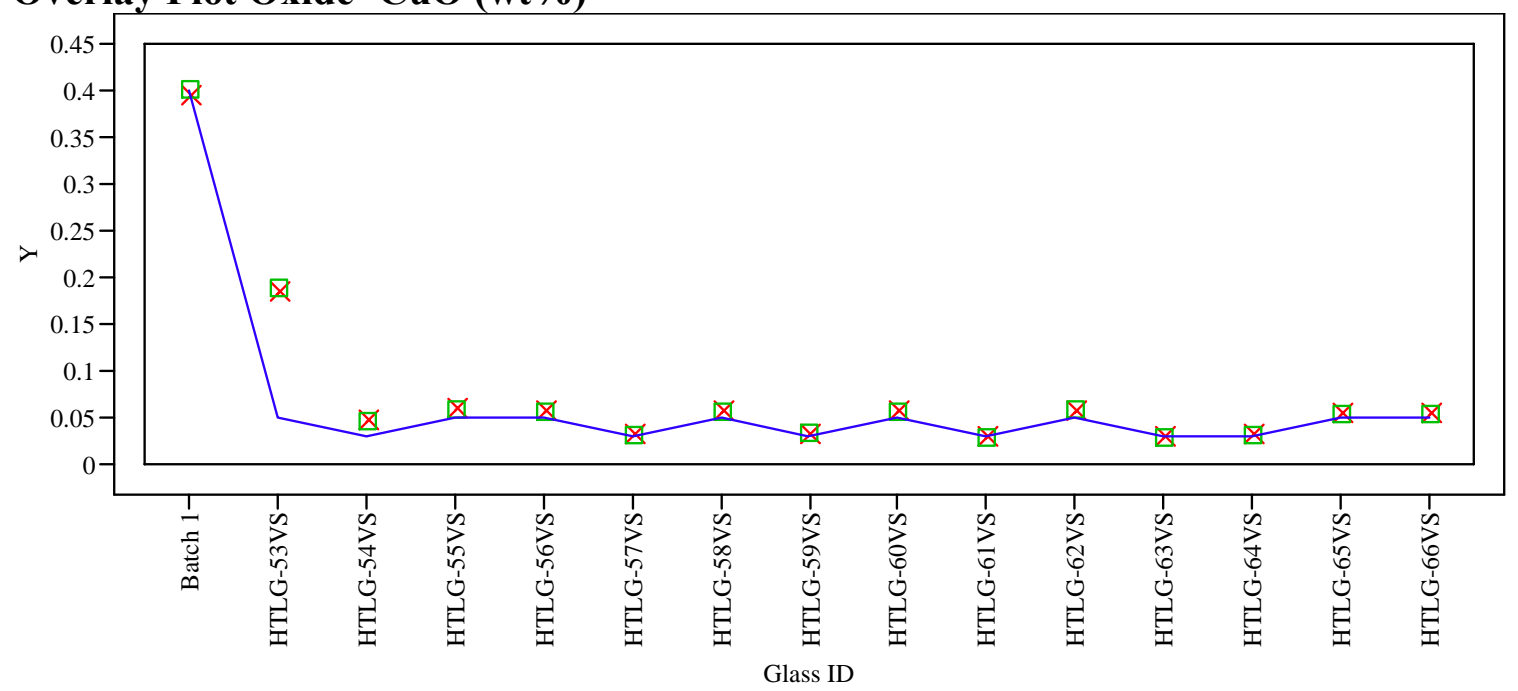

Overlay Plot Oxide $=$ Fe2O3 $(w t \%)$

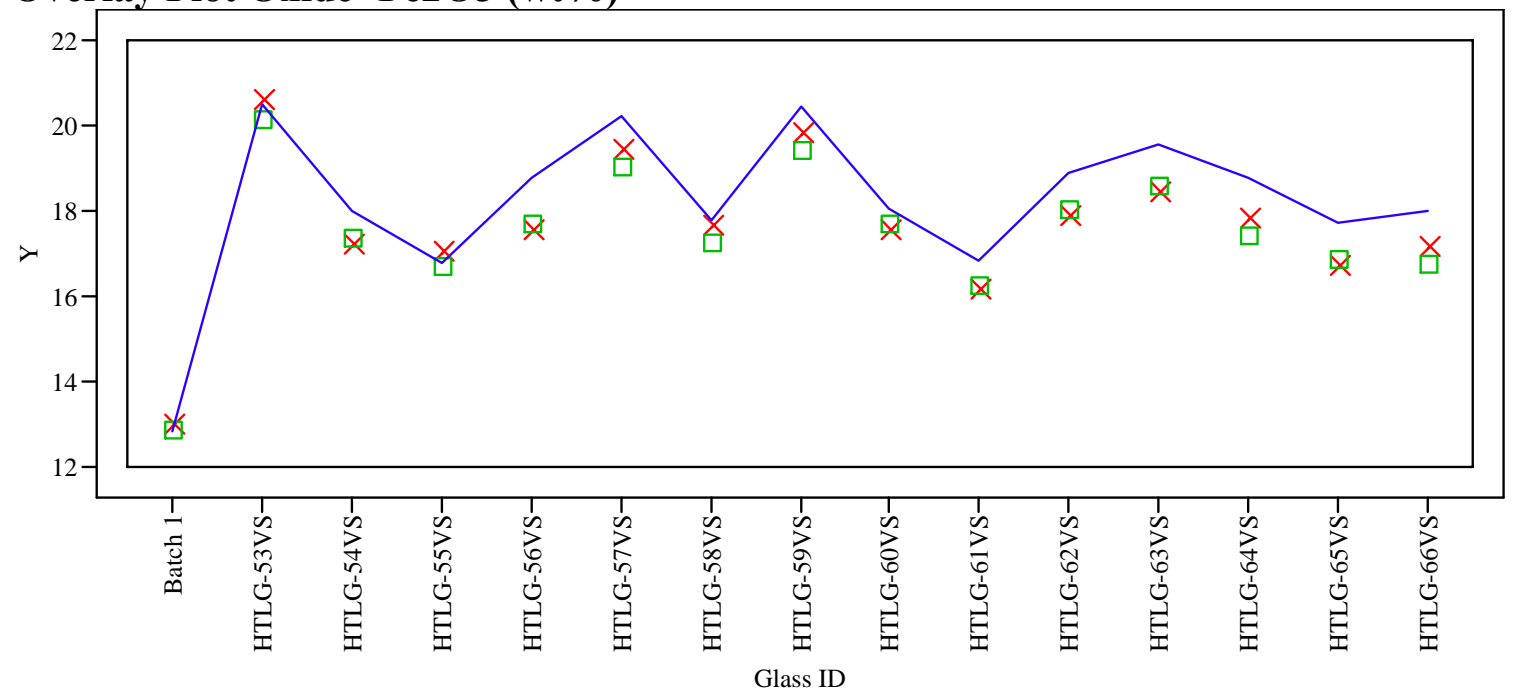

Y $\times$ Measured $\square$ Measured bc — Targeted 


\section{Exhibit A13. Average Measured and Bias-Corrected (bc) Versus Targeted Compositions by Glass ID by Analyte for the "P" Series}

Overlay Plot Oxide $=$ K2O (wt \%)

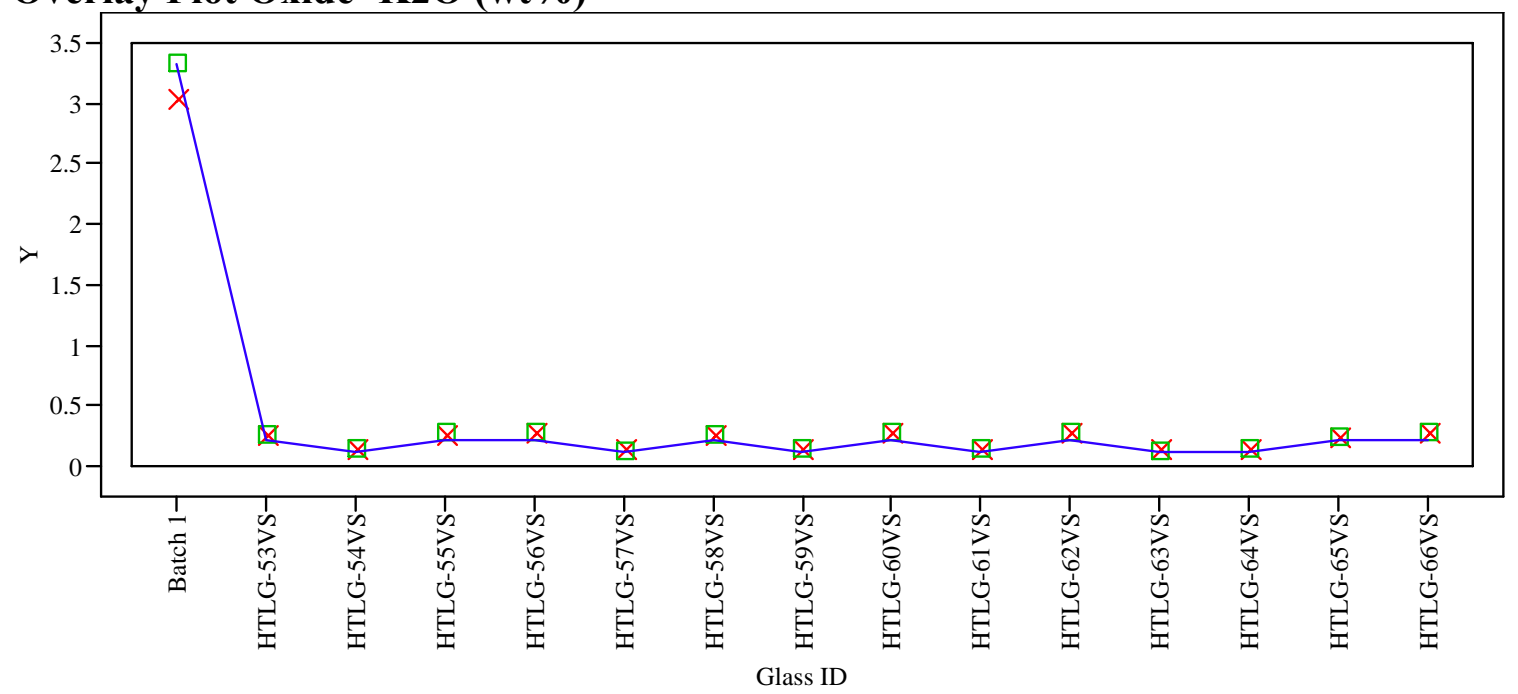

Overlay Plot Oxide $=\mathbf{L i 2 O}(\mathbf{w t} \%)$

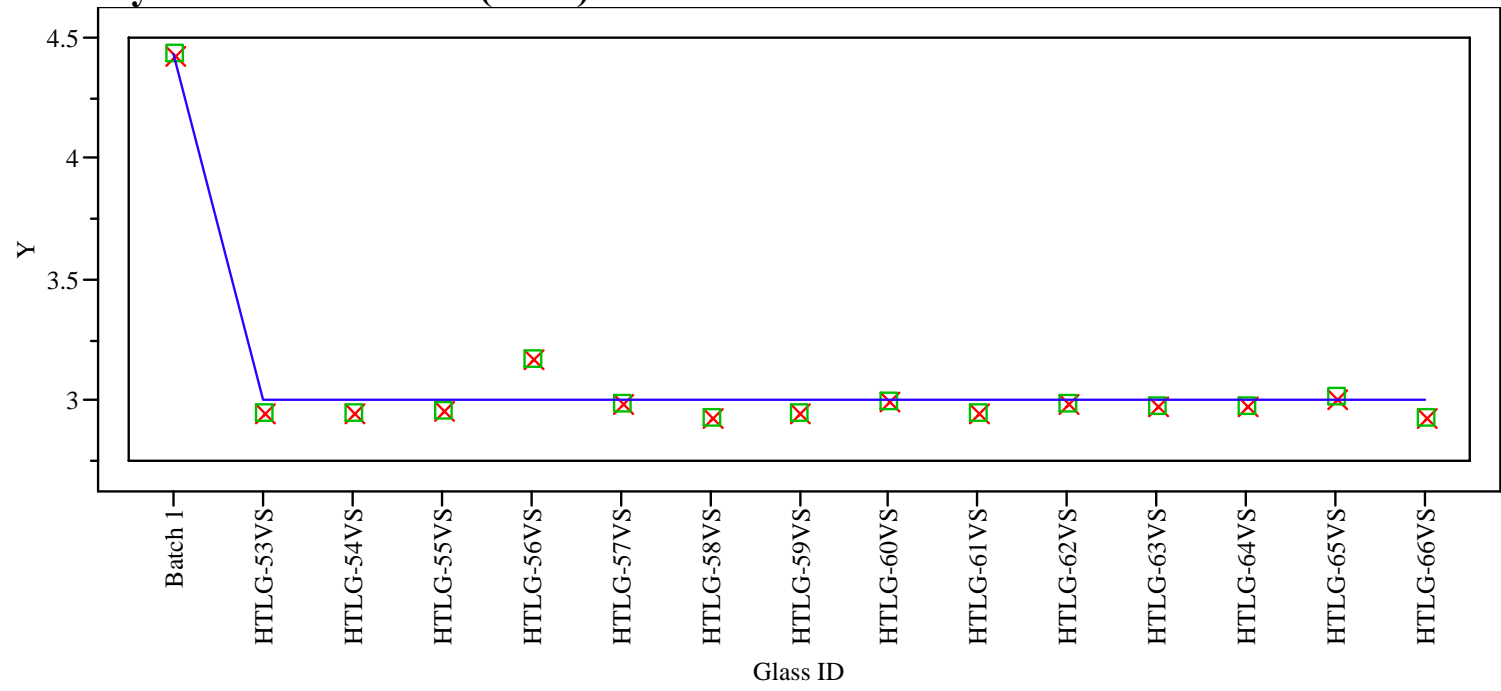

Y $\times$ Measured

- Measured bc — Targeted 


\section{Exhibit A13. Average Measured and Bias-Corrected (bc) Versus Targeted Compositions by Glass ID by Analyte for the "P" Series}

Overlay Plot Oxide $=$ MgO $($ wt \%)

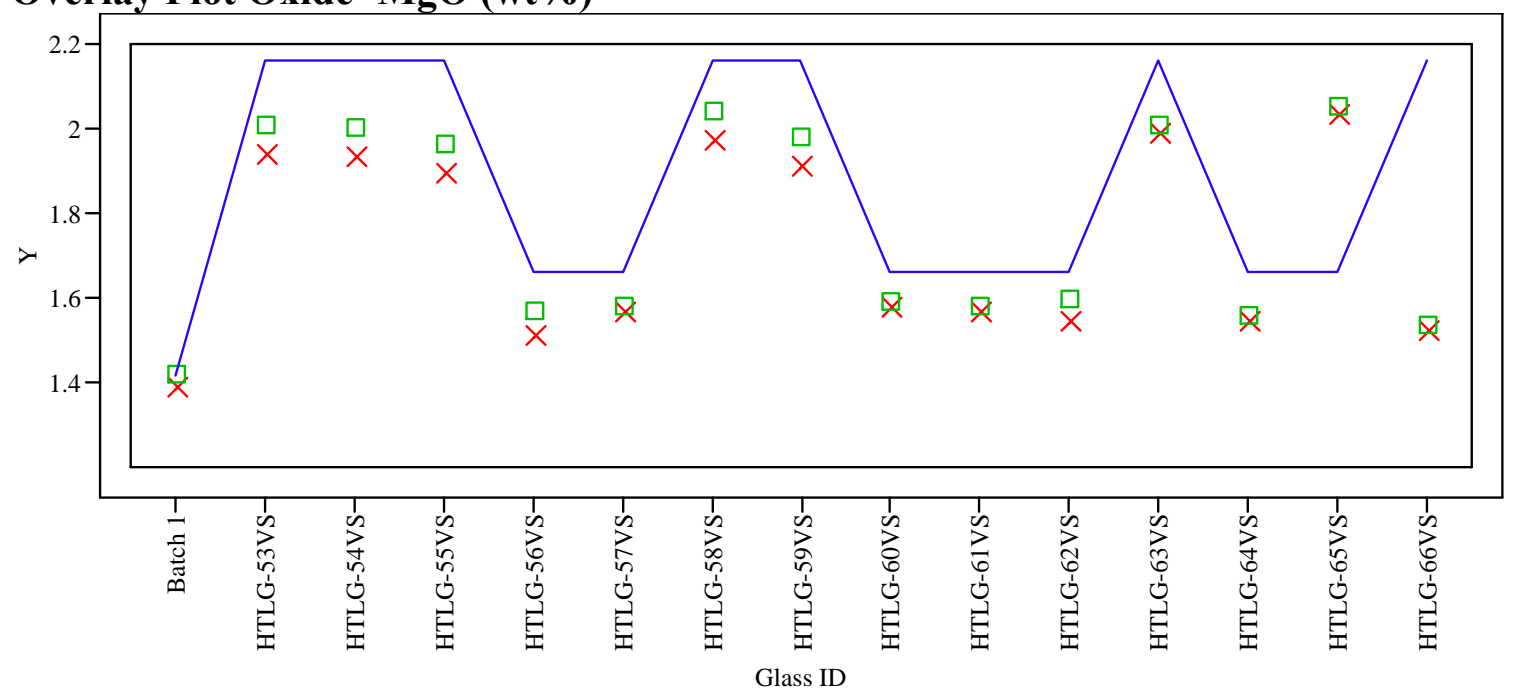

Overlay Plot Oxide $=\mathrm{MnO}\left(\mathrm{wt}_{\mathrm{t}} \mathrm{o}\right)$

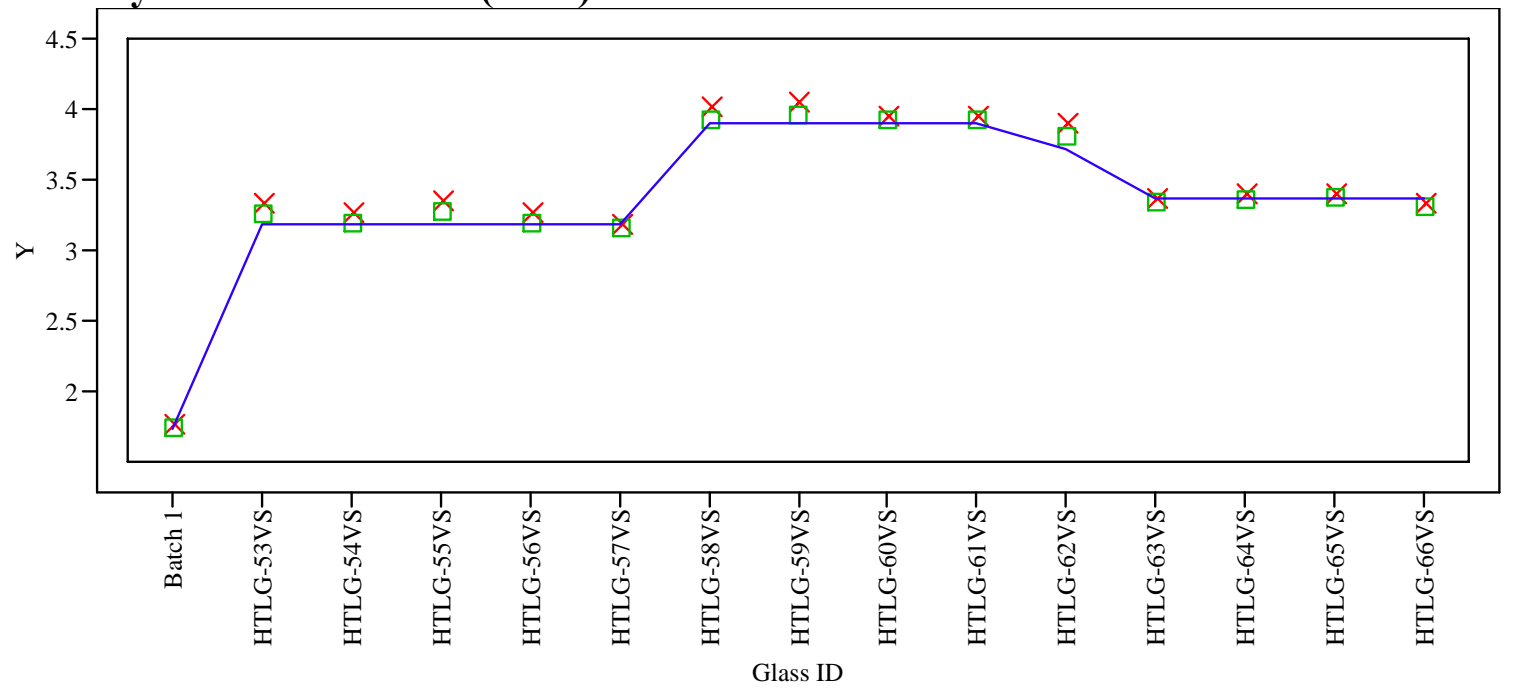

Y $\times$ Measured

- Measured bc — Targeted 
Exhibit A13. Average Measured and Bias-Corrected (bc) Versus Targeted Compositions by Glass ID by Analyte for the "P" Series

Overlay Plot Oxide=Na2O (wt \%)

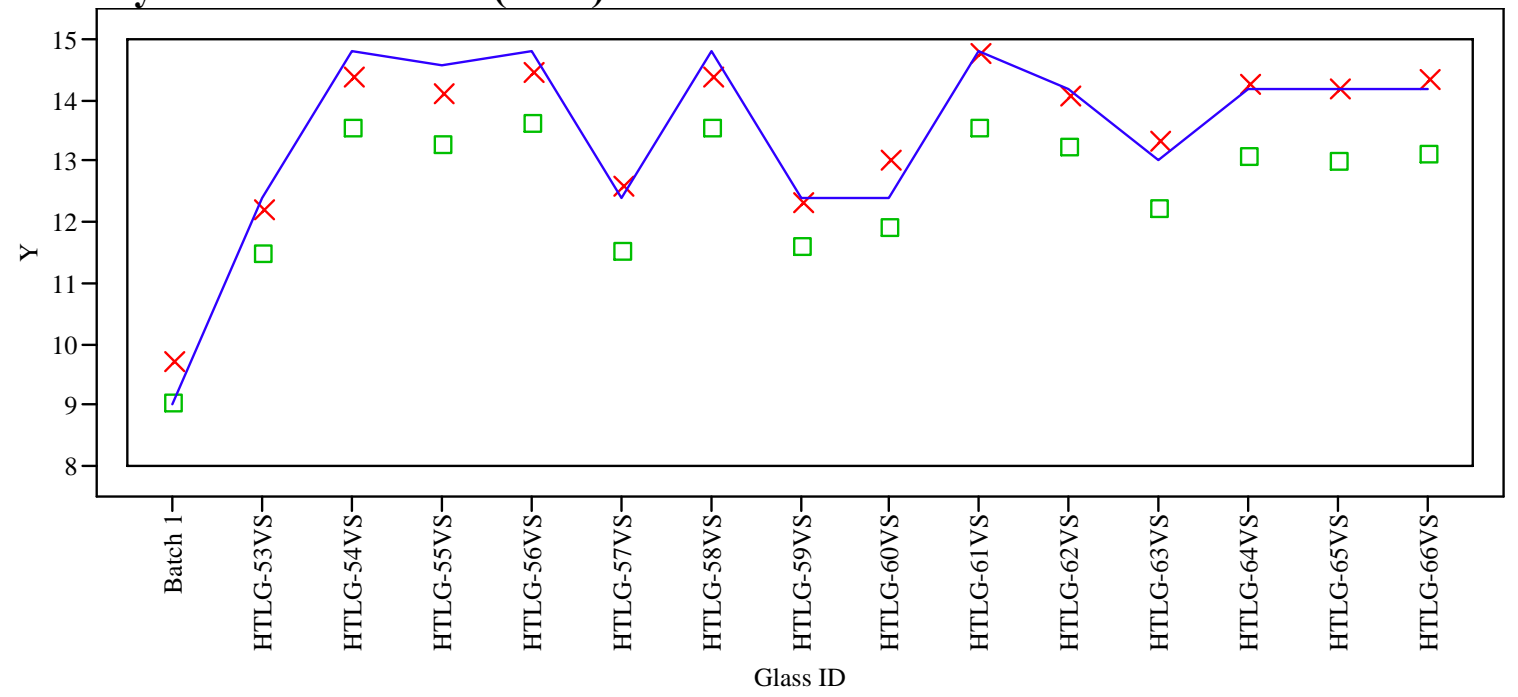

Overlay Plot Oxide $=\mathrm{NiO}\left(\mathrm{wt}^{\mathrm{O}} \%\right)$

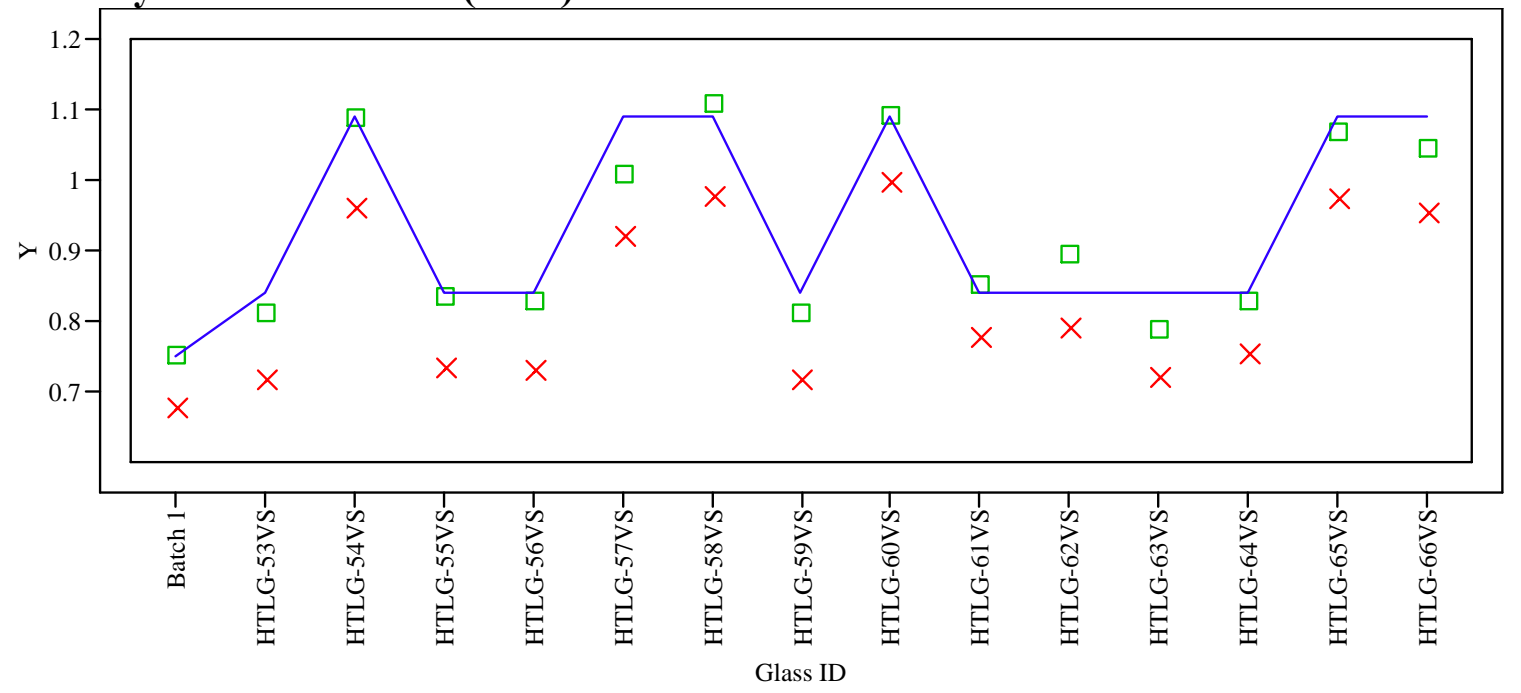

Y $\times$ Measured

Measured bc - Targeted 


\section{Exhibit A13. Average Measured and Bias-Corrected (bc) Versus Targeted Compositions by Glass ID by Analyte for the "P" Series}

Overlay Plot Oxide $=\mathrm{SiO} 2($ wt $\%)$

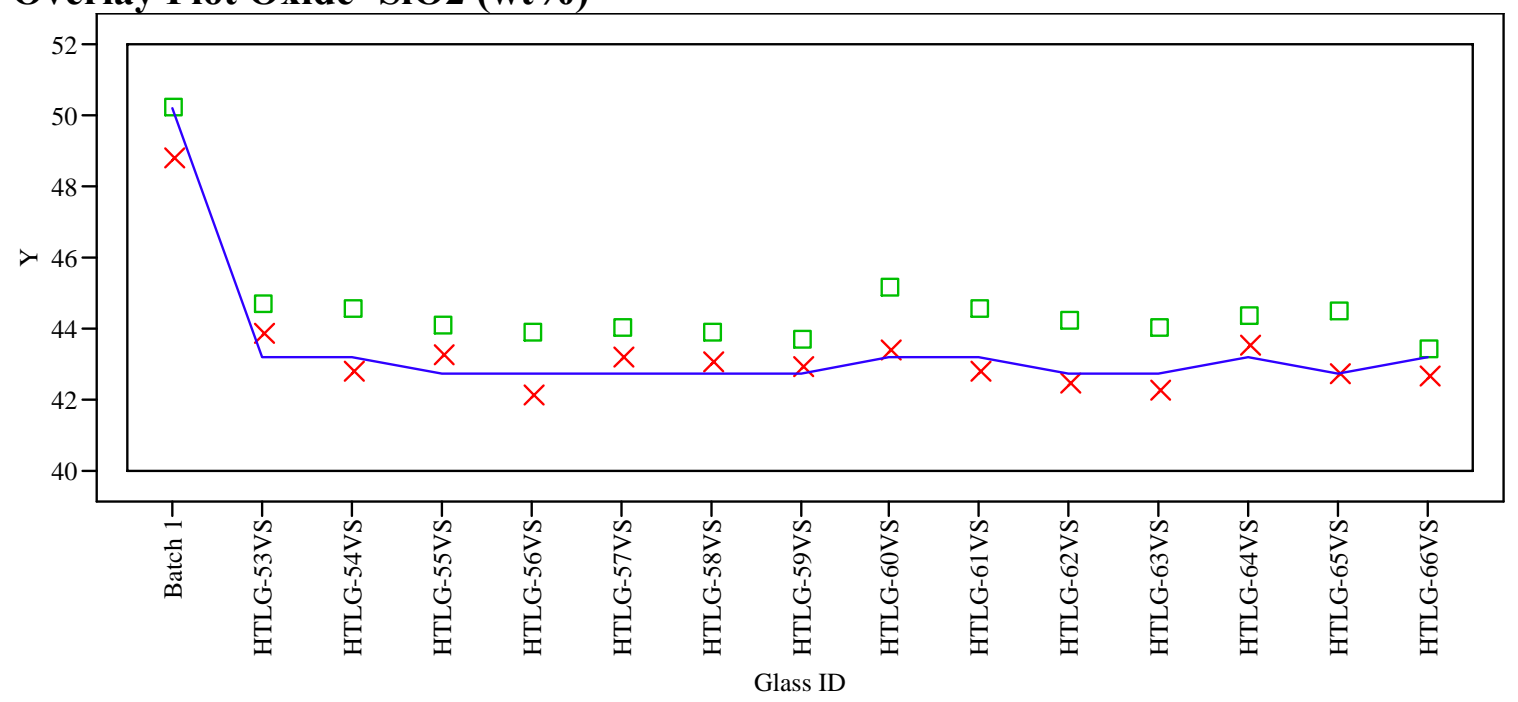

\section{Overlay Plot Oxide=SO4 (wt \%)}

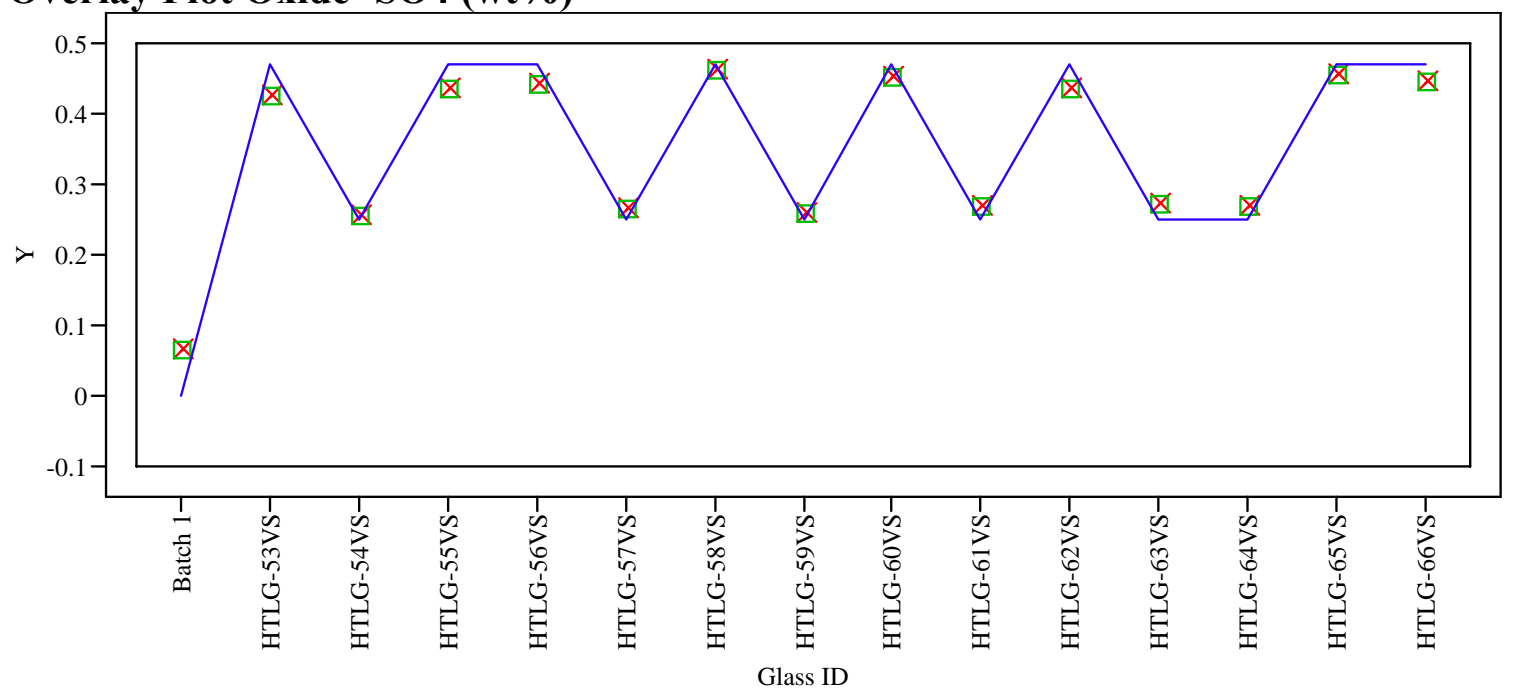

Y $\times$ Measured

- Measured bc Targeted 


\section{Exhibit A13. Average Measured and Bias-Corrected (bc) Versus Targeted Compositions by Glass ID by Analyte for the "P" Series}

Overlay Plot Oxide $=\mathrm{TiO} 2(\mathbf{w t} \%)$

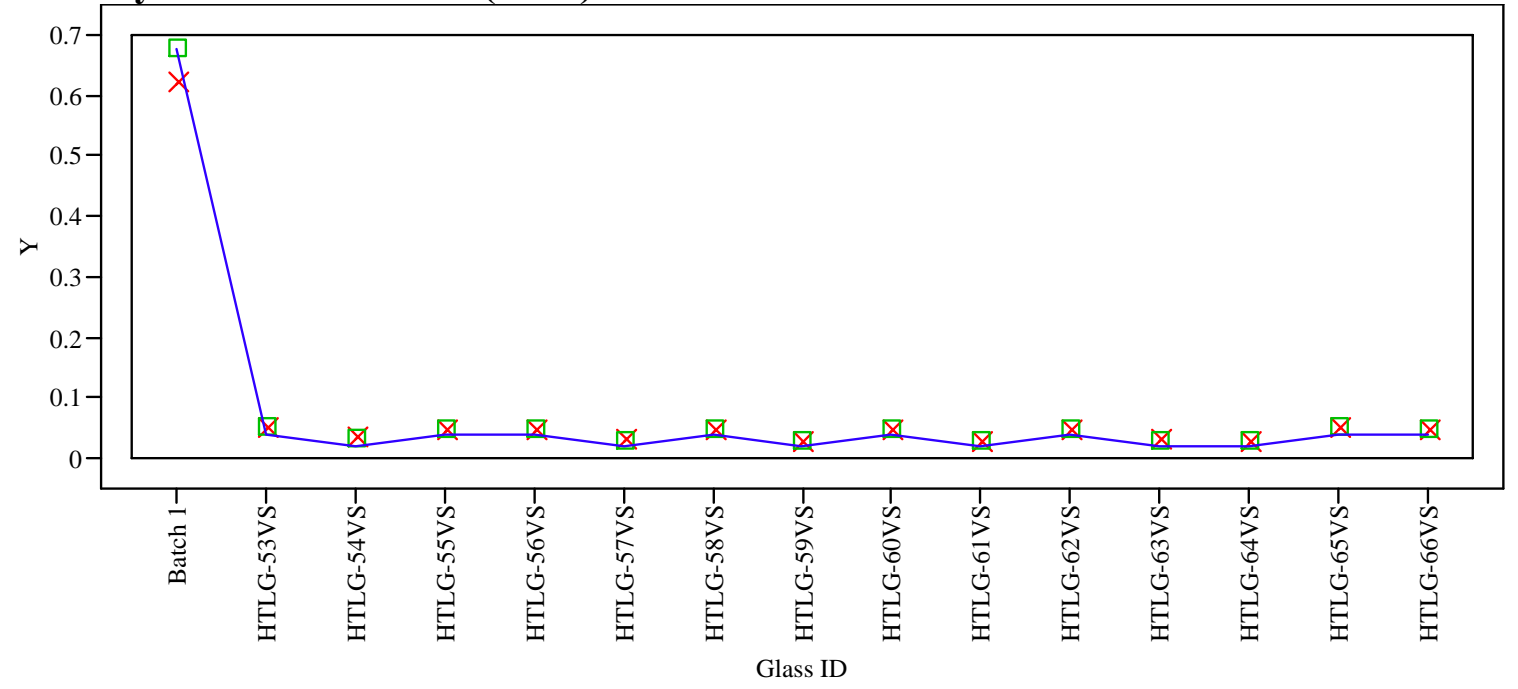

Overlay Plot Oxide $=\mathrm{ZrO2}(\mathrm{wt} \%)$

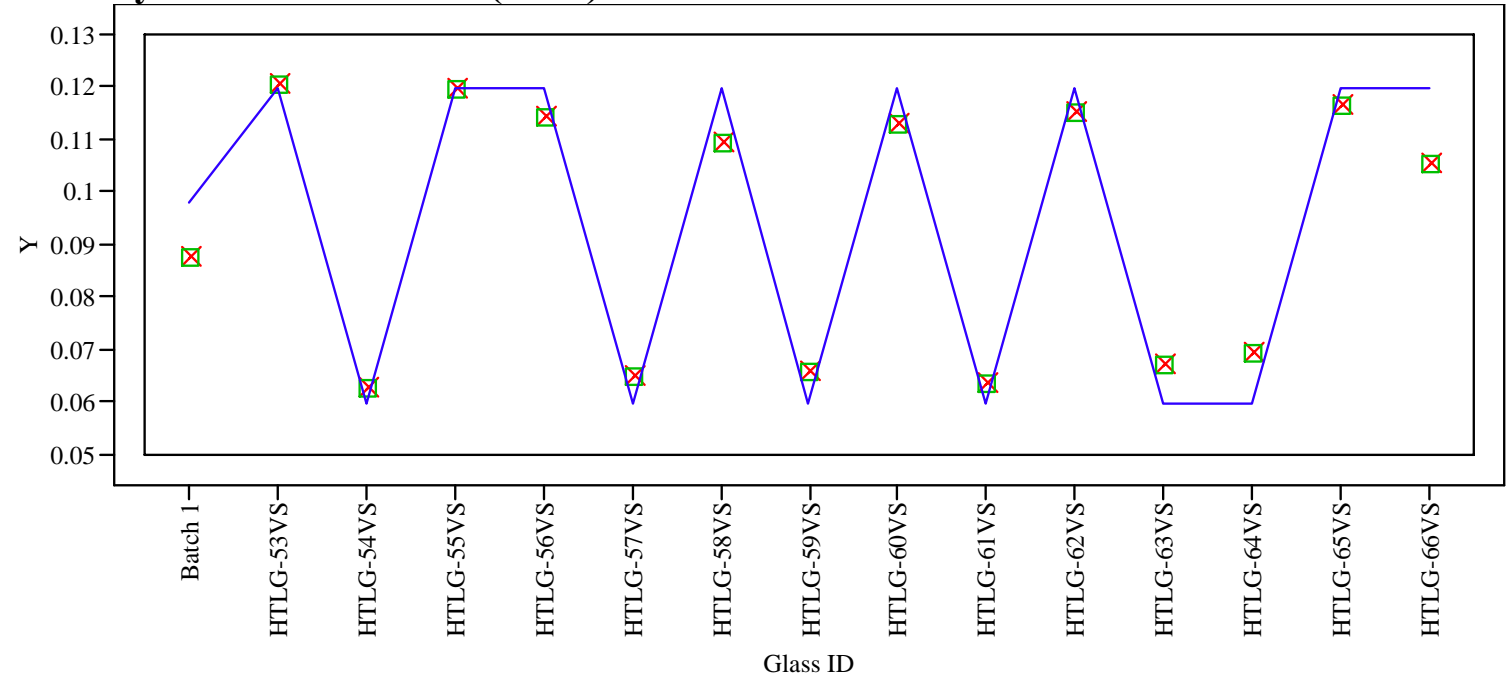

Y $\times$ Measured

- Measured bc

Targeted 
Exhibit A13. Average Measured and Bias-Corrected (bc) Versus Targeted Compositions by Glass ID by Analyte for the "P" Series

Overlay Plot Oxide=Sum of Oxides $\left(\mathrm{wt}^{\mathrm{t}} \%\right)$

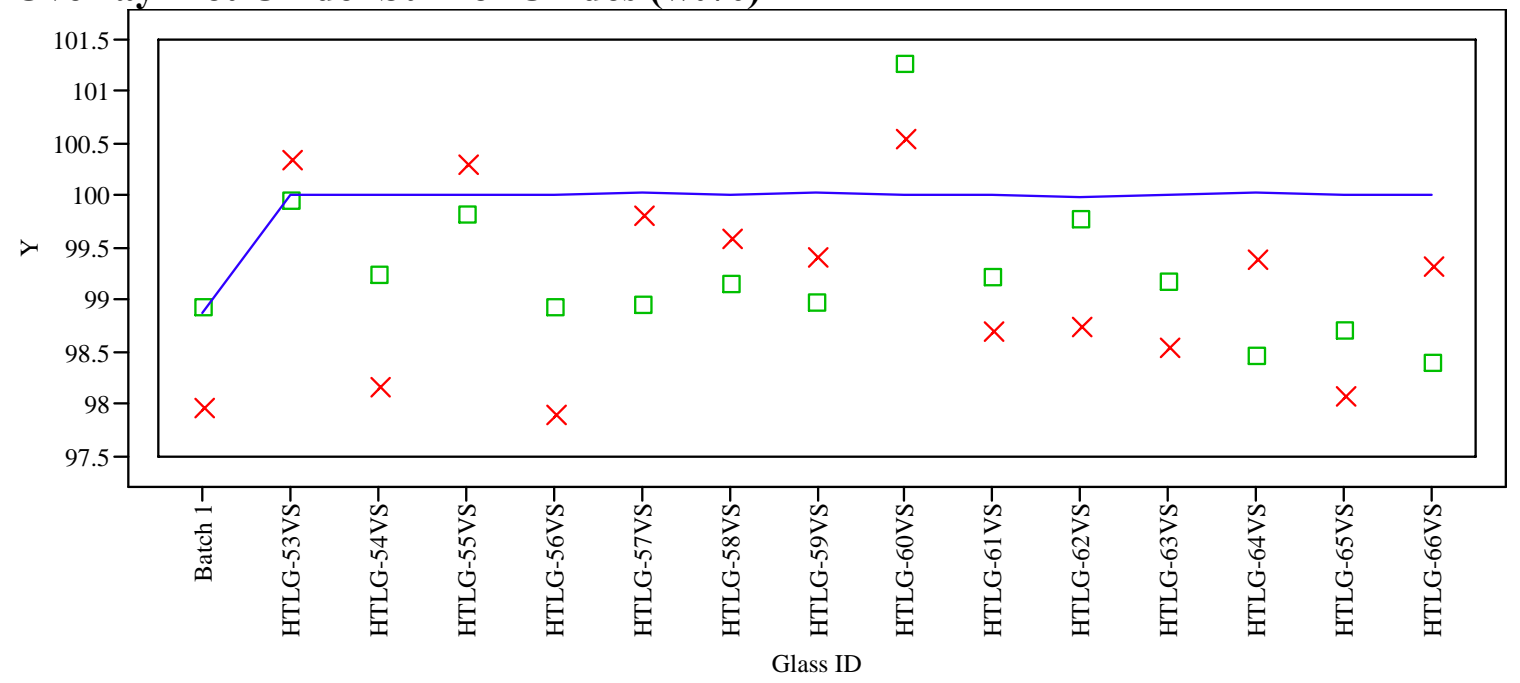

Y $\times$ Measured $\square$ Measured bc — Targeted 


\section{Exhibit A14. Average Measured and Bias-Corrected (bc) Versus Targeted} Compositions by Glass ID by Analyte for the "Q" Series

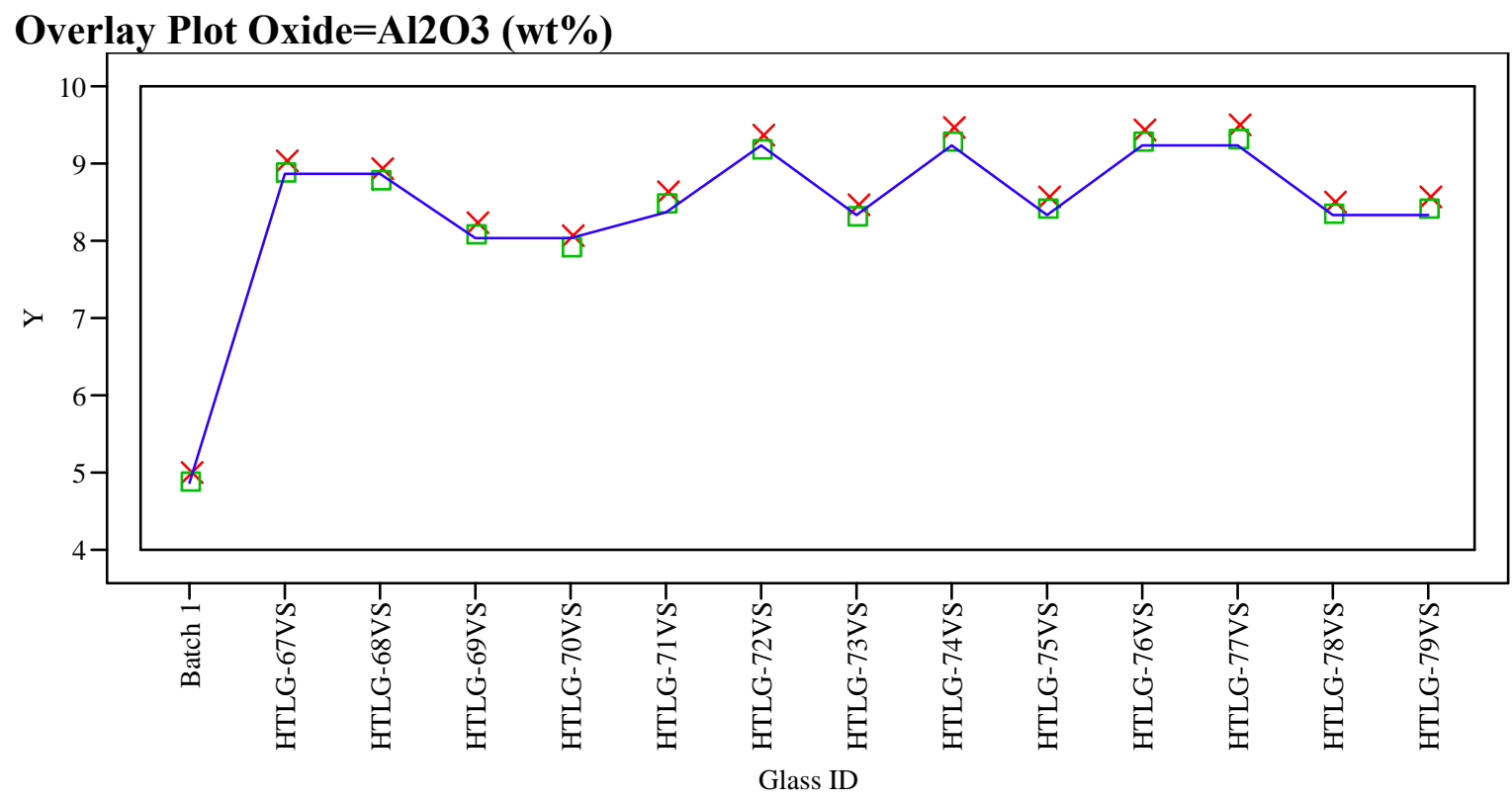

Overlay Plot Oxide $=$ B2O3 $($ wt $\%)$

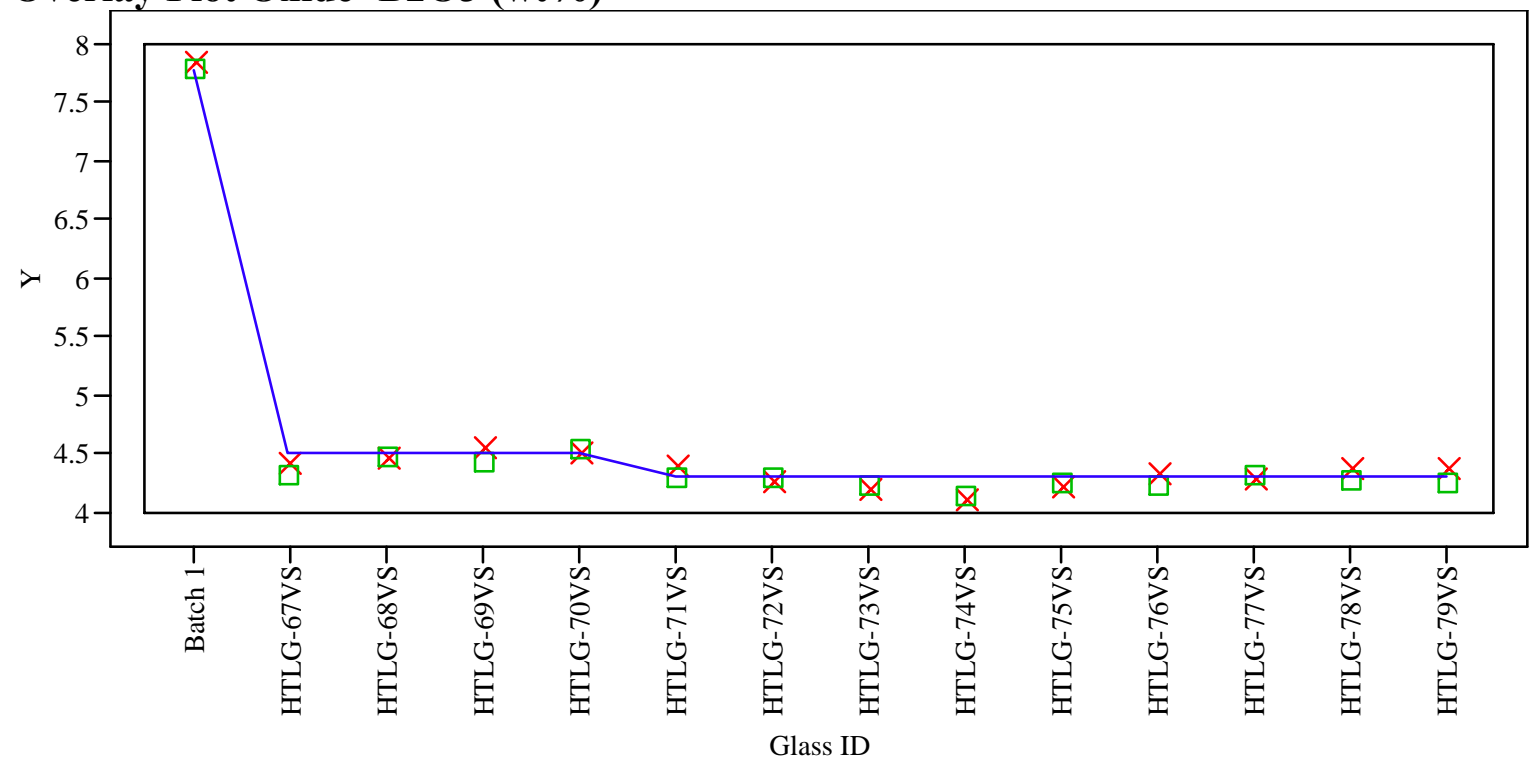

Y $\times$ Measured

回 Measured bc - Targeted 


\section{Exhibit A14. Average Measured and Bias-Corrected (bc) Versus Targeted Compositions by Glass ID by Analyte for the "Q" Series}

Overlay Plot Oxide $=\mathrm{CaO}(\mathrm{wt} \%)$

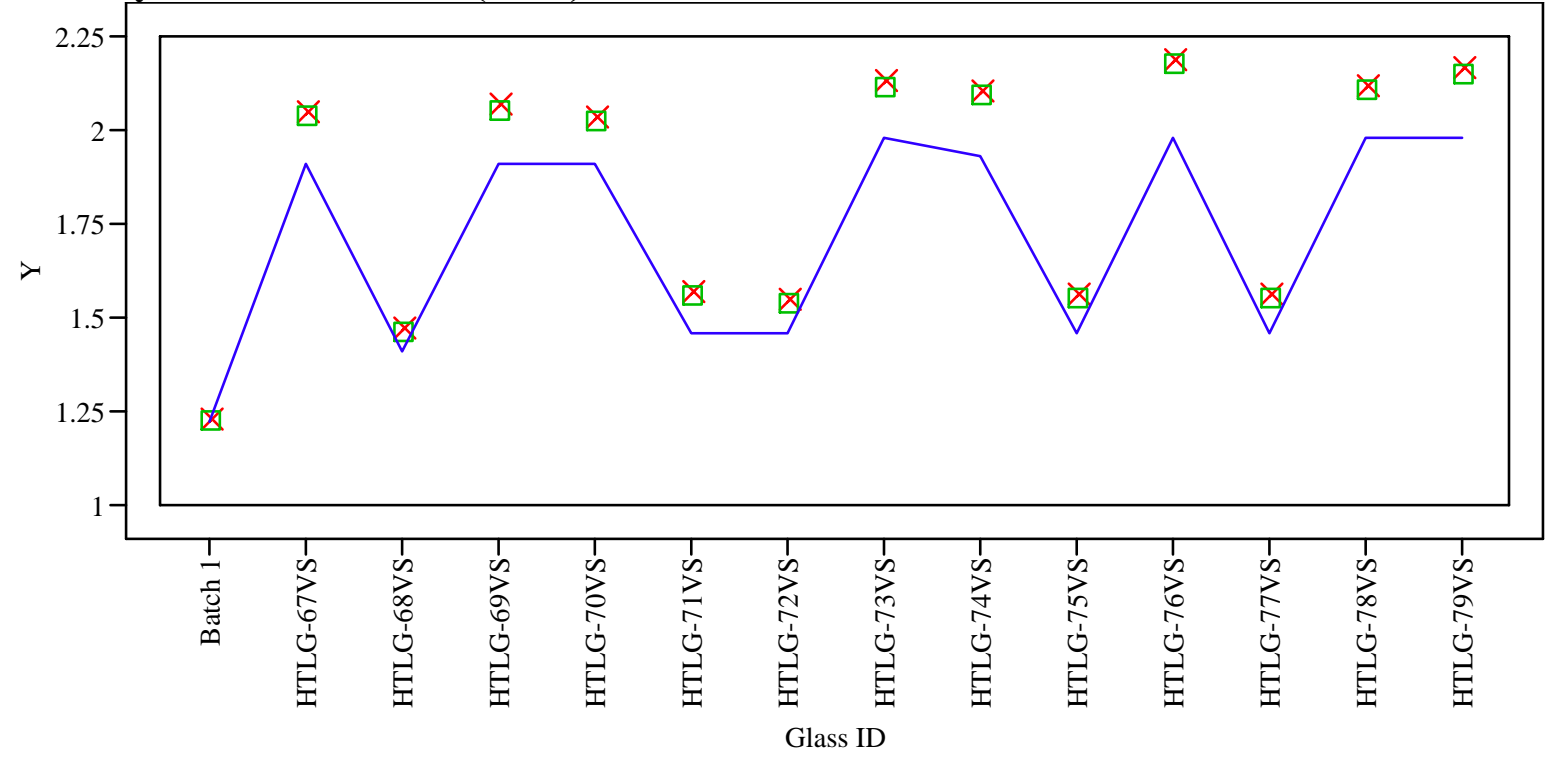

\section{Overlay Plot Oxide $=\mathrm{Cr} 2 \mathrm{O3}(\mathrm{wt} \%)$}

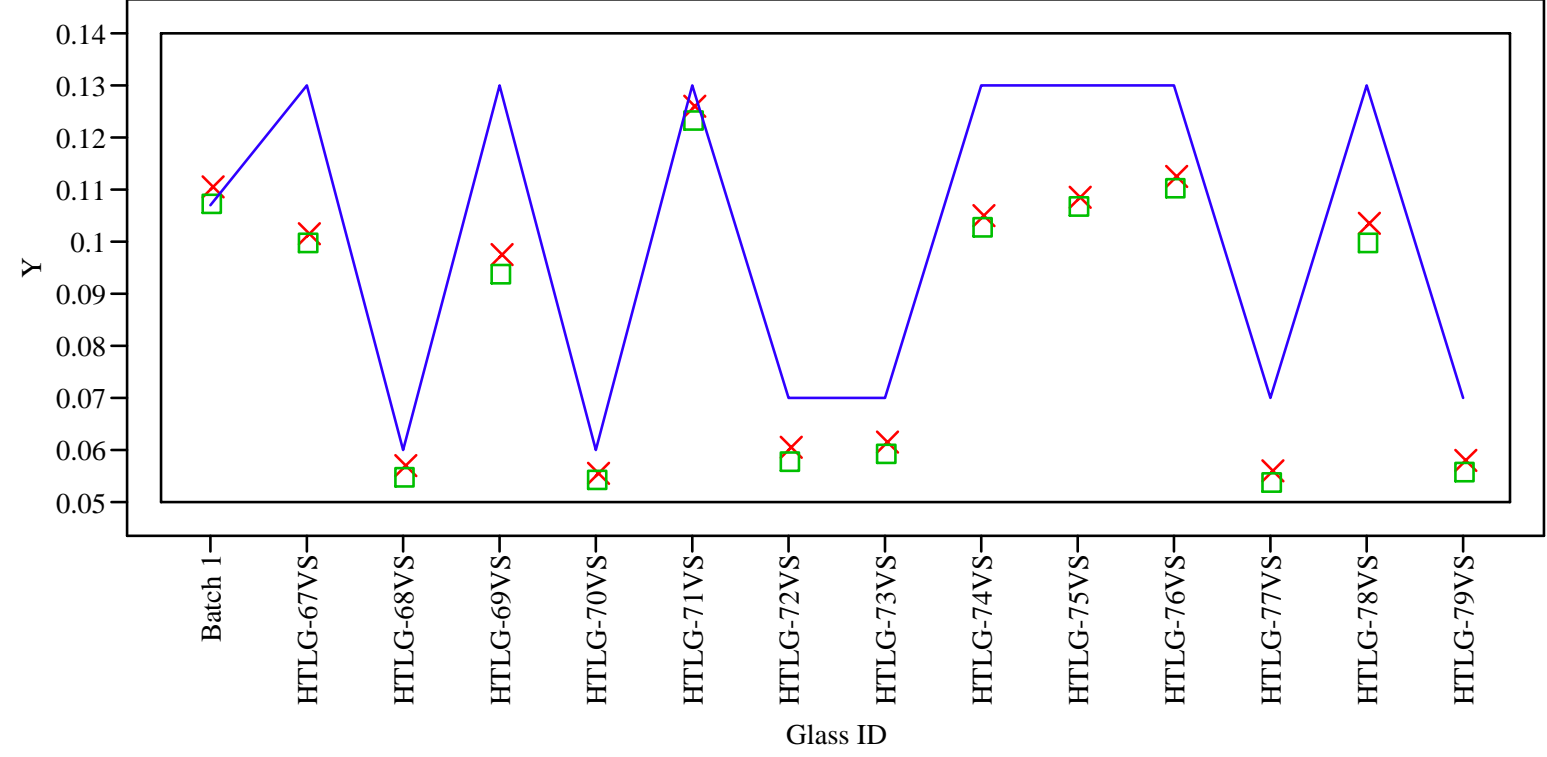

Y $\times$ Measured

- Measured bc - Targeted 


\section{Exhibit A14. Average Measured and Bias-Corrected (bc) Versus Targeted Compositions by Glass ID by Analyte for the "Q" Series}

Overlay Plot Oxide $=\mathrm{CuO}\left(\mathrm{wt}_{\mathrm{O}} \%\right)$

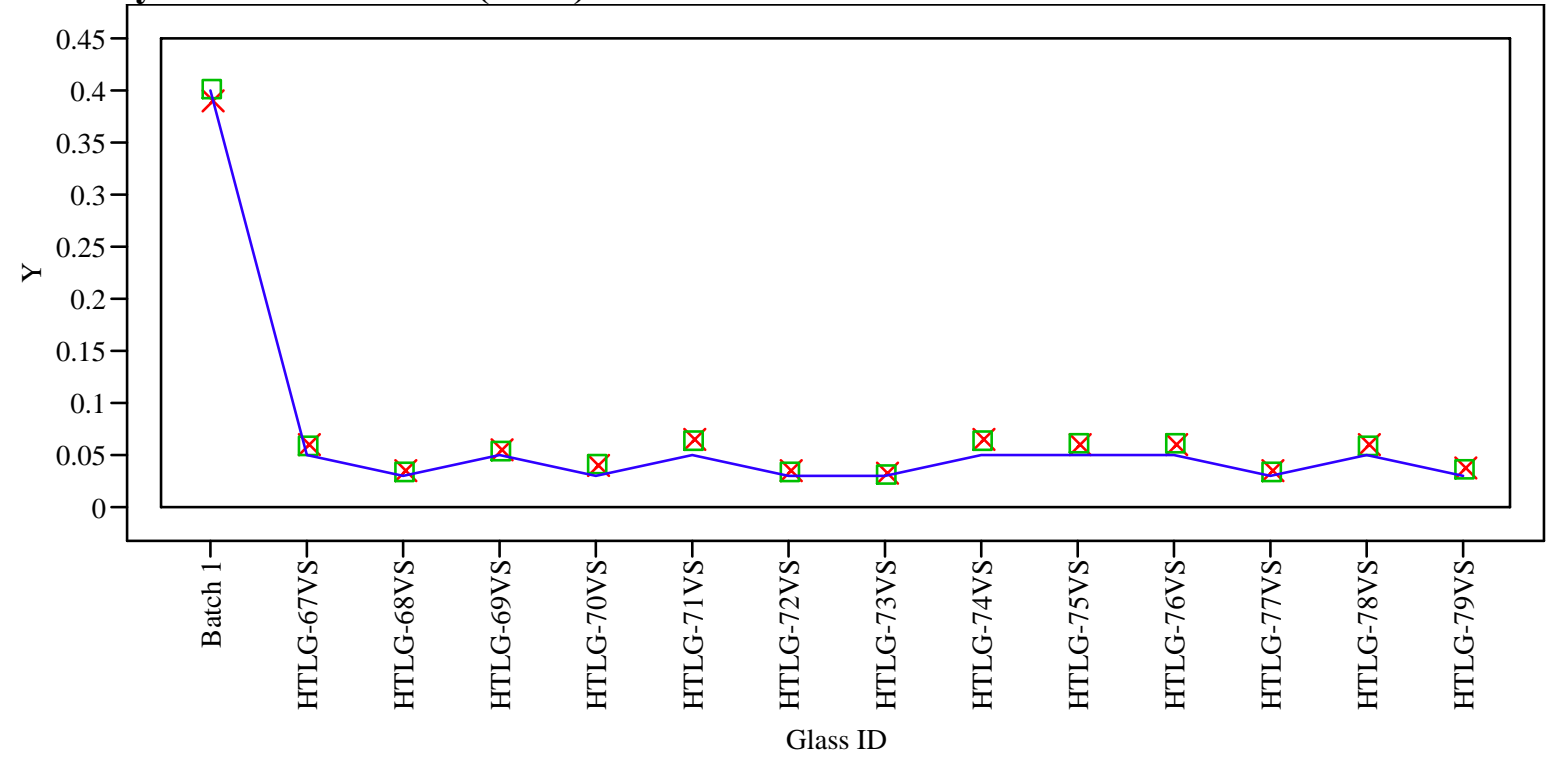

\section{Overlay Plot Oxide $=$ Fe2O3 (wt\%)}

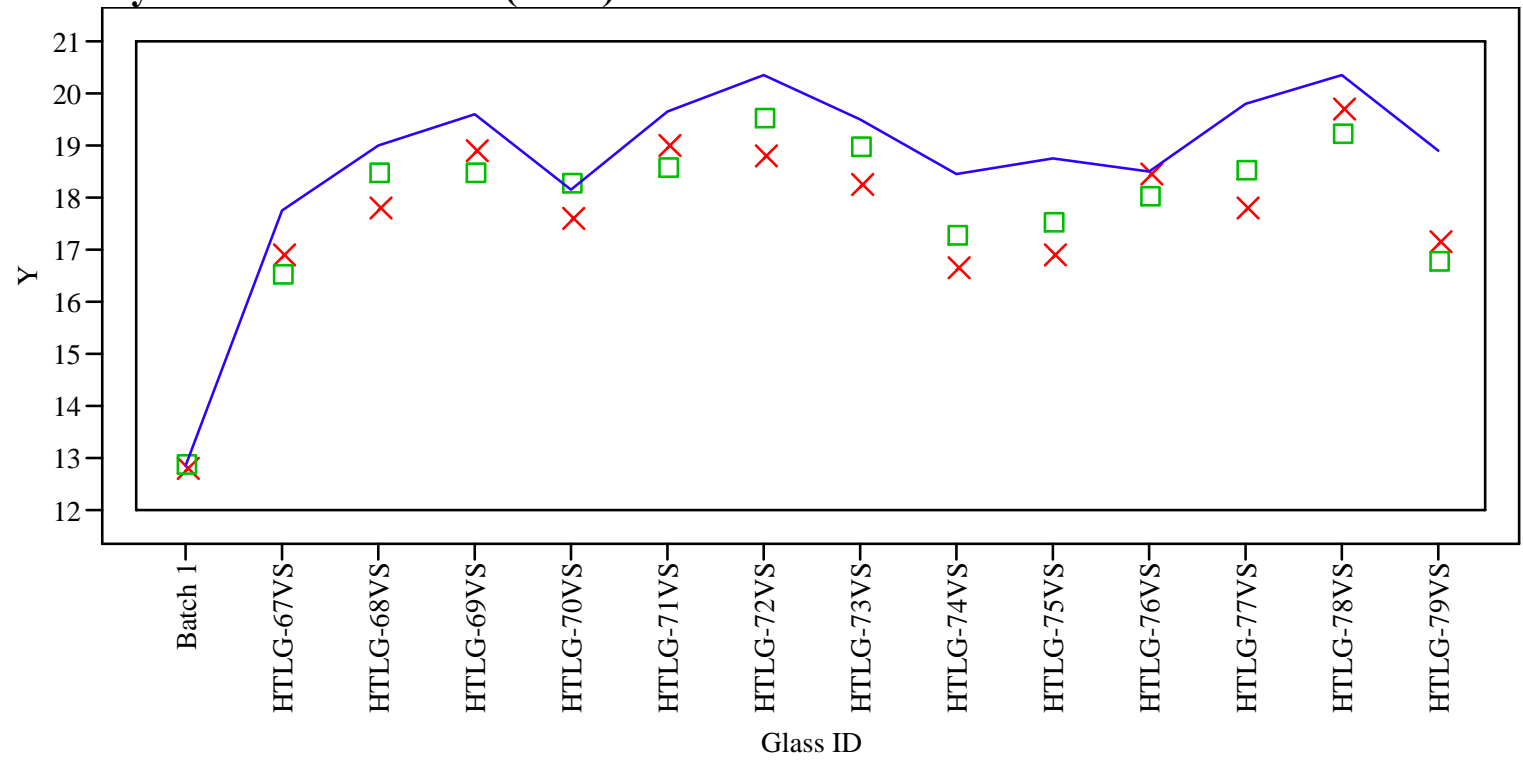

Y $\times$ Measured

- Measured bc — Targeted 


\section{Exhibit A14. Average Measured and Bias-Corrected (bc) Versus Targeted Compositions by Glass ID by Analyte for the "Q" Series}

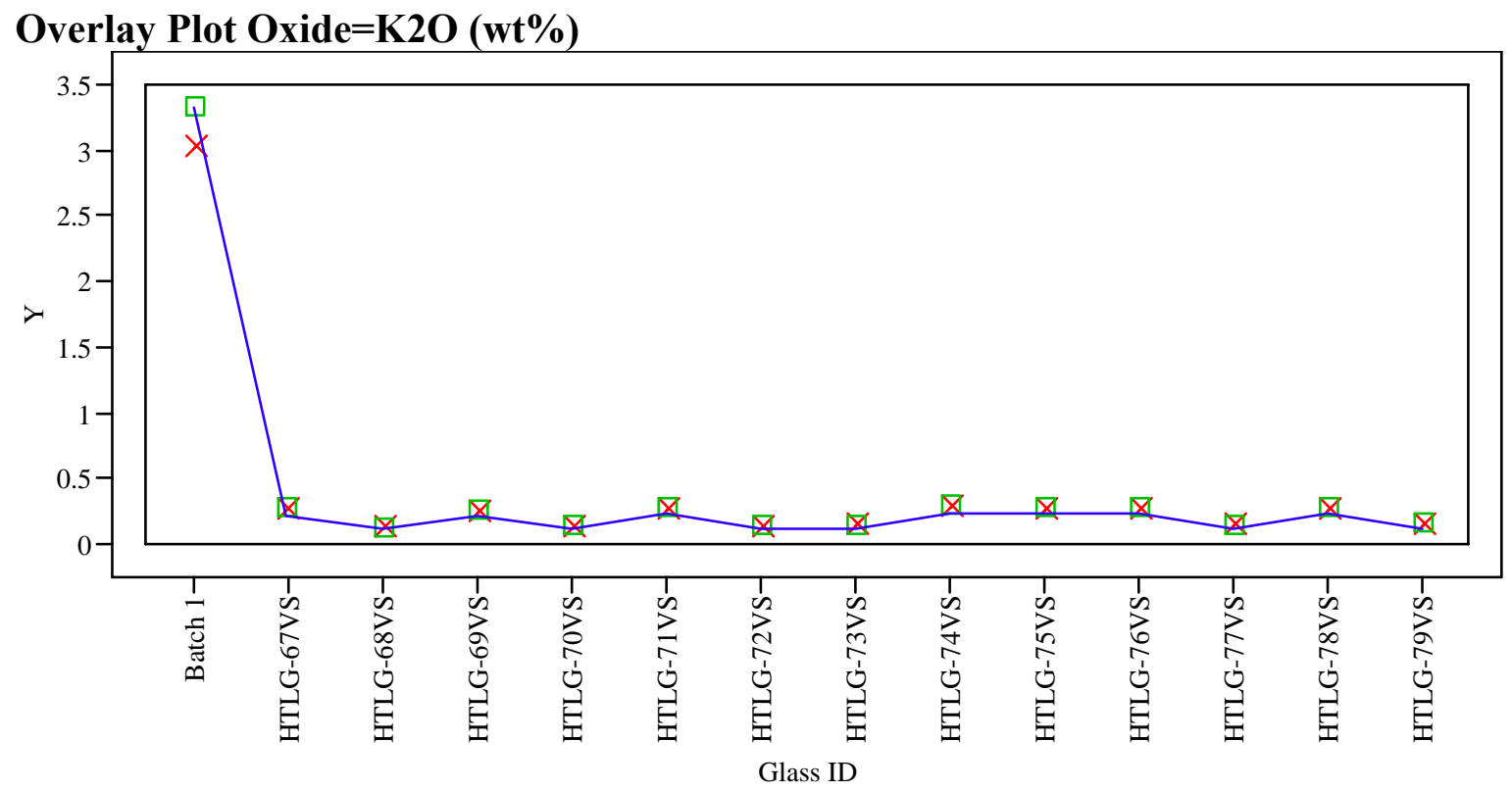

\section{Overlay Plot Oxide $=$ Li2O (wt\%)}

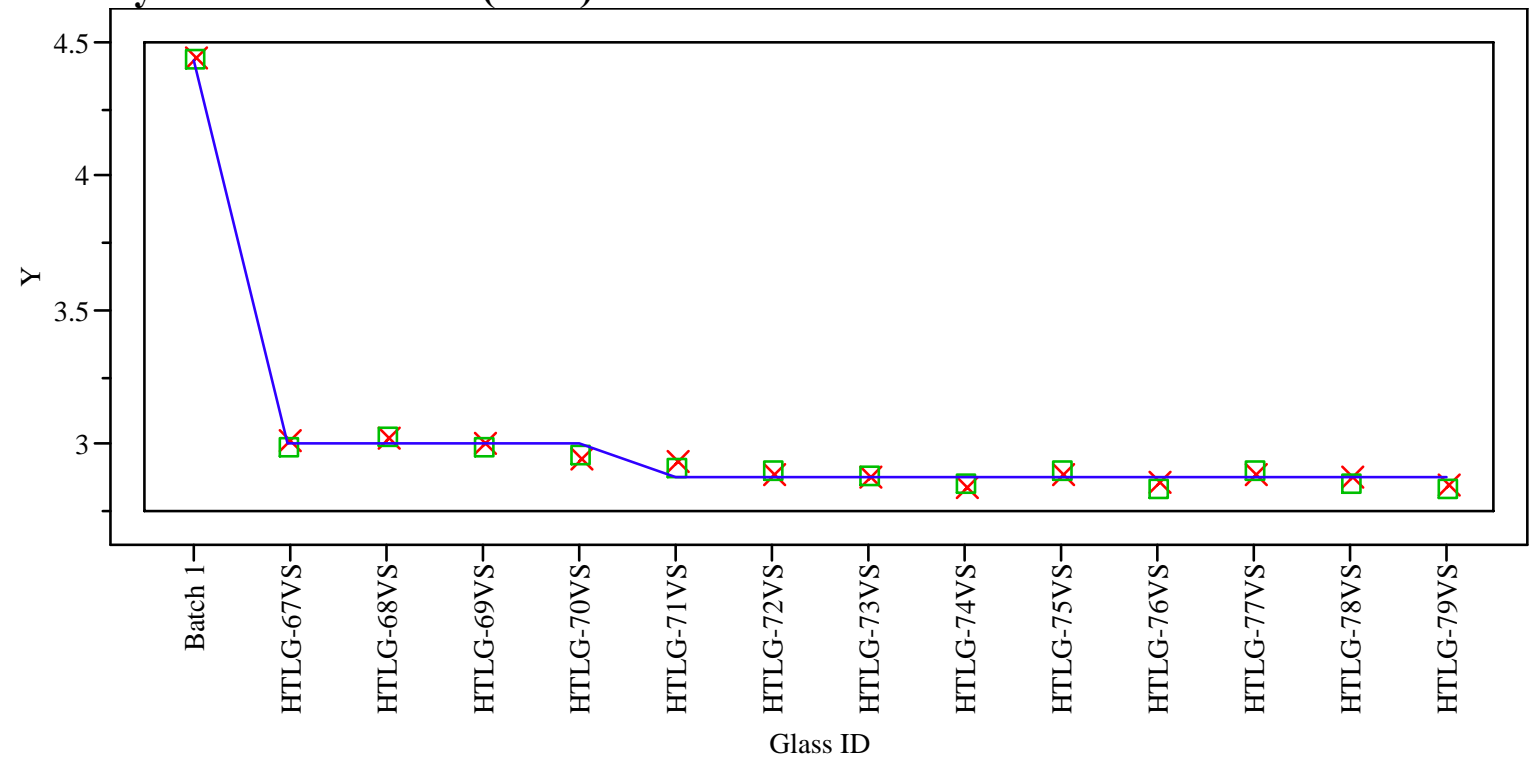

Y $\times$ Measured

- Measured bc - Targeted 


\section{Exhibit A14. Average Measured and Bias-Corrected (bc) Versus Targeted Compositions by Glass ID by Analyte for the "Q" Series}

Overlay Plot Oxide $=$ MgO $($ wt $\%)$

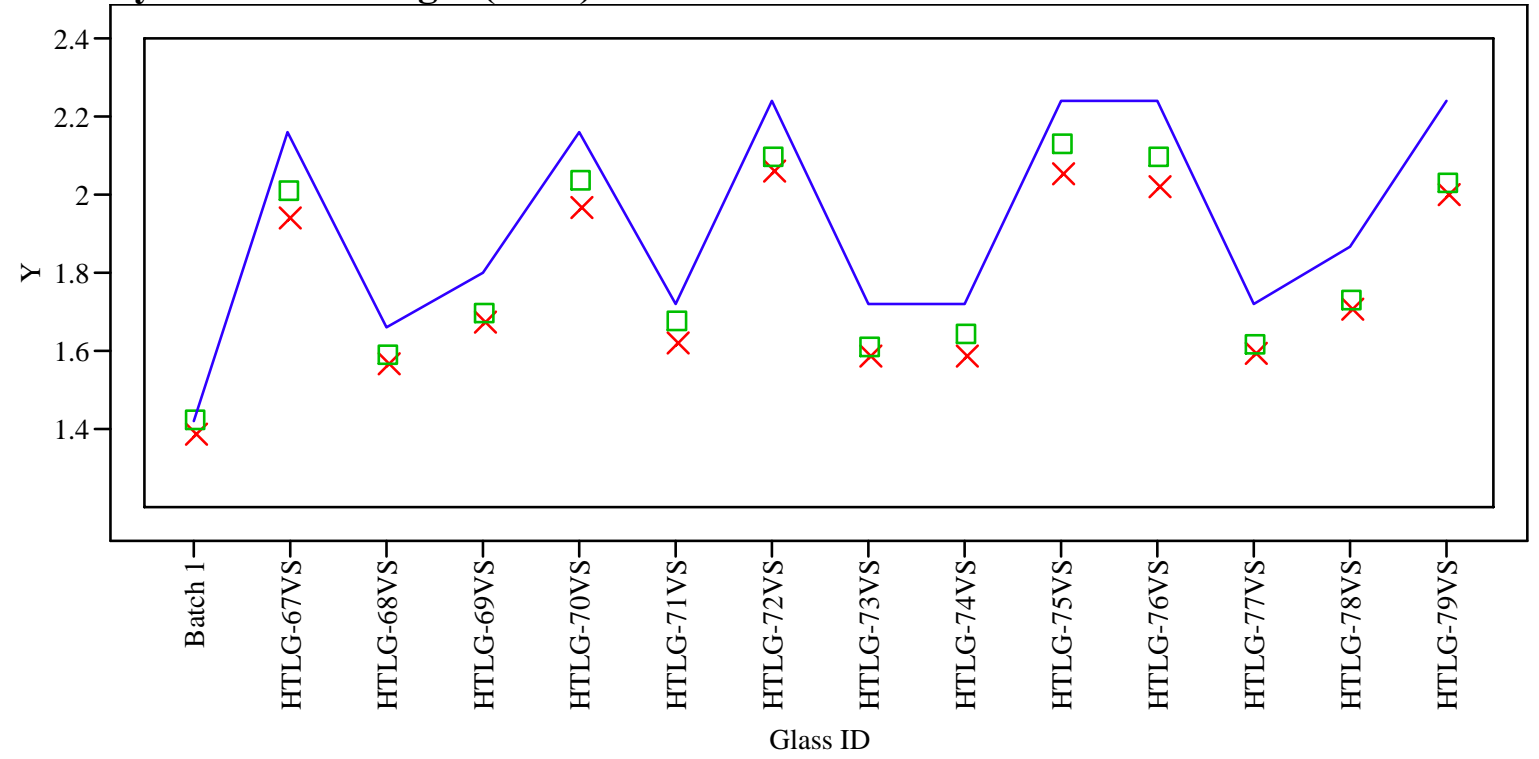

\section{Overlay Plot Oxide $=\mathrm{MnO}(\mathbf{w t} \%)$}

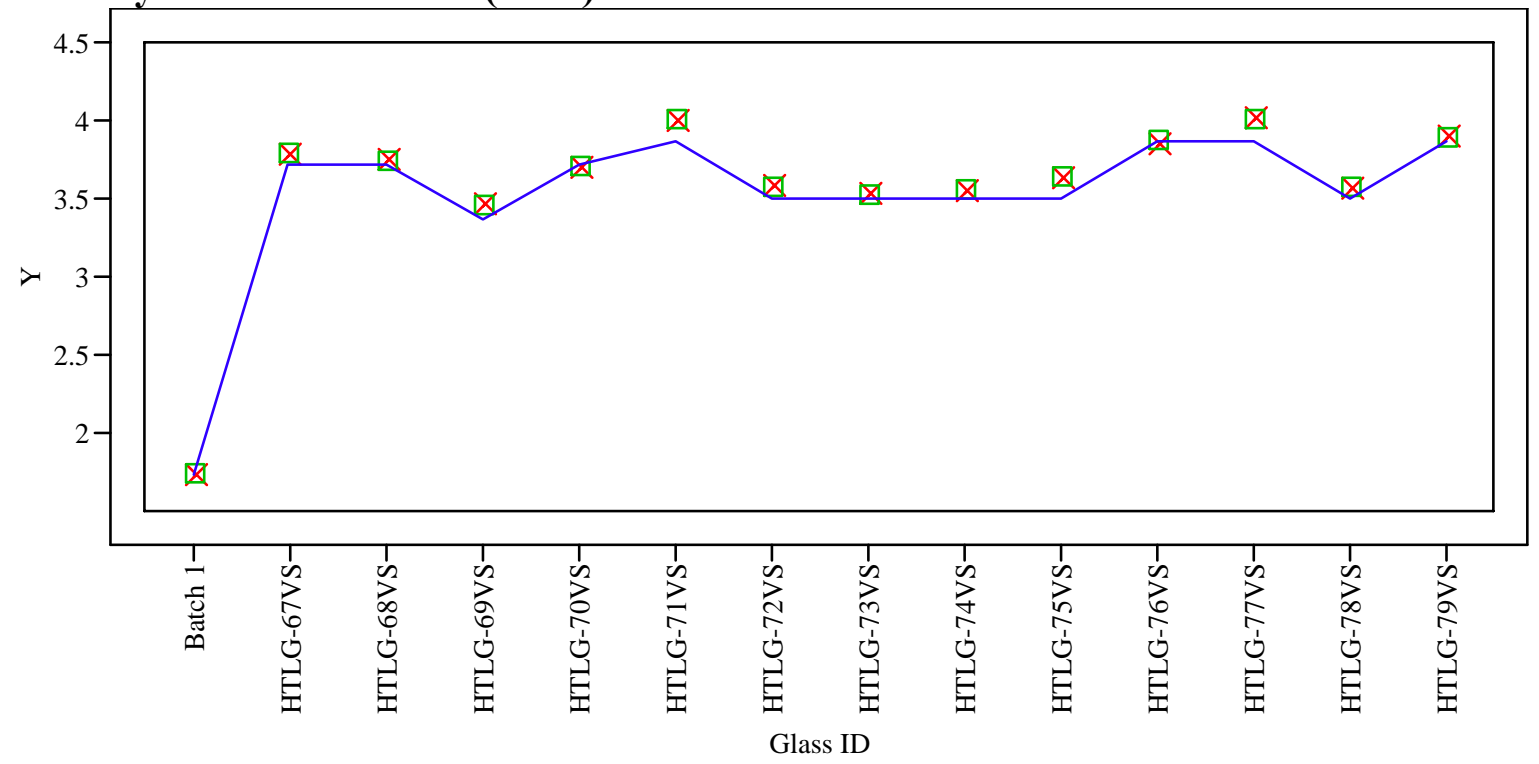

Y $\times$ Measured

- Measured bc — Targeted 


\section{Exhibit A14. Average Measured and Bias-Corrected (bc) Versus Targeted Compositions by Glass ID by Analyte for the " $Q$ " Series}

Overlay Plot Oxide=Na2O (wt\%)

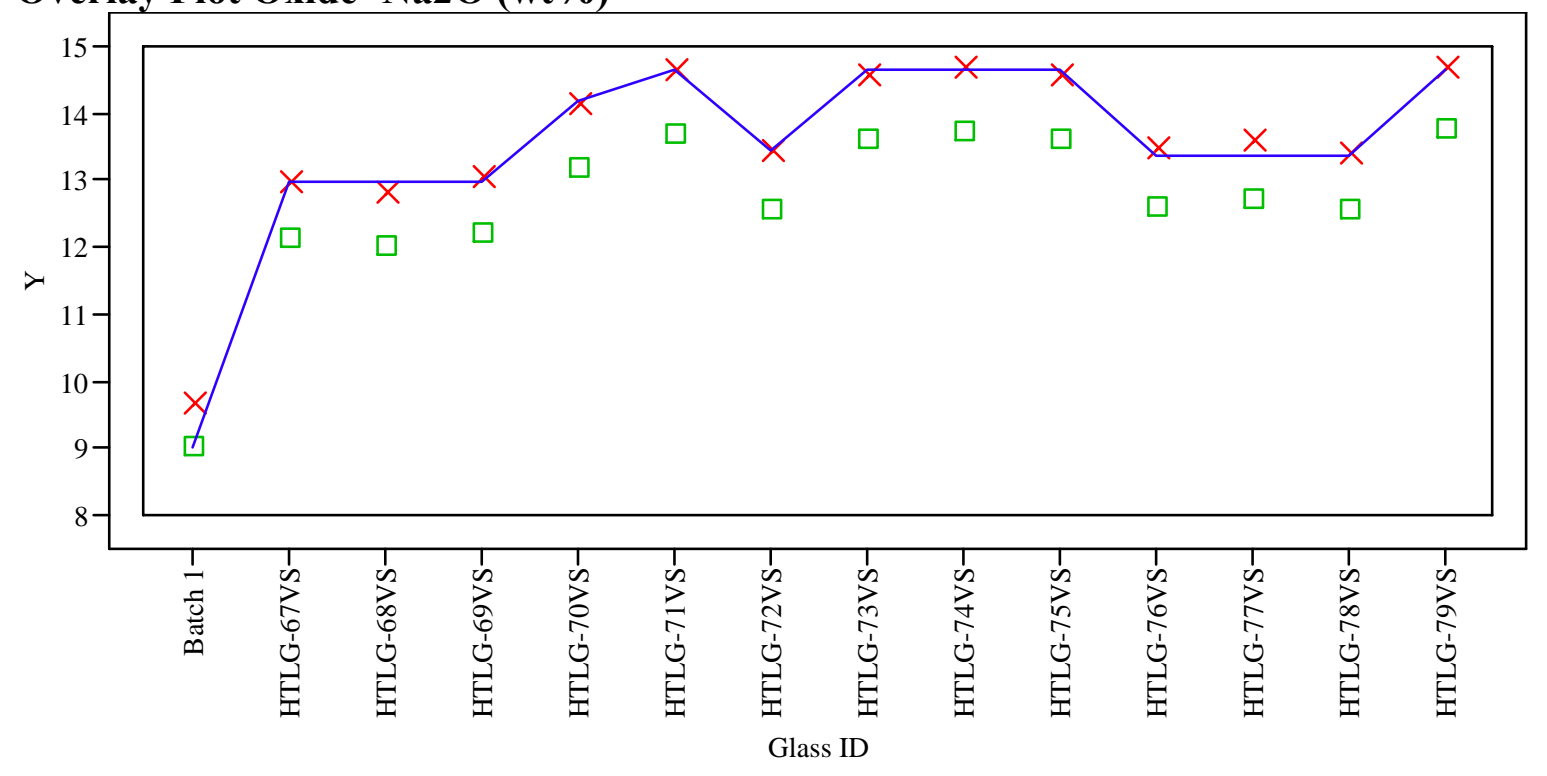

\section{Overlay Plot Oxide $=\mathrm{NiO}\left(\mathrm{wt}_{\mathrm{t}} \mathrm{o}\right)$}

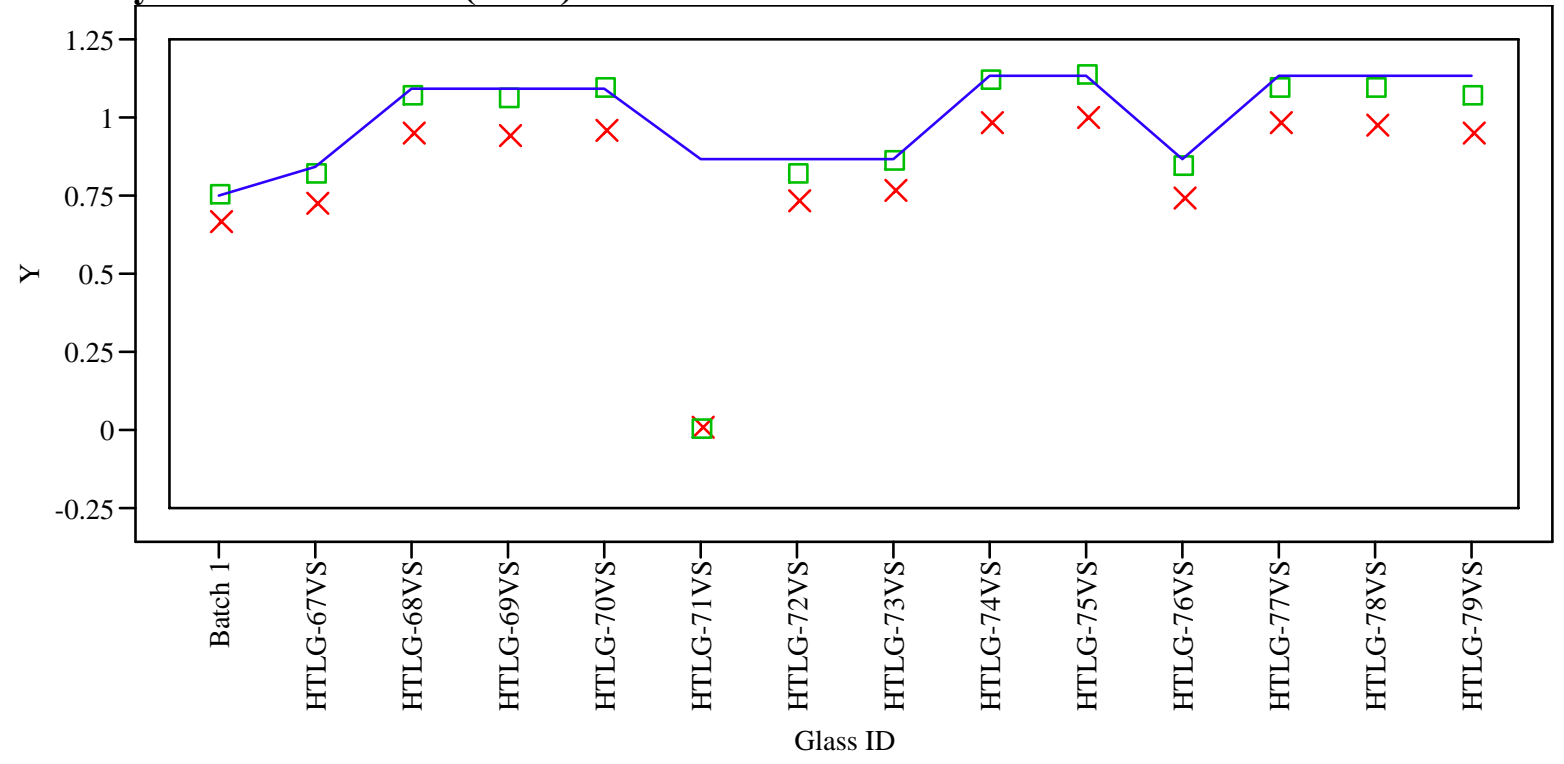

Y $\times$ Measured

- Measured bc — Targeted 


\section{Exhibit A14. Average Measured and Bias-Corrected (bc) Versus Targeted Compositions by Glass ID by Analyte for the "Q" Series}

Overlay Plot Oxide $=\mathrm{SiO} 2(\mathrm{wt} \%)$

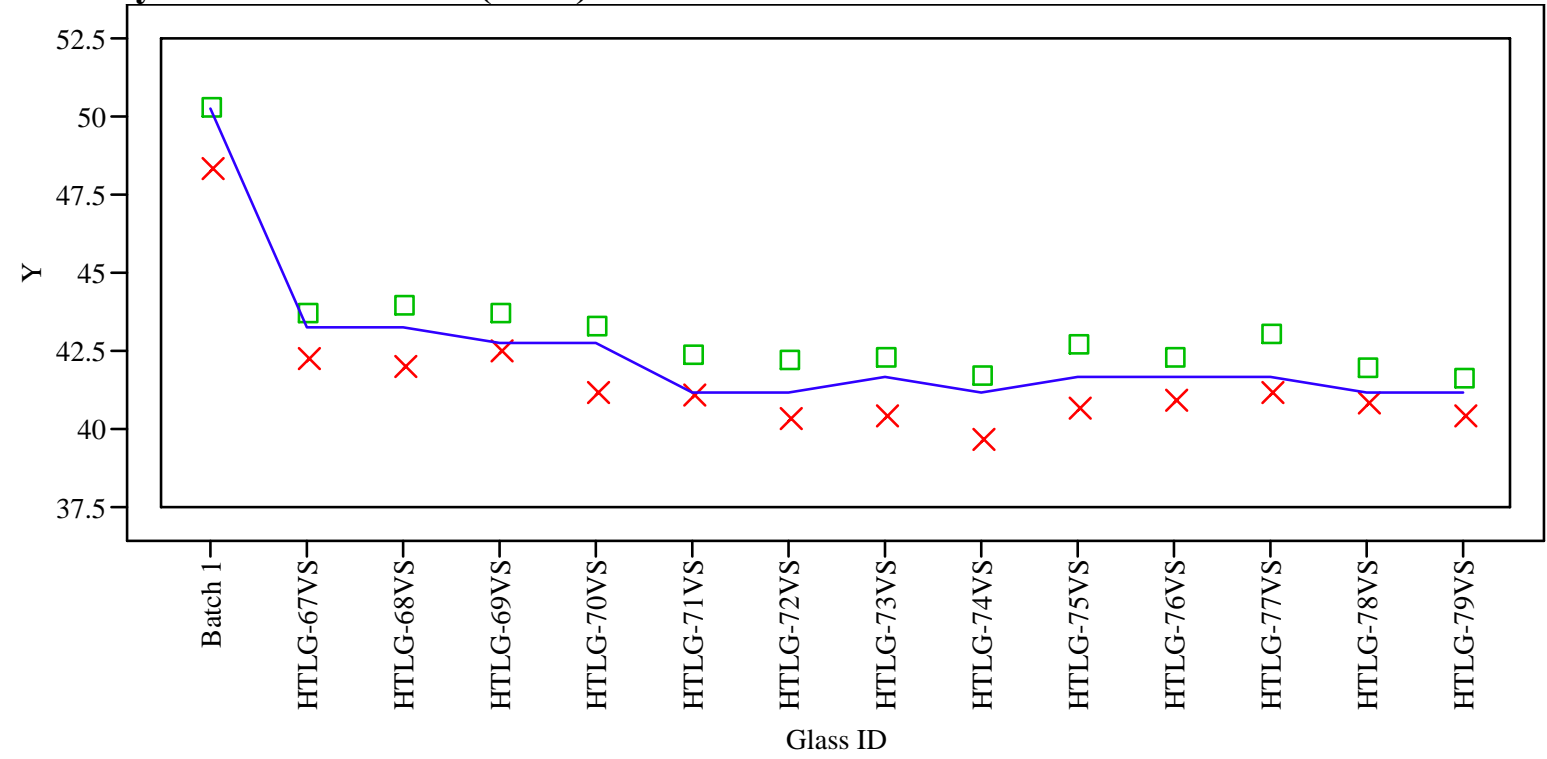

\section{Overlay Plot Oxide $=\mathrm{SO} 4(\mathrm{wt} \%)$}

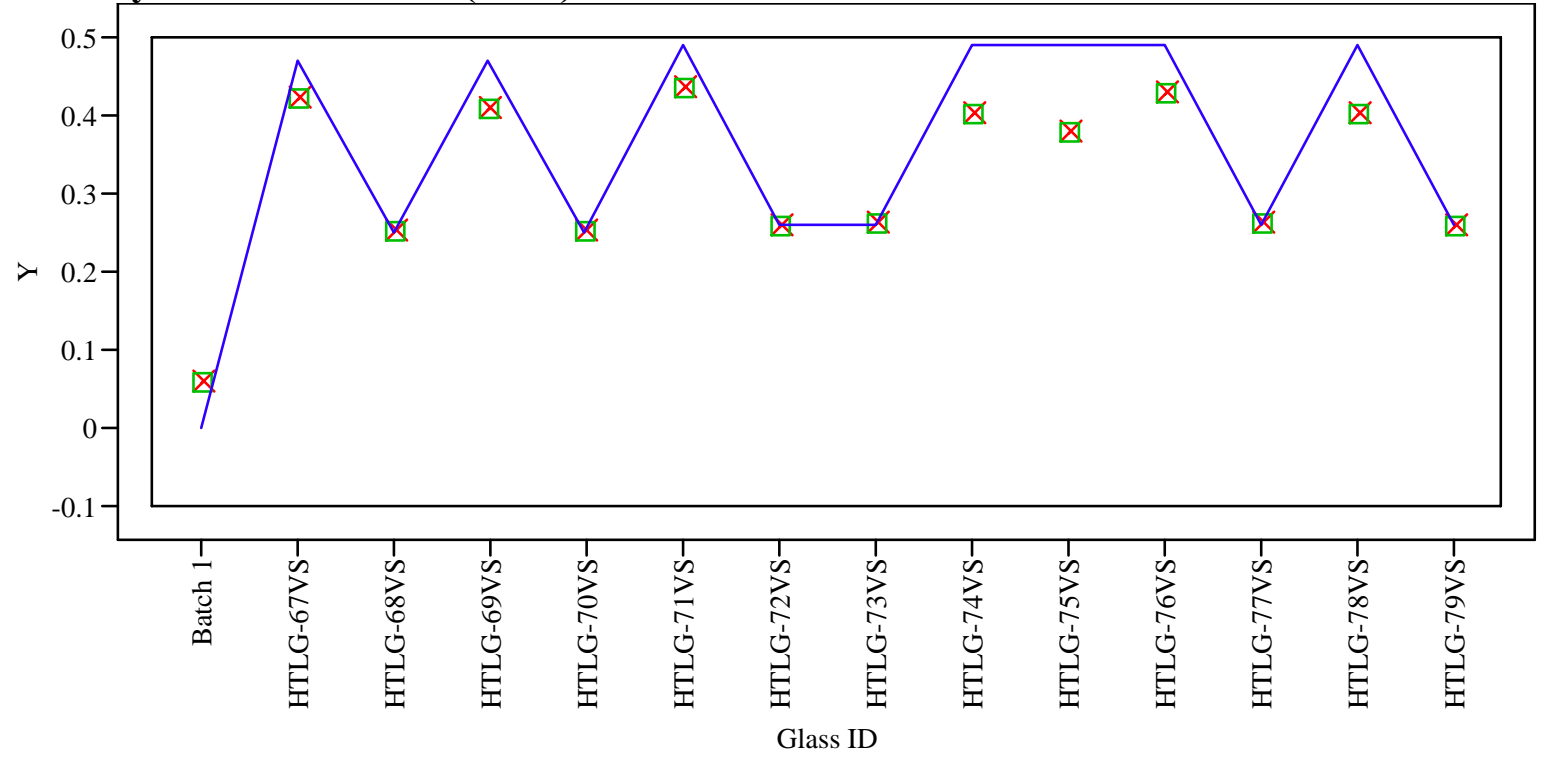

Y $\times$ Measured

- Measured bc — Targeted 


\section{Exhibit A14. Average Measured and Bias-Corrected (bc) Versus Targeted Compositions by Glass ID by Analyte for the "Q" Series}

Overlay Plot Oxide $=\mathrm{TiO} 2(\mathbf{w t} \%)$

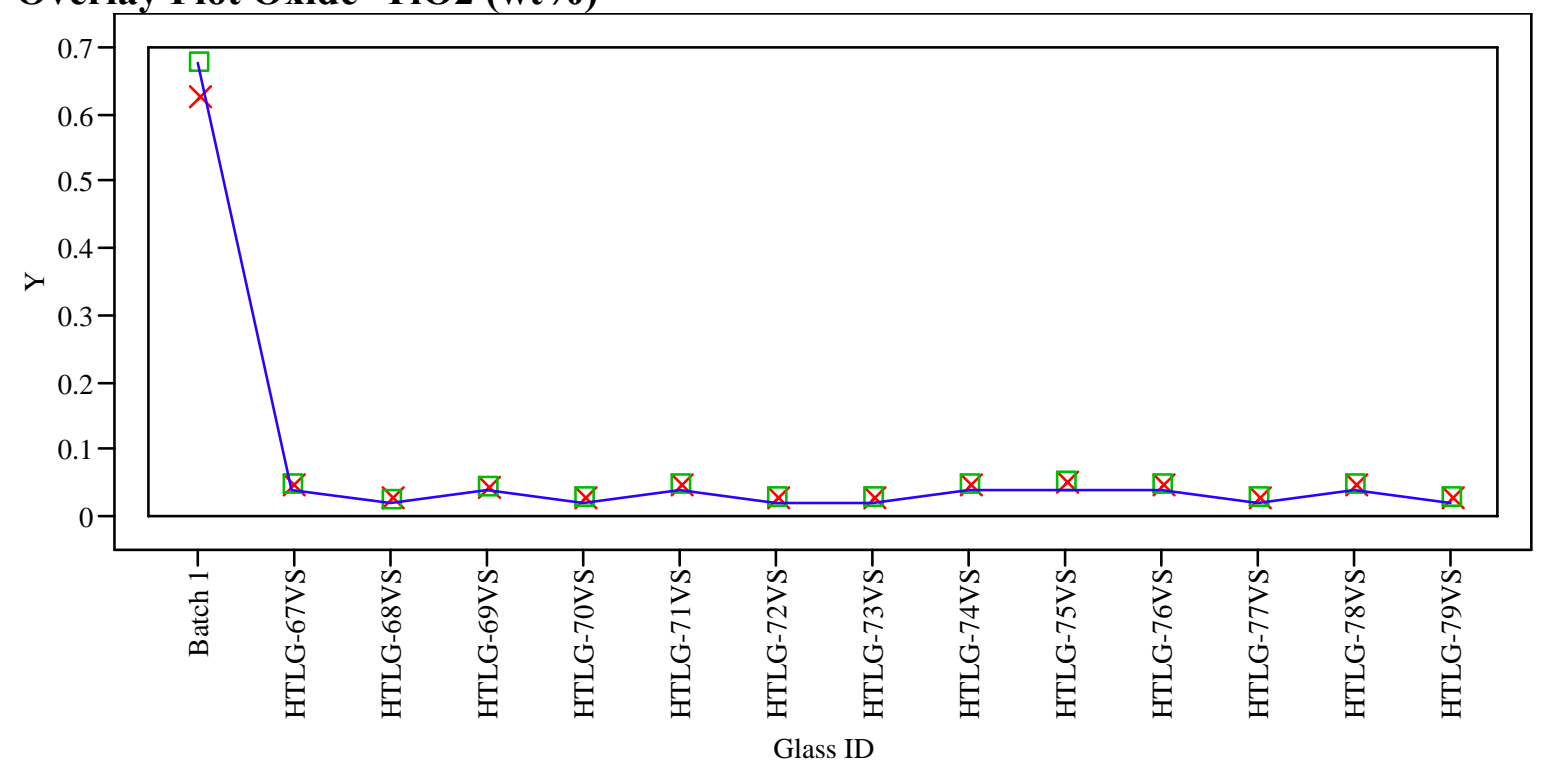

\section{Overlay Plot Oxide $=\mathrm{ZrO2}(\mathbf{w t} \%)$}

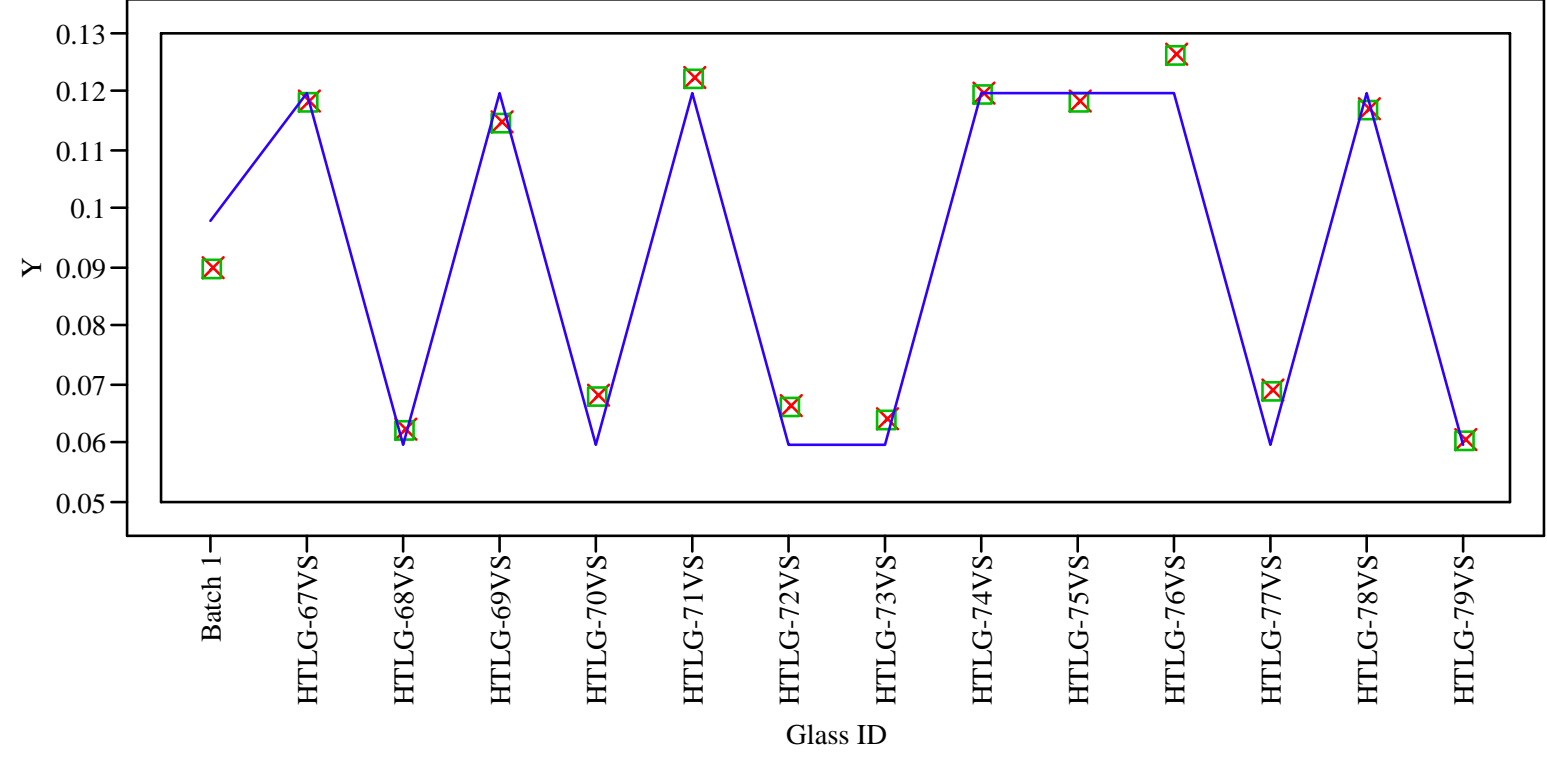

Y $\times$ Measured

- Measured bc — Targeted 
Exhibit A14. Average Measured and Bias-Corrected (bc) Versus Targeted Compositions by Glass ID by Analyte for the " $Q$ " Series

Overlay Plot Oxide=Sum of Oxides $(\mathbf{w t} \%)$

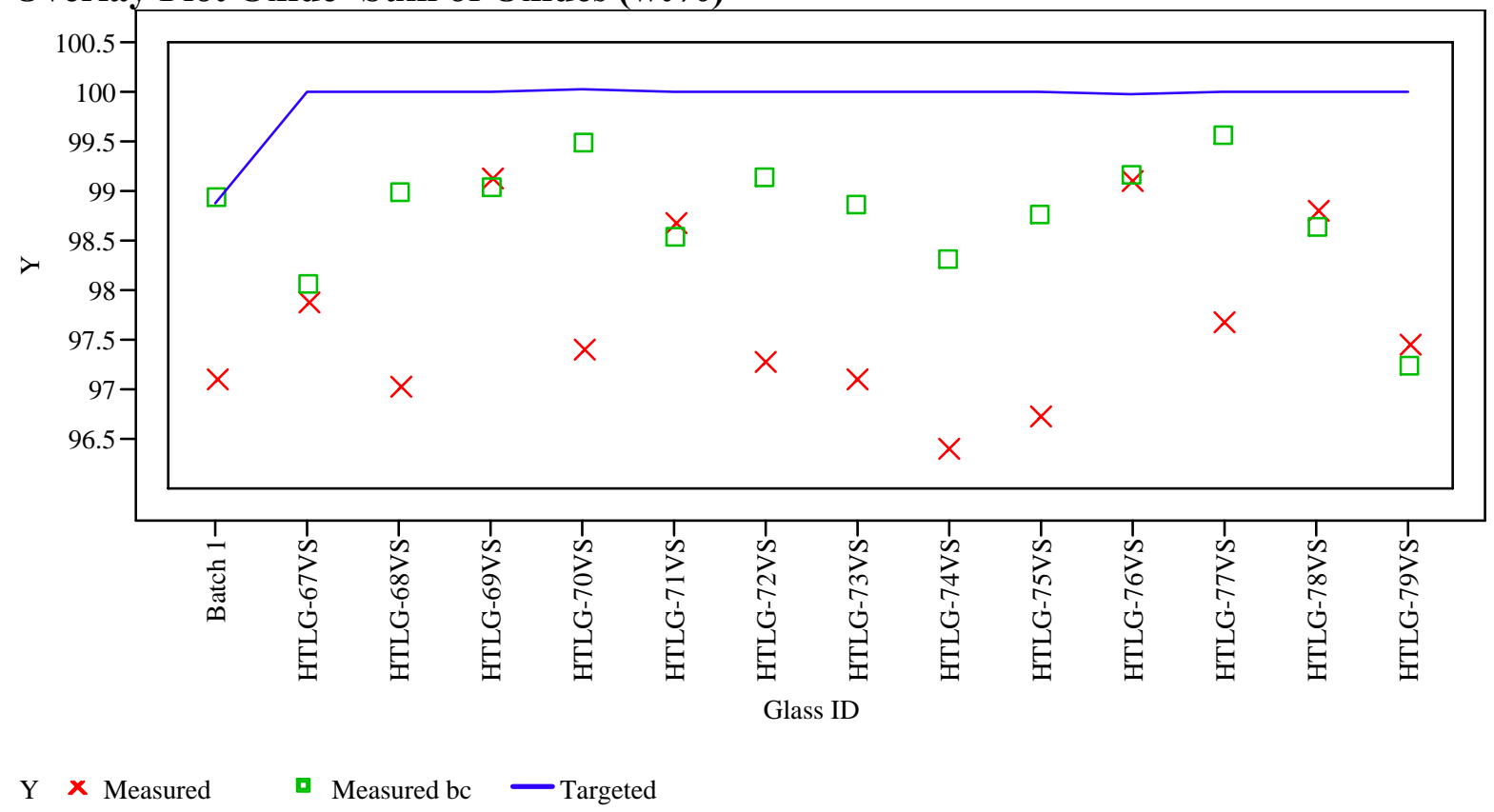


WSRC-STI-2007-00616

Revision 0

This page intentionally left blank. 


\section{Appendix B}

\section{Tables and Exhibits Supporting the Review of the PCT Measurements}


Table B1. PSAL Measurements of the PCT Solutions for the HTL Study Glasses As Reported (ar) and After the Appropriate Adjustments (in parts per million, ppm)

\begin{tabular}{|c|c|c|c|c|c|c|c|c|c|c|c|c|c|c|c|c|c|}
\hline Set & Glass ID & $\begin{array}{c}\text { Heat } \\
\text { Treatment }\end{array}$ & Block & Seq & $\begin{array}{c}\text { SRTC-ML } \\
\text { ID }\end{array}$ & $\mathrm{Al} \mathrm{ar}$ & $\mathrm{B}$ ar & $\mathrm{Fe}$ ar & $\mathrm{Li}$ ar & $\mathrm{Na}$ ar & Si ar & $\begin{array}{c}\mathrm{Al} \\
(\mathrm{ppm})\end{array}$ & $\begin{array}{c}\mathrm{B} \\
(\mathrm{ppm})\end{array}$ & $\begin{array}{c}\mathrm{Fe} \\
(\mathrm{ppm})\end{array}$ & $\begin{array}{c}\mathrm{Li} \\
(\mathrm{ppm})\end{array}$ & $\begin{array}{c}\mathrm{Na} \\
(\mathrm{ppm})\end{array}$ & $\begin{array}{c}\mathrm{Si} \\
(\mathrm{ppm})\end{array}$ \\
\hline 1 & Std Soln & & 1 & 1 & STD-11-1 & 3.64 & 21.4 & 3.89 & 9.98 & 83.4 & 50.6 & 3.64 & 21.40 & 3.89 & 9.98 & 83.40 & 50.60 \\
\hline 1 & HTLG-61VS & CCC & 1 & 2 & F23 & 13.4 & 7.33 & 6.37 & 7.84 & 63.3 & 65.1 & 22.33 & 12.22 & 10.62 & 13.07 & 105.50 & 108.50 \\
\hline 1 & HTLG-64VS & quenched & 1 & 3 & F28 & 10.5 & 9.53 & 4.22 & 8.59 & 73.3 & 67.7 & 17.50 & 15.88 & 7.03 & 14.32 & 122.17 & 112.84 \\
\hline 1 & HTLG-64VS & CCC & 1 & 4 & F65 & 11.7 & 55.1 & 14.5 & 46.7 & 198 & 169 & 19.50 & 91.84 & 24.17 & 77.83 & 330.01 & 281.67 \\
\hline 1 & HTLG-56VS & CCC & 1 & 5 & F36 & 20.3 & 22.5 & 7.21 & 22.3 & 124 & 119 & 33.83 & 37.50 & 12.02 & 37.17 & 206.67 & 198.34 \\
\hline 1 & HTLG-53VS & quenched & 1 & 6 & F82 & 9.28 & 8.8 & 4.21 & 7.77 & 56.1 & 59.2 & 15.47 & 14.67 & 7.02 & 12.95 & 93.50 & 98.67 \\
\hline 1 & HTLG-60VS & CCC & 1 & 7 & F33 & 12.7 & 5.38 & 2.72 & 6.44 & 41 & 51.6 & 21.17 & 8.97 & 4.53 & 10.73 & 68.33 & 86.00 \\
\hline 1 & HTLG-53VS & CCC & 1 & 8 & F37 & 14.2 & 6.93 & 3.46 & 7.85 & 54 & 60.6 & 23.67 & 11.55 & 5.77 & 13.08 & 90.00 & 101.00 \\
\hline 1 & HTLG-65VS & quenched & 1 & 9 & F14 & 9.6 & 8.88 & 3.72 & 7.85 & 68.4 & 63.4 & 16.00 & 14.80 & 6.20 & 13.08 & 114.00 & 105.67 \\
\hline 1 & Std Soln & & 1 & 10 & STD-11-2 & 3.81 & 19 & 3.75 & 9.9 & 83.9 & 48.9 & 3.81 & 19.00 & 3.75 & 9.90 & 83.90 & 48.90 \\
\hline 1 & HTLG-65VS & CCC & 1 & 11 & F17 & 11.5 & 8.2 & 3.77 & 9.16 & 66.2 & 66.6 & 19.17 & 13.67 & 6.28 & 15.27 & 110.34 & 111.00 \\
\hline 1 & EA & & 1 & 12 & F90 & $<1.00$ & 36.5 & $<1.00$ & 11.4 & 103 & 54.7 & 8.33 & 608.33 & 8.33 & 190.00 & 1716.67 & 911.67 \\
\hline 1 & blank & & 1 & 13 & F81 & $<1.00$ & $<1.00$ & 1.84 & $<1.00$ & 1.4 & $<1.00$ & 0.83 & 0.83 & 3.07 & 0.83 & 2.33 & 0.83 \\
\hline 1 & HTLG-57VS & CCC & 1 & 14 & F85 & 10.6 & 4.78 & 1.69 & 5.73 & 39.7 & 49.5 & 17.67 & 7.97 & 2.82 & 9.55 & 66.17 & 82.50 \\
\hline 1 & HTLG-61VS & quenched & 1 & 15 & F35 & 12.6 & 7.9 & 4.34 & 7.29 & 67.1 & 61.6 & 21.00 & 13.17 & 7.23 & 12.15 & 111.84 & 102.67 \\
\hline 1 & HTLG-60VS & quenched & 1 & 16 & F12 & 10.8 & 6.63 & 3.71 & 7 & 47.4 & 53.6 & 18.00 & 11.05 & 6.18 & 11.67 & 79.00 & 89.34 \\
\hline 1 & HTLG-57VS & quenched & 1 & 17 & F74 & 12 & 6.66 & 5.36 & 7.1 & 48.2 & 56.6 & 20.00 & 11.10 & 8.93 & 11.83 & 80.33 & 94.34 \\
\hline 1 & HTLG-56VS & quenched & 1 & 18 & F54 & 11.9 & 10.7 & 8.42 & 9.68 & 84.9 & 74.4 & 19.83 & 17.83 & 14.03 & 16.13 & 141.50 & 124.00 \\
\hline 1 & Std Soln & & 1 & 19 & STD-11-3 & 3.97 & 19.4 & 3.96 & 10 & 84.3 & 50.7 & 3.97 & 19.40 & 3.96 & 10.00 & 84.30 & 50.70 \\
\hline 1 & Std Soln & & 2 & 1 & STD-12-1 & 3.37 & 21.1 & 3.69 & 9.65 & 79 & 49 & 3.37 & 21.10 & 3.69 & 9.65 & 79.00 & 49.00 \\
\hline 1 & HTLG-53VS & quenched & 2 & 2 & F41 & 8.75 & 9.1 & 4.58 & 7.68 & 51.8 & 59.6 & 14.58 & 15.17 & 7.63 & 12.80 & 86.34 & 99.34 \\
\hline 1 & HTLG-60VS & quenched & 2 & 3 & F06 & 10.5 & 7.06 & 3.53 & 6.77 & 49.4 & 52.6 & 17.50 & 11.77 & 5.88 & 11.28 & 82.33 & 87.67 \\
\hline 1 & HTLG-65VS & quenched & 2 & 4 & F92 & 9.73 & 9.3 & 3.31 & 7.88 & 69.6 & 64.3 & 16.22 & 15.50 & 5.52 & 13.13 & 116.00 & 107.17 \\
\hline 1 & HTLG-60VS & CCC & 2 & 5 & F18 & 12.8 & 5.37 & 2.12 & 6.52 & 43.7 & 52.7 & 21.33 & 8.95 & 3.53 & 10.87 & 72.83 & 87.84 \\
\hline 1 & HTLG-61VS & quenched & 2 & 6 & F16 & 11.8 & 7.88 & 3.23 & 7.11 & 63.6 & 59.2 & 19.67 & 13.13 & 5.38 & 11.85 & 106.00 & 98.67 \\
\hline 1 & EA & & 2 & 7 & F59 & $<1.00$ & 37.1 & $<1.00$ & 11.3 & 104 & 53.6 & 8.33 & 618.33 & 8.33 & 188.33 & 1733.34 & 893.34 \\
\hline 1 & HTLG-65VS & CCC & 2 & 8 & F26 & 10.3 & 7.87 & 3.89 & 8.74 & 62.1 & 65.2 & 17.17 & 13.12 & 6.48 & 14.57 & 103.50 & 108.67 \\
\hline 1 & HTLG-56VS & quenched & 2 & 9 & F68 & 9.62 & 10.6 & 4.59 & 9.31 & 81.3 & 70.9 & 16.03 & 17.67 & 7.65 & 15.52 & 135.50 & 118.17 \\
\hline 1 & Std Soln & & 2 & 10 & STD-12-2 & 3.36 & 19.3 & 3.48 & 9.71 & 78.9 & 48.2 & 3.36 & 19.30 & 3.48 & 9.71 & 78.90 & 48.20 \\
\hline 1 & HTLG-61VS & CCC & 2 & 11 & F46 & 12.5 & 7.97 & 5.93 & 7.67 & 60.6 & $\begin{array}{ll}64.4 \\
\end{array}$ & 20.83 & 13.28 & 9.88 & 12.78 & 101.00 & 107.34 \\
\hline 1 & HTLG-64VS & quenched & 2 & 12 & F80 & 9.72 & 9.56 & 4.41 & 8.24 & 68.5 & 66.1 & 16.20 & 15.93 & 7.35 & 13.73 & 114.17 & 110.17 \\
\hline 1 & HTLG-57VS & CCC & 2 & 13 & F31 & 10.5 & 4.94 & 1.86 & 5.7 & 41.6 & 50.4 & 17.50 & 8.23 & 3.10 & 9.50 & 69.33 & 84.00 \\
\hline 1 & HTLG-57VS & quenched & 2 & 14 & F83 & 11.9 & 6.88 & 5.07 & 6.99 & 45.8 & 56.6 & 19.83 & 11.47 & 8.45 & 11.65 & 76.33 & 94.34 \\
\hline 1 & HTLG-53VS & CCC & 2 & 15 & F67 & 13.7 & 7.18 & 3.55 & 7.77 & 49.2 & 62.1 & 22.83 & 11.97 & 5.92 & 12.95 & 82.00 & 103.50 \\
\hline 1 & HTLG-56VS & CCC & 2 & 16 & F15 & 9.48 & 21.4 & 7.11 & 21.8 & 117 & 116 & 15.80 & 35.67 & 11.85 & 36.33 & 195.00 & 193.34 \\
\hline 1 & HTLG-64VS & CCC & 2 & 17 & F73 & 28.8 & 58 & 17.3 & 48 & 198 & 173 & 48.00 & 96.67 & 28.83 & 80.00 & 330.01 & 288.34 \\
\hline 1 & Std Soln & & 2 & 18 & STD-12-3 & 3.35 & 20.3 & 3.75 & 9.83 & 78.5 & 49.7 & 3.35 & 20.30 & 3.75 & 9.83 & 78.50 & 49.70 \\
\hline 1 & Std Soln & & 3 & 1 & STD-13-1 & 3.77 & 20.6 & 3.58 & 9.68 & 81 & 48.6 & 3.77 & 20.60 & 3.58 & 9.68 & 81.00 & 48.60 \\
\hline 1 & HTLG-53VS & quenched & 3 & 2 & F56 & 9.31 & 9.12 & 3.39 & 7.74 & 53.5 & 59.1 & 15.52 & 15.20 & 5.65 & 12.90 & 89.17 & 98.50 \\
\hline
\end{tabular}


Table B1. PSAL Measurements of the PCT Solutions for the HTL Study Glasses As Reported (ar) and After the Appropriate Adjustments (in parts per million, ppm)

\begin{tabular}{|c|c|c|c|c|c|c|c|c|c|c|c|c|c|c|c|c|c|}
\hline Set & Glass ID & $\begin{array}{c}\text { Heat } \\
\text { Treatment }\end{array}$ & Block & Seq & $\begin{array}{c}\text { SRTC-ML } \\
\text { ID }\end{array}$ & $\mathrm{Al} \mathrm{ar}$ & $\mathrm{B}$ ar & $\mathrm{Fe}$ ar & $\mathrm{Li}$ ar & $\mathrm{Na}$ ar & Si ar & $\begin{array}{c}\mathrm{Al} \\
(\mathrm{ppm})\end{array}$ & $\begin{array}{c}\mathrm{B} \\
(\mathrm{ppm})\end{array}$ & $\begin{array}{c}\mathrm{Fe} \\
(\mathrm{ppm})\end{array}$ & $\begin{array}{c}\mathrm{Li} \\
(\mathrm{ppm})\end{array}$ & $\begin{array}{c}\mathrm{Na} \\
(\mathrm{ppm})\end{array}$ & $\begin{array}{c}\mathrm{Si} \\
(\mathrm{ppm})\end{array}$ \\
\hline 1 & HTLG-64VS & CCC & 3 & 3 & F64 & 12.1 & 55.9 & 15.9 & 46.4 & 198 & 167 & 20.17 & 93.17 & 26.50 & 77.33 & 330.01 & 278.34 \\
\hline 1 & HTLG-57VS & CCC & 3 & 4 & F03 & 10.6 & 5.65 & 1.62 & 5.56 & 37.2 & 48.5 & 17.67 & 9.42 & 2.70 & 9.27 & 62.00 & 80.83 \\
\hline 1 & HTLG-64VS & quenched & 3 & 5 & F21 & 10.2 & 9.65 & 3.73 & 8.13 & $\begin{array}{l}68.4 \\
\end{array}$ & 63.9 & 17.00 & 16.08 & 6.22 & 13.55 & 114.00 & 106.50 \\
\hline 1 & HTLG-60VS & quenched & 3 & 6 & F40 & 10.9 & 7.09 & 3.69 & 6.88 & 46.9 & 53.8 & 18.17 & 11.82 & 6.15 & 11.47 & 78.17 & 89.67 \\
\hline 1 & HTLG-60VS & CCC & 3 & 7 & F47 & 13 & 5.43 & 2.37 & 6.34 & 41.5 & 52.4 & 21.67 & 9.05 & 3.95 & 10.57 & 69.17 & 87.34 \\
\hline 1 & HTLG-56VS & quenched & 3 & 8 & F61 & 9.62 & 10.7 & 4.43 & 9.41 & 79.8 & 70.1 & 16.03 & 17.83 & 7.38 & 15.68 & 133.00 & 116.84 \\
\hline 1 & EA & & 3 & 9 & F42 & $<1.00$ & 37.6 & $<1.00$ & 11.3 & 102 & 53.8 & 8.33 & 626.67 & 8.33 & 188.33 & 1700.00 & 896.67 \\
\hline 1 & Std Soln & & 3 & 10 & STD-13-2 & 3.77 & 19.8 & 3.62 & 9.8 & 80.8 & 49 & 3.77 & 19.80 & 3.62 & 9.80 & 80.80 & 49.00 \\
\hline 1 & HTLG-61VS & quenched & 3 & 11 & F49 & 12.5 & 8.92 & 3.58 & 7.25 & 66 & 62.1 & 20.83 & 14.87 & 5.97 & 12.08 & 110.00 & 103.50 \\
\hline 1 & HTLG-56VS & CCC & 3 & 12 & F43 & 9.87 & 21.49 & 7.5 & 21.5 & 118 & 113 & 16.45 & 35.82 & 12.50 & 35.83 & 196.67 & 188.34 \\
\hline 1 & HTLG-65VS & quenched & 3 & 13 & F07 & 10.4 & 9.69 & 3.78 & 8 & 68.9 & 66.7 & 17.33 & 16.15 & 6.30 & 13.33 & 114.84 & 111.17 \\
\hline 1 & HTLG-57VS & quenched & 3 & 14 & F09 & 11.6 & 6.97 & 4.35 & 6.83 & 45.9 & 54.9 & 19.33 & 11.62 & 7.25 & 11.38 & 76.50 & 91.50 \\
\hline 1 & HTLG-65VS & CCC & 3 & 15 & F19 & 10.5 & 7.72 & 3.22 & 8.94 & 65.6 & 66.2 & 17.50 & 12.87 & 5.37 & 14.90 & 109.34 & 110.34 \\
\hline 1 & HTLG-61VS & CCC & 3 & 16 & F50 & 13 & 7.27 & 6.32 & 7.53 & 63.3 & 63.5 & 21.67 & 12.12 & 10.53 & 12.55 & 105.50 & 105.84 \\
\hline 1 & HTLG-53VS & CCC & 3 & 17 & F57 & 13.9 & 6.68 & 3.78 & 7.37 & 48.2 & 59.1 & 23.17 & 11.13 & 6.30 & 12.28 & 80.33 & 98.50 \\
\hline 1 & blank & & 3 & 18 & F94 & $<1.00$ & $<1.00$ & $<1.00$ & $<1.00$ & $<1.00$ & $<1.00$ & 0.83 & 0.83 & 0.83 & 0.83 & 0.83 & 0.83 \\
\hline 1 & Std Soln & & 3 & 19 & STD-13-3 & 3.91 & 19.9 & 3.76 & 9.88 & 84.5 & 49.7 & 3.91 & 19.90 & 3.76 & 9.88 & 84.50 & 49.70 \\
\hline 1 & Std Soln & & 4 & 1 & STD-14-1 & 3.61 & 20.2 & 3.88 & 9.63 & 80.6 & 49.1 & 3.61 & 20.20 & 3.88 & 9.63 & 80.60 & 49.10 \\
\hline 1 & HTLG-58VS & CCC & 4 & 2 & F45 & 10.6 & 7.87 & 4.15 & 7.6 & 67.8 & 67.8 & 17.67 & 13.12 & 6.92 & 12.67 & 113.00 & 113.00 \\
\hline 1 & HTLG-54VS & CCC & 4 & 3 & F10 & 12.6 & 34.5 & 15.4 & 21.5 & 137 & 123 & 21.00 & 57.50 & 25.67 & 35.83 & 228.34 & 205.00 \\
\hline 1 & HTLG-63VS & CCC & 4 & 4 & F89 & 11.9 & 5.93 & 3 & 6.25 & 45.8 & 54.5 & 19.83 & 9.88 & 5.00 & 10.42 & 76.33 & 90.84 \\
\hline 1 & HTLG-59VS & CCC & 4 & 5 & F25 & 15.4 & 15.4 & 5.59 & 16.6 & 76 & 82.3 & 25.67 & 25.67 & 9.32 & 27.67 & 126.67 & 137.17 \\
\hline 1 & HTLG-58VS & quenched & 4 & 6 & F88 & 9.25 & 10.4 & 4.27 & 8.27 & 85.5 & 69.8 & 15.42 & 17.33 & 7.12 & 13.78 & 142.50 & 116.34 \\
\hline 1 & HTLG-66VS & quenched & 4 & 7 & F01 & 10.7 & 8.13 & 3.68 & 7.14 & 66 & 58.9 & 17.83 & 13.55 & 6.13 & 11.90 & 110.00 & 98.17 \\
\hline 1 & HTLG-62VS & quenched & 4 & 8 & F91 & 9.89 & 8.75 & 3.89 & 7.48 & 64 & 61.9 & 16.48 & 14.58 & 6.48 & 12.47 & 106.67 & 103.17 \\
\hline 1 & HTLG-59VS & quenched & 4 & 9 & F75 & 8.49 & 10.1 & 4.53 & 8.47 & 63.7 & 64.1 & 14.15 & 16.83 & 7.55 & 14.12 & 106.17 & 106.84 \\
\hline 1 & Std Soln & & 4 & 10 & STD-14-2 & 3.5 & 20.3 & 4.08 & 9.81 & 83.1 & 50.2 & 3.50 & 20.30 & 4.08 & 9.81 & 83.10 & 50.20 \\
\hline 1 & HTLG-62VS & CCC & 4 & 11 & F84 & 8.17 & 20.6 & 4.39 & 17.7 & 104 & 105 & 13.62 & 34.33 & 7.32 & 29.50 & 173.34 & 175.00 \\
\hline 1 & ARM-1 & & 4 & 12 & F58 & 2.43 & 10.1 & $<1.00$ & 7.97 & 24 & 36 & 4.05 & 16.83 & 0.83 & 13.28 & 40.00 & 60.00 \\
\hline 1 & HTLG-63VS & quenched & 4 & 13 & F63 & 11.3 & 8.21 & 5.08 & 7.39 & 54.2 & 60.4 & 18.83 & 13.68 & 8.47 & 12.32 & 90.34 & 100.67 \\
\hline 1 & HTLG-55VS & CCC & 4 & 14 & F08 & 9.88 & 55.8 & 8.19 & 43.7 & 195 & 165 & 16.47 & 93.00 & 13.65 & 72.83 & 325.01 & 275.01 \\
\hline 1 & HTLG-66VS & CCC & 4 & 15 & F52 & 11.7 & 7.38 & 3.33 & 8.5 & 62.9 & 61.9 & 19.50 & 12.30 & 5.55 & 14.17 & 104.84 & 103.17 \\
\hline 1 & HTLG-55VS & quenched & 4 & 16 & F20 & 11.8 & 8.31 & 3.29 & 7.13 & 65.8 & 58.5 & 19.67 & 13.85 & 5.48 & 11.88 & 109.67 & 97.50 \\
\hline 1 & HTLG-54VS & quenched & 4 & 17 & F05 & 10.7 & 10.9 & 5.01 & 8.63 & 82 & 72.7 & 17.83 & 18.17 & 8.35 & 14.38 & 136.67 & 121.17 \\
\hline 1 & blank & & 4 & 18 & F93 & $<1.00$ & $<1.00$ & $<1.00$ & $<1.00$ & $<1.00$ & $<1.00$ & 0.83 & 0.83 & 0.83 & 0.83 & 0.83 & 0.83 \\
\hline 1 & Std Soln & & 4 & 19 & STD-14-3 & 3.45 & 20.6 & 4.24 & 9.86 & 82.2 & 51.5 & 3.45 & 20.60 & 4.24 & 9.86 & 82.20 & 51.50 \\
\hline 1 & Std Soln & & 5 & 1 & STD-15-1 & 3.86 & 21 & 3.8 & 9.91 & 86.3 & 50.3 & 3.86 & 21.00 & 3.80 & 9.91 & 86.30 & 50.30 \\
\hline 1 & HTLG-54VS & CCC & 5 & 2 & F02 & 13.1 & 34.6 & 14.1 & 21.7 & 141 & 106 & 21.83 & 57.67 & 23.50 & 36.17 & 235.00 & 176.67 \\
\hline 1 & HTLG-62VS & CCC & 5 & 3 & F27 & 8.05 & 20 & 3.9 & 17.11 & 101 & 99 & 13.42 & 33.33 & 6.50 & 28.52 & 168.34 & 165.00 \\
\hline
\end{tabular}


Table B1. PSAL Measurements of the PCT Solutions for the HTL Study Glasses As Reported (ar) and After the Appropriate Adjustments (in parts per million, ppm)

\begin{tabular}{|c|c|c|c|c|c|c|c|c|c|c|c|c|c|c|c|c|c|}
\hline Set & Glass ID & $\begin{array}{c}\text { Heat } \\
\text { Treatment }\end{array}$ & Block & Seq & $\begin{array}{c}\text { SRTC-ML } \\
\text { ID }\end{array}$ & $\mathrm{Al}$ ar & $\mathrm{B}$ ar & $\mathrm{Fe}$ ar & $\mathrm{Li}$ ar & $\mathrm{Na}$ ar & Si ar & $\begin{array}{c}\mathrm{Al} \\
(\mathrm{ppm})\end{array}$ & $\begin{array}{c}\mathrm{B} \\
(\mathrm{ppm})\end{array}$ & $\begin{array}{c}\mathrm{Fe} \\
(\mathrm{ppm})\end{array}$ & $\begin{array}{c}\mathrm{Li} \\
(\mathrm{ppm})\end{array}$ & $\begin{array}{c}\mathrm{Na} \\
(\mathrm{ppm})\end{array}$ & $\begin{array}{c}\mathrm{Si} \\
(\mathrm{ppm})\end{array}$ \\
\hline 1 & HTLG-54VS & quenched & 5 & 4 & F70 & 9.78 & 10.9 & 5.13 & 8.56 & 81 & 71.2 & 16.30 & 18.17 & 8.55 & 14.27 & 135.00 & 118.67 \\
\hline 1 & HTLG-55VS & quenched & 5 & 5 & F79 & 11.4 & 8.55 & 3.09 & 7.18 & 66.5 & 57.3 & 19.00 & 14.25 & 5.15 & 11.97 & 110.84 & 95.50 \\
\hline 1 & HTLG-59VS & CCC & 5 & 6 & F30 & 15.6 & 15.2 & 5.17 & 16.5 & 74.6 & 80 & 26.00 & 25.33 & 8.62 & 27.50 & 124.34 & 133.34 \\
\hline 1 & HTLG-62VS & quenched & 5 & 7 & F51 & 10.5 & 9.65 & 2.67 & 8 & 69.4 & 64.3 & 17.50 & 16.08 & 4.45 & 13.33 & 115.67 & 107.17 \\
\hline 1 & HTLG-59VS & quenched & 5 & 8 & F55 & 8.33 & 9.26 & 3.51 & 8.22 & 58.8 & 59.6 & 13.88 & 15.43 & 5.85 & 13.70 & 98.00 & 99.34 \\
\hline 1 & HTLG-58VS & CCC & 5 & 9 & F38 & 10.4 & 7.1 & 3.76 & 7.41 & 70.3 & 64.3 & 17.33 & 11.83 & 6.27 & 12.35 & 117.17 & 107.17 \\
\hline 1 & Std Soln & & 5 & 10 & STD-15-2 & 3.93 & 19.8 & 3.56 & 9.69 & 85.3 & $\begin{array}{lll}48.4 \\
\end{array}$ & 3.93 & 19.80 & 3.56 & 9.69 & 85.30 & 48.40 \\
\hline 1 & HTLG-63VS & CCC & 5 & 11 & F29 & 12.9 & 6.25 & 2.65 & 6.53 & 49.2 & 55.8 & 21.50 & 10.42 & 4.42 & 10.88 & 82.00 & 93.00 \\
\hline 1 & HTLG-63VS & quenched & 5 & 12 & F87 & 11 & 8.06 & 4.16 & 7.26 & 56.1 & 56.5 & 18.33 & 13.43 & 6.93 & 12.10 & 93.50 & 94.17 \\
\hline 1 & HTLG-66VS & CCC & 5 & 13 & F48 & 11.4 & 7.3 & 1.85 & 8.74 & 61.8 & 58.7 & 19.00 & 12.17 & 3.08 & 14.57 & 103.00 & 97.84 \\
\hline 1 & HTLG-55VS & CCC & 5 & 14 & F72 & 10.4 & 54.4 & 7.72 & 43.9 & 202 & 157 & 17.33 & 90.67 & 12.87 & 73.17 & 336.67 & 261.67 \\
\hline 1 & HTLG-66VS & quenched & 5 & 15 & F78 & 10.6 & 8.31 & 2.93 & 7.08 & 64.2 & 56 & 17.67 & 13.85 & 4.88 & 11.80 & 107.00 & 93.34 \\
\hline 1 & ARM-1 & & 5 & 16 & F66 & 2.51 & 9.97 & $<1.00$ & 7.98 & 22 & 35.4 & 4.18 & 16.62 & 0.83 & 13.30 & 36.67 & 59.00 \\
\hline 1 & HTLG-58VS & quenched & 5 & 17 & F44 & 9.42 & 10.2 & 3.59 & 8.16 & 79.3 & 67.5 & 15.70 & 17.00 & 5.98 & 13.60 & 132.17 & 112.50 \\
\hline 1 & Std Soln & & 5 & 18 & STD-15-3 & 3.8 & 19.3 & 3.52 & 9.62 & 83 & 47.6 & 3.80 & 19.30 & 3.52 & 9.62 & 83.00 & 47.60 \\
\hline 1 & Std Soln & & 6 & 1 & STD-16-1 & 3.74 & 20.8 & 3.39 & 9.7 & 80.2 & 50.2 & 3.74 & 20.80 & 3.39 & 9.70 & 80.20 & 50.20 \\
\hline 1 & HTLG-66VS & CCC & 6 & 2 & F62 & 11.3 & 7.32 & 1.58 & 8.74 & 58.4 & 61.7 & 18.83 & 12.20 & 2.63 & 14.57 & 97.34 & 102.84 \\
\hline 1 & HTLG-63VS & CCC & 6 & 3 & F71 & 12.5 & 6.08 & 2.4 & 6.49 & 61.2 & 57.5 & 20.83 & 10.13 & 4.00 & 10.82 & 102.00 & 95.84 \\
\hline 1 & ARM-1 & & 6 & 4 & F76 & 2.43 & 10.7 & $<1.00$ & 8.33 & 21.9 & 37 & 4.05 & 17.83 & 0.83 & 13.88 & 36.50 & 61.67 \\
\hline 1 & HTLG-62VS & quenched & 6 & 5 & F24 & 10.2 & 9.49 & 2.1 & 7.95 & 67.8 & 65.7 & 17.00 & 15.82 & 3.50 & 13.25 & 113.00 & 109.50 \\
\hline 1 & HTLG-62VS & CCC & 6 & 6 & F22 & 7.83 & 18.5 & 3.14 & 16.6 & 97.5 & 96.8 & 13.05 & 30.83 & 5.23 & 27.67 & 162.50 & 161.34 \\
\hline 1 & HTLG-63VS & quenched & 6 & 7 & F53 & 11 & 8.22 & 4.06 & 7.22 & 52.6 & 58.3 & 18.33 & 13.70 & 6.77 & 12.03 & 87.67 & 97.17 \\
\hline 1 & HTLG-66VS & quenched & 6 & 8 & F60 & 10.6 & 7.98 & 2.77 & 7.04 & 60.8 & 58.4 & 17.67 & 13.30 & 4.62 & 11.73 & 101.34 & 97.34 \\
\hline 1 & HTLG-54VS & CCC & 6 & 9 & F69 & 13.1 & 34.1 & 14.6 & 21.4 & 138 & 106 & 21.83 & 56.83 & 24.33 & 35.67 & 230.00 & 176.67 \\
\hline 1 & Std Soln & & 6 & 10 & STD-16-2 & 3.65 & 19.2 & 3.23 & 9.46 & 78.9 & 48.8 & 3.65 & 19.20 & 3.23 & 9.46 & 78.90 & 48.80 \\
\hline 1 & HTLG-55VS & CCC & 6 & 11 & F32 & 10.5 & 57.3 & 7.8 & 44.7 & 199 & 166 & 17.50 & 95.50 & 13.00 & 74.50 & 331.67 & 276.67 \\
\hline 1 & HTLG-59VS & quenched & 6 & 12 & F13 & 8.83 & 9.62 & 3.93 & 8.21 & 58.4 & 62.1 & 14.72 & 16.03 & 6.55 & 13.68 & 97.34 & 103.50 \\
\hline 1 & HTLG-58VS & quenched & 6 & 13 & F77 & 9.22 & 10.5 & 3.62 & 8.25 & 75.9 & 71 & 15.37 & 17.50 & 6.03 & 13.75 & 126.50 & 118.34 \\
\hline 1 & HTLG-58VS & CCC & 6 & 14 & F39 & 10.4 & 6.84 & 3.2 & 7.31 & 67.4 & 64.8 & 17.33 & 11.40 & 5.33 & 12.18 & 112.34 & 108.00 \\
\hline 1 & HTLG-54VS & quenched & 6 & 15 & F86 & 8.36 & 9.9 & 3.51 & 7.86 & 74.3 & 66.6 & 13.93 & 16.50 & 5.85 & 13.10 & 123.84 & 111.00 \\
\hline 1 & blank & & 6 & 16 & F34 & $<1.00$ & $<1.00$ & $<1.00$ & $<1.00$ & $<1.00$ & $<1.00$ & 0.83 & 0.83 & 0.83 & 0.83 & 0.83 & 0.83 \\
\hline 1 & HTLG-55VS & quenched & 6 & 17 & F04 & 11.6 & 7.94 & 2.85 & 7.1 & 67.3 & 57.3 & 19.33 & 13.23 & 4.75 & 11.83 & 112.17 & 95.50 \\
\hline 1 & HTLG-59VS & CCC & 6 & 18 & F11 & 15.7 & 14.8 & 4.84 & 16.2 & 73.6 & 81.1 & 26.17 & 24.67 & 8.07 & 27.00 & 122.67 & 135.17 \\
\hline 1 & $\begin{array}{l}\text { Std Soln } \\
\end{array}$ & & 6 & 19 & $\begin{array}{l}\text { STD-16-3 } \\
\end{array}$ & 3.74 & 19.4 & 3.27 & 9.56 & 80.6 & 49.1 & 3.74 & 19.40 & 3.27 & 9.56 & 80.60 & 49.10 \\
\hline 2 & Std Soln & & 1 & 1 & STD-21-1 & 4.08 & 21.5 & 4.19 & 10.2 & 79.5 & 51.2 & 4.08 & 21.50 & 4.19 & 10.20 & 79.50 & 51.20 \\
\hline 2 & HTLG-79VS & quenched & 1 & 2 & G07 & 11.3 & 11.3 & 4.2 & 8.95 & 81.7 & 70 & 18.83 & 18.83 & 7.00 & 14.92 & 136.17 & 116.67 \\
\hline 2 & HTLG-74VS & CCC & 1 & 3 & G31 & 12 & 7.4 & 3.59 & 7.03 & 60.8 & 57.1 & 20.00 & 12.33 & 5.98 & 11.72 & 101.34 & 95.17 \\
\hline 2 & blank & & 1 & 4 & G23 & $<1.00$ & $<1.00$ & $<1.00$ & $<1.00$ & $<1.00$ & $<1.00$ & 0.83 & 0.83 & 0.83 & 0.83 & 0.83 & 0.83 \\
\hline 2 & HTLG-74VS & quenched & 1 & 5 & G17 & 12.6 & 8.8 & 3.66 & 7.36 & 67.8 & 58.9 & 21.00 & 14.67 & 6.10 & 12.27 & 113.00 & 98.17 \\
\hline
\end{tabular}


Table B1. PSAL Measurements of the PCT Solutions for the HTL Study Glasses As Reported (ar) and After the Appropriate Adjustments (in parts per million, ppm)

\begin{tabular}{|c|c|c|c|c|c|c|c|c|c|c|c|c|c|c|c|c|c|}
\hline Set & Glass ID & $\begin{array}{c}\text { Heat } \\
\text { Treatment }\end{array}$ & Block & Seq & $\begin{array}{c}\text { SRTC-ML } \\
\text { ID }\end{array}$ & $\mathrm{Al} \mathrm{ar}$ & $\mathrm{B}$ ar & $\mathrm{Fe}$ ar & $\mathrm{Li}$ ar & $\mathrm{Na}$ ar & Si ar & $\begin{array}{c}\mathrm{Al} \\
\text { (ppm) }\end{array}$ & $\begin{array}{c}\mathrm{B} \\
(\mathrm{ppm})\end{array}$ & $\begin{array}{c}\mathrm{Fe} \\
(\mathrm{ppm})\end{array}$ & $\begin{array}{c}\mathrm{Li} \\
(\mathrm{ppm})\end{array}$ & $\begin{array}{c}\mathrm{Na} \\
(\mathrm{ppm})\end{array}$ & $\begin{array}{c}\mathrm{Si} \\
(\mathrm{ppm})\end{array}$ \\
\hline 2 & HTLG-73VS & CCC & 1 & 6 & G62 & 10.1 & 31.5 & 7.37 & 31.2 & 140 & 143 & 16.83 & 52.50 & 12.28 & 52.00 & 233.34 & 238.34 \\
\hline 2 & EA & & 1 & 7 & G73 & $<1.00$ & 39.8 & $<1.00$ & 11.9 & 101 & 56.1 & 8.33 & 663.33 & 8.33 & 198.33 & 1683.34 & 935.00 \\
\hline 2 & HTLG-79VS & CCC & 1 & 8 & G44 & 11.6 & 11.2 & 3.56 & 10.9 & 77.3 & 71.6 & 19.33 & 18.67 & 5.93 & 18.17 & 128.84 & 119.34 \\
\hline 2 & HTLG-72VS & quenched & 1 & 9 & G64 & 13.1 & 8.61 & 6.41 & 7.25 & 57.8 & 59.3 & 21.83 & 14.35 & 10.68 & 12.08 & 96.34 & 98.84 \\
\hline 2 & Std Soln & & 1 & 10 & STD-21-2 & 4.07 & 20.9 & 4.11 & 10.3 & 81.3 & 51.6 & 4.07 & 20.90 & 4.11 & 10.30 & 81.30 & 51.60 \\
\hline 2 & HTLG-72VS & CCC & 1 & 11 & G16 & 10.7 & 6.12 & 1.59 & 5.52 & 46 & 49.9 & 17.83 & 10.20 & 2.65 & 9.20 & 76.67 & 83.17 \\
\hline 2 & HTLG-71VS & quenched & 1 & 12 & G29 & 12.2 & 9.78 & 4.16 & 7.9 & 71.5 & 62.4 & 20.33 & 16.30 & 6.93 & 13.17 & 119.17 & 104.00 \\
\hline 2 & HTLG-70VS & quenched & 1 & 13 & G66 & 10.9 & 11.9 & 4.11 & 9.26 & 81.7 & 73.4 & 18.17 & 19.83 & 6.85 & 15.43 & 136.17 & 122.34 \\
\hline 2 & HTLG-70VS & CCC & 1 & 14 & G35 & 11.7 & 25.1 & 6.69 & 19.1 & 106 & 102 & 19.50 & 41.83 & 11.15 & 31.83 & 176.67 & 170.00 \\
\hline 2 & HTLG-71VS & CCC & 1 & 15 & G28 & 4.5 & 68.9 & 1.5 & 50.3 & 315 & 225 & 7.50 & 114.84 & 2.50 & 83.84 & 525.01 & 375.01 \\
\hline 2 & HTLG-73VS & quenched & 1 & 16 & G09 & 11.4 & 11.6 & 2.4 & 8.61 & 79.8 & 66.6 & 19.00 & 19.33 & 4.00 & 14.35 & 133.00 & 111.00 \\
\hline 2 & HTLG-75VS & quenched & 1 & 17 & G04 & 11.3 & 10.2 & 3.86 & 7.67 & 72.4 & 64 & 18.83 & 17.00 & 6.43 & 12.78 & 120.67 & 106.67 \\
\hline 2 & HTLG-75VS & CCC & 1 & 18 & G71 & 11 & 6.91 & 2.51 & 6.28 & 61 & 58.4 & 18.33 & 11.52 & 4.18 & 10.47 & 101.67 & 97.34 \\
\hline 2 & Std Soln & & 1 & 19 & STD-21-3 & 4.21 & 21.6 & 4.36 & 10.5 & 83.5 & 52.9 & 4.21 & 21.60 & 4.36 & 10.50 & 83.50 & 52.90 \\
\hline 2 & Std Soln & & 2 & 1 & STD-22-1 & 4.18 & 21.6 & 4.3 & 10.3 & 83.6 & 50.5 & 4.18 & 21.60 & 4.30 & 10.30 & 83.60 & 50.50 \\
\hline 2 & HTLG-70VS & CCC & 2 & 2 & G81 & 11.6 & 25 & 6.6 & 19 & 107 & 98 & 19.33 & 41.67 & 11.00 & 31.67 & 178.34 & 163.34 \\
\hline 2 & HTLG-72VS & quenched & 2 & 3 & G80 & 13.1 & 8.57 & 6.21 & 7.28 & 58.9 & 57.6 & 21.83 & 14.28 & 10.35 & 12.13 & 98.17 & 96.00 \\
\hline 2 & HTLG-73VS & CCC & 2 & 4 & G53 & 10.1 & 31.3 & 6.65 & 31.4 & 147 & 138 & 16.83 & 52.17 & 11.08 & 52.33 & 245.00 & 230.00 \\
\hline 2 & EA & & 2 & 5 & G01 & $<1.00$ & 38.2 & $<1.00$ & 12 & 102 & 54.2 & 8.33 & 636.67 & 8.33 & 200.00 & 1700.00 & 903.34 \\
\hline 2 & HTLG-79VS & CCC & 2 & 6 & G30 & 11.8 & 10.9 & 3.58 & 10.8 & 80.4 & 67.7 & 19.67 & 18.17 & 5.97 & 18.00 & 134.00 & 112.84 \\
\hline 2 & HTLG-71VS & CCC & 2 & 7 & G48 & 4.31 & 66.7 & 1.28 & 49.1 & 313 & 227 & 7.18 & 111.17 & 2.13 & 81.83 & 521.68 & 378.34 \\
\hline 2 & HTLG-73VS & quenched & 2 & 8 & G85 & 11 & 10.2 & 3.44 & 8.04 & 73.7 & 59.6 & 18.33 & 17.00 & 5.73 & 13.40 & 122.84 & 99.34 \\
\hline 2 & HTLG-79VS & quenched & 2 & 9 & G43 & 11.1 & 11 & 3.94 & 8.54 & 82.6 & 63.1 & 18.50 & 18.33 & 6.57 & 14.23 & 137.67 & 105.17 \\
\hline 2 & $\begin{array}{l}\text { Std Soln } \\
\end{array}$ & & 2 & 10 & STD-22-2 & 4.18 & 20.4 & 4.24 & 10.2 & 84.6 & 49.2 & 4.18 & 20.40 & 4.24 & 10.20 & 84.60 & 49.20 \\
\hline 2 & HTLG-74VS & quenched & 2 & 11 & G51 & 12.1 & 8.42 & 3.51 & 7.13 & $\begin{array}{ll}68.8 \\
\end{array}$ & 52.9 & 20.17 & 14.03 & 5.85 & 11.88 & 114.67 & 88.17 \\
\hline 2 & HTLG-74VS & CCC & 2 & 12 & G68 & 12.4 & 7.39 & 3.62 & 7.25 & 63.3 & 56.1 & 20.67 & 12.32 & 6.03 & 12.08 & 105.50 & 93.50 \\
\hline 2 & HTLG-72VS & CCC & 2 & 13 & G82 & 10.8 & 5.48 & 1.79 & 5.59 & 47.3 & 48.5 & 18.00 & 9.13 & 2.98 & 9.32 & 78.83 & 80.83 \\
\hline 2 & HTLG-71VS & quenched & 2 & 14 & G03 & 12.3 & 9.46 & 4.13 & 8.01 & 74.6 & 60.8 & 20.50 & 15.77 & 6.88 & 13.35 & 124.34 & 101.34 \\
\hline 2 & HTLG-75VS & CCC & 2 & 15 & G50 & 11.2 & 6.53 & 2.25 & 6.65 & 65.2 & 57.8 & 18.67 & 10.88 & 3.75 & 11.08 & 108.67 & 96.34 \\
\hline 2 & HTLG-70VS & quenched & 2 & 16 & G61 & 10.1 & 10.1 & 3.89 & 8.53 & 76.9 & 63.3 & 16.83 & 16.83 & 6.48 & 14.22 & 128.17 & 105.50 \\
\hline 2 & HTLG-75VS & quenched & 2 & 17 & G67 & 11.4 & 9.18 & 3.74 & 7.73 & 76.9 & 60.1 & 19.00 & 15.30 & 6.23 & 12.88 & 128.17 & 100.17 \\
\hline 2 & Std Soln & & 2 & 18 & STD-22-3 & 4.12 & 19.4 & 3.94 & 10 & 84.7 & 46.9 & 4.12 & 19.40 & 3.94 & 10.00 & 84.70 & 46.90 \\
\hline 2 & $\begin{array}{l}\text { Std Soln } \\
\end{array}$ & & 3 & 1 & STD-23-1 & 4.02 & 20.7 & 4.2 & 10.1 & 81.1 & 49.4 & 4.02 & 20.70 & 4.20 & 10.10 & 81.10 & 49.40 \\
\hline 2 & HTLG-79VS & CCC & 3 & 2 & G22 & 11.6 & 11.4 & 3.71 & 10.9 & 76.8 & 71 & 19.33 & 19.00 & 6.18 & 18.17 & 128.00 & 118.34 \\
\hline 2 & HTLG-70VS & CCC & 3 & 3 & G83 & 11.6 & 24.8 & 6.4 & 18.9 & 107 & 99.4 & 19.33 & 41.33 & 10.67 & 31.50 & 178.34 & 165.67 \\
\hline 2 & HTLG-72VS & CCC & 3 & 4 & G20 & 11.3 & 6.06 & 2.02 & 5.82 & 48.8 & 51.8 & 18.83 & 10.10 & 3.37 & 9.70 & 81.33 & 86.34 \\
\hline 2 & HTLG-70VS & quenched & 3 & 5 & G63 & 9.8 & 10.2 & 3.83 & 8.35 & 72.1 & 63.8 & 16.33 & 17.00 & 6.38 & 13.92 & 120.17 & 106.34 \\
\hline 2 & HTLG-71VS & quenched & 3 & 6 & G25 & 12.6 & 10.3 & 3.78 & 8.42 & 75.7 & 65.3 & 21.00 & 17.17 & 6.30 & 14.03 & 126.17 & 108.84 \\
\hline 2 & EA & & 3 & 7 & G72 & $<1.00$ & 37.4 & $<1.00$ & 11.8 & 105 & 53.9 & 8.33 & 623.33 & 8.33 & 196.67 & 1750.00 & 898.34 \\
\hline
\end{tabular}


Table B1. PSAL Measurements of the PCT Solutions for the HTL Study Glasses As Reported (ar) and After the Appropriate Adjustments (in parts per million, ppm)

\begin{tabular}{|c|c|c|c|c|c|c|c|c|c|c|c|c|c|c|c|c|c|}
\hline Set & Glass ID & $\begin{array}{c}\text { Heat } \\
\text { Treatment }\end{array}$ & Block & Seq & $\begin{array}{c}\text { SRTC-ML } \\
\text { ID } \\
\end{array}$ & $\mathrm{Al}$ ar & $\mathrm{B}$ ar & Fe ar & $\mathrm{Li}$ ar & $\mathrm{Na}$ ar & Si ar & $\begin{array}{c}\mathrm{Al} \\
(\mathrm{ppm})\end{array}$ & $\begin{array}{c}\mathrm{B} \\
(\mathrm{ppm})\end{array}$ & $\begin{array}{c}\mathrm{Fe} \\
(\mathrm{ppm})\end{array}$ & $\begin{array}{c}\mathrm{Li} \\
(\mathrm{ppm})\end{array}$ & $\begin{array}{c}\mathrm{Na} \\
(\mathrm{ppm})\end{array}$ & $\begin{array}{c}\mathrm{Si} \\
(\mathrm{ppm})\end{array}$ \\
\hline 2 & HTLG-75VS & CCC & 3 & 8 & G54 & 11 & 6.65 & 2.52 & 6.47 & 63.6 & 57.9 & 18.33 & 11.08 & 4.20 & 10.78 & 106.00 & 96.50 \\
\hline 2 & HTLG-72VS & quenched & 3 & 9 & G15 & 13 & 8.05 & 6.31 & 7.21 & 59.8 & 57.6 & 21.67 & 13.42 & 10.52 & 12.02 & 99.67 & 96.00 \\
\hline 2 & Std Soln & & 3 & 10 & STD-23-2 & 3.99 & 19.6 & 4.18 & 9.94 & 81.2 & 49 & 3.99 & 19.60 & 4.18 & 9.94 & 81.20 & 49.00 \\
\hline 2 & HTLG-75VS & quenched & 3 & 11 & G45 & 10.9 & 9.54 & 3.41 & 7.62 & 72.9 & 61.8 & 18.17 & 15.90 & 5.68 & 12.70 & 121.50 & 103.00 \\
\hline 2 & HTLG-74VS & CCC & 3 & 12 & G46 & 11.6 & 7.02 & 3.74 & 6.97 & 59.2 & 56.2 & 19.33 & 11.70 & 6.23 & 11.62 & 98.67 & 93.67 \\
\hline 2 & HTLG-79VS & quenched & 3 & 13 & G58 & 11.2 & 10.6 & 3.74 & 8.69 & 83.4 & 65.6 & 18.67 & 17.67 & 6.23 & 14.48 & 139.00 & 109.34 \\
\hline 2 & HTLG-73VS & quenched & 3 & 14 & G13 & 11.2 & 9.48 & 3.47 & 8.19 & 75.1 & 62.2 & 18.67 & 15.80 & 5.78 & 13.65 & 125.17 & 103.67 \\
\hline 2 & HTLG-74VS & quenched & 3 & 15 & G57 & 13 & 8.6 & 3.69 & 7.56 & 73.9 & 59.7 & 21.67 & 14.33 & 6.15 & 12.60 & 123.17 & 99.50 \\
\hline 2 & HTLG-73VS & $\mathrm{CCC}$ & 3 & 16 & G49 & 10.3 & 31.1 & 6.78 & 31.9 & 152 & 145 & 17.17 & 51.83 & 11.30 & 53.17 & 253.34 & 241.67 \\
\hline 2 & HTLG-71VS & CCC & 3 & 17 & G76 & 4.11 & 64.6 & 1.06 & 49 & 301 & 199 & 6.85 & 107.67 & 1.77 & 81.67 & 501.68 & 331.67 \\
\hline 2 & Std Soln & & 3 & 18 & STD-23-3 & 4.11 & 20.7 & 4.26 & 10.3 & 86 & 50.3 & 4.11 & 20.70 & 4.26 & 10.30 & 86.00 & 50.30 \\
\hline 2 & Std Soln & & 4 & 1 & STD-24-1 & 3.93 & 19.6 & 4.52 & 9.98 & 78.4 & 49.5 & 3.93 & 19.60 & 4.52 & 9.98 & 78.40 & 49.50 \\
\hline 2 & HTLG-68VS & quenched & 4 & 2 & G38 & 11.3 & 8.06 & 5.01 & 7.1 & 49.8 & 57.9 & 18.83 & 13.43 & 8.35 & 11.83 & 83.00 & 96.50 \\
\hline 2 & HTLG-77VS & CCC & 4 & 3 & G78 & 10.6 & 5.27 & 2.54 & 5.6 & 43.9 & 48.6 & 17.67 & 8.78 & 4.23 & 9.33 & 73.17 & 81.00 \\
\hline 2 & HTLG-69VS & CCC & 4 & 4 & G14 & 12.2 & 5.88 & 3.73 & 6.18 & 46.3 & 53.5 & 20.33 & 9.80 & 6.22 & 10.30 & 77.17 & 89.17 \\
\hline 2 & HTLG-67VS & CCC & 4 & 5 & G60 & 7.05 & 78.2 & 3.1 & 63.2 & 246 & 197 & 11.75 & 130.34 & 5.17 & 105.34 & 410.01 & 328.34 \\
\hline 2 & HTLG-76VS & CCC & 4 & 6 & G12 & 11.8 & 12.6 & 3.41 & 8.94 & 62.7 & 60 & 19.67 & 21.00 & 5.68 & 14.90 & 104.50 & 100.00 \\
\hline 2 & HTLG-68VS & CCC & 4 & 7 & G37 & 12.1 & 5.76 & 3.61 & 5.8 & 42.7 & 53.2 & 20.17 & 9.60 & 6.02 & 9.67 & 71.17 & 88.67 \\
\hline 2 & HTLG-78VS & quenched & 4 & 8 & G24 & 10.9 & 8.33 & 6.02 & 7.52 & 61.9 & 58 & 18.17 & 13.88 & 10.03 & 12.53 & 103.17 & 96.67 \\
\hline 2 & HTLG-69VS & quenched & 4 & 9 & G19 & 9.5 & 8.17 & 4.4 & 7.55 & 56.5 & 56.6 & 15.83 & 13.62 & 7.33 & 12.58 & 94.17 & 94.34 \\
\hline 2 & Std Soln & & 4 & 10 & STD-14-2 & 4.1 & 20 & 4.77 & 10.3 & 83.1 & 50.7 & 4.10 & 20.00 & 4.77 & 10.30 & 83.10 & 50.70 \\
\hline 2 & ARM-1 & & 4 & 11 & G36 & 2.98 & 10.2 & $<1.00$ & 8.11 & 21 & 35.8 & 4.97 & 17.00 & 0.83 & 13.52 & 35.00 & 59.67 \\
\hline 2 & HTLG-78VS & CCC & 4 & 12 & G77 & 10.4 & 6.07 & 2.41 & 6.88 & 52.8 & 53.6 & 17.33 & 10.12 & 4.02 & 11.47 & 88.00 & 89.34 \\
\hline 2 & HTLG-67VS & quenched & 4 & 13 & G84 & 10.1 & 7.8 & 3.03 & 7.2 & 53.2 & 53.4 & 16.83 & 13.00 & 5.05 & 12.00 & 88.67 & 89.00 \\
\hline 2 & HTLG-76VS & quenched & 4 & 14 & G40 & 11.4 & 7.91 & 4.32 & 7.16 & 57.5 & 54.6 & 19.00 & 13.18 & 7.20 & 11.93 & 95.84 & 91.00 \\
\hline 2 & HTLG-77VS & quenched & 4 & 15 & G52 & 12.9 & 7.53 & 4.83 & 7.18 & 57.8 & 57.9 & 21.50 & 12.55 & 8.05 & 11.97 & 96.34 & 96.50 \\
\hline 2 & Std Soln & & 4 & 16 & STD-24-3 & 4.06 & 20.2 & 4.26 & 10.3 & 82.5 & 50.7 & 4.06 & 20.20 & 4.26 & 10.30 & 82.50 & 50.70 \\
\hline 2 & Std Soln & & 5 & 1 & STD-25-1 & 4.03 & 20.4 & 4.22 & 10.1 & 83.4 & 49.2 & 4.03 & 20.40 & 4.22 & 10.10 & 83.40 & 49.20 \\
\hline 2 & HTLG-68VS & quenched & 5 & 2 & G02 & 11.2 & 8.05 & 4.46 & 6.98 & 49.9 & 54.3 & 18.67 & 13.42 & 7.43 & 11.63 & 83.17 & 90.50 \\
\hline 2 & HTLG-69VS & CCC & 5 & 3 & G69 & 11.7 & 5.89 & 3.34 & 5.81 & 44.6 & 49.1 & 19.50 & 9.82 & 5.57 & 9.68 & 74.33 & 81.83 \\
\hline 2 & HTLG-78VS & quenched & 5 & 4 & G47 & 11.2 & 8.59 & 5.56 & 7.65 & 64 & 57.6 & 18.67 & 14.32 & 9.27 & 12.75 & 106.67 & 96.00 \\
\hline 2 & HTLG-68VS & CCC & 5 & 5 & G55 & 12 & 5.32 & 3.13 & 5.64 & 43.2 & 50.2 & 20.00 & 8.87 & 5.22 & 9.40 & 72.00 & 83.67 \\
\hline 2 & ARM-1 & & 5 & 6 & G86 & 2.96 & 10.2 & $<1.00$ & 8.12 & 22.4 & 34.8 & 4.93 & 17.00 & 0.83 & 13.53 & 37.33 & 58.00 \\
\hline 2 & HTLG-76VS & CCC & 5 & 7 & G26 & 12.3 & 11.9 & 3.12 & 9.13 & 66.8 & 59.8 & 20.50 & 19.83 & 5.20 & 15.22 & 111.34 & 99.67 \\
\hline 2 & HTLG-67VS & quenched & 5 & 8 & G74 & 10.3 & 7.67 & 2.67 & 7.15 & 53.7 & 52.2 & 17.17 & 12.78 & 4.45 & 11.92 & 89.50 & 87.00 \\
\hline 2 & HTLG-67VS & CCC & 5 & 9 & G10 & 8.45 & 73.5 & 4.55 & 61.2 & 246 & 202 & 14.08 & 122.50 & 7.58 & 102.00 & 410.01 & 336.67 \\
\hline 2 & Std Soln & & 5 & 10 & STD-15-2 & 4.05 & 20.6 & 4.28 & 10.2 & 82.8 & 49 & 4.05 & 20.60 & 4.28 & 10.20 & 82.80 & 49.00 \\
\hline 2 & HTLG-77VS & quenched & 5 & 11 & G11 & 13.3 & 8.69 & 4.8 & 7.32 & 59.8 & 58.7 & 22.17 & 14.48 & 8.00 & 12.20 & 99.67 & 97.84 \\
\hline 2 & HTLG-77VS & CCC & 5 & 12 & G59 & 10.9 & 5.44 & 2.22 & 5.59 & 45.8 & 47.7 & 18.17 & 9.07 & 3.70 & 9.32 & 76.33 & 79.50 \\
\hline
\end{tabular}


Table B1. PSAL Measurements of the PCT Solutions for the HTL Study Glasses As Reported (ar) and After the Appropriate Adjustments (in parts per million, ppm)

\begin{tabular}{|c|c|c|c|c|c|c|c|c|c|c|c|c|c|c|c|c|c|}
\hline Set & Glass ID & $\begin{array}{c}\text { Heat } \\
\text { Treatment }\end{array}$ & Block & Seq & $\begin{array}{c}\text { SRTC-ML } \\
\text { ID }\end{array}$ & $\mathrm{Al}$ ar & $\mathrm{B}$ ar & $\mathrm{Fe}$ ar & Li ar & $\mathrm{Na}$ ar & Si ar & $\begin{array}{c}\mathrm{Al} \\
(\mathrm{ppm})\end{array}$ & $\begin{array}{c}\mathrm{B} \\
(\mathrm{ppm})\end{array}$ & $\begin{array}{c}\mathrm{Fe} \\
(\mathrm{ppm})\end{array}$ & $\begin{array}{c}\mathrm{Li} \\
(\mathrm{ppm})\end{array}$ & $\begin{array}{c}\mathrm{Na} \\
(\mathrm{ppm})\end{array}$ & $\begin{array}{c}\mathrm{Si} \\
(\mathrm{ppm})\end{array}$ \\
\hline 2 & HTLG-76VS & quenched & 5 & 13 & G27 & 11.5 & 7.81 & 3.63 & 7.1 & 68.3 & 51.9 & 19.17 & 13.02 & 6.05 & 11.83 & 113.84 & 86.50 \\
\hline 2 & $\begin{array}{l}\text { HTLG-69VS } \\
\end{array}$ & quenched & 5 & 14 & G41 & 9.56 & 8.05 & 3.77 & 7.45 & 58.1 & 54.5 & 15.93 & 13.42 & 6.28 & 12.42 & 96.84 & 90.84 \\
\hline 2 & HTLG-78VS & CCC & 5 & 15 & G65 & 10.5 & 5.81 & 2.29 & 6.79 & 55.5 & 51.4 & 17.50 & 9.68 & 3.82 & 11.32 & 92.50 & 85.67 \\
\hline 2 & Std Soln & & 5 & 16 & STD-25-3 & 4.15 & 19.5 & 4.27 & 10 & 83.2 & 48.2 & 4.15 & 19.50 & 4.27 & 10.00 & 83.20 & 48.20 \\
\hline 2 & Std Soln & & 6 & 1 & STD-26-1 & 4.16 & 21.7 & 4.55 & 10.3 & 81.9 & 50.8 & 4.16 & 21.70 & 4.55 & 10.30 & 81.90 & 50.80 \\
\hline 2 & HTLG-67VS & quenched & 6 & 2 & G18 & 9.78 & 8.3 & 2.58 & 6.93 & 49.9 & 51 & 16.30 & 13.83 & 4.30 & 11.55 & 83.17 & 85.00 \\
\hline 2 & HTLG-67VS & CCC & 6 & 3 & G32 & 8.04 & 75.1 & 4.21 & 61.3 & 239 & 206 & 13.40 & 125.17 & 7.02 & 102.17 & 398.34 & 343.34 \\
\hline 2 & blank & & 6 & 4 & G39 & $<1.00$ & 1.16 & $<1.00$ & $<1.00$ & $<1.00$ & $<1.00$ & 0.83 & 1.93 & 0.83 & 0.83 & 0.83 & 0.83 \\
\hline 2 & HTLG-78VS & quenched & 6 & 5 & G08 & 11.3 & 9.22 & 4.73 & 8 & 67.1 & 59.7 & 18.83 & 15.37 & $\begin{array}{l}7.88 \\
\end{array}$ & 13.33 & 111.84 & 99.50 \\
\hline 2 & HTLG-68VS & CCC & 6 & 6 & G21 & $\begin{array}{ll}11.8 \\
\end{array}$ & 5.68 & 3.12 & 5.68 & 43 & 50.9 & 19.67 & 9.47 & 5.20 & 9.47 & 71.67 & 84.84 \\
\hline 2 & $\begin{array}{l}\text { HTLG-77VS } \\
\end{array}$ & quenched & 6 & 7 & G42 & 13.4 & 7.77 & 5.84 & 7.05 & 58.9 & 57.4 & 22.33 & 12.95 & 9.73 & 11.75 & 98.17 & 95.67 \\
\hline 2 & $\begin{array}{l}\text { HTLG-69VS } \\
\end{array}$ & quenched & 6 & 8 & G34 & 9.72 & 8.43 & 4.27 & 7.52 & 56.4 & 55.8 & 16.20 & 14.05 & 7.12 & 12.53 & 94.00 & 93.00 \\
\hline 2 & $\begin{array}{l}\text { HTLG-69VS } \\
\end{array}$ & CCC & 6 & 9 & G70 & 12.6 & 5.96 & 3.51 & 6.19 & 48.6 & 51.7 & 21.00 & 9.93 & 5.85 & 10.32 & 81.00 & 86.17 \\
\hline 2 & Std Soln & & 6 & 10 & STD-26-2 & 3.92 & 20.4 & 4.59 & 10.3 & 82.6 & 50.1 & 3.92 & 20.40 & 4.59 & 10.30 & 82.60 & 50.10 \\
\hline 2 & HTLG-77VS & CCC & 6 & 11 & G56 & 11.3 & 5.84 & 2.57 & 5.81 & 45.8 & 50.4 & 18.83 & 9.73 & 4.28 & 9.68 & 76.33 & 84.00 \\
\hline 2 & HTLG-76VS & CCC & 6 & 12 & G05 & 13.4 & 13.1 & 4.72 & 9.71 & 68.8 & 65.8 & 22.33 & 21.83 & 7.87 & 16.18 & 114.67 & 109.67 \\
\hline 2 & ARM-1 & & 6 & 13 & G75 & 3.05 & 11.2 & $<1.00$ & 8.54 & 22.3 & 36.8 & 5.08 & 18.67 & 0.83 & 14.23 & 37.17 & 61.33 \\
\hline 2 & HTLG-76VS & quenched & 6 & 14 & G79 & 11.6 & 8.36 & 4.27 & 7.24 & 57.5 & 55.7 & 19.33 & 13.93 & 7.12 & 12.07 & 95.84 & 92.84 \\
\hline 2 & HTLG-68VS & quenched & 6 & 15 & G33 & 11.7 & 7.6 & 5.23 & 7.1 & 53.3 & 56.1 & 19.50 & 12.67 & 8.72 & 11.83 & 88.84 & 93.50 \\
\hline 2 & HTLG-78VS & $\mathrm{CCC}$ & 6 & 16 & G06 & 10.1 & 5.58 & 3.08 & 6.51 & 51.2 & 50.2 & 16.83 & 9.30 & 5.13 & 10.85 & 85.34 & 83.67 \\
\hline 2 & Std Soln & & 6 & 17 & STD-26-3 & 3.93 & 20.5 & 4.51 & 10.2 & 82.7 & 50.2 & 3.93 & 20.50 & 4.51 & 10.20 & 82.70 & 50.20 \\
\hline
\end{tabular}




\section{Exhibit B1. Laboratory PCT Measurements in Analytical Sequence for Quenched Study Glasses with and without EA, ARM, Blanks, and Solution Standards}
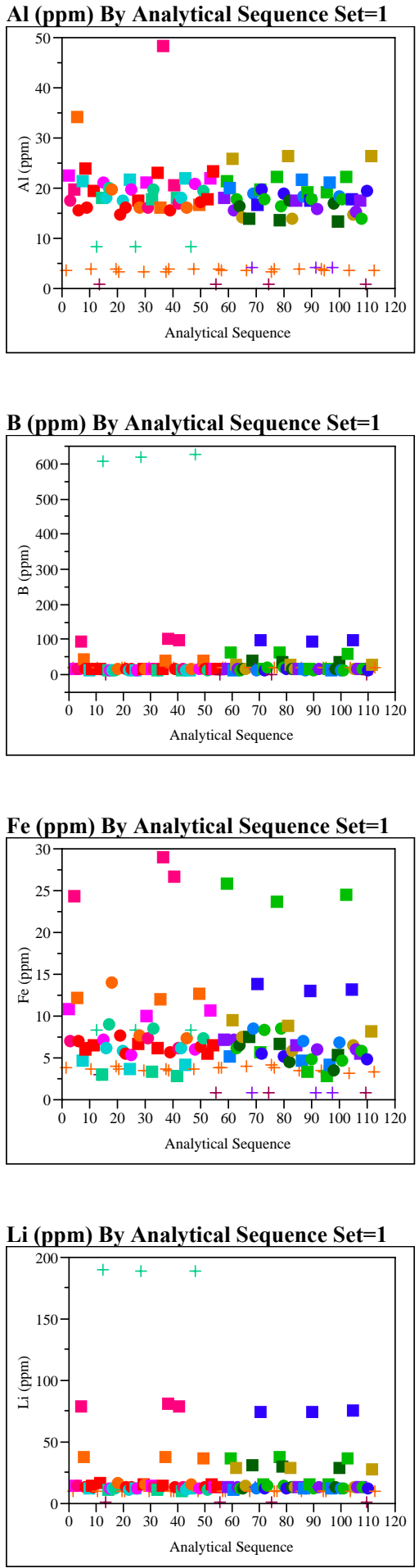
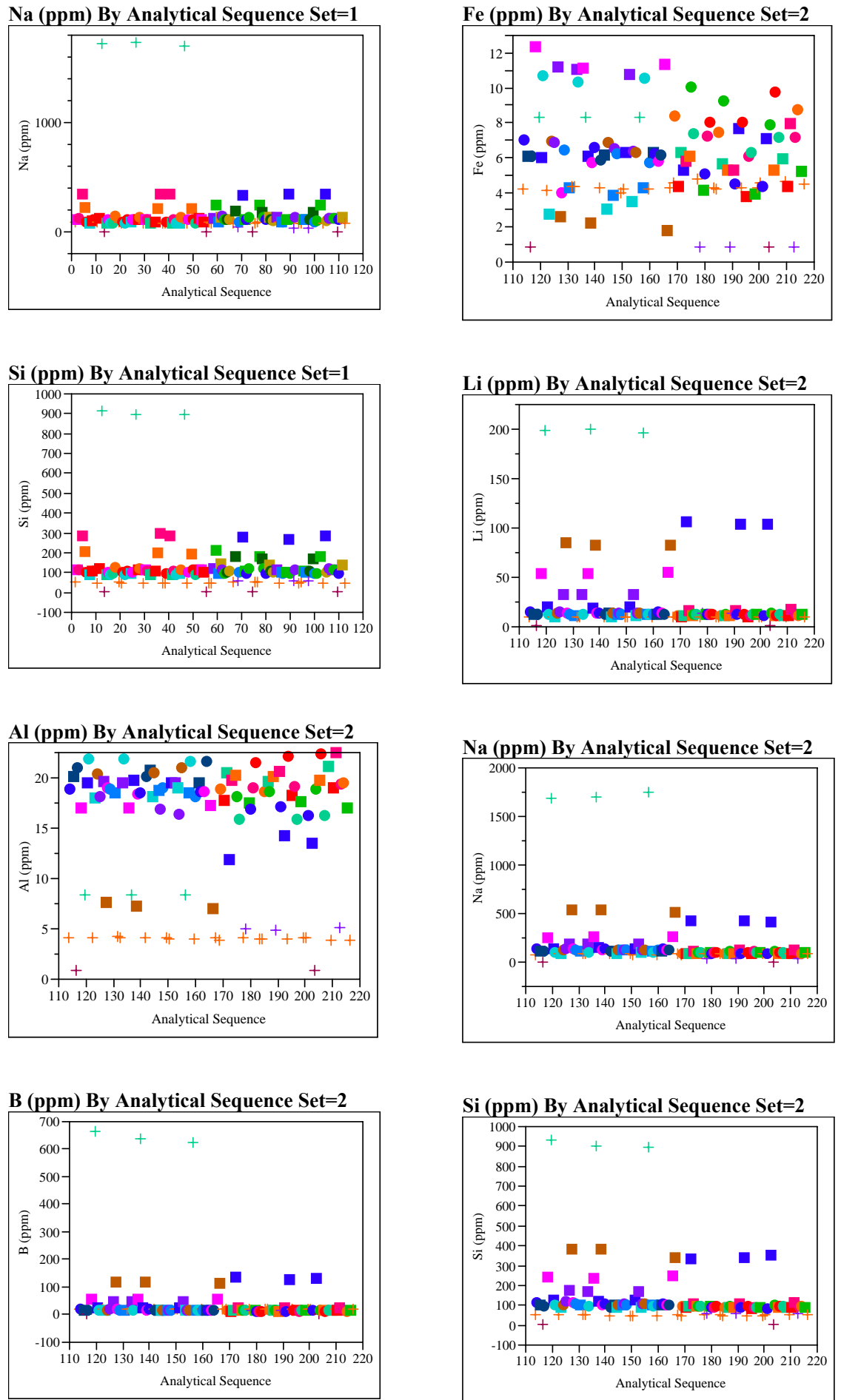

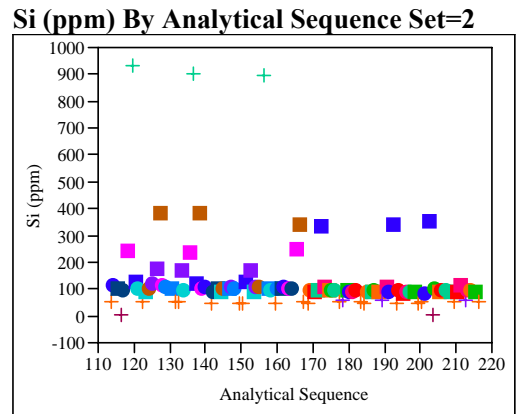




\section{Exhibit B2. Laboratory PCT Measurements in Analytical Sequence for CCC Study Glasses with and without EA, ARM, Blanks, and Solution Standards}
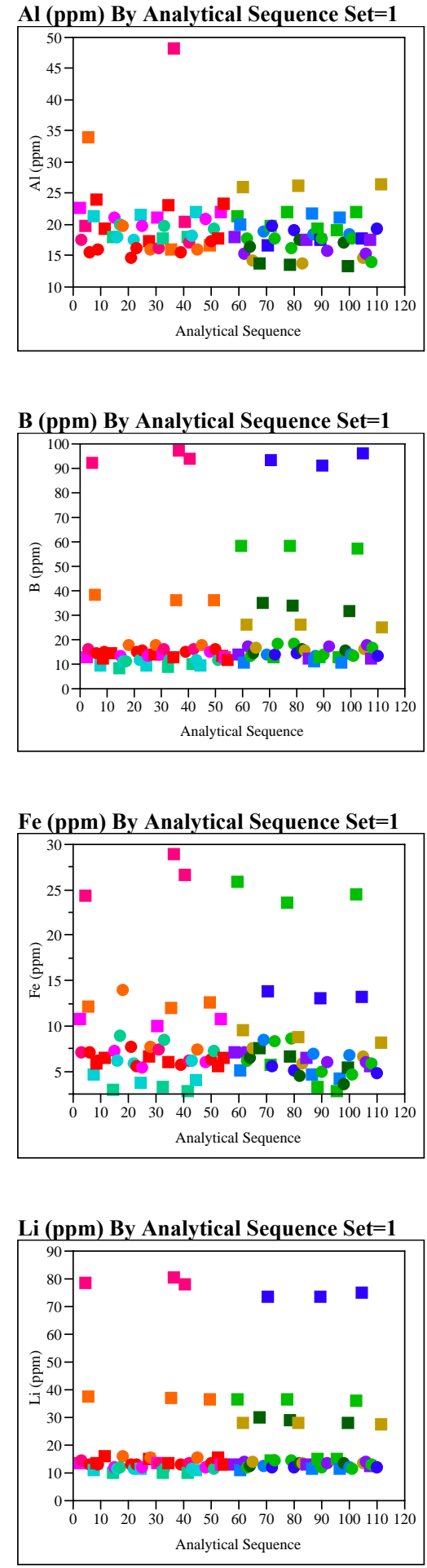
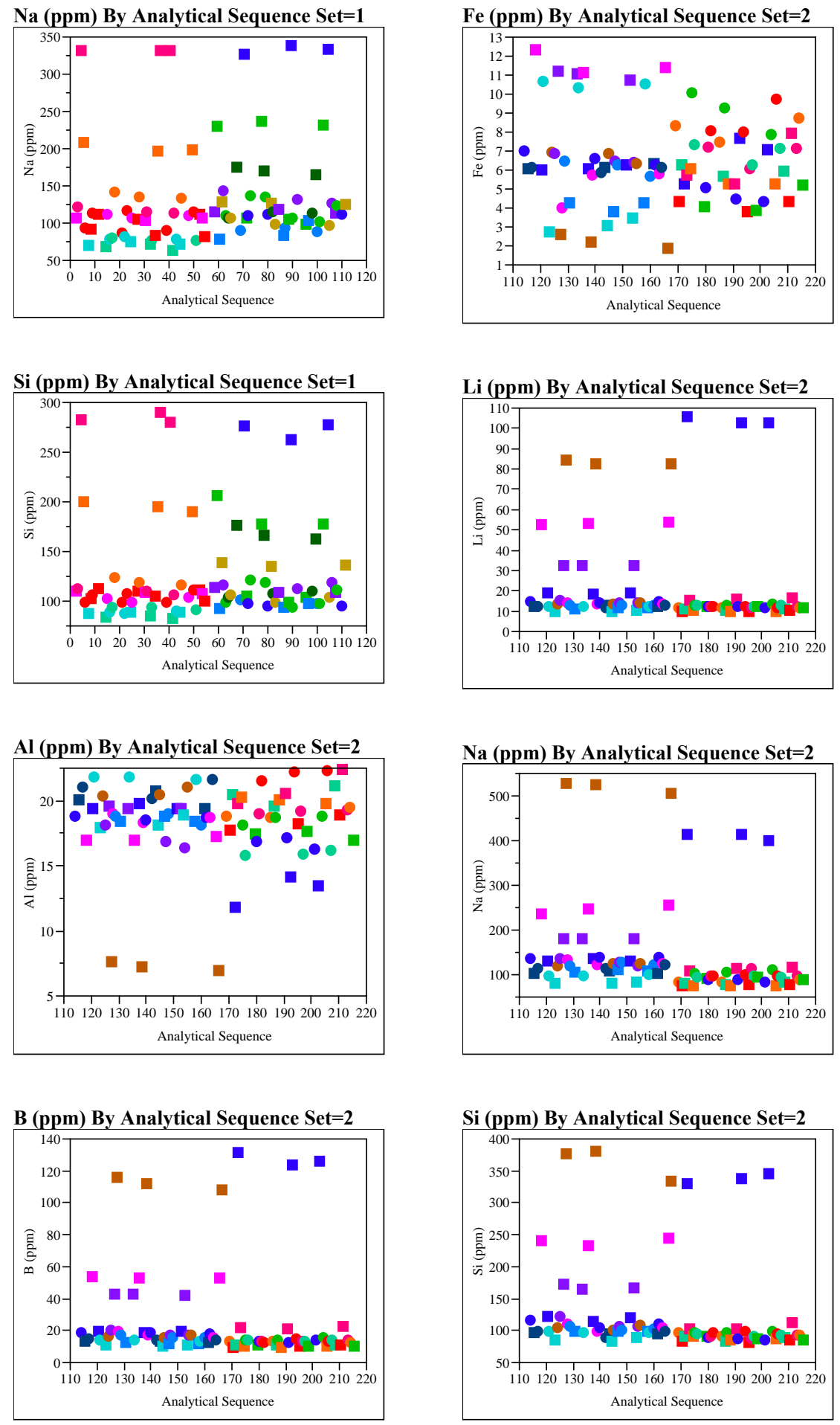

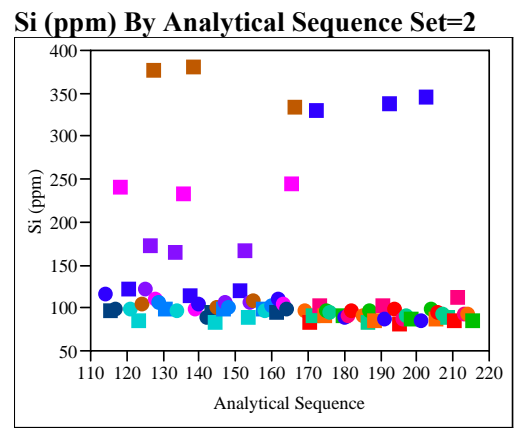


Exhibit B3. PSAL PCT Measurements byHeat Treatment by Study Glass \#

Variability Chart for $\log [\mathrm{B}$ ppm]

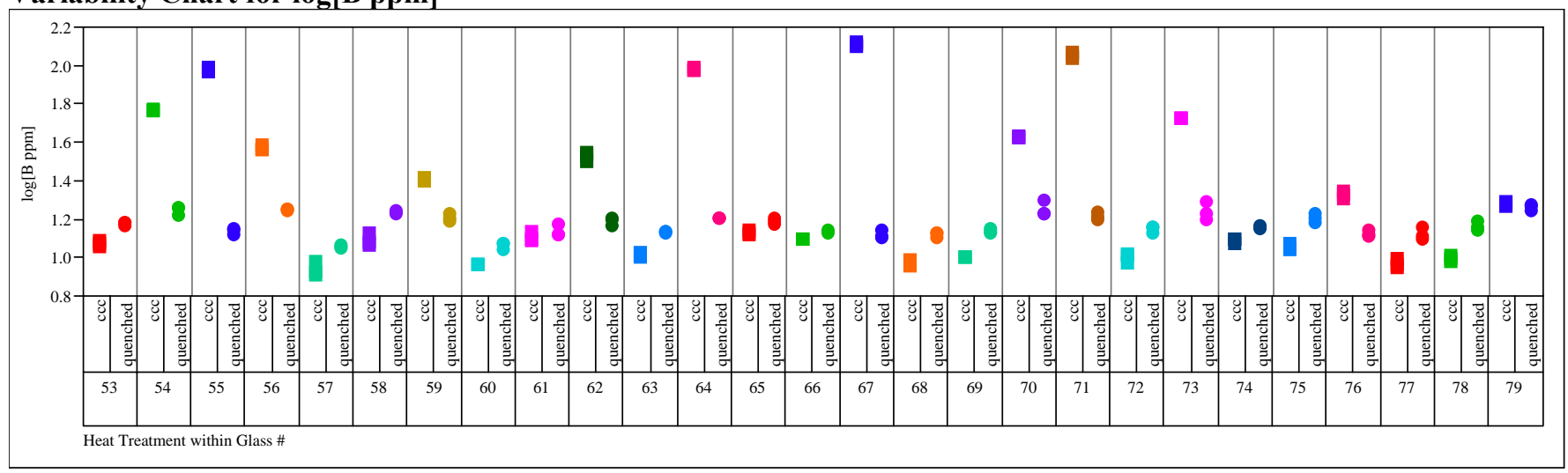

\section{Variability Chart for $\log [\mathrm{Li}$ ppm]}

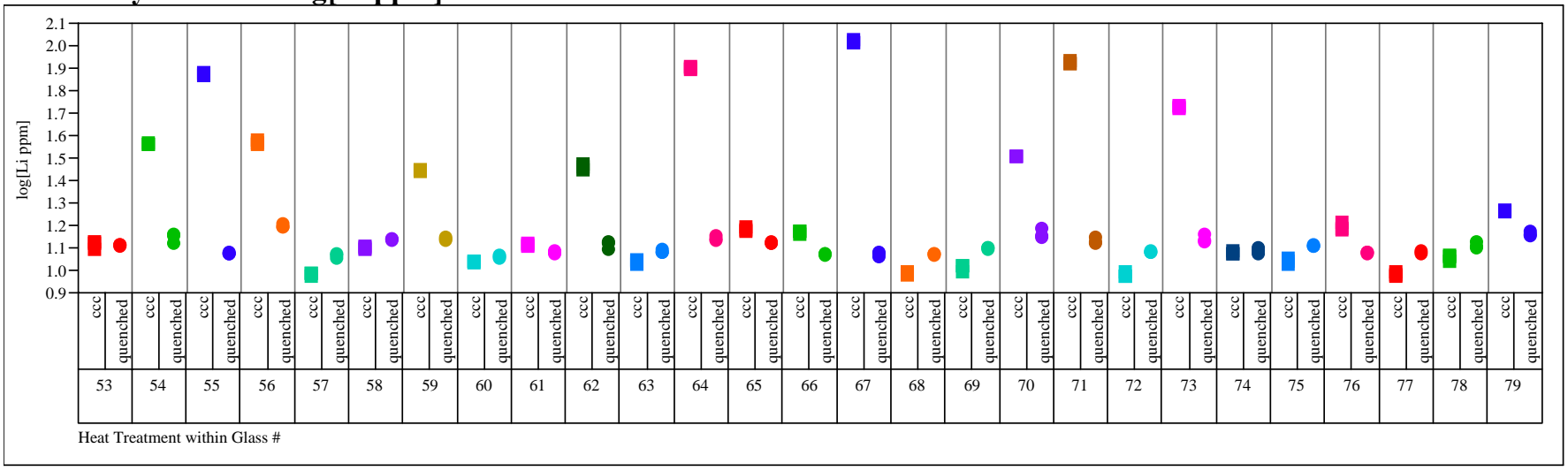


Exhibit B3. PSAL PCT Measurements byHeat Treatment by Study Glass \#

Variability Chart for log[Na ppm]

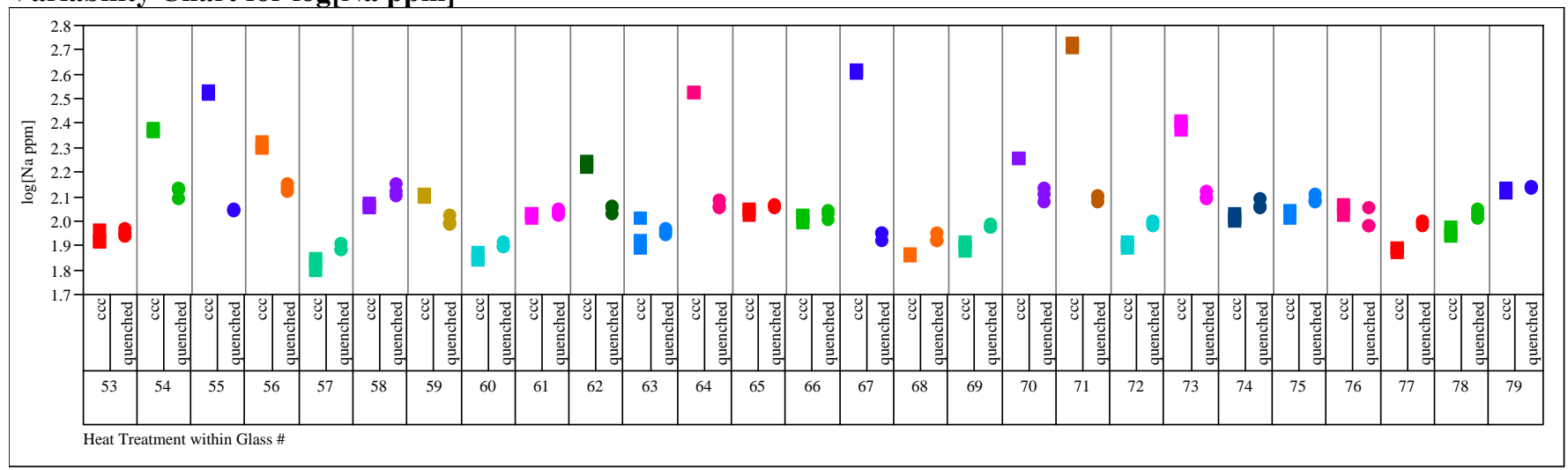

Variability Chart for log[Si ppm]

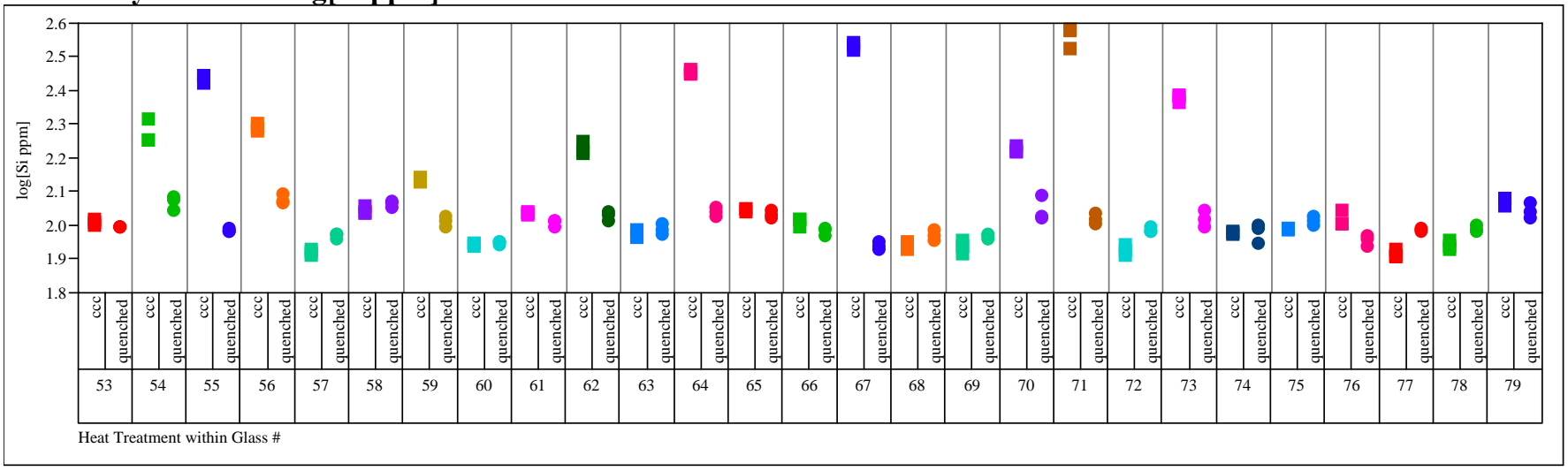


Exhibit B4. Measurements of the Multi-Element Solution Standard for Each PCT Set and Element by ICP-AES Calibration Block

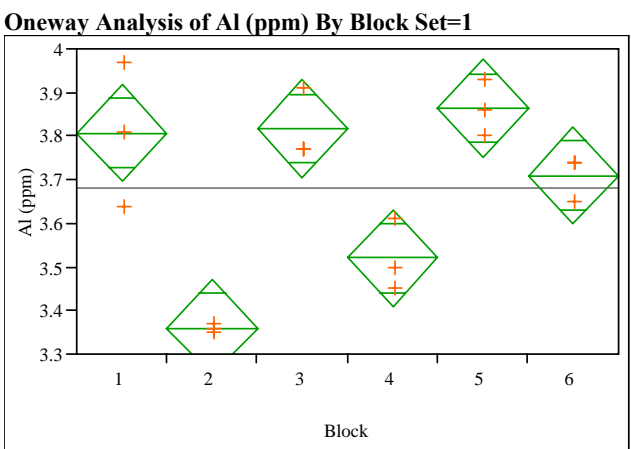

Oneway Anova

Summary of Fit

Rsquare

0.861656

Root Mean Square Enor $\quad 0.804013$

Mean of Response 3.679444

Observations (or Sum Wgts)

$$
18
$$

Analysis of Variance

Source DF Sum of Squares Mean Square F Ratio Prob > F $\begin{array}{lllllll}\text { Block } & 5 & 0.59169444 & 0.118339 & 14.9481 & <.0001\end{array}$ $\begin{array}{llll}\text { Error } & 12 & 0.09500000 & 0.007917\end{array}$

C. Total $17 \quad 0.68669444$

Means for Oneway Anova

Level Number Mean Std Error Lower 95\% Upper 95\%

$\begin{array}{llrrrr}1 & 3 & 3.80667 & 0.05137 & 3.6947 & 3.9186\end{array}$

$\begin{array}{llllll}2 & 3 & 3.36000 & 0.05137 & 3.2481 & 3.4719 \\ 3 & 3 & 3.81667 & 0.05137 & 3.7047 & 3.9286\end{array}$

$\begin{array}{llllll}3 & 3 & 3.81667 & 0.05137 & 3.7047 & 3.9286 \\ 4 & 3 & 3.52000 & 0.05137 & 3.4081 & 3.6319\end{array}$

$\begin{array}{llllll}5 & 3 & 3.86333 & 0.05137 & 3.7514 & 3.9753 \\ 6 & 3 & 3.71000 & 0.05137 & 3.5981 & 3.8219\end{array}$

Std Error uses a pooled estimate of error variance

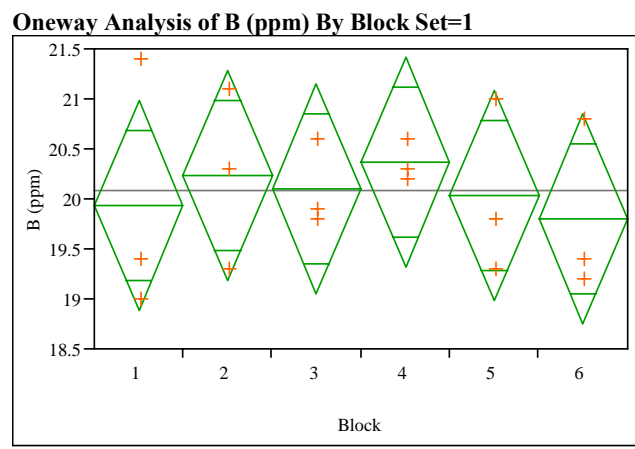

\section{Oneway Anova}

Summary of Fit

Rsquare

$\begin{array}{lr}\text { Rsquare } & 0.068839 \\ \text { Adj Rsquare } & -0.31915 \\ \text { Root Mean Square Error } & 0.838981\end{array}$

Mean of Response $\quad 20.07778$

20.07778
$\quad 18$

Analysis of Variance

Source DF Sum of Squares Mean Square F Ratio Prob > F $\begin{array}{lrrrrr}\text { Block } & 5 & 0.6244444 & 0.124889 & 0.1774 & 0.9659\end{array}$ $\begin{array}{llll}\text { Error } & 12 & 8.4466667 & 0.703889\end{array}$

C. Total $17 \quad 9.0711111$

\section{Means for Oneway Anova}

Level Number Mean Std Error Lower 95\% Upper 95\%

$\begin{array}{llllrr}1 & 3 & 19.9333 & 0.48439 & 18.878 & 20.989 \\ 2 & 3 & 20.2333 & 0.48439 & 19.178 & 21.289\end{array}$

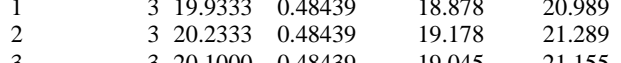

$\begin{array}{llllll}3 & 3 & 20.1000 & 0.48439 & 19.045 & 21.155 \\ 4 & 3 & 20.3667 & 0.48439 & 19.311 & 21.422\end{array}$

$\begin{array}{llllll}4 & 3 & 20.3667 & 0.48439 & 19.311 & 21.422 \\ 5 & 3 & 20.0333 & 0.48439 & 18.978 & 21.089\end{array}$

$\begin{array}{llllll}6 & 3 & 19.8000 & 0.48439 & 18.745 & 20.855\end{array}$

Std Error uses a pooled estimate of error variance

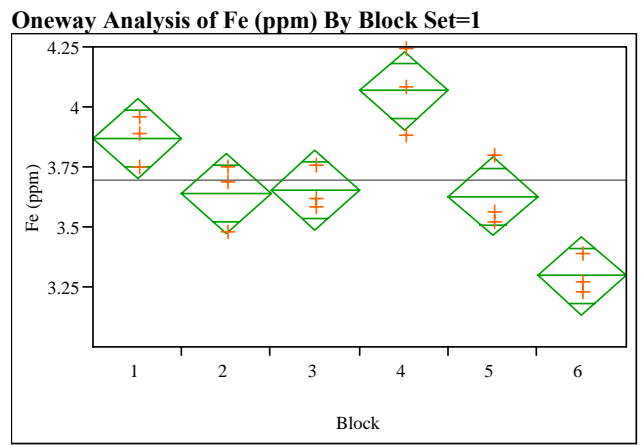

Oneway Anova

Summary of Fit

Rsquare

0.830344

Adj Rsquare

0.759654
0.130937
3.691667

Root Mean Square 3.691667

Observations (or Sum Wgts)

Analysis of Variance

Analysis of DF Sum of Squares Mean Square F Ratio Prob > F $\begin{array}{lrrrrr}\text { Block } & 5 & 1.0069167 & 0.201383 & 11.7463 & 0.0003\end{array}$ $\begin{array}{llll}\text { Error } & 12 & 0.2057333 & 0.017144\end{array}$

$\begin{array}{lll}\text { C. Total } & 17 & 1.2126500\end{array}$

Means for Oneway Anova

Level Number Mean Std Error Lower 95\% Upper 95\%

$\begin{array}{llllll}1 & 3 & 3.86667 & 0.07560 & 3.7020 & 4.0314 \\ 2 & 3 & 3.64000 & 0.07560 & 3.4753 & 3.8047\end{array}$

$\begin{array}{llllll}2 & 3 & 3.64000 & 0.07560 & 3.4753 & 3.8047 \\ 3 & 3 & 3.65333 & 0.07560 & 3.4886 & 3.8180\end{array}$

$\begin{array}{llllll}3 & 3 & 3.65333 & 0.07560 & 3.4886 & 3.8180 \\ 4 & 3 & 3.06667 & 0.07560 & 3.9020 & 4.2314\end{array}$

$\begin{array}{llllll}5 & 3 & 3.62667 & 0.07560 & 3.4620 & 3.7914 \\ 6 & 3 & 3.29667 & 0.07560 & 3.1320 & 3.4614\end{array}$

Std Error uses a pooled estimate of error variance 
Exhibit B4. Measurements of the Multi-Element Solution Standard for Each PCT Set and Element by ICP-AES Calibration Block

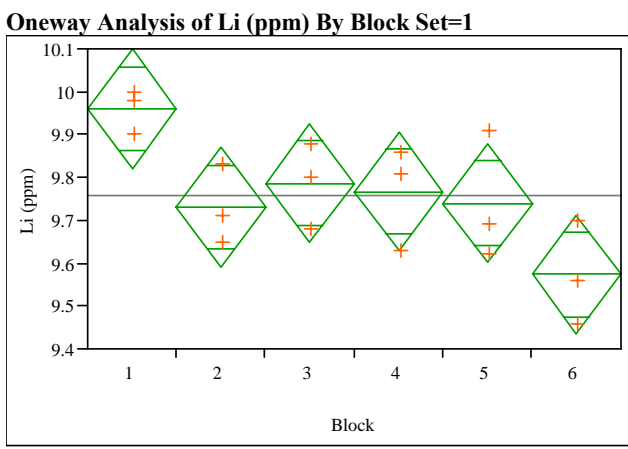

\section{Oneway Anova}

Summary of

Adj Rsquare $\quad 0.449087$

Mean of Response $\quad 0.110604$

Observations (or Sum Wgts)

Analysis of Variance

Source DF Sum of Squares Mean Square F Ratio Prob $>$ F

$\begin{array}{llllll}\text { Block } & 5 & 0.23069444 & 0.046139 & 3.7716 & 0.0277\end{array}$

\begin{tabular}{lll} 
Error & 12 & 0.14680000 \\
\hline
\end{tabular}

0.012233

Means for Oneway Anova
Level Number Mean Std Error Lower 95\% Upper 95\%

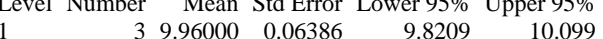

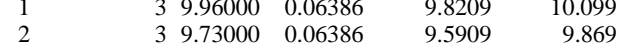

$\begin{array}{llllll}1 & 3 & 9.73000 & 0.06386 & 9.5909 & 9.869 \\ 3 & 3 & 9.78667 & 0.06386 & 9.6475 & 9.926 \\ 4 & 3 & 9.76667 & 0.06386 & 9.6275 & 9.906\end{array}$

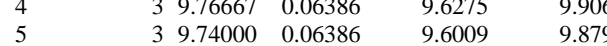

$\begin{array}{lllll}6 & 39.57333 & 0.06386 & 9.4342 & 9.712\end{array}$

Std Error uses a pooled estimate of error variance

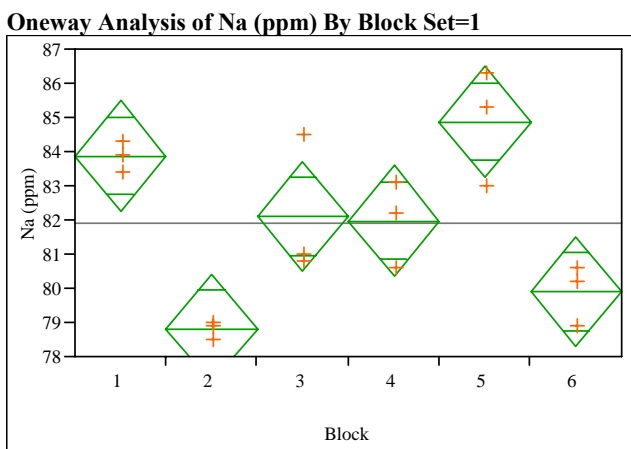

\section{Oneway Anova}

Summary of Fit

$\begin{array}{lr}\text { Rsquare } & 0.800172 \\ \text { Adj Rsquare } & 0.716911 \\ \text { Root Mean Square Error } & 1.281926 \\ \text { Mean of Response } & 81.91667 \\ \text { Observations (or Sum Wgts) } & 18\end{array}$

Analysis of Variance

Source DF Sum of Squares Mean Square F Ratio Prob > F $\begin{array}{llllll}\text { Block } & 5 & 78.965000 & 15.7930 & 9.6103 & 0.0007\end{array}$ $\begin{array}{lll}\text { Error } & 12 & 19.720000\end{array}$ 1.6433

\section{Total 1798.685000}

Means for Oneway Anova

Level Number Mean Std Error Lower 95\% Upper 95\%

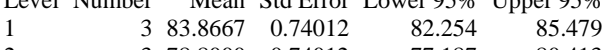

$\begin{array}{llllll}2 & 3 & 78.8000 & 0.74012 & 77.187 & 80.413 \\ 3 & 3 & 82.1000 & 0.74012 & 80.487 & 83.713\end{array}$

$\begin{array}{llllll}4 & 3 & 81.9667 & 0.74012 & 80.354 & 83.579\end{array}$

$\begin{array}{llllll}5 & 3 & 84.8667 & 0.74012 & 83.254 & 86.479 \\ 6 & 3 & 79.9000 & 0.74012 & 78.287 & 81.513\end{array}$

Std Error uses a pooled estimate of error variance

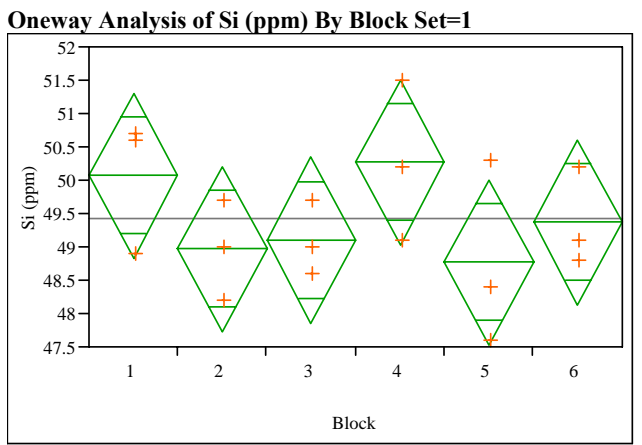

Oneway Anova

Summary of Fit

Rsquare

0.326025

Adj Rsquare

Root Mean Square Error $\quad 0.983757$

Mean of Response 49.42222

Observations (or Sum Wgts)

Analysis of Variance

Source DF Sum of Squares Mean Square F Ratio Prob > F $\begin{array}{llllll}\text { Block } & 5 & 5.617778 & 1.12356 & 1.1610 & 0.3825\end{array}$

$\begin{array}{lll}\text { Error } & 12 & 11.613333\end{array}$

Means for Oneway Anova

Level Number Mean Std Error Lower 95\% Upper 95\% $\begin{array}{llrrrr}1 & 3 & 50.0667 & 0.56797 & 48.829 & 51.304\end{array}$ $\begin{array}{llllll}1 & 3 & 50.0667 & 0.56797 & 48.829 & 51.304 \\ 2 & 3 & 48.9667 & 0.56797 & 47.729 & 50.204\end{array}$ $\begin{array}{llllll}2 & 3 & 48.9667 & 0.56797 & 47.729 & 50.204 \\ 3 & 3 & 49.1000 & 0.56797 & 47.862 & 50.338 \\ 4 & 3 & 50.2667 & 0.56797 & 49.029 & 51.504\end{array}$ $\begin{array}{llllll}3 & 3 & 50.2667 & 0.56977 & 49.029 & 51.504 \\ 5 & 3 & 48.7667 & 0.56797 & 47.529 & 50.004\end{array}$

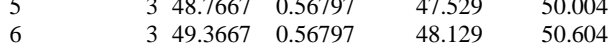

Std Error uses a pooled estimate of error variance 
Exhibit B4. Measurements of the Multi-Element Solution Standard for Each PCT Set and Element by ICP-AES Calibration Block

Fit $Y$ by $X$ Group

Oneway Analysis of Al (ppm) By Block Set=2

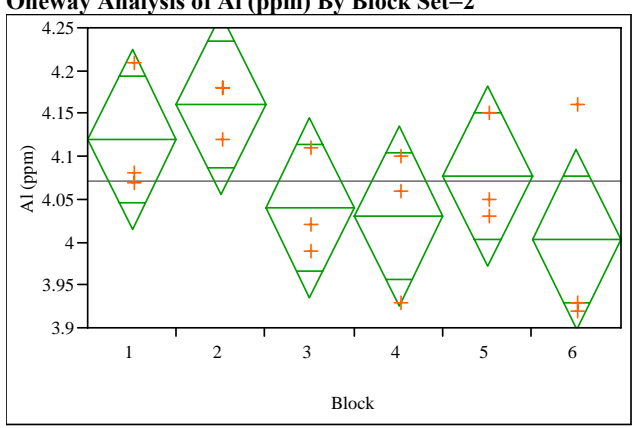

Oneway Anova

Summary of

$\begin{array}{lr}\text { Rsquare } & 0.38748 \\ \text { Adj Rsquare } & 0.132263 \\ \text { Root Mean Square Error } & 0.083333 \\ \text { Mean of Response } & 4.071667 \\ \text { Observations (or Sum Wgts) } & 18\end{array}$

Analysis of Variance

Source DF Sum of Squares Mean Square F Ratio Prob > F

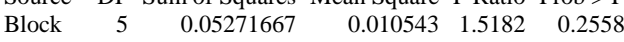
$\begin{array}{llll}\text { Error } & 12 & 0.08333333 & 0.006944\end{array}$

C. Total $17 \quad 0.13605000$

Means for Oneway Anova

Level Number Mean Std Error Lower 95\% Upper 95\%

$\begin{array}{llllll}1 & 3 & 4.12000 & 0.04811 & 4.0152 & 4.2248\end{array}$

$\begin{array}{llllll}2 & 3 & 4.16000 & 0.04811 & 4.0552 & 4.2648 \\ 3 & 3 & 4.04000 & 0.04811 & 3.352 & 4.1448\end{array}$

$\begin{array}{llllll} & 3 & 4.04000 & 0.04811 & 3.9352 & 4.1448 \\ 4 & 3 & 4.03000 & 0.04811 & 3.9252 & 4.1348\end{array}$

$\begin{array}{llllll}5 & 3 & 4.03000 & 0.04811 & 3.9252 & 4.1348 \\ 6 & 3 & 4.07667 & 0.04811 & 3.9718 & 4.1815\end{array}$

$\begin{array}{lllll}3 & 4.00333 & 0.04811 & 3.8985 & 4.1082\end{array}$

Std Error uses a pooled estimate of error variance

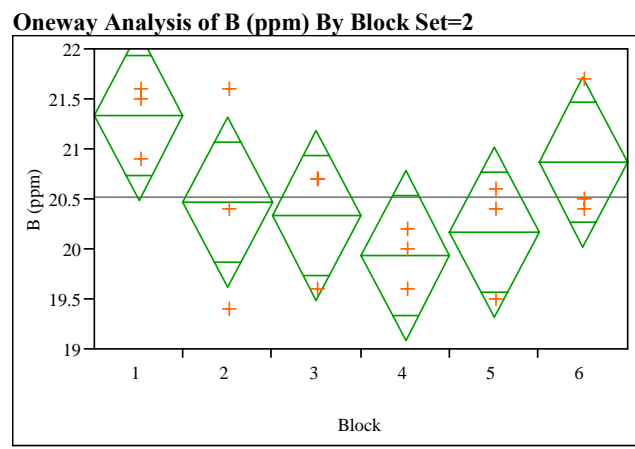

\section{Oneway Anova}

Summary of Fit

$\begin{array}{lr}\text { Rsquare } & 0.415368 \\ \text { Adj Rsquare } & 0.171771 \\ \text { Root Mean Square Error } & 0.6733 \\ \text { Mean of Response } & 20.51667 \\ \text { Observations (or Sum Wgts) } & 18\end{array}$

Analysis of Variance

Source DF Sum of Squares Mean Square F Ratio Prob > F $\begin{array}{lrrrrr}\text { Block } & 5 & 3.8650000 & 0.773000 & 1.7051 & 0.2079\end{array}$ $\begin{array}{lrrr}\text { Error } & 12 & 5.4400000 & 0.453333\end{array}$

$\begin{array}{lll}\text { C. Total } & 17 & 9.3050000\end{array}$

Means for Oneway Anova
Level Number Mean Std Error Lower 95\% Upper 95\%

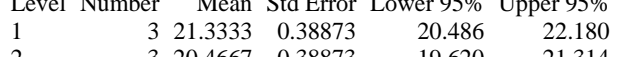

$\begin{array}{llllll}1 & 3 & 21.3333 & 0.38873 & 20.486 & 22.180 \\ 2 & 3 & 20.4667 & 0.38873 & 19.620 & 21.314 \\ 3 & 3 & 20.3333 & 0.38873 & 19.486 & 21.180\end{array}$

$\begin{array}{llllll}3 & 3 & 20.3333 & 0.38873 & 19.486 & 21.180 \\ 4 & 3 & 19.9333 & 0.38873 & 19.086 & 20.780\end{array}$

$\begin{array}{llllll}4 & 3 & 19.9333 & 0.38873 & 19.086 & 20.780 \\ 5 & 3 & 20.1667 & 0.38873 & 19.320 & 21.014 \\ 6 & 3 & 20.8667 & 0.38873 & 20.020 & 21.714\end{array}$

Std Error uses a pooled estimate of error variance

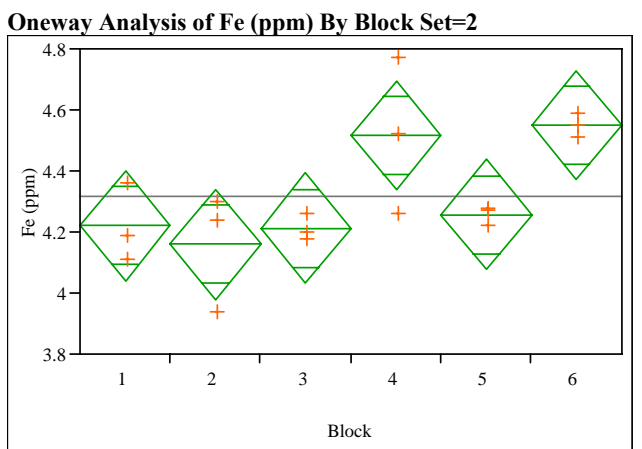

Oneway Anova

Summary of Fit

Rsquare

Adj Rsquare $\quad 0.635038$

$\begin{array}{ll}\text { Adj Rsquare } & 0.48297 \\ \text { Rot Mean Square Error } & 0.14312\end{array}$

$\begin{array}{lr}\text { Root Mean Square Error } & 0.14312 \\ \text { Mean of Response } & 4.319444\end{array}$

Observations (or Sum Wgts)

Analysis of Variance

Source DF Sum of Squares Mean Square F Ratio Prob > F $\begin{array}{lrrrrr}\text { Block } & 5 & 0.42769444 & 0.085539 & 4.1760 & 0.0198\end{array}$ $\begin{array}{llll}\text { Error } & 12 & 0.24580000 & 0.020483\end{array}$

C. Tot $17-0.67349444$

Means for Oneway Anova

Level Number Mean Std Error Lower 95\% Upper 95\% $\begin{array}{lllllr}1 & 3 & 4.22000 & 0.08263 & 4.0400 & 4.4000\end{array}$ $\begin{array}{llllll}1 & 3 & 4.16000 & 0.08263 & 3.9800 & 4.3400 \\ 3 & 3 & 4.21333 & 0.08263 & 4.0333 & 43934\end{array}$ $\begin{array}{llllll}4 & 3 & 4.51667 & 0.08263 & 4.3366 & 4.6967\end{array}$ $\begin{array}{llllll}5 & 3 & 4.25667 & 0.08263 & 4.0766 & 4.4367 \\ 6 & 3 & 4.55000 & 0.08263 & 4.3700 & 4.7300\end{array}$

Std Error uses a pooled estimate of error variance 
Exhibit B4. Measurements of the Multi-Element Solution Standard for Each PCT Set and Element by ICP-AES Calibration Block

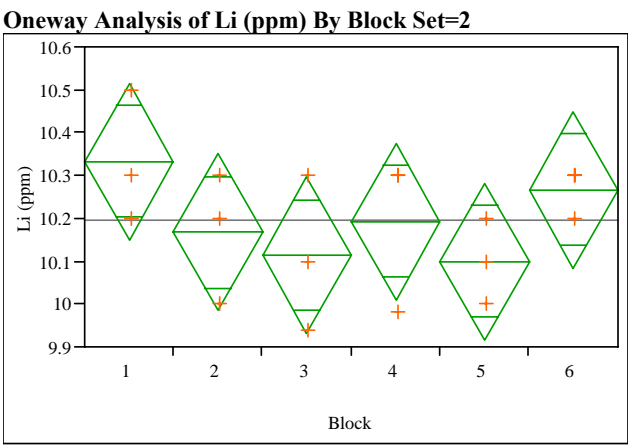

\section{Oneway Anova}

Summary

Rsquare

Adj Rsquare 0.325603

Root Mean Square Error $\quad 0.145297$

Mean of Response 10.19556

Observations (or Sum Wgts)

18

Analysis of Variance

Source DF Sum of Squares Mean Square F Ratio Prob $>$ F $\begin{array}{llllll}\text { Block } & 5 & 0.12231111 & 0.024462 & 1.1587 & 0.3835\end{array}$

\begin{tabular}{lll} 
Error & 12 & 0.25333333 \\
\hline
\end{tabular}

Means for Oneway Anova

Level Number Mean Std Error Lower 95\% Upper 95\%

$\begin{array}{llllll}1 & 3 & 10.3333 & 0.08389 & 10.151 & 10.516\end{array}$

$\begin{array}{rrrrrr}1 & 3 & 10.3333 & 0.08389 & 10.151 & 10.516 \\ 2 & 3 & 10.1667 & 0.08389 & 9.984 & 10.349\end{array}$

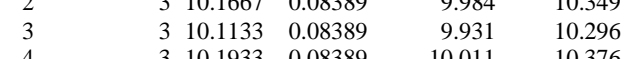

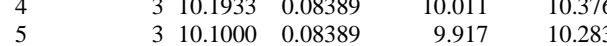

$\begin{array}{rrrrrr}5 & 3 & 10.1000 & 0.08389 & 9.917 & 10.283 \\ 6 & 3 & & & & \end{array}$

Std Error uses a pooled estimate of error variance

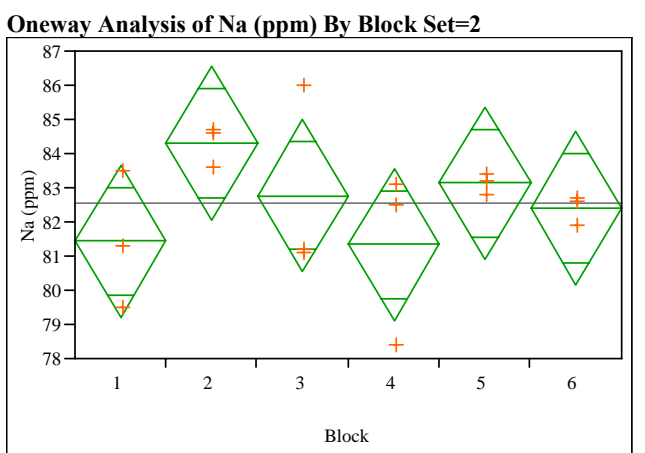

\section{Oneway Anova}

Summary of Fit

Adj Rsquare

Root Mean Square

Mean of Response

0.327958

0.04794

1.782009

Observations (or Sum Wgts)

18

Analysis of Variance

Source DF Sum of Squares Mean Square F Ratio Prob > F $\begin{array}{llllll}\text { Block } & 5 & 18.596111 & 3.71922 & 1.1712 & 0.3781\end{array}$ $\begin{array}{lll}\text { Error } & 12 & 38.106667\end{array}$ $\begin{array}{lll}3.71922 & 1.1712 & 0.3781 \\ 3.17556 & & \end{array}$

C. Total 6.702778

Means for Oneway Anova

Level Number Mean Std Error Lower 95\% Upper 95\%

$\begin{array}{llllll}1 & 3 & 81.4333 & 1.0288 & 79.192 & 83.675 \\ 2 & 3 & 84.3000 & 1.0288 & 82.058 & 86.542\end{array}$

$\begin{array}{llllll}2 & 3 & 84.3000 & 1.0288 & 82.058 & 86.542 \\ 3 & 3 & 82.7667 & 1.0288 & 80.525 & 85.008\end{array}$

$\begin{array}{llllll}4 & 3 & 81.3333 & 1.0288 & 79.092 & 83.575 \\ 5 & 3 & 83.1333 & 1.0288 & 80.892 & 85.375\end{array}$

$\begin{array}{llllll}5 & 3 & 83.1333 & 1.0288 & 80.892 & 85.375 \\ 6 & 3 & 82.4000 & 1.0288 & 80.158 & 84.642\end{array}$

Std Error uses a pooled estimate of error variance

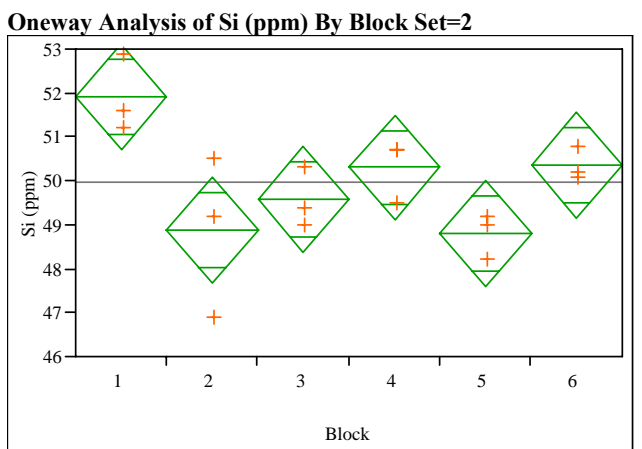

Oneway Anova

Summary of Fi

Rsquare $\quad 0.649326$

Adj Rsquare $\quad 0.503211$

Root Mean Square Error $\quad 0.953939$

$\begin{array}{lr}\text { Mean of Response } & 49.96667 \\ \text { Observations (or Sum Wgts) } & 18\end{array}$

Analysis of Variance

Source DF Sum of Squares Mean Square F Ratio Prob > F $\begin{array}{lrrrrr}\text { Block } & 5 & 20.220000 & 4.04400 & 4.4440 & 0.0160\end{array}$ Error $12 \quad 10.920000$ 0.91000

\section{Means for Oneway Anova}

Level Number Mean Std Error Lower 95\% Upper 95\% $\begin{array}{lrrrrr}1 & 3 & 51.9000 & 0.55076 & 50.700 & 53.100\end{array}$

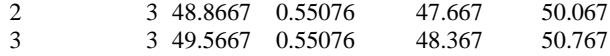
$\begin{array}{llllll}4 & 3 & 50.3000 & 0.55076 & 49.100 & 51.500\end{array}$ $\begin{array}{llllll}5 & 3 & 48.8000 & 0.55076 & 47.600 & 50.000 \\ 6 & 3 & 50.3667 & 0.55076 & 49.167 & 51.567\end{array}$

Std Error uses a pooled estimate of error variance 
WSRC-STI-2007-00616

Revision 0

\section{Exhibit B5. Correlations of Leachate Responses for Each PCT Set}

First Set of PCTs

\section{Correlations}

$\log \mathrm{NL}[\mathrm{B}(\mathrm{g} / \mathrm{L})]$

$\log N L[\operatorname{Li}(g / L)]$

$\log \mathrm{NL}[\mathrm{Na}(\mathrm{g} / \mathrm{L})]$

$\log \mathrm{NL}[\mathrm{Si}(\mathrm{g} / \mathrm{L})]$

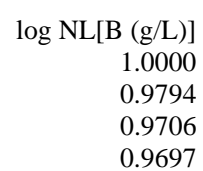

$\log \mathrm{NL}[\mathrm{Li}(\mathrm{g} / \mathrm{L})]$
0.9794
1.0000
0.9442
0.9660

$\log \mathrm{NL}[\mathrm{Na}(\mathrm{g} / \mathrm{L})]$
0.9706
0.9442
1.0000
0.9825

$\log \mathrm{NL}[\mathrm{Si}(\mathrm{g} / \mathrm{L})]$

0.9697

0.9660

0.9825

0.9697

1.0000

\section{Scatterplot Matrix}

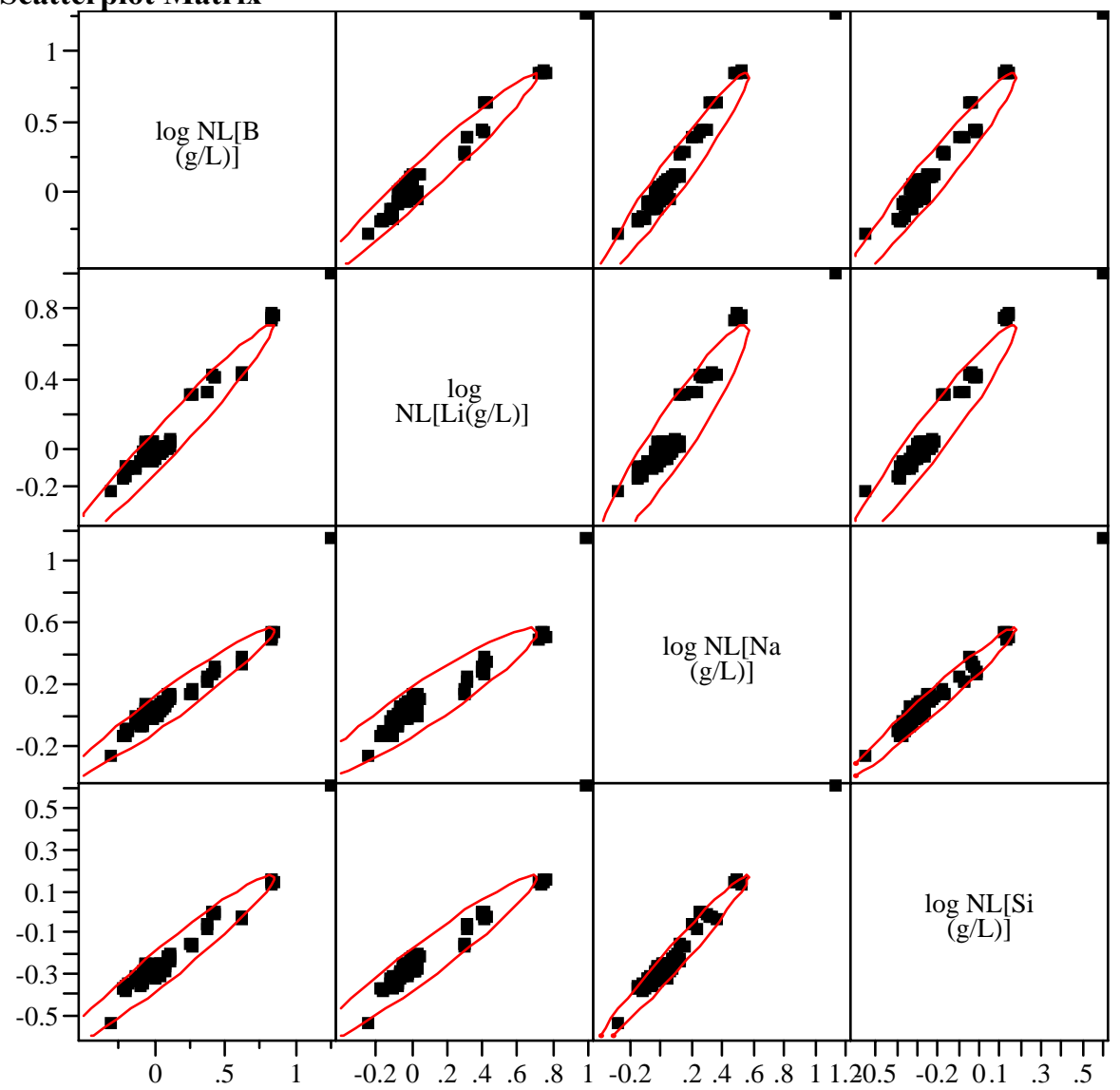




\section{Exhibit B5. Correlations of Leachate Responses for Each PCT Set}

\section{Second Set of PCTs}

\section{Correlations}

$\log \mathrm{NL}[\mathrm{B}(\mathrm{g} / \mathrm{L})]$

$\log \mathrm{NL}[\mathrm{Li}(\mathrm{g} / \mathrm{L})]$

$\log \mathrm{NL}[\mathrm{Na}(\mathrm{g} / \mathrm{L})]$

$$
\begin{array}{r}
\log \mathrm{NL}[\mathrm{B}(\mathrm{g} / \mathrm{L})] \\
1.0000 \\
0.9890 \\
0.9789 \\
0.9807
\end{array}
$$

$\log \mathrm{NL}[\mathrm{Li}(\mathrm{g} / \mathrm{L})]$
0.9890
1.0000
0.9657
0.9808

$\log \mathrm{NL}[\mathrm{Na}(\mathrm{g} / \mathrm{L})]$
0.9789
0.9657
1.0000
0.9858

$\log \mathrm{NL}[\mathrm{Si}(\mathrm{g} / \mathrm{L})]$

0.9807

0.9808

0.9858

1.0000

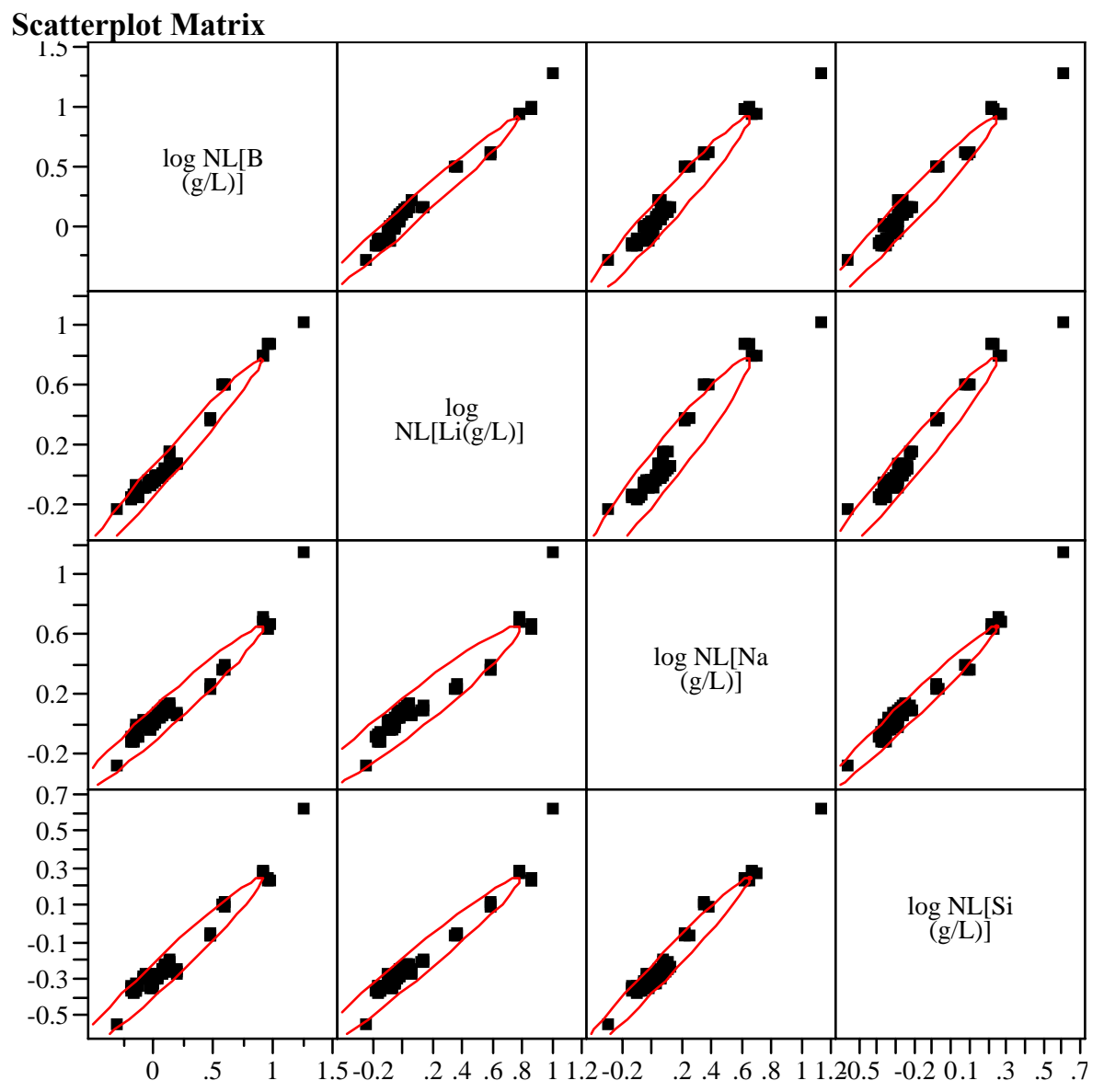




\section{Exhibit B6. PSAL PCT Measurements for Quenched and CCC Versions of Each Study Glass by Glass Series (“P” and "Q")}

Series=P — Variability Chart for $\log \mathrm{NL}[\mathrm{B}(\mathrm{g} / \mathrm{L})]$

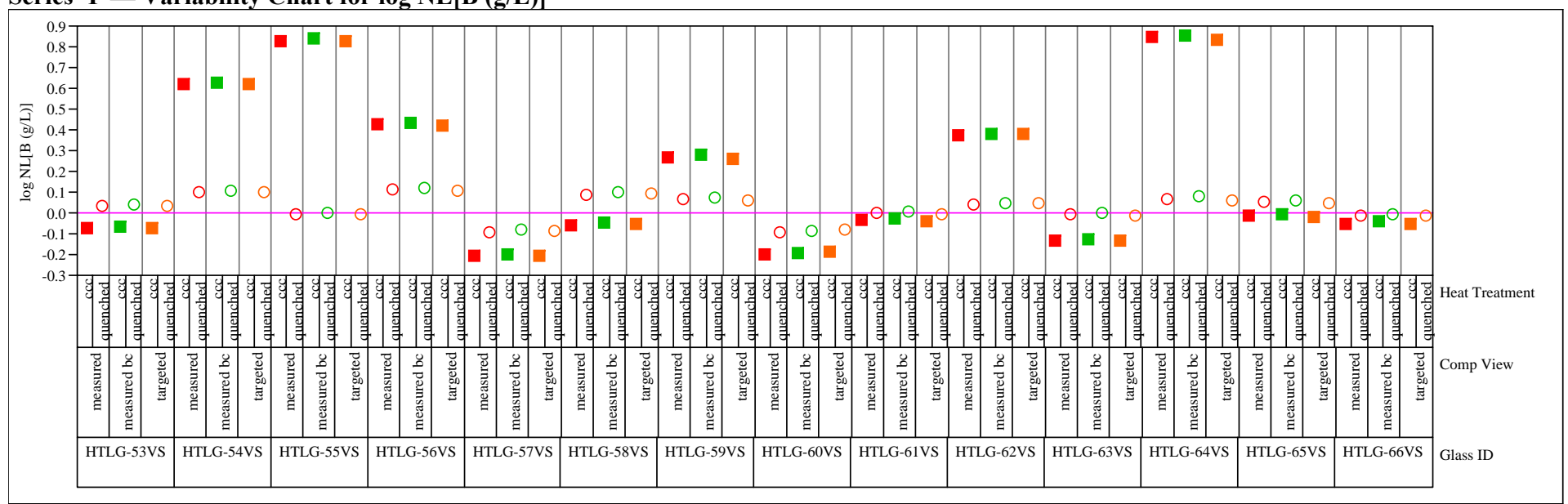

Series $=\mathrm{P}$ — Variability Chart for $\log \mathrm{NL}[\mathrm{Li}(\mathrm{g} / \mathrm{L})]$

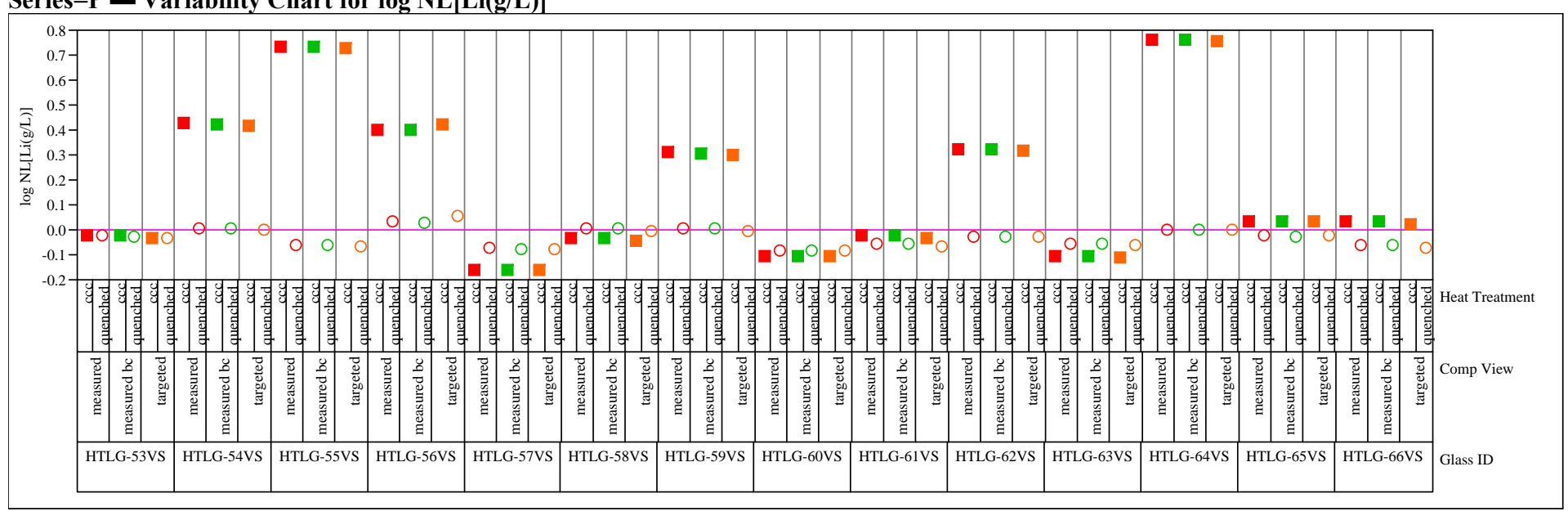




\section{Exhibit B6. PSAL PCT Measurements for Quenched and CCC Versions of Each Study Glass by Glass Series (“P” and “Q”)}

Series $=\mathrm{P}$ - Variability Chart for $\log \mathrm{NL}[\mathrm{Na}(\mathrm{g} / \mathrm{L})]$

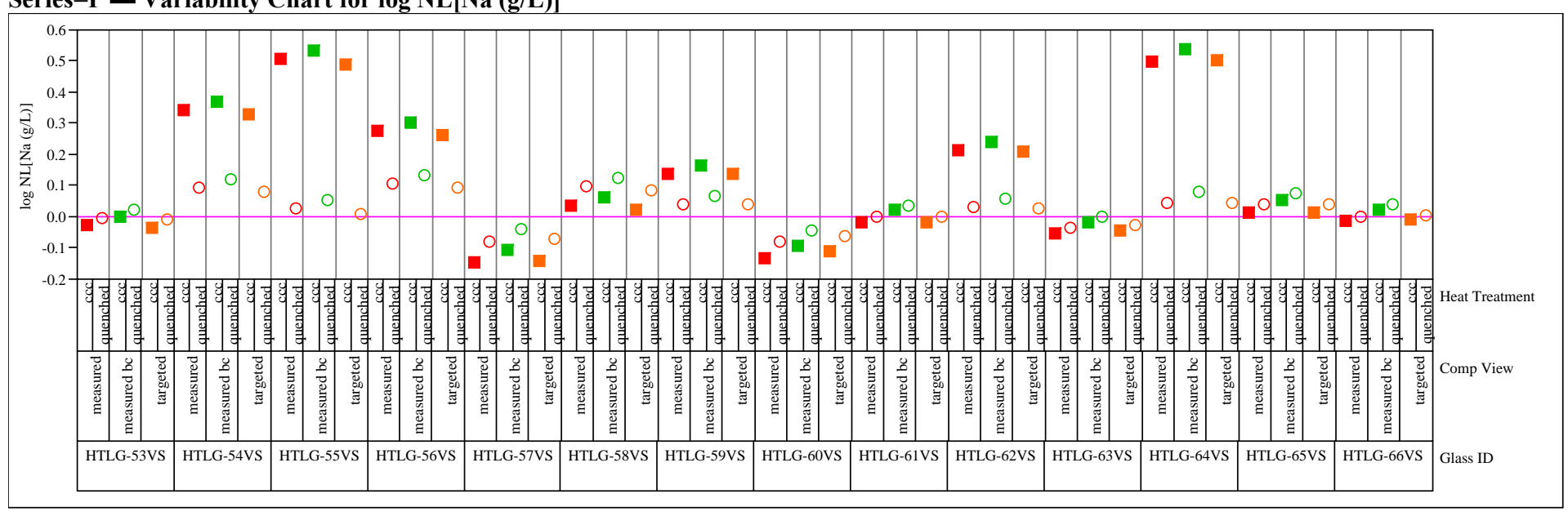

Series $=\mathrm{P}-$ Variability Chart for $\log \mathrm{NL}[\mathrm{Si}(\mathrm{g} / \mathrm{L})]$

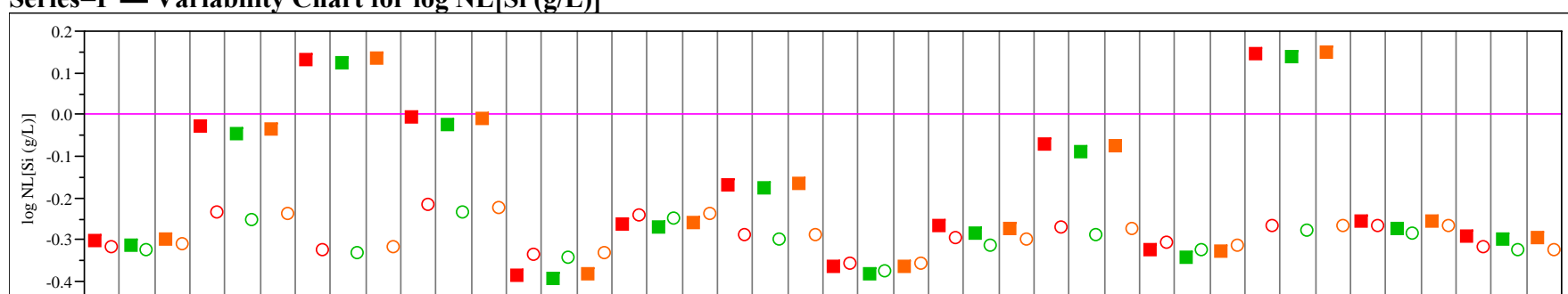

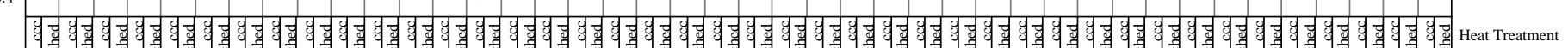
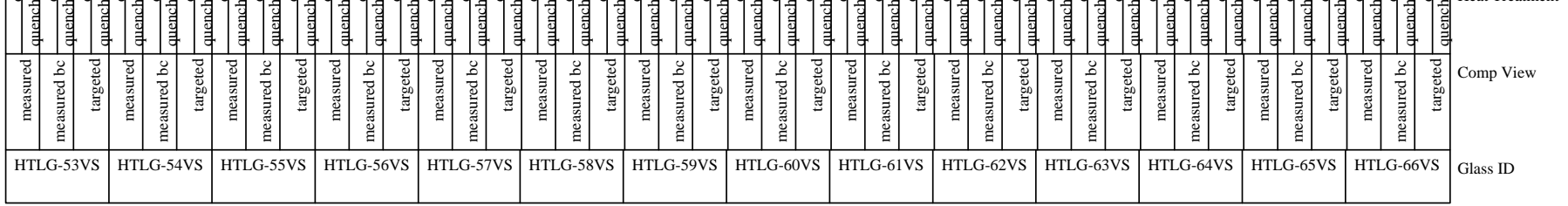


\section{Exhibit B6. PSAL PCT Measurements for Quenched and CCC Versions of Each Study Glass by Glass Series (“P” and "Q")}

Series $=P$ - Variability Chart for NL B(g/L)

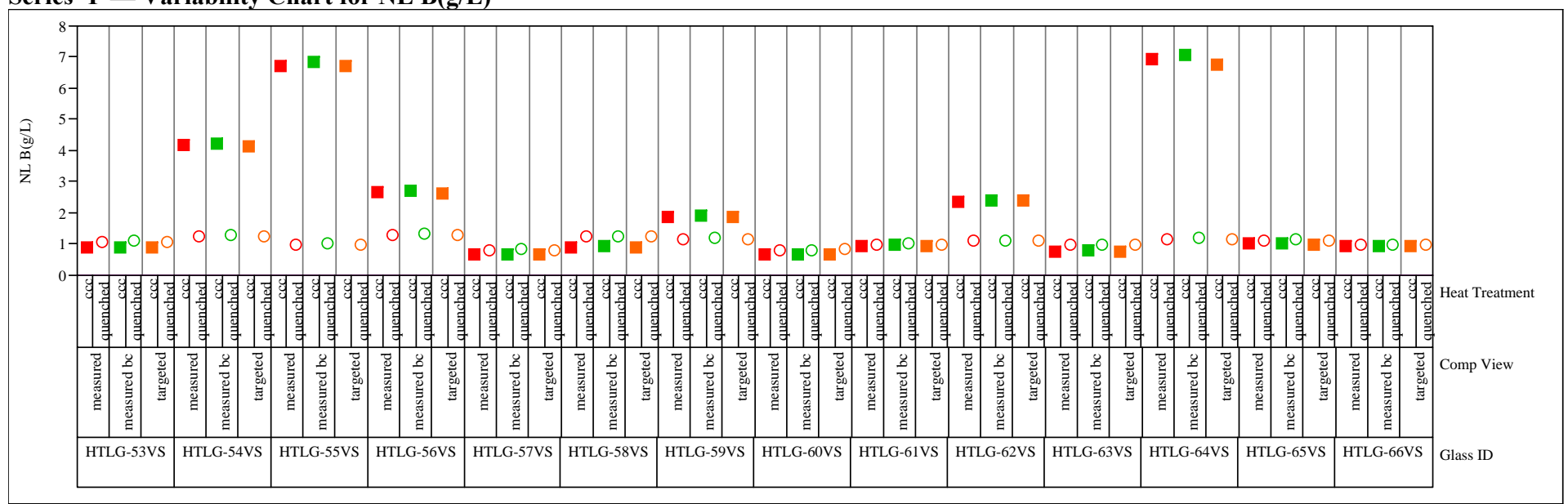

Series=P — Variability Chart for NL Li (g/L)

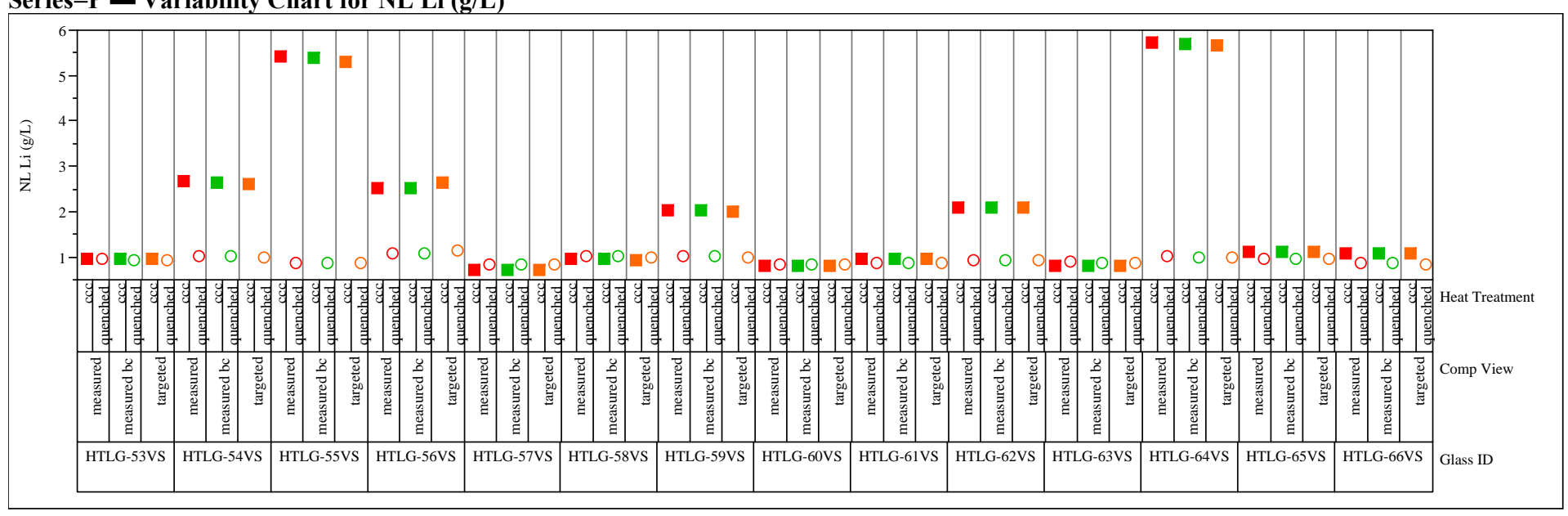




\section{Exhibit B6. PSAL PCT Measurements for Quenched and CCC Versions of Each Study Glass by Glass Series (“P” and "Q")}

Series $=\mathrm{P}$ - Variability Chart for NL Na (g/L)

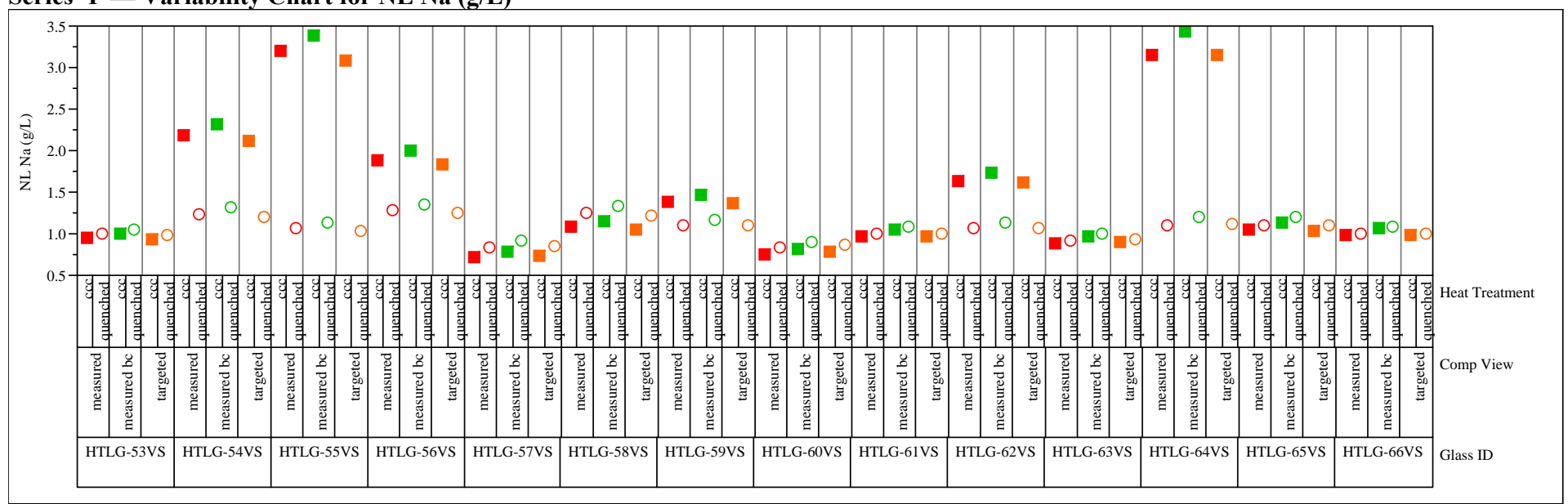

Series=P — Variability Chart for NL Si (g/L)
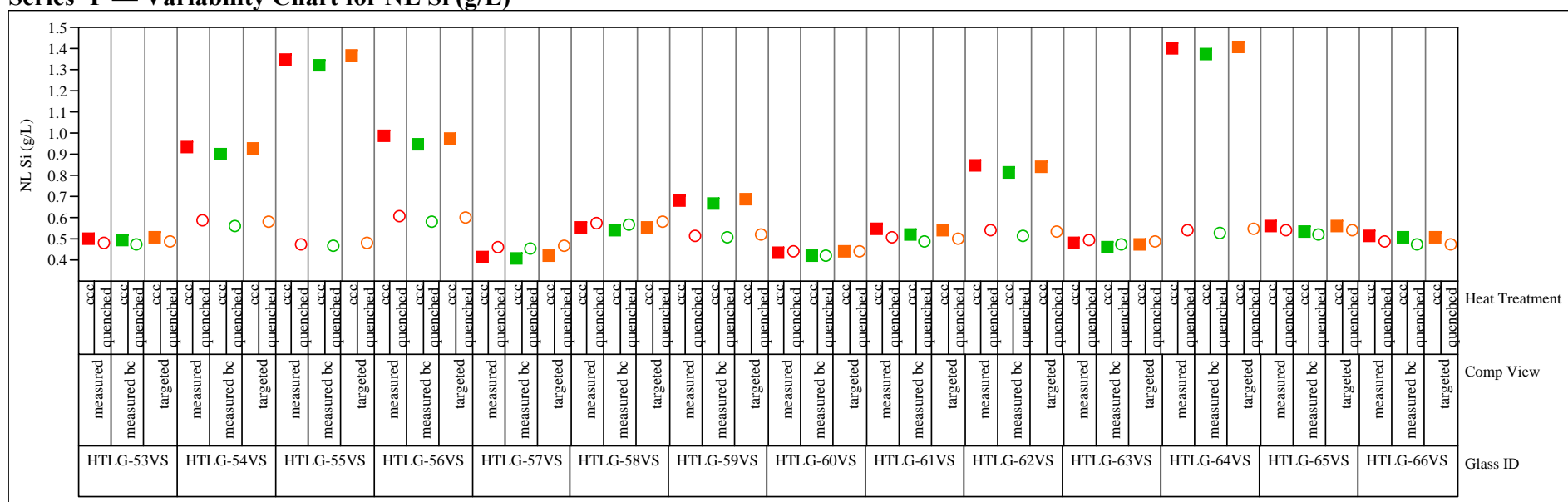


\section{Exhibit B6. PSAL PCT Measurements for Quenched and CCC Versions of Each Study Glass by Glass Series ("P” and "Q")}

Series $=Q$ — Variability Chart for $\log N L[B(g / L)]$

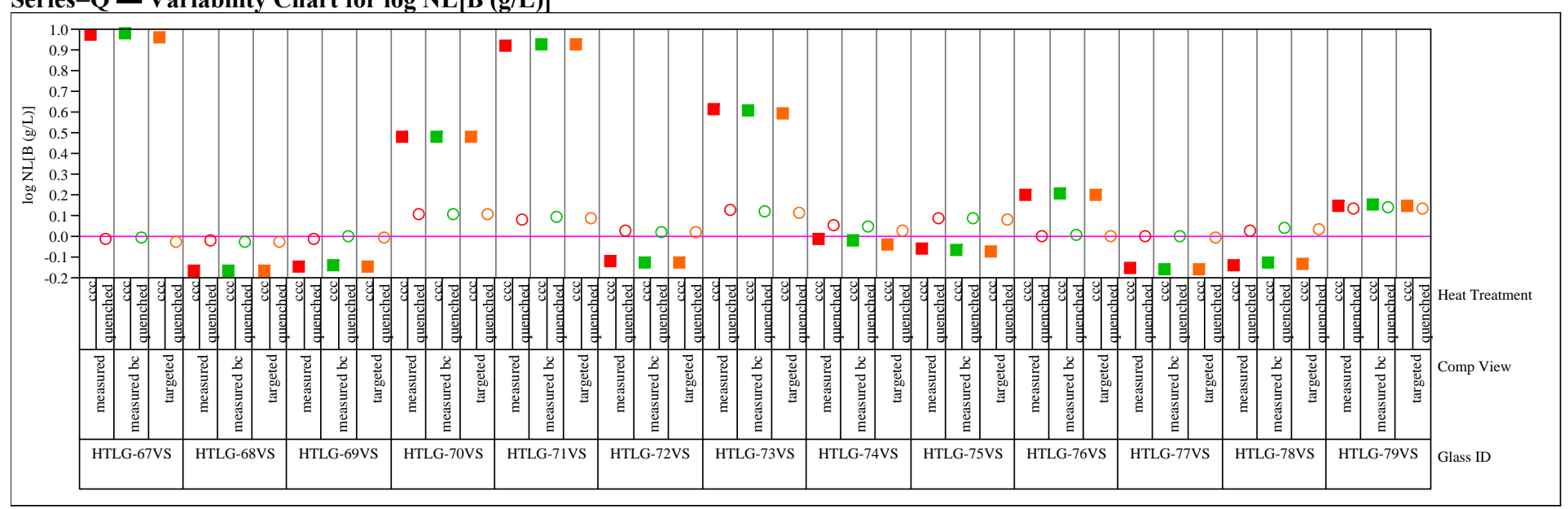

\section{Series $=\mathrm{Q}-$ Variability Chart for $\log \mathrm{NL}[\mathrm{Li}(\mathrm{g} / \mathrm{L})]$}

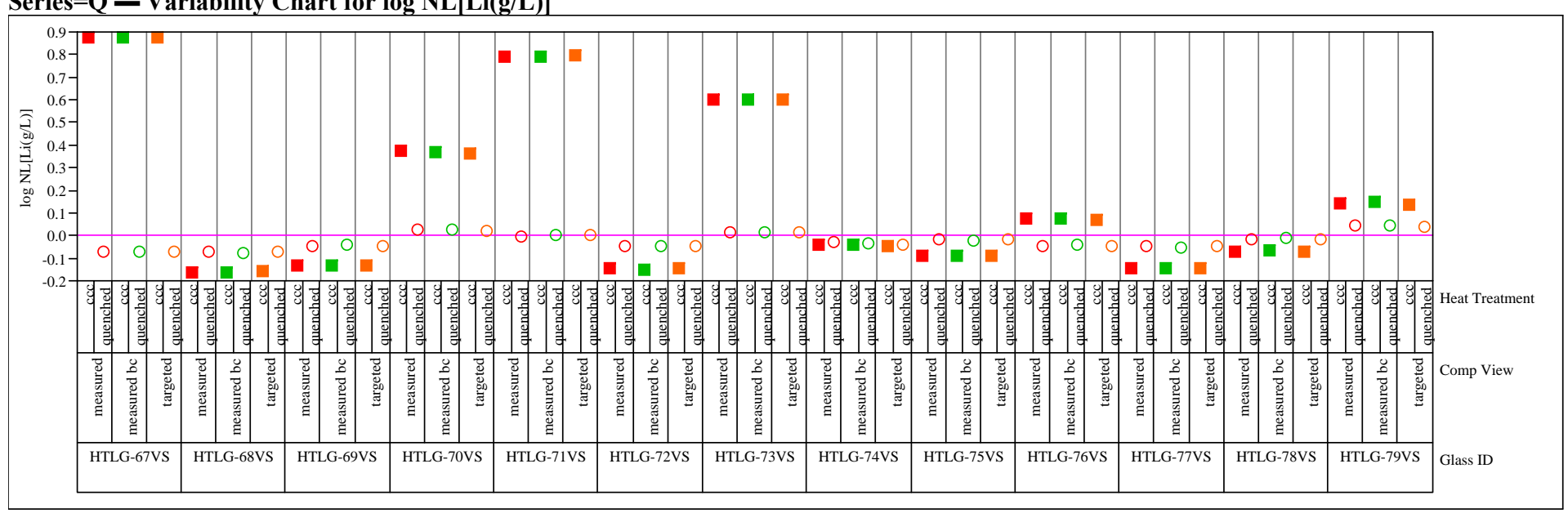




\section{Exhibit B6. PSAL PCT Measurements for Quenched and CCC Versions of Each Study Glass by Glass Series (“P” and "Q")}

Series $=\mathrm{Q}-$ Variability Chart for $\log \mathrm{NL}[\mathrm{Na}(\mathrm{g} / \mathrm{L})]$

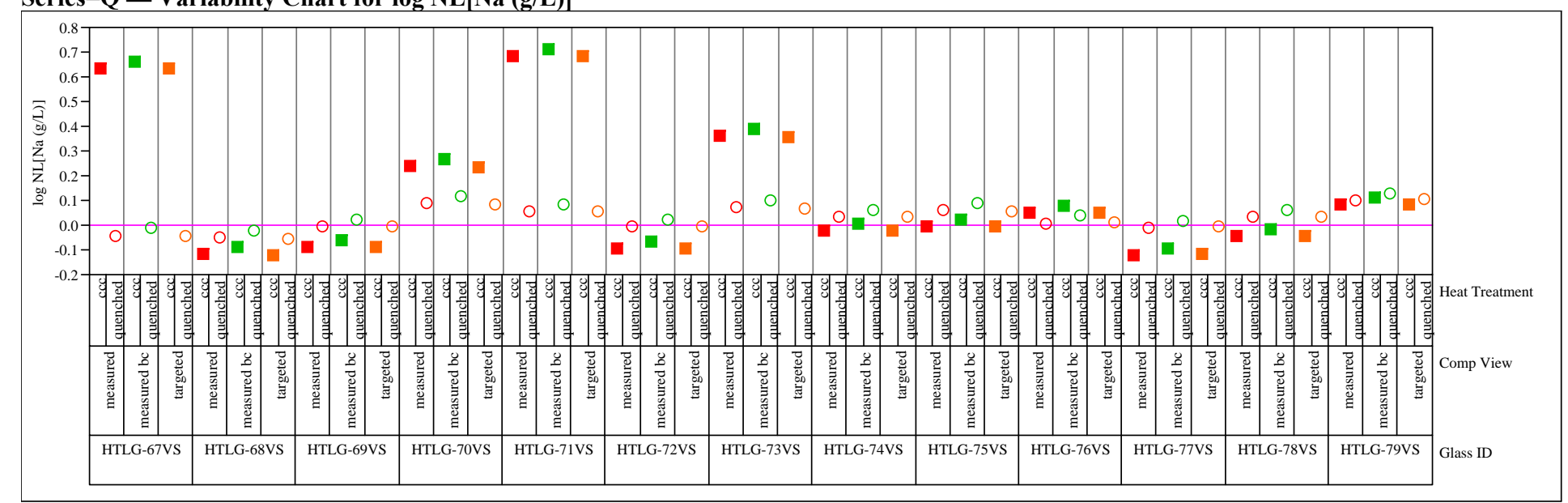

Series $=\mathrm{Q}$ - Variability Chart for $\log \mathrm{NL}[\mathrm{Si}(\mathrm{g} / \mathrm{L})]$

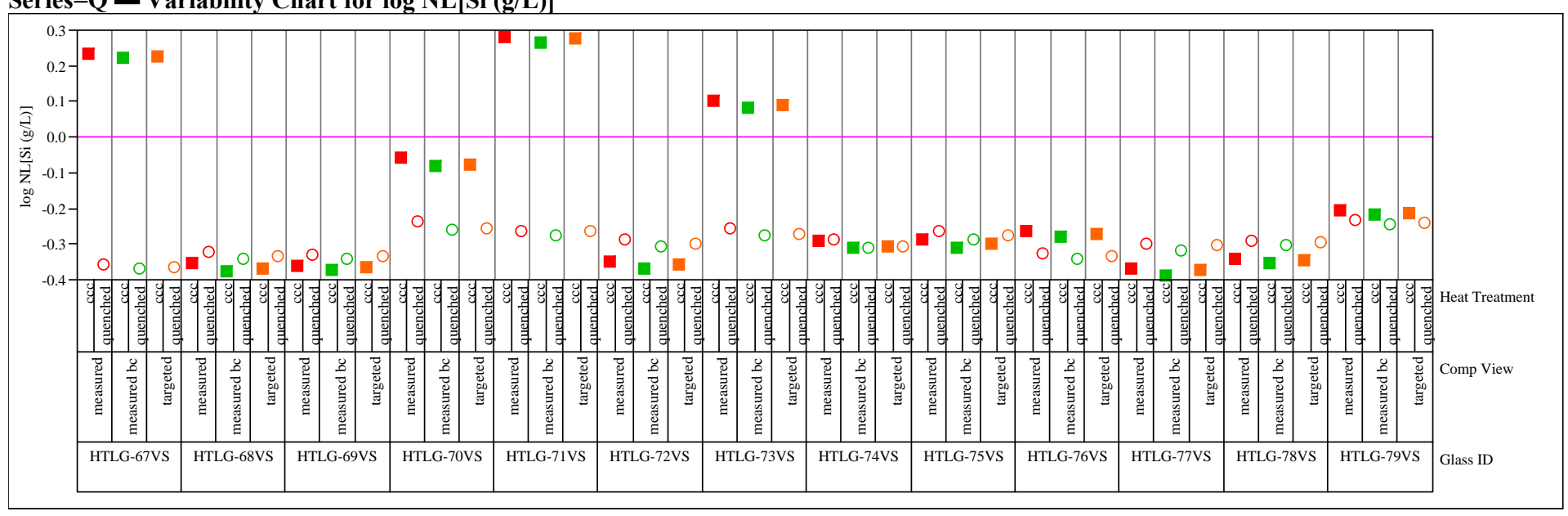




\section{Exhibit B6. PSAL PCT Measurements for Quenched and CCC Versions of Each Study Glass by Glass Series (“P” and "Q")}

Series $=\mathrm{Q}-$ Variability Chart for NL $B(g / L)$

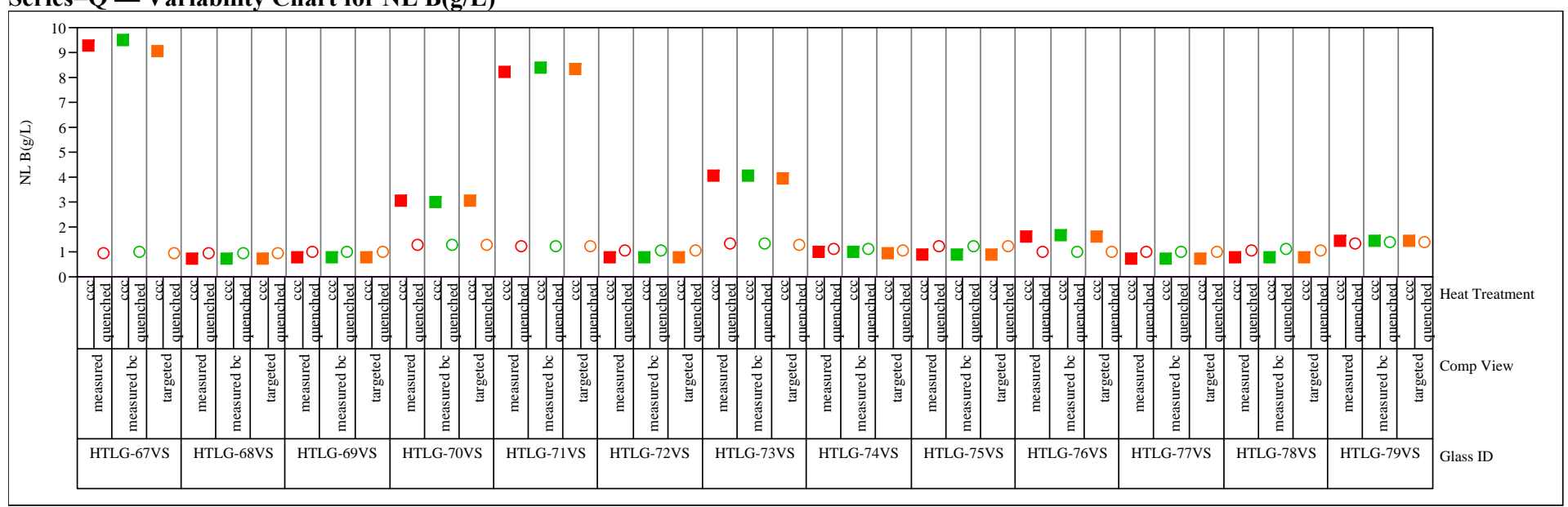

Series $=\mathbf{Q}-$ Variability Chart for $\mathbf{N L ~} \mathbf{L i}(\mathrm{g} / \mathrm{L})$

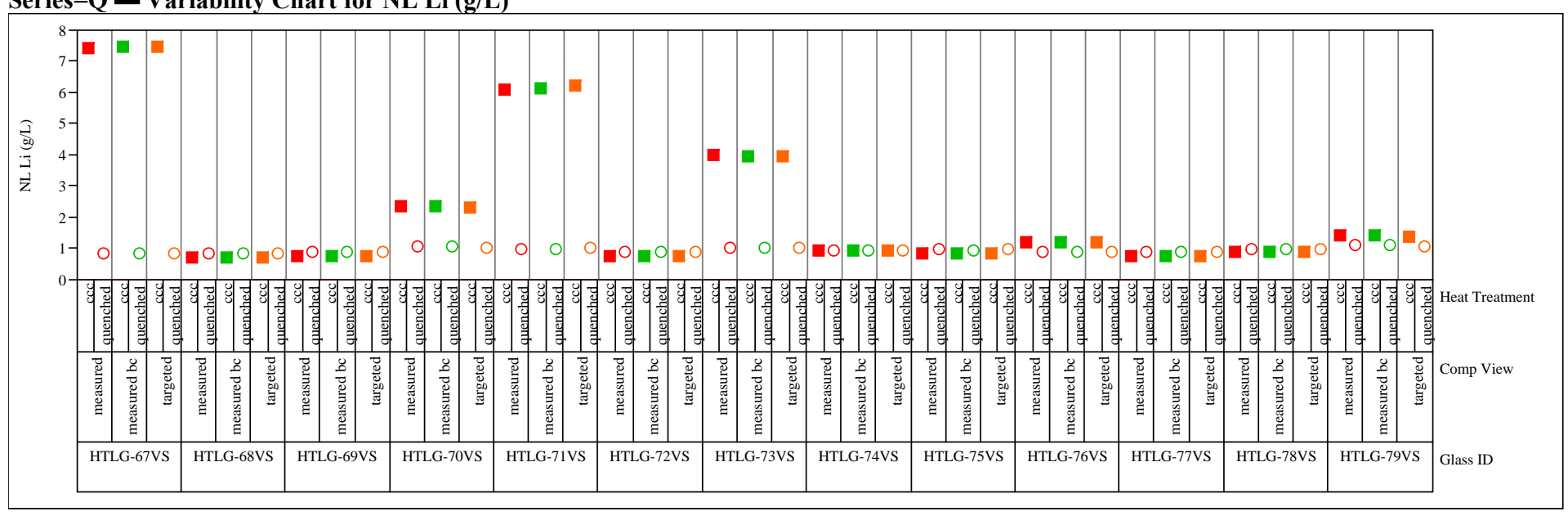




\section{Exhibit B6. PSAL PCT Measurements for Quenched and CCC Versions of Each Study Glass by Glass Series (“P” and "Q")}

Series $=Q-$ Variability Chart for NL Na $(g / L)$

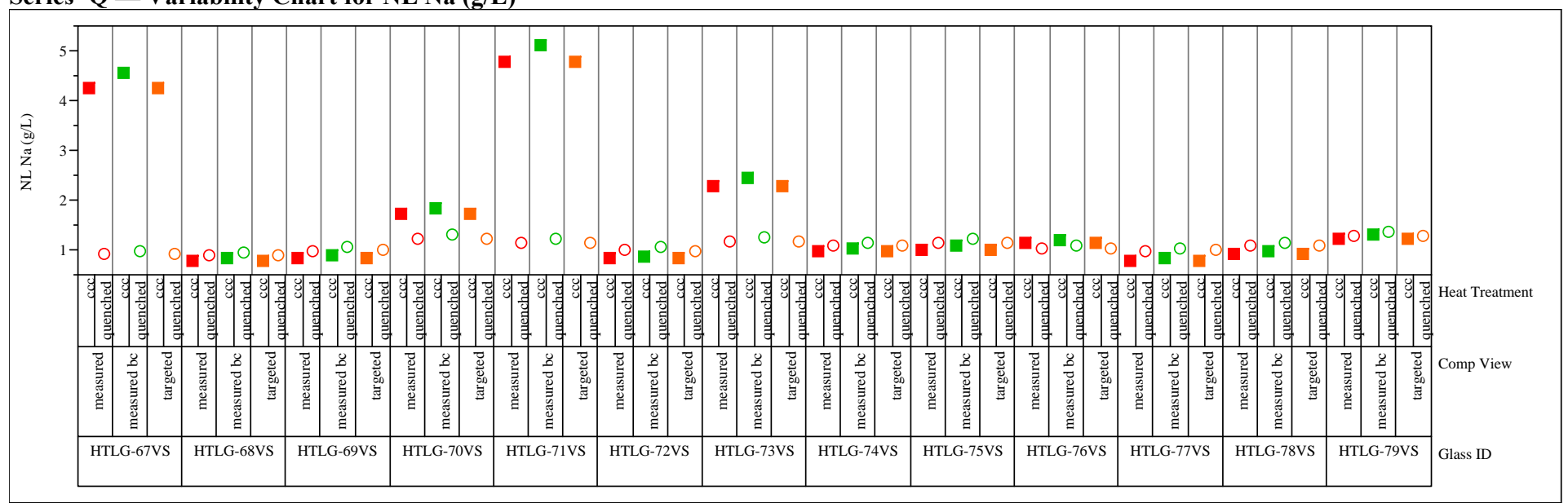

Series $=Q$ - Variability Chart for NL Si $(g / L)$

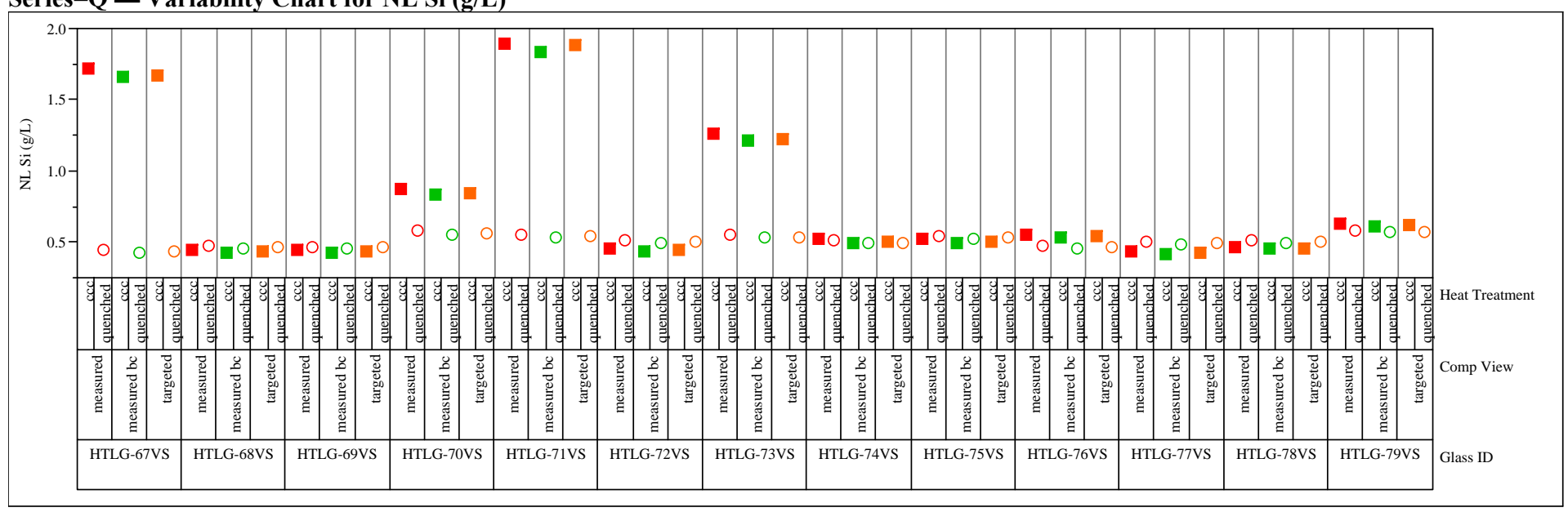




\section{Distribution:}

J.E. Marra, SRNL

R.E. Edwards, SRNL

D.A. Crowley, 999-W

C.C. Herman, 999-W

S.L. Marra, SRNL

A.B. Barnes, 704-30S

T.B. Edwards, 999-W

K.M. Fox, 999-W

I.A. Reamer, 999-W

M.E. Smith, 773-42A

M.E. Stone, 999-W

R.J. Workman, 999-W 